\title{
VTool: A Method for Predicting and Understanding the Energy Flow and Losses in Advanced Vehicle Powertrains
}

\author{
Robert Jesse Alley
}

Thesis submitted to the faculty of the Virginia Polytechnic Institute and State University in partial fulfillment of the requirements for the degree of

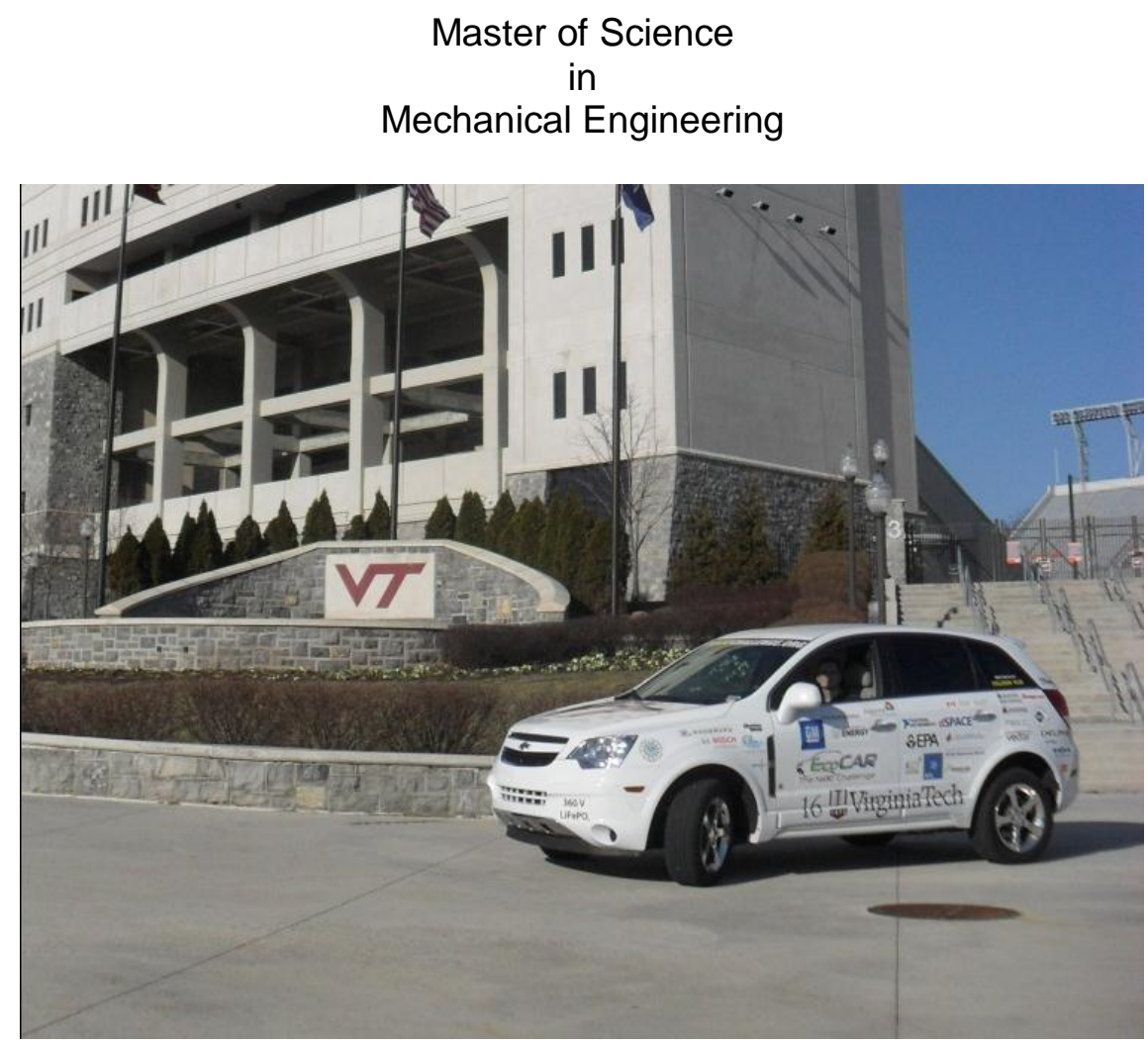

Chair: Douglas J. Nelson

Robert L. West

Scott T. Huxtable

June 15, 2012

Blacksburg, VA

Keywords: hybrid electric vehicle, plug-in hybrid electric vehicle, electric vehicle, environment, greenhouse gases, petroleum, fuel economy, powertrain modeling

Copyright 2012, R. Jesse Alley 


\title{
VTool: A Method for Predicting and Understanding the Energy Flow and Losses in Advanced Vehicle Powertrains
}

\author{
Robert Jesse Alley
}

\begin{abstract}
As the global demand for energy increases, the people of the United States are increasingly subject to high and ever-rising oil prices. Additionally, the U.S. transportation sector accounts for $27 \%$ of total nationwide Greenhouse Gas (GHG) emissions. In the U.S. transportation sector, light-duty passenger vehicles account for about $58 \%$ of energy use. Therefore incremental improvements in light-duty vehicle efficiency and energy use will significantly impact the overall landscape of energy use in America.
\end{abstract}

A crucial step to designing and building more efficient vehicles is modeling powertrain energy consumption. While accurate modeling is indeed key to effective and efficient design, a fundamental understanding of the powertrain and auxiliary systems that contribute to energy consumption for a vehicle is equally as important if not more important. This thesis presents a methodology that has been packaged into a tool, called VTool, that can be used to estimate the energy consumption of a vehicle powertrain. The method is intrinsically designed to foster understanding of the vehicle powertrain as it relates to energy consumption while still providing reasonably accurate results. VTool explicitly calculates the energy required at the wheels of the vehicle to complete a prescribed drive cycle and then explicitly applies component efficiencies to find component losses and the overall energy consumption for the drive cycle. In calculating component efficiencies and losses, VTool offers several tunable parameters that can be used to calibrate the tool for a particular vehicle, compare powertrain architectures, or simply explore the tradeoffs and sensitivities of certain parameters.

In this paper, the method is fully and explicitly developed to model Electric Vehicles (EVs), Series Hybrid Electric Vehicles (HEVs) and Parallel HEVs for various different drive cycles. VTool has also been validated for use in UDDS and HwFET cycles using on-road test results from the 2011 EcoCAR competition. By extension, the method could easily be extended for use in other cycles. The end result is a tool that can predict fuel consumption to a reasonable degree of accuracy for a variety of powertrains, calculate J1711 Utility Factor weighted energy consumption for Extended Range Electric Vehicles (EREVs) and determine the Well-to-Wheel impact of a given powertrain or fuel. VTool does all of this while performing all calculations explicitly and calculating all component losses to allow the user maximum access which promotes understanding and comprehension of the fundamental dynamics of automotive fuel economy and the powertrain as a system. 


\section{Acknowledgements}

The contributions of the research, modeling, and results from the HEVT design team are gratefully acknowledged. I would like to thank General Motors, the U.S. Department of Energy, Argonne National Labs, and the rest of the sponsors of the EcoCAR and EcoCAR 2 competitions. Finally I would like to thank HEVT Faculty Advisor Dr. Doug Nelson, and all of the team members from the 2010-2012 Hybrid Electric Vehicle Team.

Photo on the title page of the VT REX in front of Lane Stadium by author, 2012. 


\section{Table of Contents}

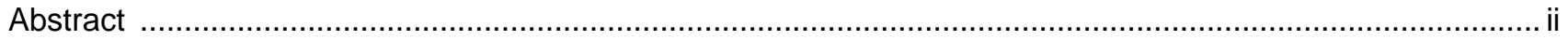

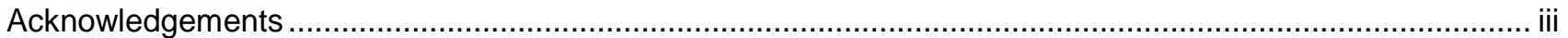

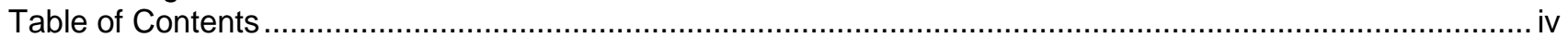

List of Multimedia Objects ............................................................................................................... viii

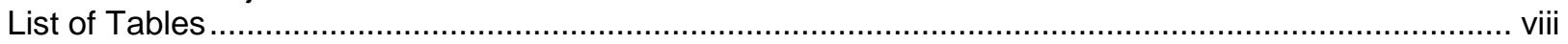

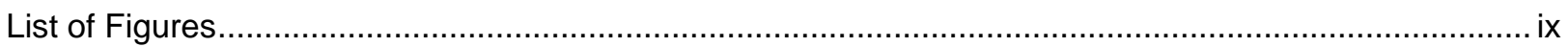

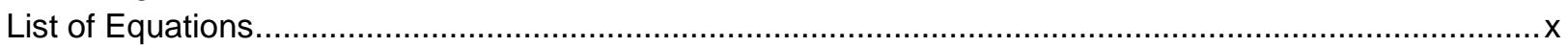

List of Nomenclature: Energy and Power Quantities .................................................................... xvii

List of Nomenclature: Component and Powertrain Efficiencies........................................................... xix

List of Nomenclature: Vehicle Parameters and Sovran Drive Cycle Coefficients ..................................xi

List of Nomenclature: Drive Cycle Case Indicator Superscript ...........................................................xxii

List of Abbreviations ................................................................................................................

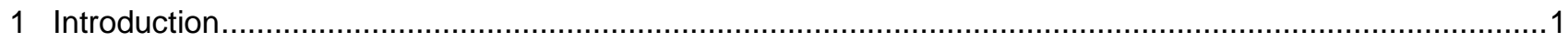

1.1 Background and Motivation for the Development of Advanced Technology Vehicles ......................1

1.1.1 Implications of Petroleum Energy Use and Greenhouse Gas Emissions ...............................1

1.1.2 American Driving Habits Pertaining to Electric Vehicle Utility: The Case for PHEVs................2

1.2 Hybrid Vehicle Powertrain Architecture Background ....................................................................

1.2.1 Effect of Electrification: HEVs, PHEVs, EREVs, and EVs .............................................

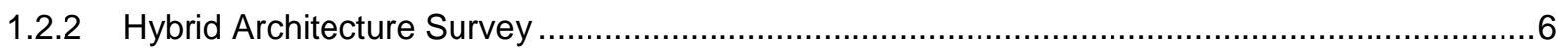

1.3 HEVT Participation in EcoCAR: The NeXt Challenge …………...........................................

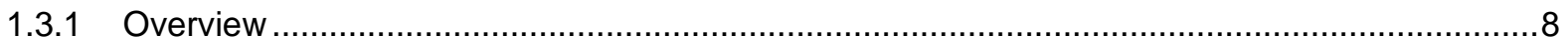

1.3.2 Vehicle Modeling and Fuel Selection ............................................................................

1.3.3 Component Sizing and Selection...........................................................................

1.3.4 Competition Results and Application to This Work..........................................................11

1.4 HEVT Participation in EcoCAR 2: Plugging in to the Future .....................................................12

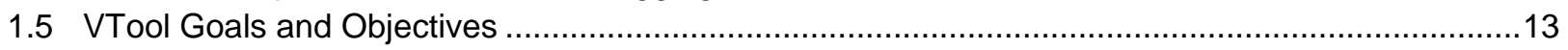

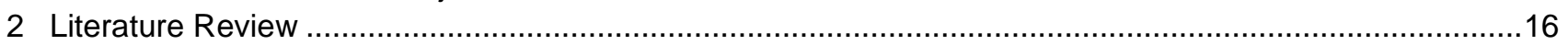

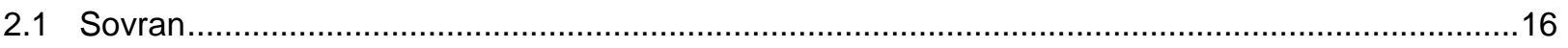

2.1.1 A Contribution to Understanding Automotive Fuel Economy and Its Limits [21] ....................16

2.1.2 Quantifying the Potential Impacts of Regenerative Braking on a Vehicle's Tractive-Fuel

Consumption for the U.S., European, and Japanese Driving Schedules [23] ....................................17

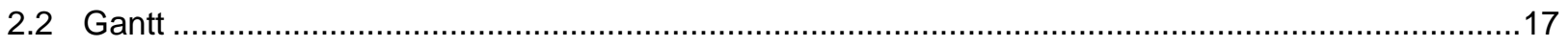

2.2.1 Energy Losses for Propelling and Braking an Electric Vehicle [24]....................................17

2.2.2 Regenerative Brake Energy Analysis for the $\mathrm{VT}_{\mathrm{REX}}$ Plug-in Hybrid Electric Vehicle [25].........17

2.3 Hochgraf, Douba: What if the Prius Wasn't a Hybrid? What if the Corolla Were? An Analysis

Based on Vehicle Limited Fuel Consumption and Powertrain and Braking Efficiency [26] .....................18

2.4 Yanni: Impact and Sensitivity of Vehicle Design Parameters on Fuel Economy Estimates [27].......18

2.5 Duoba: Calculating Results and Performance Parameters for PHEVs [28] ...................................19

2.6 Baglione: Vehicle System Energy Analysis Methodology and Tool for Determining Vehicle

Subsystem Energy Supply and Demand [29] ……………………………………………….....20

2.7 Park: Real-Time Powertrain Control Strategy for Series-Parallel Hybrid Electric Vehicles [30].......20

2.8 Boyd: Hybrid Electric Vehicle Control Strategy Based on Power Loss Calculations [31] ..................21

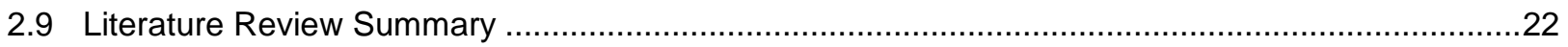

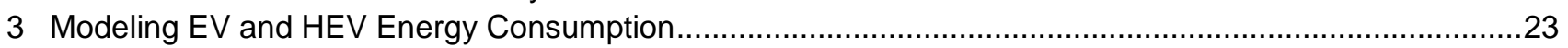

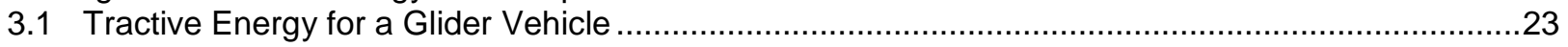

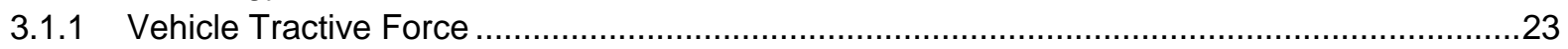

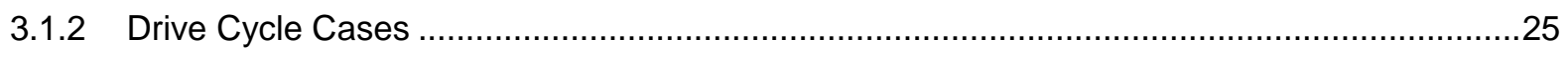

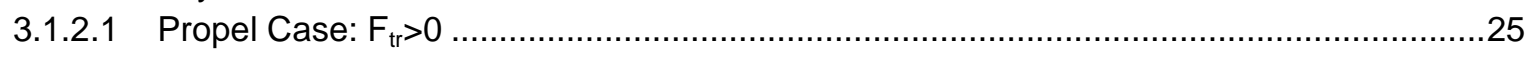




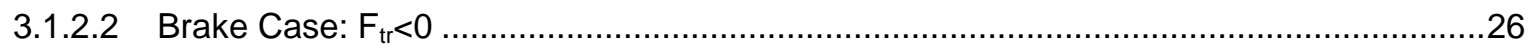

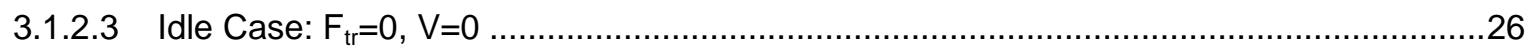

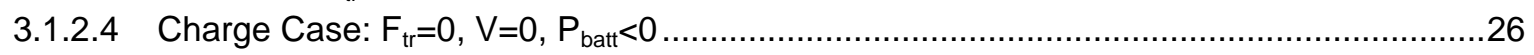

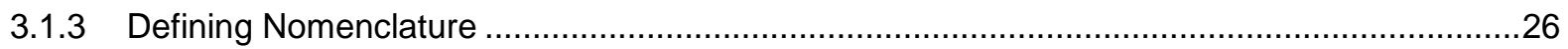

3.1.4 Calculating Tractive Energy for a Glider Vehicle Using the Sovran Coefficients ...................27

3.1.4.1 Benefits, Challenges and Underlying Assumptions of the Sovran Method .....................27

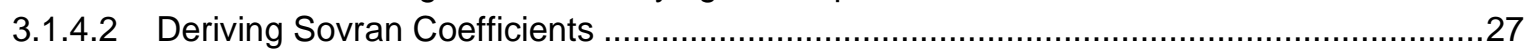

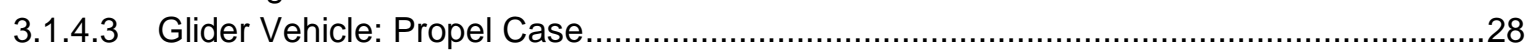

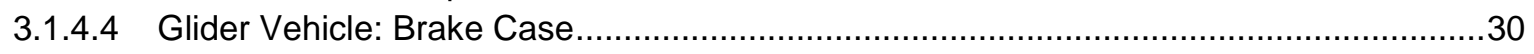

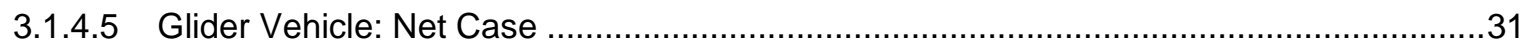

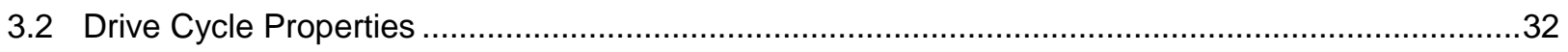

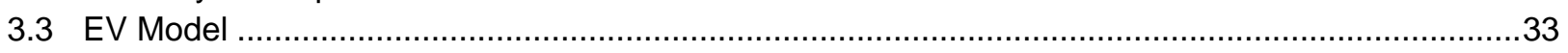

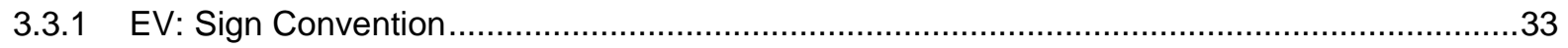

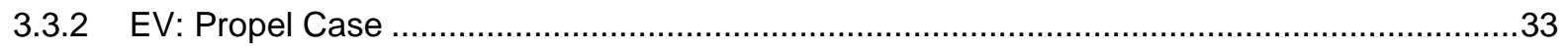

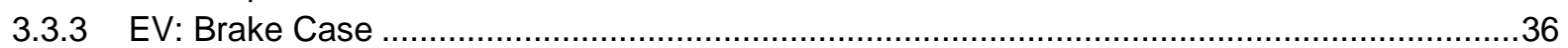

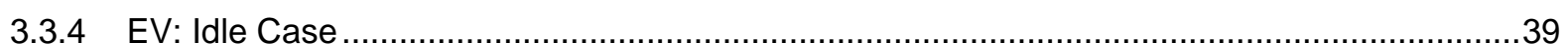

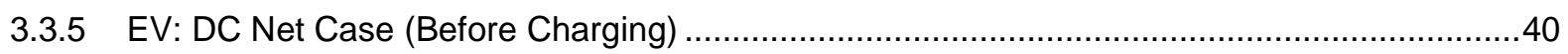

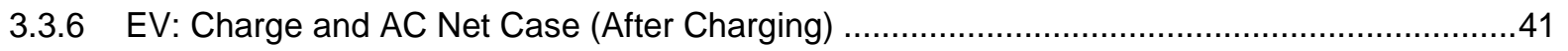

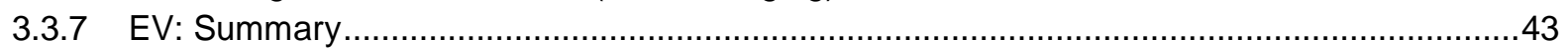

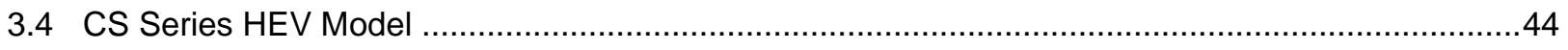

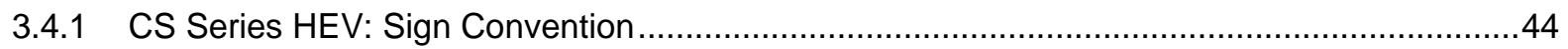

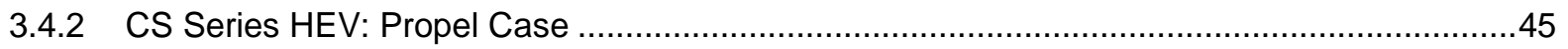

3.4.2.1 CS Series HEV: Propel Case - Equilibrium Subcase …...........................................46

3.4.2.2 CS Series HEV: Propel Case - Store Subcase ......................................................... 47

3.4.2.3 CS Series HEV: Propel Case - Use Subcase .........................................................47

3.4.2.4 CS Series HEV: Propel Case - Power Split Fraction ..................................................48

3.4.2.5 CS Series HEV: Propel Case - Energy Consumption ..............................................50

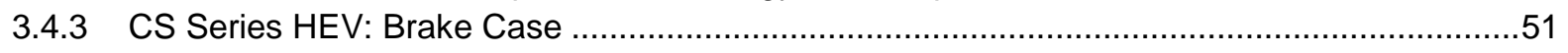

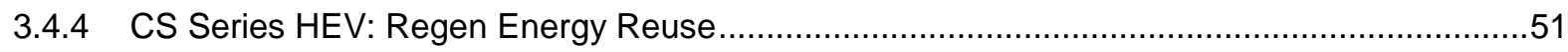

3.4.5 CS Series HEV: Fuel-Equivalent Regen Energy Credit ..................................................53

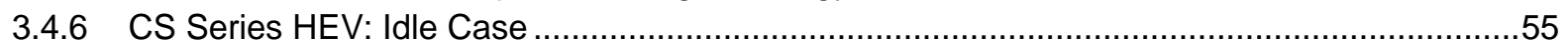

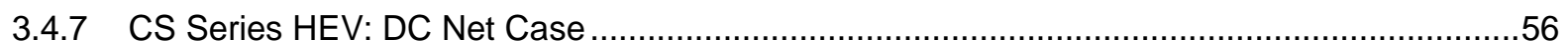

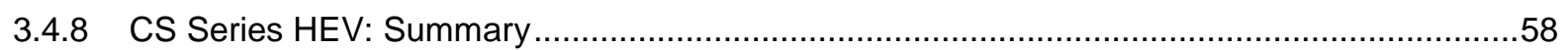

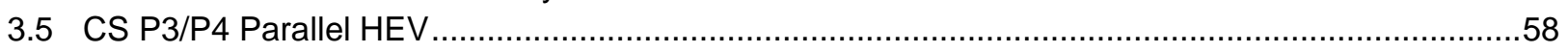

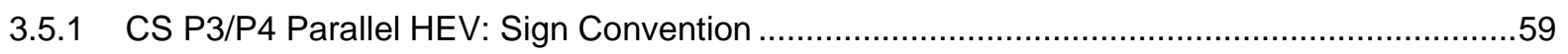

3.5.2 CS P3/P4 Parallel HEV: Propel Case ............................................................................59

3.5.2.1 CS P3/P4 Parallel HEV: Propel Case - Tractive Energy Subcase .................................60

3.5.2.2 CS P3/P4 Parallel HEV: Propel Case - Accessory Energy Subcase ............................64

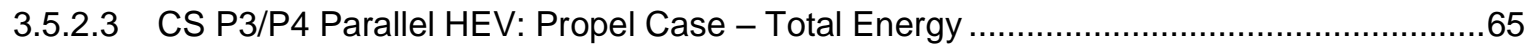

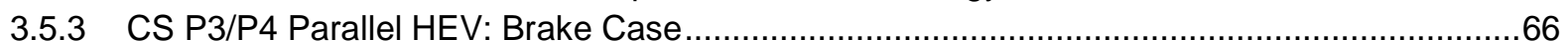

3.5.4 CS P3/P4 Parallel HEV: Regen Energy Reuse ............................................................6

3.5.5 CS P3/P4 Parallel HEV: Fuel-Equivalent Regen Energy Credit.........................................67

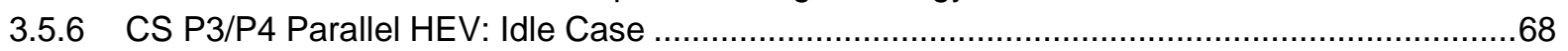

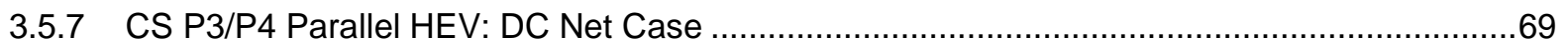

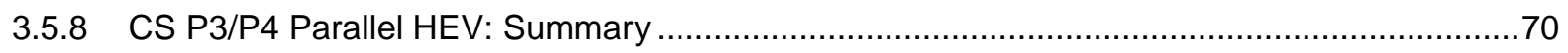

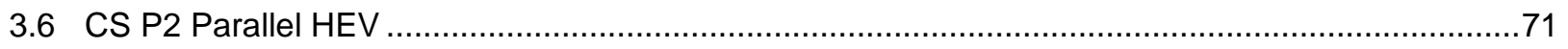

3.6.1 CS P2 Parallel HEV: Sign Convention ........................................................................ 72

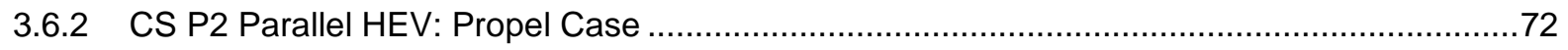

3.6.2.1 CS P3/P4 Parallel HEV: Propel Case - Tractive Energy Subcase ...............................72 
3.6.2.2 CS P2 Parallel HEV: Propel Case - Accessory Energy Subcase ..................................75

3.6.2.3 CS P2 Parallel HEV: Propel Case - Total Energy ......................................................76

3.6.3 CS P2 Parallel HEV: Brake Case ...............................................................................

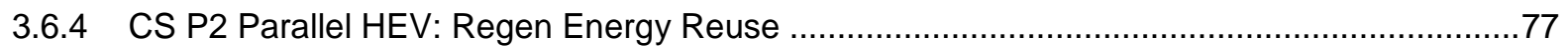

3.6.5 CS P2 Parallel HEV: Fuel-Equivalent Regen Energy Credit .............................................78

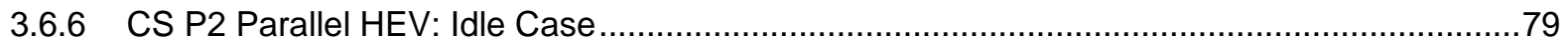

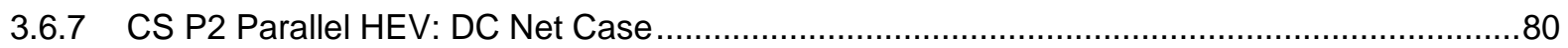

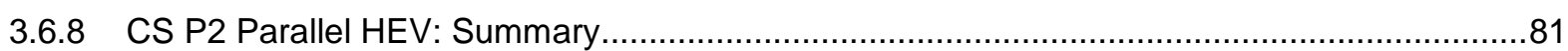

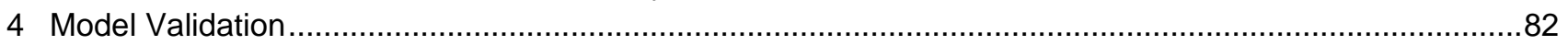

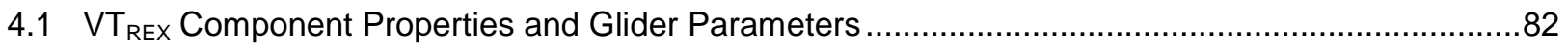

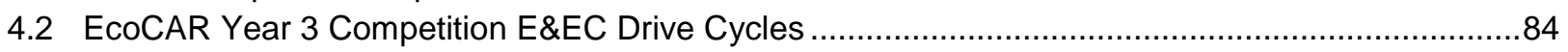

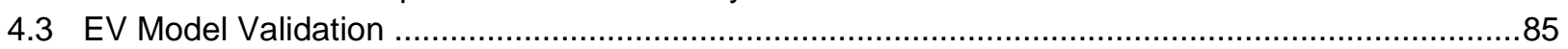

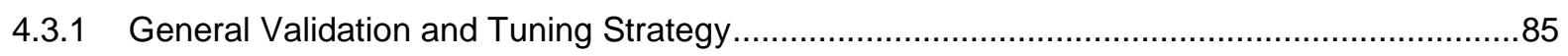

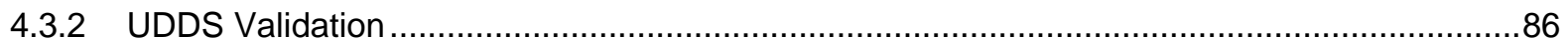

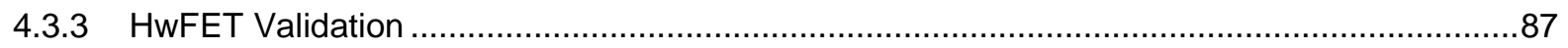

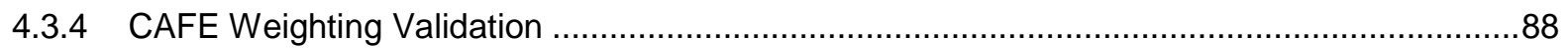

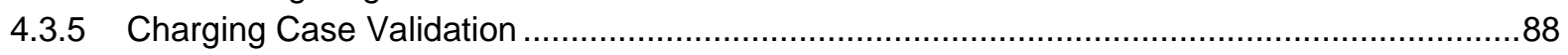

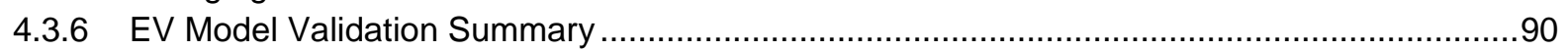

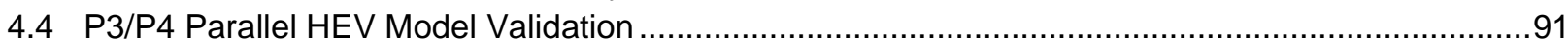

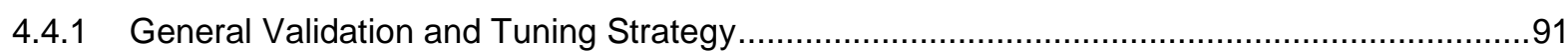

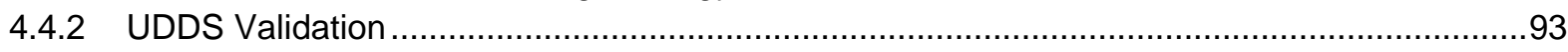

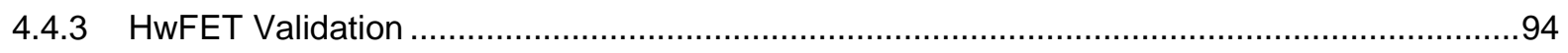

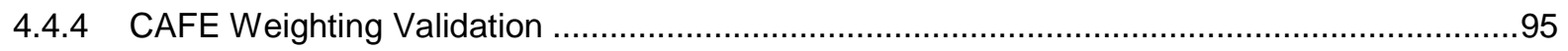

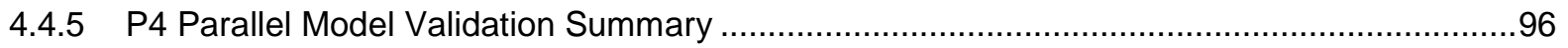

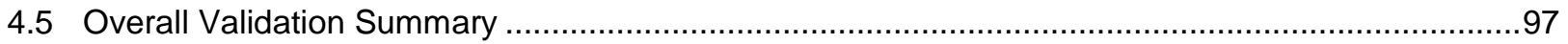

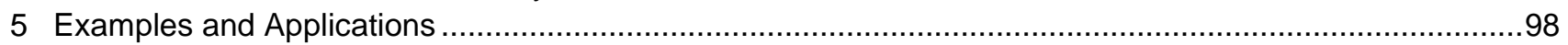

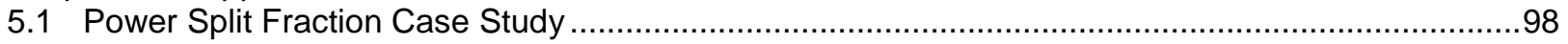

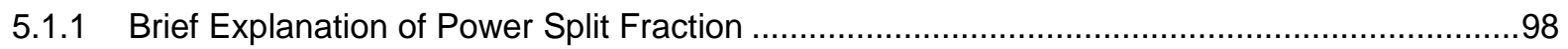

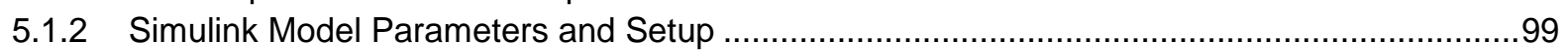

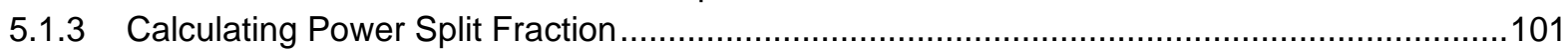

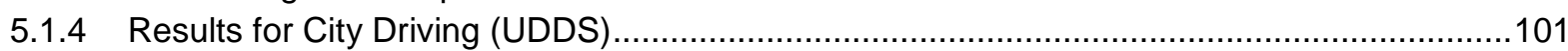

5.1.5 Results for Highway Driving (HwFET) .....................................................................104

5.2 Calculating Cycle-Weighted WTW Energy Consumption for PHEVs and EREVs ......................105

5.2.1 CAFE and 4-Cycle Energy Consumption for an EV ......................................................105

5.2.2 CAFE and 4-Cycle Energy Consumption for a P3/P4 Parallel HEV ...................................106

5.2.3 UF-Weighted CAFE and 4-Cycle Energy Consumption for a P3/P4 Parallel EREV ..............108

5.2.4 Impact of Fuel Selection on WTW UF-Weighted Energy Consumption ................................109

5.3 Using VTool to Compare Powertrain Architectures ..............................................................110

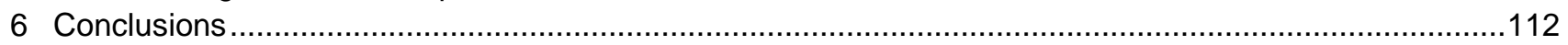

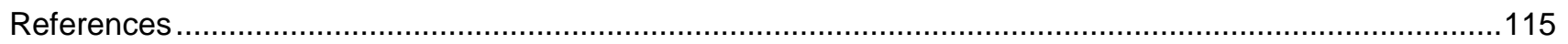

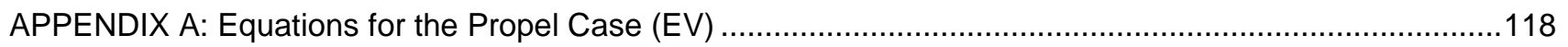

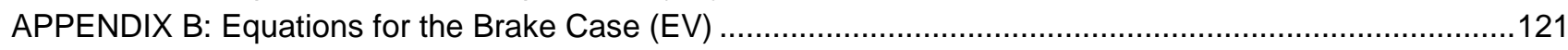

APPENDIX C: Equations for the Idle Case (EV) ..............................................................................124

APPENDIX D: Equations for the DCnet Case (EV) ..........................................................................126

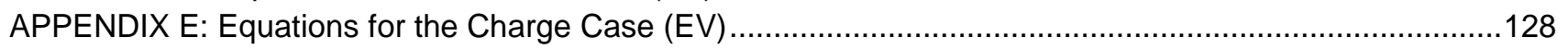

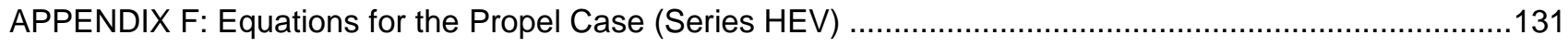

APPENDIX G: Equations for the Brake Case (Series HEV) ............................................................137

APPENDIX H: Equations for Regen Energy Reuse (Series HEV) …..................................................138

APPENDIX I: Equations for Fuel-Equivalent Regen Energy Credit (Series HEV) ......................................140 
APPENDIX J: Equations for the Idle Case (Series HEV)

APPENDIX K: Equations for the DCnet Case (Series HEV)

APPENDIX L: Equations for Tractive Energy in the Propel Case (P3/P4 Parallel HEV).....

APPENDIX M: Equations for Accessory Energy in the Propel Case (P3/P4 Parallel HEV)

APPENDIX N: Equations for Total Energy in the Propel Case (P3/P4 Parallel HEV) ................................157

APPENDIX O: Equations for the Brake Case (P3/P4 Parallel HEV) .......................................................159

APPENDIX P: Equations for Regen Energy Reuse (P3/P4 Parallel HEV) .................................................160

APPENDIX Q: Equations for Fuel-Equivalent Regen Energy Credit (P3/P4 Parallel HEV) .........................162

APPENDIX R: Equations for the Idle Case (P3/P4 Parallel HEV) ..........................................................166

APPENDIX S: Equations for the DCnet Case (P3/P4 Parallel HEV) ......................................................169

APPENDIX T: Equations for Tractive Energy in the Propel Case (P2 Parallel HEV) .................................172

APPENDIX U: Equations for Accessory Energy in the Propel Case (P2 Parallel HEV) ..............................178

APPENDIX V: Equations for Total Energy in the Propel Case (P2 Parallel HEV) .....................................180

APPENDIX W: Equations for the Brake Case (P2 Parallel HEV) .......................................................182

APPENDIX X: Equations for Regen Energy Reuse (P2 Parallel HEV) ....................................................183

APPENDIX Y: Equations for Fuel-Equivalent Regen Energy Credit (P2 Parallel HEV) ..............................186

APPENDIX Z: Equations for the Idle Case (P2 Parallel HEV) ..............................................................190

APPENDIX AA: Equations for the DCnet Case (P2 Parallel HEV) ......................................................192

APPENDIX BB: Power Split Fraction Case Study Model Parameters ....................................................195

APPENDIX CC: Power Split Fraction Case Study Model Parameters …................................................197

APPENDIX DD: Summary of Tunable Parameters Used in VTool.........................................................198

APPENDIX EE: Summary of VTool Results for EV and P3/P4 Parallel HEV models .................................199

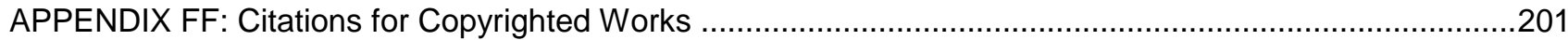




\section{List of Multimedia Objects}

\section{List of Tables}

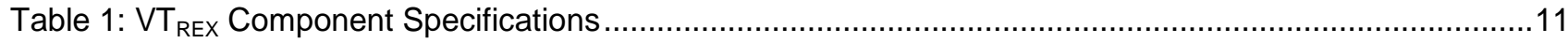

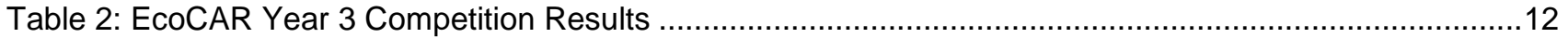

Table 3: Summary of EcoCAR 1 CAFE and EcoCAR 2 4-cycle weightings .............................................14

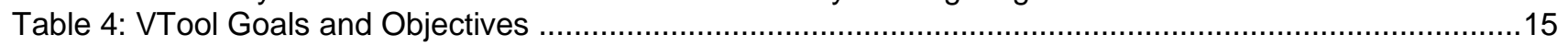

Table 5: Vehicle glider parameters used to derive Sovran coefficients ..................................................28

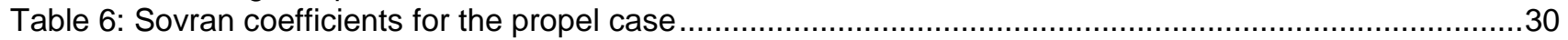

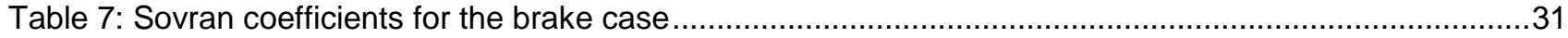

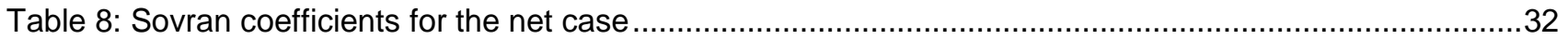

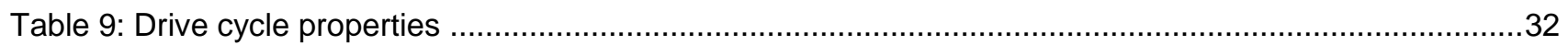

Table 10: Description of energy quantities key to the Power Split Fraction for the Series HEV ....................49

Table 11: Parallel HEV Motor position 'P' terminology summary .............................................................58

Table 12: Description of energy quantities key to the Power Split Fraction for the P3/P4 Parallel HEV ...........61

Table 13: Description of energy quantities key to the Power Split Fraction for the P2 Parallel HEV ................74

Table 14: Glider properties from 2009 Hybrid Saturn Vue used for model validation ....................................83

Table 15: Summary of EV model tunable parameters and tuning strategy ..............................................85

Table 16: Summary of EV test data and final parameter values for the UDDS cycle..................................87

Table 17: Summary of EV test data and final parameter values for the HwFET cycle ..................................88

Table 18: Summary of EV energy consumption modeling for UDDS, HwFET and CAFE ............................88

Table 19: Summary of EV model tunable parameters and tuning strategy for the charging case ...................89

Table 20: Summary of EV test data and final parameter values for the CAFE weighting ...............................90

Table 21: Summary of EV test data and final parameter values for the CAFE weighting ..............................90

Table 22: Summary of VTool modeling results and comparison to actual data for EV mode ........................91

Table 23: Summary of P4 Parallel model tunable parameters and tuning strategy ....................................92

Table 24: Summary of P4 Parallel HEV test data and final parameter values for the UDDS cycle .................93

Table 25: Summary of P4 Parallel HEV test data and final parameter values for the HwFET cycle................94

Table 26: Summary of P4 Parallel HEV energy consumption modeling for UDDS, HwFET and CAFE ...........95

Table 27: Summary of results for EV, P4 Parallel HEV and P4 Parallel EREV using UF method ...................97

Table 28: Summary of cases for Power Split Fraction case study ...........................................................98

Table 29: Summary and explanation of hybrid controller parameters varied in case study .........................100

Table 30: Summary of hybrid control strategy parameters for each case in the study ...............................100

Table 31: Power Split Fraction case study results for city driving (UDDS) ...............................................103

Table 32: Case 4 repeated with the addition of an engine start fuel penalty .............................................104

Table 33: Power Split Fraction case study results for highway driving (HwFET) .......................................104

Table 34: Summary of WTW CAFE-weighted energy consumption for the EV model................................105

Table 35: Summary of WTW 4-Cycle-weighted energy consumption for the EV model .............................106

Table 36: Comparison of CAFE and 4-Cycle weightings for the EV model.............................................106

Table 37: Summary of WTW CAFE-weighted energy consumption for the P3/P4 Parallel HEV model .........107

Table 38: Summary of WTW 4-Cycle-weighted energy consumption for the P3/P4 Parallel HEV model ......107

Table 39: Comparison of CAFE and 4-Cycle weightings for the P3/P4 Parallel HEV model .........................107

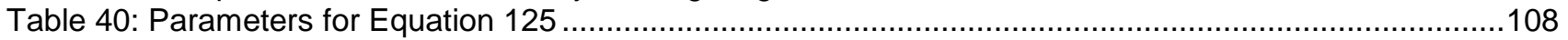

Table 41: Summary of UF calculations based on the EV model .........................................................109

Table 42: Summary of UF-weighted energy consumption results from P3/P4 Parallel EREV .......................109

Table 43: Comparison of fuels for P3/P4 Parallel EREV ......................................................................110

Table 44: Results of Series HEV and P4 Parallel HEV Comparative Analysis .........................................111

Table 45: Vehicle glider properties and other properties .....................................................................196

Table 46: Summary of all VTool tunable parameters calibrated using E\&EC data and other data ................198

Table 47: Summary of propel and DCnet case results for the EV model ..................................................199

Table 48: Summary of results for the brake case for the P3/P4 Parallel HEV model.....................................199

Table 49: Summary of propel and DCnet case results for the P3/P4 Parallel HEV model ..........................200

Table 50: Summary of results for brake case and Power Split Fraction for the P3/P4 Parallel HEV model ...200 


\section{List of Figures}

Figure 1: U.S. transportation energy use and CO2 emissions, (public domain [1]) .....................................

Figure 2: Trends in U.S. consumption, production, and net imports of liquid fuels (public domain [2]) ..............2

Figure 3: Typical one-way commute of the average U.S. resident, (public domain [3]) .................................

Figure 4: Average daily mileage distribution, (used with permission of Elsevier [4]) ....................................4

Figure 5: Fraction of the fleet appropriate for varying vehicle ranges, (used with permission of Elsevier [4]) ....4

Figure 6: Vehicle designs compared in terms of the level of electrification .................................................6

Figure 7: Series hybrid architecture design and characteristics (public domain [9]) ....................................

Figure 8: Parallel hybrid architecture design and characteristics (public domain [9]) ...................................

Figure 9: Series-Parallel hybrid architecture design and characteristics (public domain [9]) ...........................7

Figure 10: EcoCAR VT REX $_{\text {E } 85}$ Split Parallel EREV powertrain architecture ..............................................10

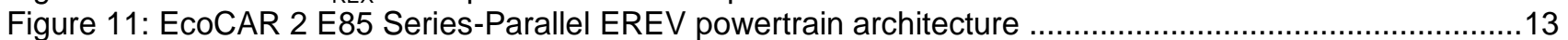

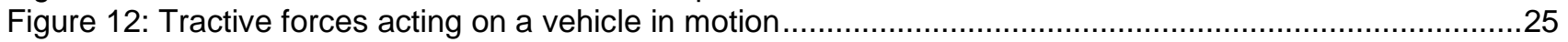

Figure 13: Nomenclature key for energy terms used throughout the model ..............................................27

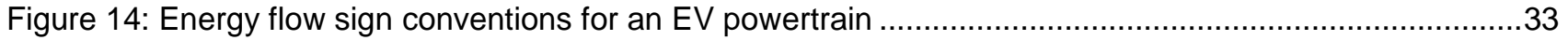

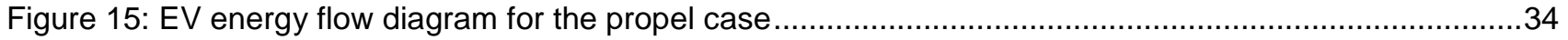

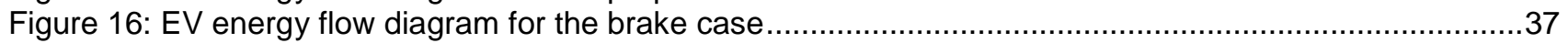

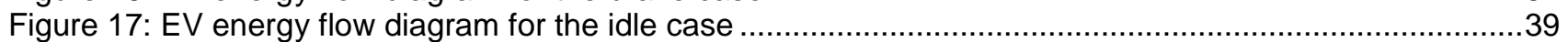

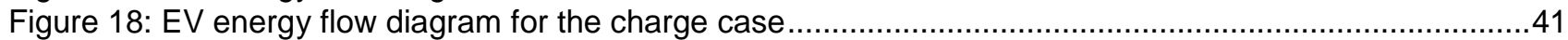

Figure 19: Energy flow sign conventions for a CS Series HEV powertrain ..................................................45

Figure 20: CS Series HEV energy flow diagram for the propel case .........................................................45

Figure 21: CS Series HEV energy flow diagram for the propel case during an equilibrium condition...............46

Figure 22: CS Series HEV energy flow diagram for the propel case during the 'store' subcase .....................47

Figure 23: CS Series HEV energy flow diagram for the propel case during the 'use' subcase.......................47

Figure 24: Energy balance at the HV bus for the Power Split Fraction ......................................................48

Figure 25: CS Series HEV energy flow diagram for regen energy reuse ..................................................52

Figure 26: CS Series HEV energy flow diagram for regen fuel credit......................................................54

Figure 27: CS Series HEV energy flow diagram for the idle case ...........................................................5

Figure 28: Energy flow sign conventions for a CS P3/P4 Parallel HEV powertrain.......................................59

Figure 29: CS P3/P4 Parallel HEV energy flow diagram for the tractive energy in the propel case ................60

Figure 30: Energy balance at the drive axle for the Power Split Fraction ...................................................61

Figure 31: CS P3/P4 Parallel HEV energy flow diagram for the accessory energy in the propel case.............64

Figure 32: CS P3/P4 Parallel HEV energy flow diagram for regen energy reuse .......................................66

Figure 33: CS P3/P4 Parallel HEV energy flow diagram for regen fuel credit .............................................67

Figure 34: CS P3/P4 Parallel HEV energy flow diagram for the idle case ..................................................69

Figure 35: Energy flow sign conventions for a CS P2 Parallel HEV powertrain ..........................................72

Figure 36: CS P2 Parallel HEV energy flow diagram for the tractive energy in the propel case ......................73

Figure 37: Energy balance at the pre-trans Node for the Power Split Fraction ............................................73

Figure 38: CS P2 Parallel HEV energy flow diagram for the accessory energy in the propel case ..................75

Figure 39: CS P2 Parallel HEV energy flow diagram for regen energy reuse ............................................77

Figure 40: CS P2 Parallel HEV energy flow diagram for regen fuel credit ...................................................78

Figure 41: CS P2 Parallel HEV energy flow diagram for the idle case ...................................................79

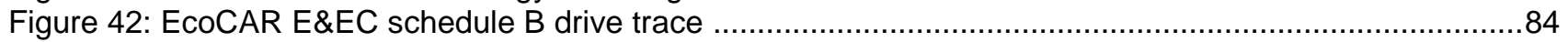

Figure 43: Illustration of power recirculation during CS operation ..........................................................96

Figure 44: Power Split Fraction case study, UDDS, case 1, near load-following, $\xi=0.88 \ldots \ldots \ldots \ldots \ldots \ldots \ldots \ldots \ldots . . .101$

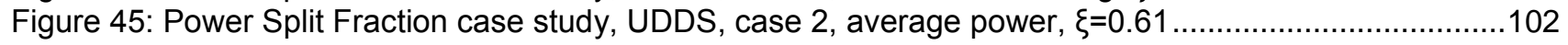

Figure 46: Power Split Fraction case study, UDDS, case 3, realistic strategy, $\xi=0.44 \ldots \ldots \ldots \ldots \ldots \ldots \ldots \ldots \ldots \ldots . . .102$

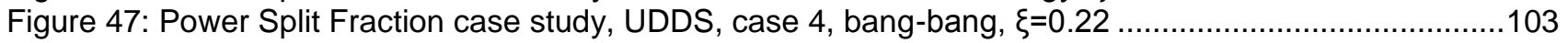

Figure 48: Top-level diagram of the Simulink model used in the Power Split Fraction case study .................195

Figure 49: Power Split Fraction case study, HwFET, case 1, near load-following, $\xi=0.96 \ldots \ldots \ldots \ldots \ldots \ldots \ldots \ldots . . . .197$

Figure 50: Power Split Fraction case study, HwFET, case 2 and case 3, average power, $\xi=0.81$................197

Figure 51: Power Split Fraction case study, HwFET, case 4, average power, $\xi=0.59 \ldots \ldots \ldots \ldots \ldots \ldots \ldots \ldots \ldots \ldots \ldots . . .197$ 


\section{List of Equations}

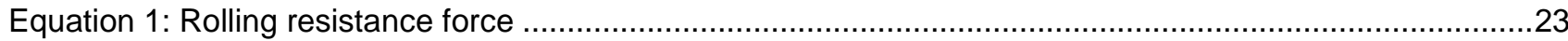

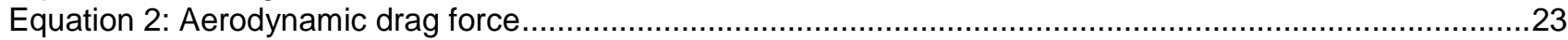

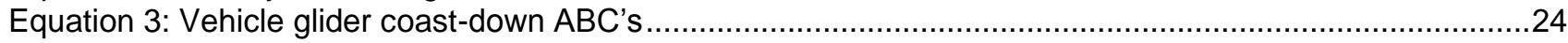

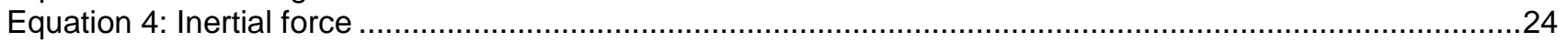

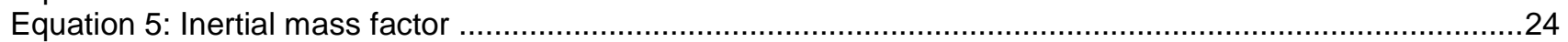

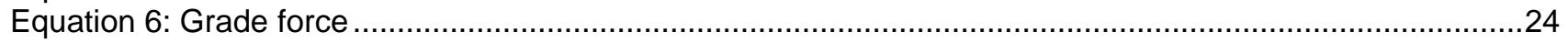

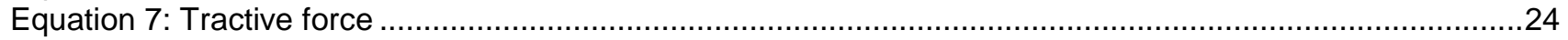

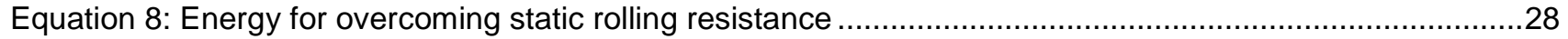

Equation 9: Energy for overcoming velocity-proportional rolling resistance .............................................29

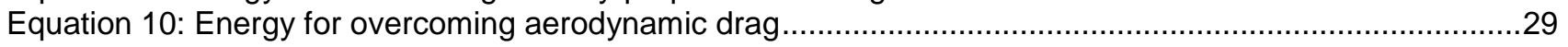

Equation 11: Energy to produce increases in vehicle kinetic energy .......................................................29

Equation 12: Energy to produce increases in gravitational potential energy ..............................................29

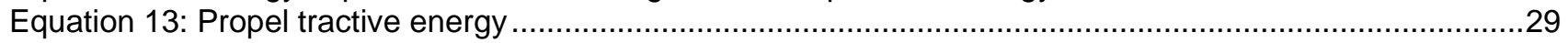

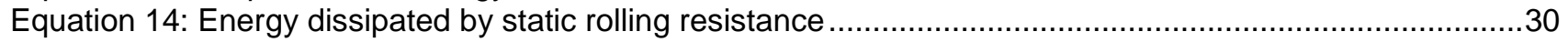

Equation 15: Energy dissipated by velocity-proportional rolling resistance ..............................................30

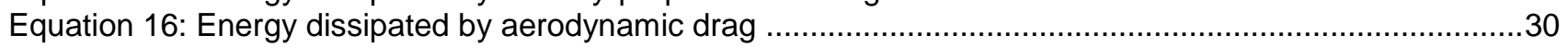

Equation 17: Energy to produce decreases in vehicle kinetic energy ........................................................30

Equation 18: Energy to produce decreases in gravitational potential energy ............................................31

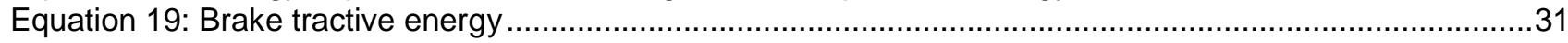

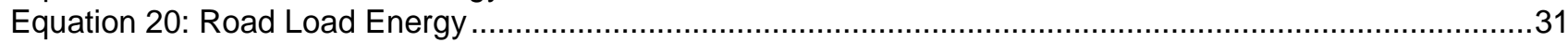

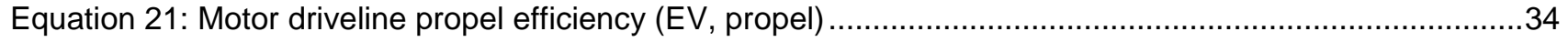

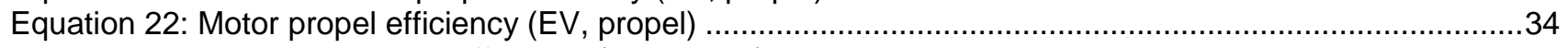

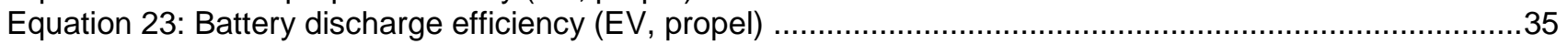

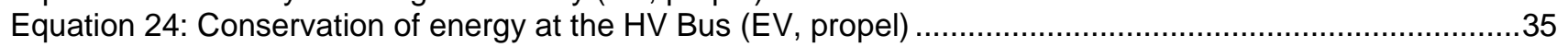

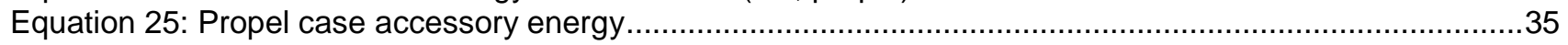

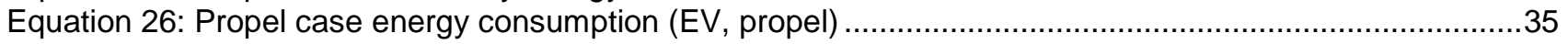

Equation 27: Propel case energy consumption in terms of losses (EV, propel) ............................................36

Equation 28: Propel case powertrain efficiency (EV, propel) .....................................................................

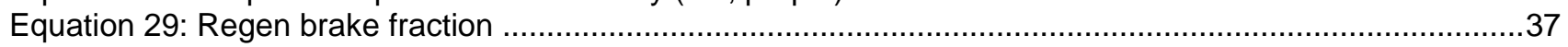

Equation 30: Conservation of energy at the HV Bus (EV, brake) .........................................................38

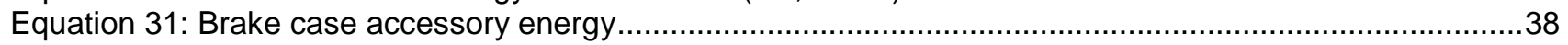

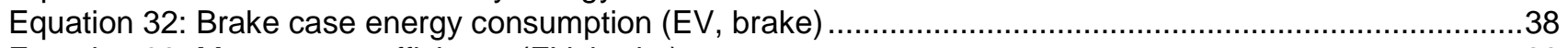

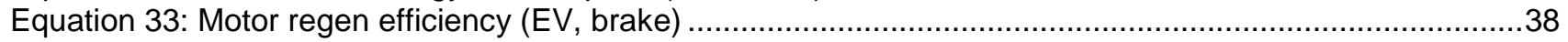

Equation 34: Brake case energy consumption in terms of losses (EV, brake) ...........................................39

Equation 35: Brake case powertrain efficiency (EV, brake) ..................................................................39

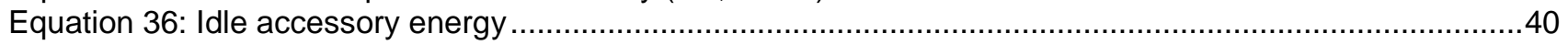

Equation 37: Idle case energy consumption (EV, idle) .................................................................... 40

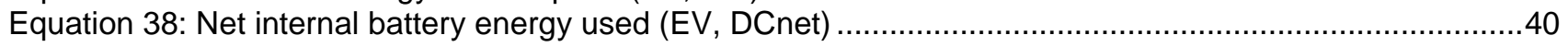

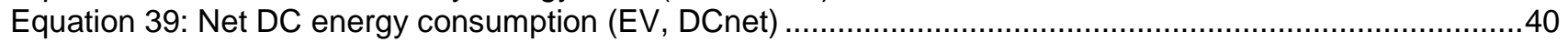

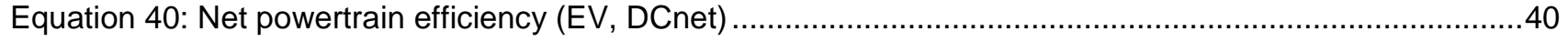

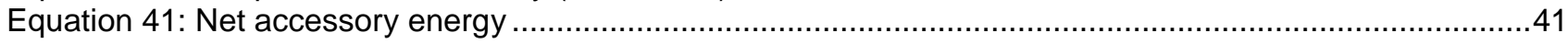

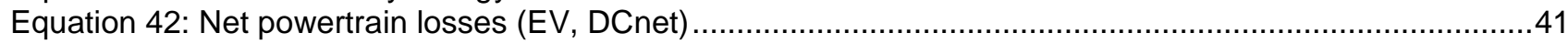

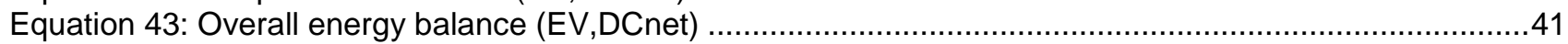

Equation 44: Battery internal energy to be replenished (EV, charge) …..................................................42

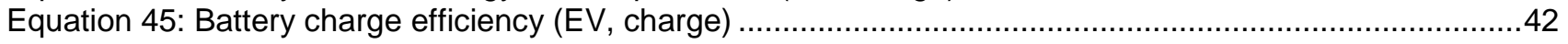

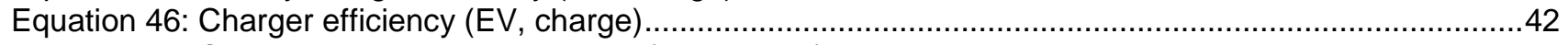

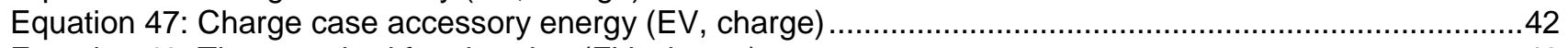

Equation 48: Time required for charging (EV, charge) .....................................................................42

Equation 49: Charging efficiency (EV, charge) .............................................................................. 42

Equation 50: Charge case energy consumption (EV, charge) ............................................................43

Equation 51: Charge case energy consumption using charging process efficiency (EV, charge) ..................43

Equation 52: Charge case energy consumption in terms of losses (EV, charge) ........................................43

Equation 53: Conservation of energy in the battery to maintain charge balance (Series, propel) ..................48

Equation 54: Power Split Fraction, genset energy directly used (Series, propel) ..........................................49

Equation 55: Power Split Fraction, genset energy recirculated (Series, propel) ….......................................49 
Equation 56: Conservation of energy at the HV bus (Series, propel) …..................................................50

Equation 57: Energy from the battery into the HV bus during the propel case (Series, propel) ......................50

Equation 58: Energy supplied by the generator (Series, propel) ............................................................50

Equation 59: Propel case fuel energy consumption (Series, propel) .......................................................51

Equation 60: Propel case powertrain efficiency (Series, propel) ..........................................................51

Equation 61: Conservation of energy for regen energy capture and reuse (Series, regen reuse) ...................52

Equation 62: Motor propel efficiency (Series, regen reuse) .................................................................52

Equation 63: Equivalent tractive energy 'credit' from regen braking (Series, regen reuse) ...........................53

Equation 64: Equivalent tractive energy 'credit' from regen in terms of losses (Series, regen reuse) ..............53

Equation 65: Powertrain efficiency for regen energy reuse (Series, regen reuse) .......................................53

Equation 66: Conservation of energy for regen fuel credit (Series, regen fuel credit) ...................................54

Equation 67: Fuel-equivalent regen energy 'credit' (Series, regen fuel credit) .............................................55

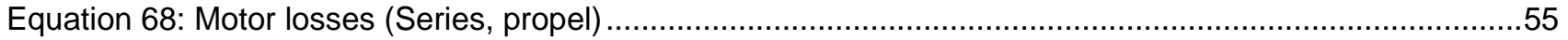

Equation 69: Motor losses (Series, regen fuel credit) ….....................................................................5

Equation 70: Idle case energy consumption (Series, idle) ...............................................................5

Equation 71: Total fuel energy calculated by case (Series, DCnet) ….................................................57

Equation 72: Total fuel energy calculated from overall energy balance (Series, DCnet) ..............................57

Equation 73: Net battery losses (Series, DCnet) …….........................................................................5

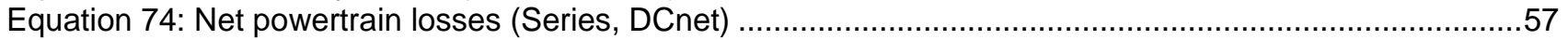

Equation 75: Net powertrain efficiency (Series, DCnet) …...............................................................5

Equation 76: Net powertrain efficiency for combined propel operation (Series, DCnet) ...............................57

Equation 77: Energy balance for overall charge balance (Series, DCnet) ...............................................58

Equation 78: Power Split Fraction, engine energy directly used (P3 Parallel, propel) ..................................62

Equation 79: Power Split Fraction, engine energy recirculated (P3 Parallel, propel) ...................................62

Equation 80: Energy stored in the battery during times of surplus power generation (P3 Parallel, propel) ......62

Equation 81: Energy used from the battery during shortages of power generation (P3 Parallel, propel) .........62

Equation 82: Conservation of energy in the battery to maintain charge balance (P3 Parallel, propel) ............62

Equation 83: Energy from the motor onto the drive axle during the propel case (P3 Parallel, propel) ..............63

Equation 84: Power Split Fraction energy recirculation efficiency (P3 Parallel, propel) ...............................63

Equation 85: Conservation of energy 'inside' the drive axle (P3 Parallel, propel) ........................................63

Equation 86: Energy supplied by the engine during the propel case (P3 Parallel, propel) ............................63

Equation 87: Propel case tractive fuel energy consumption (P3 Parallel, propel) ........................................63

Equation 88: Propel case powertrain efficiency for tractive energy (P3 Parallel, propel) .............................63

Equation 89: Propel case accessory fuel energy consumption (P3 Parallel, propel) .....................................64

Equation 90: Propel case powertrain accessory efficiency for accessory load (P3 Parallel, propel) ................65

Equation 91: Total fuel energy required for the propel case (P3 Parallel, propel) ......................................65

Equation 92: Propel case total fuel energy consumption (P3 Parallel, propel) ............................................65

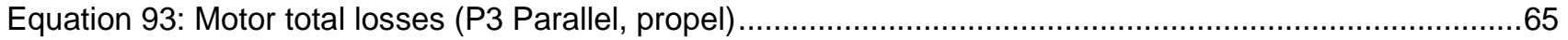

Equation 94: Total powertrain losses for accessory load (P3 Parallel, propel) ..........................................65

Equation 95: Propel case ICE powertrain efficiency (P3 Parallel, propel) ..................................................65

Equation 96: Equivalent tractive energy 'credit' from regen braking (P3 Parallel, regen reuse) ....................67

Equation 97: Powertrain efficiency for regen energy reuse (P3 Parallel, regen reuse) ..................................67

Equation 98: Fuel-equivalent regen energy ‘credit' (P3 Parallel, regen fuel credit).......................................68

Equation 99: Idle case energy consumption (P3 Parallel, idle) ................................................................69

Equation 100: Total fuel energy calculated by case (P3 Parallel, DCnet) ..................................................69

Equation 101: Total fuel energy calculated from overall energy balance (P3 Parallel, DCnet) ......................69

Equation 102: Net powertrain losses (P3 Parallel, DCnet) ................................................................ 70

Equation 103: Net powertrain efficiency (P3 Parallel, DCnet) …............................................................70

Equation 104: Net powertrain efficiency for combined propel operation (P3 Parallel, DCnet) .......................70

Equation 105: Power Split Fraction, engine energy directly used (P2 Parallel, propel) .................................74

Equation 106: Power Split Fraction, engine energy recirculated (P2 Parallel, propel) ..................................74

Equation 107: Propel case tractive fuel energy consumption (P2 Parallel, propel) ......................................75

Equation 108: Power Split Fraction energy recirculation efficiency (P2 Parallel, propel) ......................................75

Equation 109: Propel case powertrain efficiency for tractive energy (P2 Parallel, propel) ............................75

Equation 110: Propel case accessory fuel energy consumption (P2 Parallel, propel) ................................76

Equation 111: Propel case powertrain accessory efficiency for accessory load (P2 Parallel, propel) ..............76 
Equation 112: Propel case total fuel energy consumption (P2 Parallel, propel) ……....................................76

Equation 113: Propel case powertrain efficiency (P2 Parallel, propel) ….................................................76

Equation 114: Propel case accessory fuel energy consumption (P2 Parallel, brake) ...................................77

Equation 115: Equivalent tractive energy 'credit' from regen braking (P2 Parallel, regen reuse) ...................77

Equation 116: Powertrain efficiency for regen energy reuse (P2 Parallel, regen reuse) .................................78

Equation 117: Fuel-equivalent regen energy ‘credit' (P2 Parallel, idle) .........................................................79

Equation 118: Idle case energy consumption (P2 Parallel, idle) ...........................................................80

Equation 119: Total fuel energy calculated by case (P2 Parallel, DCnet) .................................................80

Equation 120: Total fuel energy calculated from overall energy balance (P2 Parallel, DCnet) .......................80

Equation 121: Net powertrain losses (P2 Parallel, DCnet) .................................................................. 80

Equation 122: Net powertrain efficiency (P2 Parallel, DCnet) …..........................................................81

Equation 123: Net powertrain efficiency for combined propel operation (P2 Parallel, DCnet) ........................81

Equation 124: Propel case tractive fuel energy consumption (P3 Parallel, propel) ......................................92

Equation 125: Utility Factor curve .............................................................................................. 108

Equation 126: Utility factor weighted AC energy consumption ........................................................108

Equation 127: Utility factor weighted fuel consumption .................................................................108

Equation 128: Utility factor weighted total energy consumption ..........................................................108

Equation 129: Motor driveline losses (EV, propel) ............................................................................118

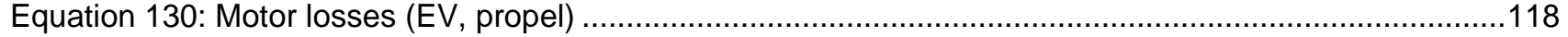

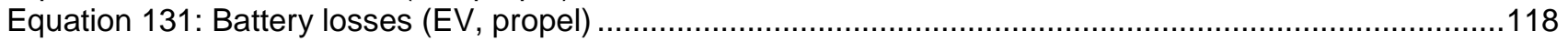

Equation 132: Propel powertrain losses (EV, propel) ….................................................................119

Equation 133: Motor driveline regen efficiency (EV, brake) ..............................................................121

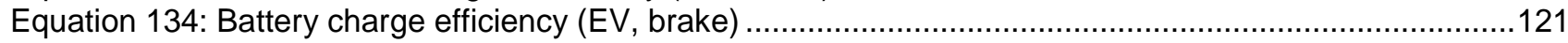

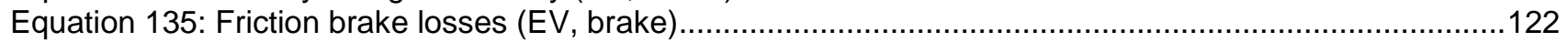

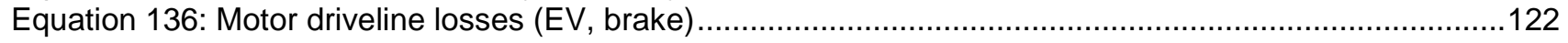

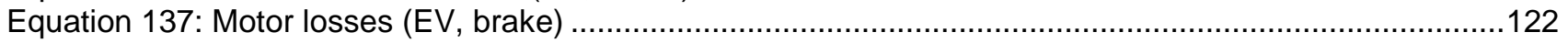

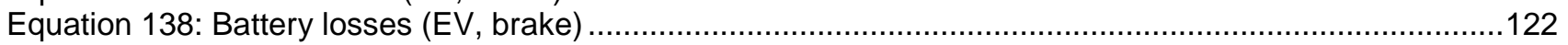

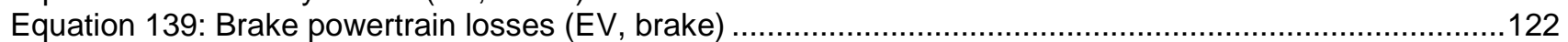

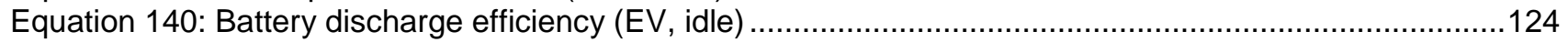

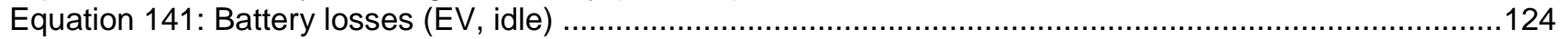

Equation 142: Conservation of energy at the HV Bus (EV, idle) .........................................................124

Equation 143: Idle case energy consumption in terms of losses (EV, idle) ..........................................125

Equation 144: Net friction brake losses (EV, DCnet) ....................................................................127

Equation 145: Net motor driveline losses (EV, DCnet) ................................................................. 127

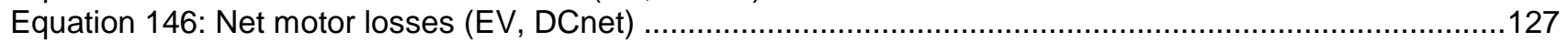

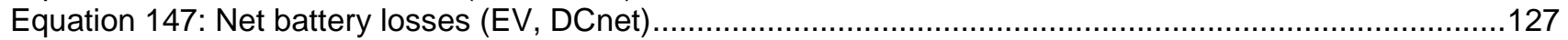

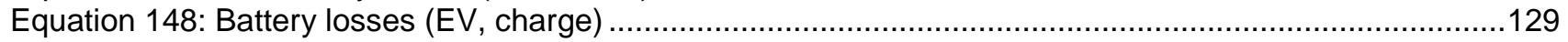

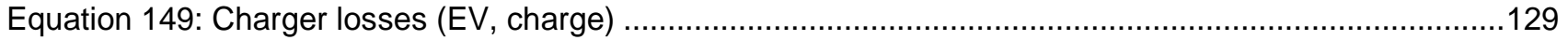

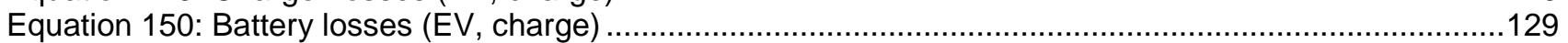

Equation 151: Conservation of energy at the HV Bus (EV, charge) .....................................................129

Equation 152: Motor driveline propel efficiency (Series, propel) .........................................................131

Equation 153: Motor propel efficiency (Series, propel) ......................................................................131

Equation 154: Battery charge efficiency (Series, propel) …...............................................................132

Equation 155: Battery discharge efficiency (Series, propel) …............................................................132

Equation 156: Generator propel efficiency (Series, propel) …............................................................132

Equation 157: Engine propel efficiency (Series, propel) …............................................................132

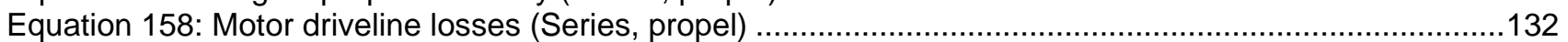

Equation 159: Battery charge losses (Series, propel) …................................................................132

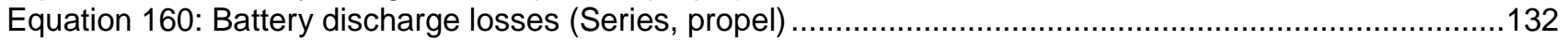

Equation 161: Total battery losses (Series, propel) ….........................................................................

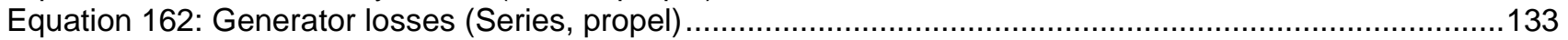

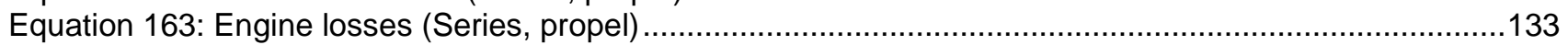

Equation 164: Propel powertrain losses (Series, propel) ................................................................... 133

Equation 165: Conservation of energy for the Power Split Fraction (Series, propel) ................................133

Equation 166: Energy stored in the battery during times of surplus power generation (Series, propel) .........133

Equation 167: Energy used from the battery during shortages of genset power generation (Series, propel).134 
Equation 168: Energy supplied by the generator in terms of losses (Series, propel) ..................................135

Equation 169: Propel case fuel energy consumption in terms of losses (Series, propel) ............................136

Equation 170: Motor driveline efficiency (Series, regen reuse) ............................................................138

Equation 171: Battery discharge efficiency (Series, regen reuse) ........................................................138

Equation 172: Motor driveline losses (Series, regen reuse) ................................................................138

Equation 173: Motor losses (Series, regen reuse) …........................................................................138

Equation 174: Battery losses (Series, regen reuse) ......................................................................138

Equation 175: Propel powertrain losses (Series, regen reuse) ..........................................................139

Equation 176: Conservation of energy at the HV bus (Series, regen reuse) ............................................139

Equation 177: Motor driveline losses (Series, regen fuel credit) .........................................................141

Equation 178: Total battery losses (Series, regen fuel credit) ...........................................................141

Equation 179: Generator losses (Series, regen fuel credit) ..............................................................141

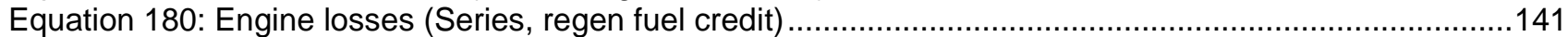

Equation 181: Propel powertrain losses (Series, regen fuel credit) .......................................................142

Equation 182: Fuel-equivalent regen energy 'credit' (P3 Parallel, regen fuel credit)...................................142

Equation 183: Generator efficiency (Series, idle) ..........................................................................143

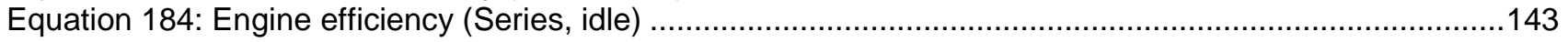

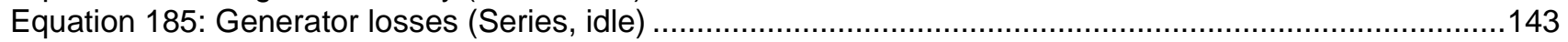

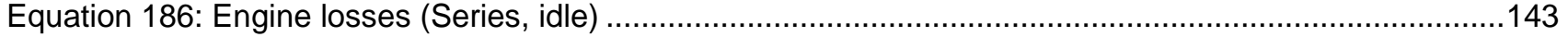

Equation 187: Conservation of energy at the HV Bus (EV, idle) .........................................................143

Equation 188: Idle case energy consumption in terms of losses (Series, idle) .......................................144

Equation 189: Net motor driveline losses (Series, DCnet) ................................................................146

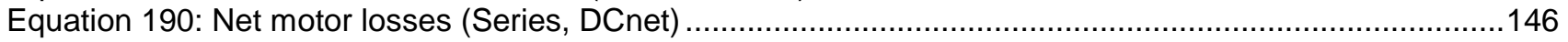

Equation 191: Net generator losses (Series, DCnet) .......................................................................146

Equation 192: Net engine losses (Series, DCnet) …......................................................................146

Equation 193: Engine propel efficiency (P3 Parallel, propel) ...............................................................148

Equation 194: Engine driveline propel efficiency (P3 Parallel, propel) .....................................................149

Equation 195: Motor driveline brake efficiency (P3 Parallel, propel) ...................................................... 149

Equation 196: Motor brake efficiency (P3 Parallel, propel) .................................................................149

Equation 197: Battery charge efficiency (P3 Parallel, propel) …..........................................................149

Equation 198: Battery discharge efficiency (P3 Parallel, propel) ..........................................................149

Equation 199: Motor propel efficiency (P3 Parallel, propel) ................................................................149

Equation 200: Motor driveline propel efficiency (P3 Parallel, propel) .......................................................149

Equation 201: Engine losses for tractive energy (P3 Parallel, propel) …...............................................150

Equation 202: Engine driveline losses for tractive energy (P3 Parallel, propel) .......................................150

Equation 203: Motor driveline generate losses for tractive energy (P3 Parallel, propel) .............................150

Equation 204: Motor generate losses for tractive energy (P3 Parallel, propel) …....................................150

Equation 205: Battery charge losses for tractive energy (P3 Parallel, propel) …......................................150

Equation 206: Battery discharge losses for tractive energy (P3 Parallel, propel) .....................................150

Equation 207: Motor propel losses for tractive energy (P3 Parallel, propel) ............................................150

Equation 208: Motor driveline propel losses for tractive energy (P3 Parallel, propel) .................................150

Equation 209: Motor driveline total losses for tractive energy (P3 Parallel, propel) .....................................150

Equation 210: Motor total losses for tractive energy (P3 Parallel, propel) ................................................150

Equation 211: Battery total losses for tractive energy (P3 Parallel, propel) …...........................................150

Equation 212: Powertrain losses for tractive energy (P3 Parallel, propel) ...............................................150

Equation 213: Conservation of energy at or 'outside' the drive axle (P3 Parallel, propel) ..........................151

Equation 214: Conservation of energy at the HV bus while charging (P3 Parallel, propel) .........................151

Equation 215: Conservation of energy at the HV bus while discharging (P3 Parallel, propel) ......................151

Equation 216: Conservation of energy for the Power Split Fraction (P3 Parallel, propel) ............................151

Equation 217: Engine propel efficiency for accessory load (P3 Parallel, propel) ........................................154

Equation 218: Engine driveline propel efficiency for accessory load (P3 Parallel, propel) ...........................154

Equation 219: Motor driveline brake efficiency for accessory load (P3 Parallel, propel) ..............................154

Equation 220: Motor brake efficiency for accessory load (P3 Parallel, propel) ............................................154

Equation 221: Engine losses for accessory load (P3 Parallel, propel) .....................................................155

Equation 222: Engine driveline losses for accessory load (P3 Parallel, propel) ......................................155

Equation 223: Motor driveline generate losses for accessory load (P3 Parallel, propel) ............................155 
Equation 224: Motor generate losses for accessory load (P3 Parallel, propel) .........................................155

Equation 225: Powertrain losses for accessory load (P3 Parallel, propel) ..............................................155

Equation 226: Conservation of energy at the drive axle for accessory load (P3 Parallel, propel) ..................155

Equation 227: Conservation of energy at the HV bus for accessory load (P3 Parallel, propel) .....................155

Equation 228: Engine total losses (P3 Parallel, propel) ........................................................................157

Equation 229: Engine driveline total losses (P3 Parallel, propel) …........................................................157

Equation 230: Motor driveline total losses (P3 Parallel, propel) ..........................................................157

Equation 231: Battery total losses (P3 Parallel, propel) .....................................................................157

Equation 232: Motor driveline efficiency (P3 Parallel, regen reuse) ….................................................160

Equation 233: Motor propel efficiency (P3 Parallel, regen reuse) ..........................................................160

Equation 234: Battery discharge efficiency (P3 Parallel, regen reuse) ....................................................160

Equation 235: Motor driveline losses (P3 Parallel, regen reuse) ..........................................................160

Equation 236: Motor losses (P3 Parallel, regen reuse) ....................................................................160

Equation 237: Battery losses (P3 Parallel, regen reuse) ....................................................................160

Equation 238: Propel powertrain losses (P3 Parallel, regen reuse) ......................................................161

Equation 239: Conservation of energy at the HV bus (P3 Parallel, regen reuse) ....................................161

Equation 240: Conservation of energy for regen energy capture and reuse (P3 Parallel, regen reuse) ........161

Equation 241: Equivalent tractive energy 'credit' from regen in terms of losses (P3 Parallel, regen reuse) ...161

Equation 242: Engine losses for tractive energy (P3 Parallel, propel) .....................................................164

Equation 243: Engine driveline losses for tractive energy (P3 Parallel, propel) .........................................164

Equation 244: Motor driveline generate losses for tractive energy (P3 Parallel, propel) ...............................164

Equation 245: Motor generate losses for tractive energy (P3 Parallel, propel) ...........................................164

Equation 246: Battery charge losses for tractive energy (P3 Parallel, propel) ….......................................164

Equation 247: Battery discharge losses for tractive energy (P3 Parallel, propel) ........................................164

Equation 248: Motor propel losses for tractive energy (P3 Parallel, propel) ...............................................164

Equation 249: Motor driveline propel losses for tractive energy (P3 Parallel, propel) .................................164

Equation 250: Motor driveline total losses for tractive energy (P3 Parallel, propel) ....................................164

Equation 251: Motor total losses for tractive energy (P3 Parallel, propel) .................................................164

Equation 252: Battery total losses for tractive energy (P3 Parallel, propel) .............................................164

Equation 253: Powertrain losses for tractive energy (P3 Parallel, propel) ................................................164

Equation 254: Conservation of energy for regen fuel credit (P3 Parallel, propel) ........................................164

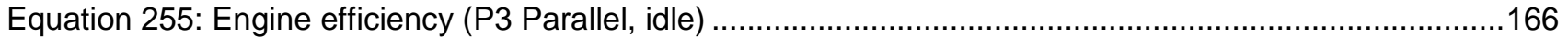

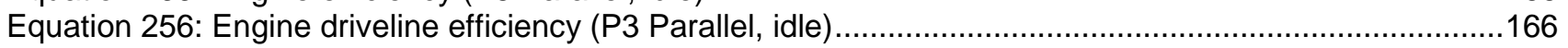

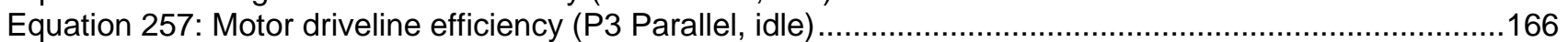

Equation 258: Motor efficiency (P3 Parallel, idle) ............................................................................166

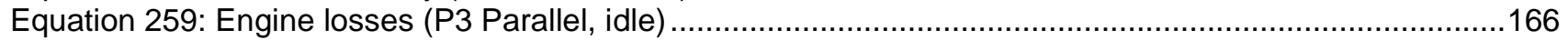

Equation 260: Engine driveline losses (P3 Parallel, idle) .................................................................166

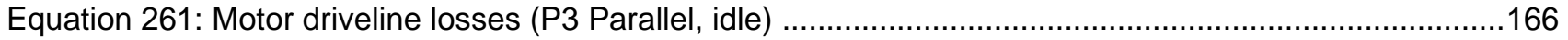

Equation 262: Motor losses (P3 Parallel, idle) ............................................................................167

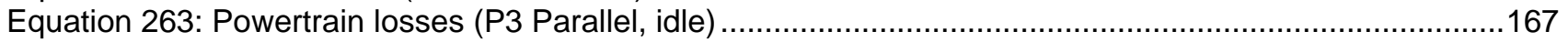

Equation 264: Conservation of energy at the HV Bus (P3 Parallel, idle) ................................................167

Equation 265: Conservation of energy at the drive axle (P3 Parallel, idle) ...............................................167

Equation 266: Idle case energy consumption in terms of losses (P3 Parallel, idle) ..................................168

Equation 267: Net engine driveline losses (P3 Parallel, DCnet) ..............................................................170

Equation 268: Net motor driveline losses (P3 Parallel, DCnet) …........................................................170

Equation 269: Net motor losses (P3 Parallel, DCnet) .....................................................................170

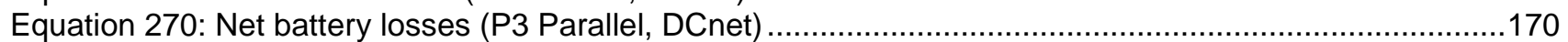

Equation 271: Net engine losses (P3 Parallel, DCnet) .....................................................................170

Equation 272: Energy balance for overall charge balance (P3 Parallel, DCnet) .......................................170

Equation 273: Engine propel efficiency (P2 Parallel, propel) .................................................................172

Equation 274: Engine driveline propel efficiency (P2 Parallel, propel) ….................................................172

Equation 275: Motor driveline brake efficiency (P2 Parallel, propel) …...................................................173

Equation 276: Motor brake efficiency (P2 Parallel, propel) ...............................................................173

Equation 277: Battery charge efficiency (P2 Parallel, propel) …..........................................................173

Equation 278: Battery discharge efficiency (P2 Parallel, propel) ….....................................................173

Equation 279: Motor propel efficiency (P2 Parallel, propel) .................................................................173 
Equation 280: Motor driveline propel efficiency (P2 Parallel, propel) ........................................................173

Equation 281: Engine losses for tractive energy (P2 Parallel, propel) ..................................................174

Equation 282: Engine driveline losses for tractive energy (P2 Parallel, propel) ........................................174

Equation 283: Motor driveline generate losses for tractive energy (P2 Parallel, propel) .............................174

Equation 284: Motor generate losses for tractive energy (P2 Parallel, propel) ............................................174

Equation 285: Battery charge losses for tractive energy (P2 Parallel, propel) ….........................................174

Equation 286: Battery discharge losses for tractive energy (P2 Parallel, propel) .......................................174

Equation 287: Motor propel losses for tractive energy (P2 Parallel, propel) ...............................................174

Equation 288: Motor driveline propel losses for tractive energy (P2 Parallel, propel) ..................................174

Equation 289: Motor driveline total losses for tractive energy (P2 Parallel, propel) ....................................174

Equation 290: Motor total losses for tractive energy (P2 Parallel, propel) ..................................................174

Equation 291: Battery total losses for tractive energy (P2 Parallel, propel) ...............................................174

Equation 292: Powertrain losses for tractive energy (P2 Parallel, propel) ...............................................174

Equation 293: Conservation of energy 'inside' the pre-trans node (P2 Parallel, propel) ..............................175

Equation 294: Conservation of energy at or 'outside' the pre-trans node (P2 Parallel, propel) .....................175

Equation 295: Conservation of energy at the HV bus while charging (P2 Parallel, propel) .........................175

Equation 296: Conservation of energy at the HV bus while discharging (P2 Parallel, propel) .......................175

Equation 297: Conservation of energy for the Power Split Fraction (P2 Parallel, propel) ............................175

Equation 298: Conservation of energy in the battery to maintain charge balance (P2 Parallel, propel) .........175

Equation 299: Energy stored in the battery during times of surplus power generation (P2 Parallel, propel) ...175

Equation 300: Energy used from the battery during shortages of power generation (P2 Parallel, propel) .....176

Equation 301: Energy from the motor onto the drive axle during the propel case (P2 Parallel, propel) .........176

Equation 303: Engine propel efficiency for accessory load (P2 Parallel, propel) .......................................178

Equation 304: Motor driveline brake efficiency for accessory load (P2 Parallel, propel) .............................178

Equation 305: Motor brake efficiency for accessory load (P2 Parallel, propel) ............................................178

Equation 306: Engine losses for accessory load (P2 Parallel, propel) …................................................178

Equation 307: Motor driveline generate losses for accessory load (P2 Parallel, propel) .............................178

Equation 308: Motor generate losses for accessory load (P2 Parallel, propel) ...........................................179

Equation 309: Powertrain losses for accessory load (P2 Parallel, propel) ..............................................179

Equation 310: Conservation of energy at the pre-trans node for accessory load (P2 Parallel, propel)..........179

Equation 311: Conservation of energy at the HV bus for accessory load (P2 Parallel, propel) .....................179

Equation 312: Engine total losses (P2 Parallel, propel) .....................................................................180

Equation 313: Engine driveline total losses (P2 Parallel, propel) …................................................180

Equation 314: Motor driveline total losses (P2 Parallel, propel) ......................................................... 180

Equation 315: Motor total losses (P2 Parallel, propel) ...................................................................180

Equation 316: Battery total losses (P2 Parallel, propel) …..............................................................180

Equation 317: Powertrain losses for accessory load (P2 Parallel, propel) ...............................................180

Equation 318: Total fuel energy required for the propel case (P2 Parallel, propel) ...................................181

Equation 319: Propel case accessory fuel energy consumption (P2 Parallel, brake) ..................................182

Equation 320: Engine driveline efficiency (P2 Parallel, regen reuse) ....................................................183

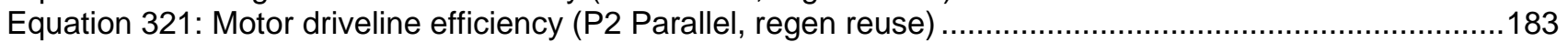

Equation 322: Motor propel efficiency (P2 Parallel, regen reuse) ….....................................................183

Equation 323: Battery discharge efficiency (P2 Parallel, regen reuse) ...................................................183

Equation 324: Engine driveline losses (P2 Parallel, regen reuse) ........................................................184

Equation 325: Motor driveline losses (P2 Parallel, regen reuse) ...........................................................184

Equation 326: Motor losses (P2 Parallel, regen reuse) ....................................................................184

Equation 327: Battery losses (P2 Parallel, regen reuse) ................................................................. 184

Equation 328: Propel powertrain losses (P2 Parallel, regen reuse) ….................................................184

Equation 329: Conservation of energy at the HV bus (P2 Parallel, regen reuse) ......................................184

Equation 330: Conservation of energy for regen energy capture and reuse (P2 Parallel, regen reuse) .........184

Equation 331: Conservation of energy at the pre-trans node (P2 Parallel, regen reuse) .............................184

Equation 332: Equivalent tractive energy 'credit' from regen in terms of losses (P2 Parallel, regen reuse) ... 185

Equation 333: Engine losses for tractive energy (P2 Parallel, propel) ......................................................188

Equation 334: Engine driveline losses for tractive energy (P2 Parallel, propel) ........................................188

Equation 335: Motor driveline generate losses for tractive energy (P2 Parallel, propel) ...........................188

Equation 336: Motor generate losses for tractive energy (P2 Parallel, propel) ..........................................188 
Equation 337: Battery charge losses for tractive energy (P2 Parallel, propel) ….......................................188

Equation 338: Battery discharge losses for tractive energy (P2 Parallel, propel) ......................................188

Equation 339: Motor propel losses for tractive energy (P2 Parallel, propel) ...............................................188

Equation 340: Motor driveline propel losses for tractive energy (P2 Parallel, propel) .................................188

Equation 341: Motor driveline total losses for tractive energy (P2 Parallel, propel) ...................................188

Equation 342: Motor total losses for tractive energy (P2 Parallel, propel) ..................................................188

Equation 343: Battery total losses for tractive energy (P2 Parallel, propel) .............................................188

Equation 344: Powertrain losses for tractive energy (P2 Parallel, propel) ................................................188

Equation 345: Conservation of energy for regen fuel credit (P2 Parallel, propel) ........................................188

Equation 346: Engine efficiency (P2 Parallel, idle) .........................................................................190

Equation 347: Motor driveline efficiency (P2 Parallel, idle) ....................................................................190

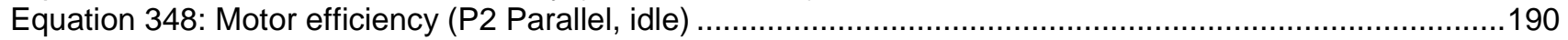

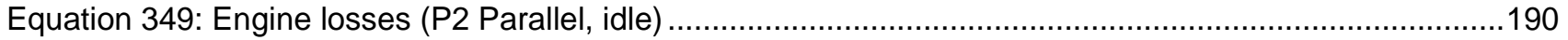

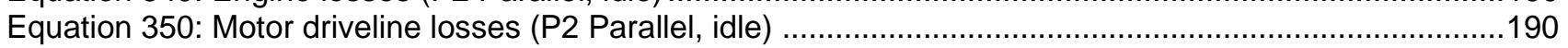

Equation 351: Motor losses (P2 Parallel, idle) ...............................................................................190

Equation 352: Powertrain losses (P2 Parallel, idle) .......................................................................190

Equation 353: Conservation of energy at the HV Bus (P2 Parallel, idle) ................................................191

Equation 354: Conservation of energy at the pre-trans node axle (P2 Parallel, idle) ..................................191

Equation 355: Idle case energy consumption in terms of losses (P2 Parallel, idle) ..................................191

Equation 356: Net engine driveline losses (P2 Parallel, DCnet) .............................................................193

Equation 357: Net motor driveline losses (P2 Parallel, DCnet) ............................................................193

Equation 358: Net motor losses (P2 Parallel, DCnet) .........................................................................193

Equation 359: Net battery losses (P2 Parallel, DCnet) ......................................................................193

Equation 360: Net engine losses (P2 Parallel, DCnet) ....................................................................193

Equation 361: Energy balance for overall charge balance (P2 Parallel, DCnet) .......................................193 
List of Nomenclature: Energy and Power Quantities

\begin{tabular}{|c|c|c|}
\hline \multicolumn{3}{|c|}{ Nomenclature for Energy and Power Quantities } \\
\hline Term & $\begin{array}{l}\text { Inherent } \\
\text { Sign }\end{array}$ & Definition \\
\hline$\left(E_{a c c}\right)^{-}$ & + & Electrical energy siphoned off the HV bus for accessory loads during brake case \\
\hline$\left(\mathrm{E}_{\mathrm{acc}}\right)^{+}$ & + & Electrical energy siphoned off the HV bus for accessory loads during propel case \\
\hline$\left(\mathrm{E}_{\mathrm{acc}}\right)^{0}$ & + & Electrical energy siphoned off the HV bus for accessory loads during idle case \\
\hline$\left(E_{\mathrm{acc}}\right)^{\mathrm{C}}$ & + & Electrical energy siphoned off the HV bus for accessory loads during charge case \\
\hline$\left(E_{\text {batt,int }}\right)^{-}$ & - & Electrical energy inside the battery captured during the brake case \\
\hline$\left(\mathrm{E}_{\text {batt,tint }}\right)^{\text {-EqFC }}$ & + & Theoretical electrical energy inside the battery. Analog to $\left(E_{\text {batt,int }}\right)^{+g e n}$ \\
\hline$\left(E_{\text {batt,int }}\right)^{-1+}$ & + & Net electrical energy inside the battery available to be reused for propulsion \\
\hline$\left(E_{\text {batt,int }}\right)^{+}$ & + & Electrical energy inside the battery used during the propel case \\
\hline$\left(\mathrm{E}_{\text {batt,tint }}\right)^{+\mathrm{EqFC}}$ & \begin{tabular}{|l|l}
- \\
\end{tabular} & Theoretical electrical energy inside the battery. Analog to $\left(E_{\text {batt,int }}\right)^{+ \text {prop }}$ \\
\hline$\left(E_{\text {batt,int }}\right)^{+g e n}$ & - & Electrical energy inside the battery stored from excess generating \\
\hline$\left(E_{\text {batt,int }}\right)^{+ \text {prop }}$ & + & Electrical energy inside the battery to be used in propulsion \\
\hline$\left(E_{\text {batt,int }}\right)^{0}$ & + & Electrical energy inside the battery used during the idle case \\
\hline$\left(E_{\text {batt,int }}\right)^{C}$ & - & Electrical energy required to replenish the battery pack after depletion \\
\hline$\left(E_{\text {batt, int }}\right)^{\text {DCnet }}$ & + & Net electrical energy inside the battery for a given drive cycle \\
\hline$\left(E_{\text {batt, },)^{-}}\right.$ & - & Electrical energy in to the battery or at the terminals during the brake case \\
\hline$\left(E_{\text {batt }, T}\right)^{-1+}$ & + & Net electrical at the battery terminals available to be reused for propulsion \\
\hline$\left(E_{\text {batt }, T}\right)^{+}$ & + & Electrical energy out of the battery or at the terminals during the propel case \\
\hline$\left(E_{\text {batt }, T}\right)^{+E q F C}$ & - & Theoretical electrical energy at the battery terminals. Analog to $\left(\mathrm{E}_{\text {batt, } \mathrm{T}}\right)^{+ \text {prop }}$ \\
\hline$\left(E_{\text {batt }, T}\right)^{+ \text {gen }}$ & - & Electrical energy in to the battery at the terminals to be stored \\
\hline$\left(E_{\text {batt }, T}\right)^{+ \text {prop }}$ & + & Electrical energy out of the battery at the terminals used for propel \\
\hline$\left(E_{\text {batt }, T}\right)^{0}$ & + & Electrical energy out of the battery or at the terminals during idle case \\
\hline$\left(E_{\text {batt }, T}\right)^{c}$ & - & Electrical energy out of the battery or at the terminals during the charging case \\
\hline$\left(E_{\text {batt }, T}\right)^{\text {DCnet }}$ & + & Net electrical energy at the terminals for a given drive cycle \\
\hline$\left(E_{\text {batt, }, T}\right)^{- \text {EqFC }}$ & + & Theoretical electrical energy at the battery terminals. Analog to $\left(\mathrm{E}_{\mathrm{batt}, \mathrm{T}}\right)^{+\mathrm{gen}}$ \\
\hline$\left(E_{\text {batt, use }}\right)^{+ \text {prop }}$ & + & Electrical energy at the terminals to be used in propulsion \\
\hline$\left(\mathrm{E}_{\text {batt, }} \text {,use }\right)^{\mathrm{EqFC}}$ & - & Theoretical electrical energy in to the battery. Analog to $\left(E_{\text {batt,use }}\right)^{+p r o p}$ \\
\hline$\left(E_{\text {chrg,AC }}\right)^{A C n e t}$ & + & Electrical energy $(\mathrm{AC})$ from the grid in to the battery charger, \\
\hline$\left(E_{\text {chrg, DC }}\right)^{\mathrm{C}}$ & + & Electrical energy (DC) out of the battery charger \\
\hline$\left(\mathrm{E}_{\mathrm{dleng}, \mathrm{tr}}\right)^{+}$ & + & Tractive energy out of the engine driveline to the drive axle for the propel case \\
\hline$\left(E_{\text {dleng,tr }}\right)^{+a c c}$ & + & Tractive energy out of the engine driveline to be used for accessory power \\
\hline$\left(E_{\text {dleng,tr }}\right)^{0}$ & + & Tractive energy out of the engine driveline for the idle case \\
\hline$\left(E_{\text {dleng,tr }}\right)^{\text {EqrC }}$ & - & Theoretical tractive energy in to the engine driveline to be used as fuel credit \\
\hline$\left(E_{d l m o t, t r}\right)^{-1+}$ & + & Surplus tractive energy out of the motor $\mathrm{D} / \mathrm{L}$ from the drive axle to be reused \\
\hline$\left(E_{d l m o t, t r}\right)^{+a c c}$ & - & Tractive energy in to the motor driveline to be used for accessory power \\
\hline$\left(\mathrm{E}_{\mathrm{dlmot}, \mathrm{tr}}\right)^{+\mathrm{EqFC}}$ & - & Theoretical tractive energy out of the motor driveline. Analog to $\left(\mathrm{E}_{\mathrm{dlmot}, \mathrm{tr}}\right)^{+\mathrm{prop}}$ \\
\hline$\left(E_{\text {dlmot,tr }}\right)^{+g e n}$ & - & Excess tractive energy in to the motor driveline to be stored \\
\hline$\left(\mathrm{E}_{\text {dllmotitritut }}\right)^{+ \text {prop }}$ & + & Surplus tractive energy out of the motor driveline to be used for propel \\
\hline$\left(E_{\text {dlmotitr }}\right)^{0}$ & - & Tractive energy in to the motor driveline during the idle case \\
\hline$\left(E_{\text {dlmot,tr }}\right)^{- \text {EqFC }}$ & + & Theoretical tractive energy out of the motor driveline. Analog to $\left(\mathrm{E}_{\mathrm{dlmot}, \mathrm{tr}}\right)^{+\mathrm{gen}}$ \\
\hline$\left(E_{e \& m, \text { mech }}\right)^{-1+}$ & + & Sum of Mechanical energy from engine and motor in to engine driveline \\
\hline$\left(E_{e \& m, \text { mech }}\right)^{+}$ & + & Sum of mechanical energy from engine and motor in to engine driveline \\
\hline$\left(E_{e \& m, m e c h}\right)^{E q F C}$ & + & Theoretical mechanical energy in from engine driveline to be used as fuel credit \\
\hline$\left(E_{\text {enq,fuel }}\right)^{+}$ & + & Fuel energy in to the engine during the propel case \\
\hline$\left(E_{\text {eng,fuel }}\right)^{\text {tacc }}$ & + & Fuel energy in to the engine used for accessory power \\
\hline$\left(E_{\text {eng,fuel }}\right)^{+t r}$ & + & Fuel energy in to the engine used for tractive power during the propel case \\
\hline$\left(E_{\text {eng,fuel }}\right)^{0}$ & + & Fuel energy in to the engine during the idle case \\
\hline$\left(\mathrm{E}_{\text {eng,fuel }}\right)^{\text {EqFC }}$ & - & Theoretical fuel energy out of the engine to be used as fuel credit \\
\hline$\left(\mathrm{E}_{\text {eng,mech }}\right)^{+}$ & + & Mechanical energy out of the engine during the propel case \\
\hline$\left(\mathrm{E}_{\text {eng,mech }}\right)^{+\mathrm{acc}}$ & + & Mechanical energy out of the engine to be used for accessory power \\
\hline
\end{tabular}




\begin{tabular}{|c|c|c|}
\hline \multicolumn{3}{|c|}{ Nomenclature for Energy and Power Quantities } \\
\hline Term & $\begin{array}{l}\text { Inherent } \\
\text { Sign }\end{array}$ & Definition \\
\hline$\left(\mathrm{E}_{\text {eng,mech }}\right)^{0}$ & + & Mechanical energy out of the engine during the idle case \\
\hline$\left(\mathrm{E}_{\text {eng,mech }}\right)^{\mathrm{EqFC}}$ & - & Theoretical mechanical energy in to the engine to be used as fuel credit \\
\hline$\left(E_{\text {eng,store }}\right)^{+}$ & - & Tractive energy from the engine stored before being used for propulsion \\
\hline$\left(E_{\text {eng,use }}\right)^{+}$ & + & Tractive energy from the engine directly used for propulsion \\
\hline$\left(E_{\text {gen,elec }}\right)^{+}$ & + & Electrical energy out of the generator during the propel case \\
\hline$\left(E_{\text {gen,elec }}\right)^{0}$ & + & Electrical energy out of the generator during the idle case \\
\hline$\left(\mathrm{E}_{\text {gen,elec }}\right)^{\mathrm{EqFC}}$ & - & Theoretical electrical energy in to the generator to be used as fuel credit \\
\hline$\left(\mathrm{E}_{\text {gen, store }}\right)^{+\mathrm{gen}}$ & - & Electrical generator energy stored before being used for propulsion \\
\hline$\left(E_{\text {gen,store }}\right)^{\mathrm{EFCC}}$ & + & Theoretical electrical energy in to HV bus. Analog to $\left(\mathrm{E}_{\text {gen,store }}\right)^{+ \text {gen }}$ \\
\hline$\left(E_{\text {gen,use }}\right)^{+}$ & + & Electrical generator energy directly used for propulsion \\
\hline$\left(\mathrm{E}_{\text {mot,elec }}\right)^{-}$ & - & Electrical energy out of the motor for the brake case \\
\hline$\left(\mathrm{E}_{\text {mot,elec }}\right)^{-1+}$ & + & Electrical energy in to the motor to be reused for propulsion \\
\hline$\left(\mathrm{E}_{\text {mot,elec }}\right)^{+}$ & + & Electrical energy in to the motor for the propel case \\
\hline$\left(E_{\text {mot,elec }}\right)^{\text {+acc }}$ & - & Electrical energy out of the motor to be used for accessory power \\
\hline$\left(E_{\text {mot,elec }}\right)^{+E q F C}$ & - & Theoretical electrical energy in to the motor. Analog to $\left(E_{\text {motelec }}\right)^{+ \text {prop }}$ \\
\hline$\left(E_{\text {mot.elec }}\right)^{+g e n}$ & - & Electrical energy out of the motor to be stored \\
\hline$\left(\mathrm{E}_{\text {mot,elec }}\right)^{+ \text {prop }}$ & + & Electrical energy in to the motor to be used \\
\hline$\left(\mathrm{E}_{\text {mot,elec }}\right)^{0}$ & - & Electrical energy out of the motor during the idle case \\
\hline$\left(E_{\text {mot,elec }}\right)^{\mathrm{EqFC}}$ & - & Theoretical electrical energy out of the motor to be used as fuel credit \\
\hline$\left(\mathrm{E}_{\text {mot,elec }}\right)^{\text {-EqFC }}$ & + & Theoretical electrical energy in to the motor. Analog to $\left(\mathrm{E}_{\text {motelec }}\right)^{+\mathrm{gen}}$ \\
\hline$\left(E_{\text {mot,mech }}\right)^{-}$ & - & Mechanical energy in to the motor for the brake case \\
\hline$\left(E_{\text {mot,mech }}\right)^{-1 /}$ & + & Mechanical energy out of the motor to be reused for propulsion \\
\hline$\left(E_{\text {mot,mech }}\right)^{+}$ & + & Mechanical energy out of the motor for the propel case \\
\hline$\left(\mathrm{E}_{\mathrm{mot}, \mathrm{mech}}\right)^{+\mathrm{acc}}$ & - & Mechanical energy in to the motor to be used for accessory power \\
\hline$\left(E_{\text {mot,mech }}\right)^{+\mathrm{EqFC}}$ & - & Theoretical mechanical energy out of the motor. Analog to $\left(E_{\text {mot,mech }}\right)^{+p r o p}$ \\
\hline$\left(\mathrm{E}_{\text {mot,mech }}\right)^{+\mathrm{gen}}$ & - & Mechanical energy in to the motor to be stored for future propulsion \\
\hline$\left(\mathrm{E}_{\mathrm{mot}, \mathrm{mech}}\right)^{+ \text {prop }}$ & + & Mechanical energy out of the motor to be used for propulsion \\
\hline$\left(E_{\text {mot,mech }}\right)^{0}$ & - & Mechanical energy in to the motor during the idle case \\
\hline$\left(\mathrm{E}_{\text {mot,mech }}\right)^{\mathrm{EqFC}}$ & - & Theoretical mechanical energy in to the motor to be used as fuel credit \\
\hline$\left(E_{\text {mot,mech }}\right)^{\text {-EqFC }}$ & + & Theoretical mechanical energy out of the motor. Analog to $\left(E_{\text {mot,mech }}\right)^{+g e n}$ \\
\hline$\left(E_{t r}\right)^{-}$ & - & Tractive energy at the wheels for the brake case \\
\hline$\left(\mathrm{E}_{\mathrm{tr}}\right)^{+}$ & + & Tractive energy at the wheels for the propel case \\
\hline$\left(E_{t r}\right)^{D C n e t}$ & + & Net tractive energy at the wheels for a given drive cycle (road load) \\
\hline$\left(E_{\text {tr,credit }}\right)^{-/ t}$ & + & Tractive energy at the wheels from stored regen energy reused for propulsion \\
\hline$\left(E_{\text {tr,credit }}\right)^{E-G F C}$ & - & Theoretical tractive energy in to the wheels to be used as fuel credit \\
\hline$\left(E_{t r, \zeta}\right)^{-}$ & - & Tractive energy in to driveline after effect of regen fraction \\
\hline$\left(E_{\alpha}\right)^{-}$ & + & Energy dissipated by rolling resistance for the brake case \\
\hline$\left(E_{\alpha}\right)^{+}$ & + & Energy for overcoming rolling resistance for the propel case \\
\hline$\left(E_{\alpha}\right)^{D C n e t}$ & + & Net energy for overcoming rolling resistance for a given drive cycle \\
\hline$\left(E_{\beta}\right)^{-}$ & + & Energy dissipated by aerodynamic drag for the brake case \\
\hline$\left(E_{\beta}\right)^{+}$ & + & Energy for overcoming aerodynamic drag for the propel case \\
\hline$\left(E_{\beta}\right)^{\text {DCnet }}$ & + & Net energy for overcoming aerodynamic drag for a given drive cycle \\
\hline$\left(E_{\gamma}\right)^{-}$ & - & Required decrease in vehicle kinetic energy for the brake case \\
\hline$\left(E_{\mathrm{V}}\right)^{+}$ & + & Energy to produce increases in vehicle kinetic energy for the propel case \\
\hline$\left(E_{V}\right)^{D C n e t}$ & + & Net energy for overcoming accelerating vehicle inertia for a given drive cycle \\
\hline$\left(E_{\Delta}\right)^{-}$ & + & Energy to produce change in altitude for the propel case \\
\hline$\left(\mathrm{E}_{\Delta}\right)^{+}$ & + & Energy to produce change in altitude for the propel case \\
\hline$\left(\mathrm{E}_{\Delta}\right)^{\mathrm{DCnet}}$ & + & Net energy for overcoming change in altitude for a given drive cycle \\
\hline$\left(E_{\lambda}\right)^{-}$ & + & Energy dissipated by velocity-proportional elements for the brake case \\
\hline$\left(E_{\lambda}\right)^{+}$ & + & Energy for overcoming velocity-proportional elements for the propel case \\
\hline$\left(E_{\lambda}\right)^{D C n e t}$ & + & Net energy for overcoming velocity-proportional elements for a given drive cycle \\
\hline
\end{tabular}




\begin{tabular}{|l|l|l|}
\hline \multicolumn{3}{|l|}{ Nomenclature for Energy and Power Quantities } \\
\hline Term & $\begin{array}{l}\text { Inherent } \\
\text { Sign }\end{array}$ & Definition \\
\hline$\left(\mathrm{P}_{\mathrm{acc}}\right)^{+}$ & + & Accessory power during the propel case (the same during brake and idle) \\
\hline$\left(\mathrm{P}_{\mathrm{acc}}\right)^{-}$ & + & Accessory power during the brake case (the same during propel and idle) \\
\hline$\left(\mathrm{P}_{\mathrm{acc}}\right)^{+}$ & + & Accessory power during the idle case (the same during propel and brake) \\
\hline$\left(\mathrm{P}_{\mathrm{acc}}\right)^{C}$ & + & Accessory power during the charge case \\
\hline$\left(\mathrm{P}_{\mathrm{charger}}\right)^{C}$ & + & Charger power. This is a component parameter. \\
\hline
\end{tabular}

\section{List of Nomenclature: Component and Powertrain Efficiencies}

For maximum flexibility, a unique component efficiency could be defined for every case of every powertrain. However, this would be exceptionally burdensome. Some components are bi-directional (like a motor) while others are uni-directional (like an engine). As such, it makes sense to define a single efficiency for uni-directional components, which will always be for the propel direction of energy flow. For bi-directional components, two efficiencies will be defined, one for the propel energy flow and one for the brake energy flow. While unique component efficiencies may be cited in the derivation of the energy consumption equation for a given case, this efficiency will be equivalent to either the propel or brake component efficiency.

\begin{tabular}{|l|c|l|}
\hline \multicolumn{2}{|l|}{ Nomenclature for Component and Powertrain Efficiencies } \\
\hline Term & Duplicates & Definition \\
\hline$\tilde{\eta}_{b a t t}^{-}$ & & Cycle average battery efficiency for the brake case \\
\hline$\tilde{\eta}_{b a t t}^{-/+}$ & $\tilde{\eta}_{b a t t}^{+}$ & Cycle average battery efficiency for the regen energy reuse case. \\
\hline$\tilde{\eta}_{b a t t}^{+}$ & & Cycle average battery efficiency for the propel case \\
\hline$\tilde{\eta}_{b a t t}^{+g e n}$ & $\tilde{\eta}_{b a t t}^{-}$ & $\begin{array}{l}\text { Cycle average battery efficiency for the propel case when the battery is receiving } \\
\text { energy }\end{array}$ \\
\hline$\tilde{\eta}_{b a t t}^{+ \text {prop }}$ & $\tilde{\eta}_{b a t t}^{+}$ & $\begin{array}{l}\text { Cycle average battery efficiency for the propel case when the battery is discharging } \\
\text { energy }\end{array}$ \\
\hline$\tilde{\eta}_{b a t t}^{0}$ & $\tilde{\eta}_{b a t t}^{+}$ & Cycle average battery efficiency for the idle case \\
\hline$\tilde{\eta}_{b a t t}^{c}$ & $\tilde{\eta}_{b a t t}^{-}$ & Average battery efficiency for the charging case \\
\hline$\tilde{\eta}_{c h r g n}^{c}$ & & $\begin{array}{l}\text { Average charging efficiency for the charging case. This includes charging Acc'y load } \\
\text { and battery efficiency }\end{array}$ \\
\hline$\tilde{\eta}_{c h r g r}^{c}$ & & Average charger efficiency for the charging case. This is just a component efficiency. \\
\hline$\tilde{\eta}_{d l, e n g}^{-/+}$ & $\tilde{\eta}_{d l, e n g}^{+}$ & Cycle average engine driveline efficiency for the regen energy reuse case. \\
\hline$\tilde{\eta}_{\text {dl,eng }}^{+}$ & & Cycle average engine driveline efficiency for the propel case \\
\hline$\tilde{\eta}_{d l, e n g}^{0}$ & $\tilde{\eta}_{d l, e n g}^{+}$ & Average engine driveline efficiency for the idle case \\
\hline$\tilde{\eta}_{\overline{d l}, \text { mot }}^{-}$ & & Cycle average motor driveline efficiency for the brake case \\
\hline
\end{tabular}




\begin{tabular}{|c|c|c|}
\hline \multicolumn{3}{|c|}{ Nomenclature for Component and Powertrain Efficiencies } \\
\hline Term & Duplicates & Definition \\
\hline$\tilde{\eta}_{d l, m o t}^{-/+}$ & $\tilde{\eta}_{d l, m o t}^{+}$ & $\begin{array}{l}\text { Cycle average motor driveline efficiency for the regen energy reuse case. Identical to } \\
\text { EV propel motor driveline efficiency. }\end{array}$ \\
\hline$\tilde{\eta}_{d l, m o t}^{+}$ & & Cycle average motor driveline efficiency for the propel case \\
\hline$\tilde{\eta}_{d l, m o t}^{+g e n}$ & $\tilde{\eta}_{\overline{d l}, \mathrm{mot}}^{-}$ & $\begin{array}{l}\text { Cycle average motor driveline efficiency for the propel case when the motor is } \\
\text { generating. }\end{array}$ \\
\hline$\tilde{\eta}_{d l, m o t}^{+ \text {prop }}$ & $\tilde{\eta}_{d l, m o t}^{+}$ & Cycle average motor efficiency for the propel case when the motor is propelling. \\
\hline$\tilde{\eta}_{d l, m o t}^{0}$ & $\tilde{\eta}_{d l, m o t}^{-}$ & Average motor driveline efficiency for the idle case \\
\hline$\tilde{\eta}_{\text {eng }}^{+}$ & & Cycle average engine efficiency for the propel case \\
\hline$\tilde{\eta}_{e n g}^{0}$ & $\tilde{\eta}_{e n g}^{+}$ & Average engine efficiency for the idle case \\
\hline$\tilde{\eta}_{\text {gen }}^{+}$ & & Cycle average generator efficiency for the propel case \\
\hline$\tilde{\eta}_{g e n}^{0}$ & $\tilde{\eta}_{g e n}^{+}$ & Average generator efficiency for the idle case \\
\hline$\tilde{\eta}_{m o t}^{-}$ & & Cycle average motor efficiency for the brake case \\
\hline$\tilde{\eta}_{m o t}^{-/+}$ & $\tilde{\eta}_{m o t}^{+}$ & Cycle average motor efficiency for the regen energy reuse case. \\
\hline$\tilde{\eta}_{m o t}^{+}$ & & Cycle average motor efficiency for the propel case \\
\hline$\tilde{\eta}_{m o t}^{+g e n}$ & $\tilde{\eta}_{m o t}^{-}$ & Cycle average motor efficiency for the propel case when the motor is generating. \\
\hline$\tilde{\eta}_{m o t}^{+p r o p}$ & $\tilde{\eta}_{m o t}^{+}$ & Cycle average motor efficiency for the propel case when the motor is propelling. \\
\hline$\tilde{\eta}_{m o t}^{0}$ & $\tilde{\eta}_{m o t}^{-}$ & Average motor efficiency for the idle case \\
\hline$\tilde{\eta}_{p t, E V}^{+}$ & & $\begin{array}{l}\text { Cycle average powertrain efficiency during the propel case for the regen energy } \\
\text { reuse flow path. This is effectively EV mode efficiency. }\end{array}$ \\
\hline$\tilde{\eta}_{p t, e n g}^{+}$ & & $\begin{array}{l}\text { Cycle average powertrain efficiency during the propel case for the fuel energy flow } \\
\text { path }\end{array}$ \\
\hline$\tilde{\eta}_{p t}^{-}$ & & Cycle average powertrain efficiency for the brake case. Includes brake Acc'y load. \\
\hline$\tilde{\eta}_{p t}^{-/+}$ & & $\begin{array}{l}\text { Cycle average powertrain efficiency for the regen energy reuse case. Does not } \\
\text { include any Acc'y energy. }\end{array}$ \\
\hline$\tilde{\eta}_{p t}^{+}$ & & Cycle average powertrain efficiency for the propel case. Includes propel Acc'y load. \\
\hline$\tilde{\eta}_{p t}^{+a c c}$ & & Cycle average powertrain efficiency to supply Acc'y load for the propel case. \\
\hline$\tilde{\eta}_{p t}^{+t r}$ & & $\begin{array}{l}\text { Cycle average powertrain efficiency for the propel case. Includes tractive energy only } \\
\text { and no Acc'y load. }\end{array}$ \\
\hline
\end{tabular}




\begin{tabular}{|l|l|l|}
\hline \multicolumn{2}{|l|}{ Nomenclature for Component and Powertrain Efficiencies } \\
\hline Term & Duplicates & Definition \\
\hline$\tilde{\eta}_{p t}^{D C n e t}$ & & $\begin{array}{l}\text { Cycle average net powertrain efficiency. Includes total Acc'y load, compares to road } \\
\text { load. }\end{array}$ \\
\hline$\tilde{\eta}_{\text {recirc }}^{+}$ & $\begin{array}{l}\text { Cycle average efficiency to recirculate energy to the battery and back out during CS } \\
\text { mode. This is the apparent or marginal efficiency associated with recirculating energy } \\
\text { to load level the engine. }\end{array}$ \\
\hline$\tilde{\zeta}$ & $\begin{array}{l}\text { Cycle average regenerative braking fraction. Expresses fraction of brake energy } \\
\text { passed to the motor driveline and not dissipated by friction brakes }\end{array}$ \\
\hline$\tilde{\xi}$ & $\begin{array}{l}\text { This is the fraction of energy produced by the engine that can be directly used by the } \\
\text { drivetrain without being stored in the battery first. } \xi=1 \text { means all energy produced by } \\
\text { the engine is directly used for traction. } \\
\text { Indicates energy weighted cycle average quantity }\end{array}$ \\
\hline$\sim$ & ${ }^{*}$ script ${ }^{*}$ &
\end{tabular}

\section{List of Nomenclature: Vehicle Parameters and Sovran Drive Cycle Coefficients}

\begin{tabular}{|l|l|l|}
\hline \multicolumn{3}{|l|}{ Nomenclature for Vehicle Parameters and Sovran Drive Cycle Coefficients } \\
\hline Term & Units & Definition \\
\hline$A$ & {$\left[\mathrm{~m}^{2}\right]$} & Frontal area \\
\hline$C_{d}$ & {$[-]$} & Coefficient of drag \\
\hline$C_{r r, 0}$ & {$[-]$} & Tire rolling resistance \\
\hline$c_{r r, 1}$ & {$[1 /(\mathrm{m} / \mathrm{s})]$} & Velocity-proportional frictive element \\
\hline$I_{w}$ & {$\left[\mathrm{~kg}-\mathrm{m}^{2}\right]$} & Polar moment of inertia of a wheel \\
\hline$M$ & {$[\mathrm{~kg}]$} & Vehicle test mass \\
\hline$M_{i}$ & {$[\mathrm{~kg}]$} & Vehicle inertial mass \\
\hline$r_{\mathrm{w}}$ & {$[\mathrm{m}]$} & Radius of a wheel \\
\hline$S$ & {$[\mathrm{~km}]$} & Drive cycle distance \\
\hline$T$ & {$[\mathrm{~s}]$} & Drive cycle time \\
\hline$t_{\text {charge }}$ & {$[\mathrm{s}]$} & Time in seconds required to replenish the battery energy expended over a cycle \\
\hline$t_{i d l e}$ & {$[\mathrm{~s}]$} & Cycle idle time \\
\hline$\tau$ & {$[\mathrm{s}]$} & Powered driving time \\
\hline$\alpha$ & {$\left[\mathrm{m} / \mathrm{s}^{2}\right]$} & Sovran coefficient for rolling resistance during the propel case \\
\hline$\alpha^{\prime}$ & {$\left[\mathrm{m} / \mathrm{s}^{2}\right]$} & Sovran coefficient for rolling resistance during the brake case \\
\hline$\beta$ & {$\left[\mathrm{kg} / \mathrm{m}^{2} \mathrm{~s}^{2}\right]$} & Sovran coefficient for aerodynamic drag during the propel case \\
\hline$\beta^{\prime}$ & {$\left[\mathrm{kg} / \mathrm{m}^{2} \mathrm{~s}^{2}\right]$} & Sovran coefficient for aerodynamic drag during the brake case \\
\hline $\mathrm{Y}$ & {$\left[\mathrm{m} / \mathrm{s}^{2}\right]$} & Sovran coefficient for inertial energy during the propel case \\
\hline $\mathrm{Y}^{\prime}$ & {$\left[\mathrm{m} / \mathrm{s}^{2}\right]$} & Sovran coefficient for inertial energy during the brake case \\
\hline$\Delta$ & {$\left[\mathrm{m}^{2} / \mathrm{s}^{2}\right]$} & Sovran coefficient for potential energy during the propel case \\
\hline$\Delta^{\prime}$ & {$\left[\mathrm{m}^{2} / \mathrm{s}^{2}\right]$} & Sovran coefficient for potential energy during the brake case \\
\hline$\lambda$ & {$\left[\mathrm{m} / \mathrm{s}^{2}\right]$} & Sovran coefficient for velocity proportional energy during the propel case \\
\hline$\lambda^{\prime}$ & {$\left[\mathrm{m} / \mathrm{s}^{2}\right]$} & Sovran coefficient for velocity proportional energy during the brake case \\
\hline
\end{tabular}




\section{List of Nomenclature: Drive Cycle Case Indicator Superscript}

\begin{tabular}{|l|l|}
\hline \multicolumn{2}{|l|}{ Nomenclature for Case Indicator Superscript } \\
\hline Superscript & Definition \\
\hline+ & Propel case: $\mathrm{F}_{\mathrm{tr}}>0$ \\
\hline- & Brake case: $\mathrm{F}_{\mathrm{tr}}<0$ \\
\hline 0 & Idle case: $\mathrm{F}_{\mathrm{tr}}=0, \mathrm{v}=0$ \\
\hline $\mathrm{C}$ & Charging case: $\mathrm{F}_{\mathrm{tr}}=0, \mathrm{P}_{\mathrm{batt}, \mathrm{T}}<0$ \\
\hline DCnet & Pre Charge Net Case \\
\hline ACnet & Post Charge Net Case \\
\hline$-/+$ & Regen Energy Reuse Case \\
\hline+ prop & Power Split Region - designates energy from the battery to be used for traction \\
\hline+ gen & Power Split Region - designates energy to be stored in battery for future use \\
\hline EqFC & Theoretical Fuel-Equivalent Energy Credit Case \\
\hline+ EqFC & Theoretical Fuel-Equivalent Energy Credit Case: Analog to +prop case \\
\hline- EqFC & Theoretical Fuel-Equivalent Energy Credit Case: Analog to +gen case \\
\hline+ tr & Propel case, only tractive energy considered \\
\hline+ acc & Propel case, only accessory energy considered \\
\hline
\end{tabular}

The figure below is included here for convenience, but is duplicated as Figure 13 in Section 3.1.3: Defining Nomenclature.

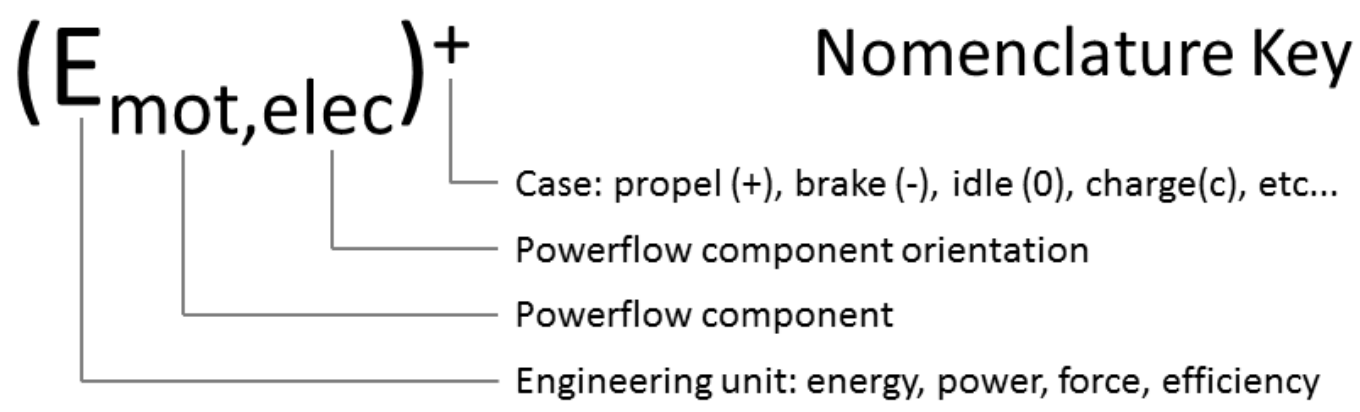


List of Abbreviations

\begin{tabular}{|l|l|}
\hline Abbreviation & Definition \\
\hline A/C & Air Conditioning \\
\hline ANL & Argonne National Labs \\
\hline AVTC & Advanced Vehicle Technology Competition \\
\hline BAS & Belted Alternator Starter \\
\hline BEV & Battery Electric Vehicle \\
\hline CAFE & Corporate Average Fuel Economy \\
\hline CAN & Controller Area Network \\
\hline CD & Charge Depleting \\
\hline Cl & Compression Ignited \\
\hline CS & Charge Sustaining \\
\hline DL & Driveline (for a motor or an engine) \\
\hline DOE & Department of Energy \\
\hline E\&EC & Emissions and Energy Consumption \\
\hline ECM & Engine Control Module \\
\hline EMI & Electro-Magnetic Interference \\
\hline EPA & Environmental Protection Agency \\
\hline EPA & Environmental Protection Agency \\
\hline EREV & Extended Range Electric Vehicle \\
\hline ESS & Energy Storage System \\
\hline EV & Electric Vehicle \\
\hline EVSE & Electric Vehicle Supply Equipment \\
\hline FPGA & Field Programmable Gate Array \\
\hline FTP & Federal Test Procedure \\
\hline GHG & Greenhouse Gas \\
\hline GM & General Motors \\
\hline GREET & Greenhouse Gas, Regulated Emissions and Energy Use in Transportation \\
\hline GVWR & Gross Vehicle Weight Rating \\
\hline HEV & Hybrid Electric Vehicle \\
\hline HEVT & Hybrid Electric Vehicle Team \\
\hline HIL & Hardware-in-the-Loop \\
\hline HV & High Voltage \\
\hline HVSC & Hybrid Vehicle Supervisory Controller \\
\hline HwFET & Highway Fuel Economy Test \\
\hline MSD & Manual Service Disconnect \\
\hline NVFEL & EPA National Vehicle and Fuel Emissions Laboratory \\
\hline OPEC & Organization of Petroleum Exporting Countries \\
\hline PEU & Petroleum Energy Use \\
\hline PHEV & Plug-in Hybrid Electric Vehicle \\
\hline PSAT & Powertrain System Analysis Toolkit \\
\hline RESS & Rechargeable Energy Storage System \\
\hline RTM & Rear Traction Motor \\
\hline SI & Spark Ignited \\
\hline SOC & State of Charge \\
\hline UDDS & Urban Dynamometer Drive Schedule \\
\hline UF & Utility Factor \\
\hline VDP & Vehicle Development Process \\
\hline VTS & Vehicle Technical Specifications \\
\hline WTP & Well to Pump \\
\hline WTW & Well to Wheels \\
\hline & \\
\hline
\end{tabular}




\section{Introduction}

\subsection{Background and Motivation for the Development of Advanced Technology Vehicles}

\subsubsection{Implications of Petroleum Energy Use and Greenhouse Gas Emissions}

The various implications of Petroleum Energy Usage (PEU) and emissions generation strongly support the need for the continued development and advancement of hybrid electric vehicles. Greenhouse gas (GHG) emissions generated by the transportation sector in the U.S. account for $27 \%$ of the nation's total GHG emissions. Worldwide, the US transportation sector also accounts for $30 \%$ of the GHG emissions in the global transportation sector [1].

There is a direct correlation between energy use and GHG emissions. Figure 1 shows that light-duty vehicles account for more than half of U.S. transportation energy use and $\mathrm{CO}_{2}$ emissions, meaning that efficiency improvements in this market segment will make a large impact on the overall energy use and GHG emissions in the U.S. [1]. The high level of energy usage in the U.S., and thus high level of GHG emissions like $\mathrm{CO}_{2}$ and criteria emissions like $\mathrm{CO}$, is a major cause of pollution. These emissions can also result in unhealthy air quality, especially in cities, and global climate change. The use of nonrenewable fossil fuels, such as coal, also contributes to these implications. Energy usage and emissions must be reduced in order to preserve the environment, minimize health concerns and achieve sustainability.
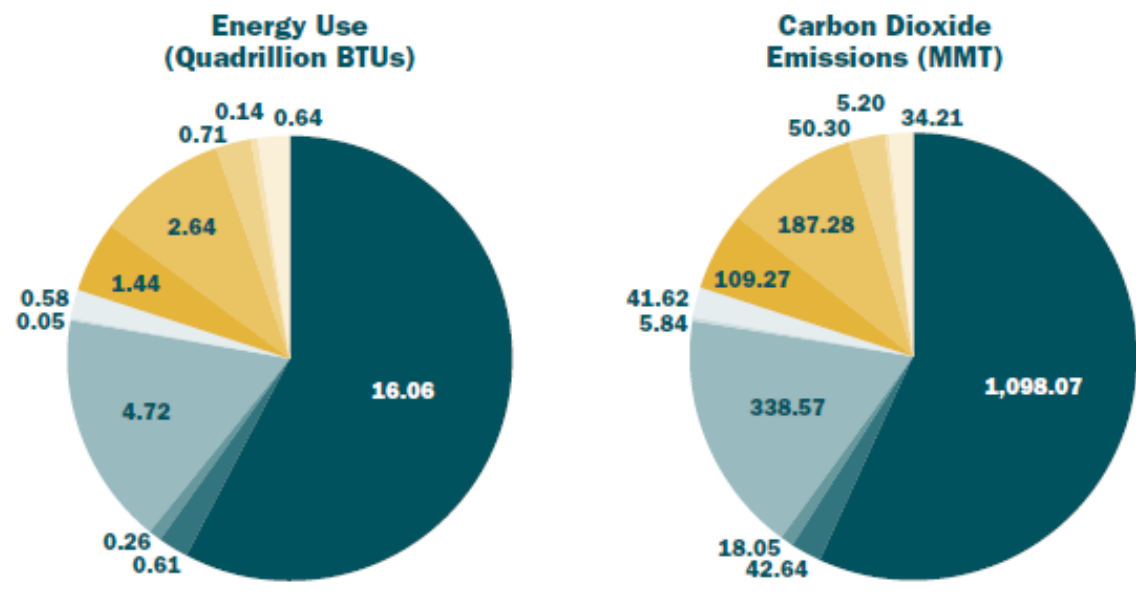

Light-Duty Vehicles Commercial Light Trucks Bus Transportation Freight Trucks Passenger Rail Freight Rail Ships Air Military Use Lubricants Pipeline

Source: EIA, 2010

Figure 1: U.S. transportation energy use and CO2 emissions, (public domain [1])

For the U.S. to maintain economic growth and avoid a national security crisis, the difficult task of developing renewable energy sources must be overcome. As shown in Figure 2, the U.S. currently consumes about 19 million barrels of petroleum per day of which about $60 \%$ is imported (as of January 2011). About $40 \%$ of these imports come from the Organization of Petroleum Exporting Countries (OPEC). Much of the oil that comes from OPEC is produced in regions and countries that are subject to disruptions and whose strategic interests can differ from those of the U.S. Additionally, the liquid fuel imported to the U.S. accounts for $70 \%$ of the national trade deficit, which is about $\$ 1$ billion per day [2]. 


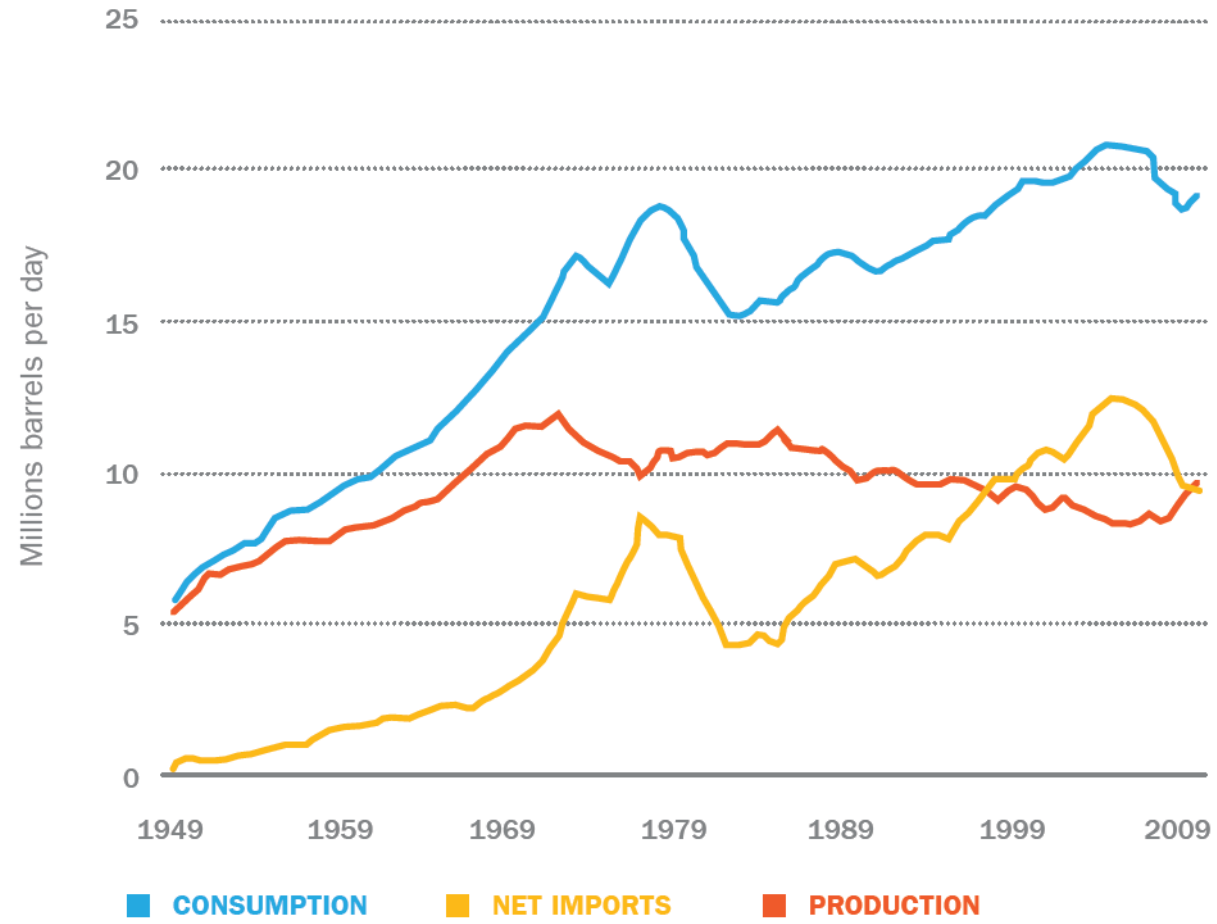

Figure 2: Trends in U.S. consumption, production, and net imports of liquid fuels (public domain [2])

By 2035, if current energy policies are not changed and no new renewable energy sources garner success, U.S. transportation GHG emissions could increase by about $10 \%$ and oil could reach $\$ 200$ per barrel [2]. As of April 16, 2012, the price of oil is $\$ 100$ per barrel. The Obama administration seeks to reduce oil imports by one third before 2025, deploy one million electric vehicles on the road before 2015, and reduce $\mathrm{GHG}$ emissions by $17 \%$ before 2020 and $83 \%$ before 2050 [2].

Overall, it is clear that improvements in Well-to-Wheel (WTW) PEU, WTW GHG and criteria emissions, and overall energy efficiency in the U.S. light duty vehicle segment will make a big impact on the U.S. in many ways. Such improvements will reduce criteria emissions, improving air quality at the local and regional levels and reduce GHG emissions which are a global contributor to global climate change. Additionally, reducing petroleum energy consumption will ease national security and balance of trade concerns. This thesis presents a method realized as a tool that aides in the modeling, development, evaluation and design of advanced technology vehicles that can help meet PEU and GHG reduction goals.

\subsubsection{American Driving Habits Pertaining to Electric Vehicle Utility: The Case for PHEVs}

A U.S. Department of Transportation study from 2003 surveyed 127.3 million adult U.S. residents concerning their daily driving habits. The participants were asked how far they commute (one way) to their job on a typical day. Figure 3 shows that $78 \%$ of American adults commute less than 40 miles (round trip) on a typical day, and $85 \%$ of adults commute less than 50 miles per day (round trip). 


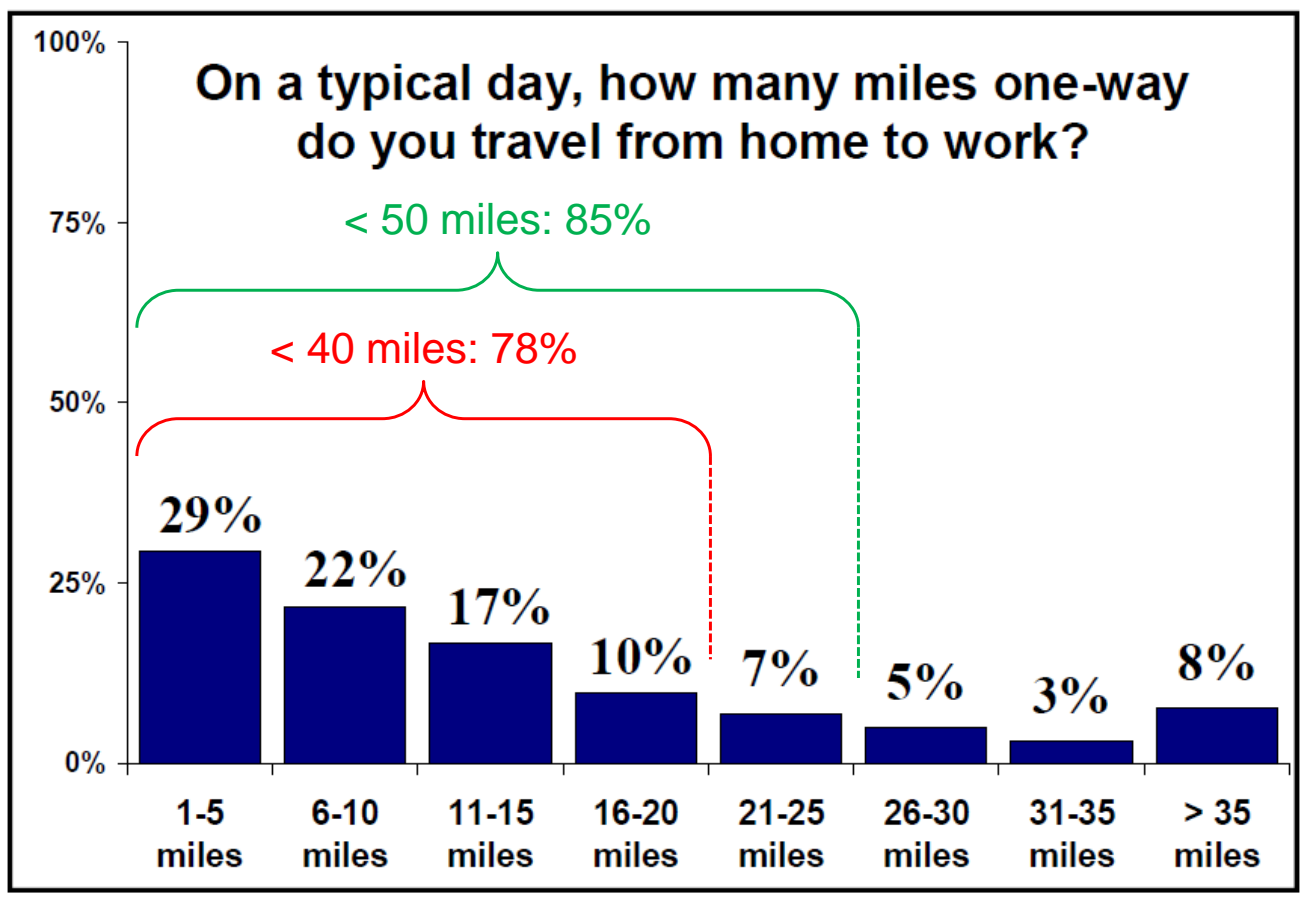

Source: US Department of Transportation, Bureau of Transportation Statistics, Omnibus Household Survey. Aggregated data cover activities for the month prior to the survey.

Figure 3: Typical one-way commute of the average U.S. resident, (public domain [3])

A more recent study was conducted 2010 in the Atlanta, Georgia area with 484 vehicles that were instrumented with data acquisition systems to track their driving habits for an entire year. The focus of this experiment was to gain knowledge on what range an EV would need for American citizens to travel completely electrically for a normal day. It should be noted that in annual vehicle miles traveled per capita, Georgia ranks 7th behind primarily rural states, accumulating 12,124 miles per person, 20\% more than the national average of 10,067 . This increase is because points of interest are more spread out in rural states, making the average annual driving distance larger [4].

Figure 4 shows a histogram of the daily driving of the monitored vehicles, broken in down into 4 mile/day bins. This figure illustrates that the vast majority of daily driving range need is in the 0-50 mile range. Excluding days of zero driving, the mean daily driving range is 44.7 miles and the median is 29.9 miles. The most common daily distance range is $12-16$ miles, represented by the highest bar in Figure 4 . In this figure, bars indicate 4 miles/day bins. The black line shows the sum of days per year where a given daily mileage is exceeded. This data shows that an EV with a limited range can still meet the majority of transportation needs [4]. 


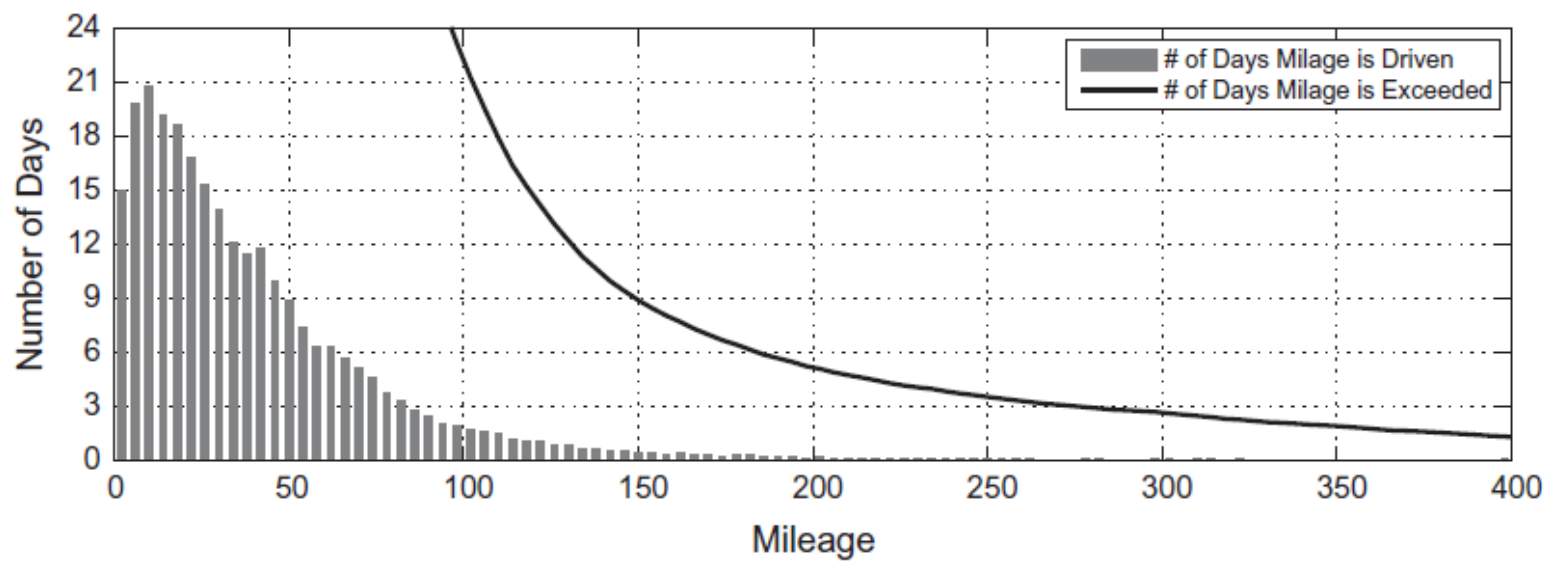

Figure 4: Average daily mileage distribution, (used with permission of Elsevier [4])

The study also found that a very large EV range would be necessary to meet the daily range requirements of all drivers all the time. Figure 5 shows that a 200 mile Battery Electric vehicle (BEV) would only satisfy the driving requirements of $30 \%$ of drivers without the need for an adaptation to their normal driving routine. An adaptation constitutes renting, purchasing, or borrowing a second vehicle, or charging the vehicle away from home. The plot shows that as drivers are more willing to make adaptations, the required range of their BEV will decrease. This means, for example, that if a driver is willing to own a second conventional vehicle that can handle the longer trips, the driver then requires less range from their BEV.

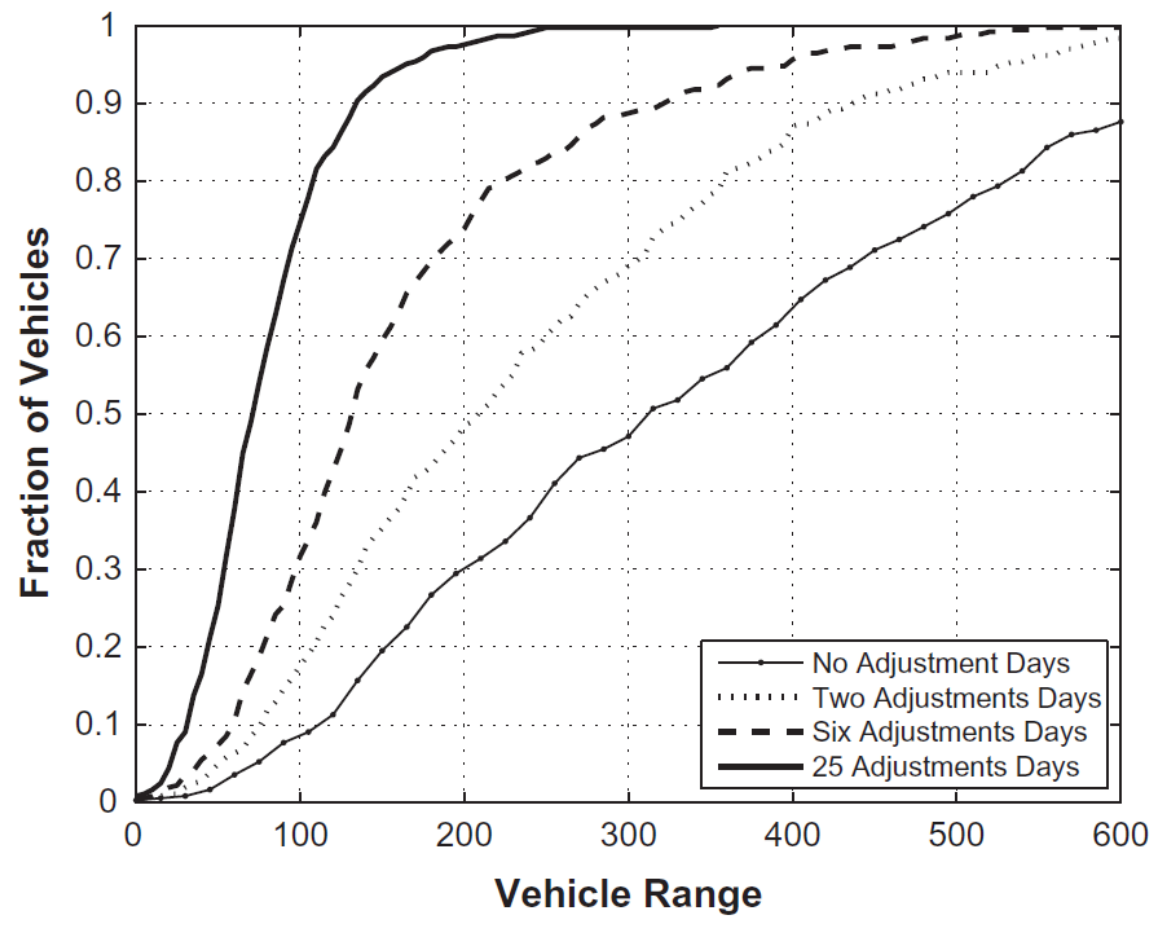

Figure 5: Fraction of the fleet appropriate for varying vehicle ranges, (used with permission of Elsevier [4]) 
The results of these two studies show that a BEV or an Extended Range Electric Vehicle (EREV) with a limited range can still meet the majority of transportation needs. Additionally, a BEV with a very large range will not meet the needs of all of the population all of the time. However, the more a driver is willing to adapt his driving habits, the smaller the range he requires of a BEV. Thus, a consumer could own a BEV which would cover the majority of his trips and a second conventional vehicle that would cover the small number of trips that require extended range beyond the capabilities of the BEV. Furthermore, a range extender could be added to a BEV to eliminate the need for a second vehicle, while optimizing the electric utility of the vehicle without requiring adaptation from the driver. For this reason, EREVs make a lot of sense for the average consumer. The tool developed in this thesis has the capability to model a variety of EREV powertrains and is designed to aid in the design and development of advanced technology vehicles such as EREVs.

\subsection{Hybrid Vehicle Powertrain Architecture Background}

\subsubsection{Effect of Electrification: HEVs, PHEVs, EREVs, and EVs}

Electrifying a vehicle means modifying it to be driven, whether partially or fully, by electrical energy to improve energy efficiency. The level of electrification of a vehicle is often a good estimate of its fuel consumption and WTW emissions. In order of increasing electrification, most electric vehicles can be classified as a mild hybrid electric vehicle (HEV), strong HEV, plug-in hybrid electric vehicle (PHEV), EREV, or BEV. These different classifications with respect to the level of electrification can be seen in Figure 6. Note that higher levels of electrification are not always desired as each classification has its own set of benefits and challenges.

Charge sustaining HEVs are the smallest step away from conventional vehicles in terms of electrification but can still reduce emissions by up to $30 \%$ compared to a conventional vehicle [5]. These vehicles have at least two Energy Storage Systems (ESS), normally a tank of fuel and a battery pack. The ESSs can either provide propulsion power together or independently [6]. A mild HEV generally has an electric drive system rated at less than $20 \mathrm{~kW}$ of power and an engine power rating high enough to provide satisfactory vehicle performance when electrical power is exhausted. In contrast, a strong HEV has an electric drive system rated at a relatively high power (usually $>50 \mathrm{~kW}$ ) allowing for the engine to be downsized. HEVs don't stray far from conventional vehicles in terms of technologies used and thus allow for an increase in energy efficiency while limiting the increase in system complexity and mass compared to a PHEV [7].

A PHEV can store and use grid electrical energy from a Rechargeable Energy Storage System (RESS) in conjunction with a traditional engine. With an onboard charger, a PHEV can charge the RESS with grid power when not in use. Compared to conventional vehicles, PHEVs powered by petroleum fuels can reduce GHG emissions by $10 \%$ to $60 \%$ and PHEVs powered by E85 can reduce GHG emissions by $20 \%$ to $80 \%$. The utility factor (UF) weighted fuel economy of a PHEV can be as high as $100 \mathrm{mpgge}$ with over $75 \%$ of the energy powering the vehicle coming from grid power. PHEVs can be blended, where the engine and motor work together to propel the vehicle, or have a distinct electric only mode like an EREV [7].

An EREV is a PHEV that can operate in electric-only mode with nearly full performance. Once the RESS is depleted of energy, the vehicle can switch to engine power for additional driving. This ability, in addition to ability to be charged overnight, makes an EREV extremely versatile. EREVs also have high consumer appeal because they allow drivers to perform their usual daily commute solely with electrical power but also make the occasional longer trip with the help of a fuel-powered engine. EREVs and other PHEVs result in minimal fuel consumption and emissions, but are very challenging to design due to the complex control schemes necessary to operate the vehicle effectively. 


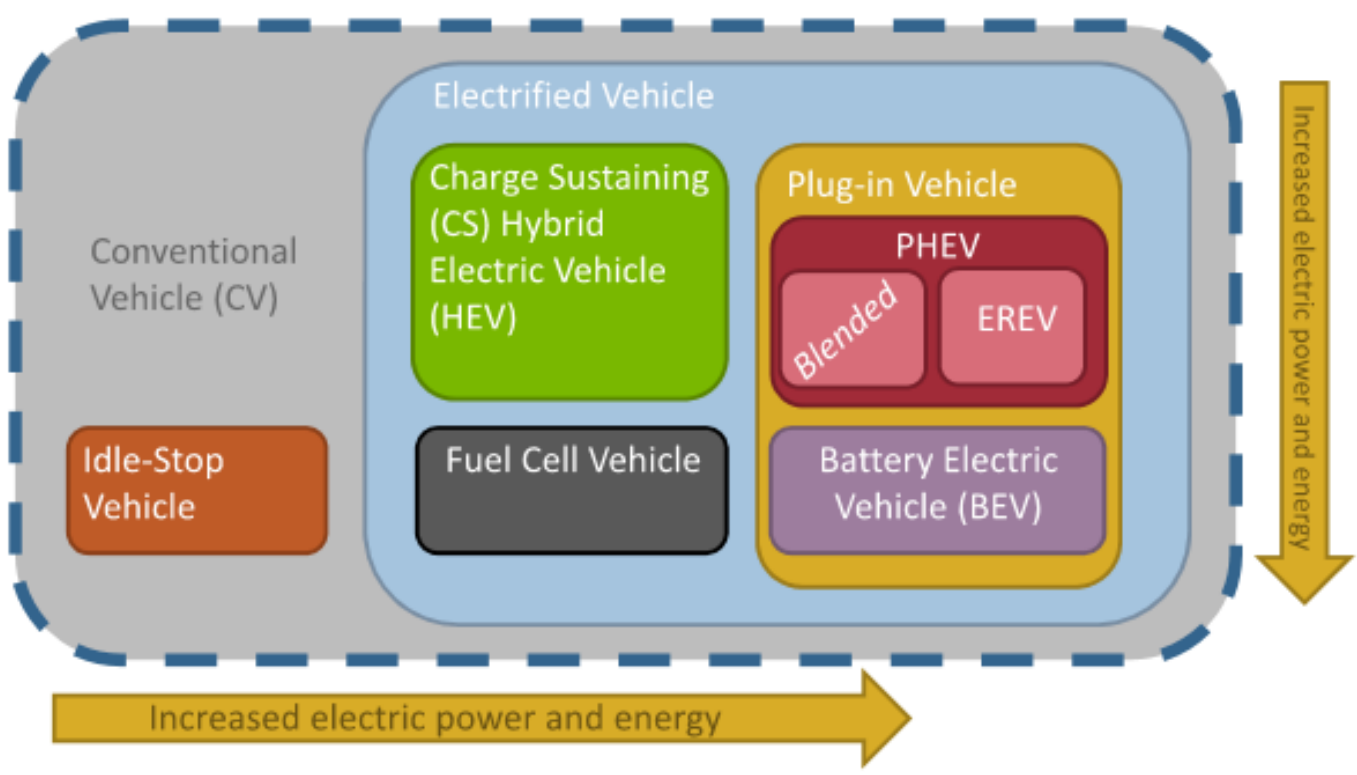

Figure 6: Vehicle designs compared in terms of the level of electrification

The most electrified vehicles are BEVs. These vehicles are not hybrids at all; they do not contain fuelpowered engines. The wheels are propelled solely by electrical power from the vehicle RESS. As a result, BEVs usually have a limited driving range [6]. However, by using very large lithium-ion battery packs, a BEV can attain driving ranges of up to $150 \mathrm{mi}$ with acceleration performance comparable or better than that of conventional vehicles. The limited range of BEVs along with expensive nature of the large batteries required result in lower consumer appeal [8]. The long battery recharge times ( $>6$ hours) using power levels available in typical homes (level 2, 6.6 kW) also limits consumer acceptance of BEVs.

Overall, increasing levels of electrification will bring increasing improvements in fuel consumption, but will also introduce added system complexity, mass and cost. However, the substantial benefits of PHEVs and EREVs warrant the additional complexity and mass. Additionally, electrification inevitably increases consumer appeal by improving the drive quality of the vehicle and making it fun to drive.

This thesis will present a method for modeling EVs and Charge Sustaining (CS) hybrids. EREVs have distinct Charge Depleting (CD) and CS modes, so they can also be modeled by coupling the results from the EV powertrain to a desired CS HEV powertrain. The EV model with the properly sized Energy Storage System (ESS) will become the CD mode of the EREV. PHEVs can be more difficult to model if they blend battery and fuel energy. Blended PHEVs do not have a distinct CD mode. Consequently, they are difficult to model using this method and will not be considered in the scope of this thesis.

\subsubsection{Hybrid Architecture Survey}

There are currently many different architecture designs for hybrid electric vehicles. The more common designs can normally be classified as Series, Parallel, or Series-Parallel, which are shown in Figure 7, Figure 8, and Figure 9, respectively. Each design differs in its control scheme, the size and number of batteries or Energy Storage Systems (ESS), and inclusion of other components. Regardless of the architecture selected, all hybrid electric vehicles are designed to have lower fuel consumption and lower emissions when compared to conventional vehicles powered only by internal combustion engines. 


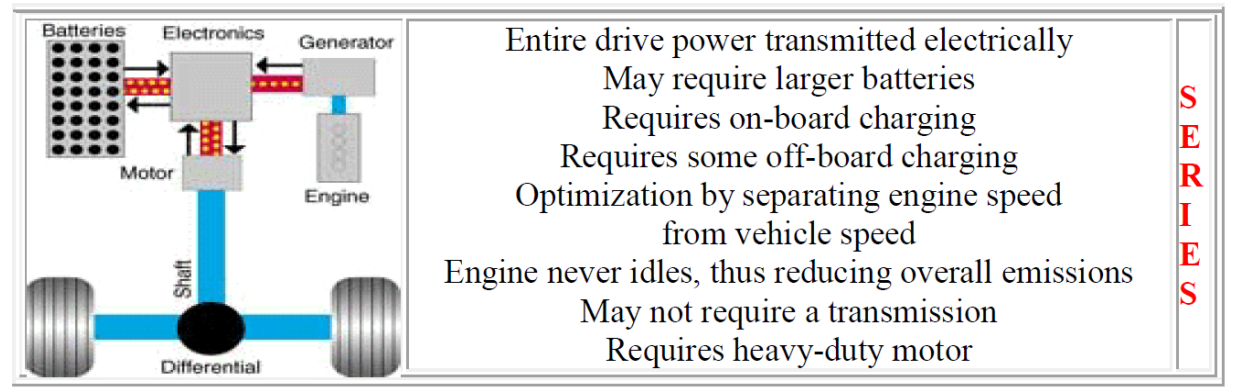

Figure 7: Series hybrid architecture design and characteristics (public domain [9])

\begin{tabular}{|c|c|c|}
\hline Batteries & Electric motor and engine both coupled & P \\
\hline
\end{tabular}

Figure 8: Parallel hybrid architecture design and characteristics (public domain [9])

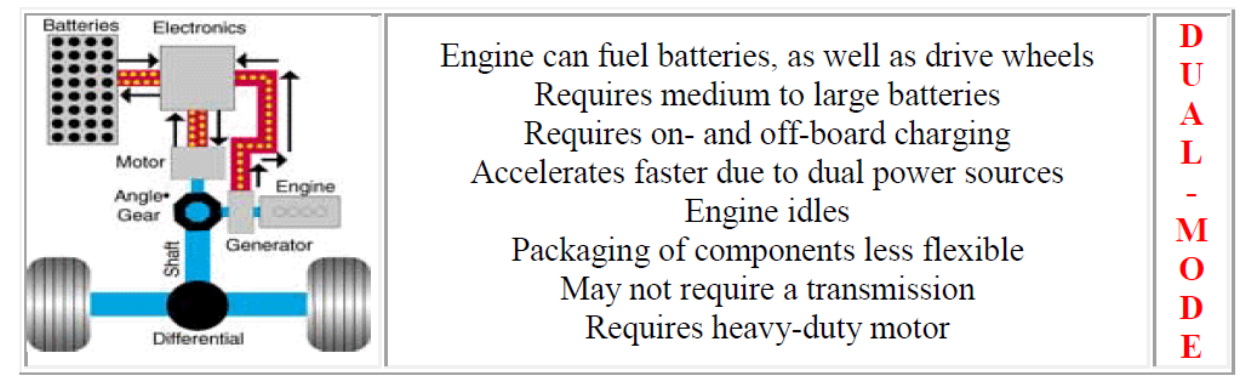

Figure 9: Series-Parallel hybrid architecture design and characteristics (public domain [9])

The main advantage of a Series hybrid architecture is that vehicle speed is independent of engine speed and only the electric motor propels the vehicle. The engine, with help from the generator, transfers energy to the batteries or motor and does not need to drastically speed up or slow down as the load varies. Consequently, the engine never idles and can run at or near its highest efficiency, greatly improving fuel economy and reducing emissions, especially during city driving. While this system configuration greatly improves engine efficiency, overall system efficiency is hindered because of the lengthy energy path from engine to wheels. Additionally, the electric motor is the only component connected directly to the drive axle, so a Series design requires a large motor for performance. For sustained highway gradeability, the generator must have a high continuous power rating [9].

In a Parallel hybrid architecture, the electric drive motor and engine can power the vehicle simultaneously. Therefore, it is possible to downsize the engine which leads to increased fuel economy. When compared to a Series architecture, an equivalent Parallel architecture will be less efficient for city driving, but more efficient for highway driving due to the direct engine torque path to the wheels. Additionally, all power from both propulsion sources can be utilized for acceleration and performance [9]. 
Series-Parallel and powersplit hybrid architectures split engine power between two different power paths. One path sends power to the generator to produce electricity and the other sends power to the mechanical gear system to drive the vehicle. Like a Parallel architecture, a Series-Parallel architecture can have high acceleration performance when the battery is used with the engine for extra power. Unlike a Parallel architecture, however, a Series-Parallel architecture has the capability to energize the HV system via a series mode. Series-Parallel also has the best overall efficiency, maintaining a good balance between city and highway driving. Due to the large number of components involved with this design, packaging everything within the car can be difficult [9]. A Series-Parallel hybrid architecture allows for quick transitions between these electric-only, powersplit, Series, and Parallel modes [10].

In summary, a Series architecture is relatively simple and improves average powertrain efficiency but all energy most flow in a series path. Because of this path, the engine and generator cannot contribute to acceleration performance (given a sufficiently powerful battery). A Parallel hybrid can sum all power to the wheels and is generally more efficient during highway driving but less efficient during city driving compared to the series design. A Series-Parallel architecture offers the best of both worlds at the cost of additional system complexity and mass. Both Series and Parallel powertrains are included in the methodology laid out in this thesis. Additional variability is possible with Parallel hybrids by varying the position of the electric motor. While the position of the electric motor is very important for integration, it is relatively unimportant for powertrain modeling and all versions of a Parallel hybrid are very similar for modeling purposes.

\subsection{HEVT Participation in EcoCAR: The NeXt Challenge}

\subsubsection{Overview}

The Hybrid Electric Vehicle Team of Virginia Tech (HEVT) participated in the 2009 - 2011 EcoCAR: The NeXt Challenge Advanced Vehicle Technology Competition (AVTC) series organized by Argonne National Lab (ANL), and sponsored by General Motors Corporation (GM), and the U.S. Department of Energy (DOE). Following GM's Vehicle Development Process (VDP), HEVT established team goals that meet or exceed the competition requirements for EcoCAR in the design of a plug-in extended range hybrid electric vehicle. The competition requires participating teams to re-engineer a stock crossover utility vehicle donated by GM. The result of this design process is an EREV that uses grid electric energy and E85 fuel for propulsion.

\subsubsection{Vehicle Modeling and Fuel Selection}

The first step in selecting the hybrid architecture was the selection of an energy source for the vehicle. The selection was based upon basic models constructed with Powertrain Systems Analysis Toolkit (PSAT), developed by Argonne National Lab (ANL) [11]. These models calculated the energy usage of the stock vehicle and compared the effects of various fuel sources on the energy used by the vehicle. In short, the models predict the overall vehicle efficiency specific to a given fuel, and then quantify the amount of petroleum energy that was expended by the vehicle for the given fuel. Similarly, vehicle emissions for a given fuel were also quantified based on fuel-specific vehicle energy consumption. To consider the total life cycle of the fuel, the analysis is performed on a well-to-wheels basis. To consider the upstream well-to-pump (WTP) factors for GHG and criteria emissions as well as petroleum energy use, ANL's GREET model $[12,13]$ is used to calculate total Well-toPump (WTP) energy, GHG emissions and criteria emissions released for the fuel. EcoCAR teams are given WTP numbers specific to North America for different candidate fuels: gasoline (E10), ethanol (E85), biodiesel (B20), electricity and hydrogen $(\mathrm{H} 2)$. The last factor that affected the selection of fuel was the practicality of the components that would be used to convert fuel to kinetic energy. 
Considering the factors outlined above with team goals in mind, HEVT selected electricity and E85 as its competition fuels [14]. Using electricity as a fuel enables an all-electric mode, which is a consumer delighter and displaces a substantial amount of petroleum energy. Though electricity has more WTW GHG and criteria emissions per unit energy than petroleum based fuels, an electric powertrain is substantially more efficient and uses less energy. Thus, the utilization of electricity in a vehicle also has the potential to reduce WTW GHG and criteria emissions. HEVT selected E85 as the range-extending fuel primarily for its ability to displace petroleum energy, but it also offers advantages over B20 and E10 in WTW GHG emissions. An additional consideration was the integration challenges associated with each fuel. The integration challenges of a fuel cell system are daunting and there is currently no established refueling infrastructure to support a fuel cell vehicle (either on the Virginia Tech campus, or nationwide). Likewise, the complexity of designing an aftermarket exhaust treatment device for a small diesel engine to meet tailpipe emissions was viewed as too complex of a task for the timeline given for student vehicle development and integration. With the combination of grid electricity and E85, HEVT predicts a $90 \%$ reduction in petroleum energy use and a 30\% reduction in WTW GHG emissions compared to the stock vehicle. This number will improve as the US energy grid becomes more renewable and cellulosic E85 becomes available. For a more detailed discussion on the fuel selection for the VTREX see [14].

\subsubsection{Component Sizing and Selection}

To meet the goal of a large electric-only range, HEVT chose to design and build a $20 \mathrm{kWh}$ ESS with materials and modules donated by A123 Systems. This was the larger of two Lithium Iron Phosphate battery packs offered by $\mathrm{A} 123$ to the teams in the EcoCAR Challenge. The pack has a nominal voltage of $352 \mathrm{~V}$ and is rated at a peak discharge rate of $600 \mathrm{~A}$ and a peak charging rate of $300 \mathrm{~A}$. The main traction motor used on the vehicle is a UQM $125 \mathrm{~kW}$ liquid-cooled permanent magnet motor, which is paired with a BorgWarner 3103 single-speed transmission with a gear reduction of 7.17:1. The motor has a top powered speed of 8,000 RPM, which gives a top vehicle speed of $89 \mathrm{mph}$. The ESS is charged using an on-board Brusa $3.3 \mathrm{~kW}$ charger that can operate on either 120 or $240 \mathrm{~V}$. A complete recharge of $93 \%$ State of Charge (SOC) swing (100\% - 7\%) takes 6 hours from a $240 \mathrm{~V} 20$ A household circuit. Figure 10 shows a high-level schematic of the components used in the vehicle. 


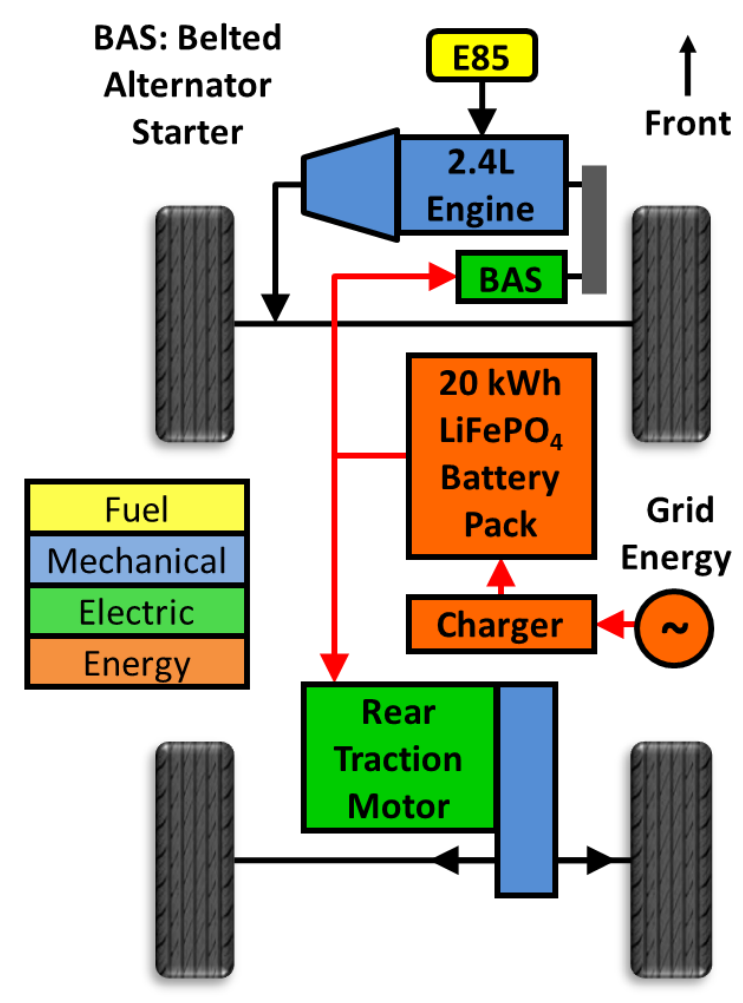

Figure 10: EcoCAR VT REX E85 Split Parallel EREV powertrain architecture

The stock engine is replaced with a GM 2009 LE9 (4-cylinder 2.4 L) FlexFuel engine. This FlexFuel engine enables the use of E85, which was chosen to meet the goal of reducing PEU. The engine will act as the primary source of propulsion in CS mode. Belted to the engine is a custom Kollmorgen $9 \mathrm{~kW}$ (peak) liquid-cooled AC induction motor that serves as a belted alternator starter (BAS). This motor replaces the alternator and starter and improves vehicle efficiency by enabling engine idle start/stop, and engine load leveling which increases the average operating efficiency of the engine. The engine is coupled with the GM ME7 4T45 4-speed automatic transmission from GM's BAS hybrid system. The transmission has an auxiliary fluid pump that keeps transmission lines pressurized while the engine is off to allow smooth, quick takeoff as soon as the engine is restarted. Since the BAS motor provides load leveling, which increases the operating efficiency of the engine, and the rear traction motor (RTM) provides electric launch, the efficiency benefits offered by a 6-speed transmission were not as significant. Thus the team instead chose the 4-speed transmission to ease the integration challenges and reduce weight. Table 1 shows an overview of the components on board the $\mathrm{VT}_{\mathrm{REX}}$. 
Table 1: $\mathrm{VT}_{\mathrm{REX}}$ Component Specifications

\begin{tabular}{|l|l|l|}
\hline Architecture & \multicolumn{3}{|l|}{ E85 Range Extended Split Parallel Architecture } \\
\hline Components & Size & Type \\
\hline Engine & $130 \mathrm{~kW}$ peak & $\begin{array}{l}\text { GM LE9 2.4 L ECOTEC VVT } \\
\text { DOHC 16V I4 FlexFuel SI }\end{array}$ \\
\hline Transmission & - & $\begin{array}{l}\text { ME7 4T45 hybrid 4-speed } \\
\text { automatic FWD }\end{array}$ \\
\hline BAS & $9 \mathrm{~kW}$ peak & $\begin{array}{l}\text { Kollmorgen custom AC induction } \\
\text { motor }\end{array}$ \\
\hline RTM & $125 \mathrm{~kW}$ peak & $\begin{array}{l}\text { UQM liquid cooled permanent } \\
\text { magnet motor }\end{array}$ \\
\hline $\begin{array}{l}\text { RTM } \\
\text { Transaxle }\end{array}$ & - & $\begin{array}{l}\text { BorgWarner 3103, gear } \\
\text { reduction: 7.17:1 }\end{array}$ \\
\hline ESS & $352 \mathrm{~V}$ & $\begin{array}{l}\text { A123 Systems custom built } \\
\text { prismatic pack, 18.7 kWh useable }\end{array}$ \\
\hline 12 V Supply & $1 \mathrm{~kW}$ cont. & $\begin{array}{l}\text { Delphi DC/DC Converter, from } \\
352 \text { V to 13.8 V }\end{array}$ \\
\hline A/C System & $10 \mathrm{~kW}$ & $\begin{array}{l}\text { High voltage electric drive, high } \\
\text { efficiency, variable speed }\end{array}$ \\
\hline ESS Charger & $3.3 \mathrm{~kW}$ cont. & $\begin{array}{l}\text { Brusa 120/240 V 50/60 Hz AC, } \\
\text { integrated on-board }\end{array}$ \\
\hline $\begin{array}{l}\text { Supervisory } \\
\text { Controller }\end{array}$ & - & $\begin{array}{l}\text { NI CompactRIO with field } \\
\text { programmable gate array (FPGA) }\end{array}$ \\
\hline
\end{tabular}

Since there are large amounts of time when the vehicle operates with the engine off, an electric High Voltage $(\mathrm{HV})$ air conditioning $(\mathrm{A} / \mathrm{C})$ compressor is installed on the vehicle to keep consumers comfortable regardless of propulsion mode. A Delphi DC/DC converter provides power for the $12 \mathrm{~V}$ system directly from the HV A123 battery pack. As expected, this converter is a necessity for CD mode, but it is also necessary for CS mode because there is not a traditional $13.8 \mathrm{~V}$ alternator on the engine.

\subsubsection{Competition Results and Application to This Work}

In year 3 of EcoCAR, the Virginia Tech vehicle, affectionately known as the $\mathrm{VT}_{\text {REX }}$ (Virginia Tech Extended Range X-over) placed first overall. The EcoCAR year 3 competition was held at GM's Milford Proving Grounds in Milford, MI. Each EcoCAR school was required to run various dynamic testing events to evaluate the vehicle's acceleration, braking, lateral handling, drive quality, towing capability, emissions and energy consumption. The $\mathrm{VT}_{\mathrm{REX}}$ completed all events and scored exceptionally in most of them. The team placed highly in every category and won several competition events. The results from year 3 competition are summarized in Table 2 alongside the final VTS predictions. The vehicle design achieved an SAE J1711 utility factor corrected fuel consumption of $2.9 \mathrm{~L}(\mathrm{ge}) / 100 \mathrm{~km}$ (82 mpgge) with an all-electric range of $87 \mathrm{~km}$ (54 miles) [15]. Using E85 fuel (corn-based in North America for the 2015 timeframe), the well-to-wheels petroleum energy use and greenhouse gas emissions are reduced by $91 \%$ and $18 \%$ respectively when compared to the stock 4-cylinder gasoline-fueled vehicle. The $\mathrm{VT}_{\mathrm{REX}}$ and the results of the EcoCAR year 3 competition are discussed in greater detail in a 2012 SAE paper written by this author et al. [16] and in a 2012 EVS paper also written by this author et al. [17]. 
Table 2: EcoCAR Year 3 Competition Results

\begin{tabular}{|l|l|l|}
\hline HEVT VTS & Prediction & Y3 Actual \\
\hline EV Range & 50 miles $^{*}$ & 54 miles* $^{*}$ \\
\hline $\begin{array}{l}\text { Fuel Economy: } \\
\text { CAFE Unadjusted, } \\
\text { Combined, UF weighted }\end{array}$ & $90 \mathrm{mpgge}{ }^{*}$ & $82 \mathrm{mpgge}^{*}$ \\
\cline { 2 - 3 } & $2.6 \mathrm{I}(\mathrm{ge}) / 100 \mathrm{~km}^{*}$ & $2.9 \mathrm{I}(\mathrm{ge}) / 100 \mathrm{~km}^{*}$ \\
\hline CS Fuel Economy & $30 \mathrm{mpgge}$ & $24 \mathrm{mpgge}^{*}$ \\
\cline { 2 - 3 } & $7.8 \mathrm{I}(\mathrm{ge}) / 100 \mathrm{~km}^{*}$ & $9.8 \mathrm{I}(\mathrm{ge}) / 100 \mathrm{~km}^{*}$ \\
\hline $0-60 \mathrm{mph}$ Acceleration & $7.3 \mathrm{~s}$ & $6.7 \mathrm{~s}$ \\
\hline $50-70 \mathrm{mph}$ Acceleration & $5.0 \mathrm{~s}$ & $3.12 \mathrm{~s}$ \\
\hline $60-0 \mathrm{mph}$ Braking & $43 \mathrm{~m}$ & $40 \mathrm{~m}$ \\
\hline Curb Weight & $2138 \mathrm{~kg}$ & $2123 \mathrm{~kg}$ \\
\hline Total Range & $205 \mathrm{mi}$ & $204 \mathrm{mi}$ \\
\hline
\end{tabular}

The most important event conducted during EcoCAR competition is the emissions and energy consumption (E\&EC) event. During this event, the vehicle is driven on road in a manner that approximates UF weighted CAFE fuel economy. Three schedules, A, B and C, were created at lengths of 20, 40 and 100 miles respectively to approximate the J1711 method for calculating UF weighted fuel consumption. The development of the UF approximation of the E\&EC cycles is discussed in Duoba's 2012 SAE paper [18]. Each schedule contained elements of city and highway driving that approximated the UDDS and HwFET cycles, respectively. The drive trace was much simplified compared to the actual certification cycles due to the need to perform the test on road. The development of these cycles and their impact on fuel economy is discussed in an EVS paper written by this author and Doug Nelson et al. [19]. This on-road approximation proved to be a reasonable approximation of the individual UDDS and HwFET cycles, but an excellent approximation of the overall CAFE weighting. The data from EcoCAR year 3 E\&EC testing will be used to calibrate and validate the tool created by this thesis.

\subsection{HEVT Participation in EcoCAR 2: Plugging in to the Future}

HEVT is now participating in the 2012-2014 EcoCAR 2: Plugging in to the Future competition. EcoCAR 2 is the latest competition in a rich 26 year history of DOE Advanced Vehicle Technology Competitions. EcoCAR 2 shares many similarities with its predecessor, EcoCAR. EcoCAR 2 is sponsored by GM and DOE. The goals of the competition are to reduce WTW PEU, WTW GHGs and WTW criteria emissions while maintaining vehicle performance, consumer acceptability and safety. The competition follows the three-year VDP that leads teams to design, build and the refine an advanced technology vehicle based on a 2013 Chevy Malibu. There are 15 teams in EcoCAR 2 across the United States and Canada including 9 universities that were also in EcoCAR. The competition is currently coming to the close of its first year, the design year.

In this year of the competition, each team must first select their competition fuel and powertrain architecture, develop component integration schemes, and develop vehicle supervisory control code among other important and detailed tasks. The team first considered available fuels on a WTW basis and then examined three distinct and unique architectures: a BEV, a Series EREV, and an Series-Parallel EREV. Each architecture was modeled in Autonomie and the advantages and challenges were examined and considered. The results of the analysis were then entered into a design matrix to evaluate the effectiveness of each architecture in the context of the EcoCAR 2 competition, and the best powertrain architecture was selected. The powertrain selected as a result of this process was an E85 Series-Parallel 
EREV shown in Figure 11. For a deeper discussion of the design process used by HEVT in the first year of EcoCAR 2, see the 2012 SAE paper written by this author et al. [20].

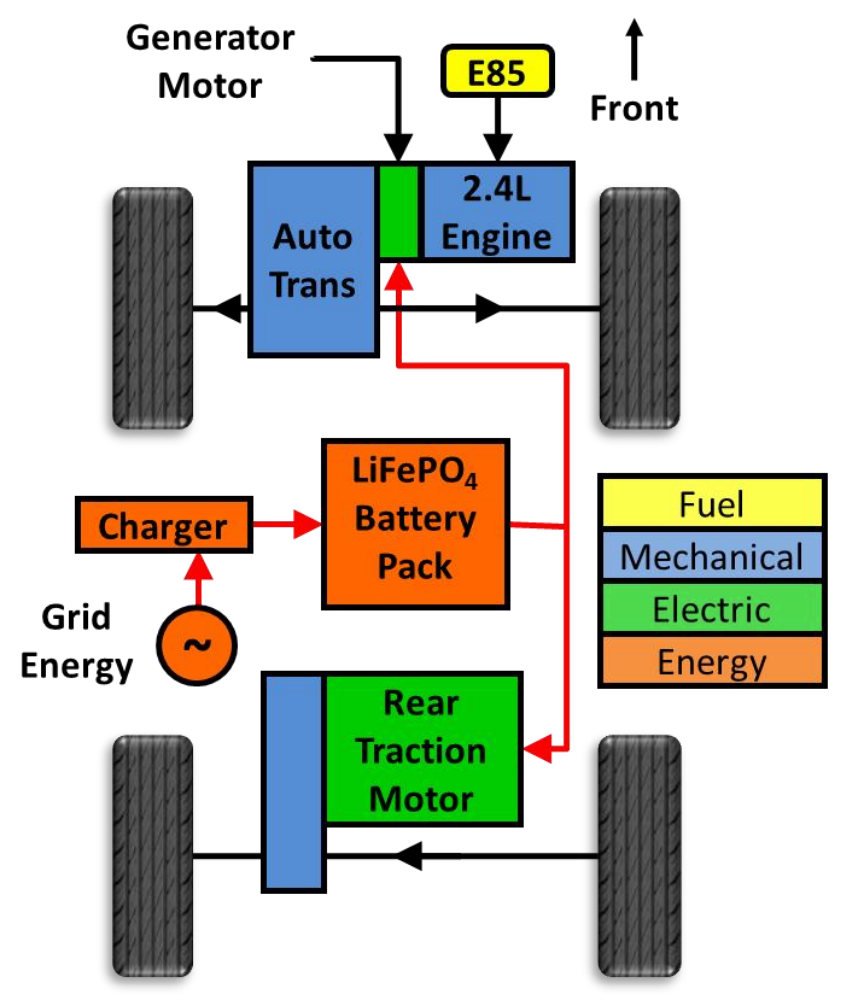

Figure 11: EcoCAR 2 E85 Series-Parallel EREV powertrain architecture

The design process used in year 1 of EcoCAR 2 is an ideal application of VTool. Per competition requirements, the team compared 3 distinct and unique powertrain architectures in a very short timeframe. The goal of this process was to choose the powertrain that was the best compromise between energy consumption, emissions, performance, consumer acceptability and feasibility (the potential for a student design team to successfully implement the architecture). Autonomie was used to perform the powertrain modeling, but was not the ideal tool because of the complexity required in the models. The goal of the exercise was to judge the potential of each powertrain. As such, the accuracy of the models was not paramount. In fact, this author doubts that it is possible to achieve a high level of confidence in the autonomie models in the timeframe allowed. Therefore, a more appropriate modeling tool would have required less time to set up while still producing reasonable results, which is exactly what VTool is designed to do. Thus, VTool is the perfect modeling tool for the architecture selection process.

\subsection{VTool Goals and Objectives}

The key objective of this thesis is to develop and present a methodology for predicting the drive cycle energy consumption of a vehicle powertrain with the main goal of developing an understanding of the system being modeled. This methodology has also been implemented as a tool, called VTool, to demonstrate the functionality of the method. There are already various powertrain modeling tools available, many of which have the capability to produce results very accurately. Hence, the purpose of VTool is not high-fidelity modeling, but rather to develop a deeper comprehension of the system. The following section discusses the goals and objectives of this thesis, which are summarized in Table 4. 
VTool separately calculates propel and brake tractive energy required at the wheels based on a set of "Sovran" coefficients [21] that characterize a given drive cycle and the vehicle glider properties. A glider vehicle is a vehicle without a powertrain. Hence, vehicle glider properties describe a vehicle independent of the powertrain and characterize several aspects of the vehicle: mass, frontal area, coefficient of drag, coefficient of rolling resistance, etc. Propel and brake energy are separated to properly account for brake energy recaptured through regenerative braking. VTool will then apply component efficiencies up the powertrain for both propel and brake cases to find battery or fuel energy required to complete the cycle. If appropriate, VTool will also calculate the energy required to recharge the battery. The tool can perform these calculations for the following: an EV, a CS Series Hybrid, and a CS Parallel Hybrid. To model a PHEV, the results from the EV model are coupled with the results from one of the CS powertrains and SAE J1711 UF weighted results can be calculated. Results from all drive cycles are all available simultaneously, so comparing between cycles is easy and VTool is able to calculate cycle weightings such as CAFE or EcoCAR 2 4-cycle. The EcoCAR 2 4-cycle weighting is an approximation of EPA 5cycle weighting that can be performed through on road testing during the week-long EcoCAR 2 competition testing. This cycle weighting is weighted as follows: $29 \% 505,12 \%$ HwFET, $14 \%$ US06 City, 45\% US06 Highway. Table 3 summarizes the characteristics of the EcoCAR 1 CAFE weighting and EcoCAR 2 4-cycle weighting. With WTW PEU and GHG information available for several fuels including grid electricity, the WTW effects of a given architecture can be calculated and compared against a different fuel or a different architecture.

Table 3: Summary of EcoCAR 1 CAFE and EcoCAR 2 4-cycle weightings

\begin{tabular}{|c|c|c|c|c|c|}
\hline Cycle & $\begin{array}{l}\text { Time (Idle } \\
\text { Time) (s) }\end{array}$ & $\begin{array}{c}\text { Weight } \\
(\%)\end{array}$ & $\begin{array}{l}\text { Max Speed } \\
\text { (mph) }\end{array}$ & $\begin{array}{c}\text { Avg Run } \\
\text { Speed (mph) }\end{array}$ & Peak Accel $\left(\mathrm{m} / \mathrm{s}^{2}\right)$ \\
\hline UDDS & $1369(241)$ & $55 \%$ & 56.7 & 23.8 & 1.48 \\
\hline HwFET & $765(4)$ & $45 \%$ & 59.9 & 48.5 & 1.43 \\
\hline \multicolumn{2}{|c|}{ EC1 CAFE weighting: } & $100 \%$ & 59.9 & 30.9 & 1.48 \\
\hline 505 & $505(94)$ & $67 \%$ & 56.7 & 31.5 & 1.48 \\
\hline US06 City & $231(27)$ & $33 \%$ & 70.7 & 31.3 & 3.76 \\
\hline \multicolumn{2}{|c|}{ EC2 4-cycle City weighting: } & $43 \%$ & 70.7 & 31.4 & 3.76 \\
\hline HwFET & $765(4)$ & $22 \%$ & 59.9 & 48.5 & 1.43 \\
\hline US06 Highway & $365(8)$ & $78 \%$ & 80.3 & 62.9 & 3.08 \\
\hline \multicolumn{2}{|c|}{ EC2 4-cycle Highway weighting: } & $57 \%$ & 80.3 & 59.0 & 3.08 \\
\hline \multicolumn{2}{|c|}{ EC2 4-cycle weighting: } & $100 \%$ & 80.3 & 42.8 & 3.76 \\
\hline
\end{tabular}

VTool also has several tunable parameters including glider properties and component efficiencies that allow the user maximum flexibility in defining the vehicle powertrain. These features provide the adaptability to perform 'What if?' scenarios to examine the effects of certain aspects of the powertrain, such as regenerative braking. These parameters also give the freedom to study the effect and sensitivity of component efficiencies on the overall system. Having a realistic sense of component efficiencies, the user can also develop an understanding of the upper limits of fuel economy and system efficiency. VTool even gives enough freedom to set component efficiencies at unrealistically high levels to study the results. For more complex hybrid powertrains, such as a Series or Parallel HEV, the tradeoffs of basic energy management strategies can be examined before it is necessary to develop a hybrid control strategy. For example, the effect of recirculating energy in a Series hybrid vehicle may be examined and compared to a similar process for a Parallel vehicle. This process would certainly also nurture an understanding of the advantages and disadvantages of each powertrain. 
While VTool is designed primarily to enhance the learning process, it is still capable of producing results with a reasonable level of accuracy. It is a simple model, but it can quickly and easily be used as a reality check against other models or test data to validate efficiency claims as reasonable. Because of this and it's many tunable parameters, it is exceptionally well suited for judging the potential of various powertrains for comparison. This capability makes it ideal for use as a design tool for powertrain architecture selection as well as component sizing and selection.

Table 4: VTool Goals and Objectives

(1) Provide a method packaged into a tool that can be used to develop a deeper understanding of the function of a vehicle powertrain.

(2) Develop a deeper understanding of:

a. Limits of fuel economy in a powertrain and the potential of a given architecture

b. Effect and sensitivity of component losses and efficiencies in the powertrain

c. Proper accounting of regenerative energy capture and utilization

d. Various tradeoffs involved in the powertrain design and component sizing process

e. The effects and tradeoffs of some basic energy management strategies for Parallel and Series vehicles

f. The effect of drive cycles on powertrain energy consumption and efficiency

(3) Provide a simple model that can be used as a reality check for other models or efficiency claims by doing the following:

a. Calculate tractive energy at the wheels for propel, brake and idle cases based on a set of drive cycle coefficients and vehicle glider parameters

b. Calculate the energy consumption and losses for each component in the powertrain, both before and after charging

c. Offer several tunable parameters (like component efficiencies) that can be used to tune the model or to examine the effect of the parameter on the model

d. Do the above for various different drive cycles

(4) Summarize results and calculate CAFE, EcoCAR 2 4-Cycle, and 5-Cycle weightings

(5) Calculate WTW effects of a given architecture for given cycle or cycle weighting. 


\section{Literature Review}

\subsection{Sovran}

\subsubsection{A Contribution to Understanding Automotive Fuel Economy and Its Limits [21]}

In 2003, Sovran published a paper that presents the physics of vehicle fuel economy in an accurate concise and understandable form. One of the key goals is to outline a method by which fuel consumption may be systematically calculated in such a way that the factors contributing to fuel consumption may be understood. The paper is specifically focused on predicting UDDS, HwFET, and ultimately CAFE fuel economy. This thesis will extend the method to any drive cycle. Sovran uses his work to show that improvements in individual component efficiencies may not significantly impact overall vehicle fuel consumption if the component does not process much energy over the course of operation.

This work starts by defining the forces acting on a vehicle at any given speed. These forces are broken down into road load forces and inertial forces. Road load forces are rolling resistance and aerodynamic drag. In this paper, Sovran uses only a single coefficient to model rolling resistance. In an earlier SAE paper published in 1981 [22], Sovran also used a velocity-proportional coefficient. Road load forces are non-conservative and must always be overcome. The vehicle inertial force, however, is conservative and can be recovered if possible. This set of three forces defines the tractive force required to propel the vehicle. Sovran then segregates all driving scenarios into three cases: propel $\left(F_{t r}>0\right)$, brake $\left(F_{t r}<0\right)$, and idle $\left(F_{\mathrm{tr}}=0\right)$. Each case has its own contribution to fuel consumption. For a conventional vehicle, the brake and idle cases are simple, but for a HEV or EV the brake case can become more complicated with the introduction of regenerative braking. This thesis will fully develop the brake case for EVs and HEVs. For an EV, there is now a distinct powertrain active during the brake case that should be accounted for. Thus, the energy consumption equation for the brake case should be developed as fully as it already has been for the propel case. For an HEV, there is the additional complexity of maintaining charge balance: all energy captured through regen braking must be expended from the battery since the energy source of an HEV is the fuel tank.

The next step in this paper is to define a set of coefficients specific to a drive cycle that can be used in conjunction with vehicle glider properties to find the tractive energy required to complete a prescribed drive cycle. These Sovran coefficients characterize the drive cycle, while the vehicle glider properties characterize the vehicle of interest. The paper goes on to show that the Sovran coefficients are a weak function of vehicle glider properties. Hence, the same set of parameters can be used for varying sets of glider properties within reason. Knowing tractive energy at the wheels, powertrain components for a conventional vehicle are added. Component efficiencies are applied up the drivetrain to find the fuel energy required to meet the tractive energy requirements of a given cycle. These component efficiencies are applied as energy-weighted cycle averages. This is a crucial concept for Sovran's paper as well as this paper because it allows for a simple and direct calculation of the energy flow through a component.

Sovran mainly focuses on conventional vehicle powertrains but also includes a brief discussion of the benefits of HEVs and the ways that they can improve vehicle fuel consumption. The goal of the Sovran paper is the same as this thesis: explain energy consumption of the vehicle in a way that allows for complete understanding of the powertrain losses and powertrain efficiencies. Achieving this goal in a way that allows for discussion leading to improvements in vehicle design is also important. As previously mentioned, this thesis will also fully develop the powertrain efficiency for the brake case and define a powertrain efficiency for the brake case. VTool will extend the method to EVs and HEVs across several drive cycles. The tool will also have the capability to calculate energy consumption for PHEVs. 


\subsubsection{Quantifying the Potential Impacts of Regenerative Braking on a Vehicle's Tractive-Fuel Consumption for the U.S., European, and Japanese Driving Schedules [23]}

The 2006 Sovran paper expands on his 2003 work to include the effects of regenerative braking and highlight its potential for reducing fuel consumption. While the propel case is meticulously laid out to explicitly consider all losses in the propel powertrain, the brake case takes a shortcut by applying a single efficiency term to brake tractive energy. This term accounts for the losses required to capture brake energy, store it in a hybrid battery pack and then reuse it at some future point in time. The energy could be used for propulsion, or to supply accessory loads during idle or various other ways depending on the specific vehicle system. While this single term is an acceptable simplification and will still model regen braking relatively accurately, it partially undercuts the goal of the Sovran method, which is to understand the system. It is, however, a step in the right direction. This thesis takes into account regen braking but does so by developing the energy consumption for the brake case to the same level of detail as the propel case. Sovran also demonstrates the application of the Sovran coefficients to additional drive cycles such as European and Japanese cycles. VTool will further expand on this to include various drive cycles for design flexibility.

\subsection{Gantt}

\subsubsection{Energy Losses for Propelling and Braking an Electric Vehicle [24]}

This is Gantt's thesis and includes all of the concepts covered in the above paper but in much greater depth. In addition, it also defines two additional Sovran parameters: one for the effects of grade and another to include a velocity-proportional rolling resistance term. Primarily focused on an EV, the energy flow path of the powertrain is carefully developed for both propel and regen brake conditions with accessory load carefully accounted for. Energy-weighted cycle average efficiencies are defined for the battery, motor and driveline, and simple models for calculating these average efficiencies are outlined for the battery and motor. The paper also provides guidance for predicting the losses in the charging process. The paper then validates the proposed model with real dynamometer test data gather at EPA's National Vehicle Fuels and Emissions Lab in Ann Arbor, Michigan. This data, as concede by Gantt, was of questionable quality due to noise in the data stream and inaccuracies involved in setting up the road load of the dynamometer.

Gantt's work is crucial to this author as this paper largely picks up where Gantt finished. Gantt cites as future work to expand the EV model to include more complex hybrid powertrains, to include more drive cycles and to validate the method with more accurate test data. This paper will accomplish all of these things. The methodology used, however, will differ slightly. Gantt used a $1 \mathrm{~Hz}$ spreadsheet model to predict energy consumption. While he did derive the Sovran coefficients for UDDS, HwFET and US06 cycles, he did not use them to calculate tractive energy. This author will rely solely on the Sovran coefficients to calculate tractive energy. Hence, the model used by Gantt is capable of deriving some component efficiencies, while VTool is not. It will instead leverage component efficiencies as tunable parameters and rely on other tools to establish reasonable energy-weighted cycle average component efficiencies.

\subsubsection{Regenerative Brake Energy Analysis for the VT ${ }_{\mathrm{REX}}$ Plug-in Hybrid Electric Vehicle [25]}

This work by Gantt is largely based upon the Sovran method and reuses Sovran's concepts of tractive forces, propel, brake and idle cases, and energy-weighted drive cycle average efficiencies. Instead of a conventional vehicle, however, the paper analyzes the electric-only mode of a PHEV, the Virginia Tech entry for the 2010-2011 EcoCAR Challenge. Like this thesis, Gantt's paper develops the energy flow for both the propel and brake cases, defining separate component efficiencies for both energy flow directions. Gantt's paper shows that for an EV the propel energy out of the battery and the brake energy into the battery can be summed to find total tractive energy required to complete the cycle. Accessory 
load is carefully accounted for in each case and will increase the amount of propel energy required out of the battery while reducing the amount of brake energy stored in the battery. An additional case (charging) is also defined to account for the losses required to recharge the battery. The effects of regenerative braking are specifically discussed including the effects of real-world application in a test vehicle. One conclusion reached is that a regen brake fraction parameter must be established to account for brake energy dissipated by the friction brakes of the vehicle.

\subsection{Hochgraf, Douba: What if the Prius Wasn't a Hybrid? What if the Corolla Were? An Analysis Based on Vehicle Limited Fuel Consumption and Powertrain and Braking Efficiency [26]}

In 2010, Hochgraf and Douba presented a paper on vehicle limited fuel consumption and powertrain net efficiency. Hochgraf takes into account powertrain and non-powertrain related improvements made to vehicles. He also examines the impact that these improvements have on the combined propel and brake efficiency of the powertrain. Hochgraf defines powertrain propel and brake efficiency as the vehicle limited fuel consumption over the vehicle fuel consumption. Vehicle limited fuel consumption is defined as the net energy required at the wheels to complete a drive cycle, or the road load energy. This concept is similar to the method Sovran uses to calculate tractive forces at the wheels. Rather than using the vehicle specific parameters, however, Hochgraf uses the coast down data provided by EPA for their dynamometer target coefficients (A, B, and C). Hochgraf's approach assumes $100 \%$ efficiency for the regenerative braking powertrain, meaning that all energy spent to accelerate the vehicle was recaptured and used to reduce fuel consumption.

The concept of vehicle limited fuel consumption provides valuable insight into understanding the limits of fuel economy. As defined by Hochgraf, vehicle limited fuel consumption is essentially the road load energy required to propel the vehicle and is equivalent to a $100 \%$ efficient vehicle. Thus, this parameter defines the absolute best fuel consumption a given vehicle is capable of achieving for a given cycle. VTool also considers the concepts of powertrain propel and braking efficiencies, but will separate the two cases as Sovran suggests. As previously mentioned, vehicle operation will be broken down into propel, brake and idle cases. The energy consumption for the propel and brake cases will be kept distinct instead of looking only at net energy consumption. In this way, the user will be able to examine the losses along the energy flow path and understand why less than $100 \%$ of brake energy can be captured and reused.

\subsection{Yanni: Impact and Sensitivity of Vehicle Design Parameters on Fuel Economy Estimates [27]}

Similar to Sovran, Yanni has developed a set of equations that can be used to predict vehicle energy consumption without the need for a dynamic second-by-second model. PSAT is cited as a useful tool for vehicle fuel economy analysis after the vehicle powertrain has been designed. However, PSAT [11] can be quite overwhelming in the concept selection stage when the designer may be examining several component or architecture configuration variations. In his paper, Yanni attempts to create a modeling tool better suited for powertrain design. Like Sovran, he is able to show the effect of regen braking and provide a breakdown of force (and ultimately energy) that is attributed to rolling resistance, aerodynamic and inertia. He does, however, makes some assumptions to simplify his model including a propel powertrain efficiency of $50 \%$. He also assumes that propel and brake efficiencies are the same. For some powertrain components, like a transmission, this is a reasonable assumption. For others, like motors and some battery chemistries, this is rarely true.

The method laid out in this paper shares many of the same goals as Yanni's method but offers some additional functionality. This author's method will be capable of applying different component efficiencies for propel and brake cases and will also be applicable to Series and Parallel HEV architectures as well as 
EV and PHEV powertrains. The user will also be able to examine the effect of changes in component efficiencies on overall powertrain efficiency. This promotes comprehension and understanding of the powertrain as a system, which is one of the key goals of VTool.

\subsection{Duoba: Calculating Results and Performance Parameters for PHEVs [28]}

As previously stated, VTool has been validated in part with data from on-road testing during EcoCAR year 3 competition at GM's Milford Proving Grounds. The on-road testing was designed to emulate Utility Factor (UF) weighted CAFE testing, which is 55\% UDDS and 45\% HwFET. In his paper, Douba explains the five drive cycles used for EPA emissions certification: UDDS, HwFET, US06, Cold FTP and SC03. UDDS and HwFET cycles are the classic city and highway cycles that have traditionally been used for fuel economy certification. The first 505 seconds of the UDDS are often used as a separate cycle to capture cold start dynamics of an engine. The US06 cycle has both city and highway elements and features higher speeds and accelerations to better simulation actual driver behavior. This cycle is often separated into city and highway portions which can be used as separate cycles. The procedure for the FTP (Federal Test Procedure) is to run a UDDS cycle followed by a 505. The Cold FTP is simply an FTP run at $-7^{\circ} \mathrm{C}$. The SC03 is run at $35^{\circ} \mathrm{C}$ with solar load and $\mathrm{A} / \mathrm{C}$ running. Combinations of these 5 cycles can be used to achieve an average fuel economy to represent desired driving behavior. Common combinations include CAFE (UDDS and HwFET), 5-cycle (all 5 above) and EcoCAR 4-cycle (505, HwFET, US06 City, US06 Highway) which is an approximation of the 5-cycle weighting.

Douba authored another paper not available for review at the time this thesis was written that explains the development of the Utility Factor approximation for the on-road EcoCAR cycles. Three schedules, A, B and $\mathrm{C}$, were created at lengths of 20,40 and 100 miles respectively to approximate the $\mathrm{J} 1711$ method for calculating UF weighted fuel consumption. The development of the UF approximation of the E\&EC cycles is discussed in Duoba's 2012 SAE paper [18]. Each schedule contained elements of city and highway driving that approximated the UDDS and HwFET cycles, respectively. The drive trace was much simplified compared to the actual certification cycles due to the need to perform the test on road. The development of these cycles and their impact on fuel economy is discussed in an EVS paper written by this author and Doug Nelson et al. [19]. This on-road approximation proved to be a reasonable approximation of the individual UDDS and HwFET cycles, but an excellent approximation of the overall CAFE weighting. The data from EcoCAR year 3 E\&EC testing will be used to calibrate and validate the tool created by this thesis.

Fuel economy testing procedures are well-established for conventional vehicles and single fuel hybrids. EVs, PHEVs and EREVs, however, pose a new challenge for a variety of reasons. Because of the nonlinear nature of batteries, EVs must be tested over the entire range of the battery to properly capture the full range of powertrain characteristics. Range evaluation, for example, must be performed by fully depleting the battery instead of testing for energy consumption and then extrapolating to find range. Testing a EREV or PHEV is more complicated still. An EREV will typically have distinct CD and CS modes, but a PHEV may leverage blended operation. Utility Factor is calculated based upon EV range, and PHEVs with blended operation do not have a true EV range. Hence it can be very difficult to calculate UF and therefore overall fuel economy for PHEVs. VTool has the capability to model EV and CS HEV powertrains which can be combined to calculate UF weighted fuel economy for a variety of EREV architectures. However, the tool is not configured to calculate fuel economy for the blended operation of a PHEV. 


\subsection{Baglione: Vehicle System Energy Analysis Methodology and Tool for Determining Vehicle Subsystem Energy Supply and Demand [29]}

Baglione developed a vehicle powertrain model similar to Gantt's thesis while working at Chrysler, but for a conventional vehicle. The goal of Baglione's work was to predict fuel consumption for a given drive cycle and correlate the real test data. Baglione's paper focuses specifically on improvements made to a power steering pump and the resulting impacts on fuel economy, but the concept is valid and can be applied to other powertrain or accessory components. His model is much more complex than other models reviewed (Sovran, Yanni, Gantt), but one key goal is common between all of them: correctly predicting the fuel economy of the vehicle based upon incremental component improvements. Baglione uses a SIMULINK model, while Gantt uses an Excel time-based spreadsheet model. Neither Yanni nor Sovran even use second-by-second calculations and deal only with 'lumps' of energy as a simplification of their method. A key advantage Baglione has over other authors is access to test data with which to calibrate more complicated and higher fidelity models. His model for rolling resistance, for example, takes into account several parameters including tire pressure and tire load. This stands in contrast to the rolling resistance model used by Sovran, Gantt and this author which leverages only 2 static coefficients of rolling resistance. For Baglione's purpose, the extra complexity is justified since his model is geared at predicting fuel economy improvements that result from relatively small improvements in individual components. The overall fuel economy improvements are likely to be very slight and below the noise floor for most testing data. A well-calibrated model, therefore, is very useful for quantifying the effects of these marginal component improvements.

This level of detail is not necessary for VTool. In fact, it stands in contradiction to the goal of understanding the powertrain as a system. VTool is instead a simple model with relatively few parameters that are easy to set up. It also explicitly calculates energy consumption and losses for each component in the powertrain. This lays out the entire energy consumption analysis to be examined and comprehended by the user.

\subsection{Park: Real-Time Powertrain Control Strategy for Series-Parallel Hybrid Electric Vehicles [30]}

Park of Hyundai Motor Co. presented a paper in 2007 explaining the development of a real-time control strategy for a Series-Parallel HEV. While Park's paper is focused on control strategy development, he touches on some concepts important to this thesis. In an HEV powertrain there is the capability to raise average engine operating efficiency by using an electric motor to apply additional load to the engine and store the extra energy in a hybrid battery pack for later use. This process is referred to as recirculating energy. The catch is that the extra energy must be reused at some future point under unknown operating conditions. Real-time control strategy decisions concerning the amount of power to recirculate are complicated and must consider several factors including engine load, battery SOC, and recirculation efficiency (which is the efficiency to store and then reuse excess engine energy). Hence, it is essential to know the system energy recirculation efficiency. Park includes two parameters of this nature in his analysis. The first, system discharge efficiency, calculated the equivalent fuel power of the electrical battery power used in propulsion. This parameter is used to determine how much energy would be required at a later time to replenish battery energy used for propulsion. The second, system charge efficiency, served the opposite purpose: it determines the amount of tractive energy that could be discharged from a given amount of battery power. Each of the parameters uses a cycle average efficiency for either discharging energy from the battery to the wheels or charging the battery with fuel energy. Together, the parameters constitute a round-trip recirculating efficiency.

Park uses a single lumped efficiency and does not derive the efficiency term from the components in the system. The analysis is also limited to a powersplit Series-Parallel architecture. This concept of a future- 
looking recirculation efficiency is used in this thesis but explored in greater depth. The recirculation efficiency is derived explicitly from the energy flow path and illustrates the effects of different architecture configurations on the efficiency of recirculating fuel energy. An additional parameter (referred to as the Power Split Fraction) is also introduced in this thesis to determine the amount of energy that is recirculated. This parameter allows basic energy management strategies to be explored without the need for developing a hybrid control strategy.

\subsection{Boyd: Hybrid Electric Vehicle Control Strategy Based on Power Loss Calculations} [31]

In 2008, Boyd authored an SAE paper based on his thesis concerning the development of a control strategy for a Split-Parallel Architecture (SPA) HEV that was designed and built for the 2005-2008 ChallengeX competition. Boyd's paper did an excellent job of explicitly defining and calculating losses for each powertrain component. This method makes it easier for the reader to see and comprehend where losses occur and how they affect the overall system efficiency. Like Park, Boyd also defined a term to account for the effect of recirculating energy called the conversion efficiency. This conversion efficiency is the effective system efficiency to convert fuel energy to electrical energy and store it in the ESS. He also defines a 'marginal' efficiency which is essentially an energy-weighted cycle average conversion efficiency. This marginal efficiency is necessary for the concept to be used in a real-time controller. Unlike Park, this marginal efficiency only represents the increase in fuel needed above engine-only mode to store energy in the ESS. The marginal efficiency can be used to judge the value of placing an additional load on the engine above the load that is already placed on the engine from road load. However, the marginal efficiency only accounts for one part of the round-trip recirculation path and is similar to Park's system discharge efficiency. Boyd also defines the other half of the round-trip recirculation path separately in his paper.

In short, the effectiveness of a given mode (such as motor assist mode or electric only mode) is evaluated by comparing the amount of fuel energy required to produce an equivalent power in engine only mode to the amount of energy required from the ESS for the motor. To compare apples-to-apples, the conversion efficiency is used to convert battery energy to fuel energy by determining the amount of fuel energy that would be required to store the specified amount of electrical energy in the battery. Boyd also includes consideration for regenerative braking. In his model, regen braking energy will artificially inflate the conversion efficiency. This is because for the same amount of fuel burned there will be more energy stored in the battery. A higher conversion efficiency means less 'fuel-equivalent' energy is required from the ESS for a given motor assist case, which would make discharging energy from the ESS a more attractive option to the vehicle controller.

Like Park's paper, Boyd's paper is focused on control strategy development, which is not of direct usefulness to this thesis. However, Boyd does introduce some useful concepts. Once again, the concept of explicitly calculating component losses for clarity is highly valuable for promoting the understanding of the system. Also, the idea of a conversion efficiency or a marginal efficiency for recirculating energy will be necessary for this thesis when considering HEV architectures. One concept not explored by any of the other papers reviewed is the effect of regen braking on the overall system, and particularly on the conversion efficiency. While Boyd lumps the effects of regen braking with conversion efficiency, VTool will keep these two energy paths separate. In practice, they are necessarily linked. But for a modeling tool like VTool, they should be decoupled so that the user can see the effects of each energy flow path separately and understand the effect of each without the influence of the other. The concept of fuelequivalent battery energy is also intriguing and will be reused by VTool to calculate fuel-equivalent regen brake energy and accessory energy. This will allow an apples-to-apples comparison of regen braking or accessory load to the overall tractive energy from the engine. 


\subsection{Literature Review Summary}

Several methods for calculating fuel consumption have already been established by many different authors. Sovran presents a method for calculating fuel economy by separating vehicle operation into propel, brake and idle cases. He also presents a set of 'Sovran' coefficients for calculating tractive energy at the wheels for a given drive cycle and glider vehicle. Gantt expands on this method by developing the brake powertrain as thoroughly as the propel powertrain for an EV. Gantt, however, uses a second-bysecond approach and does not use Sovran coefficients. Other methods, like those developed by Hochgraf and Yanni, make simplifying assumptions like using a smeared powertrain efficiency or assuming identical component efficiencies for the propel and brake case or by considering only the net case without separating into propel and brake instances. These methods seek to be simple enough to be useful as design and learning tools. Yet other modeling methods, like Baglione's method or PSAT/Autonomie, are much more complex and are meant for high-fidelity fuel consumption modeling. These methods are extraordinarily useful, but also exceptionally cumbersome for powertrain design or architecture selection.

This thesis will build directly off of the work done by Sovran and Gantt. Like both works, this thesis also separates vehicle operation into propel, brake and idle cases. VTool also uses the Sovran coefficients to calculate tractive energy at the wheels and fully develops the regenerative braking powertrain like Gantt. Expanding on these works, VTool will also define propel, brake and idle fuel consumption for Series and Parallel HEV powertrains. For these powertrains, additional subcases are defined to clearly and correctly develop the equations for fuel economy. Like the work done by Sovran, Gantt, Hochgraf and Yanni, this thesis is geared toward the understanding and comprehension of fuel consumption and of the powertrain. As such, it is necessarily a lower fidelity model than PSAT or Autonomie or the model developed by Baglione. Having said this, VTool is still capable of producing fuel-consumption predictions to a reasonable level of accuracy for its intended purpose.

Fuel consumption models are also useful (and often necessary) for use in real-time hybrid controllers for HEVs. Both Park and Boyd present papers detailing real-time hybrid supervisory controllers that leverage fuel-consumption modeling to improve real-time fuel consumption. The key concept used by both authors is that any energy stored in the battery of the HEV must eventually be reused to maintain charge balance. Park defines system charge and discharge efficiencies that are used as part of larger equations to govern vehicle operation. Somewhat differently, Boyd defines a marginal efficiency which is an energy-weighted cycle-average efficiency that represents the increase in fuel needed above engine-only mode to store energy in the ESS. While this marginal efficiency only accounts for one half of the energy recirculation path, Boyd does define the discharge path separately.

This thesis is not concerned with real-time control strategies, but does take into account the effect of energy recirculation for both Series and Parallel HEVs. VTool defines and utilizes a Power Split Fraction which is a tunable parameter that controls the fraction of energy that is used directly for propulsion. As this fraction converges to one, more of the energy produced by the engine will go directly to the wheels and less energy will be stored in the ESS first. The losses for each component in the energy recirculation path are explicitly calculated and rolled into a recirculation efficiency. This method clearly demonstrates that recirculating energy incurs extra component losses, but has the potential to raise the engine operating efficiency and therefore the overall powertrain efficiency. The method also allows the user to explore the benefits and tradeoffs of simple energy management strategies before needing a complex hybrid controller. This thesis will also present some examples to demonstrate the relationship between Power Split Fraction and engine efficiency as well as some natural limits on the Power Split Fraction. 


\section{Modeling EV and HEV Energy Consumption}

This section outlines the methodology and governing equations behind VTool. First, the equations for calculating tractive energy at the wheels are presented. These equations pertain to a glider vehicle and are the starting point for further energy consumption calculations for a particular powertrain architecture. Four architectures will be examined: EV, CS Series HEV, CS P3/P4 Parallel HEV, and CS P2 Parallel HEV. For each powertrain, the propel, brake and idle cases will be presented in addition to any other subcases that are necessary.

\subsection{Tractive Energy for a Glider Vehicle}

The first step in the process is independent of vehicle powertrain and depends solely upon vehicle glider parameters and the prescribed drive cycle. Vehicle operation is broken into propel, brake and idle cases and the tractive energy is calculated for both propel and brake cases. The calculated tractive energy will include non-conservative road load forces as well as the conservative effects of vehicle inertia.

\subsubsection{Vehicle Tractive Force}

Any vehicle in motion will experience a set of simple forces that resist the motion of the vehicle. These forces come from tire rolling resistance $\left(F_{\text {rolling }}\right)$, aerodynamic drag $\left(F_{\text {aero }}\right)$, accelerating the inertia of the vehicle $\left(F_{\text {inertia }}\right)$ and the grade of the driving surface $\left(F_{\text {grade }}\right)$. These forces are expressed in Equations 1-4 below and illustrated by Figure 12. The force due to rolling resistance, expressed in Equation 1, includes the vehicle mass $(\mathrm{M})$, gravitational acceleration $(\mathrm{g})$, vehicle velocity $(\mathrm{V})$ and two coefficients of rolling resistance $\left(\mathrm{C}_{\text {rro }}\right.$ and $\left.\mathrm{C}_{\mathrm{rr} 1}\right)$. The first coefficient $\left(\mathrm{C}_{\mathrm{rr} 0}\right)$ captures the frictional effect of the tire-road interface and is not dependent upon vehicle speed. The second coefficient $\left(C_{r r 1}\right)$ represents velocity-proportional frictional effects such as the friction in wheel bearings. Data, however, is rarely available to establish $\mathrm{C}_{\mathrm{rr} 1}$ for a given vehicle, so $\mathrm{C}_{\text {rro }}$ is often used alone. In this scenario, $\mathrm{C}_{\text {rro }}$ is slightly bigger than coefficient of rolling resistance quoted for the tires.

$$
\frac{\text { Equation 1: Rolling resistance force }}{F_{\text {rolling }}=c_{r r_{0}} M g+c_{r r_{1}} M g V}
$$

The aerodynamic drag force, expressed in Equation 2, includes the density of air ( $\rho$ ), coefficient of aerodynamic drag $\left(C_{d}\right)$, the frontal area of the vehicle $(A)$ and the square of vehicle velocity.

$$
\begin{gathered}
\text { Equation 2: Aerodynamic drag force } \\
F_{\text {aero }}=\frac{1}{2} \rho C_{d} A V^{2}
\end{gathered}
$$

Often, the glider properties of a vehicle will be experimentally determined in a coast-down test. In such a test, the vehicle is brought up to a given speed on a flat road then the transmission is shifted into neutral and the vehicle velocity is measured as the vehicle coasts down to near zero speed. Acceleration is derived from this trace and the $\mathrm{A}, \mathrm{B}$ and $\mathrm{C}$ coefficients form a polynomial, shown in Equation 3 . It is easy to see that the $C$ coefficient can be used to find $C_{d}$ and $A_{f}$ while $A$ and $B$ would be used to derive $c_{r r o}$ and $\mathrm{c}_{\mathrm{rr} 1}$. As previously stated, $\mathrm{c}_{\mathrm{rr} 0}$ and $\mathrm{C}_{\mathrm{d}}$ will be different in the absence of $\mathrm{c}_{\mathrm{rr} 1}$ because $\mathrm{c}_{\mathrm{rr} 0}$ and $\mathrm{C}_{\mathrm{d}}$ must now also account for the $B$ coefficient also. Also note that the $A B C$ coefficients are the result of a polynomial curve fit, so it is entirely possible to have a negative ' $B$ ' term. 


\section{Equation 3: Vehicle glider coast-down ABC's}

$$
A+B V+C V^{2}=M a
$$

The inertial force, expressed in Equation 4, includes vehicle inertial mass $\left(\mathrm{M}_{\mathrm{i}}\right)$, and the time derivative of velocity, which is acceleration. The inertial vehicle mass, expressed in Equation 5, accounts for the rotating inertia of components such as the wheels of the vehicle. $I_{w}$ is the rotational inertia of the wheels, and $r_{w}$ is the radius of the wheels. While it is easy to find information concerning the wheel radius of a vehicle, it is typically much more difficult to find information about the rotational inertia of the wheels of the vehicle. In the absence of such information, $M_{i}$ is usually approximated as $1.04 \mathrm{M}$.

\section{Equation 4: Inertial force}

$$
F_{\text {inertia }}=M_{i} \frac{d V}{d t}
$$

$$
\begin{aligned}
& \text { Equation } 5: \text { Inertial mass factor } \\
& M_{i}=\left(1+\frac{4 I_{w}}{M r_{w}^{2}}\right) M \approx 1.04 M
\end{aligned}
$$

The grade force is expressed in Equation 6. The angle $\theta$ is the angle of the driving surface. For small angles, however, this equation can be linearized and $\sin (\theta)$ can be approximated as the grade of the road (expressed as a percentage). However, standardized drive cycles used by the EPA for fuel economy certification have no grade and therefore always begin and end at the same elevation. Hence, grade force is rarely accounted for in fuel consumption modeling, but is often a criterion for engine or motor sizing.

$$
\begin{gathered}
\text { Equation 6: Grade force } \\
F_{\text {grade }}=M g \sin (\theta) \approx M g * \text { grade }
\end{gathered}
$$

The total force required to move the vehicle at any given speed and acceleration is the sum of these forces and is referred to as tractive force $\left(F_{t r}\right)$. The equation for tractive force is given in Equation 7 below. Figure 12 illustrates the application of these forces to a vehicle. The operation of a vehicle is often expressed as a time-based velocity trace such as with the various certification cycles used by the EPA. Such drive cycles include UDDS, HwFET, US06, SC03, LA92 and many others. Given a prescribed drive schedule, the equation for tractive force then describes the tractive force required at the wheels of the vehicle at any given instant.

$$
F_{\text {tr }}=F_{\text {grade }}+F_{\text {inertia }}+F_{\text {aero }}+F_{\text {rolling }}
$$

As is described in following sections, vehicle operation may be segregated into propel, brake and idle cases based upon the state of tractive force $\left(F_{t r}>0, F_{t r}<0, F_{t r}=0\right)$. Tractive force is used to find tractive power and the instantaneous tractive power may be integrated over a drive cycle to find the total propel or brake energy (at the wheels) required to complete the cycle. Alternatively the total propel or brake energy may be calculated using the Sovran coefficient method described in Section 3.1.4. 


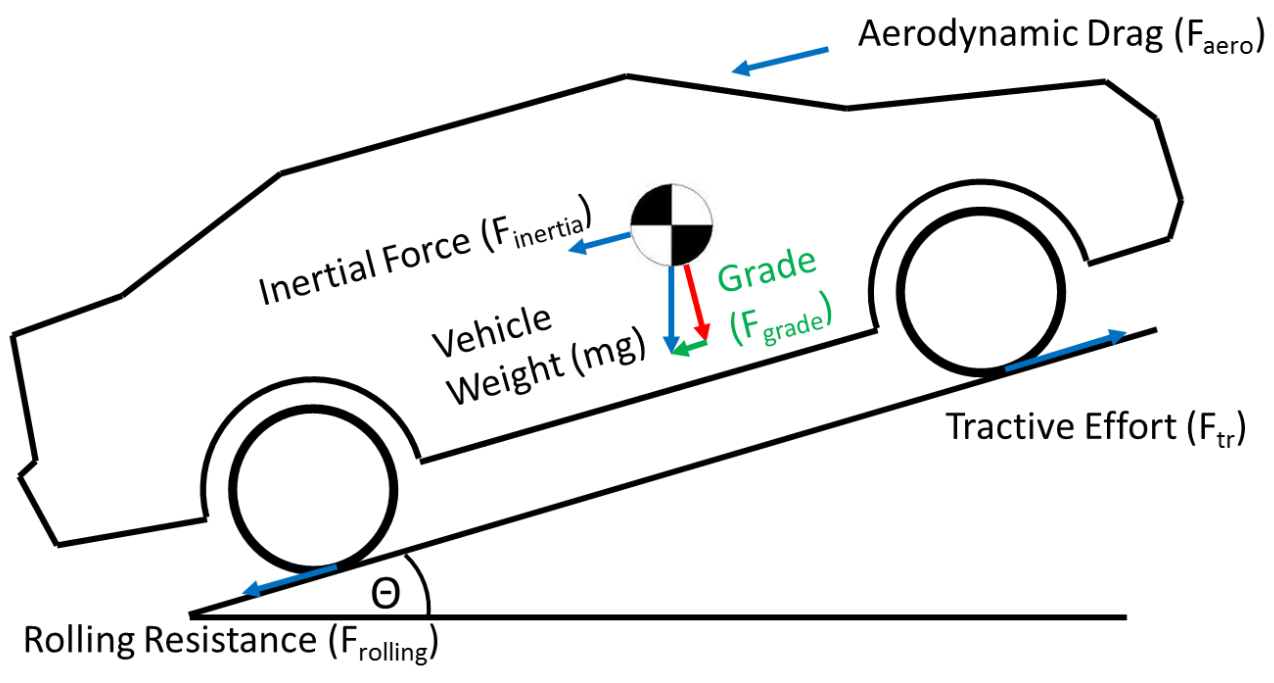

Figure 12: Tractive forces acting on a vehicle in motion

Tractive force describes the force required at the wheels to move the vehicle, but does not include any upstream considerations of the powertrain. Hence, the force produced by an engine or motor will necessarily be greater than positive tractive force in order to overcome the losses in all the powertrain components. Likewise, the fuel (or battery) power or energy required to move the vehicle will be greater than the tractive power or energy, respectively. From another perspective, tractive energy is the minimum energy required to propel the vehicle and would represent a $100 \%$ efficient vehicle with ideal powertrain components. Thus, tractive energy establishes a lower bound on the fuel consumption of the vehicle.

\subsubsection{Drive Cycle Cases}

The operation of any vehicle can be divided into 3 distinct cases: propel, brake and idle. These 3 cases are defined based upon tractive force and vehicle speed. For an Electric Vehicle (EV) or a Plug-in Hybrid Electric Vehicle (PHEV), there is an additional case: charge. A charge or refuel case is not defined for an HEV or conventional vehicle because refueling losses are negligible. The charge case is unique since the powertrain is disabled, but energy is still flowing into the battery. It also does not depend on a prescribed drive cycle (except to define the amount of battery energy to replenish). These 4 cases are used to segregate the energy use (and recovery) for the vehicle to properly understand and account for the losses in the powertrain.

\subsubsection{Propel Case: $\mathrm{F}_{\mathrm{tr}}>\mathbf{0}$}

In the propel case, tractive force is greater than zero and positive energy is required from the powertrain to propel the vehicle. In this case, energy flows from the powertrain energy source (either fuel tank or battery) to the wheels. As previously stated, the force required to propel the vehicle is greater at the engine or motor than at the wheels.

A nuance of the propel case is a powered deceleration. In this nuance, the vehicle is decelerating but still requires positive tractive force. This is demonstrated by an underpowered vehicle climbing a grade, such as a heavily loaded freight truck. The vehicle is applying maximum tractive effort but is slowing down because of the grade forces. Alternatively, this case could be encountered by a vehicle that is slowing down for a stop light, and would come to a stop before desired. Thus, the driver would request power while the vehicle is slowing down to avoid stopping short. 


\subsubsection{Brake Case: $F_{t \underline{t}} \leq 0$}

In the brake case, tractive force is less than zero and negative energy is required from the powertrain to slow the vehicle. For this methodology, brake energy is considered inherently negative. This stands in contrast to Sovran [1] who considers both propel and brake energy as positive. Either method is valid, however, since the energy balance merely depends upon the sign convention. A conventional vehicle does not have the ability to recapture and reuse brake energy, but EVs and HEVs do. The only energy available for recovery is stored in the moving inertia of the vehicle or any change in elevation and the vehicle must still overcome rolling resistance and drag. Thus, when calculating tractive brake force, the inertial force will be inherently negative while rolling resistance and drag will be positive.

The main reason for separating propel and brake cases is to properly account for recaptured regenerative braking energy. As brake energy is recaptured, some energy is lost along the way due to component losses and the energy stored in the battery will always be less than the tractive brake energy available at the wheels. This is the inverse of the propel case where propel battery energy is greater than propel tractive energy. Thus, propel and brake cases must be considered separately or the losses for the brake case power flow will not be considered correctly.

\subsubsection{Idle Case: $F_{t r}=0, V=0$}

In the idle case, the vehicle is at rest and tractive force is zero. Power is still required, however, for pumps, fans, headlights, control modules, air conditioning and other accessories. Hence, the power required to maintain all of these auxiliary systems is called the accessory load. For a conventional vehicle, the engine will remain running to avoid restarting the engine. In almost all HEVs and PHEVs, however, the engine is deactivated and the accessory load is supplied from battery energy. This is the same as an EV where all accessory energy necessarily comes from the battery. For prescribed drive cycles, idle time is a property of the drive cycle and does not change with vehicle glider properties or powertrain components.

\subsubsection{Charge Case: $F_{t t}=0, V=0, P_{\text {batt }} \leq 0$}

The last case, valid only for PHEVs and EVs, is the charge case. This is where battery recharging is accounted for. For this case, the vehicle is at rest and tractive force is zero, but there is still energy flowing into the battery. Per the sign convention (which will be discussed later), this energy is inherently negative. While energy for the propel or brake case is typically reported as $\mathrm{DC} \mathrm{wh} / \mathrm{mi}$, the total energy required to charge the battery after the cycle is cited as $A C$ wh/mi. This reflects the source of the energy: AC grid energy.

\subsubsection{Defining Nomenclature}

Several different energy flow cases are presented throughout this thesis, so it is of the utmost importance to establish a clear nomenclature convention to properly identify energy quantities. Figure 13 illustrates the convention that will be used consistently throughout this thesis. The first letter indicates the engineering unit (energy, power, force, etc.). Almost exclusively, however, this model will work with energy quantities. The two subscripts are used to specify the location within the system. The first subscript refers to a specific component while the second subscript indicates on which "side" (input or output) of the component. The superscript indicates the case of interest. As described in the previous section, there are at least 4 cases for every powertrain (propel, brake, idle, charge). However, more subcases may be defined depending on the powertrain. A list of these cases and the corresponding superscripts is located in the front matter of this thesis. 


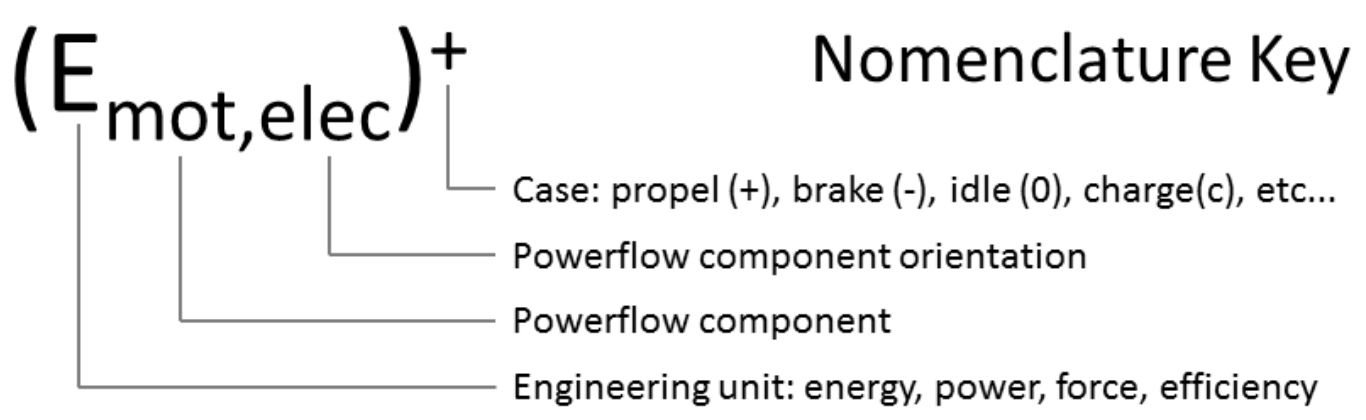

Figure 13: Nomenclature key for energy terms used throughout the model

As an example, the Figure 13 refers to the energy at the node on the electrical side of the motor specifically for the propel case. It is important to note that the motor has a mechanical side and an electrical side, either of which could be an input or an output. So instead of using input or output which is a relative term, the nomenclature uses a fixed reference point on the component. This author cannot overstress the importance of using the nomenclature in combination with powertrain diagrams. A diagram is not provided here as this is a general case, but diagrams will be provided for each subcase of each powertrain. It is also important to note that a positive sign indicates energy is flowing toward the wheels while a negative sign indicates that energy is flowing in the opposite direction toward the battery.

\subsubsection{Calculating Tractive Energy for a Glider Vehicle Using the Sovran Coefficients}

The tractive energy at the wheels of the vehicle required to complete a drive cycle can be found in a variety of different ways. This paper uses the concept laid out by Sovran in [21], which leverages a set of Sovran coefficients to characterize a given drive cycle.

\subsubsection{Benefits, Challenges and Underlying Assumptions of the Sovran Method}

Using the Sovran method means dealing with 'lumps' of energy and does not consider instantaneous power use. This is a fundamental assumption and presents both benefits and challenges. The main benefit is reducing the size and scope of the model. Instead of dealing with dynamic time-stepped models that can become large and burdensome, the initial calculations to find tractive energy become easy. In the process, however, model fidelity and detail is lost. This is an acceptable tradeoff because the goal of the methodology is to promote understanding and comprehension of the system rather than to predict energy consumption to a high degree of accuracy.

Another challenge with this approach is estimating component efficiencies. In a second-by-second model like the one used by Gantt [24], component efficiencies can be calculated within the model. When dealing only with lumps of energy, however, the detail required to model component efficiencies is no longer present. Consequently, the user must rely on other tools, test data or estimates to determine component efficiencies. The benefit to the situation is being able to directly and independently change all component efficiencies. Because component efficiencies are no longer implicitly calculated within the model, they essentially become 'knobs' with which to experiment with the model and to explore the effect of certain efficiencies on the overall system. Again, this flexibility also lends itself understanding and comprehension. Other nuances of using the Sovran method will be discussed throughout this thesis when appropriate.

\subsubsection{Deriving Sovran Coefficients}

A key concept in the Sovran model is that the Sovran coefficients characterize the drive cycles while the vehicle glider parameters characterize the vehicle. Thus, a combination of Sovran coefficients and vehicle 
glider parameters will yield tractive energy for a given glider vehicle over a given drive cycle. As Sovran noted in his 2003 paper [21], the Sovran coefficients do vary slightly with changes in certain vehicle glider properties like test mass, coefficient of drag or coefficient of rolling resistance. The coefficients presented in this paper were derived from a second-by-second model representative of the 2013 Chevy Malibu that will be used during the EcoCAR 2 competition. These coefficients are documented in Table 5. The N/V parameter is not actually used by Sovran but is included here for convenience. Likewise, the rotating inertia factor was not originally used by Sovran but is used here because the rotational inertia of the wheels $\left(I_{w}\right)$ is not known. The correlation between Sovran coefficients and vehicle glider properties is weak, so it can be safely assumed that a single set of Sovran coefficients is valid for any set of glider properties that does not vary too much from the coefficients listed below.

Table 5: Vehicle glider parameters used to derive Sovran coefficients

\begin{tabular}{|l|l|}
\hline Vehicle & EC2 Malibu \\
\hline$C_{r r 0}(1 /(\mathrm{m} / \mathrm{s}))$ & 0.00592 \\
\hline$C_{r r 1}$ & 0.000225 \\
\hline$C_{d}$ & 0.30 \\
\hline$A\left(m^{2}\right)$ & 2.3 \\
\hline$C_{d} A\left(m^{2}\right)$ & 0.69 \\
\hline$M(k g)$ Curb Mass) & 2100 \\
\hline$M i(k g)$ & 2184 \\
\hline Rotating Inertia Factor & 1.04 \\
\hline$R_{w}(\mathrm{~m})$ & 0.324 \\
\hline$N / V(r p m / m p h)$ & 94.0 \\
\hline
\end{tabular}

Sovran's original set of coefficients include considerations for rolling resistance, aerodynamic drag and vehicle inertia. This thesis expands the original set of 3 coefficients to also include grade and a velocityproportional rolling resistance. All of the following energy quantities are expressed in $\mathrm{kJ}$ assuming the units of all the parameters used in the equation are kept consistent with the units laid out in the list of vehicle parameters and coefficients in the front matter.

\subsubsection{Glider Vehicle: Propel Case}

The first Sovran coefficient, $\alpha$, corresponds to static rolling resistance. Equation 8 below expresses the energy spent to overcome static rolling resistance for the propel case. Rolling resistance is a nonconservative force and must always be overcome to propel the vehicle. $\mathrm{S}$ is the distance of the prescribed drive cycle (in km).

\section{Equation 8: Energy for overcoming static rolling resistance}

$$
\left(E_{\alpha}\right)^{+}=\alpha c_{r r 0} M S
$$

The second Sovran coefficient, $\lambda$, corresponds to velocity-proportional rolling resistance. Equation 9 expresses the energy spent to overcome velocity-proportional frictional forces for the propel case. Together, Equation 8 and Equation 9 represent the total energy needed to overcome non-conservative frictional forces for the propel case. As previously mentioned, this coefficient is often neglected due to a lack of data. It is typically a relatively small contributor to overall rolling resistance energy, so this simplifying assumption is acceptable. However, if $\lambda$ and $c_{r r 1}$ are neglected, the model becomes a twoparameter model and $\mathrm{C}_{\mathrm{rro}}$ and $\mathrm{C}_{\mathrm{d}}$ must consequently change. 


\section{Equation 9: Energy for overcoming velocity-proportional rolling resistance}

$$
\left(E_{\lambda}\right)^{+}=\lambda c_{r r 1} M S
$$

The third Sovran coefficient, $\beta$, corresponds to aerodynamic drag. Equation 10 below expresses the energy spent to overcome aerodynamic drag for either the propel or brake case. Like rolling resistance, aero drag is a non-conservative force and must always be overcome to propel the vehicle so the equations for propel and brake are the same.

\section{Equation 10: Energy for overcoming aerodynamic drag}

$$
\left(E_{\beta}\right)^{+}=\beta\left(\frac{C_{d} A}{M}\right) M S
$$

The fourth Sovran coefficient, $\gamma$, corresponds to inertial effects. Equation 11 below expresses the energy spent to produce increases in vehicle kinetic energy during the propel case. This force is conservative and there is opportunity to recover some of this energy through regenerative braking. Hence, the effects of decelerations are accounted for during the brake case. The inertial mass is used here because rotational inertia matters in this case where previously only linear inertia or normal force was relevant.

\section{Equation 11: Energy to produce increases in vehicle kinetic energy}

$$
\left(E_{\gamma}\right)^{+}=\gamma\left(1+\frac{4 I_{w}}{M r_{w}^{2}}\right) M S=\gamma \frac{M_{i}}{M} M S=\gamma M_{i} S
$$

The fifth Sovran coefficient, $\Delta$, corresponds to changes in elevation. Equation 12 below expresses the energy spent to produce increases in elevation, or potential energy. This force is also conservative and there is opportunity to recover some of this energy though the method for recovering gravitational potential energy is not as straightforward as recovering kinetic energy. Note that cycle distance is not present in this term. This is because the elevation depends only on longitudinal distance and is decoupled from cycle distance. This term is rarely used because all certification cycles contain zero grade. However, it is entirely possible to gain net elevation during the propel case while maintaining an overall net zero grade for the cycle.

\section{Equation 12: Energy to produce increases in gravitational potential energy}

$$
\left(E_{\Delta}\right)^{+}=\frac{\Delta}{S} M S=\Delta M
$$

These equations can be summed to find the overall tractive energy required for the propel case, as shown in Equation 13.

\section{Equation 13: Propel tractive energy}

$$
\left(E_{t r}\right)^{+}=\left(E_{\alpha}\right)^{+}+\left(E_{\lambda}\right)^{+}+\left(E_{\beta}\right)^{+}+\left(E_{\gamma}\right)^{+}+\left(E_{\Delta}\right)^{+}
$$

Restated:

$$
\frac{\left(E_{t r}\right)^{+}}{M S}=\alpha c_{r r 0}+\lambda c_{r r 1}+\beta\left(\frac{C_{d} A}{M}\right)+\gamma \frac{M_{i}}{M}+\frac{\Delta}{S}
$$


All of the Sovran coefficients used in VTool for the propel case are documented in Table 6 below. Note that the grade coefficient is zero for every cycle because none of the cycles have any grade characteristics during the propel case.

Table 6: Sovran coefficients for the propel case

\begin{tabular}{|c|r|r|r|r|r|}
\hline \multicolumn{6}{|c|}{ Propel Case } \\
\hline & \multicolumn{1}{|c|}{$\alpha\left[\mathrm{m} / \mathrm{s}^{2}\right]$} & \multicolumn{1}{c|}{$\lambda\left[\mathrm{m} / \mathrm{s}^{2}\right]$} & $\beta\left[\mathrm{kg} / \mathrm{m}-\mathrm{s}^{2}\right]$ & \multicolumn{1}{c|}{$\left[\mathrm{m} / \mathrm{s}^{2}\right]$} & \multicolumn{1}{c|}{$\Delta\left[\mathrm{m}^{2} / \mathrm{s}^{2}\right]$} \\
\hline & Rolling Res & Velocity Prop & Aero Drag & Inertial & \multicolumn{1}{c|}{ Grade } \\
\hline UDDS & 7.061 & 101.3 & 103.4 & 0.1651 & 0 \\
\hline & 7.375 & 134.2 & 161.9 & 0.1557 & 0 \\
\hline HwFET & 6.770 & 70.8 & 49.0 & 0.1738 & 0 \\
\hline US06 & 8.859 & 202.0 & 287.1 & 0.0463 & 0 \\
\hline US06 City & 7.799 & 219.6 & 386.4 & 0.1783 & 0 \\
\hline US06 Hwy & 5.501 & 112.4 & 158.2 & 0.4623 & 0 \\
\hline SC03 & 8.452 & 250.0 & 451.2 & 0.0975 & 0 \\
\hline LA92 & 6.875 & 100.4 & 102.5 & 0.1939 & 0.2138 \\
\hline
\end{tabular}

\subsubsection{Glider Vehicle: Brake Case}

The tractive energy equations for the brake case are the same as for the propel case except for sign. Non-conservative forces retain a positive sign because they must always be overcome. Hence, the vehicle is always expending energy to overcome these forces. Conservative forces switch sign because the kinetic or potential energy stored in the mass of the vehicle can be recovered as the vehicle slows down or falls in elevation. Thus, energy may be recovered from conservative forces through regenerative braking, but not until the non-conservative forces are first overcome. This reinforces the sign convention briefly mentioned earlier: positive energy is flowing out to the wheels while negative energy is flowing in through the wheels. The following equations establish the tractive energy contributors for the brake case. Note that the Sovran coefficients now carry a 'prime' marker (e.g. $\alpha$ ') to distinguish propel coefficients from brake coefficients. Also note that the time spent by the vehicle in the brake case will be different from the time spent in the propel case. Additionally, the total tractive energy for the brake will be different than (and less than) total tractive energy for the propel case.

Equation 14: Energy dissipated by static rolling resistance

$$
\left(E_{\alpha}\right)^{-}=\alpha^{\prime} c_{r r 0} M S
$$

Equation 15: Energy dissipated by velocity-proportional rolling resistance

$$
\left(E_{\lambda}\right)^{-}=\lambda^{\prime} c_{r r 1} M S
$$

Equation 16: Energy dissipated by aerodynamic drag

$$
\left(E_{\beta}\right)^{-}=\beta^{\prime}\left(\frac{C_{d} A}{M}\right) M S
$$

Equation 17: Energy to produce decreases in vehicle kinetic energy

$$
\left(E_{\gamma}\right)^{-}=-\gamma^{\prime}\left(1+\frac{4 I_{w}}{M r_{w}^{2}}\right) M S=-\gamma M_{i} S
$$


Equation 18: Energy to produce decreases in gravitational potential energy

$$
\left(E_{\Delta}\right)^{-}=-\Delta^{\prime} M
$$

Equation 19: Brake tractive energy

$$
\left(E_{t r}\right)^{-}=\left(E_{\alpha}\right)^{-}+\left(E_{\lambda}\right)^{-}+\left(E_{\beta}\right)^{-}+\left(E_{\gamma}\right)^{-}+\left(E_{\Delta}\right)^{-}
$$

Restated:

$$
\frac{\left(E_{t r}\right)^{-}}{M S}=\alpha^{\prime} c_{r r 0}+\lambda^{\prime} c_{r r 1}+\beta^{\prime}\left(\frac{C_{d} A}{M}\right)-\gamma^{\prime} \frac{M_{i}}{M}-\frac{\Delta^{\prime}}{S}
$$

All of the Sovran coefficients used in VTool for the brake case are documented in Table 7 below. Again, the grade coefficient is zero for every cycle because none of the cycles have any grade characteristics

\begin{tabular}{|c|c|c|c|c|c|}
\hline \multicolumn{6}{|c|}{ Brake Case } \\
\hline & $\alpha^{\prime}\left[\mathrm{m} / \mathrm{s}^{2}\right]$ & $\lambda^{\prime}\left[\mathrm{m} / \mathrm{s}^{2}\right]$ & $\beta^{\prime}\left[\mathrm{kg} / \mathrm{m}-\mathrm{s}^{2}\right]$ & $\mathrm{y}^{\prime}\left[\mathrm{m} / \mathrm{s}^{2}\right]$ & $\Delta^{\prime}\left[\mathrm{m}^{2} / \mathrm{s}^{2}\right]$ \\
\hline & Rolling Res & Velocity Prop & Aero Drag & Inertial & Grade \\
\hline UDDS & 2.749 & 32.75 & 28.09 & 0.1651 & 0 \\
\hline 505 & 2.435 & 34.83 & 37.28 & 0.1557 & $\underline{0}$ \\
\hline 867 & 3.040 & 30.81 & 19.50 & 0.1738 & $\underline{0}$ \\
\hline HwFET & 0.951 & 18.92 & 23.30 & 0.0463 & 0 \\
\hline US06 & 2.011 & 45.86 & 75.60 & 0.1783 & 0 \\
\hline US06 City & 4.309 & 81.15 & 107.47 & 0.4623 & $\underline{0}$ \\
\hline US06 Hwy & 1.358 & 35.83 & 66.50 & 0.0975 & 0 \\
\hline SC03 & 2.935 & 41.63 & 37.10 & 0.1939 & $\underline{0}$ \\
\hline LA92 & 2.890 & 45.07 & 49.70 & 0.2138 & 0 \\
\hline
\end{tabular}
during the brake case.

Table 7: Sovran coefficients for the brake case

\subsubsection{Glider Vehicle: Net Case}

An interesting observation is that summing propel and brake tractive energy will yield road load, which is simply the sum of all the energy expended to meet non-conservative forces. Equation 20 below gives the expression for road load. The superscript for road load is DCnet, meaning a net case before charging. This will be discussed more in the EV modeling section.

$$
\begin{gathered}
\frac{\text { Equation 20: Road Load Energy }}{\left(E_{t r}\right)^{\text {DCnet }}=\left(E_{t r}\right)^{+}+\left(E_{t r}\right)^{-}} \\
\text {Restated: } \\
\frac{\left(E_{t r}\right)^{\text {DCnet }}}{M S}=c_{r r 0}\left(\alpha+\alpha^{\prime}\right)+c_{r r 1}\left(\lambda+\lambda^{\prime}\right)+\left(\frac{C_{d} A}{M}\right)\left(\beta+\beta^{\prime}\right)
\end{gathered}
$$

Examine the net Sovran coefficients, which are given below in Table 8, provides interesting insights. The net values for the static rolling resistance coefficient should always be 9.81 , which is the acceleration of gravity. This makes sense because the force of rolling resistance is simply the product of vehicle test mass and the acceleration of gravity. Also, the inertia coefficients should always sum to zero. This indicates that all the energy used to accelerate the vehicle is eventually used to decelerate the vehicle - 
ie, the vehicle begins and ends the drive cycle at the same speed (usually zero). The same notion holds true for the grade coefficient. For any cycle that starts and ends at the same elevation, the sum of the propel and brake grade coefficients will equal zero. While acceleration and grade always sum to zero for standard drive cycles, the average grade and average acceleration for the propel and brake cases may not necessarily be equal. These three phenomena can be used as sanity checks to validate that the Sovran coefficients are properly conserving energy. Unfortunately, the other two coefficients do not lend themselves to such sanity checks. Interesting trends can be spotted, however. The net values for both of these coefficients are higher for cycles with higher average speeds - ie, the net coefficient for aero drag is higher for the HwFET than for the UDDS. This is because the aerodynamic drag force is dependent upon the square of velocity.

Table 8: Sovran coefficients for the net case

\begin{tabular}{|c|r|r|r|r|r|}
\hline \multicolumn{7}{|c|}{ Net Case } \\
\hline & \multicolumn{1}{|c|}{$\alpha^{\text {net }}\left[\mathrm{m} / \mathrm{s}^{2}\right]$} & \multicolumn{1}{c|}{$\lambda^{\text {net }}\left[\mathrm{m} / \mathrm{s}^{2}\right]$} & $\beta^{\text {net }}\left[\mathrm{kg} / \mathrm{m}-\mathrm{s}^{2}\right]$ & $\gamma^{\text {net }}\left[\mathrm{m} / \mathrm{s}^{2}\right]$ & $\Delta^{\text {net }}\left[\mathrm{m}^{2} / \mathrm{s}^{2}\right]$ \\
\hline & Rolling Res & Velocity Prop & Aero Drag & Inertial & \multicolumn{1}{c|}{ Grade } \\
\hline UDDS & 9.81 & 134.1 & 131.5 & 0.00 & 0.00 \\
\hline HwFET & 9.81 & 169.0 & 199.2 & 0.00 & 0.00 \\
\hline US06 & 9.81 & 101.6 & 68.5 & 0.00 & 0.00 \\
\hline US06 City & 9.81 & 220.9 & 310.4 & 0.00 & 0.00 \\
\hline US06 Hwy & 9.81 & 265.4 & 462.0 & 0.00 & 0.00 \\
\hline SC03 & 9.81 & 193.6 & 265.7 & 0.00 & 0.00 \\
\hline LA92 & 9.81 & 285.8 & 517.7 & 0.00 & 0.00 \\
\hline
\end{tabular}

\subsection{Drive Cycle Properties}

Most drive cycle properties are purely functions of the prescribed drive cycle, but one parameter depends on the glider properties of the vehicle: the amount of time that the vehicle is propelling, referred to as powered time $(\tau)$. Sovran discusses this parameter in his 2003 paper [21]. This parameter along with total cycle time and cycle idle time will be used to determine accessory energy for the propel, brake and idle cases. Note that the time spent by the vehicle in the brake case may be calculated by subtracting idle time and powered time from cycle time. These cycle times along with cycle distance and some other cycle parameters are given in Table 9 below.

Table 9: Drive cycle properties

\begin{tabular}{|c|c|c|c|c|c|c|c|}
\hline & $\mathrm{S}[\mathrm{km}]$ & $\mathrm{T}[\mathrm{s}]$ & $\mathrm{t}[\mathrm{s}]$ & $\tau[s]$ & $\mathrm{V}_{\max }[\mathrm{m} / \mathrm{s}]$ & $\mathrm{V}_{\mathrm{avg}}[\mathrm{m} / \mathrm{s}]$ & $a_{\max }\left[\mathrm{m} / \mathrm{s}^{2}\right]$ \\
\hline Drive Cycle & Distance & $\begin{array}{l}\text { Cycle } \\
\text { Time }\end{array}$ & $\begin{array}{l}\text { Idle } \\
\text { Time }\end{array}$ & $\begin{array}{l}\text { Powered } \\
\text { Time }\end{array}$ & $\begin{array}{l}\text { Max } \\
\text { Speed }\end{array}$ & $\begin{array}{c}\text { Average } \\
\text { Running Speed }\end{array}$ & $\begin{array}{c}\text { Max } \\
\text { Acceleration }\end{array}$ \\
\hline UDDS & 12.0 & 1372 & 244 & 753 & 25.3 & 10.6 & 1.48 \\
\hline 505 & 5.8 & 505 & 94 & 286 & 25.3 & 14.1 & 1.48 \\
\hline 867 & 6.2 & 867 & 150 & 467 & 15.3 & 8.6 & 1.48 \\
\hline HwFET & 16.5 & 765 & 4 & 669 & 26.8 & 21.7 & 1.43 \\
\hline US06 & 12.8 & 596 & 35 & 411 & 35.9 & 22.8 & 3.76 \\
\hline US06 City & 2.8 & 236 & 33 & 108 & 31.6 & 13.8 & 3.76 \\
\hline US06 Hwy & 10.0 & 360 & 2 & 303 & 35.9 & 27.9 & 3.08 \\
\hline SC03 & 5.8 & 596 & 106 & 318 & 24.5 & 11.8 & 2.28 \\
\hline LA92 & 15.8 & 1435 & 217 & 775 & 30.0 & 13.0 & 3.08 \\
\hline
\end{tabular}




\subsection{EV Model}

The following section outlines the equations and methodology used to predict EV energy consumption.

\subsubsection{EV: Sign Convention}

The sign convention used for the EV powertrain is illustrated in Figure 14. The dashed lines represent positive energy flow. In general, positive energy flow is moving to the wheels or some other destination where it will be consumed (like accessory loads). This means, for example, that energy into the battery is inherently negative. Obeying the sign convention is especially important when writing conservation of energy equations. For an example of how to follow the sign convention when summing energy at a node (like the HV bus below) see Section 3.3.3 below which outlines the brake case for the EV powertrain.

\section{Sign Conventions}

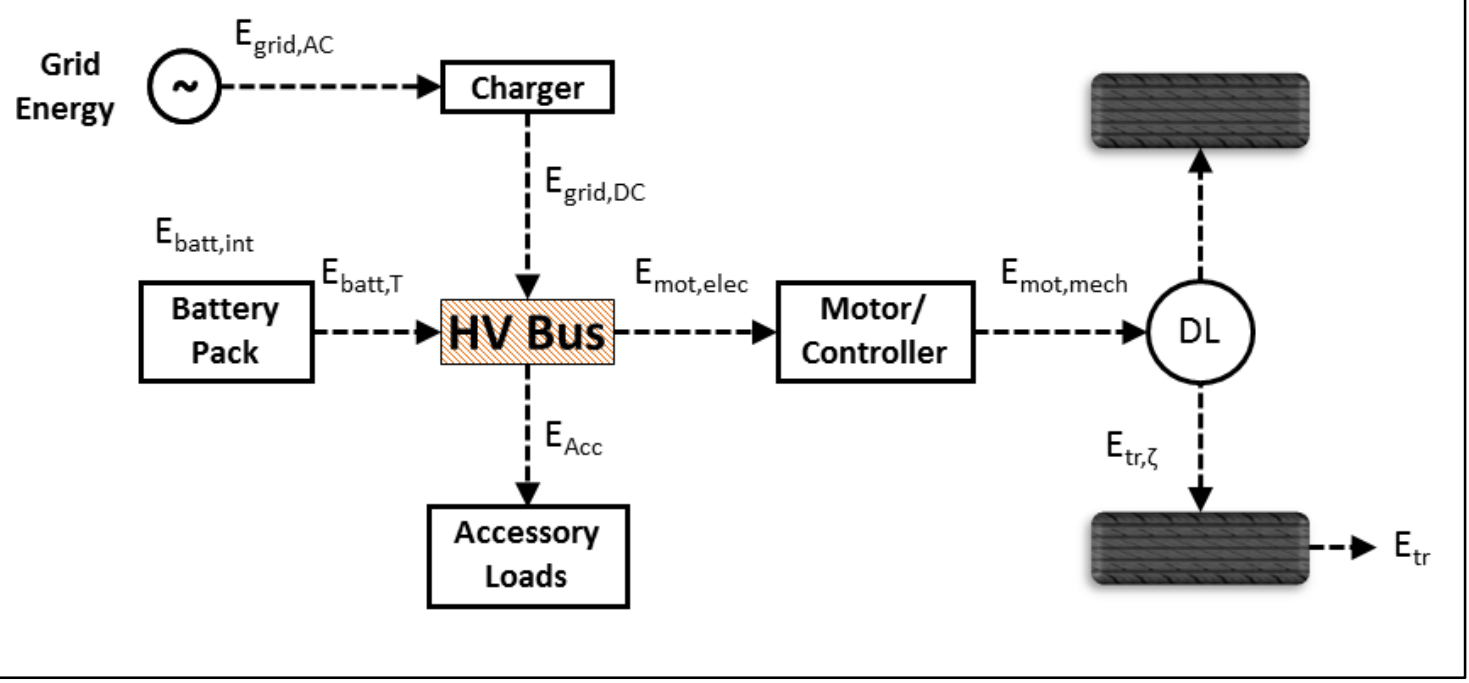

Figure 14: Energy flow sign conventions for an EV powertrain

\subsubsection{EV: Propel Case}

The energy flow paths are shown in blue in Figure 15. Energy flows from the battery to the wheels. For clarity, these paths are shown in juxtaposition to the dashed lines indicating the sign convention. For consistency, all of the arrows showing energy flow paths for a given case will remain the same color regardless of direction. Losses for each component are shown in red and are always inherently positive. 


\section{Propel: $\mathrm{F}_{\mathrm{Tr}}>0$}

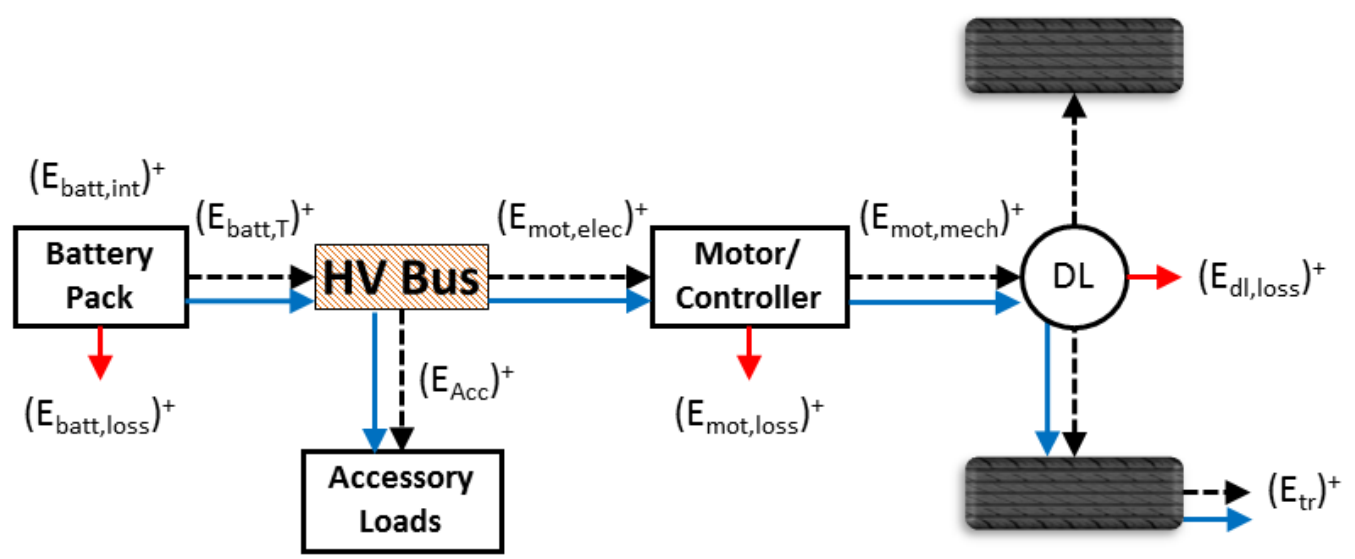

Figure 15: EV energy flow diagram for the propel case

The energy quantity of interest for this case is internal battery energy, $\left(E_{\text {batt,int }}\right)^{+}$. As an example, the equation for propel energy consumption is derived below. The first step is to establish efficiency equations for each component. While driving, energy will flow from the battery to the wheels and will diminish as losses are incurred by each component in the powertrain. The component efficiencies, defined in Equation 21 through Equation 23, reflect this. The motor driveline efficiency is tractive energy over mechanical energy output from the motor (which is the same as mechanical energy input to the driveline). Remember that this efficiency term carries the tilde accent which indicates an energy weighted cycle average efficiency. Mechanical motor output energy must be larger than tractive energy, so the driveline efficiency must be less than one, which makes sense. Battery energy is routinely referred to in one of two states: at the terminals or internal to the battery. Energy at the battery terminals is much easier to measure and is therefore commonly used and easily correlated to test data. Internal energy is the true measure of how much energy was used but is nearly impossible to measure. Usually this quantity must be estimated based upon energy at the terminals. For definitions of other energy quantities and efficiencies, refer to the list of nomenclature in the front matter.

Equation 21: Motor driveline propel efficiency (EV, propel)

$$
\tilde{\eta}_{d l, m o t}^{+}=\frac{\left(E_{t r}\right)^{+}}{\left(E_{\text {mot }, \text { mech }}\right)^{+}}
$$

\section{Equation 22: Motor propel efficiency (EV, propel)}

$$
\tilde{\eta}_{m o t}^{+}=\frac{\left(E_{\text {mot }, \text { mech }}\right)^{+}}{\left(E_{\text {mot }, \text { elec }}\right)^{+}}
$$




\section{Equation 23: Battery discharge efficiency (EV, propel)}

$$
\tilde{\eta}_{\text {batt }}^{+}=\frac{\left(E_{\text {batt }, T}\right)^{+}}{\left(E_{\text {batt }, \text { int }}\right)^{+}}
$$

The next step is to begin building the equation for energy consumption. The main idea is to start at one end of the powertrain and work down the energy flow path to the other, applying efficiencies and finding losses to move from one node of the system to the next. Rearranging Equation 23, we have:

$$
\left(E_{\text {batt }, \text { int }}\right)^{+}=\frac{\left(E_{\text {batt }, T}\right)^{+}}{\tilde{\eta}_{\text {batt }}^{+}}
$$

Internal battery energy is equal to battery energy at the terminals plus the losses incurred to move the energy to the battery internals. To move forward, we now consider the energy at the battery terminals and we must perform an energy balance at the HV bus, given in Equation 24. Because all energy is inherently positive based on the sign convention, the energy balance is straightforward.

\section{Equation 24: Conservation of energy at the HV Bus (EV, propel)}

$$
\left(E_{\text {batt }, T}\right)^{+}=\left(E_{\text {mot }, e l e c}\right)^{+}+\left(E_{\text {Acc }}\right)^{+}
$$

Accessory energy (in kJ) for the propel case, shown in Equation 25, is found with by multiplying an average accessory power (in $\mathrm{kW}$ ) by $\tau$, the duration of powered driving for the cycle.

\section{Equation 25: Propel case accessory energy}

$$
\left(E_{A c c}\right)^{+}=\tau\left(P_{A c c}\right)^{+}
$$

Substituting Equation 24 for $\left(\mathrm{E}_{\mathrm{batt}, \mathrm{T}}\right)^{+}$, we have:

$$
\left(E_{\text {batt }, \text { int }}\right)^{+}=\frac{1}{\tilde{\eta}_{\text {batt }}^{+}}\left\{\left(E_{\text {Acc }}\right)^{+}+\left(E_{\text {mot }, e l e c}\right)^{+}\right\}
$$

The equation now includes accessory load. To continue down the energy flow path, we now consider $\left(E_{\text {mot,elec }}\right)^{+}$. Rearranging Equation 22 and substituting, we have:

$$
\left(E_{\text {batt }, \text { int }}\right)^{+}=\frac{1}{\tilde{\eta}_{\text {batt }}^{+}}\left\{\left(E_{\text {Acc }}\right)^{+}+\left[\frac{\left(E_{\text {mot }, \text { mech }}\right)^{+}}{\tilde{\eta}_{\text {mot }}^{+}}\right]\right\}
$$

Finally, rearranging Equation 23 and substituting, we arrive at Equation 26, the energy consumption equation for the propel case.

\section{Equation 26: Propel case energy consumption (EV, propel)}

$$
\left(E_{\text {batt }, \text { int }}\right)^{+}=\frac{1}{\tilde{\eta}_{\text {batt }}^{+}}\left\{\left(E_{\text {Acc }}\right)^{+}+\left[\frac{\left(E_{t r}\right)^{+}}{\left(\tilde{\eta}_{\text {mot }}^{+}\right)\left(\tilde{\eta}_{d l, m o t}^{+}\right)}\right]\right\}
$$

A different way to express this equation is in terms of losses, which is also shown below in Equation 27. This equation shows that the internal battery energy must meet propel tractive energy plus the accessory load and the losses for all the components. This equation is also very intuitive to derive and provides a complimentary perspective on the operation of the vehicle. The derivation is not provided here for brevity, 
but a full step-by-step derivation is documented in Appendix A. While efficiencies depend upon the direction of energy flow, losses do not and are always inherently positive. As a result, the energy consumption equation in terms of losses is nearly identical for the propel case and the brakes case. However, efficiencies are usually required to calculate losses, so the two equations are most useful when used to complement each other (ie as sanity checks).

\section{Equation 27: Propel case energy consumption in terms of losses (EV, propel)}

$$
\left(E_{\text {batt }, \text { int }}\right)^{+}=\left(E_{\text {tr }}\right)^{+}+\left(E_{\text {Acc }}\right)^{+}+\left(E_{\text {batt }, \text { loss }}\right)^{+}+\left(E_{\text {mot }, \text { loss }}\right)^{+}+\left(E_{\text {dlmot }, \text { loss }}\right)^{+}
$$

Knowing $\left(\mathrm{E}_{\mathrm{batt}, \text { int }}\right)^{+}$, we can easily find $\left(\mathrm{E}_{\mathrm{batt}, \mathrm{T}}\right)^{+}$and define the $\mathrm{EV}$ powertrain efficiency for the propel case in Equation 28. This equation represents the powertrain efficiency for the propel case. It is expressed at the battery terminals because this is where energy consumption is commonly measured during testing. In real operating scenarios, $\left(\mathrm{E}_{\text {batt,int }}\right)^{+}$is never truly known and only estimated, therefore battery energy is expressed at the terminals to provide a correlation to a quantity that can be experimentally determined. Also note that this efficiency includes propel accessory load.

\section{Equation 28: Propel case powertrain efficiency (EV, propel)}

$$
\tilde{\eta}_{p t}^{+}=\frac{\left(E_{t r}\right)^{+}}{\left(E_{b a t t, T}\right)^{+}}
$$

This derivation process and all the equations and definitions necessary are presented in Appendix A. In this illustration, the energy consumption equation was derived by beginning at the battery and working down the energy flow path to the wheels. The equation could have been derived in the opposite order and the calculations in VTool work in this order, from the wheels to the battery. Either method is valid. The energy consumption equation may also be derived in terms of losses, as illustrated by Equation 27 . This derivation as well as the equations for all the individual component losses is also provided in Appendix $A$.

\subsubsection{EV: Brake Case}

The energy flow paths are shown in green in Figure 16. Energy flows from the wheels back to the battery. For the brake case, there is now a new loss for the friction brakes. The electric motor is inadequate for all braking scenarios so a mechanical braking system remains on the vehicle. Because of this, some fraction of total braking energy applied over the entire brake case comes from mechanical brakes. All of this energy is discarded as heat in the brake rotors. 


\section{Braking: $\mathrm{F}_{\mathrm{Tr}}<0$}

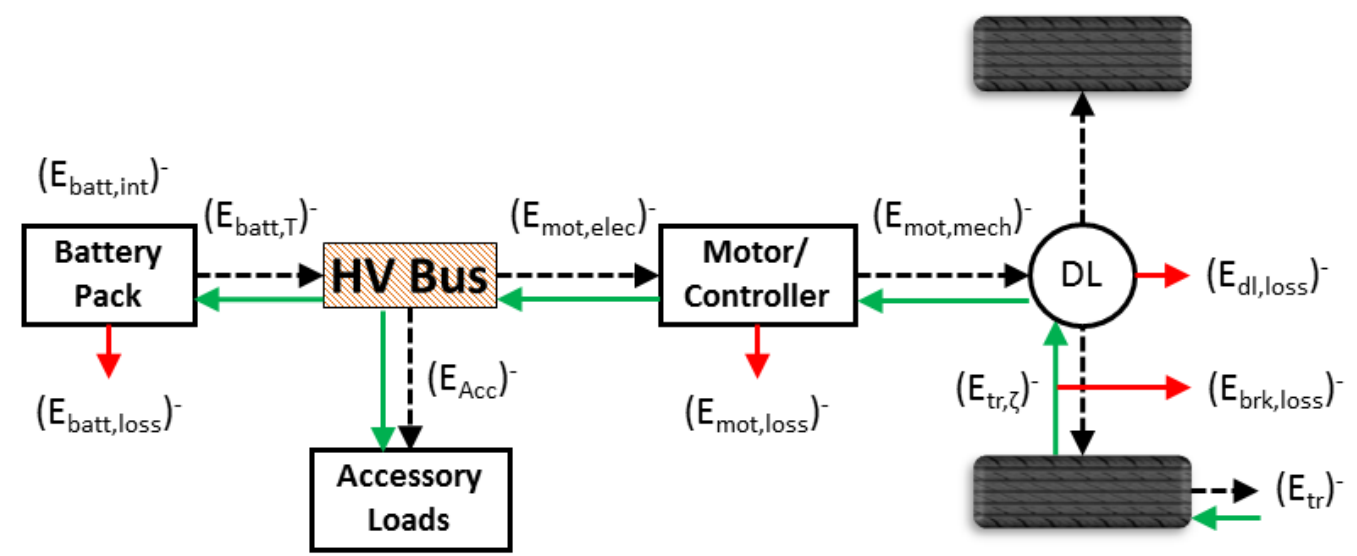

Figure 16: EV energy flow diagram for the brake case

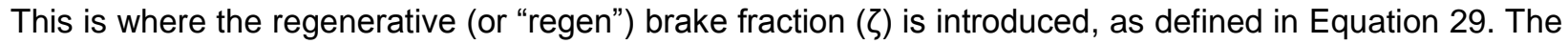
regen brake fraction varies between 1 and 0 with $\zeta=1$ meaning that all brake energy at the wheels is passed on to the driveline. Inversely, $\zeta=0$ means that all brake energy at the wheels is dissipated in friction braking. The value of $\zeta$ will vary depending upon the particular powertrain and vehicle system. Some hybrids are configured with 'push-through' braking systems where the hydraulic brakes are totally bypassed during the first bit of brake pedal travel. The axle of integration will also make a difference. Integrating the traction motor on the rear axle will lessen the amount of braking force available to the motor due to weight transfer to the front axle while braking. Other energy consumption models could potentially add depth to regen energy capture by including dynamic effects such as minimum speed or maximum power limits. However, the end result is that some fraction of braking energy available at the wheels is passed along to the driveline to be eventually recovered in the battery. This is the simplest approach and is the best approach for this model because all dynamic effects are washed out as a consequence of using Sovran coefficients to calculate tractive energy at the wheels.

$$
\begin{gathered}
\text { Equation 29: Regen brake fraction } \\
\tilde{\zeta}=\frac{\left(E_{t r, \zeta}\right)^{-}}{\left(E_{t r}\right)^{-}}
\end{gathered}
$$

Another subtle but important point to note is the direction of the energy flow to the accessory loads. All the other energy quantities for this case are inherently negative because of the sign convention. However, accessory energy, $\left(E_{A c c}\right)^{-}$, flows in the same direction as in the propel case and is inherently positive. Now the sign convention must be used to achieve the proper energy balance. As an example, the process for leveraging the sign convention when writing conservation of energy equations is demonstrated below. The first step is a simple sum at the HV Bus node. This is done in accordance with the energy flow in Figure 16 (in green), and does not account for the sign convention.

$$
\left(E_{\text {mot }, e l e c}\right)^{-}=\left(E_{\text {batt }, T}\right)^{-}+\left(E_{\text {Acc }}\right)^{-}
$$


Now the sign of each energy quantity must be noted according to the sign convention. Referring to the energy flow diagram in Figure 16, accessory energy flows with the sign convention and therefore inherits a positive sign. Battery energy at the terminals and motor energy both flow against the sign convention and therefore inherit negative signs. Applying the signs as previously described, we have:

$$
(-1)\left(E_{\text {mot }, \text { elec }}\right)^{-}=(-1)\left(E_{\text {batt }, T}\right)^{-}+(+1)\left(E_{\text {Acc }}\right)^{-}
$$

Rearranging this equation, we arrive at Equation 30, the conservation of energy expression at the HV Bus node. As it turns out, this equation is identical to the equivalent equation for the propel case.

\section{Equation 30: Conservation of energy at the HV Bus (EV, brake)}

$$
\left(E_{\text {batt }, T}\right)^{-}=\left(E_{\text {mot }, e l e c}\right)^{-}+\left(E_{\text {Acc }}\right)^{-}
$$

Similar to the propel case, the accessory load for the brake is simply accessory power multiplied by time. This time value is found by subtracting powered driving time and idle time from the total cycle time. Hence, accessory energy for the brake case is given by

\section{Equation 31: Brake case accessory energy}

$$
\left(E_{\text {Acc }}\right)^{-}=\left(T-\tau-t_{\text {idle }}\right)\left(P_{\text {Acc }}\right)^{-}
$$

The derivation of the energy consumption equation is similar to the propel case and is documented in Appendix $B$ along with all the definitions and equations necessary for the brake case. Equation 32 gives the energy consumption equation for the brake case. This equation shows that the internal battery energy recovered from regen braking will be less than the tractive brake energy. Bear in mind that $\left(E_{\text {batt }}\right)^{-}$is inherently negative as is $\left(E_{t r}\right)^{-}$. The accessory load, however, is still inherently positive. Hence, the accessory load is reducing the amount of energy stored in the battery by making the numbers less negative.

$$
\begin{gathered}
\text { Equation 32: Brake case energy consumption (EV, brake) } \\
\left(E_{\text {batt }, \text { int }}\right)^{-}=\tilde{\eta}_{\text {batt }}^{-}\left\{\left(E_{t r}\right)^{-}(\tilde{\zeta})\left(\tilde{\eta}_{d l, m o t}^{-}\right)\left(\tilde{\eta}_{m o t}^{-}\right)+\left(E_{A c c}\right)^{-}\right\}
\end{gathered}
$$

An important nuance to note is how efficiencies depend on the direction of powerflow. As an example, examine the drive cycle average efficiency of the motor for the propel and brake cases, shown in Equation 22 and Equation 33, respectively. In the propel case, the energy flows from the battery to the wheels, so energy on the electrical side of the motor is necessarily larger than on the mechanical side. Hence, mechanical energy is divided by the efficiency term and electrical energy is indeed larger. The opposite is true for the brake case. Electrical energy must be smaller than mechanical energy because energy is flowing from the wheels to the battery. Thus, mechanical energy is multiplied by the efficiency term.

Equation 22: Motor propel efficiency (EV, propel)

$$
\left(E_{\text {mot }, e l e c}\right)^{+}=\frac{\left(E_{\text {mot }, \text { mech }}\right)^{+}}{\tilde{\eta}_{\text {mot }}^{+}}
$$

Equation 33: Motor regen efficiency (EV, brake)

$$
\left(E_{\text {mot }, \text { elec }}\right)^{-}=\tilde{\eta}_{\text {mot }}^{-}\left(E_{\text {mot }, \text { mech }}\right)^{-}
$$


Equation 34 gives the energy consumption for the brake case in terms of component losses. This equation illustrates that the powertrain pays all the component losses and the accessory load and then keeps the rest of the tractive brake energy from the wheels. Note that losses due to non-conservative forces such as tire friction are already accounted for in $\left(E_{t r}\right)^{-}$.

\section{Equation 34: Brake case energy consumption in terms of losses (EV, brake)}

$$
\left(E_{\text {batt }, \text { int }}\right)^{-}=\left(E_{t r}\right)^{-}+\left(E_{\text {Acc }}\right)^{-}+\left(E_{\text {batt }, \text { loss }}\right)^{-}+\left(E_{\text {mot }, \text { loss }}\right)^{-}+\left(E_{\text {dlmot }, l o s s}\right)^{-}+\left(E_{\text {brk,loss }}\right)^{-}
$$

Like the propel case, the brake case powertrain efficiency references battery energy at the terminals and includes the accessory load. This efficiency, defined in Equation 35, is also likely to be lower than the propel case because of the effect of the regen brake fraction.

Equation 35: Brake case powertrain efficiency (EV, brake)

$$
\tilde{\eta}_{p t}^{-}=\frac{\left(E_{b a t t, T}\right)^{-}}{\left(E_{t r}\right)^{-}}
$$

\subsubsection{EV: Idle Case}

The energy flow paths are shown in gray in Figure 17. Energy flows from the battery directly to the accessory load. This is a relatively simple case involving only component loss, the battery.

\section{Idle: $\mathrm{F}_{\mathrm{Tr}}=0$}

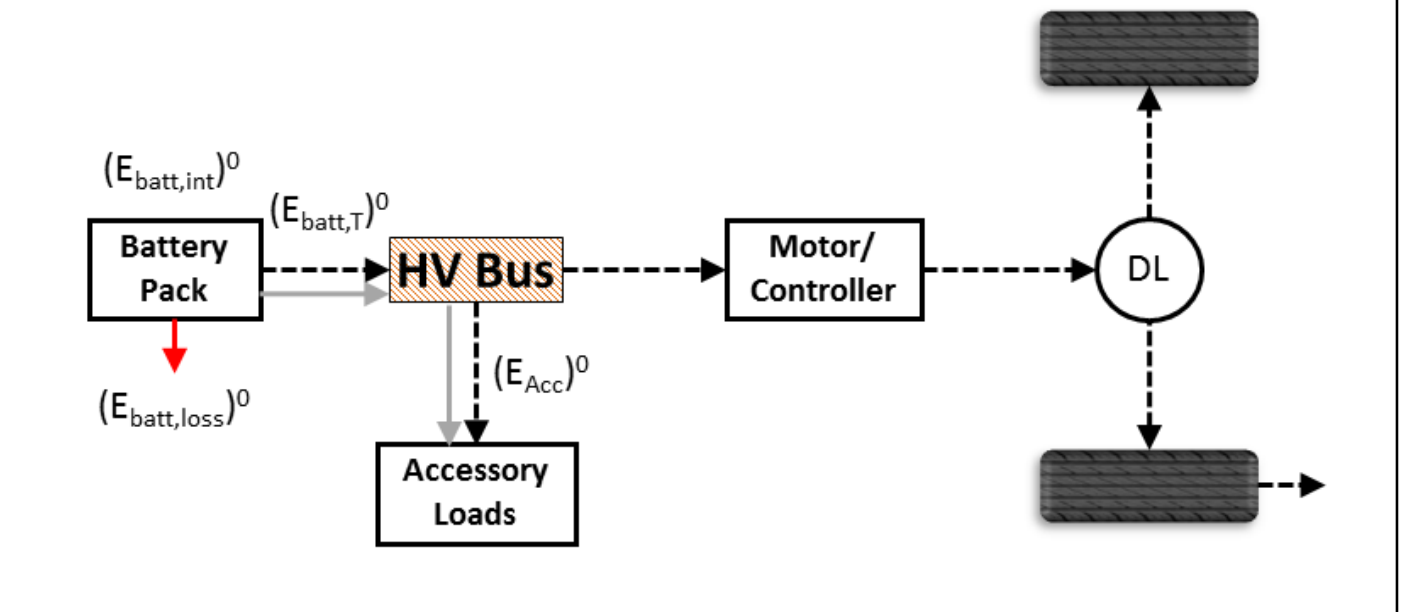

Figure 17: EV energy flow diagram for the idle case

Accessory energy during idle is defined by Equation 36 as accessory power times the prescribed cycle idle time. Idle case energy consumption, defined by Equation 37 is simply idle accessory energy plus battery losses. 
Equation 36: Idle accessory energy

$$
\left(E_{\text {Acc }}\right)^{0}=\left(t_{\text {idle }}\right)\left(P_{\text {Acc }}\right)^{0}
$$

\section{Equation 37: Idle case energy consumption (EV, idle)}

$$
\left(E_{\text {batt }, \text { int }}\right)^{0}=\frac{\left(E_{\text {Acc }}\right)^{0}}{\tilde{\eta}_{\text {batt }}^{0}}
$$

\subsubsection{EV: DC Net Case (Before Charging)}

Because an EV is a single energy source vehicle, energy consumption from the three cases (propel, brake and idle) may be summed outright. This case is called the DCnet case because it describes the DC electrical energy required from the battery to complete the drive cycle. This is in contrast to the AC energy required to recharge the battery after completing the cycle. Energy from the brake case will be inherently negative and will reduce the total energy consumption as expected (assuming there is more regen energy captured than accessory energy spent during the brake case). Net internal battery energy is given by Equation 38 and is related to the change in battery SOC. As previously mentioned, energy consumption for an EV is more commonly expressed as battery energy at the terminals of the battery as shown in Equation 39. If the battery pack is efficient, however, the discrepancy between internal battery energy and energy at the terminals will be small.

\section{Equation 38: Net internal battery energy used (EV, DCnet)}

$$
\left(E_{\text {batt }, \text { int }}\right)^{\text {DCnet }}=\left(E_{\text {batt }, \text { int }}\right)^{+}+\left(E_{\text {batt }, \text { int }}\right)^{-}+\left(E_{\text {batt }, \text { int }}\right)^{0}
$$

Equation 39: Net DC energy consumption (EV, DCnet)

$$
\left(E_{\text {batt }, T}\right)^{\text {DCnet }}=\left(E_{\text {batt }, T}\right)^{+}+\left(E_{\text {batt }, T}\right)^{-}+\left(E_{\text {batt }, T}\right)^{0}
$$

Now, a net powertrain efficiency may be defined. There are various ways to define net powertrain efficiency, but Equation 40 gives the definition used for this tool. Included in $\left(E_{\text {batt, }, T}\right)^{\text {DCnet }}$ is the energy expended in propulsion, the energy recovered in regen braking, but also the total accessory load. Thus, $\left(E_{\text {batt }, T}\right)^{\text {DCnet }}$ represents the total energy required from the battery terminals to operate the vehicle over the entire duration of the drive cycle. This quantity is compared to $\left(E_{t r}\right)^{\text {DCnet }}$, which is of course road load and represents all of the non-conservative forces that had to be overcome. This powertrain efficiency, then, is essentially a combination of propel and brake powertrain efficiencies, but does not include battery losses. As a result, the net powertrain efficiency will certainly be lower than either propel or brake powertrain efficiency on its own. While this makes sense and it properly represents the system efficiency, it misrepresents the value of regen braking because the propel powertrain efficiency is higher than the net powertrain efficiency.

\section{Equation 40: Net powertrain efficiency (EV, DCnet)}

$$
\tilde{\eta}_{p t}^{D C n e t}=\frac{\left(E_{t r}\right)^{\text {DCnet }}}{\left(E_{\text {batt }, T}\right)^{\text {DCnet }}}=\frac{\left(E_{t r}\right)^{+}+\left(E_{t r}\right)^{-}}{\left(E_{\text {batt }, T}\right)^{+}+\left(E_{\text {batt }, T}\right)^{-}+\left(E_{\text {batt }, T}\right)^{0}}
$$

To examine the apparent benefit of regen braking, $\left(E_{\text {batt, } T}\right)^{\text {DCnet }}$ could be compared to propel tractive energy. Propulsion is the end goal and it is what the driver is 'paying' for, so it could makes sense to compare what was actually paid to what was paid for. It is also useful to determine total accessory energy used and the total powertrain losses as shown in Equation 41 and Equation 42. The net accessory 
energy can serve as a sanity check since $\left(E_{A c c}\right)^{\text {DCnet }}$ should equal accessory power times the prescribed cycle time. The total losses in the powertrain are a good way to judge the effectiveness of the powertrain. When normalized to cycle distance, total losses can be used to compare powertrain performance between different cycles.

$$
\begin{gathered}
\text { Equation 41: Net accessory energy } \\
\left(E_{A C C}\right)^{\text {DCnet }}=\left(E_{A C C}\right)^{+}+\left(E_{A C C}\right)^{-}+\left(E_{A C C}\right)^{0}=P_{A C C} * T \\
\text { Equation 42: Net powertrain losses (EV, DCnet) } \\
\left(E_{p t, \text { loss }}\right)^{\text {DCnet }}=\left(E_{p t, l o s s}\right)^{+}+\left(E_{p t, l o s s}\right)^{-}+\left(E_{\text {batt }, \text { loss }}\right)^{0}
\end{gathered}
$$

Finally, an overall energy balance is assessed as a sanity check. This energy balance, defined in Equation 43 , shows that the net battery energy required to complete the cycle must equal the sum of road load (the non-conservative forces), the total accessory load and all of the powertrain losses. These and other support equations are provided in Appendix $D$ as a summary of the net case.

$$
\begin{gathered}
\text { Equation 43: Overall energy balance (EV,DCnet) } \\
\left(E_{\text {batt } \text { int }}\right)^{\text {DCnet }}=\left(E_{t r}\right)^{\text {DCnet }}+\left(E_{\text {Acc }}\right)^{\text {DCnet }}+\left(E_{p t, l o s s}\right)^{\text {DCnet }}
\end{gathered}
$$

\subsubsection{EV: Charge and AC Net Case (After Charging)}

In the charge case, energy discharged from the battery is replenished by grid energy, as shown in Figure 18. The vehicle is connected to the grid via an on-board charging unit that converts AC current to DC current. For the $\mathrm{VT}_{\mathrm{REX}}$, a $3.3 \mathrm{~kW}$ charger is used that is compatible with input voltages from $100 \mathrm{~V}$ to 264 $\mathrm{V}$, which covers the US standard $110 \mathrm{~V}$ and $220 \mathrm{~V}$ outlets. There are many different charging power levels as defined by the SAE J1772 standard. The key equations and definitions for the charging case are laid out in this section, but all of the necessary equations and derivations are contained in Appendix $\mathrm{E}$.

\section{Charging: $\mathrm{F}_{\mathrm{Tr}}=0, \mathrm{P}_{\text {batt }, \mathrm{T}}<0$}

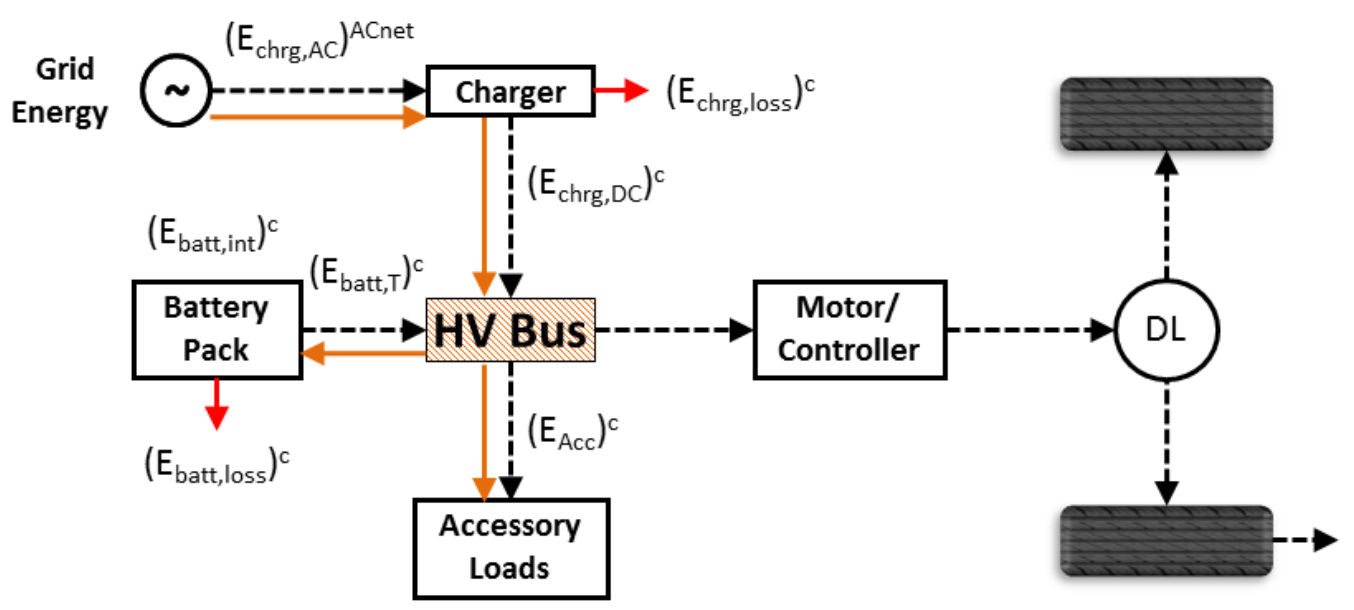

Figure 18: EV energy flow diagram for the charge case 
To conserve energy, all the energy used during the cycle, $\left(E_{\text {batt,int }}\right)^{D C n e t}$, must be replaced during the charging phase in addition to any charging losses and accessory loads. To be consistent with the sign convention, the internal battery energy replaced during charging, $\left(E_{\text {batt,int }}\right)^{C}$, must be inherently negative which yields Equation 44:

\section{Equation 44: Battery internal energy to be replenished (EV, charge)}

$$
\left(E_{\text {batt }, \text { int }}\right)^{c}=-\left(E_{\text {batt }, \text { int }}\right)^{\text {DCnet }}
$$

Because of losses and accessory load, more energy is required from the grid than in the battery internals. The component losses for the charge case include battery losses, and charger losses. The battery charge efficiency for the charge case, defined by Equation 45, is the same efficiency used for all battery charging as a simplifying approximation. The charger efficiency, defined by Equation 46, describes the component efficiency of the charger and does not consider any additional losses of the accessory load.

\section{Equation 45: Battery charge efficiency (EV, charge)}

$$
\tilde{\eta}_{\text {batt }}^{c}=-\frac{\left(E_{\text {batt }, \text { int }}\right)^{c}}{\left(E_{\text {batt }, T}\right)^{c}}=\frac{\left(E_{\text {batt }, \text { int }}\right)^{\text {DCnet }}}{\left(E_{\text {batt }, T}\right)^{c}} \approx \tilde{\eta}_{\text {batt }}^{-}
$$

\section{Equation 46: Charger efficiency (EV, charge)}

$$
\tilde{\eta}_{\text {chrgr }}^{c}=\frac{\left(E_{\text {chrg,DC }}\right)^{c}}{\left(E_{\text {chrg,AC }}\right)^{A C n e t}}
$$

The accessory load present during charging will be different than the normal accessory load present during vehicle operation and can be expected to be around $100 \mathrm{~W}$. This accessory load is to supply power to coolant pumps, controllers, battery contactors and other systems necessary for charging. The accessory energy required during charging is defined by Equation 47 . The time required for charging is defined in Equation 48 and is dependent upon the amount of energy required to recharge the pack and the power level of the charger.

\section{Equation 47: Charge case accessory energy (EV, charge)}

$$
\left(E_{\text {Acc }}\right)^{C}=\left(t_{\text {charge }}\right)\left(P_{\text {Acc }}\right)^{C}
$$

\section{Equation 48: Time required for charging (EV, charge)}

$$
\left(t_{\text {charge }}\right)=\frac{\left(E_{\text {batt }, \text { int }}\right)^{\text {DCnet }}}{\left(P_{\text {charger }}\right)^{C}}
$$

An additional efficiency that is often useful is the charging efficiency, which is defined in Equation 49. This is different from the charger component efficiency and represents the overall efficiency of the entire charging process, including battery losses, charger losses and accessory load.

\section{Equation 49: Charging efficiency (EV, charge)}

$$
\tilde{\eta}_{\text {chrgn }}^{c}=-\frac{\left(E_{\text {batt }, \text { int }}\right)^{c}}{\left(E_{\text {chrg }, A C}\right)^{A C n e t}}=\frac{\left(E_{\text {batt }, \text { int }}\right)^{\text {DCnet }}}{\left(E_{\text {chrg }, A C}\right)^{A C n e t}}
$$


Finally, the $A C$ energy required to replace the $D C$ energy consumed during the cycle is described in Equation 50. This energy quantity is expressed as $A C$ net because it is the net $A C$ grid energy required for the vehicle to complete the cycle. Alternatively, this quantity can be expressed using the charging process efficiency as given by Equation 51 . Charging process efficiency is calculated relatively easily by measuring energy on the $\mathrm{AC}$ side of the charger and at the battery terminals. Using the charging process efficiency simplifies the calculation and makes the energy consumption equation more applicable to realworld test data that can be acquired, which makes the model more useful. This efficiency is also a very good way to view the overall charging efficiency and is akin to propel or brake powertrain efficiency. An important nuance to $A C$ net energy is the location where the AC energy into the HV charger is physically measured. Energy could be measured, for example, either upstream or downstream of the Electric Vehicle Supply Equipment (EVSE) and therefore may or may not include EVSE losses. Often, AC energy is measured at the breaker panel where it is most convenient. However, if the panel is not close to the EVSE, significant wire losses could be incurred from the length of the wire between the panel and the EVSE. Each test environment will likely be different, so it is important to be aware of the potential losses that will be inherently included in the measurements.

Equation 50: Charge case energy consumption (EV, charge)

$$
\left(E_{\text {chrg }, A C}\right)^{A C n e t}=\frac{1}{\tilde{\eta}_{\text {chrgr }}^{c}}\left[\left(E_{\text {Acc }}\right)^{c}+\frac{\left(E_{\text {batt }, \text { int }}\right)^{\text {DCnet }}}{\tilde{\eta}_{\text {batt }}^{c}}\right]
$$

Equation 51: Charge case energy consumption using charging process efficiency (EV, charge)

$$
\left(E_{\text {chrg }, A C}\right)^{A C n e t}=\frac{\left(E_{\text {batt }, \text { int }}\right)^{\text {DCnet }}}{\tilde{\eta}_{\text {chrgn }}^{C}}
$$

This energy consumption equation can also be expressed in terms of losses as in Equation 52. From this perspective, it is clear that the required cycle net AC grid energy is the net $D C$ energy consumed during the cycle plus charging accessory energy, charger losses and battery losses.

Equation 52: Charge case energy consumption in terms of losses (EV, charge)

$$
\left(E_{\text {chrg }, A C}\right)^{\text {ACnet }}=\left(E_{\text {batt }, \text { int }}\right)^{\text {DCnet }}+\left(E_{\text {Acc }}\right)^{c}+\left(E_{\text {chrg }, \text { loss }}\right)^{c}+\left(E_{\text {batt }, \text { loss }}\right)^{c}
$$

\subsubsection{EV: Summary}

The EV powertrain is the simplest and most straightforward of the powertrains examined in this thesis. There is only one energy source (the battery) so a simple sum of the cases may be performed to find energy consumption. This sum is performed once before charging to find DC energy consumption and again after charging to find $A C$ energy consumption. DC energy consumption is useful for evaluating the powertrain and sizing the battery or finding EV range. Because it is easy to measure energy at the terminals of the battery, DC energy consumption is quoted as energy at the terminals of the battery even though battery losses are still calculated. AC energy consumption represents the total grid energy consumed by the vehicle to complete the drive cycle and is useful for overall energy consumption to compare with other vehicles. This measure of energy consumption is also useful for calculating the WTW impact of the vehicle.

The Charge Sustaining (CS) Series and Parallel HEVs presented in the following sections will reuse the concepts presented for the EV powertrain. HEVs also have the ability to perform regen braking, but the 
brake powertrain will be identical (or nearly identical) to the EV powertrain. Thus, the brake case presented here will be reused for both Series and Parallel HEV architectures.

PHEV and EREV powertrains may also be considered by coupling the results from the EV powertrain with the results of the desired CS HEVs. This is straightforward for PHEVs that have a fully capable CD mode and a distinct transition to CS mode. Blended PHEVs pose more of a challenge, however, and are not covered in the scope of this thesis, but a similar analysis could be used to understand them by combining electric and fuel consumption.

\subsection{CS Series HEV Model}

The following section outlines the equations and methodology used to predict fuel energy consumption for a Charge Sustaining (CS) Series HEV. A vehicle is effectively a charge sustaining vehicle if it uses an ESS during operation but the net energy out of the ESS during extended operation is nearly zero. Also, a $\mathrm{HEV}$ is necessarily charge sustaining unless it has the means to recharge the ESS from the grid (in which case it would be a PHEV). It is rarely operationally advantageous for an HEV to be charge balanced over a drive cycle. An HEV control strategy is not designed to maintain charge balance, but rather to use the ESS to maximize operational efficiency within certain bounds (such as ESS power or SOC). However, a truly charge sustaining or charge balanced vehicle is desirable for testing or modeling purposes. If the net energy out of the ESS is not zero for testing or modeling, the overall fuel consumption of the vehicle can be significantly impacted. If the vehicle completes the cycle at a lower SOC than it began the cycle, it would have used some battery energy to complete the cycle in addition to the fuel used to complete the cycle. And because fuel use is the measure of fuel consumption, the test results would show that the vehicle had an artificially low fuel consumption (the vehicle would appear to perform better than it actually performed). For this reason, net battery energy into (or out of) the battery must be $<1 \%$ of fuel energy consumed over the drive cycle for a fuel economy test to be a valid. The same effect is applicable to modeling exercises. Hence, it is necessary to model HEVs as charge balanced.

\subsubsection{CS Series HEV: Sign Convention}

The sign convention used for the Series powertrain is illustrated in Figure 19. Like the EV powertrain, the dashed lines represent positive energy flow. As mentioned previously, positive energy flow generally means energy is moving to the wheels or some other destination where it will be consumed (like accessory loads). The key difference between the Series HEV and EV powertrains is the addition of an engine and generator and the lack of a HV battery charger. For this model, the Series powertrain is considered to be charge sustaining and therefore does not need a charger. However, the results for the CS Series HEV can be coupled with the results from the EV to examine to effects of a Series PHEV or EREV. 


\section{Sign Conventions}

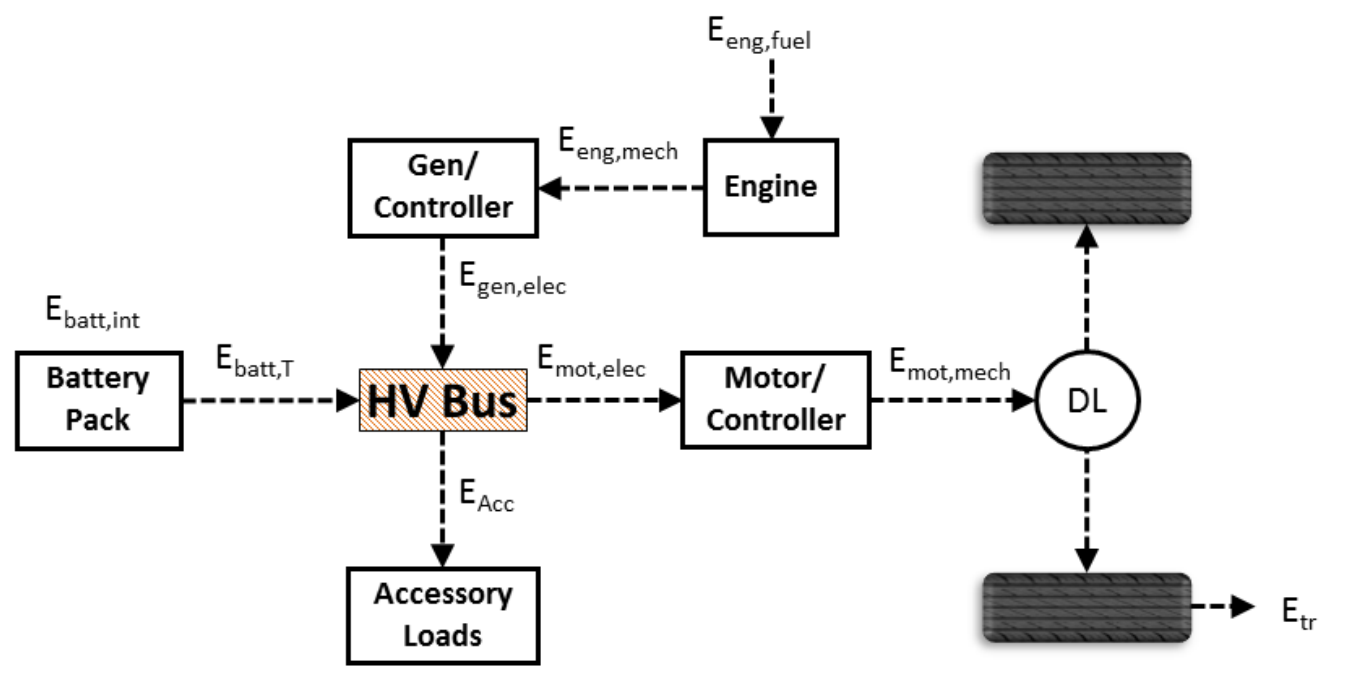

Figure 19: Energy flow sign conventions for a CS Series HEV powertrain

\subsubsection{CS Series HEV: Propel Case}

The energy flow paths are shown in blue in Figure 20. Energy (shown in blue) flows from the engine to the wheels. Losses for each component are shown also in red and are inherently positive.

\section{Propel: $\mathrm{F}_{\mathrm{Tr}}>0$}

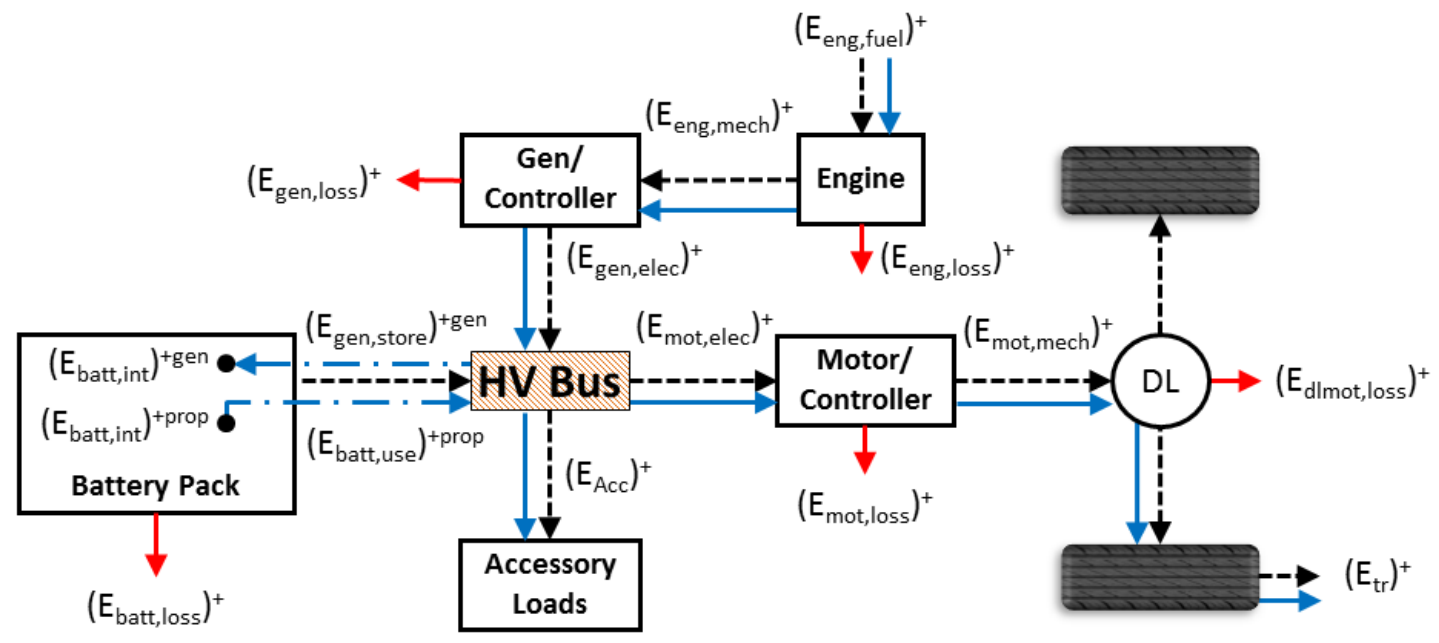

Figure 20: CS Series HEV energy flow diagram for the propel case 
The dashed blue lines indicate that power may flow along that path, but will not always flow along the path. Because the engine and generator (or genset) are decoupled from the wheels and the traction motor propels the vehicle, the genset is not constrained to output the exact amount of power required for propulsion. As such, the genset may produce more power, less power, or exactly the amount of power required for traction. The propel case may then be subdivided into three corresponding subcases: store, use and equilibrium. Any of these subcases may occur at any time during vehicle operation (depending upon component power limits and battery SOC). In fact, the powertrain is likely to transition between states frequently during actual operation.

\subsubsection{CS Series HEV: Propel Case - Equilibrium Subcase}

This first subcase examined is the equilibrium subcase where the genset produces the exact amount of energy required for traction. As illustrated by Figure 21, all energy (or power) created by the genset goes directly to supplying tractive power and accessory loads. There is no surplus or shortage of power, so no power is put into or removed from the battery. It is theoretically possible for the vehicle to operate solely in this subcase. This mode of operation is referred to as a pure load following strategy. Theoretically, a pure load following Series HEV would not require a battery. In practice, however, maintaining the genset at exactly the right power level proves to be exceptionally difficult. Additionally, regen braking would not be possible without a battery and operating the genset in this manner is relatively inefficient. For these reasons, a true pure load following strategy is impractical and unwise.

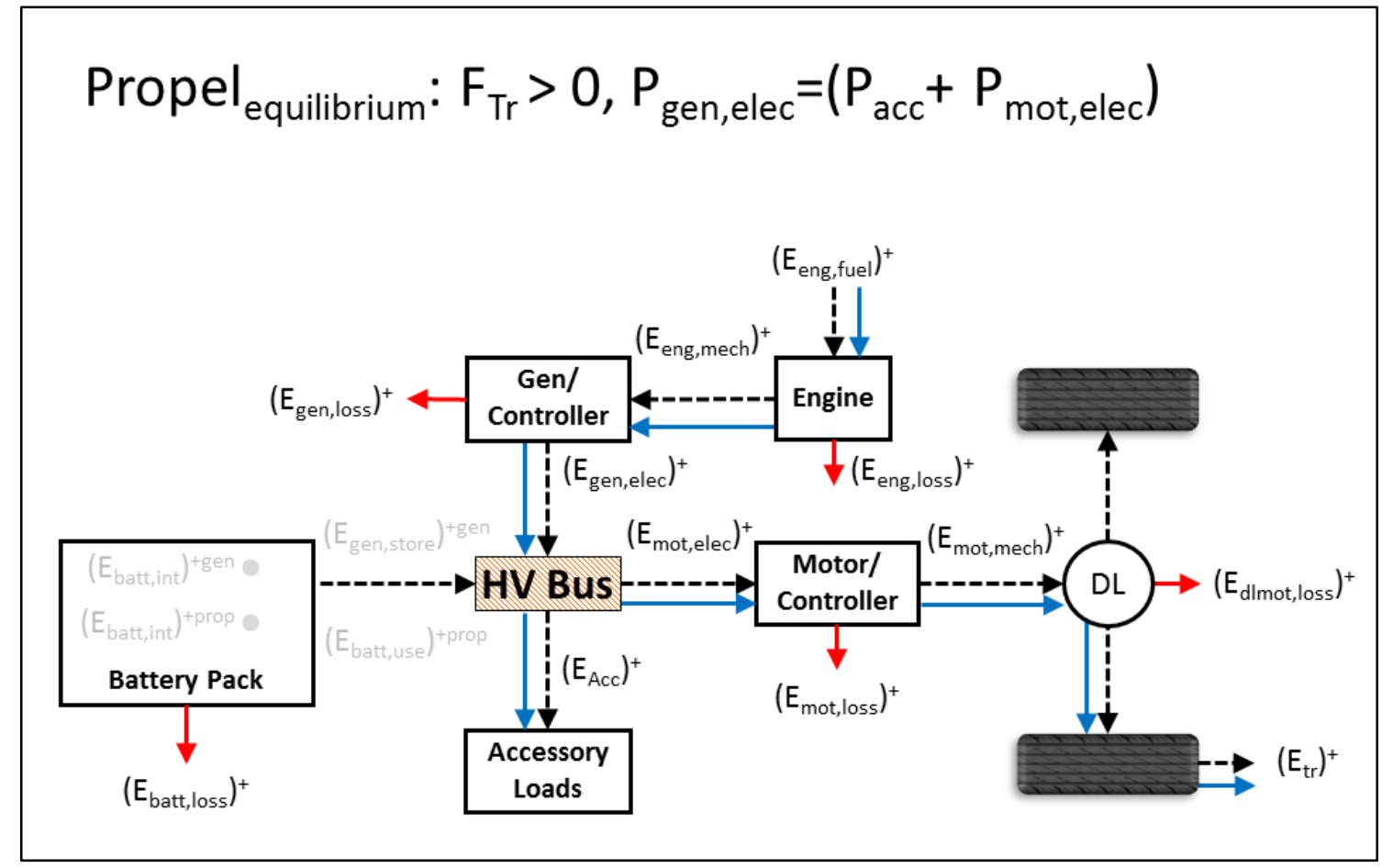

Figure 21: CS Series HEV energy flow diagram for the propel case during an equilibrium condition 


\subsubsection{CS Series HEV: Propel Case - Store Subcase}

The second subcase is the 'Store' subcase. In this subcase, more power is generated than is required at the HV bus and the excess power is stored in the battery as shown in Figure 22.

$$
\text { Propel }_{\text {store }}: F_{T r}>0, P_{\text {gen,elec }}>\left(P_{\text {acc }}+P_{\text {mot,elec }}\right)
$$

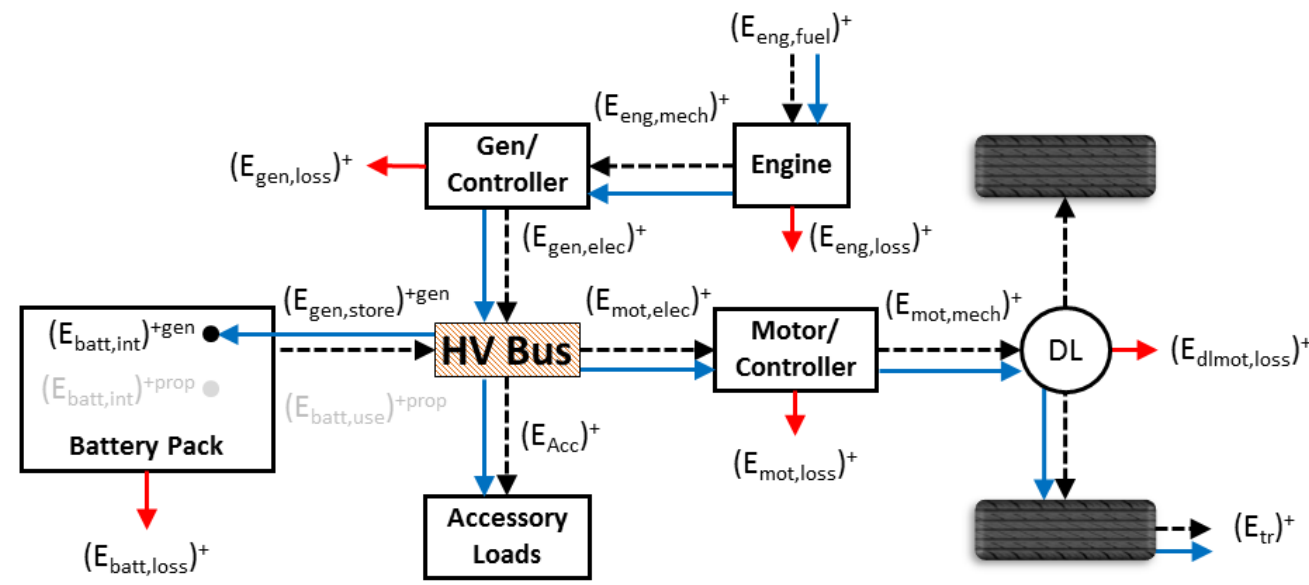

Figure 22: CS Series HEV energy flow diagram for the propel case during the 'store' subcase

\subsubsection{CS Series HEV: Propel Case - Use Subcase}

The third subcase is the 'Use' subcase. In this subcase, the energy stored during the 'Store' subcase is reused to meet the electrical power load at the HV bus. Figure 23 illustrates this case.

$$
\text { Propel }{ }_{\text {use }}: F_{\text {Tr }}>0, P_{\text {gen,elec }}<\left(P_{\text {acc }}+P_{\text {mot,elec }}\right)
$$

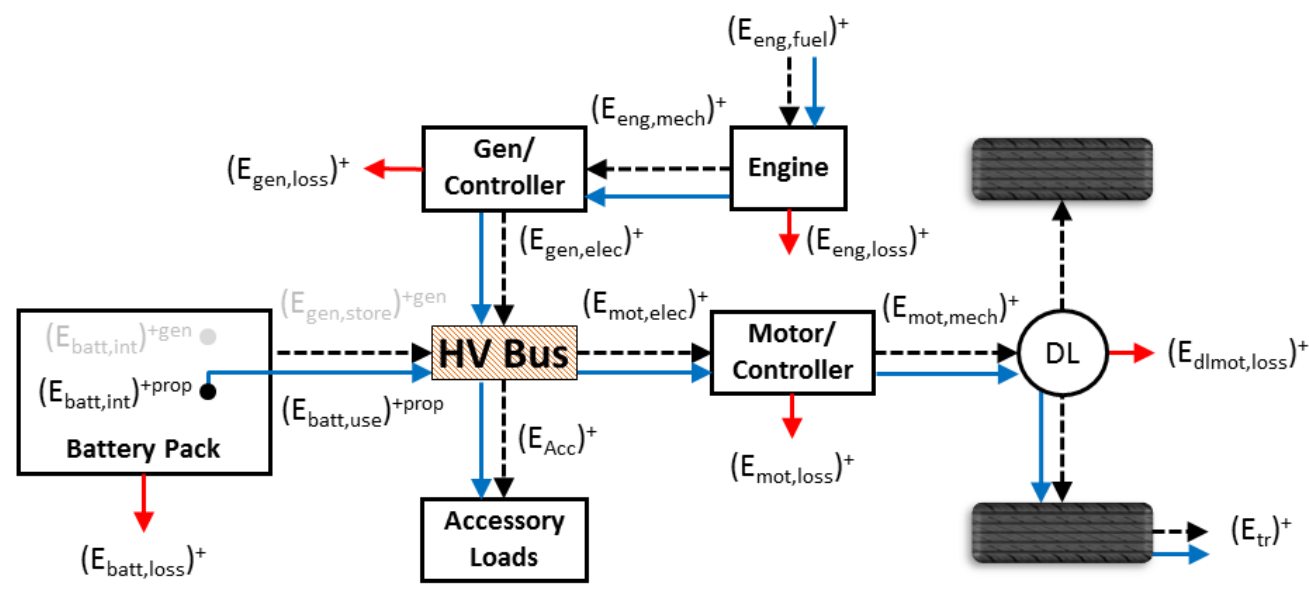

Figure 23: CS Series HEV energy flow diagram for the propel case during the 'use' subcase 
For a CS HEV, all the energy stored during the 'Store' subcase must be expended during the 'Use' subcase. This brings us to Equation 53, the conservation of energy equation that is necessary for charge balance over the propel case. The superscripts '+prop' and '+gen' correspond to the 'Use' and 'Store' subcases. Also note that the energy stored during the Store subcase is inherently negative due to the sign convention.

Equation 53: Conservation of energy in the battery to maintain charge balance (Series, propel)

$$
\left(E_{\text {batt }, \text { int }}\right)^{+ \text {prop }}=-\left(E_{\text {batt }, \text { int }}\right)^{+ \text {gen }}
$$

\subsubsection{CS Series HEV: Propel Case - Power Split Fraction}

Summing power over an entire drive cycle, some amount of energy was used directly to meet the load at the HV bus, while the rest was stored in the battery first before being used. A sum of energies at the HV bus is illustrated by Figure 24.

\section{Power Split Fraction - Energy Balance at HV Bus}

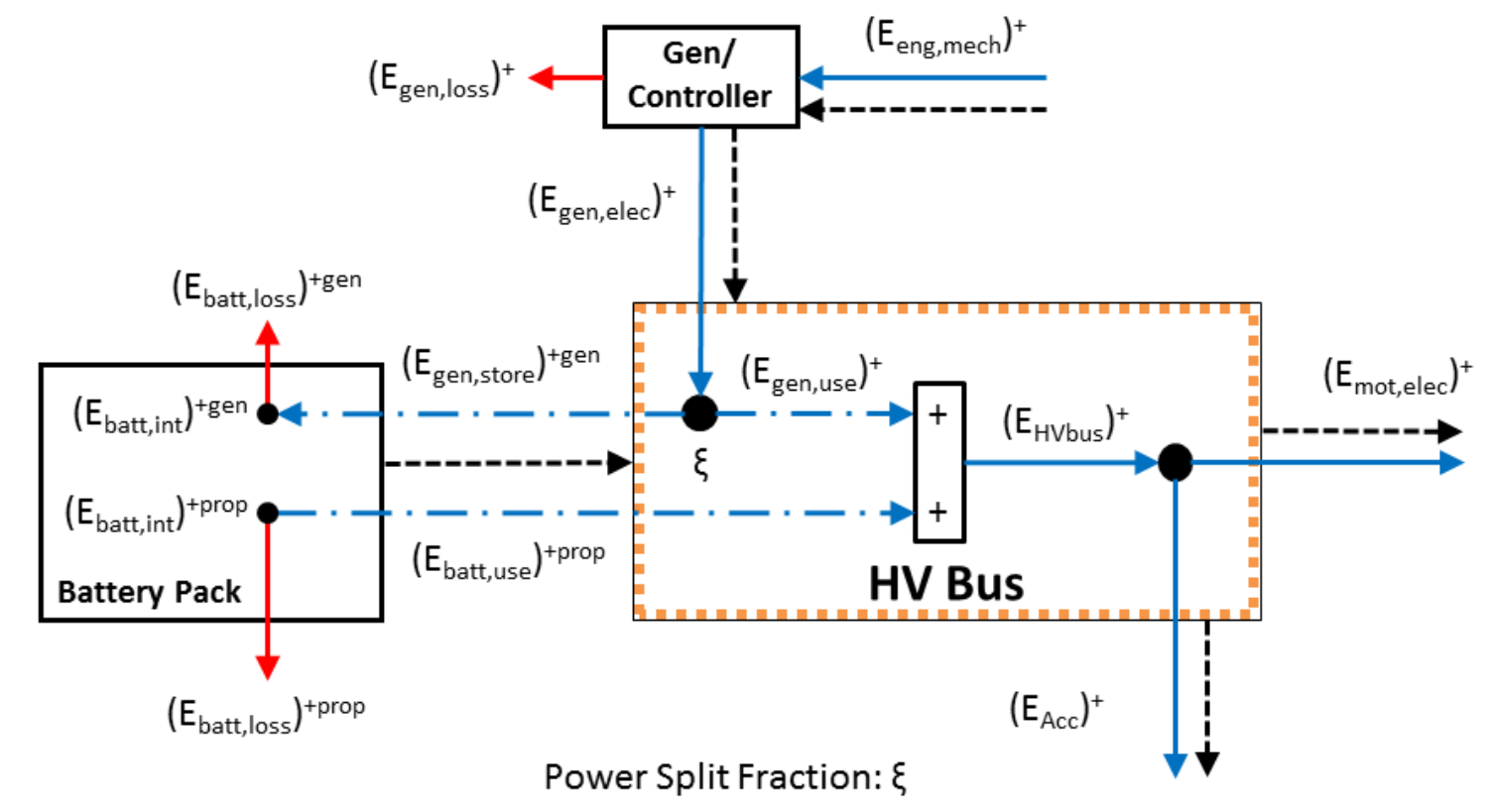

Figure 24: Energy balance at the HV bus for the Power Split Fraction

The four key parameters for analyzing the Power Split Fraction are $\left(E_{\text {gen,elec }}\right)^{+},\left(E_{\text {gen,use }}\right)^{+},\left(E_{\text {gen,store }}\right)^{+ \text {gen }}$, and $\left(E_{\text {batt,use }}\right)^{+ \text {prop }}$. These parameters are defined in the list of nomenclature in the front matter of this thesis, but a more thorough description is provided in Table 10. 
Table 10: Description of energy quantities key to the Power Split Fraction for the Series HEV

\begin{tabular}{|l|l|l|}
\hline Term & $\begin{array}{l}\text { Inherent } \\
\text { Sign }\end{array}$ & Definition \\
\hline$\left(\mathrm{E}_{\text {gen,elec }}\right)^{+}$ & + & $\begin{array}{l}\text { Electrical energy out of the generator during the propel case. This is the total } \\
\text { energy required from the generator to meet tractive energy requirements along } \\
\text { with all losses and the accessory load. }\end{array}$ \\
\hline$\left(\mathrm{E}_{\text {gen,use }}\right)^{+}$ & + & $\begin{array}{l}\text { Electrical generator energy directly used for propulsion. As } \xi \text { goes to } 1 \text {, this } \\
\text { quantity converges to }\left(\mathrm{E}_{\text {gen,elec }}\right)^{+}\end{array}$ \\
\hline$\left(\mathrm{E}_{\text {gen,store }}\right)^{+\mathrm{gen}}$ & - & $\begin{array}{l}\text { Electrical generator energy stored before being used for propulsion. This energy } \\
\text { is recirculated before being used to meet the load at the HV bus. }\end{array}$ \\
\hline$\left(\mathrm{E}_{\text {batt,use }}\right)^{+ \text {prop }}$ & + & $\begin{array}{l}\text { Electrical energy at the terminals to be used in propulsion. This energy was first } \\
\text { stored in the battery and this is the second half of the recirculation path. The sum } \\
\left.\text { of }\left(\mathrm{E}_{\text {batt,use }}\right)^{+ \text {prop }} \text { and ( } \mathrm{E}_{\text {gen,use }}\right)^{+} \text {must meet the energy required at the HV bus. This } \\
\text { does not include the energy reused from stored regen braking energy. That energy } \\
\text { is accounted for in a separate case for clarity. }\end{array}$ \\
\hline
\end{tabular}

The amount of energy that is directly used is described by the Power Split Fraction $(\xi)$ as defined by Equation 54 . For $\xi=1$, all energy created by the genset goes directly to meeting the load at the HV bus and $\left(E_{\text {gen,use }}\right)^{+}=\left(E_{\text {gen,elec }}\right)^{+}$.

Equation 54: Power Split Fraction, genset energy directly used (Series, propel)

$$
\begin{gathered}
\tilde{\xi}=\frac{\left(E_{\text {gen,use }}\right)^{+}}{\left(E_{\text {gen }, \text { elec }}\right)^{+}} \\
\text {Restated: } \\
\left(E_{\text {gen }, \text { use }}\right)^{+}=\tilde{\xi}\left(E_{\text {gen,elec }}\right)^{+}
\end{gathered}
$$

Related to Equation 54, the amount of energy stored in the battery before being used is defined in Equation 55. As the Power Split Fraction drops, more energy is recirculated through the battery before being used. For $\xi=0$, all energy produced by the genset is stored in the battery before being used. As soon as the cycle begins, accessory loads are present meaning there is a load at the HV bus. And if there is any load on the HV bus, some of the power produced by the genset will go directly to meeting that load. Therefore for Power Split Fraction to truly be zero, the genset would have to deposit all of the energy required for the cycle into the battery at the initial instant of the cycle. This is obviously not possible. So we see that the upper and lower limits of $\xi$ cannot be feasibly reached in actual operation.

\section{Equation 55: Power Split Fraction, genset energy recirculated (Series, propel)}

$$
\left(E_{\text {gen }, \text { store }}\right)^{+}=(-1)(1-\tilde{\xi})\left(E_{\text {gen }, \text { elec }}\right)^{+}
$$

As $\xi$ falls, more energy is cycled through the battery and the genset has more freedom to operate. This usually translates to a higher cycle average genset efficiency (not necessarily higher system efficiency). The tradeoff is the extra losses incurred by storing energy in the battery and then taking it back out. If the extra increase in genset efficiency does not offset the extra recirculation losses, then $\xi$ is too low and more genset energy should be used directly for propulsion. For a Series HEV, however, the recirculation path is very short and only incurs two extra losses (battery charge and discharge losses). 
As the battery is used to buffer more energy, $\xi$ will decrease and the genset efficiency will increase. There is obviously a correlation between $\xi$ and the genset efficiency, but it is unclear what exactly that correlation is. This correlation is explored and discussed in greater detail later in this thesis.

\subsubsection{CS Series HEV: Propel Case - Energy Consumption}

The energy consumption equation is built in the same manner described previously in the EV section. Instead of battery energy, though, the Series HEV uses fuel energy so the equation is developed starting at the fuel input to the engine. Applying engine and generator efficiencies, we have the following equation for genset output:

$$
\left(E_{\text {eng,fuel }}\right)^{+}=\frac{\left(E_{\text {gen }, \text { elec }}\right)^{+}}{\left(\tilde{\eta}_{\text {eng }}^{+}\right)\left(\tilde{\eta}_{\text {gen }}^{+}\right)}
$$

Next, an expression for the Power Split Fraction is required. This expression must relate to genset output power, accessory load and motor input power and include the Power Split Fraction. To develop this expression we begin with Equation 56, the conservation of energy equation for the HV bus:

\section{Equation 56: Conservation of energy at the HV bus (Series, propel)}

$$
\left(E_{\text {gen }, \text { use }}\right)^{+}+\left(E_{\text {batt }, \text { use }}\right)^{+ \text {prop }}=\left(E_{\text {mot }, \text { elec }}\right)^{+}+\left(E_{\text {Acc }}\right)^{+}
$$

We must now relate $\left(E_{\text {batt,use }}\right)^{+ \text {prop }}$ back to $\left(E_{\text {gen,elec }}\right)^{+}$. We do this by starting with Equation 53 and applying battery charge and discharge efficiencies to arrive at:

$$
\frac{\left(E_{\text {batt }, \text { use }}\right)^{+}}{\tilde{\eta}_{\text {batt }}^{+ \text {prop }}}=\left(E_{\text {gen,store }}\right)^{+}\left(\tilde{\eta}_{\text {batt }}^{+ \text {gen }}\right)
$$

Substituting Equation 55, and rearranging we arrive at Equation 57, the equation for energy used from the battery during the propel case (not including regen braking energy that is reused).

Equation 57: Energy from the battery into the HV bus during the propel case (Series, propel)

$$
\left(E_{\text {batt }, \text { use }}\right)^{+ \text {prop }}=(1-\tilde{\xi})\left(E_{\text {gen }, \text { elec }}\right)^{+}\left(\tilde{\eta}_{\text {batt }}^{+ \text {gen }}\right)\left(\tilde{\eta}_{\text {batt }}^{+ \text {prop }}\right)
$$

Substituting this equation into Equation 56 and rearranging, we arrive at:

$$
\left(E_{\text {gen }, \text { use }}\right)^{+}=\left(E_{\text {mot }, \text { elec }}\right)^{+}+\left(E_{\text {Acc }}\right)^{+}-(1-\tilde{\xi})\left(E_{\text {gen }, \text { elec }}\right)^{+}\left(\tilde{\eta}_{\text {batt }}^{+ \text {gen }}\right)\left(\tilde{\eta}_{\text {batt }}^{+ \text {prop }}\right)
$$

Using Equation 54, we can relate $\left(E_{\text {gen,use }}\right)^{+}$back to $\left(E_{\text {gen,elec }}\right)^{+}$. After combining terms and rearranging we have Equation 58, the energy required from the genset to complete the cycle for a given $\xi$.

Equation 58: Energy supplied by the generator (Series, propel)

$$
\left(E_{\text {gen }, \text { elec }}\right)^{+}=\frac{\left(E_{\text {mot }, \text { elec }}\right)^{+}+\left(E_{\text {Acc }}\right)^{+}}{\tilde{\xi}+(1-\tilde{\xi})\left(\tilde{\eta}_{\text {batt }}^{+ \text {gen }}\right)\left(\tilde{\eta}_{\text {batt }}^{+ \text {prop }}\right)}
$$

Applying the remaining component efficiencies down the powertrain, we have Equation 59, the energy consumption equation for the propel case of the Series HEV. Notice that the Power Split Fraction only affects the battery charge and discharge efficiencies. All other component efficiencies are always present regardless of the Power Split Fraction because all the energy must flow through these components. For example, all energy required for the cycle must flow through the motor and driveline. There are no 
divergent paths to divert energy flow around these components so these component efficiencies always take full effect.

$$
\begin{gathered}
\text { Equation 59: Propel case fuel energy consumption (Series, propel) } \\
\left(E_{\text {eng,fuel }}\right)^{+}=\frac{1}{\left(\tilde{\eta}_{\text {eng }}^{+}\right)\left(\tilde{\eta}_{\text {gen }}^{+}\right)\left(\tilde{\xi}+(1-\tilde{\xi})\left(\tilde{\eta}_{\text {batt }}^{+g e n}\right)\left(\tilde{\eta}_{\text {batt }}^{+ \text {prop }}\right)\right)}\left[\frac{\left(E_{t r}\right)^{+}}{\left(\tilde{\eta}_{\text {mot }}^{+}\right)\left(\tilde{\eta}_{d l}^{+}\right)}+\left(E_{\text {Acc }}\right)^{+}\right]
\end{gathered}
$$

The powertrain efficiency for the propel case may now be defined using Equation 60 . Because all the energy required for the drive cycle now comes from the fuel tank, fuel energy is used to calculate the powertrain efficiency rather than battery energy. Note that this does not account for any energy recovered and reused via regen braking. Regen braking energy is considered separately in a different case and related back to fuel-equivalent energy. It is inherently negative (similar to internal battery energy for brake case for an EV) and is summed with idle and propel fuel use in the DCnet case to find overall fuel consumption. Appendix $\mathrm{F}$ fully documents all of the efficiencies and equations needed for the propel case for the Series HEV.

Equation 60: Propel case powertrain efficiency (Series, propel)

$$
\tilde{\eta}_{p t}^{+}=\frac{\left(E_{\text {tr }}\right)^{+}}{\left(E_{\text {eng,fuel }}\right)^{+}}
$$

\subsubsection{CS Series HEV: Brake Case}

The brake case for the Series HEV is identical to the EV because the electric powertrain is exactly the same. Therefore, the energy consumption equation for the brake case is expressed by Equation 32. Like the $\mathrm{EV}$, this energy will be reused later in propulsion. Refer to Appendix $\mathrm{B}$ for full documentation of the brake case.

$$
\frac{\text { Equation 32: Brake case energy consumption (EV, brake) }}{\left(E_{\text {batt }, \text { int }}\right)^{-}=\tilde{\eta}_{\text {batt }}^{-}\left\{\left(E_{t r}\right)^{-}(\tilde{\zeta})\left(\tilde{\eta}_{d l, m o t}^{-}\right)\left(\tilde{\eta}_{m o t}^{-}\right)+\left(E_{A c c}\right)^{-}\right\}}
$$

While it is plausible that the genset could be operating and creating power during the brake case, this case is modeled as if the engine is off for the entirety of the brake case. Because this model deals only with 'lumps' of energy as calculated by the Sovran coefficients, it does not matter if energy created by the engine is applied during the propel case or brake case. Therefore, all engine energy is accounted for separately in the propel case to maintain clarity. Keep in mind, however, that the generator could be undersized and be forced to operate during the brake case to maintain charge balance.

\subsubsection{CS Series HEV: Regen Energy Reuse}

To properly account for regen braking energy stored in the battery during the brake case, a new subcase is created and designated by the superscript $(-/+)$. This subcase is separated from the rest of the propel case to ensure charge balanced is properly maintained. Also, keeping this energy separate allows the user to directly examine the effect of regen braking on the overall fuel consumption of the vehicle. To maintain charge balance for a CS Series HEV, all energy stored in the battery from regen braking must be reused at some point in the drive cycle, as expressed in Equation 61. This expression is necessary since the energy captured during the brake case is inherently negative but must be inherently positive when reused later. 
Equation 61: Conservation of energy for regen energy capture and reuse (Series, regen reuse)

$$
\left(E_{\text {batt }, \text { int }}\right)^{-}=-\left(E_{\text {batt }, \text { int }}\right)^{-/+}
$$

Figure 25 illustrates regen brake energy being reused for propulsion. Energy works from the battery internals out to the wheels. Once at the wheels, this energy is equivalent to tractive energy and could be considered as a 'credit' against the tractive energy required to complete the cycle. This tractive energy credit would be subtracted from the tractive energy required to complete the propel case which would in turn reduce the fuel energy required to complete the cycle. This approach is valid, but this author goes one step further by taking this tractive energy credit and working back up the powertrain to the fuel tank to find the fuel-equivalent regen braking energy credit. This approach will be discussed further in the next section.

\section{Regen Energy Reuse: $\mathrm{F}_{\mathrm{Tr}}>0$}

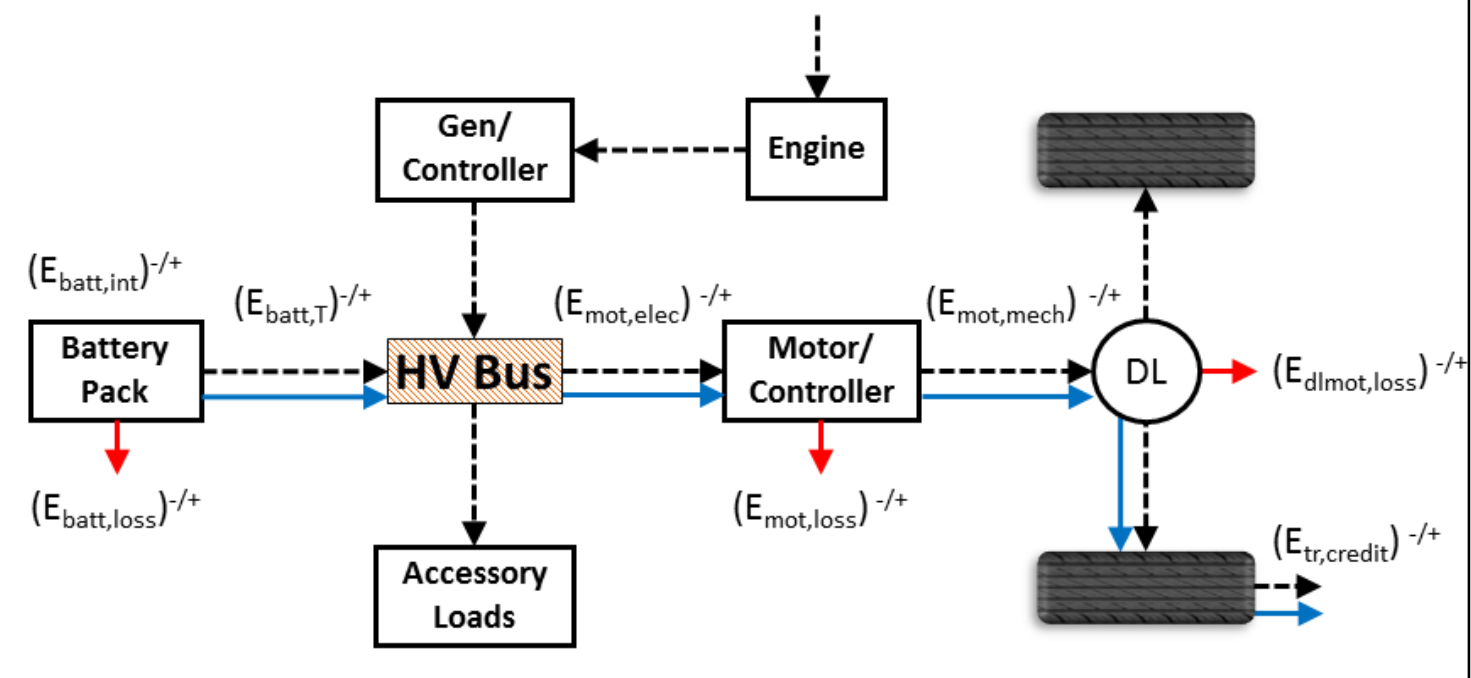

Figure 25: CS Series HEV energy flow diagram for regen energy reuse

The energy consumption equation for this case is essentially identical to the propel case for an EV. Different component efficiencies could be used, but for simplicity they are considered equivalent to the propel component efficiencies as illustrated by Equation 62.

Equation 62: Motor propel efficiency (Series, regen reuse)

$$
\tilde{\eta}_{m o t}^{-/+}=\frac{\left(E_{\text {mot }, \text { mech }}\right)^{-/+}}{\left(E_{\text {mot,elec }}\right)^{-/+}} \approx \tilde{\eta}_{\text {mot }}^{+}
$$

For the EV propel case, $\left(E_{\text {batt,int }}\right)^{+}$is of interest, but for this case $\left(E_{t r, c r e d i t}\right)^{-/ t}$ is of interest. Hence, the energy consumption equation is developed by starting at the wheels and working back up the powertrain to the battery applying component efficiencies and finding losses along the way. The same method used for 
developing previous energy consumption equations is also used here, but instead of starting at the battery or fuel tank, we start at the wheels. Equation 63 is the end result of this process and defines the equivalent tractive energy that would be saved by using regen braking. Notice that the internal battery energy term refers to the battery energy stored in the brake case, corresponding with Equation 61. Notice also that the efficiencies carry the $(-/+)$ superscript and are specific to the regen energy reuse case, but are approximated as propel component efficiencies in practice. Equation 64 also expresses this regen energy credit in terms of losses.

Equation 63: Equivalent tractive energy 'credit' from regen braking (Series, regen reuse)

$$
\left(E_{\text {tr }, \text { credit }}\right)^{-/+}=-\left(E_{\text {batt }, \text { int }}\right)^{-}\left(\tilde{\eta}_{\text {batt }}^{-/+}\right)\left(\tilde{\eta}_{\text {mot }}^{-/+}\right)\left(\tilde{\eta}_{\text {dl,mot }}^{-/+}\right)
$$

Equation 64: Equivalent tractive energy 'credit' from regen in terms of losses (Series, regen reuse)

$$
\left(E_{\text {tr,credit }}\right)^{-/+}=\left(E_{\text {batt }, \text { int }}\right)^{-/+}-\left(E_{\text {pt,loss }}\right)^{-/+}=-\left(E_{\text {batt }, \text { int }}\right)^{-}-\left(E_{\text {pt loss }}\right)^{-/+}
$$

The powertrain efficiency for this case is described by Equation 65 and should be very similar to the propel powertrain efficiency for an EV. However, there is no accessory load for this the regen energy reuse case because all propel accessory energy has already been accounted for in propel case for the CS Series HEV. Therefore, the efficiency should be slightly higher for the regen energy reuse case.

\section{Equation 65: Powertrain efficiency for regen energy reuse (Series, regen reuse)}

$$
\tilde{\eta}_{p t}^{-/+}=\frac{\left(E_{\text {tr,credit }}\right)^{-/+}}{\left(E_{\text {batt }, \text { int }}\right)^{-/+}}
$$

\subsubsection{CS Series HEV: Fuel-Equivalent Regen Energy Credit}

As previously mentioned, all regen energy captured during a given cycle must also be expended during that cycle to maintain charge balance. When accounting for the reuse of this energy, an equivalent tractive credit may be calculated to show how much tractive energy may be saved by taking advantage of regen braking. The vehicle, then, would not require fuel energy to meet this amount of tractive energy and the total fuel required for the cycle would be reduced. Thus, the tractive energy that would result from using stored regen braking energy can be 'credited' against the propel tractive energy required to complete the cycle.

Taking the concept of an energy credit one step farther, however, we can directly determine the amount of fuel energy saved by using regen braking. We can do this by calculating the fuel energy required to produce the same tractive energy credit that has already been calculated. A similar approach is also used in [32]. Thus, we define a new case for calculating the fuel-equivalent regen energy credit or regen fuel credit. This case is designated by the (EqFC) superscript, which is short for "equivalent fuel consumption". For this theoretical case, energy flows from the wheels back through the powertrain and out of the engine to produce fuel as shown in Figure 26. The end result is a negative quantity of fuel that may be summed later in the DCnet case. This approach is very similar to the EV case where propel and brake energy are summed in the DCnet case. Of course, the engine cannot produce fuel, but this approach demonstrates a parallel with the EV powertrain and shows explicitly and directly how much fuel energy is saved via regen braking.

By design, this case exactly mimics the propel case because the regen fuel credit is the amount of fuel that would otherwise be used during the propel case. Hence, the energy flow shown in Figure 26 is exactly the opposite of the propel case with the exception of accessory load. No accessory load is 
considered in the regen fuel credit case because all propel accessory load has already been considered. Because this case exactly mimics the propel case, all component efficiencies necessarily must be identical to the propel component efficiencies. Thus, all the component efficiencies documented for this case in Appendix I are direct references to propel component efficiencies.

\section{Regen Energy Reuse: Fuel-Equivalent Energy Credit}

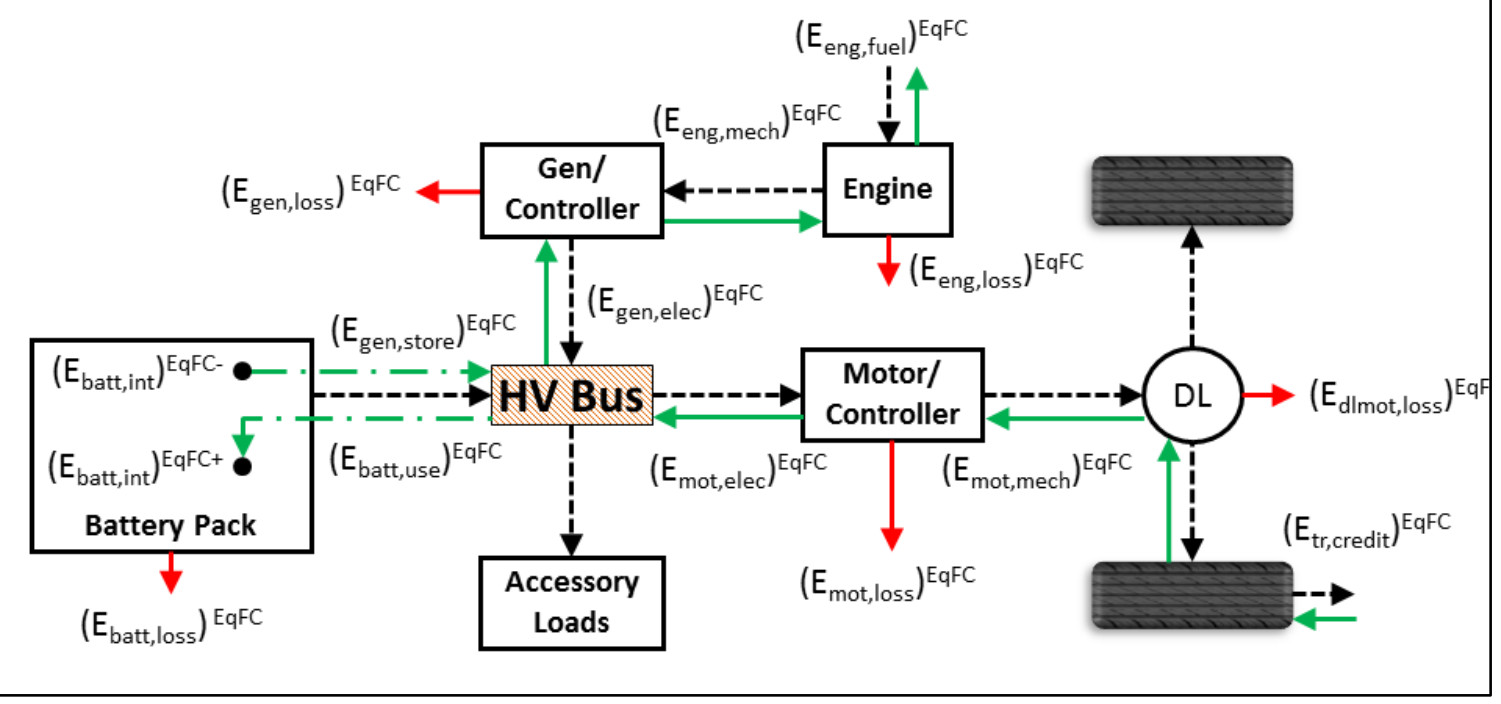

Figure 26: CS Series HEV energy flow diagram for regen fuel credit

To keep sign conventions consistent, a conservation of energy equation is written for the tractive energy credit, as shown in Equation 66. The tractive energy credit for the regen energy reuse case is inherently positive. This equation now dictates that the tractive energy credit for the regen fuel credit case will be inherently negative, as it should be.

\section{Equation 66: Conservation of energy for regen fuel credit (Series, regen fuel credit)}

$$
\left(E_{\text {tr,credit }}\right)^{E q F C}=-\left(E_{\text {tr,credit }}\right)^{-/+}
$$

Since the regen fuel credit case duplicates the propel case, we will start from the propel case to develop the energy consumption equation. Equation 59 gives the energy consumption equation for the propel case.

\section{Equation 59: Propel case fuel energy consumption (Series, propel)}

$$
\left(E_{\text {eng,fuel }}\right)^{+}=\frac{1}{\left(\tilde{\eta}_{\text {eng }}^{+}\right)\left(\tilde{\eta}_{\text {gen }}^{+}\right)\left(\tilde{\xi}+(1-\tilde{\xi})\left(\tilde{\eta}_{\text {batt }}^{+ \text {gen }}\right)\left(\tilde{\eta}_{\text {batt }}^{+ \text {prop }}\right)\right)}\left[\frac{\left(E_{\text {tr }}\right)^{+}}{\left(\tilde{\eta}_{\text {mot }}^{+}\right)\left(\tilde{\eta}_{d l}^{+}\right)}+\left(E_{\text {Acc }}\right)^{+}\right]
$$

Only minor modifications are required to adapt this equation to the regen fuel credit case. The accessory energy may be set to zero and eliminated and the fuel energy and tractive energy quantities are changed to reflect this case: 


$$
\left.\left(E_{\text {eng,fuel }}\right)^{E q F C}=\frac{1}{\left(\tilde{\eta}_{\text {eng }}^{+}\right)\left(\tilde{\eta}_{\text {gen }}^{+}\right)\left(\xi+(1-\xi)\left(\tilde{\eta}_{\text {batt }}^{+ \text {gen }}\right)\left(\tilde{\eta}_{\text {batt }}^{+ \text {prop }}\right)\right)}\left[\frac{\left(E_{\text {tr,credit }}\right)^{E q F C}}{\left(\tilde{\eta}_{\text {mot }}^{+}\right)\left(\tilde{\eta}_{\text {dl }}^{+}\right)}+\right)^{ \pm}\right]
$$

Substituting Equation 66 to put the regen fuel credit in terms of the already known tractive energy credit, we arrive at Equation 67, the energy consumption equation for the regen fuel credit. Full documentation of this case including all terms, efficiencies and equations is included in Appendix I.

Equation 67: Fuel-equivalent regen energy 'credit' (Series, regen fuel credit)

$$
\left(E_{\text {eng,fuel }}\right)^{E q F C}=\frac{1}{\left(\tilde{\eta}_{\text {eng }}^{+}\right)\left(\tilde{\eta}_{\text {gen }}^{+}\right)\left(\tilde{\xi}+(1-\tilde{\xi})\left(\tilde{\eta}_{\text {batt }}^{+ \text {gen }}\right)\left(\tilde{\eta}_{\text {batt }}^{+ \text {prop }}\right)\right)}\left[\frac{-\left(E_{\text {tr,credit }}\right)^{-/+}}{\left(\tilde{\eta}_{\text {mot }}^{+}\right)\left(\tilde{\eta}_{\text {dl }}^{+}\right)}\right]
$$

One caveat to this case is the losses. Losses are always positive except in this theoretical case where they are negative. As an example, the equations for motor losses for the propel and regen fuel credit cases are shown in Equation 68 and Equation 69. Note that the two equations are identical, but because the inherent sign is reversed, the motor loss will now be negative. Losses for the regen fuel credit case will be important to calculate correct overall losses and will be discussed further in the DCnet case section.

$$
\frac{\text { Equation 68: Motor losses (Series, propel) }}{\left(E_{m o t, l o s s}\right)^{+}=\left(E_{m o t, e l e c}\right)^{+}-\left(E_{m o t, m e c h}\right)^{+}}
$$

Equation 69: Motor losses (Series, regen fuel credit)

$$
\left(E_{\text {mot }, \text { loss }}\right)^{E q F C}=\left(E_{\text {mot,elec }}\right)^{E q F C}-\left(E_{\text {mot }, \text { mech }}\right)^{E q F C}
$$

\subsubsection{CS Series HEV: Idle Case}

Building off the idea of fuel-equivalent energy use, the accessory energy required for the idle case is also calculated as fuel-equivalent energy. Like the regen fuel credit, this approach allows the user to directly examine the impact of fuel energy. For this approach, all energy consumed by accessories during the idle case is assumed to come from the engine, as illustrated by Figure 27. For the Series HEV, all accessories are assumed to be electrical. Therefore, some fraction of the electrical energy will likely be supplied directly by the battery - possibly pulling from energy stored from regen braking. However, all energy consumed by the vehicle must ultimately come from the fuel tank. Therefore it is acceptable to make the simplifying assumption that the genset supplies all accessory energy for the idle case. 


\section{Idle: $\mathrm{F}_{\mathrm{Tr}}=0$}

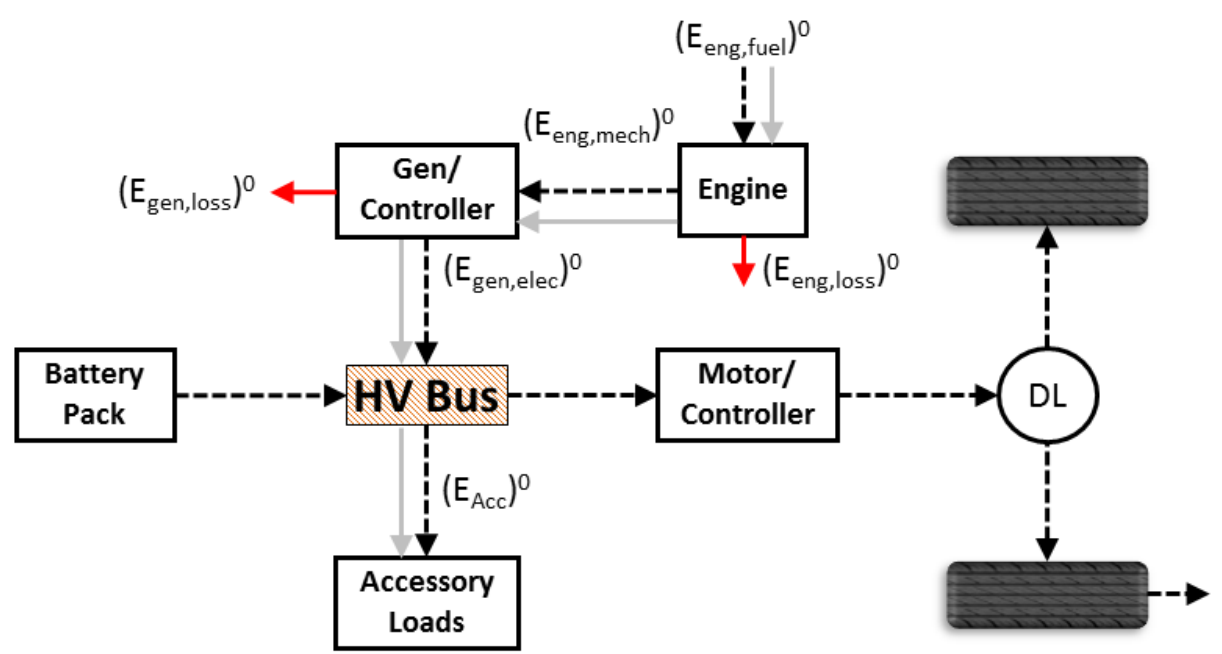

Figure 27: CS Series HEV energy flow diagram for the idle case

As before, the total idle accessory energy is defined by Equation 36, which depends on the vehicle accessory load and amount of idle time. These two parameters are characteristic of the drive cycle only and are independent of vehicle properties. The only two component efficiencies in play for the idle case are the generator and engine efficiencies. These efficiencies are specified as separate and distinct efficiencies, but are approximated with the cycle average efficiencies used for the propel case. Equation 70 gives the energy consumption equation for the idle case in terms of fuel energy.

Equation 70: Idle case energy consumption (Series, idle)

$$
\left(E_{\text {eng,fuel }}\right)^{0}=\frac{\left(E_{\text {gen }, \text { elec }}\right)^{0}}{\left(\tilde{\eta}_{\text {eng }}^{0}\right)\left(\tilde{\eta}_{\text {gen }}^{0}\right)}=\frac{\left(E_{\text {Acc }}\right)^{0}}{\left(\tilde{\eta}_{\text {eng }}^{0}\right)\left(\tilde{\eta}_{\text {gen }}^{0}\right)}
$$

\subsubsection{CS Series HEV: DC Net Case}

The terminology for the net case (DCnet) is retained for the Series HEV even though the powertrain does not have the capability to recharge and therefore does not have an ACnet case. The DCnet case is still useful to sum energy consumption from the various cases previously defined. However, the Series HEV has two energy sources (fuel tank and battery) whereas the EV has only one. For this reason, summing the cases is no longer straightforward. But since fuel-equivalent energy has been calculated for the propel brake and idle cases, the cases may be summed outright as shown by Equation 71. Instead of a direct energy benefit from the brake case as in the EV powertrain, there is now a more roundabout energy benefit from storing, then reusing regen energy in the regen credit case. The total energy consumed by the Series HEV over the cycle can also be calculated by summing road load energy, accessory energy and all the losses, as shown in Equation 72. 


$$
\frac{\text { Equation 71: Total fuel energy calculated by case (Series, DCnet) }}{\left(E_{\text {eng,fuel }}\right)^{\text {DCnet }}=\left(E_{\text {eng,fuel }}\right)^{+}+\left(E_{\text {eng,fuel }}\right)^{0}+\left(E_{\text {eng,fuel }}\right)^{E q F C}}
$$

Equation 72: Total fuel energy calculated from overall energy balance (Series, DCnet)

$$
\left(E_{\text {eng,fuel }}\right)^{\text {DCnet }}=\left(E_{\text {tr }}\right)^{\text {DCnet }}+\left(E_{\text {ACC }}\right)^{\text {DCnet }}+\left(E_{\text {pt }, \text { loss }}\right)^{\text {DCnet }}
$$

Because regen energy reuse was considered separately, the propel case consumed more energy than would actually be consumed. The difference is made up in the DCnet case where the propel energy is summed with the inherently negative regen fuel credit. However, the losses for the propel case are still too high and have not yet been corrected. Thus, the inherently negative losses for the regen fuel credit case must be summed with the losses from the propel case to find the losses that will actually be experienced by the powertrain. As an example, the net losses for the battery are shown in Equation 73. The battery incurs losses in the propel, brake, regen energy reuse and regen energy credit cases. However, the regen energy credit case is only a theoretical case and results in a negative amount of fuel energy. Likewise, the losses are also inherently negative and theoretical but must be summed with the losses from the other cases to maintain conservation of energy. The other equations for net component losses may be found in Appendix $\mathrm{K}$ with the rest of the equations used in the DCnet case for the Series HEV. The total losses from each case may be summed to find the total net losses for the entire drive cycle, as shown in Equation 74.

Equation 73: Net battery losses (Series, DCnet)

$$
\begin{gathered}
\left(E_{\text {batt }, \text { loss }}\right)^{\text {DCnet }}=\left(E_{\text {batt }, \text { loss }}\right)^{+}+\left(E_{\text {batt }, \text { loss }}\right)^{-}+\left(E_{\text {batt loss }}\right)^{-/+}+\left(E_{\text {batt,loss }}\right)^{E q F C} \\
\left(E_{p t, \text { loss }}\right)^{\text {DCnet }}= \\
\text { Equation 74: Net powertrain losses (Series, DCnet) } \\
\left(E_{p t, l o s s}\right)^{+}+\left(E_{p t, l o s s}\right)^{-}+\left(E_{p t, l o s s}\right)^{0}+\left(E_{p t, l o s s}\right)^{-/+}+\left(E_{p t, l o s s}\right)^{E q F C}
\end{gathered}
$$

Now knowing total fuel energy expended, an overall powertrain efficiency is defined in Equation 75. However, the Series HEV has two somewhat distinct powertrains. Both use the same electric motor for propulsion, but one uses the battery as an energy source while the other use the fuel tank. Hence, it is also interesting to separately examine efficiencies of each powertrain. The efficiency for series mode has already been defined during the propel case by Equation 60. Likewise, the efficiency for EV mode has been defined during the regen energy reuse case by Equation 65. Combining these two modes of operation, we now define the efficiency for the net combined propel mode with Equation 76 . This efficiency considers overall vehicle operation during propel mode including times when the genset is on and energy is coming from the fuel tank and other times when the genset is off and energy is coming from the battery.

Equation 75: Net powertrain efficiency (Series, DCnet)

$$
\tilde{\eta}_{p t}^{\text {DCnet }}=\frac{\left(E_{\text {tr }}\right)^{\text {DCnet }}}{\left(E_{\text {eng,fuel }}\right)^{\text {DCnet }}}
$$

Equation 76: Net powertrain efficiency for combined propel operation (Series, DCnet)

$$
\tilde{\eta}_{\text {pt,propel }}^{\text {DCnet }}=\frac{\left(E_{\text {tr }}\right)^{+}+\left(E_{\text {tr,credit }}\right)^{E q F C}}{\left(E_{\text {eng,fuel }}\right)^{+}+\left(E_{\text {batt }, \text { int }}\right)^{-/+}}
$$


The Series HEV powertrain is much more complex than the EV powertrain - in actual operation as well as in modeling. Therefore it is prudent to establish multiple energy balances, some of which are redundant, to provide sanity checks to the user. An example of such a check is given by Equation 77, which is an energy balance on the battery to ensure charge balance over the entire cycle. This equation is a combination of Equation 53 and Equation 64 (in Appendix $\mathrm{H}$ ) and is therefore partially redundant. The first two terms in the equation account for energy used during the propel case or during CS series mode. If the CS mode of the powertrain is truly charge balanced, these two terms should sum to zero. The other three terms account for regen energy reuse. If all the energy captured in the battery from regen braking is expended in propulsion, these three terms will sum to zero. Additional energy balances have been described in previous sections but can also be found in Appendix $\mathrm{K}$.

$$
\begin{aligned}
& \text { Equation 77: Energy balance for overall charge balance (Series, DCnet) } \\
& \left(E_{\text {batt }, \text { int }}\right)^{+ \text {prop }}+\left(E_{\text {batt }, \text { int }}\right)^{+ \text {gen }}+\left(E_{\text {batt }, \text { int }}\right)^{-}+\left(E_{\text {tr, credit }}\right)^{-/+}+\left(E_{\text {pt,loss }}\right)^{-/+}=0
\end{aligned}
$$

\subsubsection{CS Series HEV: Summary}

The Series HEV proves to be much more complicated than the EV from both an operational and modeling standpoint. Along with this additional complexity, however, comes additional freedom of operation to optimize efficiency. In actual vehicle operation, this will come into play with the hybrid control strategy and the control of the genset. In this model, the dynamic control strategy is represented by a single parameter, the Power Split Fraction.

The Series powertrain also has two energy sources compared the single energy source of the EV powertrain. This introduces challenges with maintaining charge balance as well as properly calculating total energy consumption. Thus, the concept of fuel-equivalent energy use is introduced to relate the energy used or stored in the idle and brake cases back to the propel case. A fuel-equivalent regen energy 'credit' is calculated based upon battery energy stored during the brake case. This regen credit directly shows the benefits of regen braking by relating the energy saved by recovering brake energy to useable fuel energy. Hence, the user will now know exactly how much fuel was saved by enabling regen braking. Likewise, the impact of accessory load can be more directly examined by calculating the equivalent fuel energy.

These ideas will be adapted to the Parallel powertrains presented in the next sections and demonstrated to work in different powertrain layouts. As mentioned previously, PHEV and EREV powertrains may also be considered by coupling the results from this CS powertrain with the results from the EV powertrain.

\subsection{CS P3/P4 Parallel HEV}

The following section outlines the equations and methodology used to predict fuel energy consumption for a CS P3/P4 Parallel HEV. The ' $P$ ' terminology refers to the position of the motor(s) in the powertrain architecture. Table 11 summarizes and explains the terminology and naming system. While the packaging and physical implementation of P3 and P4 parallel HEVs will be significantly different, they are schematically identical. Hence the two architectures are lumped together for energy consumption modeling.

Table 11: Parallel HEV Motor position 'P' terminology summary

\begin{tabular}{|l|l|}
\hline Name & Motor Position \\
\hline P1 & Pre-trans, on accessory side of engine (E.G. Belted Alternator Starter) \\
\hline P2 & Pre-trans, between engine and transmission \\
\hline P3 & Post-trans, sharing a drive axle with the engine \\
\hline P4 & Post-trans, on a separate a drive axle from the engine \\
\hline
\end{tabular}




\subsubsection{CS P3/P4 Parallel HEV: Sign Convention}

The sign convention used for the P3/P4 Parallel powertrain is illustrated in Figure 28. As always, the dashed lines represent positive energy flow. The key difference between the Series HEV and P3/P4 Parallel HEV powertrains is the absence of a generator and a path to the wheels for the engine. This now allows all torque sources to put torque to the wheels in contrast with the series powertrain where only one torque source may propel the vehicle. This also introduces new control dynamics and the ability to split propel torque between the engine and the motor to optimize efficiency. Additional nuances of the P3/P4 Parallel powertrain architecture will be discussed in further depth later in this section.

\section{Sign Conventions}

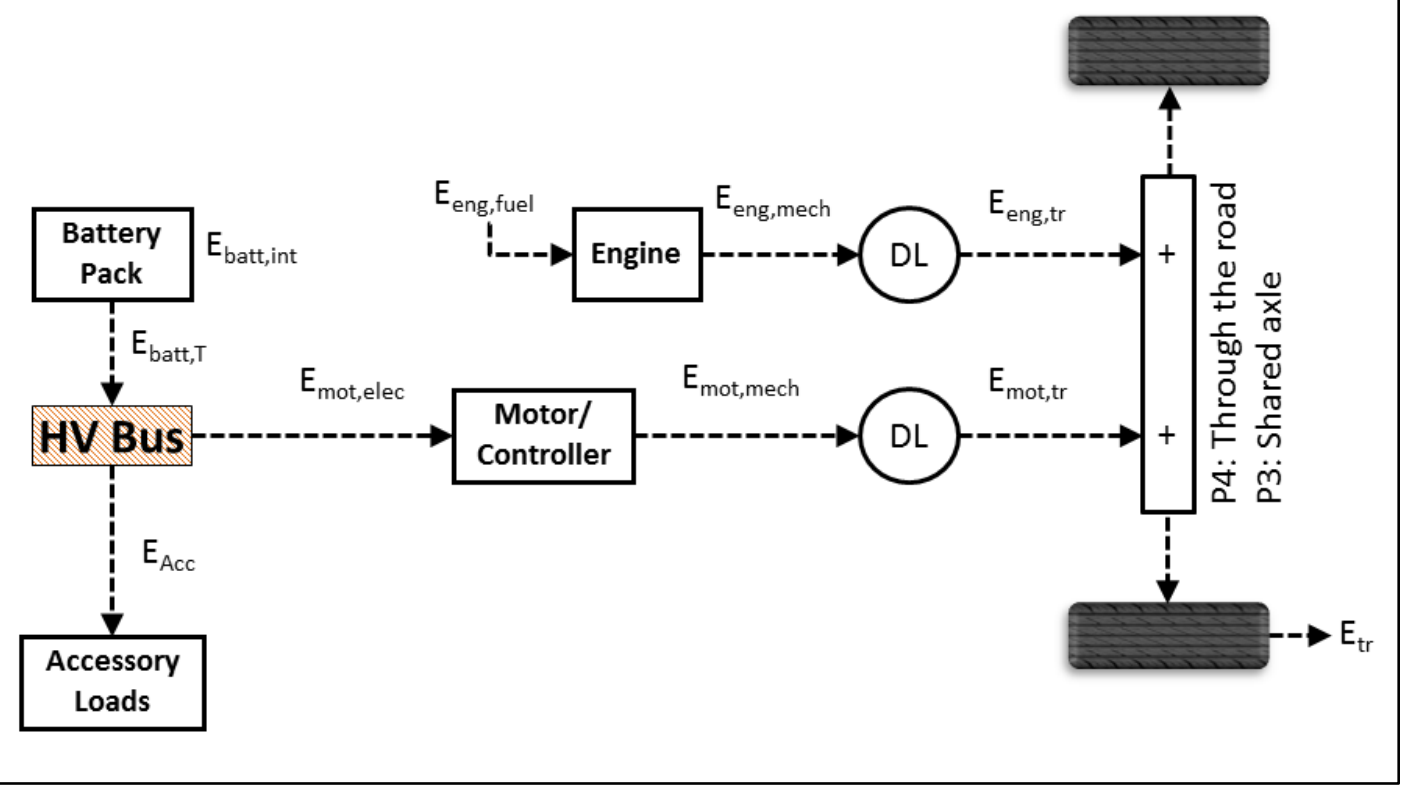

Figure 28: Energy flow sign conventions for a CS P3/P4 Parallel HEV powertrain

\subsubsection{CS P3/P4 Parallel HEV: Propel Case}

The propel case for the P3/P4 Parallel HEV is divided into two subcases: tractive energy and accessory energy. Two new superscripts are introduced to designate these subcases: (+tr) indicates the tractive energy case and (+acc) indicates the accessory energy case. This is done to isolate the accessory load from the effect of the Power Split Fraction. For a Parallel HEV, the accessory load is in the energy recirculation path. Consequently, the Power Split Fraction would affect the total amount of accessory energy consumed for the cycle. For example, if $\xi=0$ no energy would be diverted onto the recirculation path and the accessory load could not be met without depleting battery energy. In actual operation, this is feasible since captured regen energy could be used to meet the accessory load. For this method, however, regen energy is assumed to be used solely for traction. This assumption simplifies calculations and also directly shows the effect of regen energy capture on tractive effort and overall fuel consumption. Likewise, idle case accessory energy is calculated separately and then related back to fuel-equivalent energy. Thus, it makes sense to calculate the propel accessory load in a similar manner. 


\subsubsection{CS P3/P4 Parallel HEV: Propel Case - Tractive Energy Subcase}

The energy flow paths for tractive energy are shown in blue in Figure 29. Energy (shown in blue) flows from the engine to the wheels. Losses for each component are shown also in red and are inherently positive.

\section{Propel (Tractive Energy): $\mathrm{F}_{\mathrm{Tr}}>0$}

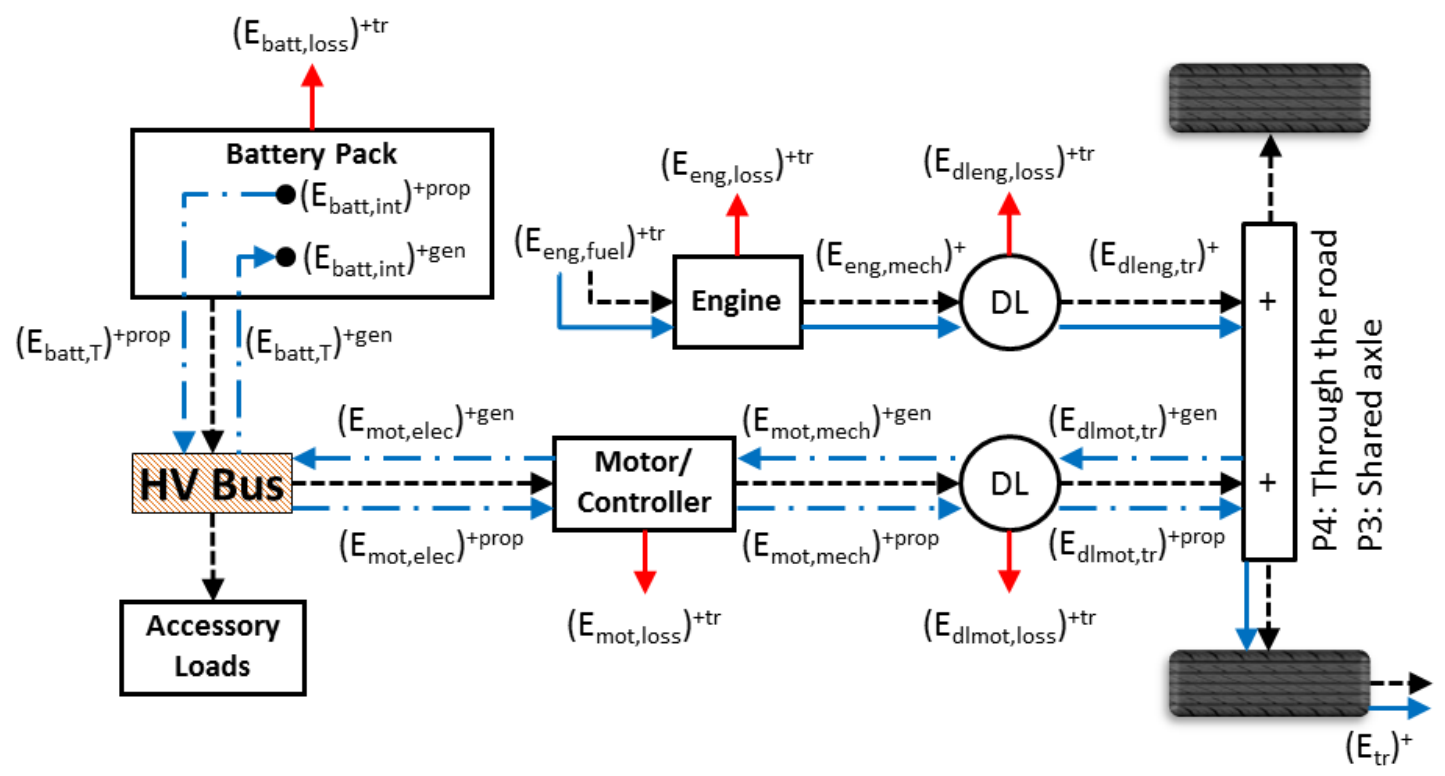

Figure 29: CS P3/P4 Parallel HEV energy flow diagram for the tractive energy in the propel case

Like the Series powertrain, the P3/P4 Parallel powertrain has the operational freedom to produce more (or less) energy than is required to propel the vehicle and use the battery as an energy buffer. With the Series powertrain, the genset is decoupled from the wheels and has 2 degrees of operational freedom (torque and speed). The genset is therefore capable of producing more (or less) electrical energy than was required at the HV bus to propel the vehicle. Thus, the power split occurs at the HV bus node. The P3/P4 Parallel powertrain has two torque sources both coupled to the wheels and both with one degree of freedom (torque). In CS mode, all energy must come from the fuel tank so the engine is the primary torque source. The torque split is achieved by using the drive motor to assist the engine or provide extra load to the engine. Thus, the power split occurs at the drive axle as shown in Figure 30. 


\section{Power Split Fraction - Energy Balance at Drive Axle}

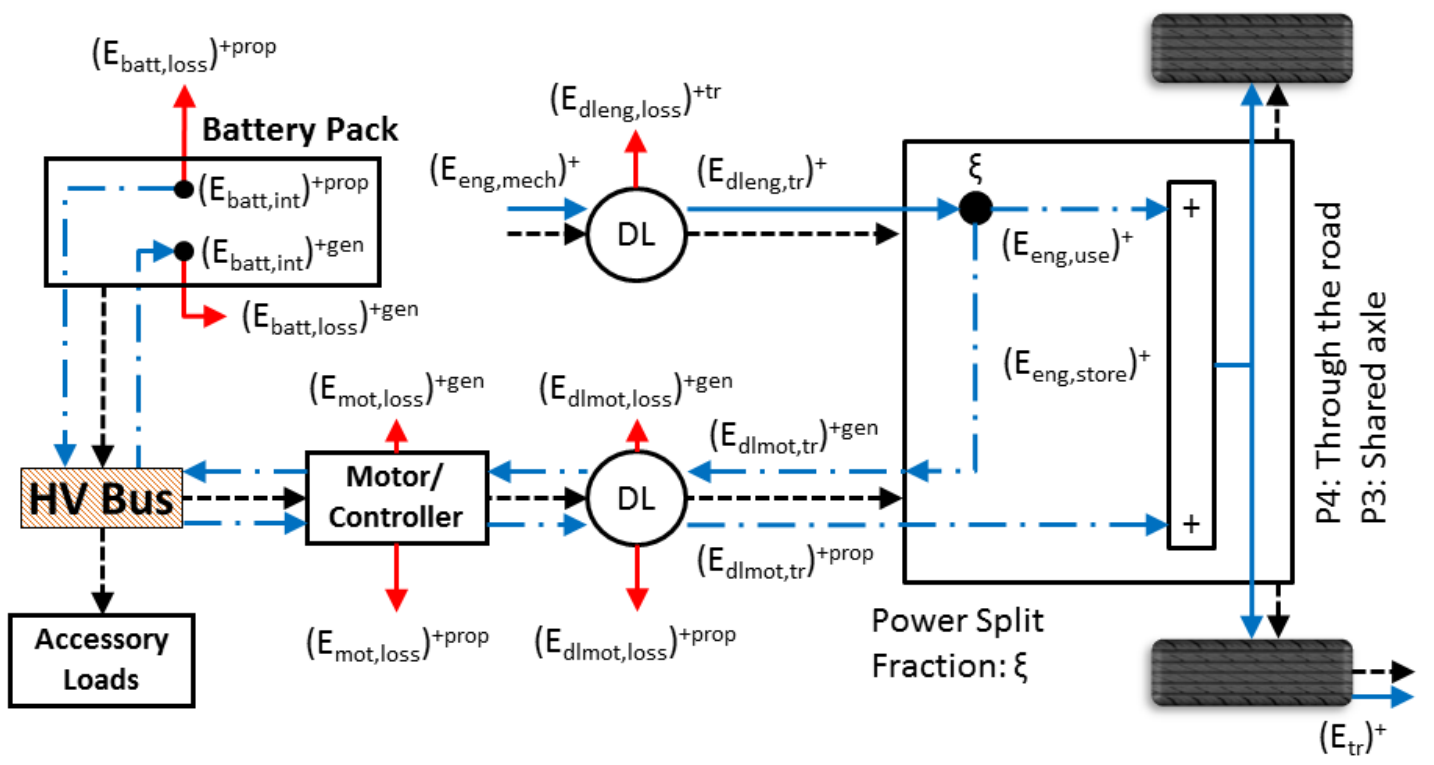

Figure 30: Energy balance at the drive axle for the Power Split Fraction

The four key parameters for analyzing the Power Split Fraction are $\left(E_{\text {dleng }}\right)^{+},\left(E_{\text {eng,use }}\right)^{+},\left(E_{d l m o t, t r}\right)^{+ \text {gen }}$, and $\left(E_{d l m o t, t r}\right)^{+p r o p}$. These parameters are defined in the list of nomenclature in the front matter of this thesis, but a more thorough description is provided in Table 12.

Table 12: Description of energy quantities key to the Power Split Fraction for the P3/P4 Parallel HEV

\begin{tabular}{|c|c|c|}
\hline Term & $\begin{array}{l}\text { Inherent } \\
\text { Sign }\end{array}$ & Definition \\
\hline$\left(\mathrm{E}_{\text {dleng }}\right)^{+}$ & + & $\begin{array}{l}\text { Mechanical energy out of the engine driveline during the propel case. This is the } \\
\text { total energy required from the engine to meet tractive energy requirements and all } \\
\text { losses except engine and engine driveline losses (not including accessory load } \\
\text { which is considered separately). }\end{array}$ \\
\hline$\left(\mathrm{E}_{\text {eng,use }}\right)^{+}$ & + & $\begin{array}{l}\text { Mechanical engine energy directly used for propulsion. As } \xi \text { goes to } 1 \text {, this } \\
\text { quantity converges to }\left(E_{\text {dleng }}\right)^{+} \text {. }\end{array}$ \\
\hline $\begin{array}{l}\left(E_{\text {dlmot,tr }}\right)^{+g e n} \\
\text {-or- } \\
\left(E_{\text {eng,store }}\right)^{+}\end{array}$ & - & $\begin{array}{l}\text { Mechanical engine energy stored before being used for propulsion. This energy is } \\
\text { recirculated before being used by the drive motor to assist in propulsion. Note that } \\
\text { none of this energy is used to meet the propel case accessory load, which is } \\
\text { considered as a separate case. }\end{array}$ \\
\hline$\left(\mathrm{E}_{\mathrm{dlmot}, \mathrm{tr}}\right)^{+ \text {prop }}$ & + & $\begin{array}{l}\text { Mechanical energy out of the motor driveline to be used in propulsion. This energy } \\
\text { was first stored in the battery and this is the result of second half of the } \\
\text { recirculation path. The sum of }\left(E_{\text {dlmot,tr }}+{ }^{+p r o p} \text { and }\left(E_{\text {eng,use }}\right)^{+} \text {must meet the tractive }\right. \\
\text { energy required at the wheels. This does not include the energy reused from } \\
\text { stored regen braking energy. Regen brake energy is accounted for in a separate } \\
\text { case for clarity. }\end{array}$ \\
\hline
\end{tabular}


The Power Split Fraction for the P3/P4 Parallel HEV is defined by Equation 78. As previously stated, all energy created by the engine goes directly to the wheels and $\left(E_{\text {eng,use }}\right)^{+}=\left(E_{\text {dleng,tr }}\right)^{+}$when $\xi=1$. Under this scenario, the vehicle operates very much like a conventional vehicle with tractive power only coming from the engine (except for regen energy that is reused). Likewise, Equation 79 describes the amount of energy that is recirculated before being used. For $\xi=0$, all energy is recirculated before being used. As previously discussed, this scenario is impossible to achieve in actual vehicle operation.

Equation 78: Power Split Fraction, engine energy directly used (P3 Parallel, propel)

$$
\begin{gathered}
\tilde{\xi}=\frac{\left(E_{\text {eng,use }}\right)^{+}}{\left(E_{\text {dleng,tr }}\right)^{+}} \\
\text {Restated: } \\
\left(E_{\text {eng }, \text { use }}\right)^{+}=\tilde{\xi}\left(E_{\text {dleng,tr }}\right)^{+}
\end{gathered}
$$

Equation 79: Power Split Fraction, engine energy recirculated (P3 Parallel, propel)

$$
\left(E_{\text {eng }, \text { store }}\right)^{+}=\left(E_{\text {dlmot }, \text { tr }}\right)^{+ \text {gen }}=(-1)(1-\xi)\left(E_{\text {dleng,tr }}\right)^{+}
$$

To build the energy consumption equation for this case, we must first establish an expression for the Power Split Fraction. We accomplish this by fist considering the total amount of energy stored in the battery via the Power Split Fraction, given by Equation 80. On the way to the battery from the drive axle, three losses are incurred: motor driveline, motor and battery losses.

Equation 80: Energy stored in the battery during times of surplus power generation (P3 Parallel, propel)

$$
\left(E_{\text {batt }, \text { int }}\right)^{+ \text {gen }}=(-1)(1-\xi)\left(E_{\text {dleng,tr }}\right)^{+}\left(\tilde{\eta}_{\text {dl,mot }}^{+g e n}\right)\left(\tilde{\eta}_{\text {mot }}^{+ \text {gen }}\right)\left(\tilde{\eta}_{\text {batt }}^{+g e n}\right)
$$

Next, we consider the total amount of energy used from the battery over the course of the cycle (not including regen energy use) given by Equation 81. Again, three losses are incurred as the energy flows from the battery internals back out to the drive axle: battery, motor and motor driveline losses.

Equation 81: Energy used from the battery during shortages of power generation (P3 Parallel, propel)

$$
\left(E_{\text {batt }, \text { int }}\right)^{+ \text {prop }}=\frac{\left(E_{d l m o t, t r}\right)^{+ \text {prop }}}{\left(\tilde{\eta}_{\text {batt }}^{+ \text {prop }}\right)\left(\tilde{\eta}_{\text {mot }}^{+ \text {prop }}\right)\left(\tilde{\eta}_{d l, m o t}^{+ \text {prop }}\right)}
$$

To be charge balanced over the entire cycle, we introduce Equation 82 . This equation asserts that all energy stored in the battery during the propel case must also be used during the propel case. Note that there is a sign difference between the two quantities - this is because they are equal and opposite and $\left(E_{\text {batt,int }}\right)^{+ \text {gen }}$ is inherently negative.

Equation 82: Conservation of energy in the battery to maintain charge balance (P3 Parallel, propel)

$$
\left(E_{\text {batt }, \text { int }}\right)^{+ \text {prop }}=-\left(E_{\text {batt }, \text { int }}\right)^{+ \text {gen }}
$$

Substituting Equation 80 and Equation 81 into Equation 82 and rearranging we arrive at Equation 83, which describes the total amount of energy the drive motor output to the drive axle for the propel case. 
The recirculation efficiency, $\eta_{\text {recirc }}$, describes the round-trip efficiency of the recirculation path and is defined in Equation 84. For the Parallel powertrain, $\eta_{\text {recirc }}$ includes six terms meaning that the recirculation path is relatively long. The recirculation efficiency was not defined for the Series powertrain because the path is relatively short including only battery charge and discharge losses.

Equation 83: Energy from the motor onto the drive axle during the propel case (P3 Parallel, propel)

$$
\left(E_{\text {dlmot }, t r}\right)^{+ \text {prop }}=\left(E_{\text {dleng,tr }}\right)^{+}(1-\xi)\left(\tilde{\eta}_{\text {recirc }}^{+}\right)
$$

Equation 84: Power Split Fraction energy recirculation efficiency (P3 Parallel, propel)

$$
\tilde{\eta}_{\text {recirc }}^{+}=\left(\tilde{\eta}_{d l, m o t}^{+g e n}\right)\left(\tilde{\eta}_{\text {mot }}^{+ \text {gen }}\right)\left(\tilde{\eta}_{\text {batt }}^{+ \text {gen }}\right)\left(\tilde{\eta}_{\text {batt }}^{+ \text {prop }}\right)\left(\tilde{\eta}_{\text {mot }}^{+ \text {prop }}\right)\left(\tilde{\eta}_{d l, \text { mot }}^{+ \text {prop }}\right)
$$

Next, we introduce Equation 85, a conservation of energy equation for the drive axle:

Equation 85: Conservation of energy 'inside' the drive axle (P3 Parallel, propel)

$$
\left(E_{\text {tr }}\right)^{+}=\left(E_{\text {eng }, \text { use }}\right)^{+}+\left(E_{\text {dlmot }, t r}\right)^{+ \text {prop }}
$$

Substituting Equation 78 and Equation 83 and rearranging we arrive at Equation 86 which describes the total energy required from the engine driveline to complete the propel portion of the drive cycle. Applying engine driveline and engine losses, we arrive at Equation 87, the energy consumption equation for the P3/P4 Parallel powertrain during the propel case.

Equation 86: Energy supplied by the engine during the propel case (P3 Parallel, propel)

$$
\left(E_{\text {dleng }, t r}\right)^{+}=\frac{\left(E_{\text {tr }}\right)^{+}}{\xi+(1-\xi)\left(\tilde{\eta}_{\text {recirc }}^{+}\right)}
$$

Equation 87: Propel case tractive fuel energy consumption (P3 Parallel, propel)

$$
\left(E_{\text {eng,fuel }}\right)^{+t r}=\frac{\left(E_{\text {tr }}\right)^{+}}{\left[\xi+(1-\xi)\left(\tilde{\eta}_{\text {recirc }}^{+}\right)\right]\left(\tilde{\eta}_{\text {eng }}^{+}\right)\left(\tilde{\eta}_{\text {dl,eng }}^{+}\right)}
$$

The tractive propel powertrain efficiency may now be defined by Equation 88. This propel powertrain efficiency is similar to previously defined powertrain efficiencies for other powertrains with the key distinction that this powertrain efficiency does not include accessory load.

Equation 88: Propel case powertrain efficiency for tractive energy (P3 Parallel, propel)

$$
\tilde{\eta}_{p t}^{+t r}=\frac{\left(E_{\text {tr }}\right)^{+}}{\left(E_{\text {eng,fuel }}\right)^{+t r}}
$$

Comparing the two powertrain architectures, a notable difference is the length of the recirculation path. The Series powertrain has only bi-directional battery losses while the Parallel powertrain has bidirectional battery, motor and motor driveline losses. Thus, the recirculation path is much longer and less efficient for the Parallel powertrain. This makes it less advantageous to recirculate energy and means that a comparatively higher Power Split Fraction will optimize system efficiency. However, the path from fuel tank to the wheels is shorter for the Parallel powertrain. Taking $\xi=1$ and sending all energy directly to the wheels (and thereby bypassing the recirculation path), we can see from Equation 87 that the Parallel powertrain has only two losses - engine and engine driveline losses. In contrast, however, the Series powertrain has 4 full-time losses: engine, generator, motor and motor driveline losses. It is reasonable to expect motor driveline and engine driveline losses to be similar, so the Series powertrain essentially has 
two additional full-time losses compared to the Parallel powertrain. But the Series powertrain also has the additional operational freedom of the engine being decoupled from the wheels which allows the engine to operate at a higher cycle-average efficiency.

\subsubsection{CS P3/P4 Parallel HEV: Propel Case - Accessory Energy Subcase}

As previously stated, the energy required for the accessory load is handled separately in a distinct case to prevent modeling conflicts with the mechanics of the Power Split Fraction. Figure 31 below describes the energy flow of the powertrain to supply accessory energy during the propel case.

\section{Propel (Accessory Energy): $\mathrm{F}_{\mathrm{Tr}}>0$}

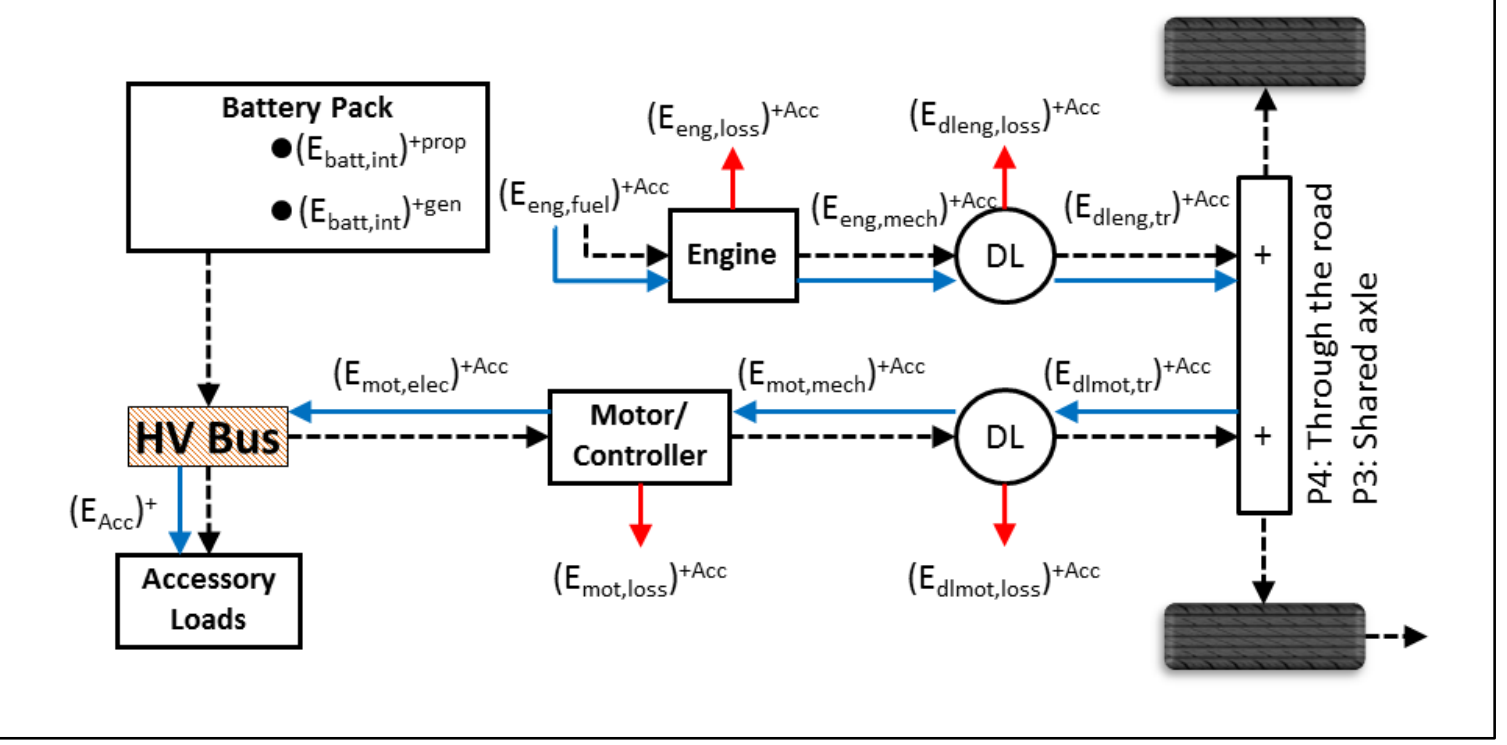

Figure 31: CS P3/P4 Parallel HEV energy flow diagram for the accessory energy in the propel case

As the figure shows, the energy path incurs 4 losses and goes through the drive axle. The energy consumption equation for this case, Equation 89, is developed in the same way as other energy consumption equations by starting at the engine and working down the energy flow path applying component efficiencies along the way. It is important to note that propel component efficiencies are used for this case instead of distinct efficiencies. This reduces complexity and simplifies calculations, but is also a more realistic representation of actual operation. Since the accessory load is applied during propel conditions and accessory is usually a small fraction of tractive power, the powertrain components should be expected to operate at or near propel efficiency to supply the extra energy for the accessory load.

Equation 89: Propel case accessory fuel energy consumption (P3 Parallel, propel)

$$
\left(E_{\text {eng,fuel }}\right)^{+a c c}=\frac{\left(E_{\text {Acc }}\right)^{+}}{\left(\tilde{\eta}_{\text {eng }}^{+}\right)\left(\tilde{\eta}_{d l, e n g}^{+}\right)\left(\tilde{\eta}_{d l, m o t}^{+}\right)\left(\tilde{\eta}_{\text {mot }}^{+}\right)}
$$

The powertrain efficiency for accessory energy is defined by Equation 90. This powertrain accessory efficiency is unique in that it evaluates the efficiency of the powertrain in meeting the propel accessory 
load. While accessory load is usually considered to be a parasitic load, this efficiency sheds a different perspective and paints the accessory load as useful energy.

Equation 90: Propel case powertrain accessory efficiency for accessory load (P3 Parallel, propel)

$$
\tilde{\eta}_{p t}^{+a c c}=\frac{\left(E_{\text {Acc }}\right)^{+}}{\left(E_{\text {eng,fuel }}\right)^{+a c c}}
$$

\subsubsection{CS P3/P4 Parallel HEV: Propel Case - Total Energy}

Having established separate cases for tractive energy and accessory energy consumption, a net case is now necessary to determine the total energy required to complete the propel case. Equation 91 defines the conservation of energy equation that links tractive energy and accessory energy and describes the total energy consumption for the propel case. Inserting the energy consumption equations for tractive energy and accessory energy, we arrive at Equation 92, the overall energy consumption equation for the propel case.

Equation 91: Total fuel energy required for the propel case (P3 Parallel, propel)

$$
\left(E_{\text {eng,fuel }}\right)^{+}=\left(E_{\text {eng,fuel }}\right)^{+t r}+\left(E_{\text {eng,fuel }}\right)^{+ \text {acc }}
$$

Equation 92: Propel case total fuel energy consumption (P3 Parallel, propel)

$$
\left(E_{\text {eng,fuel }}\right)^{+}=\frac{\left(E_{\text {tr }}\right)^{+}}{\left[\xi+(1-\xi)\left(\tilde{\eta}_{\text {recirc }}^{+}\right)\right]\left(\tilde{\eta}_{\text {eng }}^{+}\right)\left(\tilde{\eta}_{\text {dl,eng }}^{+}\right)}+\frac{\left(E_{\text {Acc }}\right)^{+}}{\left(\tilde{\eta}_{\text {eng }}^{+}\right)\left(\tilde{\eta}_{\text {dl,eng }}^{+}\right)\left(\tilde{\eta}_{\text {dl,mot }}^{+}\right)\left(\tilde{\eta}_{\text {mot }}^{+}\right)}
$$

The losses incurred while meeting both tractive and accessory energy must also be considered. All components except the battery incurred losses during both cases. Total motor losses are given in Equation 93 as an example. The equation for total powertrain losses during the propel case are defined in Equation 94. The equations for other component losses along with all equations necessary for this case are provided in Appendix N.

Equation 93: Motor total losses (P3 Parallel, propel)

$$
\left(E_{\text {mot }, l o s s}\right)^{+}=\left(E_{m o t, l o s s}\right)^{+t r}+\left(E_{m o t, l o s s}\right)^{+a c c}
$$

Equation 94: Total powertrain losses for accessory load (P3 Parallel, propel)

$$
\left(E_{\text {pt }, \text { loss }}\right)^{+}=\left(E_{\text {eng,loss }}\right)^{+}+\left(E_{\text {dleng,loss }}\right)^{+}+\left(E_{\text {dlmot }, \text { loss }}\right)^{+}+\left(E_{\text {mot }, \text { loss }}\right)^{+}+\left(E_{\text {bat }, \text { loss }}\right)^{+}
$$

The propel powertrain efficiency, defined in Equation 95, is the equivalent of the propel powertrain efficiencies for the Series and EV powertrains because it includes accessory load. It is now designated as the 'ICE' powertrain efficiency because the powertrain now has two distinct powertrains that can propel the vehicle. The first is the ICE powertrain powered by the engine that is essentially the same as a conventional vehicle powertrain. The second is the EV powertrain which is identical to the powertrain used in the EV. The powertrain efficiency for the EV powertrain will be defined later. The two powertrain efficiencies are kept separate to allow for comparison between the two powertrains.

Equation 95: Propel case ICE powertrain efficiency (P3 Parallel, propel)

$$
\tilde{\eta}_{p t, I C E}^{+}=\frac{\left(E_{\text {tr }}\right)^{+}}{\left(E_{\text {eng,fuel }}\right)^{+}}
$$




\subsubsection{CS P3/P4 Parallel HEV: Brake Case}

The brake case for the P3/P4 Parallel HEV is identical to the EV because the electric powertrain is exactly the same. Therefore, the energy consumption equation for the brake case is expressed by Equation 32. Like the EV, this energy will be reused later in propulsion. Refer to Appendix B for full documentation of the brake case and Appendix $\mathrm{O}$ for a summary of the brake case for this powertrain.

$$
\begin{gathered}
\text { Equation 32: Brake case energy consumption (EV, brake) } \\
\left(E_{\text {batt }, \text { int }}\right)^{-}=\tilde{\eta}_{b a t t}^{-}\left\{\left(E_{t r}\right)^{-}(\tilde{\zeta})\left(\tilde{\eta}_{d l, m o t}^{-}\right)\left(\tilde{\eta}_{m o t}^{-}\right)+\left(E_{A c c}\right)^{-}\right\}
\end{gathered}
$$

\subsubsection{CS P3/P4 Parallel HEV: Regen Energy Reuse}

Like the Series powertrain, a separate case is used to account for the reuse of all captured regen brake energy. The energy flow path for this case is illustrated in Figure 32. All equations for the regen energy reuse case are documented in Appendix $P$.

\section{Regen Energy Reuse: $\mathrm{F}_{\mathrm{Tr}}>0$}

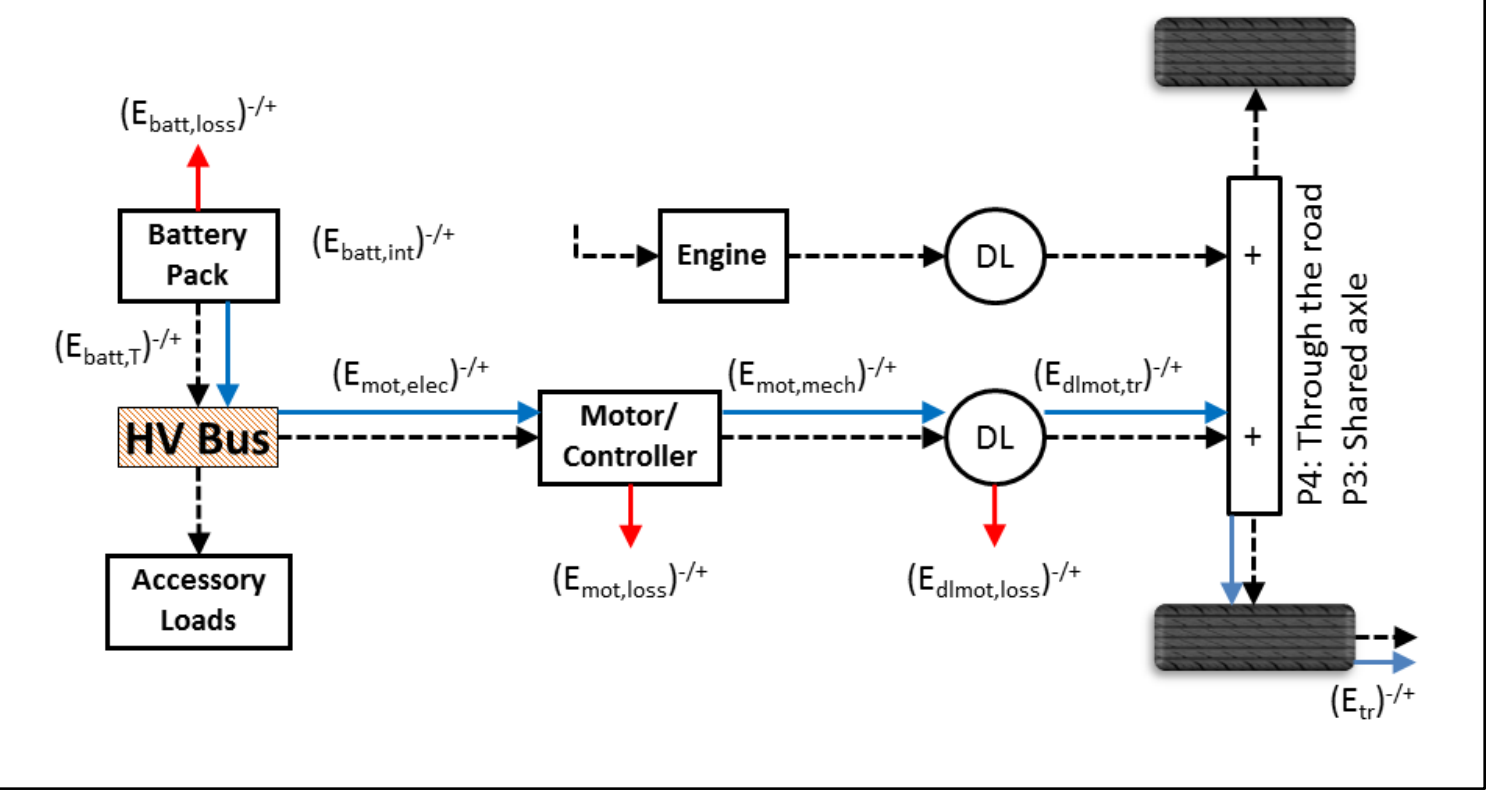

Figure 32: CS P3/P4 Parallel HEV energy flow diagram for regen energy reuse

The energy consumption equation for this case, given by Equation 96, is developed in the same manner as for the Series powertrain. In fact, the equation is identical to the energy consumption equation for the Series HEV. Keep in mind that component efficiencies unique to the regen reuse case are used in the equation but these efficiencies are approximated to be the respective propel efficiencies. This assumption simplifies calculations and eases the burden of determining an additional unique component efficiency. 
Equation 96: Equivalent tractive energy 'credit' from regen braking (P3 Parallel, regen reuse)

$$
\left(E_{\text {tr }, \text { credit }}\right)^{-/+}=-\left(E_{\text {batt }, \text { int }}\right)^{-}\left(\tilde{\eta}_{\text {batt }}^{-/+}\right)\left(\tilde{\eta}_{\text {mot }}^{-/+}\right)\left(\tilde{\eta}_{\text {dl,mot }}^{-/+}\right)
$$

The powertrain efficiency for this case is given by Equation 97 . This powertrain efficiency also represents the powertrain efficiency for the EV powertrain. As previously mentioned, the ICE and EV powertrain efficiencies are calculated separately for comparison. Later, a combined powertrain efficiency will also be calculated.

Equation 97: Powertrain efficiency for regen energy reuse (P3 Parallel, regen reuse)

$$
\tilde{\eta}_{p t, E V}^{-/+}=\frac{\left(E_{\text {tr,credit }}\right)^{-/+}}{\left(E_{\text {batt }, \text { int }}\right)^{-/+}}
$$

\subsubsection{CS P3/P4 Parallel HEV: Fuel-Equivalent Regen Energy Credit}

As in the Series HEV, the tractive energy 'credit' received from reusing stored regen energy is calculated as fuel-equivalent energy. Like before, this calculation is accomplished by starting with the tractive energy credit calculated in Equation 96 and then working back through a theoretical backward-flowing powertrain that is identical to the propel powertrain (except for the powerflow direction). The result of this calculation is the engine 'producing' fuel. This of course is not actually possible and is only a theoretical calculation. The fuel energy produced by the engine is inherently negative and will be summed later with the fuel use incurred during the propel case, thus reducing the total fuel energy used to complete the cycle. The energy flow for this case is illustrated by Figure 33. Again, it is identical to the (+tr) propel case for the P3/P4 Parallel powertrain except that the energy now flows in the opposite direction. All the equations for this case are fully documented in Appendix Q.

\section{Regen Energy Reuse: Fuel-Equivalent Energy Credit}

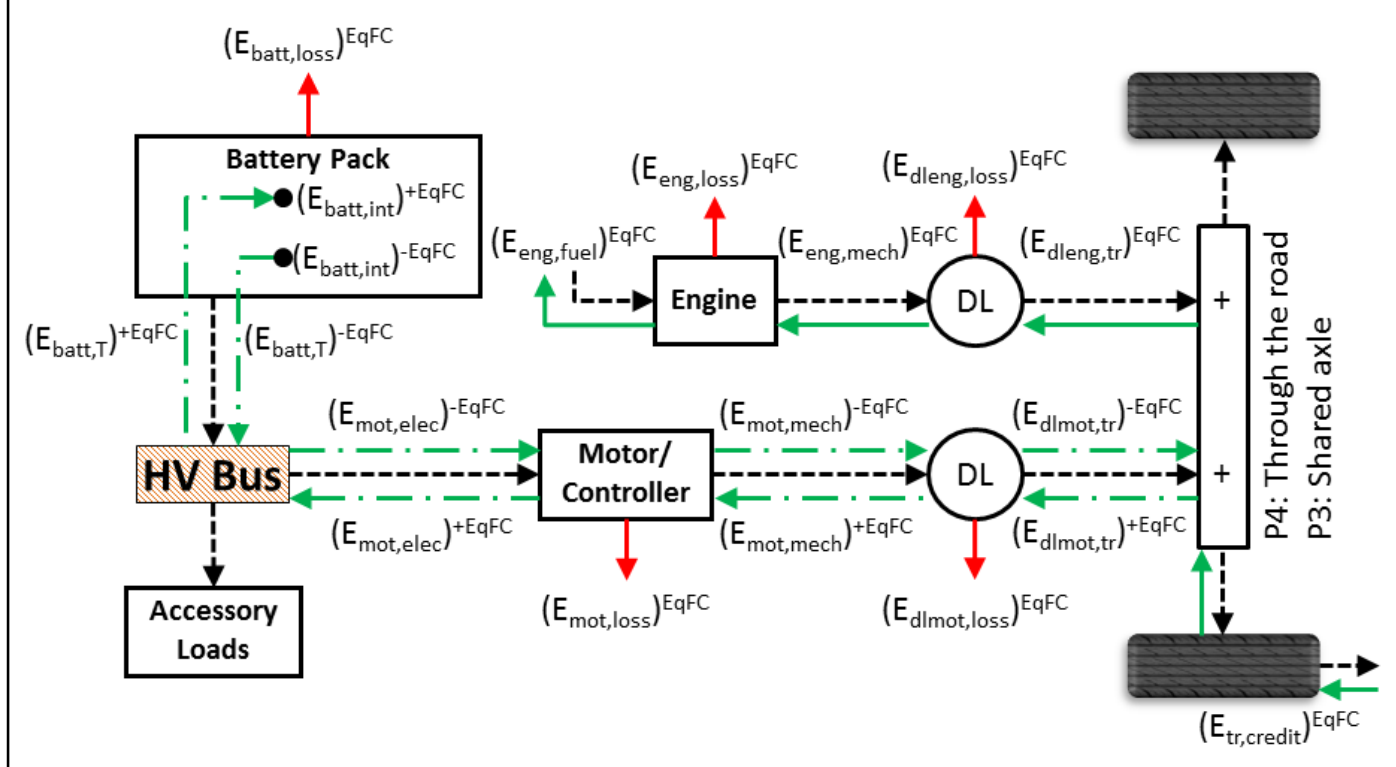

Figure 33: CS P3/P4 Parallel HEV energy flow diagram for regen fuel credit 
The energy consumption equation for this case is defined in Equation 98. This equation represents the fuel-equivalent credit received from performing regen braking. The equation is essentially identical to Equation 87, the energy consumption equation for the (+tr) propel case. This is as expected since the (+tr) case exempts accessory load just like this case.

Equation 98: Fuel-equivalent regen energy 'credit' (P3 Parallel, regen fuel credit)

$$
\left(E_{\text {eng,fuel }}\right)^{E q F C}=\frac{-\left(E_{\text {tr,credit }}\right)^{E q F C}}{\left[\xi+(1-\xi)\left(\tilde{\eta}_{\text {recirc }}^{+}\right)\right]\left(\tilde{\eta}_{\text {eng }}^{+}\right)\left(\tilde{\eta}_{\text {dl,eng }}^{+}\right)}
$$

Bear in mind that the losses for this case are negative as explained previously in Section 3.4.5. Also, other cases use the propel component efficiencies to estimate the unique component efficiencies for that case. The regen reuse case, for example, uses propel component efficiencies to estimate the unique component efficiencies for the regen reuse case. This is an assumption and an approximation to reduce complexity. The component efficiencies used in regen fuel-credit case are necessarily exactly the same as in the propel case. This is because this case seeks to exactly replicate the propel case except in the opposite direction.

\subsubsection{CS P3/P4 Parallel HEV: Idle Case}

As before, the accessory energy required for the idle case is also calculated as fuel-equivalent energy. Like the regen fuel credit, this approach allows the user to directly examine the impact on fuel energy. For this approach, all energy consumed by accessories during the idle case is assumed to come from the engine, as illustrated by Figure 34. As always, all accessories are assumed to be electrical and not engine driven. This is a reasonable assumption since even conventional vehicles are switching to more electrically driven accessory systems for efficiency. All the equations for this case are fully documented in Appendix R.

\section{Idle: $\mathrm{F}_{\mathrm{Tr}}=0$}

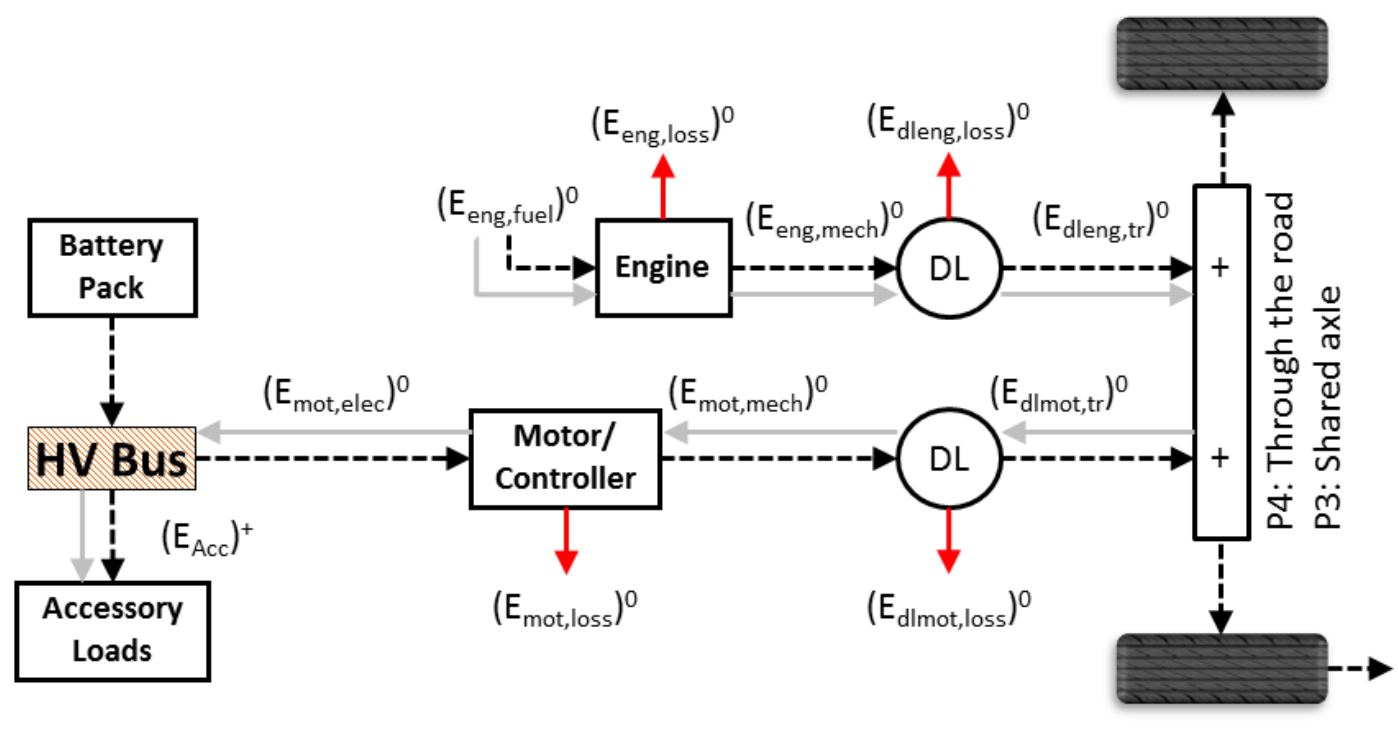


Figure 34: CS P3/P4 Parallel HEV energy flow diagram for the idle case

As before, the total idle accessory energy is defined by Equation 36, which depends on the vehicle accessory load and amount of idle time. These two parameters are characteristic of the drive cycle only and are independent of vehicle properties. The path to supply the accessory load for the P3/P4 Parallel HEV (4 losses) is quite long compared to the Series HEV (2 losses). Equation 99 gives the energy consumption equation for the idle case. Remember that component efficiencies unique to the idle case are given here, but are actually approximated by already established propel component efficiencies for simplicity.

$$
\begin{gathered}
\text { Equation 99: Idle case energy consumption (P3 Parallel, idle) } \\
\left(E_{\text {eng,fuel }}\right)^{0}=\frac{-\left(E_{\text {mot,elec }}\right)^{0}}{\left(\tilde{\eta}_{\text {eng }}^{0}\right)\left(\tilde{\eta}_{d l, e n g}^{0}\right)\left(\tilde{\eta}_{d l, m o t}^{0}\right)\left(\tilde{\eta}_{m o t}^{0}\right)}=\frac{\left(E_{\text {Acc }}\right)^{0}}{\left(\tilde{\eta}_{\text {eng }}^{0}\right)\left(\tilde{\eta}_{d l, e n g}^{0}\right)\left(\tilde{\eta}_{d l, m o t}^{0}\right)\left(\tilde{\eta}_{m o t}^{0}\right)}
\end{gathered}
$$

It is important to note that energy must be transmitted through the drive axle to reach the HV bus and therefore the accessory load. This arrangement could prove problematic at idle. For the P4 configuration, the drive motor is on a separate axle which means that the vehicle must be moving to transmit power through the motor. For a P3 configuration, the engine and motor share a drive axle which also means that power cannot be transmitted unless the drive axle is moving. This will not cause problems for modeling purposes, but can present undesirable situations in actual implementation. For example, if the vehicle were stuck in traffic for an extended period of time, the only way to supply the accessory load would be to drain the HV battery. For a limited time, this is an acceptable mode of operation. Long term, however, the battery would be depleted and accessories would no longer be able to function. In extreme cases, the HV battery could potentially be drained to a point to where it could no longer energize the $12 \mathrm{~V}$ bus through a DC-DC converter. This would result in a total system failure because any vehicle with a HV battery depends on the battery contactors which in turn depend on $12 \mathrm{~V}$ power to remain closed. There are also other systems and controllers that depend on proper $12 \mathrm{~V}$ system voltage levels to remain operational.

\subsubsection{CS P3/P4 Parallel HEV: DC Net Case}

As before, the DCnet case is used to summarize all of the cases presented thus far even though this powertrain has no HV battery charger and therefore no ACnet case. The total fuel energy consumption for a given cycle may be calculated in two different ways. The first, given by Equation 100, simply sums the energy consumed during the propel, idle and regen fuel-credit cases.

\section{Equation 100: Total fuel energy calculated by case (P3 Parallel, DCnet)}

$$
\left(E_{\text {eng,fuel }}\right)^{\text {DCnet }}=\left(E_{\text {eng,fuel }}\right)^{+}+\left(E_{\text {eng,fuel }}\right)^{0}+\left(E_{\text {eng,fuel }}\right)^{\text {EqFC }}
$$

The second, given by Equation 101, can be used as a sanity check against the first and sums the road load, all accessory energy for the cycle and all the losses for the cycle. This equation asserts that the total fuel required to complete the cycle must meet road load, plus the accessory load, plus all the losses incurred during operation.

Equation 101: Total fuel energy calculated from overall energy balance (P3 Parallel, DCnet)

$$
\left(E_{\text {eng, fuel }}\right)^{\text {DCnet }}=\left(E_{\text {tr }}\right)^{\text {DCnet }}+\left(E_{A C C}\right)^{\text {DCnet }}+\left(E_{\text {pt }, \text { loss }}\right)^{\text {DCnet }}
$$

Road load and total accessory load may be calculated independent of the powertrain, but the total losses are dependent on the specific powertrain and component efficiencies. The total losses may be calculated using Equation 102. 


$$
\left(E_{p t, \text { loss }}\right)^{\text {DCnet }}=\begin{aligned}
& \text { Equation 102: Net powertrain losses (P3 Parallel, DCnet) } \\
& =\left(E_{p t, \text { loss }}\right)^{+}+\left(E_{p t, l o s s}\right)^{-}+\left(E_{p t, l o s s}\right)^{-/+}+\left(E_{p t, l o s s}\right)^{0}+\left(E_{p t, l o s s}\right)^{E q F C}
\end{aligned}
$$

The net powertrain efficiency for this powertrain is defined in Equation 103. This measure of powertrain efficiency compares road load against the total fuel used to complete the cycle. This efficiency will be lower than the propel powertrain efficiency (Equation 95) or the combined propel powertrain efficiency, which is defined in Equation 104. The combined propel powertrain efficiency includes the losses of both the EV and the ICE powertrains and will also be lower than the propel ICE powertrain efficiency. This is because the propel ICE powertrain efficiency only includes losses from the ICE powertrain. Likewise, the net powertrain efficiency includes all losses from all cases during operation and is therefore lower than the combined propel powertrain efficiency. This nuance in powertrain efficiencies is also discussed in detail in Section 3.3.5 and more supporting equations for this case can be found in Appendix S.

Equation 103: Net powertrain efficiency (P3 Parallel, DCnet)

$$
\tilde{\eta}_{p t}^{D C n e t}=\frac{\left(E_{\text {tr }}\right)^{\text {DCnet }}}{\left(E_{\text {eng,fuel }}\right)^{\text {DCnet }}}
$$

Equation 104: Net powertrain efficiency for combined propel operation (P3 Parallel, DCnet)

$$
\tilde{\eta}_{\text {pt,propel }}^{\text {DCnet }}=\frac{\left(E_{\text {tr }}\right)^{+}+\left(E_{\text {tr,credit }}\right)^{E q F C}}{\left(E_{\text {eng,fuel }}\right)^{+}+\left(E_{\text {bat }, \text { int }}\right)^{-/+}}
$$

\subsubsection{CS P3/P4 Parallel HEV: Summary}

The P3/P4 Parallel powertrain introduces new challenges, complexities, nuances as well as benefits and drawbacks compared to the Series powertrain. The engine no longer has 2 degrees of freedom (torque and speed). Because it has been coupled to the wheels it now has only 1 degree of freedom (torque). Hence, the engine still has some operational freedom that can be used to increase average efficiency but not as much freedom as in the Series powertrain. However, the engine may now also contribute to vehicle performance whereas the Series engine and generator may not put torque to the wheels to contribute to acceleration performance.

The concept of the Power Split Fraction is again used for this powertrain. The power split occurs at the drive axle instead of the HV bus, where it occurs for the Series HEV. For modeling purposes, the mechanics of the Power Split Fraction are very similar, but the recirculation paths are very different. The Series recirculation path only includes battery losses while the Parallel recirculation path includes battery, motor and motor driveline losses. Thus the Parallel recirculation path is much longer meaning that it is now less advantageous to recirculate energy. However, the Parallel HEV has a shorter path from the fuel tank to the wheels and only has 2 full-time losses which are always present regardless of the Power Split Fraction. The Series HEV has 4 full-time losses. So while the Parallel HEV is more restricted in operation, it has a much more direct full-time energy transmission path.

The concepts of regen energy reuse and fuel-equivalent energy consumption are also reused for this powertrain. These concepts allow the benefits of regen braking to be directly related to fuel energy. Likewise, the effects of idle accessory energy can be related back to fuel-equivalent energy using this method. On additional nuance of the Parallel HEV is the path to supply the accessory load, which must 
flow through the drive axle. As a result, the engine cannot assist with the accessory load unless the vehicle is moving. This nuance does not affect the powertrain modeling but must be considered for actual operation. An additional nuance involving the accessory load is its location in the recirculation path. As a result, accessory energy must be accounted for in a separate case so that the Power Split Fraction will not skew the effect of accessory load. Therefore two new subcases were created within the propel case: one for tractive energy (+tr) and another for accessory energy (+acc).

\subsection{CS P2 Parallel HEV}

The following section outlines the equations and methodology used to predict fuel energy consumption for a CS P2 Parallel HEV. Referring to Table 11, the P2 position indicates a pre-trans motor between the engine and transmission. The P2 powertrain is very similar to the P3 or P4 powertrains and contains all of the same powertrain components. The motor driveline, however, now has some additional considerations. The motor connects to the drivetrain between the engine and transmission, which can be accomplished in several different ways. A direct drive motor that is placed in-line with the engine drive shaft with no gear reduction would have an efficiency of essentially $100 \%$. If it were belt-driven, however, the efficiency would be somewhat lower, certainly less than $100 \%$.

The only other difference between the three powertrains is the packaging and arrangement of components. The P3 and P4 powertrains are schematically the same, but the P2 powertrain is slightly different. For the P2 powertrain, the drive motor now contributes power before the engine driveline at a 'pre-trans' node. This effectively shifts the summation node from right after the engine driveline (posttrans) to right before the engine driveline (pre-trans). This small change can have some notable operational impacts. The regen energy capture and reuse path now includes an extra driveline loss. Additionally, the path from the engine to the HV bus now no longer goes through the drive axle. Therefore the HV bus may now be energized without requiring the vehicle to be in motion. Additional nuances of the P2 Parallel powertrain architecture will be discussed in further depth later in this section. 


\subsubsection{CS P2 Parallel HEV: Sign Convention}

The sign convention used for the P2 Parallel powertrain is illustrated in Figure 35. As always, the dashed lines represent positive energy flow.

\section{Sign Convention}

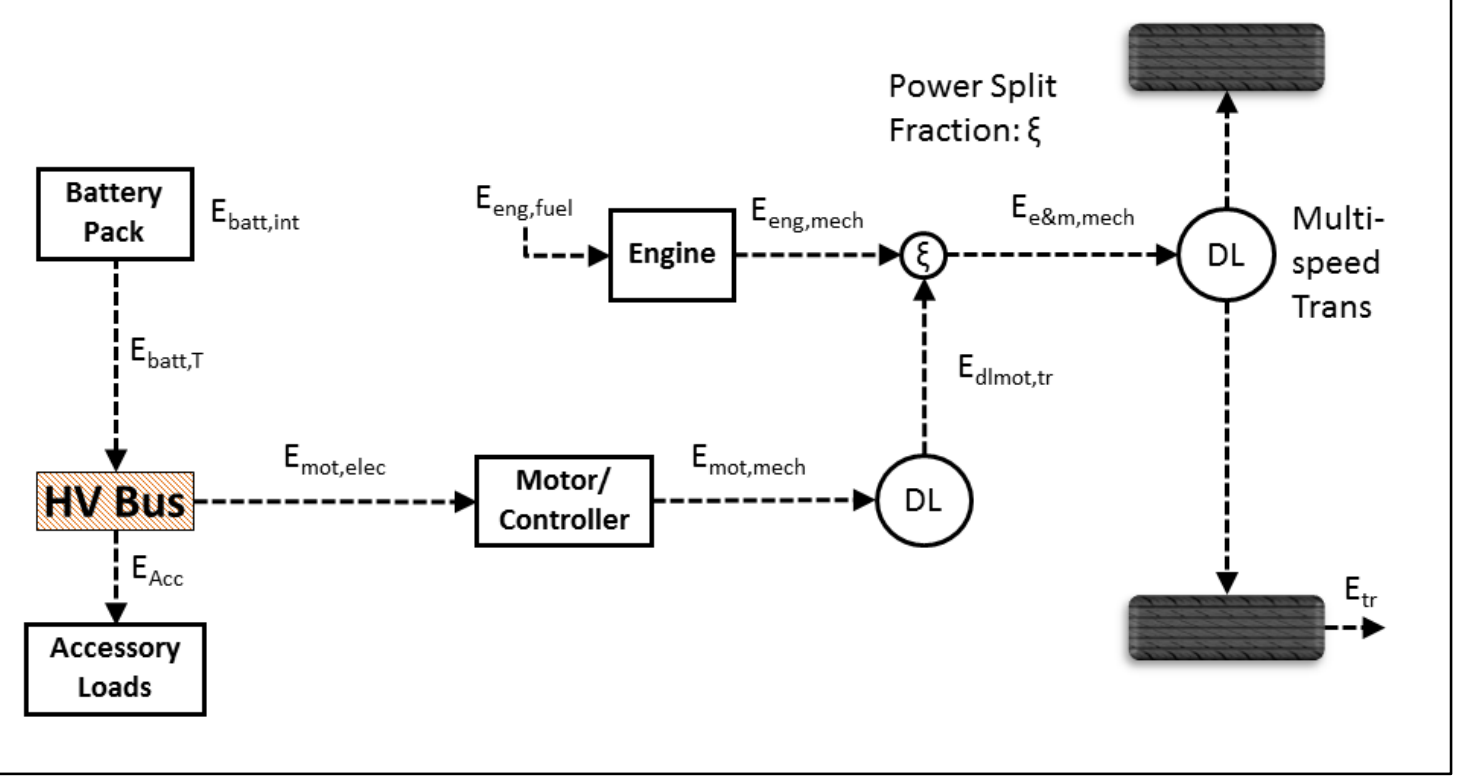

Figure 35: Energy flow sign conventions for a CS P2 Parallel HEV powertrain

\subsubsection{CS P2 Parallel HEV: Propel Case}

Like the P3/P4 Parallel HEV, the propel case is divided into two subcases: tractive energy (+tr) and accessory energy $(+\mathrm{acc})$. Like before, this is done to prevent the dynamics of the Power Split Fraction from interfering with the effects of the accessory load.

\subsubsection{CS P3/P4 Parallel HEV: Propel Case - Tractive Energy Subcase}

The energy flow paths for tractive energy are shown in blue in Figure 36. Energy (shown in blue) flows from the engine to the wheels. Losses for each component are shown also in red and are inherently positive. All the equations for this case are fully documented in Appendix T. 


\section{Propel (Tractive Energy): $\mathrm{F}_{\mathrm{Tr}}>0$}

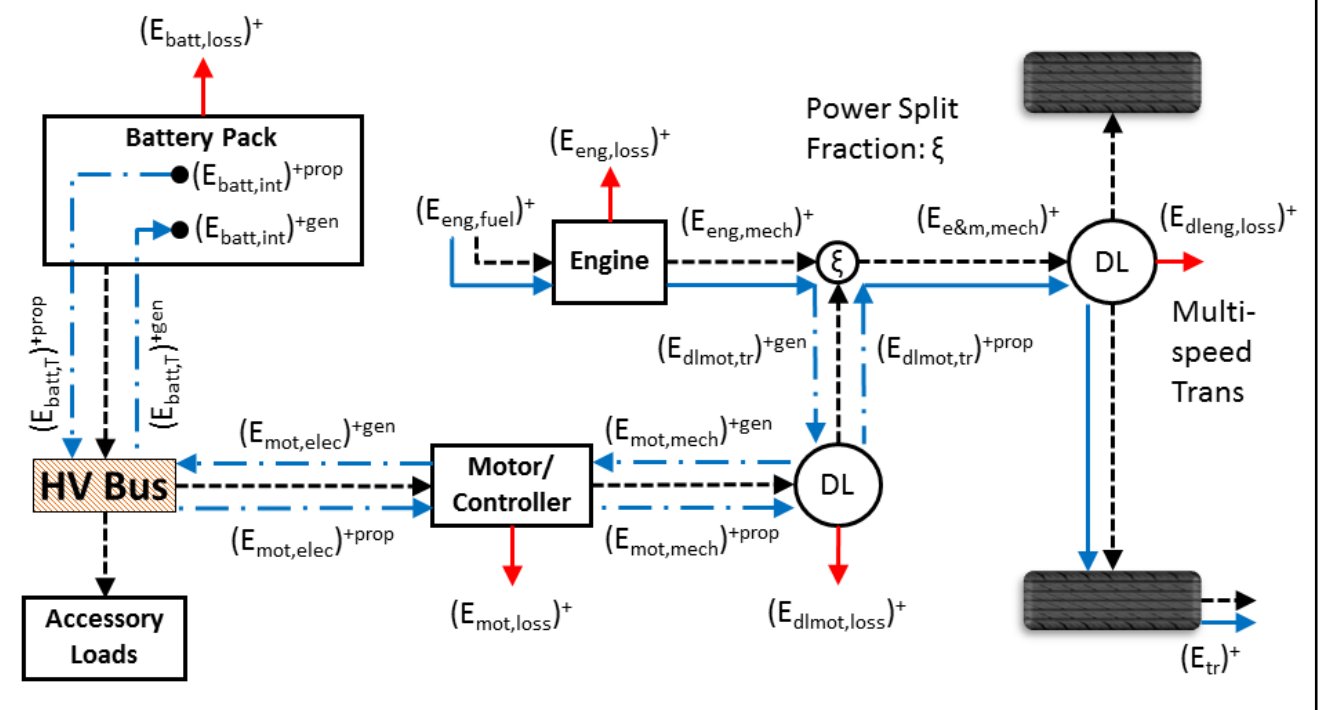

Figure 36: CS P2 Parallel HEV energy flow diagram for the tractive energy in the propel case

The P2 powertrain is nearly identical to the P3 and P4 powertrains. The only difference is that the drive motor enters the drivetrain pre-trans instead of post-trans. Therefore, the Power Split Fraction occurs at a somewhat arbitrary pre-trans node instead of at the drive axle, as shown in Figure 37 . Both the engine and the motor still have one degree of freedom each (torque) and are both kinematically constrained to vehicle speed.

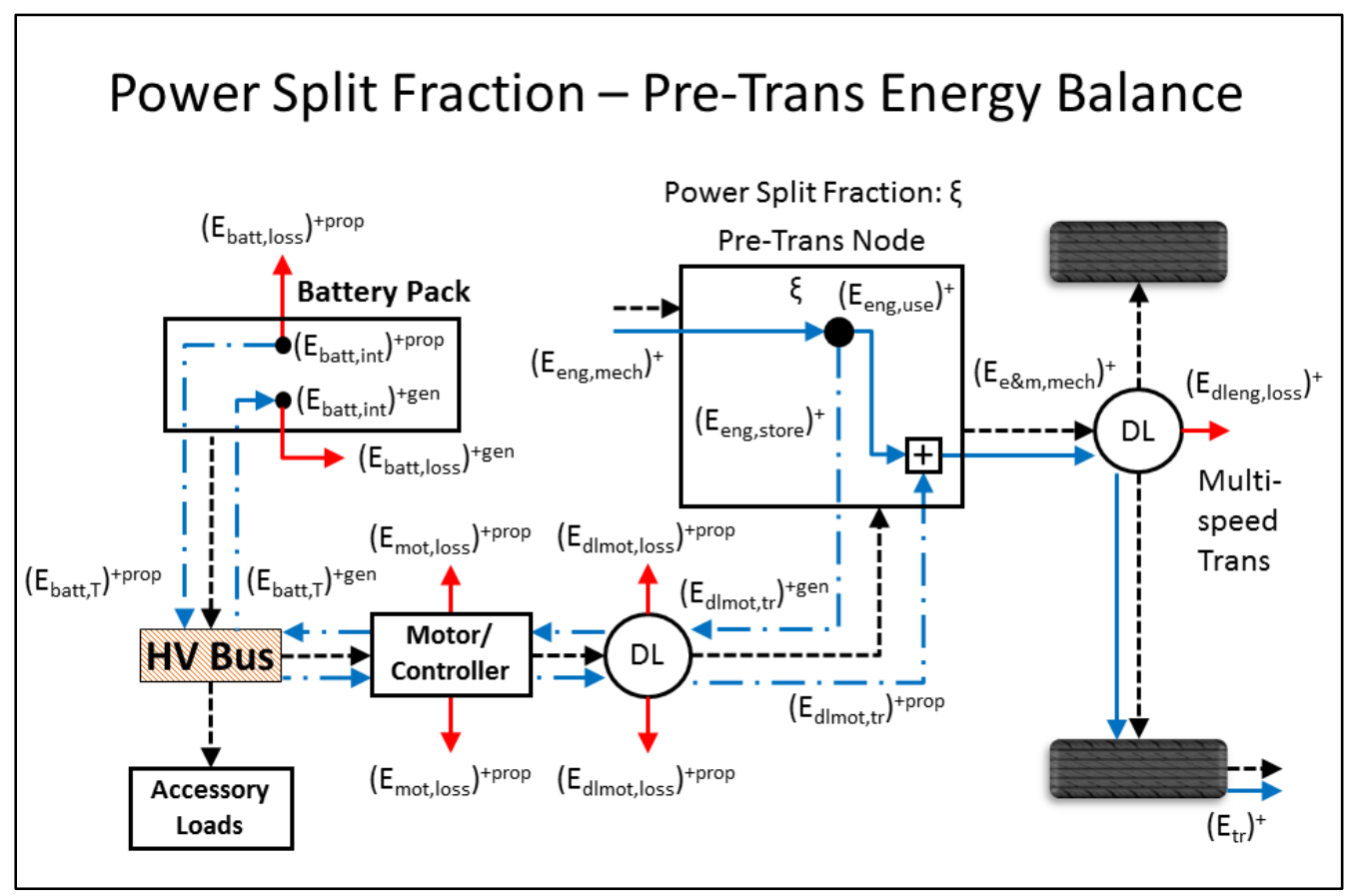

Figure 37: Energy balance at the pre-trans Node for the Power Split Fraction 
The four key parameters for analyzing the Power Split Fraction are $\left(E_{\text {eng,mech }}\right)^{+},\left(E_{\text {eng,use }}\right)^{+},\left(E_{\text {dlmot,tr }}\right)^{+ \text {ten }}$, and $\left(E_{\text {dlmot,tr }}\right)^{+ \text {prop }}$. These parameters are defined in the list of nomenclature in the front matter of this thesis, but a more thorough description is provided in Table 13.

Table 13: Description of energy quantities key to the Power Split Fraction for the P2 Parallel HEV

\begin{tabular}{|c|c|c|}
\hline Term & $\begin{array}{l}\text { Inherent } \\
\text { Sign }\end{array}$ & Definition \\
\hline$\left(\mathrm{E}_{\text {eng,mech }}\right)^{+}$ & + & $\begin{array}{l}\text { Mechanical energy out of the engine during the propel case. This is the total } \\
\text { energy required from the engine to meet tractive energy requirements and all } \\
\text { losses except engine losses (not including accessory load which is considered } \\
\text { separately). }\end{array}$ \\
\hline$\left(\mathrm{E}_{\text {eng,use }}\right)^{+}$ & + & $\begin{array}{l}\text { Mechanical engine energy directly used for propulsion. As } \xi \text { goes to } 1 \text {, this } \\
\text { quantity converges to }\left(E_{\text {enamech }}\right)^{+} \text {. }\end{array}$ \\
\hline $\begin{array}{l}\left(E_{\text {dlmot,tr }}\right)^{+g e n} \\
\text {-or- } \\
\left(E_{\text {eng,store }}\right)^{+}\end{array}$ & - & $\begin{array}{l}\text { Mechanical engine energy stored before being used for propulsion. This energy is } \\
\text { recirculated before being used by the drive motor to assist in propulsion. Note that } \\
\text { none of this energy is used to meet the propel case accessory load, which is } \\
\text { considered as a separate case. }\end{array}$ \\
\hline$\left(E_{d l m o t, t r}\right)^{+p r o p}$ & + & $\begin{array}{l}\text { Mechanical energy out of the motor driveline to be used in propulsion. This energy } \\
\text { was first stored in the battery and this is the result of second half of the } \\
\text { recirculation path. The sum of }\left(E_{\text {dlmot,tr }}\right)^{+p r o p} \text { and }\left(E_{\text {eng,use }}\right)^{+} \text {must meet the tractive } \\
\text { energy required at the wheels plus engine driveline losses. This does not include } \\
\text { the energy reused from stored regen braking energy. Regen brake energy is } \\
\text { accounted for in a separate case for clarity. }\end{array}$ \\
\hline
\end{tabular}

The Power Split Fraction for the P2 Parallel HEV is defined by Equation 105. As previously stated, all energy created by the engine goes directly to the wheels and $\left(E_{\text {eng,use }}\right)^{+}=\left(E_{\text {eng,mech }}\right)^{+}$when $\xi=1$. Under this scenario, the vehicle operates very much like a conventional vehicle with tractive power only coming from the engine (except for regen energy that is reused). Likewise, Equation 106 describes the amount of energy that is recirculated before being used. For $\xi=0$, all energy is recirculated before being used. As previously discussed, this scenario is impossible to achieve in actual vehicle operation.

Equation 105: Power Split Fraction, engine energy directly used (P2 Parallel, propel)

$$
\begin{gathered}
\xi=\frac{\left(E_{\text {eng,use }}\right)^{+}}{\left(E_{\text {eng,mech }}\right)^{+}} \\
\text {Restated: } \\
\left(E_{\text {eng }, \text { use }}\right)^{+}=\tilde{\xi}\left(E_{\text {eng, } \text { mech }}\right)^{+}
\end{gathered}
$$

Equation 106: Power Split Fraction, engine energy recirculated (P2 Parallel, propel)

$$
\left(E_{\text {eng,store }}\right)^{+}=\left(E_{\text {dlmot }, t r}\right)^{+ \text {gen }}=(-1)(1-\xi)\left(E_{\text {eng,mech }}\right)^{+}
$$

The energy consumption equation for the P2 Parallel HEV, given by Equation 107, is built in the exact same manner as the P3/P4 Parallel HEV. Even though the components have been slightly rearranged, all of the equations are identical for the (+tr) propel case. This is because the engine driveline was not a part of the recirculation path for either powertrain. Thus, the recirculation path is identical for the two powertrains and the order of components does not matter. The recirculation efficiency for the P2 Parallel $\mathrm{HEV}$ is defined in Equation 108. Notice that it has the exact same six terms as Equation 84, the 
recirculation efficiency for the P3/P4 powertrain. The rest of the equations for this case are omitted here for brevity but documented in Appendix T.

Equation 107: Propel case tractive fuel energy consumption (P2 Parallel, propel)

$$
\left(E_{\text {eng,fuel }}\right)^{+t r}=\frac{\left(E_{\text {tr }}\right)^{+}}{\left[\xi+(1-\xi)\left(\tilde{\eta}_{\text {recirc }}^{+}\right)\right]\left(\tilde{\eta}_{\text {eng }}^{+}\right)\left(\tilde{\eta}_{\text {dl,eng }}^{+}\right)}
$$

Equation 108: Power Split Fraction energy recirculation efficiency (P2 Parallel, propel)

$$
\tilde{\eta}_{\text {recirc }}^{+}=\left(\tilde{\eta}_{\text {dl,mot }}^{+ \text {gen }}\right)\left(\tilde{\eta}_{\text {mot }}^{+g e n}\right)\left(\tilde{\eta}_{\text {batt }}^{+g e n}\right)\left(\tilde{\eta}_{\text {batt }}^{+ \text {prop }}\right)\left(\tilde{\eta}_{\text {mot }}^{+ \text {prop }}\right)\left(\tilde{\eta}_{\text {dl,mot }}^{+ \text {prop }}\right)
$$

The tractive propel powertrain efficiency may now be defined by Equation 109. This propel powertrain efficiency is similar to previously defined powertrain efficiencies for other powertrains with the key distinction that this powertrain efficiency does not include accessory load.

Equation 109: Propel case powertrain efficiency for tractive energy (P2 Parallel, propel)

$$
\tilde{\eta}_{p t}^{+t r}=\frac{\left(E_{t r}\right)^{+}}{\left(E_{\text {eng,fuel }}\right)^{+}}
$$

\subsubsection{CS P2 Parallel HEV: Propel Case - Accessory Energy Subcase}

As previously stated, the energy required for the accessory load is handled separately in a distinct case to prevent modeling conflicts with the mechanics of the Power Split Fraction. Figure 38 below describes the energy flow of the powertrain to supply accessory energy during the propel case. All the equations for this case are fully documented in Appendix U.

\section{Propel (Accessory Energy): $\mathrm{F}_{\mathrm{Tr}}>0$}

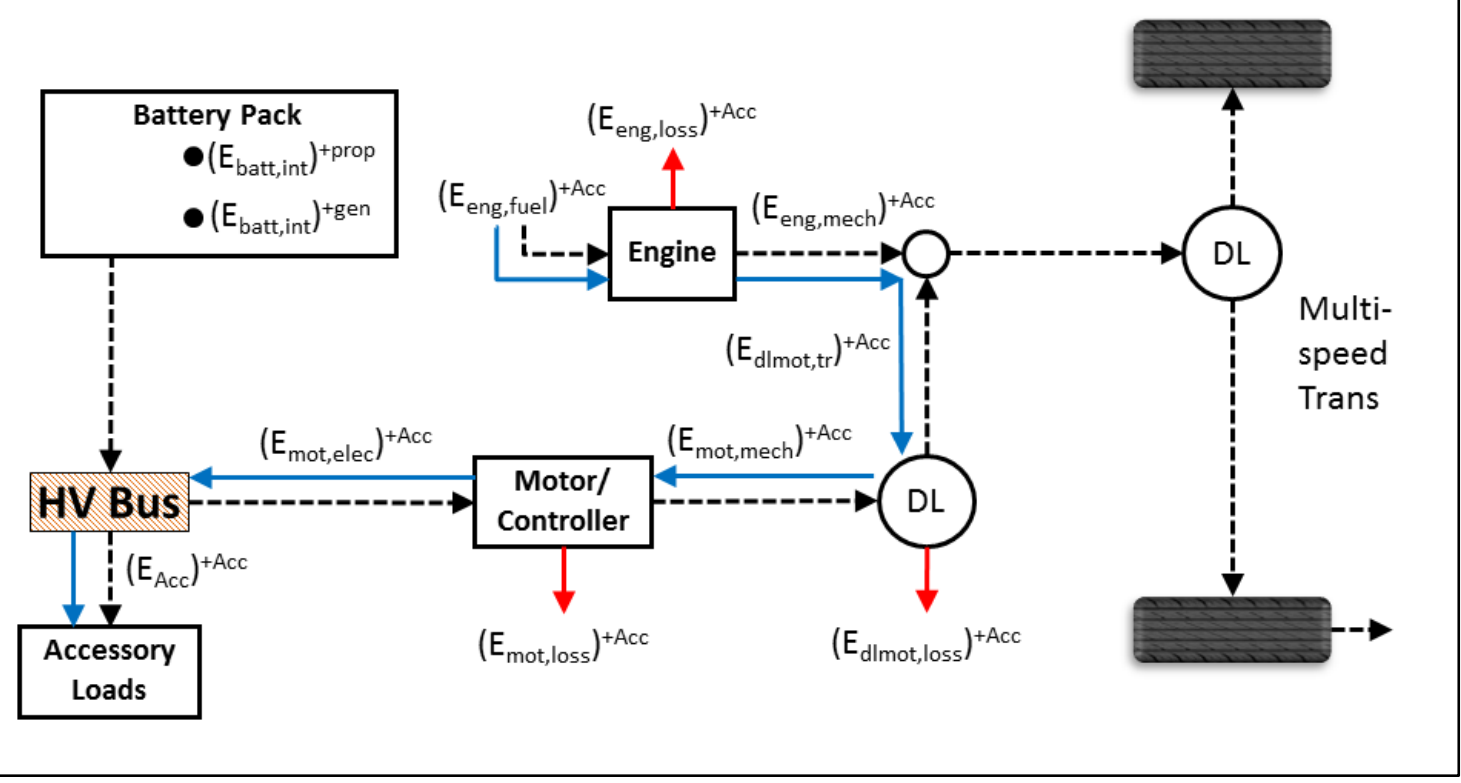

Figure 38: CS P2 Parallel HEV energy flow diagram for the accessory energy in the propel case 
As the figure shows, the energy path incurs only 3 losses and does not traverse through the drive axle like the P3 or P4 powertrains must do. The energy consumption equation for this case, Equation 110, is developed in the same way as other energy consumption equations by starting at the engine and working down the energy flow path applying component efficiencies along the way. As with previous cases, propel component efficiencies are used for this case instead of distinct efficiencies.

Equation 110: Propel case accessory fuel energy consumption (P2 Parallel, propel)

$$
\left(E_{\text {eng,fuel }}\right)^{+a c c}=\frac{\left(E_{\text {Acc }}\right)^{+}}{\left(\tilde{\eta}_{\text {eng }}^{+}\right)\left(\tilde{\eta}_{d l, m o t}^{+}\right)\left(\tilde{\eta}_{m o t}^{+}\right)}
$$

The powertrain accessory efficiency is defined by Equation 111. This powertrain accessory efficiency is identical to the equation for the P3/P4 Parallel HEV and is unique in that it evaluates the efficiency of the powertrain in meeting the propel accessory load.

\section{Equation 111: Propel case powertrain accessory efficiency for accessory load (P2 Parallel, propel)}

$$
\tilde{\eta}_{p t}^{+a c c}=\frac{\left(E_{\text {Acc }}\right)^{+}}{\left(E_{\text {eng,fuel }}\right)^{+a c c}}
$$

\subsubsection{CS P2 Parallel HEV: Propel Case - Total Energy}

Having established separate cases for tractive energy and accessory energy consumption, a net case is now necessary to determine the total energy required to complete the propel case. Combining the energy consumption equations for tractive energy and accessory energy, we arrive at Equation 112, the overall energy consumption equation for the propel case.

Equation 112: Propel case total fuel energy consumption (P2 Parallel, propel)

$$
\left(E_{\text {eng,fuel }}\right)^{+}=\frac{\left(E_{\text {tr }}\right)^{+}}{\left[\xi+(1-\xi)\left(\tilde{\eta}_{\text {recirc }}^{+}\right)\right]\left(\tilde{\eta}_{\text {eng }}^{+}\right)\left(\tilde{\eta}_{\text {dl,eng }}^{+}\right)}+\frac{\left(E_{\text {Acc }}\right)^{+}}{\left(\tilde{\eta}_{\text {eng }}^{+}\right)\left(\tilde{\eta}_{\text {dl,mot }}^{+}\right)\left(\tilde{\eta}_{\text {mot }}^{+}\right)}
$$

The propel powertrain efficiency, defined in Equation 113, is the equivalent of the propel powertrain efficiencies for the Series and EV powertrains because it includes accessory load. It is now designate as the 'ICE' powertrain efficiency because it represents the efficiency of the ICE powertrain as previously discussed in Section 3.5.2.3.

Equation 113: Propel case powertrain efficiency (P2 Parallel, propel)

$$
\tilde{\eta}_{p t, I C E}^{+}=\frac{\left(E_{\text {tr }}\right)^{+}}{\left(E_{\text {eng,fuel }}\right)^{+}}
$$

As before the losses must also be considered and combined as appropriate. The P2 powertrain is identical to the P3 and P4 powertrains except that the engine driveline no longer incurs a loss to supply accessory energy. The equations for component and total losses may be found in Appendix $V$ along with all of the other equations for this case.

\subsubsection{CS P2 Parallel HEV: Brake Case}

Unlike the Series HEV and the P3/P4 Parallel HEV, the brake case is not identical to the EV, though it is very similar. The only difference is the presence of the engine driveline in the EV powertrain. This negatively impacts regen braking performance by adding losses to the energy flow path. The energy 
consumption equation for the brake case is given by Equation 114. The extra efficiency term is highlighted for emphasis. Refer to Appendix W for documentation for the brake case for the P2 Parallel HEV.

Equation 114: Propel case accessory fuel energy consumption (P2 Parallel, brake)

$$
\left(E_{\text {batt }, i n t}\right)^{-}=\tilde{\eta}_{b a t t}^{-}\left\{\left(E_{t r}\right)^{-}(\tilde{\zeta})\left(\tilde{\eta}_{d l, m o t}^{-}\right)\left(\tilde{\eta}_{d l, e n g}^{-}\right)\left(\tilde{\eta}_{m o t}^{-}\right)+\left(E_{\text {Acc }}\right)^{-}\right\}
$$

\subsubsection{CS P2 Parallel HEV: Regen Energy Reuse}

As always, a separate case is used to account for the reuse of all captured regen brake energy. The energy flow path for this case is illustrated in Figure 39. All the equations for this case are fully documented in Appendix X.

\section{Regen Energy Reuse: $\mathrm{F}_{\mathrm{Tr}}>0$}

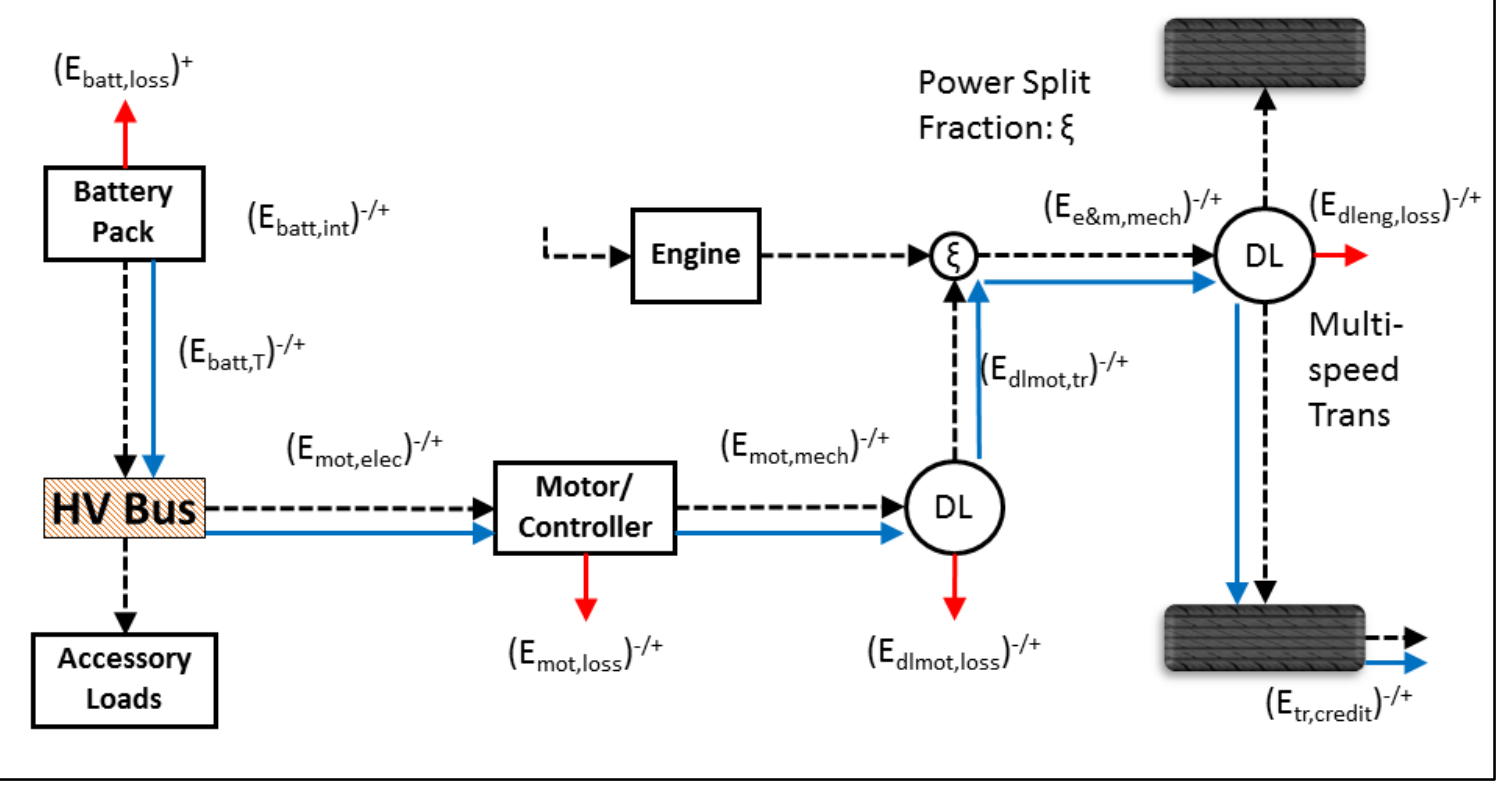

Figure 39: CS P2 Parallel HEV energy flow diagram for regen energy reuse

The energy consumption equation for this case, given by Equation 115, is developed in the same manner as before. Like the brake case, this energy consumption equation also carries an extra component loss for the engine driveline, which is highlighted for emphasis below. Keep in mind that component efficiencies unique to the regen reuse case are used in the equation but these efficiencies are approximated to be the respective propel efficiencies. This assumption simplifies calculations and eases the burden of determining an additional unique component efficiency.

Equation 115: Equivalent tractive energy 'credit' from regen braking (P2 Parallel, regen reuse)

$$
\left(E_{\text {tr,credit }}\right)^{-/+}=-\left(E_{\text {batt }, \text { int }}\right)^{-}\left(\tilde{\eta}_{\text {batt }}^{-/+}\right)\left(\tilde{\eta}_{\text {mot }}^{-/+}\right)\left(\tilde{\eta}_{d l, \text { mot }}^{-/+}\right)\left(\tilde{\eta}_{\text {dl,eng }}^{-/+}\right)
$$


The powertrain efficiency for this case is given by Equation 167. This powertrain efficiency also represents the powertrain efficiency for the EV powertrain. As previously mentioned, the ICE and EV powertrain efficiencies are calculated separately for comparison. Later, a combined powertrain will also be calculated.

\section{Equation 116: Powertrain efficiency for regen energy reuse (P2 Parallel, regen reuse)}

$$
\tilde{\eta}_{p t, E V}^{-/+}=\frac{\left(E_{\text {tr,credit }}\right)^{-/+}}{\left(E_{\text {batt }, \text { int }}\right)^{-/+}}
$$

\subsubsection{CS P2 Parallel HEV: Fuel-Equivalent Regen Energy Credit}

As in the Series HEV, the tractive energy 'credit' received from reusing stored regen energy is calculated as fuel-equivalent energy. Like before, this calculation is accomplished by starting with the tractive energy credit calculated in Equation 115 and then working back through a theoretical backward-flowing powertrain that is identical to the propel powertrain (except for the flow direction). The result of this calculation is the engine 'producing' fuel. This of course is not actually possible and is only a theoretical calculation. The fuel energy produced by the engine is inherently negative and will be summed later with the fuel use incurred during the propel case, thus reducing the total fuel energy used to complete the cycle. The energy flow for this case is illustrated by Figure 40. Again, it is identical to the (+tr) propel case for the P2 Parallel powertrain except that the energy now flows in the opposite direction.

\section{Regen Energy Reuse: Fuel-Equivalent Energy Credit}

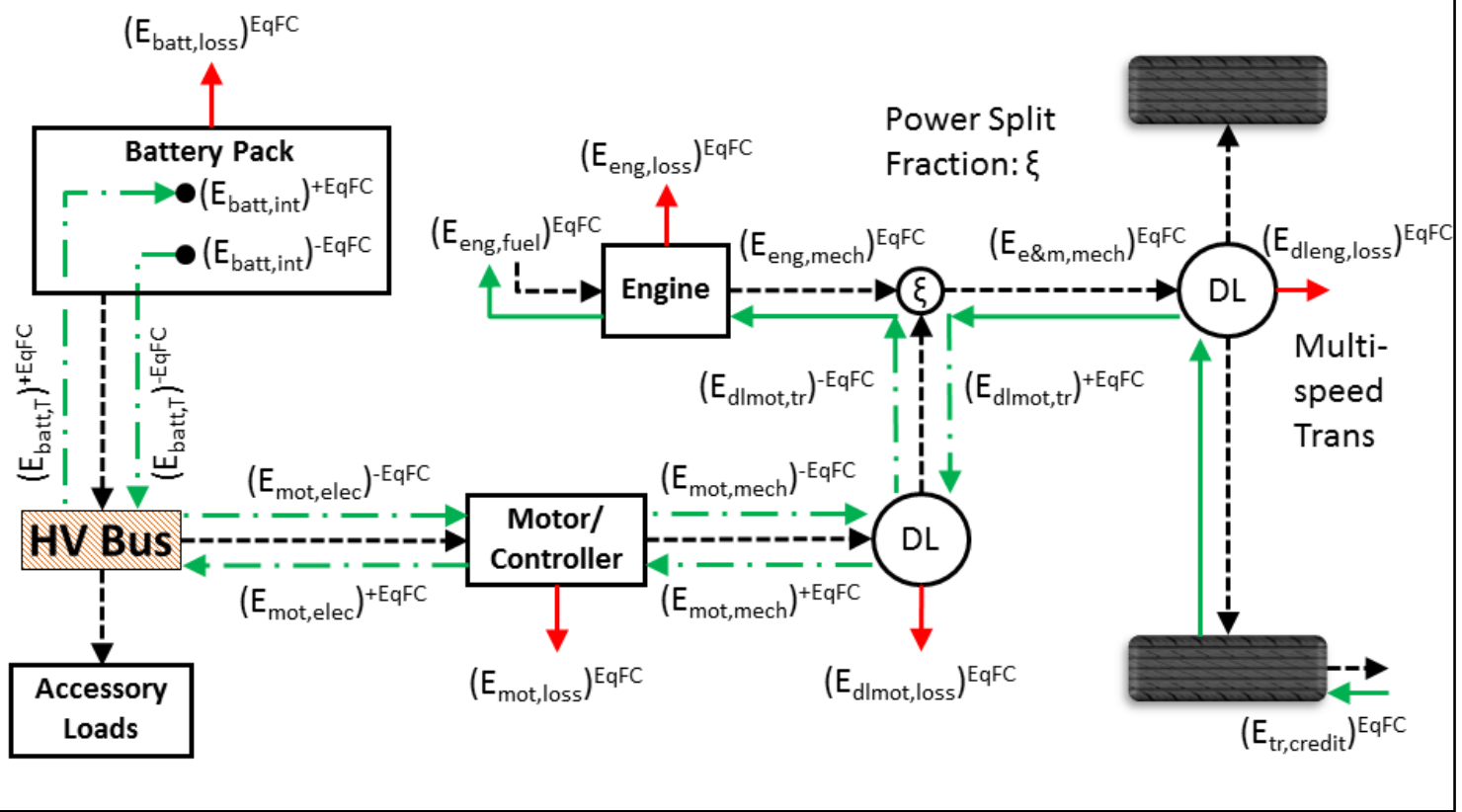

Figure 40: CS P2 Parallel HEV energy flow diagram for regen fuel credit

The energy consumption equation for this case is defined in Equation 117. This equation represents the fuel-equivalent credit received from performing regen braking. The equation is essentially identical to 
Equation 107, the energy consumption equation for the (+tr) propel case. This is as expected since the (+tr) case exempts accessory load just like this case.

Equation 117: Fuel-equivalent regen energy 'credit' (P2 Parallel, idle)

$$
\left(E_{\text {eng,fuel }}\right)^{E q F C}=\frac{-\left(E_{\text {tr,credit }}\right)^{E q F C}}{\left[\xi+(1-\xi)\left(\tilde{\eta}_{\text {recirc }}^{+}\right)\right]\left(\tilde{\eta}_{\text {eng }}^{+}\right)\left(\tilde{\eta}_{\text {dl,eng }}^{+}\right)}
$$

Bear in mind that the losses for this case are negative as explained previously in Section 3.4.5. Also, The component efficiencies used in regen fuel-credit case are necessarily exactly the same as in the propel case. This is because this case seeks to exactly replicate the propel case except in the opposite direction. All the equations for this case are fully documented in Appendix Y.

\subsubsection{CS P2 Parallel HEV: Idle Case}

As before, the accessory energy required for the idle case is also calculated as fuel-equivalent energy. For this approach, all energy consumed by accessories during the idle case is assumed to come from the engine, as illustrated by Figure 41. All the equations for this case are fully documented in Appendix $Z$.

\section{Idle: $\mathrm{F}_{\mathrm{Tr}}=0$}

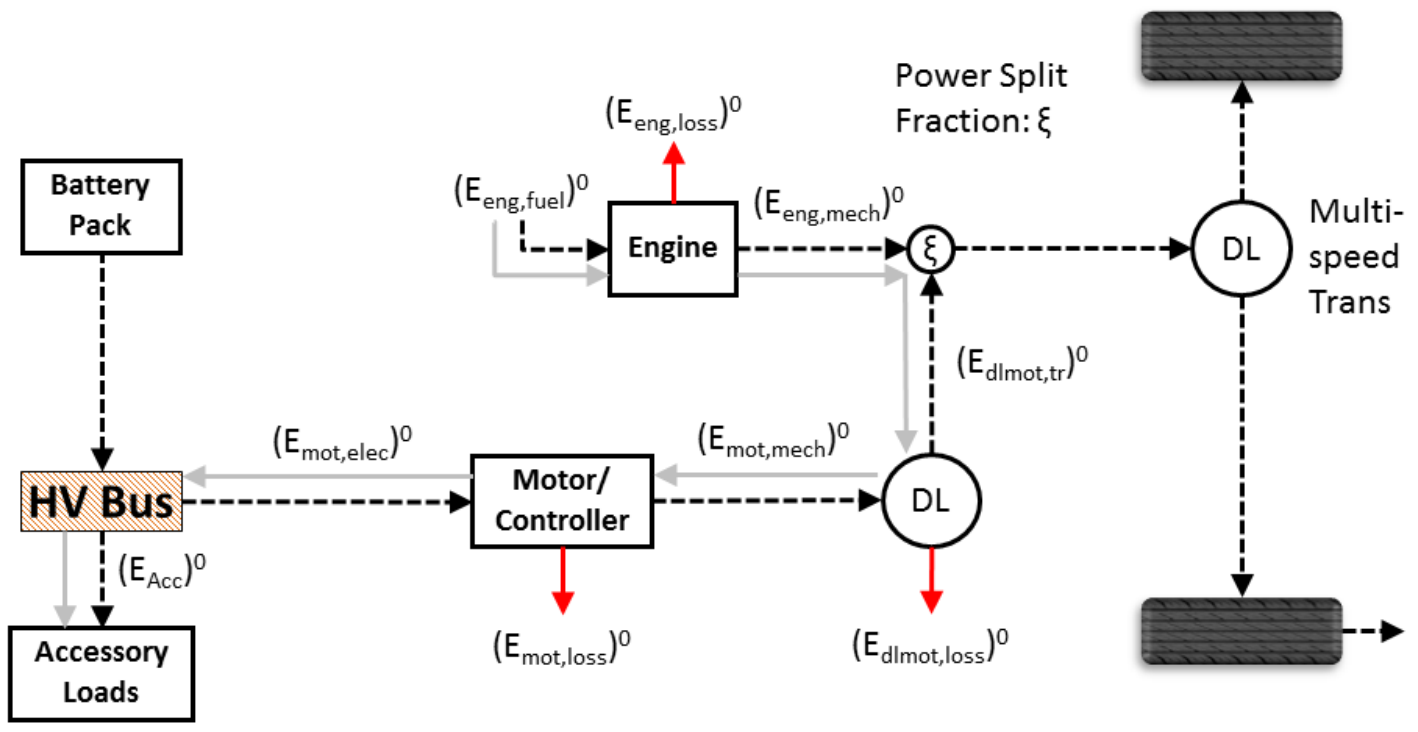

Figure 41: CS P2 Parallel HEV energy flow diagram for the idle case

As before, the total idle accessory energy is defined by Equation 36, which depends on the vehicle accessory load and amount of idle time. These two parameters are characteristic of the drive cycle only and are independent of vehicle properties. The path to supply the accessory load for the P2 Parallel HEV (3 losses) is shorter than the P3/P4 Parallel HEV (4 losses). Equation 118 gives the energy consumption equation for the idle case. Remember that component efficiencies unique to the idle case are given here, but are actually approximated by already established propel component efficiencies for simplicity. 
Equation 118: Idle case energy consumption (P2 Parallel, idle)

$$
\left(E_{\text {eng,fuel }}\right)^{0}=\frac{-\left(E_{\text {mot }, \text { elec }}\right)^{0}}{\left(\tilde{\eta}_{\text {eng }}^{0}\right)\left(\tilde{\eta}_{\text {dl,mot }}^{0}\right)\left(\tilde{\eta}_{\text {mot }}^{0}\right)}=\frac{\left(E_{\text {Acc }}\right)^{0}}{\left(\tilde{\eta}_{\text {eng }}^{0}\right)\left(\tilde{\eta}_{d l, m o t}^{0}\right)\left(\tilde{\eta}_{\text {mot }}^{0}\right)}
$$

Unlike the P3 or P4 configurations, it is no longer necessary that energy be transmitted through the drive axle to reach the HV bus and therefore the accessory load. Depending on the actual integration of the motor and engine, it may not be possible decouple either from vehicle speed. However, the engine driveline would likely have some mechanism to decouple the engine and motor from the wheels. This could be a torque converter, for example. Or transmission neutral could be used to decouple the engine and motor from the wheels. In any case, the P2 configuration would likely not experience the same problem with powering accessory loads at zero speed that the P3 or P4 configurations experience.

\subsubsection{CS P2 Parallel HEV: DC Net Case}

As before, the DCnet case is used to summarize all of the cases presented thus far even though this powertrain has no HV battery charger and therefore no ACnet case. The total fuel energy consumption for a given cycle may be calculated in two different ways. The first, given by Equation 119, simply sums the energy consumed during the propel, idle and regen fuel-credit cases.

Equation 119: Total fuel energy calculated by case (P2 Parallel, DCnet)

$$
\left(E_{\text {eng,fuel }}\right)^{\text {DCnet }}=\left(E_{\text {eng,fuel }}\right)^{+}+\left(E_{\text {eng,fuel }}\right)^{0}+\left(E_{\text {eng,fuel }}\right)^{\text {EqFC }}
$$

The second, given by Equation 120, can be used as a sanity check against the first and sums the road load, all accessory energy for the cycle and all the losses for the cycle. This equation asserts that the total fuel required to complete the cycle must meet road load, plus the accessory load, plus all the losses incurred during operation.

Equation 120: Total fuel energy calculated from overall energy balance (P2 Parallel, DCnet)

$$
\left(E_{\text {eng }, \text { fuel }}\right)^{\text {DCnet }}=\left(E_{\text {tr }}\right)^{\text {DCnet }}+\left(E_{A C C}\right)^{\text {DCnet }}+\left(E_{p t, l o s s}\right)^{\text {DCnet }}
$$

Road load and total accessory load may be calculated independent of the powertrain, but the total losses are dependent on the specific powertrain and component efficiencies. The total losses may be calculated using Equation 121.

Equation 121: Net powertrain losses (P2 Parallel, DCnet)

$$
\left(E_{p t, l o s s}\right)^{\text {DCnet }}=\left(E_{p t, l o s s}\right)^{+}+\left(E_{p t, l o s s}\right)^{-}+\left(E_{p t, l o s s}\right)^{-/+}+\left(E_{p t, l o s s}\right)^{0}+\left(E_{p t, l o s s}\right)^{E q F C}
$$


The net powertrain efficiency for this powertrain is defined in Equation 122. This measure of powertrain efficiency compares road load against the total fuel used to complete the cycle. The combined propel powertrain efficiency, is defined in Equation 123. The combined propel powertrain efficiency includes the losses of both the EV and the ICE powertrains. Nuances in powertrain efficiencies are discussed in detail in Section 3.3.5 and 3.5.7 and more supporting equations for this case can be found in Appendix AA.

Equation 122: Net powertrain efficiency (P2 Parallel, DCnet)

$$
\tilde{\eta}_{p t}^{\text {DCnet }}=\frac{\left(E_{\text {tr }}\right)^{\text {DCnet }}}{\left(E_{\text {eng,fuel }}\right)^{\text {DCnet }}}
$$

Equation 123: Net powertrain efficiency for combined propel operation (P2 Parallel, DCnet)

$$
\tilde{\eta}_{\text {pt,propel }}^{\text {DCnet }}=\frac{\left(E_{\text {tr }}\right)^{+}+\left(E_{\text {tr,credit }}\right)^{E q F C}}{\left(E_{\text {eng, fuel }}\right)^{+}+\left(E_{\text {batt }, \text { int }}\right)^{-/+}}
$$

\subsubsection{CS P2 Parallel HEV: Summary}

The P2 Parallel powertrain is very similar to the P3/P4 Parallel powertrain and contains many of the same characteristics. Both the engine and the drive motor have one operational degree of freedom each (torque). The key difference, however, is the placement of the drive motor, which is now located pretransmission instead of post-transmission. This introduces an extra loss to the EV powertrain which negatively impacts regen energy capture and reuse. However, it also removes a component loss from the accessory path and prevents energy from necessarily traveling through the drive axle to reach the HV bus from the fuel tank.

The concept of the Power Split Fraction is again used for this powertrain. The power split now occurs at a pre-trans node instead of at the drive axle or the HV bus. Compared to the P3/P4 HEV, the recirculation path is exactly the same. Additionally, the 2 full-time losses are also exactly the same. The concepts of regen energy reuse and fuel-equivalent energy consumption are also reused for this powertrain. Otherwise, no new concepts were introduced for this powertrain and it was in fact nearly identical in most cases. The one exception was the additional losses introduced to the EV powertrain by the engine driveline. This nuance is subtle, but unique to the P2 configuration. 


\section{Model Validation}

To validate the method and VTool, data was used from the Year 3 EcoCAR competition in which Virginia Tech competed against 15 other North American universities. As a part of the Year 3 competition, the EcoCAR competition vehicles were tested using various dynamic events to evaluate vehicle acceleration, braking, lateral handling, drive quality, towing capability, emissions and energy consumption. There were also static presentation events designed to evaluate the consumer acceptability, integration refinement and engineering merit of the vehicle. More information on the Year 3 competition and Virginia Tech's entry, the $\mathrm{VT}_{\mathrm{REX}}$, is contained in [14], [16], and [17].

Specifically, the on-road Emissions and Energy Consumption (E\&EC) testing data from competition is used in this thesis to validate parts of VTool and by extension the method in general. However, the entirety of VTool could not be validated using the available data. This is because VTool covers 4 different powertrains and 5 different drive cycles, while there is only test data available from one vehicle and an on-road approximations of 2 cycles (UDDS and HwFET).

This section will discuss in detail the $\mathrm{VT}_{\mathrm{REX}}$ to define the test vehicle, the drive cycles used in E\&EC testing at competition, as well as the results of the validation of VTool and the limitations present given the available data.

\section{1 $\mathrm{VT}_{\mathrm{REX}}$ Component Properties and Glider Parameters}

Before using test results to validate or calibrate VTool, the test vehicle must first be defined including: vehicle glider properties, powertrain architecture, and component properties. All of these parameters have already been defined in either the introduction or other sections. However, the tables and figures summarizing key information have been reproduced here for convenience. Figure 10 on the next page illustrates the powertrain architecture of the $\mathrm{VT}_{\mathrm{REX}}$. The $\mathrm{VT}_{\mathrm{REX}}$ is a Split-Parallel EREV - essentially a P4 Parallel powertrain with an additional P1 motor (though the P1 motor was small and only used for engine idle start/stop). Hence the test vehicle can only be used to validate the EV model and the P3/P4 Parallel model, but not the other powertrains. Table 1 on the next page summarizes the component specifications for the vehicle. For more detail on the powertrain components and overall operation, see Section 1.3.3. The glider properties of the vehicle, a 2009 Saturn Vue, are summarized in Table 14 on the next page. 


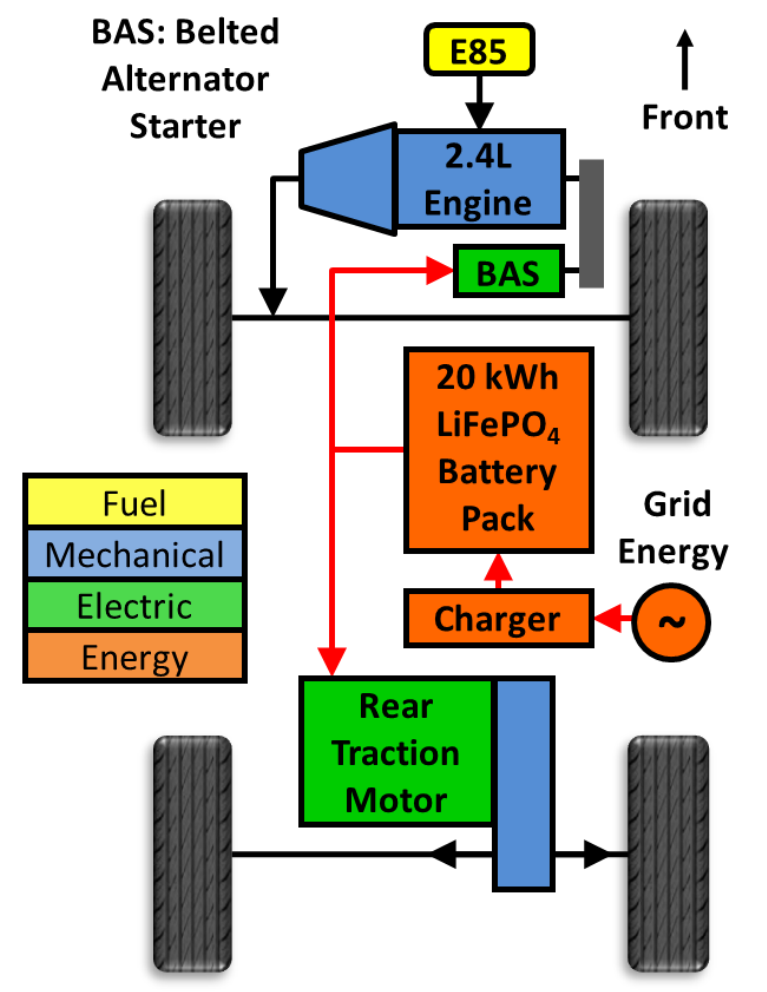

Table 14: Glider properties from 2009 Hybrid Saturn Vue used for model validation

\begin{tabular}{|l|l|}
\hline Vehicle & 2009 Saturn Vue \\
\hline $\mathrm{C}_{\text {rro }}$ & 0.009 \\
\hline $\mathrm{C}_{\mathrm{d}}$ & 0.36 \\
\hline $\mathrm{A}\left(\mathrm{m}^{2}\right)$ & 2.6 \\
\hline $\mathrm{C}_{\mathrm{d}} \mathrm{A}$ & 0.936 \\
\hline $\mathrm{M}(\mathrm{kg})$ & 2440 \\
\hline $\mathrm{Mi}(\mathrm{kg})$ & 2223 \\
\hline Rotating Inertia Factor & 1.04 \\
\hline $\mathrm{R}_{\mathrm{w}}(\mathrm{m})$ & 0.346 \\
\hline $\mathrm{N} / \mathrm{V}(\mathrm{rpm} / \mathrm{mph})$ & 89.5 \\
\hline
\end{tabular}

Figure 10: EcoCAR VT $T_{R E X}$ E85 Split Parallel EREV powertrain architecture

Table 1: VT $\mathrm{REX}$ Component Specifications

\begin{tabular}{|l|l|l|}
\hline Architecture & \multicolumn{2}{|l|}{ E85 Range Extended Split Parallel Architecture } \\
\hline Components & Size & Type \\
\hline Engine & $130 \mathrm{~kW}$ peak & $\begin{array}{l}\text { GM LE9 2.4 L ECOTEC VVT } \\
\text { DOHC 16V I4 FlexFuel SI }\end{array}$ \\
\hline Transmission & - & $\begin{array}{l}\text { ME7 4T45 hybrid 4-speed } \\
\text { automatic FWD }\end{array}$ \\
\hline BAS & $9 \mathrm{~kW}$ peak & $\begin{array}{l}\text { Kollmorgen custom AC induction } \\
\text { motor }\end{array}$ \\
\hline RTM & $125 \mathrm{~kW}$ peak & $\begin{array}{l}\text { UQM liquid cooled permanent } \\
\text { magnet motor }\end{array}$ \\
\hline $\begin{array}{l}\text { RTM } \\
\text { Transaxle }\end{array}$ & - & $\begin{array}{l}\text { BorgWarner 3103, gear } \\
\text { reduction: 7.17:1 }\end{array}$ \\
\hline ESS & $352 \mathrm{~V}$ & $\begin{array}{l}\text { A123 Systems custom built } \\
\text { prismatic pack, 18.7 kWh useable }\end{array}$ \\
\hline 12 V Supply & $1 \mathrm{~kW}$ cont. & $\begin{array}{l}\text { Delphi DC/DC Converter, from } \\
\text { 352 V to 13.8 V }\end{array}$ \\
\hline A/C System & $10 \mathrm{~kW}$ & $\begin{array}{l}\text { High voltage electric drive, high } \\
\text { efficiency, variable speed }\end{array}$ \\
\hline thermal) & $3.3 \mathrm{~kW}$ cont. & $\begin{array}{l}\text { Brusa 120/240 V 50/60 Hz AC, } \\
\text { integrated on-board }\end{array}$ \\
\hline $\begin{array}{l}\text { Supervisory } \\
\text { Controller }\end{array}$ & - & $\begin{array}{l}\text { NI CompactRIO with field } \\
\text { programmable gate array (FPGA) }\end{array}$ \\
\hline
\end{tabular}




\subsection{EcoCAR Year 3 Competition E\&EC Drive Cycles}

The drive cycles used to perform E\&EC testing at the Year 3 EcoCAR competition were on-road approximations of the EPA CAFE weighting method. The CAFE weighting includes 55\% UDDS and $45 \%$ HwFET. Hence, there is no test data available for other cycles such as US06 and LA92 that may be used to validate VTool. As such, only the UDDS and HwFET drive cycles will be calibrated and will serve as proof of concept for VTool and the method presented in this thesis.

An additional nuance to the test data is that it was performed on-road and not on a dynamometer. This requires that the cycles be simplified so that the driver can safely complete the cycles in an on-road environment where other vehicles are present. It is important to note that the circle track at GM's Milford proving grounds was used for closed-course testing instead of an open-road environment. The development was also constrained to the distance of the circle track among other things.

As discussed in Section 2.5, the E\&EC testing was broken into three schedules of different lengths $(A=20$ $\mathrm{mi}, \mathrm{B}=40 \mathrm{mi}$ and $\mathrm{C}=100 \mathrm{mi}$ ) that were used as an on-road approximation of the SAE J1711 Utility Factor weighting method [15]. Each schedule contained elements of city and highway driving (ie UDDS and HwFET) as illustrated by Figure 42. For schedule B, shown below, 4 city cycles and 3 highway cycles were completed. For schedule C, 10 city cycles and 9 highway cycles were completed. Likewise, schedule A included 2 city cycles and 1 highway cycle.

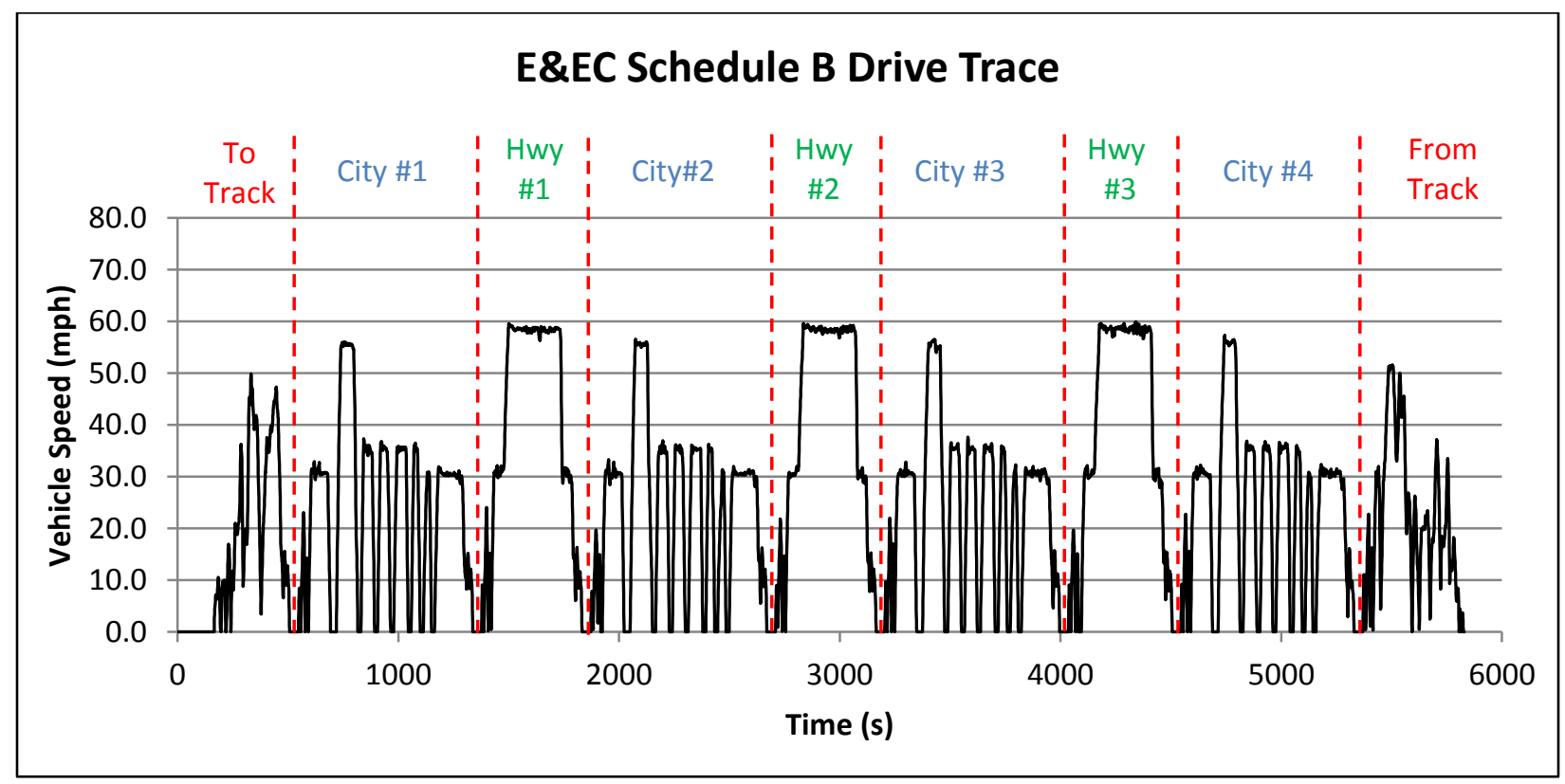

Figure 42: EcoCAR E\&EC schedule B drive trace

The simplified nature of the E\&EC drive schedules means that the UDDS and HwFET cycles are not perfectly represented. However, this on-road approximation proved to be a reasonable approximation of the individual UDDS and HwFET cycles, but an excellent approximation of the overall CAFE and UF weightings. Table 3 (taken from Section 1.5) summarizes the characteristics of the EcoCAR 1 CAFE weighting as well as the EcoCAR 2 4-cycle weighting. 
Table 3: Summary of EcoCAR 1 CAFE and EcoCAR 2 4-cycle weightings

\begin{tabular}{|c|c|c|c|c|c|}
\hline Cycle & $\begin{array}{l}\text { Time (Idle } \\
\text { Time) (s) }\end{array}$ & $\begin{array}{c}\text { Weight } \\
(\%)\end{array}$ & $\begin{array}{c}\text { Max Speed } \\
\text { (mph) }\end{array}$ & $\begin{array}{c}\text { Avg Run } \\
\text { Speed (mph) }\end{array}$ & Peak Accel $\left(\mathrm{m} / \mathrm{s}^{2}\right)$ \\
\hline UDDS & $1369(241)$ & $55 \%$ & 56.7 & 23.8 & 1.48 \\
\hline HwFET & $765(4)$ & $45 \%$ & 59.9 & 48.5 & 1.43 \\
\hline \multicolumn{2}{|c|}{ EC1 CAFE weighting: } & $100 \%$ & 59.9 & 30.9 & 1.48 \\
\hline 505 & $505(94)$ & $67 \%$ & 56.7 & 31.5 & 1.48 \\
\hline US06 City & $231(27)$ & $33 \%$ & 70.7 & 31.3 & 3.76 \\
\hline \multicolumn{2}{|c|}{ EC2 4-cycle City weighting: } & $43 \%$ & 70.7 & 31.4 & 3.76 \\
\hline HwFET & $765(4)$ & $22 \%$ & 59.9 & 48.5 & 1.43 \\
\hline US06 Highway & $365(8)$ & $78 \%$ & 80.3 & 62.9 & 3.08 \\
\hline \multicolumn{2}{|c|}{ EC2 4-cycle Highway weighting: } & $57 \%$ & 80.3 & 59.0 & 3.08 \\
\hline \multicolumn{2}{|c|}{ EC2 4-cycle weighting: } & $100 \%$ & 80.3 & 42.8 & 3.76 \\
\hline
\end{tabular}

\subsection{EV Model Validation}

The EV model in VTool was validated using data from schedule B of E\&EC testing. Schedule B was chosen because it had more data than schedule A and contained only EV data. Schedule $C$ contains approximately 54 miles ( 5 city and 5 highway cycle) of EV driving before the vehicle transitioned to CS mode. However, the presence of CS data in the results complicated post-processing. Schedule B contains 40 miles of EV driving (4 city and 3 highway cycles) which is sufficient data to produce confident results.

\subsubsection{General Validation and Tuning Strategy}

There are effectively 8 parameters that could be tuned to achieve the proper results. There are also 3 benchmarks derived from the EcoCAR E\&EC data that may be used to verify proper tuning for each cycle (propel, brake and DCnet energy consumption at the battery terminals). Table 15 summarizes the tunable parameters for the EV model. There are additional parameters to be tuned for the charging case - these parameters are presented later

Table 15: Summary of EV model tunable parameters and tuning strategy

\begin{tabular}{|c|c|c|c|c|}
\hline & Parameter & Symbol & Data available? & $\begin{array}{c}\text { Tune } \\
\text { Parameter? }\end{array}$ \\
\hline \multirow{3}{*}{ 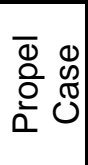 } & Battery discharge efficiency & $\tilde{\eta}_{\text {batt }}^{+}$ & Yes, A123 ICD data & No \\
\hline & Motor propel efficiency & $\tilde{\eta}_{\text {mot }}^{+}$ & Yes, but suspect & Yes \\
\hline & Motor driveline propel efficiency & $\tilde{\eta}_{\mathrm{dl}, \mathrm{mot}}^{+}$ & Yes, from Gantt [24] & If needed \\
\hline \multirow{5}{*}{ 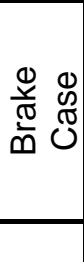 } & Battery charge efficiency & $\tilde{\eta}_{\text {batt }}^{-}$ & Yes, A123 ICD data & No \\
\hline & Motor brake efficiency & $\tilde{\eta}_{\text {mot }}^{-}$ & Yes, but suspect & Yes \\
\hline & Motor driveline brake efficiency & $\tilde{\eta}_{\mathrm{dl}, \mathrm{mot}}^{-}$ & Yes, from Gantt [24] & If needed \\
\hline & Regen brake fraction & $\tilde{\zeta}$ & No & Yes \\
\hline & Accessory load & $\mathrm{P}_{\mathrm{acc}}$ & Yes, from Perkins [33] & No \\
\hline
\end{tabular}

The model was validated by segregating the E\&EC data into city and highway portions and calibrating the UDDS and HwFET cycles separately. For each cycle, the propel, brake and overall DC energy 
consumption was calculated at the battery terminals. Additionally, the motor propel and brake efficiencies were calculated. Unfortunately, there was insufficient data present from the E\&EC drive schedules to calculate any of the other tunable parameters. However, data exists from other sources for 4 of the parameters. Data from A123 internal control documents was used to generate a confident estimate at battery charge and discharge efficiency. Likewise, data was gathered by Gantt to calculate the driveline propel and brake efficiency. This data was gathered during dynamometer testing at EPA facilities in Ann Arbor, $\mathrm{Ml}$ and is discussed in Gantt's thesis [24]. Additionally, data from other testing performed by Perkins and presented in his IEEE paper [33] was used to establish the accessory load.

The E\&EC data gives a ballpark figure for motor efficiency, but the numbers are suspect because the brake efficiency seems too high and the propel efficiency seems a little low. Knowing that the reported values for mechanical and electrical energy are estimates calculated by the inverter, the related efficiencies are used only as general ballpark guidelines.

There are 3 parameters that may be independently tuned to align the modeled propel, brake and net energy consumption with the empirically determined values. These three parameters are motor propel and brake efficiency and the regen brake fraction. The motor propel efficiency is the only unknown for the propel case so it was tuned until the propel energy consumption met the empirically derived benchmark value. The motor brake efficiency was also adjusted to bring brake energy consumption closer to the target. The regen brake fraction was used to ensure that the brake motor efficiency was in a reasonable range while still hitting the benchmark value. Each cycle was tuned independently, but the goal was to meet the CAFE net energy consumption. As a result, both the UDDS and HwFET cycles missed their benchmark targets, but the CAFE weighting hit the target, which is more important.

\subsubsection{UDDS Validation}

Table 16 summarizes the target or benchmark values derived from various datasets including the EcoCAR E\&EC dataset. The table also summarizes the tunable parameters used in the model, the results of the model and the error from the target value. The results show that the modeled values for net and propel energy consumption overestimated the target by about $4 \%$ each. This was done purposefully to meet the CAFE target, which will be presented in a later section. The motor propel efficiency was left at $84 \%$, but the brake efficiency was reduced to $86 \%$ from $91 \%$. The peak efficiency of the motor used in the $\mathrm{VT}_{\mathrm{REX}}$ is $94 \%$, so an empirical value of $91 \%$ seemed unrealistically high. The motor driveline propel and brake efficiencies were also reduced to $91 \%$ from $92 \%$ to further increase the net energy consumption to help meet the CAFE target. The regen brake fraction was set at 0.75 to bring the brake energy consumption to meet its target. 
Table 16: Summary of EV test data and final parameter values for the UDDS cycle

\begin{tabular}{|c|c|c|c|c|c|}
\hline & Parameter & Symbol & Empirical Value & Modeled Value & \% Error \\
\hline \multirow{4}{*}{$\begin{array}{l}\Phi \\
\mathbb{D} \\
0 \\
0 \\
\Phi \\
\stackrel{0}{0} \\
\stackrel{0}{0}\end{array}$} & Propel energy consumption & $\left(\mathrm{E}_{\text {batt,int }}\right)^{+}$ & $398 \mathrm{DC} \mathrm{Wh} / \mathrm{mi}$ & $411 \mathrm{DC} \mathrm{Wh} / \mathrm{mi}$ & $3.3 \%$ \\
\hline & Battery discharge efficiency & $\tilde{\eta}_{\text {batt }}^{+}$ & $99 \%$ & $99 \%$ & $0 \%$ \\
\hline & Motor propel efficiency & $\tilde{\eta}_{\text {mot }}^{+}$ & $84 \%$ & $84 \%$ & $0 \%$ \\
\hline & Motor driveline propel efficiency & $\tilde{\eta}_{\mathrm{dl}, \mathrm{mot}}^{+}$ & $92 \%$ & $91 \%$ & $1 \%$ \\
\hline \multirow{5}{*}{ 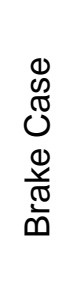 } & Brake energy consumption & $\left(E_{\text {batt,int }}\right)^{-}$ & $-77 \mathrm{DC} \mathrm{Wh} / \mathrm{mi}$ & $-77 \mathrm{DC} \mathrm{Wh} / \mathrm{mi}$ & $0 \%$ \\
\hline & Battery charge efficiency & $\tilde{\eta}_{\text {batt }}^{-}$ & $99 \%$ & $99 \%$ & $0 \%$ \\
\hline & Motor brake efficiency & $\tilde{\eta}_{\text {mot }}^{-}$ & $91 \%$ & $86 \%$ & $5.5 \%$ \\
\hline & Motor driveline brake efficiency & $\tilde{\eta}_{\mathrm{dl}, \mathrm{mot}}^{-}$ & $92 \%$ & $91 \%$ & $1 \%$ \\
\hline & Regen brake fraction & $\tilde{\zeta}$ & -- & 0.75 & -- \\
\hline \multirow{2}{*}{ U } & DC net energy consumption & $\left(E_{\text {batt,int }}\right)^{\mathrm{DCnet}}$ & $321 \mathrm{DC} \mathrm{Wh} / \mathrm{mi}$ & $334 \mathrm{DC} \mathrm{Wh} / \mathrm{mi}$ & $4 \%$ \\
\hline & Accessory load & $P_{\text {acc }}$ & $600 \mathrm{~W}$ & $600 \mathrm{~W}$ & $0 \%$ \\
\hline
\end{tabular}

\subsubsection{HwFET Validation}

Table 17 summarizes the target or benchmark values derived from various datasets including the EcoCAR E\&EC dataset. The table also summarizes the tunable parameters used in the model, the results of the model and the error from the target value. The propel energy consumption was very close to its target, but the brake energy consumption missed the target by $36 \%$. However, the net energy consumption for the HwFET was within $1.1 \%$ of the target so the error in the brake energy consumption is acceptable. This error is likely due to discrepancies between the actual HwFET cycle and the on-road approximation. In the actual HwFET cycle, there is only 1 deceleration but in on-road E\&EC cycle there are 3 decelerations for schedule $B$ (one for each repetition of highway driving).

For consistency, the motor driveline efficiency, and regen brake fraction were kept consistent with the UDDS values. As a result, the propel motor efficiency was reduced to $79 \%$ and the brake motor efficiency was reduced to $88 \%$. 
Table 17: Summary of EV test data and final parameter values for the HwFET cycle

\begin{tabular}{|c|c|c|c|c|c|}
\hline & Parameter & Symbol & Empirical Value & Modeled Value & $\%$ Error \\
\hline \multirow{4}{*}{$\begin{array}{l}0 \\
\mathscr{D} \\
\mathcal{O} \\
\bar{\Phi} \\
\stackrel{0}{0} \\
\stackrel{0}{0}\end{array}$} & Propel energy consumption & $\left(E_{\text {batt,int }}\right)^{+}$ & $384 \mathrm{DC} \mathrm{Wh} / \mathrm{mi}$ & $375 \mathrm{DC} \mathrm{Wh} / \mathrm{mi}$ & $2.3 \%$ \\
\hline & Battery discharge efficiency & $\tilde{\eta}_{\text {batt }}^{+}$ & $99 \%$ & $99 \%$ & $0 \%$ \\
\hline & Motor propel efficiency & $\tilde{\eta}_{\text {mot }}^{+}$ & $82 \%$ & $79 \%$ & $3.7 \%$ \\
\hline & Motor driveline propel efficiency & $\tilde{\eta}_{\mathrm{dl}, \mathrm{mot}}^{+}$ & $92 \%$ & $91 \%$ & $1 \%$ \\
\hline \multirow{5}{*}{$\begin{array}{l}\mathbb{0} \\
\mathbb{N} \\
0 \\
0 \\
\mathscr{D} \\
\frac{\Sigma}{\mathbb{N}} \\
\overline{0}\end{array}$} & Brake energy consumption & $\left(E_{\text {batt, int }}\right)^{-}$ & $-28 \mathrm{DC} \mathrm{Wh} / \mathrm{mi}$ & $-18 \mathrm{DC} \mathrm{Wh} / \mathrm{mi}$ & $36 \%$ \\
\hline & Battery charge efficiency & $\tilde{\eta}_{\text {batt }}^{-}$ & $99 \%$ & $99 \%$ & $0 \%$ \\
\hline & Motor brake efficiency & $\tilde{\eta}_{\text {mot }}^{-}$ & $90 \%$ & $88 \%$ & $2.2 \%$ \\
\hline & Motor driveline brake efficiency & $\tilde{\eta}_{\mathrm{dl}, \mathrm{mot}}^{-}$ & $92 \%$ & $91 \%$ & $1 \%$ \\
\hline & Regen brake fraction & $\tilde{\zeta}$ & --- & 0.75 & --- \\
\hline \multirow{2}{*}{ 仓 } & DC net energy consumption & $\left(E_{\text {batt,int }}\right)^{\text {DCnet }}$ & $357 \mathrm{DC} \mathrm{Wh} / \mathrm{mi}$ & $353 \mathrm{DC} \mathrm{Wh} / \mathrm{mi}$ & $1.1 \%$ \\
\hline & Accessory load & $\mathrm{P}_{\mathrm{acc}}$ & $600 \mathrm{~W}$ & $600 \mathrm{~W}$ & $0 \%$ \\
\hline
\end{tabular}

\subsubsection{CAFE Weighting Validation}

As previously mentioned, the primary goal of tuning the UDDS and HwFET cycles was to achieve an accurate CAFE weighted DC net energy consumption. Table 18 summarizes the modeled energy consumption for UDDS and HwFET cycles as well as the CAFE weighting. The net DC energy consumption, or energy consumption at the battery terminals, came out to $343 \mathrm{DC} \mathrm{Wh} / \mathrm{mi}$ which is within $1 \%$ of the empirical value of $345 \mathrm{DC} \mathrm{Wh} / \mathrm{mi}$. This is more than close enough considering that the on-road test data is an approximation of the CAFE weighting.

Table 18: Summary of EV energy consumption modeling for UDDS, HwFET and CAFE

\begin{tabular}{|c|c|c|c|c|c|}
\hline Parameter & Symbol & Cycle & $\begin{array}{c}\text { Empirical } \\
\text { Value }\end{array}$ & $\begin{array}{c}\text { Modeled } \\
\text { Value }\end{array}$ & \% Error \\
\hline \multirow{3}{*}{ Propel energy consumption } & $\left(E_{b a t t, T}\right)^{+}$ & UDDS & $398 \mathrm{DC} \mathrm{Wh} / \mathrm{mi}$ & $411 \mathrm{DC} \mathrm{Wh} / \mathrm{mi}$ & $3.3 \%$ \\
\hline & $\left(E_{\text {batt }, T}\right)^{+}$ & HwFET & $384 \mathrm{DC} \mathrm{Wh} / \mathrm{mi}$ & $375 \mathrm{DC} \mathrm{Wh} / \mathrm{mi}$ & $2.3 \%$ \\
\hline & $\left(E_{\text {batt }, T}\right)^{+}$ & CAFE & $405 \mathrm{DC} \mathrm{Wh} / \mathrm{mi}$ & $395 \mathrm{DC} \mathrm{Wh} / \mathrm{mi}$ & $2.5 \%$ \\
\hline \multirow{3}{*}{ Brake energy consumption } & $\left(E_{\text {batt }, T}\right)^{-}$ & UDDS & $-77 \mathrm{DC} \mathrm{Wh} / \mathrm{mi}$ & $-77 \mathrm{DC} \mathrm{Wh} / \mathrm{mi}$ & $0.0 \%$ \\
\hline & $\left(E_{\text {batt }, T}\right)^{-}$ & HwFET & $-28 \mathrm{DC} \mathrm{Wh} / \mathrm{mi}$ & $-18 \mathrm{DC} \mathrm{Wh} / \mathrm{mi}$ & $36 \%$ \\
\hline & $\left(E_{\text {batt }, T}\right)^{-}$ & CAFE & $-59 \mathrm{DC} \mathrm{Wh} / \mathrm{mi}$ & $-50 \mathrm{DC} \mathrm{Wh} / \mathrm{mi}$ & $14.5 \%$ \\
\hline \multirow{3}{*}{ DC net energy consumption } & $\left(E_{\text {batt }, T}\right)^{\text {DCnet }}$ & UDDS & $321 \mathrm{DC} \mathrm{Wh} / \mathrm{mi}$ & $334 \mathrm{DC} \mathrm{Wh} / \mathrm{mi}$ & $4.0 \%$ \\
\hline & $\left(E_{\text {batt }, T}\right)^{D C n e t}$ & HwFET & $357 \mathrm{DC} \mathrm{Wh} / \mathrm{mi}$ & $353 \mathrm{DC} \mathrm{Wh} / \mathrm{mi}$ & $1.1 \%$ \\
\hline & $\left(E_{\text {batt }, T}\right)^{\text {DCnet }}$ & CAFE & $345 \mathrm{DC} \mathrm{Wh} / \mathrm{mi}$ & $343 \mathrm{DC} \mathrm{Wh} / \mathrm{mi}$ & $0.6 \%$ \\
\hline
\end{tabular}

\subsubsection{Charging Case Validation}

For the charging case, there are 4 parameters that can be tuned to achieve the proper AC net energy consumption. However, the 4 parameters are not fully decoupled. The charging efficiency parameter is redundant with the other three. Hence, there are two methods by which AC net energy may be calculated (this is discussed in detail in Section 3.3.6). Depending upon the data available, it may be more 
advantageous to use one modeling strategy over the other. For example, it is much easier to gather empirical data on the overall charging efficiency of the system. This measurement only requires a power meter at the battery terminals and another power meter somewhere on the AC side of the system (usually at the electrical panel). The other method requires component efficiency information for the charger and the battery as well as the charging case accessory load. This data is often hard to obtain so the charging efficiency is often used instead.

Unlike the propel or brake case, the tunable parameters for the charging case are prone to change significantly depending upon the charging location and how AC energy is measured. One nuance of the charging case that is not included in this model is the effect of losses in the wire on the AC side of the system before reaching the onboard HV charger. The length of wire between the charge port on the car and the $A C$ electrical panel could change drastically depending upon the specific charge location. If $A C$ energy is always measured at the AC electrical panel, then the losses in this wire could cause significant discrepancies in $\mathrm{AC}$ energy consumption necessitating significant changes in the tunable parameters.

Table 19 summarizes the tunable parameters for this case and shows that the only parameter that needs tuning is the charging accessory load. The battery charge efficiency is kept constant from the previous case and the efficiency for the HV charger is set using data from the manufacturer (Brusa). Data for charging efficiency is available from the E\&EC data gathered during competition. Additional data is also available from charging in HEVT's on-campus facility in the Ware Lab. Thus, the only parameter requiring tuning is the charging accessory load. This means that the charging accessory load will model the actual accessory load and the wire losses from the electrical panel to the car (since the wire losses are otherwise unmodeled).

Table 19: Summary of EV model tunable parameters and tuning strategy for the charging case

\begin{tabular}{|c|c|c|c|c|}
\hline & Parameter & Symbol & Data available? & $\begin{array}{c}\text { Tune } \\
\text { Parameter? }\end{array}$ \\
\hline \multirow{4}{*}{ 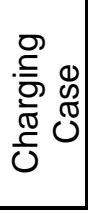 } & Battery charge efficiency & $\tilde{\eta}_{\text {batt }}^{\mathrm{C}}$ & Yes, A123 ICD data & No \\
\hline & Charging accessory load & $\left(P_{a c c}\right)^{C}$ & No & Yes \\
\hline & Charger efficiency & $\tilde{\eta}_{\text {chrgr }}^{\mathrm{C}}$ & Yes, Brusa ICD data & No \\
\hline & Charging efficiency & $\tilde{\eta}_{\text {chrgn }}^{\mathrm{C}}$ & Yes, E\&EC data & No \\
\hline
\end{tabular}

To minimize the effect of wire losses as previously described, the charging accessory load was tuned using data from on-campus Ware Lab charging where the wire run was less than 10 feet. In VTool, there are two redundant calculations for AC net energy - one using the lumped charging efficiency and the other using the other three parameters. Since the charging efficiency was well known from the data, the accessory load was tuned until the two AC net energy calculations agreed. The final parameter values are summarized in Table 20 below. The accessory load was set to $102 \mathrm{~W}$, which is much less than the normal accessory load of $600 \mathrm{~W}$. This, however, makes sense because far fewer accessory systems are active while charging. Using these numbers, the AC net energy consumption was found to be $395 \mathrm{AC}$ $\mathrm{Wh} / \mathrm{mi}$. This number is for the CAFE weighting and is based upon a DC energy consumption of $343 \mathrm{DC}$ $\mathrm{Wh} / \mathrm{mi}$ (which was found in the previous section). 
Table 20: Summary of EV test data and final parameter values for the CAFE weighting

\begin{tabular}{|c|c|c|c|}
\hline & Parameter & Symbol & Modeled Value \\
\hline \multirow{5}{*}{ 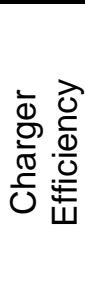 } & AC net energy consumption & $\left(E_{\text {grid, } A C}\right)^{A C n e t}$ & $395 \mathrm{AC} \mathrm{Wh} / \mathrm{mi}$ \\
\hline & Battery discharge efficiency & $\tilde{\eta}_{\text {batt }}^{\mathrm{C}}$ & $99 \%$ \\
\hline & Charging accessory load & $\left(P_{a c c}\right)^{C}$ & $102 \mathrm{~W}$ \\
\hline & Charger efficiency & $\tilde{\eta}_{\text {chrgr }}^{\mathrm{C}}$ & $92 \%$ \\
\hline & Charging efficiency & $\tilde{\eta}_{\text {chrgn }}^{\mathrm{C}}$ & $88 \%$ \\
\hline
\end{tabular}

Additionally, the AC net energy consumption was checked against data from competition. As Table 21 shows, the actual AC energy consumption at competition was $437 \mathrm{AC} \mathrm{Wh} / \mathrm{mi}$. This leads to a $9.8 \%$ error in $A C$ energy consumption. The cause of this error is the arrangement for charging used at GM's Milford Proving Grounds - the electrical panel was more than 100 feet away from the vehicles. This resulted in substantial wire losses. From E\&EC data, the lumped charging efficiency was found to be $80 \%$ on average. Using this charging efficiency instead of $88 \%$, the modeled AC energy consumption comes to $434 \mathrm{AC} \mathrm{Wh} / \mathrm{mi}$, giving an error of less than $1 \%$ which is well within reason. The charging accessory load would be 370 Watts (which is much too high) if it were tuned using a charging efficiency of $80 \%$.

Table 21: Summary of EV test data and final parameter values for the CAFE weighting

\begin{tabular}{|c|c|c|c|c|c|}
\hline Parameter & Symbol & Cycle & $\begin{array}{c}\text { Empirical } \\
\text { Value }\end{array}$ & $\begin{array}{c}\text { Modeled } \\
\text { Value }\end{array}$ & \% Error \\
\hline \multirow{3}{*}{$\begin{array}{c}\text { AC net energy consumption } \\
\text { (at 88\% charging efficiency) } \\
\text { Ware Lab charging data }\end{array}$} & $\left(E_{\text {grid, }, A C}\right)^{A C n e t}$ & UDDS & --- & $385 \mathrm{AC} \mathrm{Wh} / \mathrm{mi}$ & --- \\
\hline & $\left(E_{\text {grid, } A C}\right)^{A C n e t}$ & HwFET & --- & $406 \mathrm{AC} \mathrm{Wh} / \mathrm{mi}$ & --- \\
\hline & $\left(E_{\text {grid, }, A C}\right)^{A C n e t}$ & CAFE & $437 \mathrm{AC} \mathrm{Wh} / \mathrm{mi}$ & $395 \mathrm{AC} \mathrm{Wh} / \mathrm{mi}$ & $9.8 \%$ \\
\hline \multirow{3}{*}{$\begin{array}{c}\text { AC net energy consumption } \\
\text { (at 80\% charging efficiency) } \\
\text { E\&EC charging data }\end{array}$} & $\left(E_{\text {grid, } A C}\right)^{A C n e t}$ & UDDS & -- & $424 \mathrm{AC} \mathrm{Wh} / \mathrm{mi}$ & -- \\
\hline & $\left(E_{\text {grid, } A C}\right)^{A C n e t}$ & HwFET & --- & $446 \mathrm{AC} \mathrm{Wh} / \mathrm{mi}$ & --- \\
\hline & $\left(E_{\text {grid, } A C}\right)^{A C n e t}$ & CAFE & $437 \mathrm{AC} \mathrm{Wh} / \mathrm{mi}$ & $434 \mathrm{AC} \mathrm{Wh} / \mathrm{mi}$ & $0.7 \%$ \\
\hline
\end{tabular}

\subsubsection{EV Model Validation Summary}

Table 22 presents a summary of the modeling results produced by VTool after all parameters were tuned as described above. As the table shows, both the AC and DC energy consumption approximate the actual consumption within $1 \%$. Hence, VTool was successful in modeling energy consumption for an EV for UDDS and HwFET cycles. By extension, the method described above could be used to calibrate VTool to properly model EV mode for other cycles such as US06, SC03 or LA92. Table 22 also presents the net tractive energy (or road load) modeled by VTool and compares it to the actual road load from the E\&EC data. Comparing these two values shows how well the E\&EC drive schedule approximated the UDDS and HwFET cycles as well as the CAFE weighting. The E\&EC drive schedule overestimated the road load for UDDS by $7.4 \%$ and overestimated HwFET by $4.2 \%$ but was within $3 \%$ for the CAFE weighted road load. This shows that the E\&EC schedule was very effective in approximating the CAFE weighting. 
Table 22: Summary of VTool modeling results and comparison to actual data for EV mode

\begin{tabular}{|c|c|c|c|c|}
\cline { 2 - 5 } \multicolumn{1}{c|}{} & \multicolumn{4}{|c|}{ CAFE Weighted Results - EV Mode } \\
\cline { 2 - 5 } & Weight & $\left(\mathrm{E}_{\text {grid,AC }}\right)^{\text {ACnet }}$ & $\left(\mathrm{E}_{\text {batt, }}\right)^{\text {DCnet }}$ & $\left(\mathrm{E}_{\text {tr }}\right)^{\text {DCnet }}$ \\
\cline { 2 - 5 } & {$[\%]$} & {$[\mathrm{AC}$ Wh/mi] } & {$[\mathrm{DC}$ Wh/mi] } & {$[\mathrm{Wh} / \mathrm{mi}]$} \\
\hline \multirow{4}{*}{ UDDS } & Model & 424 & 334 & 151 \\
\cline { 2 - 5 } & Actual & --- & 321 & 163 \\
\cline { 2 - 5 } & Error & --- & $4.0 \%$ & $7.4 \%$ \\
\hline \multirow{4}{*}{ HwFET } & Model & 446 & 353 & 226 \\
\cline { 2 - 5 } & Actual & --- & 357 & 236 \\
\cline { 2 - 5 } & Error & --- & $1.1 \%$ & $4.2 \%$ \\
\hline \multirow{4}{*}{ CAFE } & Model & 434 & 343 & 185 \\
\cline { 2 - 5 } & Actual & 437 & 345 & 190 \\
\cline { 2 - 5 } & Error & $0.7 \%$ & $0.6 \%$ & $2.6 \%$ \\
\hline
\end{tabular}

\subsection{P3/P4 Parallel HEV Model Validation}

The P3/P4 model in VTool was validated using data from schedule C of E\&EC testing. Schedule $C$ was chosen because it was the only schedule that contained hybrid operation. Schedule $C$ is 104 miles long and the EV range was 54 miles leaving 50 miles of CS operation. The data was segregated so that only the final 50 miles of data were considered. This isolated CS mode for the vehicle. This is the only available test data for the vehicle, but covers a significant duration of operation and therefore can be trusted. Although the model is valid for a P3 or a P4 Parallel hybrid, the test vehicle most closely resembles a P4 Parallel hybrid.

\subsubsection{General Validation and Tuning Strategy}

Moving from the EV model to the P4 model, there are now 11 parameters that can be tuned to achieve the proper results. Eight of these parameters are carry-overs from the EV case and will not require retuning. This leaves 3 new parameters that require calibration: engine efficiency, engine driveline efficiency and the Power Split Fraction. Table 23 summarizes the tunable parameters for the P4 Parallel HEV model and notes whether or not the parameter needs tuning. 
Table 23: Summary of P4 Parallel model tunable parameters and tuning strategy

\begin{tabular}{|c|c|c|c|c|c|}
\hline & Parameter & Symbol & Data available? & $\begin{array}{l}\text { Tuned in } \\
\text { EV model }\end{array}$ & $\begin{array}{l}\text { Tune/Retune } \\
\text { Parameter? }\end{array}$ \\
\hline \multirow{6}{*}{$\begin{array}{l}\mathbb{D} \\
\mathscr{D} \\
\mathbb{O} \\
\frac{0}{\Phi} \\
\frac{0}{0} \\
\frac{0}{0}\end{array}$} & Battery discharge efficiency & $\tilde{\eta}_{\text {batt }}^{+}$ & Yes, A123 ICD data & Yes & No \\
\hline & Motor propel efficiency & $\tilde{\eta}_{\text {mot }}^{+}$ & Yes, but suspect & Yes & If needed \\
\hline & Motor driveline propel efficiency & $\tilde{\eta}_{\mathrm{dl}, \mathrm{mot}}^{+}$ & Yes, from Gantt [24] & Yes & No \\
\hline & Engine efficiency & $\tilde{\eta}_{\text {eng }}^{+}$ & Yes, E\&EC data & No & If needed \\
\hline & Engine driveline efficiency & $\begin{array}{l}\tilde{\eta}_{\mathrm{dl}, \text { eng }}^{+} \\
\end{array}$ & No & No & Yes \\
\hline & Power Split Fraction & $\tilde{\xi}$ & Yes, E\&EC data & No & Yes \\
\hline \multirow{5}{*}{ 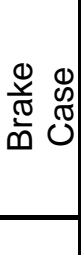 } & Battery charge efficiency & $\tilde{\eta}_{\text {batt }}^{-}$ & Yes, A123 ICD data & Yes & No \\
\hline & Motor brake efficiency & $\tilde{\eta}_{\text {mot }}^{-}$ & Yes, but suspect & Yes & No \\
\hline & Motor driveline brake efficiency & $\tilde{\eta}_{\mathrm{dl}, \mathrm{mot}}^{-}$ & Yes, from Gantt [24] & Yes & No \\
\hline & Regen brake fraction & $\tilde{\zeta}$ & No & Yes & No \\
\hline & Accessory load & $\mathrm{P}_{\mathrm{acc}}$ & Yes, from Perkins [33] & Yes & No \\
\hline
\end{tabular}

Unfortunately there are fewer benchmarks that can be extracted from the data to aid in calibration. There exists fuel consumption data from the test. However fuel consumption was measured by weight at the end of the test which means that only a CAFE fuel consumption benchmark may be extracted. There is dynamic data for engine torque and motor torque, however. Engine torque was used to find engine output power, which was summed to find total mechanical energy used for the entire cycle. This was compared to total fuel use to find a CAFE weighted average engine efficiency. Motor torque was used to calculate mechanical motor power and was segregated into propel and brake cases. The propel case was then subdivided into two cases: motor assist (+EqFC or +prop) and motor generate (-EqFC or +gen). The energy produced during the motor generate subcase was used in conjunction with engine mechanical output power to estimate the Power Split Fraction. Recalling Equation 79, the Power Split Fraction can be expressed in terms of motor driveline energy and engine driveline energy.

\section{Equation 79: Power Split Fraction, engine energy recirculated (P3 Parallel, propel)}

$$
\left(E_{\text {dlmot }, t r}\right)^{+g e n}=(-1)(1-\xi)\left(E_{\text {dleng,tr }}\right)^{+}
$$

Applying component efficiencies for the engine and motor drivelines and rearranging to solve for the Power Split Fraction we have Equation 124. This equation allows us to express the Power Split Fraction in terms of engine output energy and mechanical motor energy. This is important because both of these energy quantities can be determined empirically from the data.

Equation 124: Propel case tractive fuel energy consumption (P3 Parallel, propel)

$$
\xi=\left[\frac{\left(E_{\text {mot }, \text { mech }}\right)^{+g e n}}{\tilde{\eta}_{d l, \text { mot }}^{+g e n}} * \frac{1}{\left(E_{\text {eng,mech }}\right)^{+}\left(\tilde{\eta}_{d l, \text { eng }}^{+}\right)}\right]
$$

Having reasonable data for engine efficiency and a basis for estimating the Power Split Fraction, these two parameters were initially set at their empirically determined values. The engine driveline efficiency was then adjusted to achieve desired results. To keep the engine driveline efficiency within a reasonable range, the CAFE weighted average engine efficiency was also tweaked. This efficiency was also lowered slightly from the CAFE weighted average for the UDDS cycle and raised slightly for the HwFET cycle. 


\subsubsection{UDDS Validation}

Table 24 summarizes the target or benchmark values derived from various datasets including the EcoCAR E\&EC dataset. The table also summarizes the tunable parameters used in the model, the results of the model and the error from the target value. Due to the nature of the data, there were very few benchmarks that could be used to independently tune the parameters for the UDDS cycle. The engine efficiency was necessarily reduced by a substantial margin from what the data suggested. The efficiencies for the UDDS and HwFET cycles were assumed to be different with the HwFET being a few points higher. With a conventional vehicle, the engine is much more efficient on the highway because the road load is higher and therefore the load on the engine is higher. With the P4 Parallel powertrain, the motor can be used to recirculate energy and increase the load on the engine, thus closing the gap in engine efficiency between the two cases. The engine driveline efficiency was assumed to be similar to the motor driveline efficiency but was turned down to $88 \%$ to achieve the target fuel consumption. This parameter was held constant for both drive cycles. The Power Split Fraction was set at 0.74 in accordance with the E\&EC data.

Table 24: Summary of P4 Parallel HEV test data and final parameter values for the UDDS cycle

\begin{tabular}{|c|c|c|c|c|c|}
\hline & Parameter & Symbol & Empirical Value & Modeled Value & \% Error \\
\hline \multirow{7}{*}{ 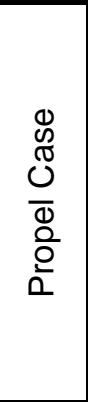 } & Propel fuel consumption & $\left(\mathrm{E}_{\text {eng,fuel }}\right)^{+}$ & --- & $1682 \mathrm{Wh} / \mathrm{mi}$ & --- \\
\hline & Battery discharge efficiency & $\tilde{\eta}_{\text {batt }}^{+}$ & $99 \%$ & $99 \%$ & $0 \%$ \\
\hline & Motor propel efficiency & $\tilde{\eta}_{\text {mot }}^{+}$ & $84 \%$ & $84 \%$ & $0 \%$ \\
\hline & Motor driveline propel efficiency & $\tilde{\eta}_{\mathrm{dl}, \mathrm{mot}}^{+}$ & $92 \%$ & $91 \%$ & $1 \%$ \\
\hline & Engine efficiency & $\tilde{\eta}_{\text {eng }}^{+}$ & $30 \%$ (CAFE) & $24 \%$ & $20 \%$ \\
\hline & Engine driveline efficiency & $\tilde{\eta}_{\mathrm{dl}, \text { eng }}^{+}$ & --- & $88 \%$ & --- \\
\hline & Power Split Fraction & $\tilde{\xi}$ & --- & 0.74 & --- \\
\hline \multirow{6}{*}{ 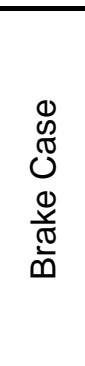 } & Fuel-equivalent regen credit & $\left(E_{\text {eng,fuel }}\right)^{\text {EqFC }}$ & -- & $-309 \mathrm{Wh} / \mathrm{mi}$ & -- \\
\hline & Brake energy consumption & $\left(E_{\text {batt,int }}\right)^{-}$ & $-77 \mathrm{DC} \mathrm{Wh} / \mathrm{mi}$ & $-77 \mathrm{DC} \mathrm{Wh} / \mathrm{mi}$ & $0 \%$ \\
\hline & Battery charge efficiency & $\tilde{\eta}_{\text {batt }}^{-}$ & $99 \%$ & $99 \%$ & $0 \%$ \\
\hline & Motor brake efficiency & $\tilde{\eta}_{\text {mot }}^{-}$ & $91 \%$ & $86 \%$ & $5.5 \%$ \\
\hline & Motor driveline brake efficiency & $\tilde{\eta}_{\mathrm{dl}, \mathrm{mot}}^{-}$ & $92 \%$ & $91 \%$ & $1 \%$ \\
\hline & Regen brake fraction & $\tilde{\zeta}$ & --- & 0.75 & --- \\
\hline \multirow{2}{*}{ ن } & DC net fuel consumption & $\left(E_{\text {eng,fuel }}\right)^{\text {DCnet }}$ & -- & $1407 \mathrm{Wh} / \mathrm{mi}$ & -- \\
\hline & Accessory load & $\mathrm{P}_{\mathrm{acc}}$ & $600 \mathrm{~W}$ & $600 \mathrm{~W}$ & $0 \%$ \\
\hline
\end{tabular}




\subsubsection{HwFET Validation}

Table 25 summarizes the target or benchmark values derived from various datasets including the EcoCAR E\&EC dataset. The table also summarizes the tunable parameters used in the model, the results of the model and the error from the target value. Like the UDDS, there are few benchmarks that can be used individually to calibrate the parameters for this cycle. The engine driveline was kept constant at $88 \%$ for consistency, but the engine efficiency was increased to $26 \%$. This is a 2 point increase compared to the UDDS cycle which is a modest improvement. This improvement reflects the fact that ICE powertrains are typically more efficient during highway driving. Like before, the Power Split Fraction was set to reflect the data, this time at 0.76 .

Table 25: Summary of P4 Parallel HEV test data and final parameter values for the HwFET cycle

\begin{tabular}{|c|c|c|c|c|c|}
\hline & Parameter & Symbol & Empirical Value & Modeled Value & \% Error \\
\hline \multirow{7}{*}{ 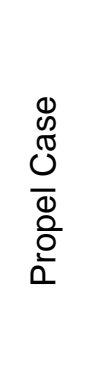 } & Propel energy consumption & $\left(\mathrm{E}_{\text {eng,fuel }}\right)^{+}$ & --- & $1324 \mathrm{Wh} / \mathrm{mi}$ & --- \\
\hline & Battery discharge efficiency & $\tilde{\eta}_{\text {batt }}^{+}$ & $99 \%$ & $99 \%$ & $0 \%$ \\
\hline & Motor propel efficiency & $\tilde{\eta}_{\text {mot }}^{+}$ & $84 \%$ & $84 \%$ & $0 \%$ \\
\hline & Motor driveline propel efficiency & $\tilde{\eta}_{\mathrm{dl}, \mathrm{mot}}^{+}$ & $92 \%$ & $91 \%$ & $1 \%$ \\
\hline & Engine efficiency & $\tilde{\eta}_{\text {eng }}^{+}$ & $30 \%$ (CAFE) & $26 \%$ & $13 \%$ \\
\hline & Engine driveline efficiency & $\tilde{\eta}_{\text {dl,eng }}^{+}$ & --- & $88 \%$ & --- \\
\hline & Power Split Fraction & $\tilde{\xi}$ & --- & 0.76 & --- \\
\hline \multirow{6}{*}{ 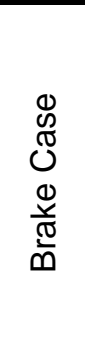 } & Fuel-equivalent regen credit & $\left(E_{\text {eng,fuel }}\right)^{E q F C}$ & --- & $-63 \mathrm{Wh} / \mathrm{mi}$ & --- \\
\hline & Brake energy consumption & $\left(E_{\text {batt,int }}\right)^{-}$ & $-28 \mathrm{DC} \mathrm{Wh} / \mathrm{mi}$ & $-18 \mathrm{DC} \mathrm{Wh} / \mathrm{mi}$ & $36 \%$ \\
\hline & Battery charge efficiency & $\tilde{\eta}_{\text {batt }}^{-}$ & $99 \%$ & $99 \%$ & $0 \%$ \\
\hline & Motor brake efficiency & $\tilde{\eta}_{\text {mot }}^{-}$ & $91 \%$ & $86 \%$ & $5.5 \%$ \\
\hline & Motor driveline brake efficiency & $\tilde{\eta}_{\mathrm{dl}, \mathrm{mot}}^{-}$ & $92 \%$ & $91 \%$ & $1 \%$ \\
\hline & Regen brake fraction & $\tilde{\zeta}$ & --- & 0.75 & --- \\
\hline \multirow{2}{*}{ U } & DC net energy consumption & $\left(E_{\text {eng,fuel }}\right)^{\text {DCnet }}$ & -- & $1261 \mathrm{Wh} / \mathrm{mi}$ & --- \\
\hline & Accessory load & $\mathrm{P}_{\mathrm{acc}}$ & $600 \mathrm{~W}$ & $600 \mathrm{~W}$ & $0 \%$ \\
\hline
\end{tabular}




\subsubsection{CAFE Weighting Validation}

Like with the EV model, the primary goal of tuning the UDDS and HwFET cycles was to achieve an accurate CAFE weighted fuel consumption. Table 18 summarizes the modeled energy consumption for UDDS and HwFET cycles as well as the CAFE weighting. The net CAFE fuel consumption came out to $1341 \mathrm{Wh} / \mathrm{mi}$, which is almost exactly equal to the empirical value, $1342 \mathrm{Wh} / \mathrm{mi}$. Unfortunately, there was no empirical basis to individually calibrate each cycle. In lieu of such data, the individual cycles were tuned to reflect expect trends. City driving is expected to be less efficient and generally requires slightly more energy. Reflecting this expectation, the net fuel consumption for the UDDS cycle was $5 \%$ higher than the CAFE fuel consumption. Similarly, highway driving is more efficient and required $6 \%$ less energy than the CAFE weighting.

Table 26: Summary of P4 Parallel HEV energy consumption modeling for UDDS, HwFET and CAFE

\begin{tabular}{|c|c|c|c|c|c|}
\hline Parameter & Symbol & Cycle & $\begin{array}{l}\text { Empirical } \\
\text { Value }\end{array}$ & $\begin{array}{l}\text { Modeled } \\
\text { Value }\end{array}$ & $\%$ Error \\
\hline \multirow{3}{*}{ Propel fuel consumption } & $\left(\mathrm{E}_{\text {eng,fuel }}\right)^{+}$ & UDDS & --- & $1682 \mathrm{Wh} / \mathrm{mi}$ & --- \\
\hline & $\left(E_{\text {eng,fuel }}\right)^{+}$ & HwFET & --- & $1324 \mathrm{Wh} / \mathrm{mi}$ & --- \\
\hline & $\left(\mathrm{E}_{\text {eng,fuel }}\right)^{+}$ & CAFE & --- & $1521 \mathrm{Wh} / \mathrm{mi}$ & --- \\
\hline \multirow{3}{*}{$\begin{array}{l}\text { Brake energy consumption } \\
\text { (Same as EV model) }\end{array}$} & $\left(E_{\text {eng,fuel }}\right)^{\text {EqFC }}$ & UDDS & $-77 \mathrm{DC} \mathrm{Wh} / \mathrm{mi}$ & $-77 \mathrm{DC} \mathrm{Wh} / \mathrm{mi}$ & $0.0 \%$ \\
\hline & $\left(\mathrm{E}_{\text {eng,fuel }}\right)^{\mathrm{EqFC}}$ & HwFET & $-28 \mathrm{DC} \mathrm{Wh} / \mathrm{mi}$ & $-18 \mathrm{DC} \mathrm{Wh} / \mathrm{mi}$ & $36 \%$ \\
\hline & $\left(E_{\text {eng,fuel }}\right)^{\text {EqFC }}$ & CAFE & $-59 \mathrm{DC} \mathrm{Wh} / \mathrm{mi}$ & $-50 \mathrm{DC} \mathrm{Wh} / \mathrm{mi}$ & $14.5 \%$ \\
\hline \multirow{3}{*}{ Fuel-equivalent regen credit } & $\left(E_{\text {eng,fuel }}\right)^{\text {DCnet }}$ & UDDS & --- & $-309 \mathrm{Wh} / \mathrm{mi}$ & --- \\
\hline & $\left(E_{\text {eng,fuel }}\right)^{D C n e t}$ & HwFET & --- & $-63 \mathrm{Wh} / \mathrm{mi}$ & --- \\
\hline & $\left(E_{\text {eng,fuel }}\right)^{\text {DCnet }}$ & CAFE & -- & $-198 \mathrm{Wh} / \mathrm{mi}$ & --- \\
\hline \multirow{3}{*}{ DC net fuel consumption } & $\left(E_{\text {eng,fuel }}\right)^{\mathrm{DCnet}}$ & UDDS & -- & $1407 \mathrm{Wh} / \mathrm{mi}$ & --- \\
\hline & $\left(E_{\text {eng,fuel }}\right)^{D C n e t}$ & HwFET & --- & $1261 \mathrm{Wh} / \mathrm{mi}$ & --- \\
\hline & $\left(E_{\text {eng,fuel }}\right)^{\text {DCnet }}$ & CAFE & $1342 \mathrm{Wh} / \mathrm{mi}$ & $1341 \mathrm{Wh} / \mathrm{mi}$ & $0.1 \%$ \\
\hline
\end{tabular}

It is interesting to note that the propel fuel consumption for the UDDS and HwFET were $11 \%$ higher and $13 \%$ lower (respectively) than the CAFE average. The propel case does not include the effect of regen braking, so the larger spread between the two fuel consumption values can be attributed to the effect of regen braking. Examining the regen fuel credit, the regen fuel credit was $309 \mathrm{Wh} / \mathrm{mi}$ for the UDDS cycle, which is $18 \%$ of the propel energy consumed. Contrastingly, only $63 \mathrm{Wh} / \mathrm{mi}$ of regen fuel credit was earned during the HwFET, which is only $5 \%$ of the propel energy consumed. This illustrates the differences between the two cycles as well as the substantial benefit offered by regen braking for city driving. 


\subsubsection{P4 Parallel Model Validation Summary}

Using the E\&EC data from the Year 3 EcoCAR competition, the P4 Parallel model of VTool was calibrated to within $1 \%$ of the measured CAFE fuel consumption. All the parameters calibrated for EV mode were untouched. This includes motor, motor driveline, and battery efficiencies as well as the regen brake fraction and accessory load. The engine driveline efficiency was set to $88 \%$ and the engine efficiency was set to $24 \%$ for the UDDS and $26 \%$ for the HwFET. The Power Split Fraction was set at 0.74 for the UDDS and 0.76 for the HwFET. These values were directly derived from the E\&EC data.

Figure 43 illustrates the dynamics of power recirculation. The motor (or RTM) power trace was created by isolating the propel case only and then subdividing into motor assist (+prop) and motor generate (+gen) cases. In this way, the effects of regen braking (which is not included in the dynamics of the Power Split Fraction) is removed from the data stream. The blue trace indicates periods where the motor was assisting the engine and using surplus battery energy. Likewise, the green trace shows periods where the motor was placing additional load on the engine and storing the extra energy in the battery.

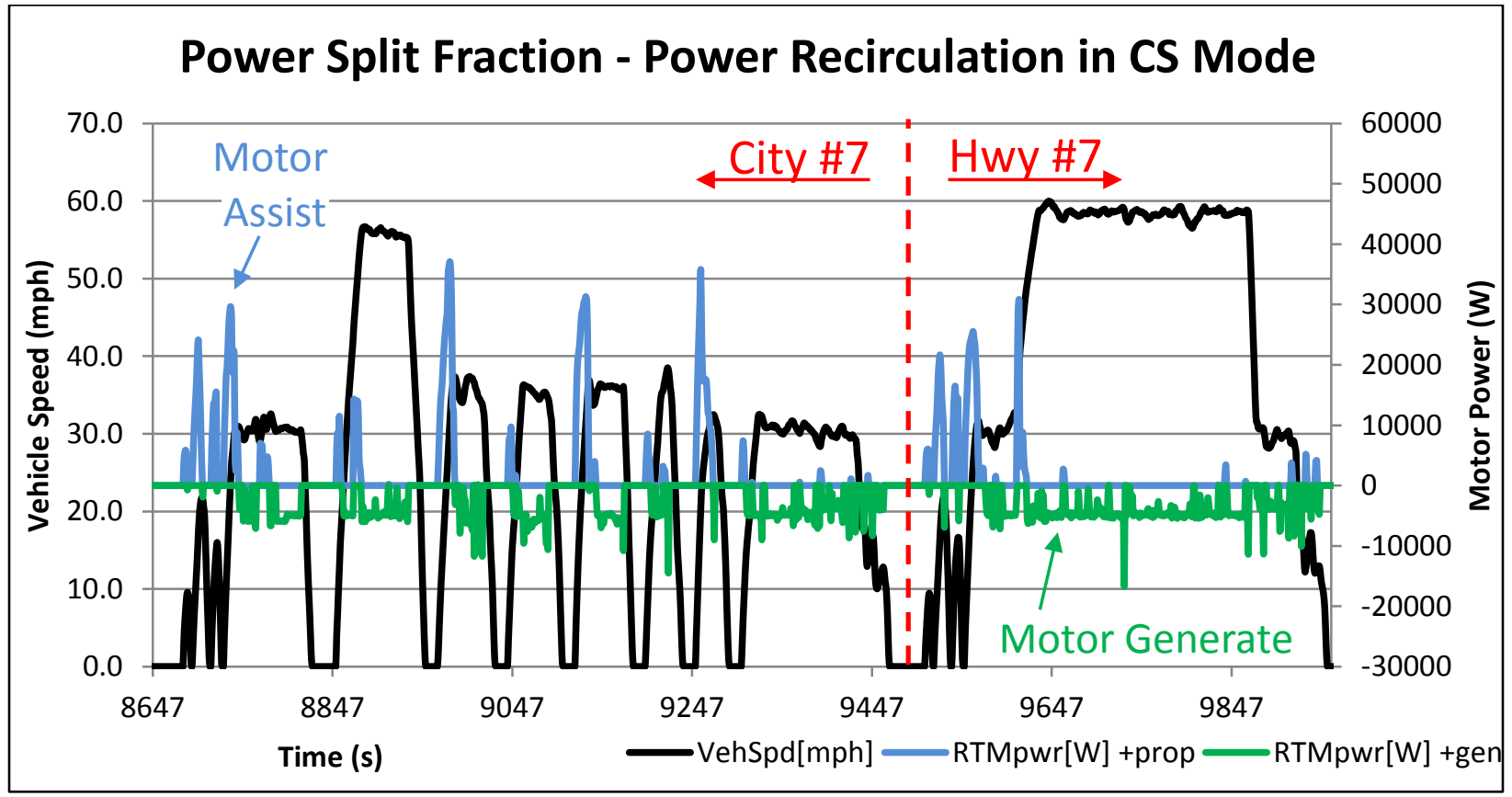

Figure 43: Illustration of power recirculation during CS operation

As the figure illustrates, the motor was operating at a near-constant $5 \mathrm{~kW}$ during the motor generate case. This would have placed an additional 6-7 kW of load on the engine (considering the two driveline efficiencies at $88 \%$ and $91 \%$ ). The benefit of recirculating power is to push the engine to a higher average operating load, which in turn increases engine efficiency. Intuitively, this author would expect more power recirculation during city driving which push the Power Split Fraction to lower values. Since the average engine load is lower, there is more benefit to be gained by recirculating power. During highway operation, the load on the engine is already relatively high without recirculating any power. Hence, it is expected to be less advantageous to recirculate power during highway driving. However, the Power Split Fraction was nearly equal for the two cycles based upon the data. The Power Split Fraction, of course, is entirely dependent upon the hybrid control strategy and the control strategy is not designed to solely optimize fuel consumption. There are also driveability concerns - especially since the motor was on the rear axle. Thus, the vehicle control strategy (and consequently the Power Split Fraction) was the result of a set of compromises to a complex engineering challenge. 


\subsection{Overall Validation Summary}

The overall results of the validation of VTool using E\&EC data and other data are summarized in Table 27 and Table 46. The final values of all tunable parameters used in the models presented previously are summarized in Table 46 in Appendix DD. The energy consumption results for the EV model (both DC and AC energy consumption) the P4 Parallel HEV model are summarized in Table 27. The net energy consumption results for the EV model and P4 Parallel model were within $1 \%$ of the corresponding empirical value showing that VTool was successfully calibrated. Also included in this table is an example of how VTool can be used to calculate UF weighted energy consumption. These results are achieved by combining the results of the EV model with the P4 Parallel HEV to create a P4 Parallel EREV. The results for UF-weighted AC energy consumption were within $1 \%$, but the results for UF-weighted fuel consumption and total energy consumption were not as accurate. Given that the E\&EC drive schedule was designed as an on-road approximation of the CAFE weighting and of the J1711 UF method, it is still acceptable to be within $5 \%$ of the empirical data. Thus, the ability of VTool to combine models to perform UF weighting has also been successfully verified. For discussion of the UF-weighting method, see Section 5.2.3.

Table 27: Summary of results for EV, P4 Parallel HEV and P4 Parallel EREV using UF method

\begin{tabular}{|c|c|c|c|c|c|}
\hline Parameter & Symbol & Cycle & $\begin{array}{l}\text { Empirical } \\
\text { Value }\end{array}$ & $\begin{array}{l}\text { Modeled } \\
\text { Value }\end{array}$ & $\%$ Error \\
\hline \multirow{3}{*}{$\begin{array}{c}\text { Electric Vehicle } \\
\text { DC net fuel consumption }\end{array}$} & $\left(E_{\text {batt }, T}\right)^{\text {DCnet }}$ & UDDS & $321 \mathrm{DC} \mathrm{Wh} / \mathrm{mi}$ & $334 \mathrm{DC} \mathrm{Wh} / \mathrm{mi}$ & $4.0 \%$ \\
\hline & $\left(E_{\text {batt }, T}\right)^{\text {DCnet }}$ & HwFET & $357 \mathrm{DC} \mathrm{Wh} / \mathrm{mi}$ & $353 \mathrm{DC} \mathrm{Wh} / \mathrm{mi}$ & $1.1 \%$ \\
\hline & $\left(E_{\text {batt }, T}\right)^{\text {DCnet }}$ & CAFE & $345 \mathrm{DC} \mathrm{Wh} / \mathrm{mi}$ & $343 \mathrm{DC} \mathrm{Wh} / \mathrm{mi}$ & $0.6 \%$ \\
\hline \multirow{3}{*}{$\begin{array}{c}\text { AC net energy consumption } \\
\text { (at 80\% charging efficiency) } \\
\text { E\&EC charging data }\end{array}$} & $\left(E_{\text {grid, } A C}\right)^{A C n e t}$ & UDDS & -- & $424 \mathrm{AC} \mathrm{Wh} / \mathrm{mi}$ & --- \\
\hline & $\left(E_{\text {grid }, A C}\right)^{A C n e t}$ & HwFET & --- & $446 \mathrm{AC} \mathrm{Wh} / \mathrm{mi}$ & --- \\
\hline & $\left(\mathrm{E}_{\text {grid, } \mathrm{AC}}\right)^{\mathrm{ACnet}}$ & CAFE & $437 \mathrm{AC} \mathrm{Wh} / \mathrm{mi}$ & $434 \mathrm{AC} \mathrm{Wh} / \mathrm{mi}$ & $0.7 \%$ \\
\hline \multirow{3}{*}{$\begin{array}{c}\text { P4 Parallel HEV } \\
\text { DC net fuel consumption }\end{array}$} & $\left(E_{\text {eng,fuel }}\right)^{\text {DCnet }}$ & UDDS & --- & $1407 \mathrm{Wh} / \mathrm{mi}$ & -- \\
\hline & $\left(E_{\text {eng,fuel }}\right)^{\text {DCnet }}$ & HwFET & --- & $1261 \mathrm{Wh} / \mathrm{mi}$ & --- \\
\hline & $\left(E_{\text {eng,fuel }}\right)^{D C n e t}$ & CAFE & $1342 \mathrm{Wh} / \mathrm{mi}$ & $1341 \mathrm{Wh} / \mathrm{mi}$ & $0.1 \%$ \\
\hline \multirow{4}{*}{$\begin{array}{c}\text { P4 Parallel EREV } \\
\text { UF-weighted fuel consumption }\end{array}$} & EV Range & CAFE & 54 miles & 54 miles & $0 \%$ \\
\hline & $\left(E_{\text {grid, } A C}\right)^{U F}$ & CAFE & $311 \mathrm{AC} \mathrm{Wh} / \mathrm{mi}$ & $310 \mathrm{AC} \mathrm{Wh} / \mathrm{mi}$ & $0.3 \%$ \\
\hline & $\left(E_{\text {eng,fuel }}\right)^{U F}$ & CAFE & $406 \mathrm{Wh} / \mathrm{mi}$ & $384 \mathrm{Wh} / \mathrm{mi}$ & $5.4 \%$ \\
\hline & $\left(E_{\text {total }}\right)^{U F}$ & CAFE & $712 \mathrm{Wh} / \mathrm{mi}$ & $694 \mathrm{Wh} / \mathrm{mi}$ & $2.5 \%$ \\
\hline
\end{tabular}




\section{Examples and Applications}

This section includes examples of the functionality of VTool.

\subsection{Power Split Fraction Case Study}

At any given time in a dynamic real-time Series HEV control strategy, the genset may be producing more or less power than is needed at the HV bus. If the genset is producing too much power, the excess will be stored in the battery and used later. If the genset produces too little, extra power from the battery is used to make up the difference. The amount of energy recirculated is defined by the Power Split Fraction, as previously described. To explore the concept of the Power Split Fraction, a case study was performed on a Series HEV using a separately developed time-based Simulink model. The purpose of the case study is to investigate the effects of the Power Split Fraction and its correlation to engine or genset efficiency. The study also aims to examine the upper and lower bounds of the Power Split Fraction. To accomplish these goals, $\xi$ will be varied and the results will be examined to determine the best hybrid operating strategy. Four cases were considered and are summarized in Table 28. Additionally, the 4 cases will also be applied to both city and highway driving to examine the effects of the drive cycle on $\xi$.

Table 28: Summary of cases for Power Split Fraction case study

\begin{tabular}{|l|l|l|l|}
\hline $\begin{array}{l}\text { Case } \\
\#\end{array}$ & $\begin{array}{l}\text { Case } \\
\text { Name }\end{array}$ & Target $\xi$ & $\begin{array}{l}\text { Case Description } \\
\text { Load } \\
\text { Following }\end{array}$ \\
\hline 1 & $\xi=1.0$ & $\begin{array}{l}\text { Load following means that the genset attempts to match the load at the } \\
\text { HV bus as if there is no battery in the system. The goal is to achieve } \xi \\
1, \text { however this may not be realistic. Hence, } \xi \text { will be driven as close to 1 } \\
\text { as possible. The advantage of load-following is that the genset can take } \\
\text { advantage of occasions when road load is already high to keep the load } \\
\text { on the engine high without the need to incur recirculation losses. }\end{array}$ \\
\hline 2 & $\begin{array}{l}\text { Average } \\
\text { Power }\end{array}$ & $\xi=\sim 0.7$ & $\begin{array}{l}\text { This case approximates operating the genset at a nearly constant power } \\
\text { level close to the cycle-average power level. While this would not be a } \\
\text { wise control strategy to implement in an actual vehicle, it is a special case } \\
\text { since the genset may be run at a near constant power and generate the } \\
\text { exact amount of energy required to complete the cycle without turning off. }\end{array}$ \\
\hline 3 & $\begin{array}{l}\text { Realistic } \\
\text { Strategy }\end{array}$ & $\xi=\sim 0.4$ & $\begin{array}{l}\text { This case would reflect a more realistic Hybrid Control strategy. It will } \\
\text { have a minimum engine power requirement and some SOC-based } \\
\text { proportional control to include some load-following dynamics. }\end{array}$ \\
\hline 4 & $\begin{array}{l}\text { Bang- } \\
\text { Bang }\end{array}$ & $\xi=0$ & $\begin{array}{l}\text { It is impossible to bring } \xi \text { all the way down to zero (this would require an } \\
\text { impulse of energy). This case will attempt to drive } \xi \text { as low as possible by } \\
\text { increasing the minimum engine power level to slightly above the } \\
\text { generator continuous power rating. This will force the genset to operate at } \\
\text { its highest possible continuous power. It is called a Bang-Bang strategy } \\
\text { because the engine turns on, runs a high constant load and then turns } \\
\text { back off. }\end{array}$ \\
\hline
\end{tabular}

\subsubsection{Brief Explanation of Power Split Fraction}

As $\xi$ falls, more energy is cycled through the battery and the genset has more freedom to operate at different power levels. This usually translates to a higher cycle average genset efficiency (not necessarily higher system efficiency). The tradeoff is the extra losses incurred by storing energy in the battery and then taking it back out. If the extra increase in genset efficiency does not offset the extra recirculation losses, then $\xi$ is too low and more genset energy should be used directly for propulsion. As the battery is used to buffer more energy, $\xi$ will decrease and the genset efficiency will increase. There is obviously a correlation between $\xi$ and the genset efficiency, but the exact correlation is unclear. Hence, this project will seek to analyze this correlation and give some basis for predicting the genset efficiency based upon $\xi$. 


\section{Power Split Fraction - Energy Balance at HV Bus}

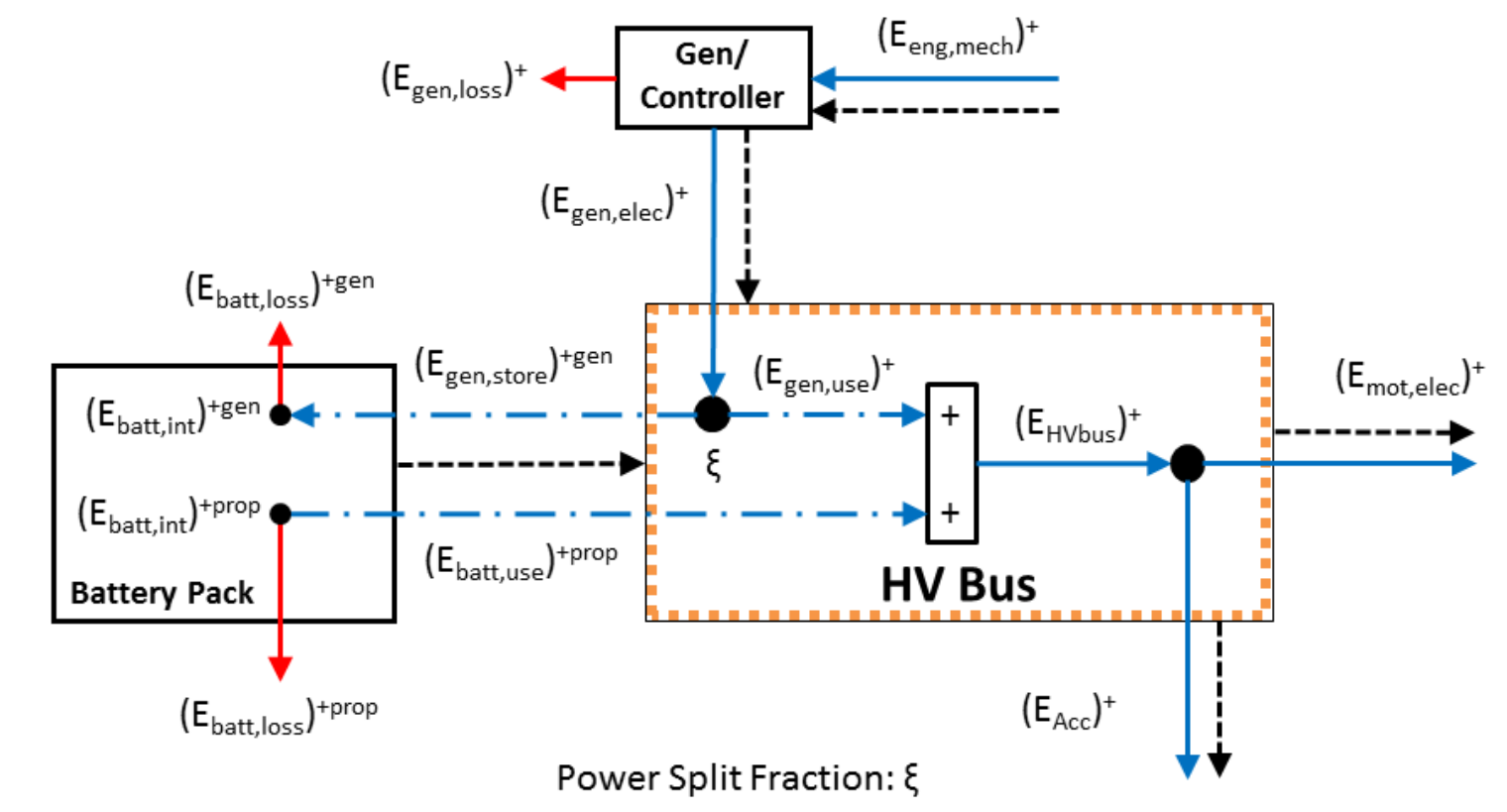

Figure 24: Energy balance at the HV bus for the Power Split Fraction

\subsubsection{Simulink Model Parameters and Setup}

The Simulink model was developed separately from VTool and is fundamentally different in that it is a dynamic model that performs second-by-second calculations. It calculates tractive force and power instantaneously and uses quasi-dynamic component models to calculate losses in each component and ultimately the power required from both the engine and the battery. This stands in contrast with VTool and the Sovran method which only deals with 'lumps' of energy and is not capable of examining instantaneous effects. The Simulink model includes component models for the battery, motor, motor driveline, engine, and generator. The model also includes a dynamic driver model and a dynamic hybrid control strategy. The high-level topology of this model is documented by Figure 48 in Appendix BB. Also in Appendix BB is Table 45 which documents the key parameters for the Simulink model.

The vehicle modeled is a 2013 Chevy Malibu and the powertrain components were modeled to be very similar to the components used in the $\mathrm{VT}_{\text {REX }}$. Specifically, the engine $(2.4 \mathrm{~L})$, motor (125 kW peak) and motor driveline are identical. The battery has similar characteristics but is about half the size ( $8.8 \mathrm{kWh})$ and the generator is $50 \mathrm{~kW}$ peak ( $25 \mathrm{~kW}$ cont). The hybrid controller in the model controls genset power output using a proportional controller that monitors battery SOC and compares it to an SOC target value. The controller also enforced a minimum on or off time of 30 seconds to prevent rapid and repeating engine starts. Likewise, a hysteresis effect is applied to battery SOC. For example, if the engine is on the 
controller will not turn it off until the battery SOC reaches $>3 \%$ above its target. This feature also prevents rapid and repeated engine starts. Additionally, the controller has a minimum engine power parameter that may be used to enforce a minimum engine-on power. The only parameters varied for the case study are summarized in Table 29.

Table 29: Summary and explanation of hybrid controller parameters varied in case study

\begin{tabular}{|l|l|}
\hline Parameter & Effect \\
\hline $\begin{array}{l}\text { Minimum } \\
\text { Levine Power }\end{array}$ & $\begin{array}{l}\text { Forces the engine to operate at a minimum power level or shut off. A higher minimum } \\
\text { engine power level will result in more engine stops and starts since the engine will be } \\
\text { forced to shut off to let the SOC drop. }\end{array}$ \\
\hline SOC gain & $\begin{array}{l}\text { Changes how aggressively the controller tries to get back to the target SOC. A higher } \\
\text { gain will lead to more aggressive spikes in genset load. }\end{array}$ \\
\hline SOC resolution & $\begin{array}{l}\text { This is a separate gain applied to the target SOC and actual SOC before the error is } \\
\text { calculated for the proportional controller. Increasing this gain gives the proportional } \\
\text { controller more resolution to follow the drive cycle demand more precisely. A higher } \\
\text { SOC resolution will lead to a more load-following strategy. }\end{array}$ \\
\hline
\end{tabular}

Table 30 summarizes the parameter configuration used for each of the 4 cases to achieve the target Power Split Fraction. The parameters presented in Table 29 were set up according to control strategy description presented in Table 30 and then tuned to achieve the desired Power Split Fraction value.

Table 30: Summary of hybrid control strategy parameters for each case in the study

\begin{tabular}{|l|l|l|l|}
\hline $\begin{array}{l}\text { Case } \\
\#\end{array}$ & $\begin{array}{l}\text { Case } \\
\text { Name }\end{array}$ & Target $\xi$ & Control strategy description \\
\hline 1 & $\begin{array}{l}\text { Pure } \\
\text { Load } \\
\text { Following }\end{array}$ & $\xi=1.0$ & $\begin{array}{l}\text { In this case the gain on the proportional controller in the Hybrid Controller } \\
\text { will be set very high and the minimum engine power level will be set to } \\
\text { zero. Hence, the proportional controller will have the responsiveness } \\
\text { needed to closely load-follow and the engine will not be forced off at low } \\
\text { power levels. }\end{array}$ \\
\hline 2 & $\begin{array}{l}\text { Average } \\
\text { Power }\end{array}$ & $\xi=\sim 0.7$ & $\begin{array}{l}\text { This will be accomplished by relaxing the minimum engine power to zero } \\
\text { and relaxing the SOC gain to lazily maintain SOC. The SOC gain } \\
\text { parameter must be iteratively tuned to the prescribed drive cycle for this } \\
\text { strategy to work. This means that it is not feasible for actual } \\
\text { implementation in a real-time Hybrid Controller. }\end{array}$ \\
\hline 3 & $\begin{array}{l}\text { Realistic } \\
\text { Strategy }\end{array}$ & $\xi=\sim 0.4$ & $\begin{array}{l}\text { In this case, the control strategy will have a low minimum engine power } \\
\text { and some SOC-based proportional control. The SOC gain will be } \\
\text { modulated to increase or decrease } \xi . \text { The higher the gain, the higher the } \\
\text { average power level, the more it reflects a bang-bang strategy and the } \\
\text { lower } \xi \text { becomes. }\end{array}$ \\
\hline 4 & $\begin{array}{l}\text { Bang- } \\
\text { Bang }\end{array}$ & $\xi=0$ & $\begin{array}{l}\text { This case will attempt to drive } \xi \text { as low as possible by increasing the } \\
\text { minimum engine power level to slightly above the generator continuous } \\
\text { power rating. This will force the genset to operate at its highest possible } \\
\text { average power whenever it is operating. Once battery SOC reaches the } \\
\text { target, the genset will turn off. }\end{array}$ \\
\hline
\end{tabular}

An additional nuance of this model is maintaining charge balance over the duration of the cycle. If the vehicle finishes the cycle with a lower battery SOC than when it started, the fuel use will be artificially low since some battery energy was used to propel the vehicle. Likewise, the fuel consumption will be artificially high if the battery SOC is higher at the end of the cycle than at the beginning since extra fuel was required to charge the battery during the cycle. Thus, the net battery energy consumed over the drive cycle must be $<1 \%$ of fuel energy consumed during the cycle. This is the requirement for a valid fuel 
economy certification test for an HEV and is a reasonable limit. In the Simulink model, charge balance was achieved by iteratively modifying the initial SOC until the simulation fell within the $1 \%$ margin. Additionally, 3 cycles were run back-to-back to increase the total fuel use and therefore increase the margin of error for the final SOC.

\subsubsection{Calculating Power Split Fraction}

As previously described, the Power Split Fraction is defined by Equation 55.

Equation 55: Power Split Fraction, genset energy recirculated (Series, propel)

$$
\left(E_{\text {gen }, \text { store }}\right)^{+ \text {gen }}=(-1)(1-\tilde{\xi})\left(E_{\text {gen,elec }}\right)^{+}
$$

Rearranged:

$$
\tilde{\xi}=1+\frac{\left(E_{\text {gen }, \text { store }}\right)^{+ \text {gen }}}{\left(E_{\text {gen,elec }}\right)^{+}}
$$

A HV bus sub-block is included in the Simulink model to properly handle and probe the energy distribution at the HV bus node. Regen braking was accounted for in this block, but is introduced in such a way that regen energy onto the bus from the motor does not enter in to this calculation. Hence, the Power Split Fraction is not affected by regen energy capture, other than indirect effects that regen braking has on the overall system.

\subsubsection{Results for City Driving (UDDS)}

This section contains the results of the 4 cases for city driving (UDDS). For these 4 cases, no fuel penalty was assessed for engine starts, which artificially favors strategies which run the genset at high power levels and start the engine often. All of the cases correspond to a regen brake fraction of 0.55 . The control strategy uses a target SOC of $45 \%$, has upper and lower bounds of $\pm 3 \%$ and will keep the engine either on or off for at least 30 seconds to prevent rapid and repeating engine starts. The results for case 1 are illustrated in Figure 44. The plot shows that the control strategy did a reasonable good job at load following. However, the vehicle was incapable of exactly load following resulting in a Power Split Fraction of only 0.88 meaning that $88 \%$ of power produced by the genset was sent directly to the HV bus. The test resulted in an engine efficiency of $29 \%$ and a fuel economy of $31.0 \mathrm{mpg}$.

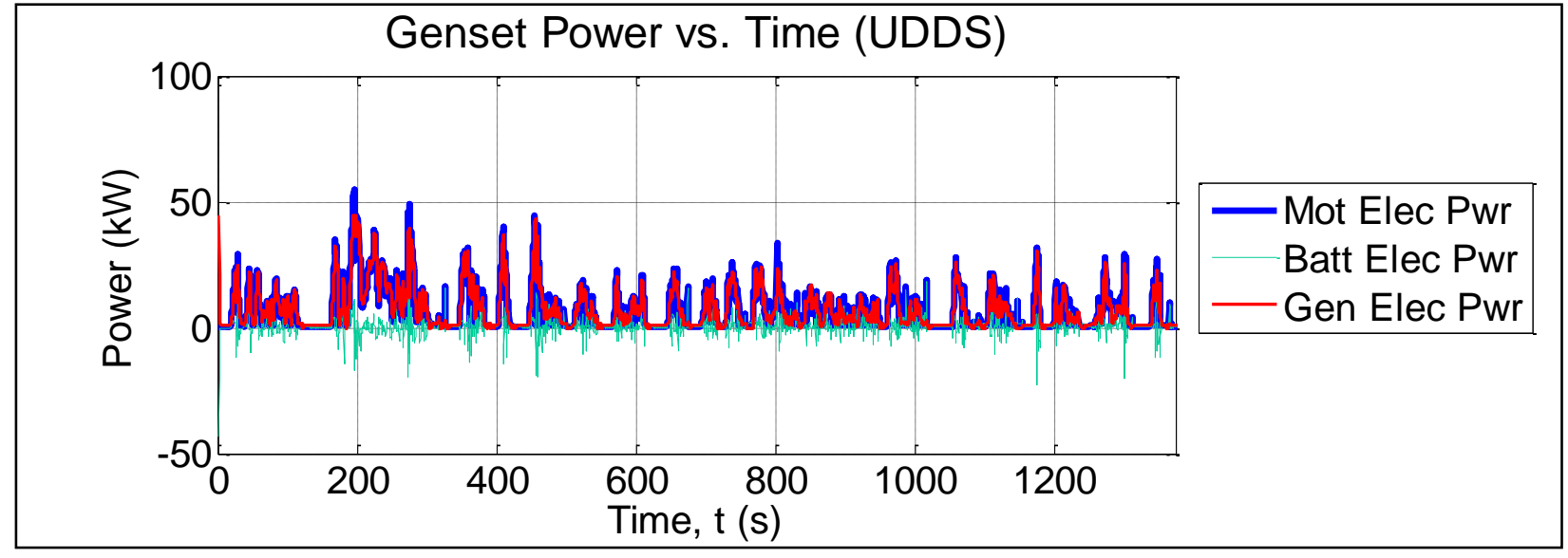

Figure 44: Power Split Fraction case study, UDDS, case 1, near load-following, $\xi=0.88$ 
The results for case 2 are illustrated in Figure 45. The plot shows that the genset operated at a near constant power level of about $5 \mathrm{~kW}$ for the duration of the cycle. The battery was needed often to assist for accelerations and times of high tractive effort but was charged during low power operation and during idle. This strategy resulted in a Power Split Fraction of 0.61 , an engine efficiency of $31 \%$ and a fuel economy of $31.4 \mathrm{mpg}$. Except for $\xi$, these results are very similar to case 1 . The genset operates at a relatively low average power level for both cases, which suggests that average engine power level has a significant impact on overall vehicle fuel economy.

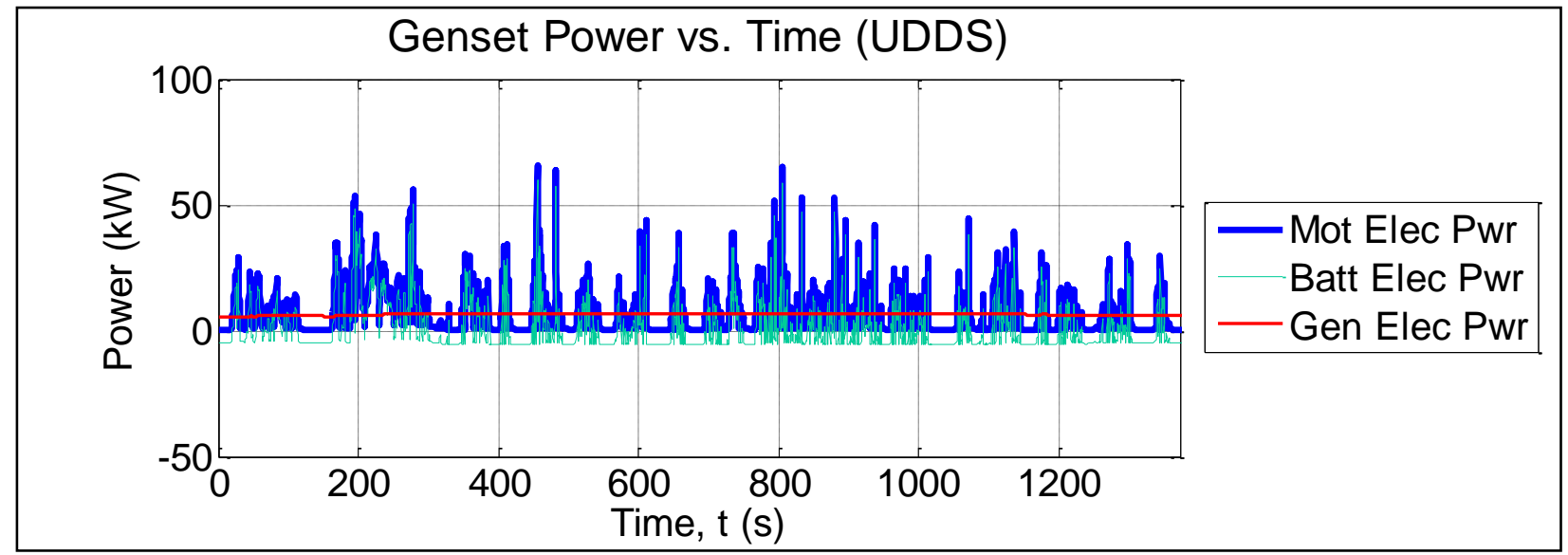

Figure 45: Power Split Fraction case study, UDDS, case 2, average power, $\xi=0.61$

The results for case 3 are illustrated in Figure 46. The plot shows that the genset operated at about 10-12 $\mathrm{kW}$ while it was on and exhibited some weak load-following behavior. The plot shows 2 UDDS cycles back-to-back to show the long-term engine cycling of the control strategy. The engine spent roughly equal amounts of time on and off. While the engine was on, the battery was still used extensively to assist the genset since the genset only operated at about 10-12 kW. Of course, the vehicle operated as an EV and the battery supplied all tractive power while the genset was off. Over the 3 back-to-back UDDS cycles, the control strategy started the engine 5 times. This strategy resulted in a Power Split Fraction of 0.44, an engine efficiency of $35 \%$ and a fuel economy of $38.9 \mathrm{mpg}$. These results show great improvement over the first two cases because the control strategy allowed vehicle to use the battery to give the genset more operational freedom to increase the average operating power level.

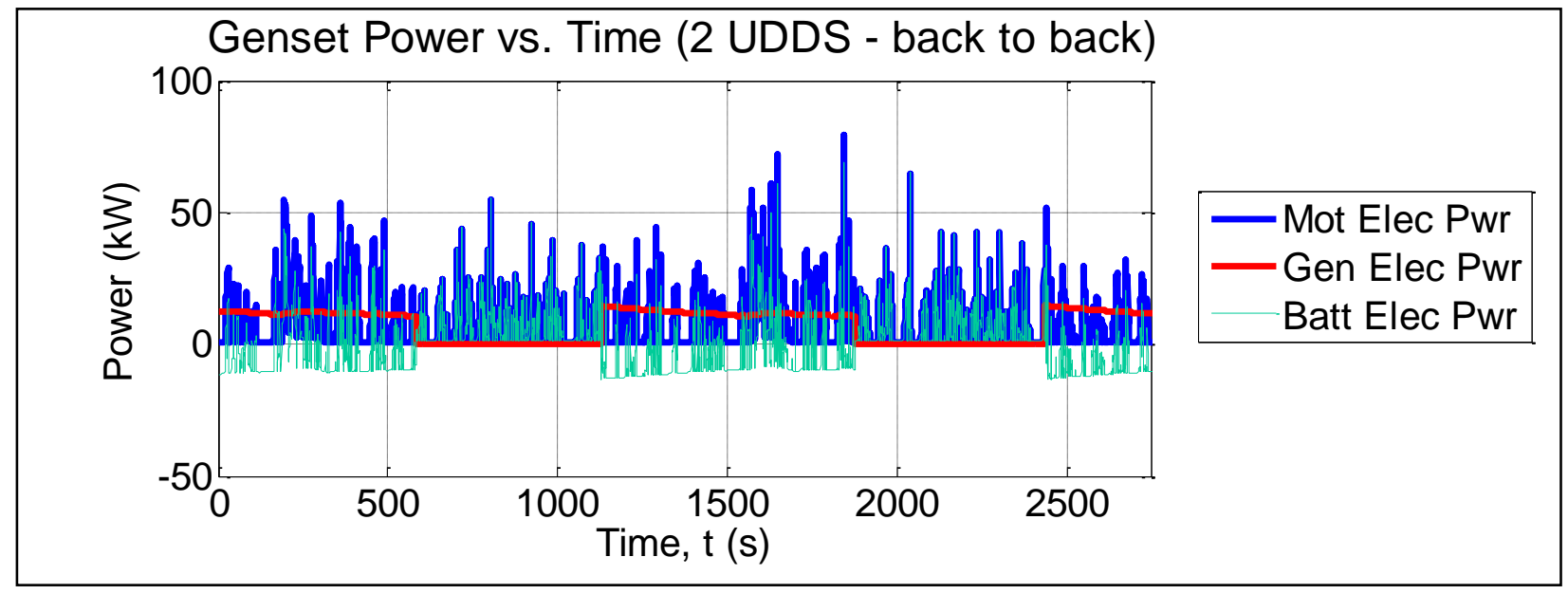

Figure 46: Power Split Fraction case study, UDDS, case 3, realistic strategy, $\xi=0.44$ 
The results for case 4 are illustrated in Figure 47. The plot shows that the genset operated at $35 \mathrm{~kW}$ while it was on, but spent a relative short amount of time running. Because the average operating power level was so high, the genset recharged the battery quickly while it was on and spent extended periods of time off while the battery was being depleted. Over the 3 back-to-back UDDS cycles, the control strategy started the engine 7 times. This strategy resulted in a Power Split Fraction of 0.22 , an engine efficiency of $38 \%$ and a fuel economy of $40.5 \mathrm{mpg}$. Even though the strategy operated the genset above its maximum continuous power rating, the vehicle was not able to achieve the goal of $\xi=0$. This is expected, but also shows that the upper limit of $\xi$ is more reachable than the lower limit. This strategy also improved engine efficiency and fuel economy compared to case 3 which is a direct result of the increased average operating power. Notice, however, that increasing from an average power of roughly $11 \mathrm{~kW}$ in case 3 to $35 \mathrm{~kW}$ in case 4 only increases engine efficiency by 3 points. Contrastingly, going from an average power of roughly $5 \mathrm{~kW}$ in case 2 to roughly $11 \mathrm{~kW}$ in case 3 resulted in a 5 point engine efficiency increase. Thus, much can be gained from an initial increase in average engine operating power and there are diminishing returns on additional increases in average engine power.

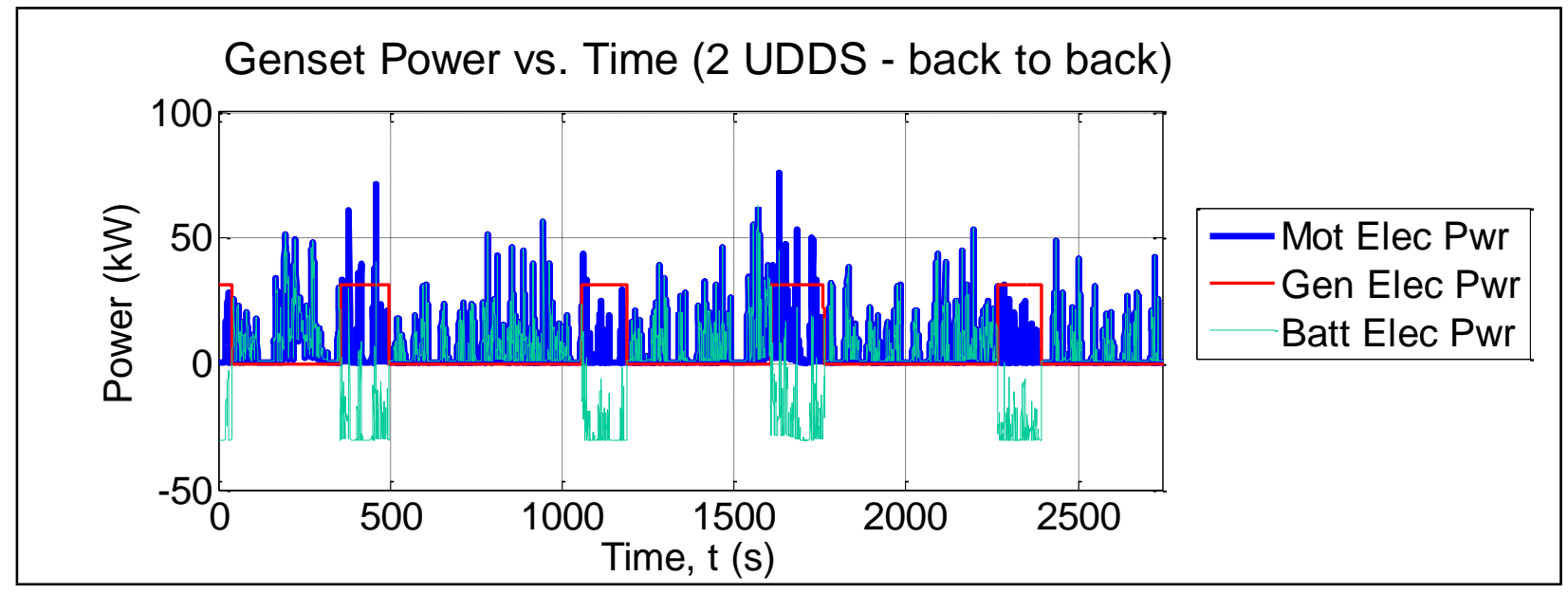

Figure 47: Power Split Fraction case study, UDDS, case 4, bang-bang, $\xi=0.22$

The results for this part of the case study are summarized in Table 31. The four cases achieved an even spread, but the limits of $\xi$ proved to be unattainable, as expected. It is important to note that the engine modeled efficiencies are generous and likely over-estimated considering that the modeled engine is a 4 cylinder spark ignited engine. Regardless, the engine efficiencies may still be compared between cases to draw conclusions about differences in the control strategies. The best strategy is the bang-bang strategy used in case 4. However, the effects of engine starts were not considered in this analysis. Additionally, other factors such as Noise Vibration and Harshness (NVH) and emissions were not modeled or considered. All of these factors would negatively affect case 4 (and case 3 to a lesser degree).

Table 31: Power Split Fraction case study results for city driving (UDDS)

\begin{tabular}{|l|l|l|l|l|l|l|l|l|l|l|l|}
\hline Case & SOC $_{\mathrm{i}}$ & $\begin{array}{l}\text { Min Eng } \\
\text { Pwr }\end{array}$ & $\begin{array}{l}\text { SOC } \\
\text { Gain }\end{array}$ & $\begin{array}{l}\text { SOC } \\
\text { Res }\end{array}$ & $\xi$ & $\eta_{\text {eng }}$ & $\eta_{\text {gen }}$ & $\eta_{\text {genset }}$ & $\begin{array}{l}\text { Fuel } \\
\text { Economy }\end{array}$ & $\begin{array}{l}\% \text { Fuel } \\
\text { Energy }\end{array}$ & $\begin{array}{l}\text { Eng } \\
\text { Starts }\end{array}$ \\
\hline$[\#]$ & {$[\%]$} & {$[\mathrm{kW}]$} & {$[---]$} & {$[---]$} & {$[---]$} & {$[\%]$} & {$[\%]$} & {$[\%]$} & mpgge & {$[\%]$} & {$[\#]$} \\
\hline 1 & 0.45 & 0 & 1000000 & 100 & 0.88 & $29 \%$ & $85 \%$ & $25 \%$ & 31.0 & $0.13 \%$ & 1 \\
\hline 2 & 0.46 & 0 & 35000 & 1 & 0.61 & $30 \%$ & $87 \%$ & $26 \%$ & 31.4 & $0.55 \%$ & 1 \\
\hline 3 & 0.47 & 10 & 80000 & 1 & 0.44 & $35 \%$ & $91 \%$ & $32 \%$ & 38.9 & $0.47 \%$ & 5 \\
\hline 4 & 0.46 & 35 & 100000 & 1 & 0.22 & $38 \%$ & $90 \%$ & $34 \%$ & 40.5 & $-0.25 \%$ & 7 \\
\hline
\end{tabular}


To examine the effect of starting the engine on the strategy, the $4^{\text {th }}$ case was re-run with an engine start fuel penalty of 2.2 grams. This case was chosen because it has the most engine starts. This gives an engine start fuel penalty of 2.2 grams, which means that an additional 2.2 grams of fuel is used every time the engine starts. This is a more realistic model and dis-incentivizes 'bang-bang' controllers which result in more engine starts. Again, note that emissions are not considered anywhere in this analysis and that strategies that are great for genset efficiency (like a 'bang-bang' strategy) are likely detrimental to tailpipe emissions.

As the results show, the fuel economy dropped from $40.5 \mathrm{mpg}$ to $40.2 \mathrm{mpg}$, a negligible decrease. For this case, $1.65 \mathrm{~kg}$ of fuel was used. This puts engine start fuel in perspective -2.2 grams per start (or 15.4 grams total) is very small compared to $1.65 \mathrm{~kg}$ of fuel used overall. The conclusion, then, is that fuel required to start the engine has a very small impact on overall fuel economy.

Table 32: Case 4 repeated with the addition of an engine start fuel penalty

\begin{tabular}{|l|l|l|l|l|l|l|l|l|l|l|l|}
\hline Case & SOC $_{\mathrm{i}}$ & $\begin{array}{l}\text { Min Eng } \\
\text { Pwr }\end{array}$ & $\begin{array}{l}\text { SOC } \\
\text { Gain }\end{array}$ & $\begin{array}{l}\text { SOC } \\
\text { Res }\end{array}$ & $\xi$ & $\eta_{\text {eng }}$ & $\eta_{\text {gen }}$ & $\eta_{\text {genset }}$ & $\begin{array}{l}\text { Fuel } \\
\text { Economy }\end{array}$ & $\begin{array}{l}\text { \% Fuel } \\
\text { Energy }\end{array}$ & $\begin{array}{l}\text { Eng } \\
\text { Starts }\end{array}$ \\
\hline$[\#]$ & {$[\%]$} & {$[\mathrm{kW}]$} & {$[---]$} & {$[---]$} & {$[--]$} & {$[\%]$} & {$[\%]$} & {$[\%]$} & mpgge & {$[\%]$} & {$[\#]$} \\
\hline 4 & 0.46 & 35 & 100000 & 1 & 0.22 & $37 \%$ & $90 \%$ & $34 \%$ & 40.2 & $-0.25 \%$ & 7 \\
\hline
\end{tabular}

\subsubsection{Results for Highway Driving (HwFET)}

The above cases were repeated for the HWFET to examine differences that occur between city and highway driving. All of the controller parameters were kept constant between the two cases to hold a baseline for comparison. The results are summarized in Table 33. Not surprisingly, the load-following type strategies were much better for highway conditions because highway cruising is necessarily already a higher-load driving condition. With city driving as in the UDDS, the average genset load is relatively low (only about $6 \mathrm{~kW}$ ). With highway driving as in the HWFET, the average genset load is higher (close to about $15 \mathrm{~kW}$ ). So for city driving, a load following strategy has poor performance because the average road load is so low. On the highway, however, the average road load is higher so a load following strategy will inherently operate at a higher load, and therefore a more efficient operating point. The difference in road load also illustrates an important point: cases 2 and 3 are identical because the average genset power was already above the specified minimum power.

Table 33: Power Split Fraction case study results for highway driving (HwFET)

\begin{tabular}{|l|l|l|l|l|l|l|l|l|l|l|l|}
\hline Case & SOC $_{\mathrm{i}}$ & $\begin{array}{l}\text { Min Eng } \\
\text { Pwr }\end{array}$ & $\begin{array}{l}\text { SOC } \\
\text { Gain }\end{array}$ & $\begin{array}{l}\text { SOC } \\
\text { Res }\end{array}$ & $\xi$ & $\eta_{\text {eng }}$ & $\eta_{\text {gen }}$ & $\eta_{\text {genset }}$ & $\begin{array}{l}\text { Fuel } \\
\text { Economy }\end{array}$ & $\begin{array}{l}\% \text { Fuel } \\
\text { Energy }\end{array}$ & $\begin{array}{l}\text { Eng } \\
\text { Starts }\end{array}$ \\
\hline$[\#]$ & {$[\%]$} & {$[\mathrm{kW}]$} & {$[---]$} & {$[---]$} & {$[---]$} & {$[\%]$} & {$[\%]$} & {$[\%]$} & mpgge & {$[\%]$} & {$[\#]$} \\
\hline 1 & 0.46 & 0 & 1000000 & 100 & 0.96 & $35 \%$ & $90 \%$ & $32 \%$ & 39.3 & $0.21 \%$ & 1 \\
\hline 2 & 0.46 & 0 & 80000 & 1 & 0.81 & $35 \%$ & $91 \%$ & $32 \%$ & 40.0 & $0.06 \%$ & 1 \\
\hline 3 & 0.47 & 10 & 80000 & 1 & 0.81 & $35 \%$ & $91 \%$ & $32 \%$ & 40.0 & $0.06 \%$ & 1 \\
\hline 4 & 0.46 & 35 & 100000 & 1 & 0.59 & $33 \%$ & $90 \%$ & $30 \%$ & 37.0 & $0.30 \%$ & 5 \\
\hline
\end{tabular}

The difference in cycles also substantially effects the Power Split Fraction. Case 1 achieved 0.96 for higway driving while only achieving 0.88 for city driving. The control strategy was able to get closer to $\xi=1$ because the HwFET is a less dynamic cycle than the UDDS, so the proportional controller was able to more closely load follow. Additionally the higher road load of the HwFET means that $\xi$ is different for identical control strategies. For example, the bang-bang strategy used in case 4 resulted in $\xi=0.59$ for the HwFET and $\xi=0.22$ for the UDDS cycle which is much lower. Thus, the strategy is exactly the same but because the road load of the HwFET is higher, more energy was forced to go directly to the HV bus and 
skip the recirculation path. Overall, there is very little difference in the fuel economy of cases 1,2 or 3 . This is because of the higher average road load of the HWFET. Contrasting with the UDDS, the worst strategy for the HWFET is case 4, the high genset power 'bang-bang' strategy. This strategy yields a lower fuel economy because additional energy was recirculated without getting the added benefit of raising the genset efficiency. Plots of the results of the four highway cases are documented in Appendix CC.

\subsection{Calculating Cycle-Weighted WTW Energy Consumption for PHEVs and EREVs}

VTool has the capability to calculate energy consumption for EV, Series HEV, and Parallel HEV powertrains for a variety of drive cycles including UDDS (as well as 505 and 867), HwFET, and US06 (as well as City and Highway portions individually). Because the tool is excel based, all of the calculations are available concurrently and a 'run' button need not be pushed. For this reason, VTool is ideal for calculating cycle weightings (such as CAFE, EPA 5-cycle and EcoCAR 2 4-cycle). The tool can also calculate UF weighted energy consumption for EREVs by combining the results from the EV model with the results from one of the HEV models. Additionally, the WTW effects of a given powertrain and fuel can be calculated within the tool using upstream factors from the GREET model [12].

\subsubsection{CAFE and 4-Cycle Energy Consumption for an EV}

A detailed summary of results for the EV model for UDDS (broken into 505 and 867 portions), HwFET, and US06 (broken into city and highway portions) is included in Appendix EE. Parameters of interest for the propel and DCnet cases are summarized in Table 47 and the results for the brake case are summarized in Table 48. These tables include energy consumption parameters as well as efficiencies of interest (such as powertrain efficiencies). A more concise summary of EV energy consumption for the UDDS and HwFET cycles and CAFE weighting is included in Table 34. Likewise, Table 35 summarizes results for the 4-Cycle weighting and the cycles involved. These tables include only AC and DC energy consumption, road load energy consumption, WTW petroleum energy use (PEU) and WTW Greenhouse Gas Emissions (GHGs). This collection of results gives a clear picture of overall vehicle operation. Road load energy consumption gives an idea of the intensity of the cycle. DC energy consumption tells how efficient the vehicle is and can be used to calculate EV range. AC grid energy consumption is the total energy used to complete the cycle and is used to calculate WTW factors (like PEU and GHGs). Because all the cycles are all concurrently present in VTool, the results merely need to be referenced and then properly weighted to calculate the proper cycle weighting. Because all the values are on a per-mile basis, a direct weighted average may be applied. A per-energy quantification of fuel consumption is referred to as fuel economy and most often expressed as miles per gallon (mpg). A gallon of gasoline can be considered an energy quantity using the energy density of gasoline at about $33.7 \mathrm{kWh} / \mathrm{gal}$. CAFEweighted fuel economy may be calculated from CAFE-weighted consumption. However, fuel economy numbers cannot be directly weighted using a weighted average - they must be inverted first so the quantity is expressed on a per-mile basis.

Table 34: Summary of WTW CAFE-weighted energy consumption for the EV model

\begin{tabular}{|l|c|r|r|r|r|r|}
\cline { 2 - 7 } \multicolumn{1}{c|}{} & \multicolumn{6}{c|}{ CAFE Weighted Results - Electric Vehicle } \\
\cline { 2 - 7 } \multicolumn{1}{c|}{} & Weight & $\left(\mathrm{E}_{\text {grid,AC }}\right)^{\text {ACnet }}$ & $\left(\mathrm{E}_{\text {batt, }}\right)^{\text {DCnet }}$ & $\left(\mathrm{E}_{\mathrm{tr}}\right)^{\text {DCnet }}$ & WTW PEU & WTW GHG \\
\cline { 2 - 7 } & {$[\%]$} & {$[\mathrm{AC}$ Wh$/ \mathrm{mi}]$} & {$[\mathrm{DC}$ Wh/mi] } & {$[\mathrm{Wh} / \mathrm{mi}]$} & {$[\mathrm{WhPE} / \mathrm{mi}]$} & {$[\mathrm{g} / \mathrm{mi}]$} \\
\hline UDDS & 0.55 & 423 & 334 & 151 & 14.40 & 274 \\
\hline HwFET & 0.45 & 446 & 353 & 226 & 15.16 & 289 \\
\hline
\end{tabular}


Table 35: Summary of WTW 4-Cycle-weighted energy consumption for the EV model

\begin{tabular}{|c|c|c|c|c|c|c|}
\hline & \multicolumn{6}{|c|}{ 4-Cycle Weighted Results - Electric Vehicle } \\
\hline & Weight & $\left(E_{\text {grid, } A C}\right)^{A C n e t}$ & $\left(E_{\text {batt }, T}\right)^{\text {DCnet }}$ & $\left(E_{t r}\right)^{\text {DCnet }}$ & WTW PEU & WTW GHG \\
\hline & [\%] & {$[\mathrm{AC} \mathrm{Wh}$ Wi] } & [DC Wh/mi] & {$[\mathrm{Wh} / \mathrm{mi}]$} & [WhPE/mi] & [g/mi] \\
\hline 505 & 0.29 & 443 & 350 & 180 & 15.08 & 287 \\
\hline US06 City & 0.14 & 746 & 586 & 207 & 25.35 & 483 \\
\hline HwFET & 0.12 & 446 & 353 & 226 & 15.16 & 289 \\
\hline US06 Hwy & 0.45 & 570 & 450 & 313 & 19.36 & 369 \\
\hline 4-Cycle V & ighted: & 543 & 428 & 249 & 18.46 & 352 \\
\hline
\end{tabular}

CAFE is weighted $55 \%$ City (UDDS) and $45 \%$ Highway (HwFET). The EcoCAR 24 -Cycle emulates the EPA 5-Cycle weighting and is weighted $43 \%$ City (29\% 505, $14 \%$ US06 City) and $57 \%$ Highway (12\% HwFET and 45\% US06 Highway). Examining road load for both cycle weightings, the CAFE weighting is much less aggressive at only $185 \mathrm{Wh} / \mathrm{mi}$ compared to $249 \mathrm{Wh} / \mathrm{mi}$, which is about a $35 \%$ increase in energy. Table 46 in Appendix DD shows that the component efficiencies for US06 City and Highway are comparable. However, the ACnet energy use is about $25 \%$ more for the 4 -Cycle weighting compared to CAFE, as shown in Table 36. This discrepancy is due to the increased road load of the 4-Cycle weighting.

Table 36: Comparison of CAFE and 4-Cycle weightings for the EV model

\begin{tabular}{|l|c|c|c|c|c|}
\cline { 2 - 7 } \multicolumn{1}{c|}{} & \multicolumn{5}{c|}{ Comparison of CAFE and 4-Cycle - Electric Vehicle } \\
\cline { 2 - 7 } & $\left(\mathrm{E}_{\text {grid, } A)^{\text {ACnet }}}\right.$ & $\left(\mathrm{E}_{\text {batt, } \mathrm{T}}\right)^{\text {DCnet }}$ & $\left(\mathrm{E}_{\mathrm{tr}}\right)^{\mathrm{DCnet}}$ & WTW PEU & WTW GHG \\
\cline { 2 - 7 } & {$[\mathrm{AC}$ Wh/mi] } & {$[\mathrm{DC}$ Wh/mi] } & {$[\mathrm{Wh} / \mathrm{mi}]$} & {$[\mathrm{WhPE} / \mathrm{mi}]$} & {$[\mathrm{g} / \mathrm{mi}]$} \\
\hline CAFE Weighted & 434 & 342 & 185 & 14.7 & 281 \\
\hline 4-Cycle Weighting & 543 & 428 & 249 & 18.5 & 352 \\
\hline \% Difference & $25 \%$ & $25 \%$ & $35 \%$ & $25 \%$ & $25 \%$ \\
\hline
\end{tabular}

\subsubsection{CAFE and 4-Cycle Energy Consumption for a P3/P4 Parallel HEV}

A detailed summary of results for the P3/P4 Parallel HEV model for UDDS (broken into 505 and 867 portions), HwFET, and US06 (broken into city and highway portions) is included in Appendix EE. Parameters of interest for the propel and DCnet cases are summarized in Table 49 and the results for the brake case are summarized in Table 50. As with the EV model, a more concise summary of energy consumption for the UDDS and HwFET cycles and CAFE weighting is included in Table 37. Likewise, Table 38 summarizes results for the P3/P4 Parallel HEV model for 4-Cycle weighting and the cycles involved. Like before, these summary tables include road load, WTW PEU and WTW GHG factors. However, instead of AC and DC energy use, total fuel use is reported in terms of fuel consumption $(\mathrm{Wh} / \mathrm{mi})$ and in terms of fuel economy $(\mathrm{mpg})$. 
Table 37: Summary of WTW CAFE-weighted energy consumption for the P3/P4 Parallel HEV model

\begin{tabular}{|l|c|c|r|r|r|r|}
\cline { 2 - 7 } \multicolumn{1}{c|}{} & \multicolumn{6}{c|}{ CAFE Weighted Results - P3/P4 Parallel HEV } \\
\cline { 2 - 7 } \multicolumn{1}{c|}{} & Weight & $\left(\mathrm{E}_{\text {eng,fuel }}\right)^{\text {DCnet }}$ & $\left(\mathrm{E}_{\text {eng,fuel }}\right)^{\text {DCnet }}$ & $\left(\mathrm{E}_{\text {tr }}\right)^{\text {DCnet }}$ & WTW PEU & WTW GHG \\
\cline { 2 - 7 } \multicolumn{1}{c|}{$[\%]$} & {$[\mathrm{Wh} / \mathrm{mi}]$} & {$[\mathrm{mpg}]$} & {$[\mathrm{Wh} / \mathrm{mi}]$} & {$[\mathrm{WhPE} / \mathrm{mi}]$} & {$[\mathrm{g} / \mathrm{mi}]$} \\
\hline UDDS & 0.55 & 1405 & 23 & 151 & 1382.2 & 452 \\
\hline HwFET & 0.45 & 1261 & 26 & 226 & 1240.6 & 406 \\
\hline
\end{tabular}

Table 38: Summary of WTW 4-Cycle-weighted energy consumption for the P3/P4 Parallel HEV model

\begin{tabular}{|l|c|c|c|r|r|r|}
\cline { 2 - 7 } \multicolumn{1}{c|}{} & \multicolumn{6}{c|}{ 4-Cycle Weighted Results - P3/P4 Parallel HEV } \\
\cline { 2 - 7 } \multicolumn{1}{c|}{} & Weight & $\left(\mathrm{E}_{\text {eng,fuel }}\right)^{\text {DCnet }}$ & $\left(\mathrm{E}_{\text {eng,fuel }}\right)^{\text {DCnet }}$ & $\left(\mathrm{E}_{\text {tr }}\right)^{\text {DCnet }}$ & WTW PEU & WTW GHG \\
\cline { 2 - 7 } \multicolumn{1}{c|}{$[\%]$} & {$[\mathrm{Wh} / \mathrm{mi}]$} & {$[\mathrm{mpg}]$} & {$[\mathrm{Wh} / \mathrm{mi}]$} & {$[$ WhPE/mi] } & {$[\mathrm{g} / \mathrm{mi}]$} \\
\hline 505 & 0.29 & 1469 & 22 & 180 & 1445 & 473 \\
\hline US06 City & 0.14 & 2205 & 15 & 207 & 2169 & 710 \\
\hline HwFET & 0.12 & 1261 & 26 & 226 & 1240 & 406 \\
\hline US06 Hwy & 0.45 & 1643 & 20 & 313 & 1616 & 529 \\
\hline \multicolumn{2}{|c|}{ 4-Cycle Weighted: } & 1625 & 20 & 249 & 1599 & 523 \\
\cline { 2 - 7 }
\end{tabular}

Comparing the CAFE and 4-Cycle weightings for the P3/P4 Parallel HEV in Table 39, the results are similar to the EV model. The road load is of course $35 \%$ greater for the 4-Cycle weighting, but the total fuel use is only $21 \%$ greater (compared to $25 \%$ for the EV). This indicates that the HEV ICE powertrain suffers relatively less from an increase in cycle road load. This is perhaps due to the nature of internal combustion engines: ICEs are more apt to operate at a higher efficiency at higher loads than are electric motors.

Table 39: Comparison of CAFE and 4-Cycle weightings for the P3/P4 Parallel HEV model

\begin{tabular}{|l|c|c|c|c|c|}
\cline { 2 - 6 } \multicolumn{1}{c|}{} & \multicolumn{4}{c|}{ Comparison of CAFE and 4-Cycle - P3/P4 Parallel HEV } \\
\cline { 2 - 6 } \multicolumn{1}{c|}{} & $\left(\mathrm{E}_{\text {eng,fuel }}\right)^{\text {DCnet }}$ & $\left(\mathrm{E}_{\text {eng,fuel }}\right)^{\text {DCnet }}$ & $\left(\mathrm{E}_{\text {tr }}\right)^{\text {DCnet }}$ & WTW PEU & WTW GHG \\
\cline { 2 - 7 } & {$[\mathrm{Wh} / \mathrm{mi}]$} & {$[\mathrm{mpg}]$} & {$[\mathrm{Wh} / \mathrm{mi}]$} & {$[\mathrm{WhPE} / \mathrm{mi}]$} & {$[\mathrm{g} / \mathrm{mi}]$} \\
\hline CAFE Weighted & 1340 & 24 & 185 & 1318.5 & 431 \\
\hline 4-Cycle Weighting & 1625 & 20 & 249 & 1599 & 523 \\
\hline \% Difference & $21 \%$ & --- & $35 \%$ & $21 \%$ & $21 \%$ \\
\hline
\end{tabular}




\subsubsection{UF-Weighted CAFE and 4-Cycle Energy Consumption for a P3/P4 Parallel EREV}

The UF-Weighted energy consumption of an EREV may be calculated by coupling the results of an EV and an HEV using the SAE J1711 Utility Factor weighting method. As discussed in Section 4.5, the Utility Factor indicates the fraction of miles spent operating as an EV. For the purposes of this exercise, the Utility Factor is a corporate Utility Factor based on 2005 NHTS corporate fleet data. This means that the Utility Factor represents the corporate average of American drivers. The Utility Factor may be approximated directly from EV range using Equation 125, with the necessary parameters summarized in Table 40.

$$
\begin{gathered}
\text { Equation 125: Utility Factor curve } \\
U F=1-e^{-\left[c_{1} *\left(\frac{\text { EVrange }}{\text { Dnorm }}\right)^{1}+c_{2} *\left(\frac{\text { EVrange }}{\text { Dnorm }}\right)^{2}+\ldots+c_{6} *\left(\frac{\text { EVrange }}{\text { Dnorm }}\right)^{6}\right]}
\end{gathered}
$$

Table 40: Parameters for Equation 125

\begin{tabular}{|l|l|}
\hline Dnorm & 399.9 \\
\hline C1 & 10.52 \\
\hline C2 & -7.282 \\
\hline C3 & -26.37 \\
\hline C4 & 79.08 \\
\hline C5 & -77.36 \\
\hline C6 & 26.07 \\
\hline
\end{tabular}

The UF-weighted AC energy consumption is calculated using Equation 126 where UF is the utility factor:

Equation 126: Utility factor weighted AC energy consumption

$$
\left(E_{\text {grid }, A C}\right)^{U F}=U F *\left(E_{\text {grid }, A C}\right)^{A C n e t}
$$

Likewise, the UF-weighted fuel consumption is calculated using Equation 127:

Equation 127: Utility factor weighted fuel consumption

$$
\left(E_{\text {eng,fuel }}\right)^{U F}=(1-U F) *\left(E_{\text {eng,fuel }}\right)^{\text {DCnet }}
$$

The total UF-weighted energy consumption is then calculated using Equation 128:

Equation 128: Utility factor weighted total energy consumption

$$
\left(E_{\text {total }}\right)^{U F}=\left(E_{\text {eng }, \text { fuel }}\right)^{U F}+\left(E_{\text {grid,AC }}\right)^{U F}
$$

Equation 127 and Equation 128 represent two separate methods to evaluate vehicle energy or fuel use depending upon the desired evaluation criteria. In some cases, fuel use is much more important than AC energy use (or total energy use). Fuel use, for example, is directly tied to petroleum energy use and grid electricity has a very low petroleum energy content. Therefore the AC energy use of a PHEV contributes little to the overall petroleum energy use of the vehicle and the UF-weighted fuel consumption is essentially a direct indicator of overall petroleum energy use. In this case, fuel consumption is the more important criteria and Equation 127 could be used to evaluate vehicle performance. However, using this method neglects AC grid energy and is essentially 'free' and unaccounted for. Therefore to get a better overall picture of vehicle efficiency and performance, Equation 128 can be used to calculate total energy 
consumption. Total energy use is a more appropriate quantity to evaluate GHG or criteria emissions since grid electricity is a significant contributor to both GHG and criteria emissions. The 2009-2011 EcoCAR challenge used UF-weighted fuel consumption as an evaluation of energy consumption while the 20122014 EcoCAR 2 challenge has moved to consider total energy use to evaluate energy consumption.

As shown in Table 41, the CAFE-weighted Utility Factor for this vehicle is calculated based on the EV range to be 0.71 . This means that the EREV will spend $71 \%$ of operational mileage as an electric vehicle on average. Notice also that the DC energy consumption is higher for the 4-Cycle weighting which means that the EV range is shorter. With a UF of 0.64 , the EV range for the 4-Cycle weighting is only 43 miles. Thus the 4-Cycle EV range is 11 miles (or 20\%) shorter than the EV range for CAFE.

Table 41: Summary of UF calculations based on the EV model

\begin{tabular}{|l|r|r|r|c|}
\cline { 2 - 5 } \multicolumn{1}{c|}{} & \multicolumn{4}{c|}{ Utility Factor Determination } \\
\cline { 2 - 5 } \multicolumn{1}{c|}{$\left(\mathrm{E}_{\text {batt, }}\right)^{\text {DCnet }}$} & Useable Batt Capacity & EV Range & Utility Factor \\
\cline { 2 - 5 } & {$[\mathrm{DC}$ Wh/mi] } & {$[\mathrm{Wh}]$} & {$[\mathrm{mi}]$} & {$[\%]$} \\
\hline CAFE & 342 & 18400 & 54 & 0.711 \\
\hline 4-cycle & 428 & 18400 & 43 & 0.640 \\
\hline
\end{tabular}

Applying the equations presented previously, the UF-weighted energy consumption results are calculated and summarized in Table 42. Comparing EV and HEV energy consumption, HEV energy consumption is about 3 times that of the EV. This comparison illustrates the benefits of a full-function electric powertrain. Because an HEV will use 3 times more energy than an EV, the benefit of a high Utility Factor and therefore EV range cannot be understated. A good illustration of this assertion is the WTW petroleum energy consumption of this EREV. WTW PEU is 49\% higher for the 4-Cycle weighting compared to the CAFE weighting. Gasoline has nearly $100 \%$ petroleum energy content while grid electricity has nearly $0 \%$. The utility factor dropped 7 points from CAFE to 4-Cycle meaning that there is more ICE operation during 4-Cycle operation and therefore more gasoline consumed. Because gasoline has nearly 100\% petroleum energy content, this small difference in UF created a big increase in PEU.

Table 42: Summary of UF-weighted energy consumption results from P3/P4 Parallel EREV

\begin{tabular}{|c|c|c|c|c|c|c|c|}
\hline & \multicolumn{7}{|c|}{ 4-Cycle Weighted Results - P3/P4 Parallel EREV } \\
\hline & EV Range & UF & $\left(E_{\text {grid }, A C}\right)^{A C n e t}$ & $\left(E_{\text {eng,fuel }}\right)^{\text {DCnet }}$ & $\left(E_{\text {total }}\right)^{U F}$ & WTW PEU & WTW GHG \\
\hline & [mi] & [\%] & [AC Wh/mi] & [Wh/mi] & [Wh/mi] & [WhPE/mi] & [g/mi] \\
\hline CAFE & 54 & 0.711 & 434 & 1340 & 696 & 392 & 324 \\
\hline 4-Cycle & 43 & 0.640 & 543 & 1625 & 862 & 585 & 368 \\
\hline$\%$ Difference & $-20 \%$ & $-10 \%$ & $25 \%$ & $21 \%$ & $24 \%$ & $49 \%$ & $14 \%$ \\
\hline
\end{tabular}

\subsubsection{Impact of Fuel Selection on WTW UF-Weighted Energy Consumption}

To illustrate the impact of fuel selection, the P3/P4 Parallel HEV model was run using E85 and B20 in addition to E10. The results are summarized in Table 43. It is important to note that no component efficiencies were changed in this case study for simplicity. This simplification will cause B20 to appear to be an especially bad fuel choice. However, B20 will necessarily come with a more efficient $\mathrm{Cl}$ diesel engine and all of the numbers will be smaller because the diesel powertrain is more efficient and will therefore consume less energy. The results do, however, illustrate the point that fuel selection has a large impact on WTW factors of interest such as petroleum energy use and Greenhouse Gas emissions. 
Table 43: Comparison of fuels for P3/P4 Parallel EREV

\begin{tabular}{|l|c|c|c|c|c|c|c|}
\cline { 2 - 8 } \multicolumn{1}{c|}{} & \multicolumn{7}{c|}{ 4-Cycle Weighted Results - P3/P4 Parallel HEV } \\
\cline { 2 - 8 } & $\begin{array}{c}\text { HEV/EV } \\
\text { WTW PEU }\end{array}$ & $\begin{array}{c}\text { UF-weighted } \\
\text { WTW PEU }\end{array}$ & $\begin{array}{c}\text { HEV/EV } \\
\text { WTW GHG }\end{array}$ & $\begin{array}{c}\text { UF-weighted } \\
\text { WTW GHG }\end{array}$ & $\begin{array}{c}\text { Energy } \\
\text { Density }\end{array}$ & $\begin{array}{c}\text { WTW PE } \\
\text { content }\end{array}$ & $\begin{array}{c}\text { WTW } \\
\text { GHGs }\end{array}$ \\
\cline { 2 - 8 } & {$[$ WhPE/mi] } & {$[$ WhPE/mi] } & {$[\mathrm{g} / \mathrm{mi}]$} & {$[\mathrm{g} / \mathrm{mi}]$} & {$[\mathrm{Wh} / \mathrm{kg}]$} & {$[\mathrm{WhPE/Wh]}$} & {$[\mathrm{g} / \mathrm{kWh}]$} \\
\hline E10 & 1599 & 585 & 523 & 368 & 11440 & $98 \%$ & 322 \\
\hline E85 & 514 & 194 & 424 & 333 & 7960 & $32 \%$ & 261 \\
\hline B20 & 1396 & 511 & 468 & 348 & 11550 & $86 \%$ & 288 \\
\hline Electricity & 18.5 & --- & 352 & --- & -- & $3 \%$ & 648 \\
\hline
\end{tabular}

E85 contains only $32 \%$ petroleum energy and will therefore reduce WTW PEU from $1599 \mathrm{WhPE} / \mathrm{mi}$ (68\%) to $514 \mathrm{WhPE} / \mathrm{mi}$ compared to the E10 Parallel HEV. Electricity offers an even greater benefit with only $3 \%$ petroleum energy content. The EV model uses only $18.5 \mathrm{WhPE} / \mathrm{mi}$, which is a $99 \%$ reduction in WTW PEU compared to the E10 Parallel HEV. This drastic reduction in WTW PEU underscores another benefit of EVs and full-function electric powertrains. Examining the GHG content of the fuels, it is clear that grid electricity is by far the worst fuel. However, the EV still produces fewer WTW GHGs than the Parallel HEV for any of the liquid fuels. This phenomenon occurs because the HEV powertrains consume 3 times more energy than the EV. Thus, electricity is 2-2.5 times more GHG dense, but EVs use about 3 times less energy resulting in a reduction in WTW GHGs.

\subsection{Using VTool to Compare Powertrain Architectures}

Another useful function of VTool is its capability to easily compare between two different powertrain architectures. This capability can be highly useful for an architecture selection process, like the process that is undertaken for the EcoCAR competition. For example, VTool could be used to make an initial comparison between a Series HEV and a Parallel HEV. This comparison could give an EcoCAR school an orientation to what to expect from each powertrain architecture and how they compare. The tool could also be used to compare a theoretical powertrain configuration to an existing configuration. The purpose of this comparison would be to determine how efficient the theoretical powertrain would need to be to improve upon the existing powertrain configuration. Take for example the CS mode of the $\mathrm{VT}_{\mathrm{REX}}$, a P4 Parallel HEV. From EcoCAR E\&EC test data, the VT REX consumed $1341 \mathrm{Wh} / \mathrm{mi}$ in CS mode and VTool was calibrated to match this value. The results of this calibration are summarized in Table 27. A 'What if?' analysis may now be performed to compare a Series HEV to the $\mathrm{VT}_{\mathrm{REX}}$. To perform this analysis, the generator efficiency was set at $89 \%$ based on the modeling presented in Section 5.1 , and the engine efficiency was modified until the energy consumption equaled the P4 Parallel HEV model that represents the $\mathrm{VT}_{\mathrm{REX}}$. All other parameters were held constant between the two models. This assumes that all components in the two powertrains of interest are identical except for the engine and/or generator. The results of this analysis are summarized in Table 44. 
Table 44: Results of Series HEV and P4 Parallel HEV Comparative Analysis

\begin{tabular}{|c|c|c|c|c|c|}
\hline & $\begin{array}{c}\text { P4 Parallel } \\
\text { HEV }\end{array}$ & \multicolumn{4}{|c|}{ Series HEV } \\
\hline & $\begin{array}{c}\text { Net Fuel } \\
\text { Consumption }\end{array}$ & $\begin{array}{c}\text { Net Fuel } \\
\text { Consumption }\end{array}$ & $\begin{array}{c}\text { Cycle Average } \\
\text { Engine Efficiency }\end{array}$ & $\begin{array}{c}\text { Cycle Average } \\
\text { Generator Efficiency }\end{array}$ & $\begin{array}{c}\text { Cycle Average } \\
\text { Genset Efficiency }\end{array}$ \\
\hline & $\left(E_{\text {eng,fuel }}\right)^{\mathrm{DCnet}}$ & $\left(E_{\text {eng,fuel }}\right)^{\mathrm{DCnet}}$ & $\left(n_{\text {eng,ser }}\right)^{+}$ & $\left(\eta_{\text {gen }}\right)^{+}$ & $\left(\eta_{\text {eng,ser }}\right)^{+*}\left(\eta_{\text {gen }}\right)^{+}$ \\
\hline & [Wh/mi] & [Wh/mi] & [\%] & [\%] & [\%] \\
\hline UDDS & 1405 & 1403 & $27 \%$ & $89 \%$ & $24 \%$ \\
\hline HwFET & 1261 & 1246 & $32 \%$ & $89 \%$ & $28 \%$ \\
\hline CAFE & 1340 & 1332 & $29 \%$ & $89 \%$ & $26 \%$ \\
\hline
\end{tabular}

As the results in Table 44 show, the engine efficiency of the Series HEV was tuned until the Series HEV consumed slightly less energy than the P4 Parallel HEV. Now, the resulting engine efficiency may be examined to determine if that engine efficiency is possible or reasonable to achieve. For the city cycle, the engine efficiency for the Series HEV only needed to be $27 \%$ to perform better than the P4 Parallel $\mathrm{HEV}$. This is due in part to poor engine efficiency for the P4 Parallel HEV during city driving. An engine efficiency of $27 \%$ is easily achievable with a series genset so it is reasonable to expect a Series HEV to perform better than a P4 Parallel HEV during city driving. The opposite is true for highway driving - the Series HEV would be required to achieve a cycle average efficiency of $32 \%$. An engine efficiency of $32 \%$ is much harder to achieve, though it is possible for a series genset configuration. Thus, the VTool user should be skeptical that a Series HEV can perform better than a P4 Parallel HEV during highway driving. Examining the CAFE-weighted results, an engine efficiency of only $29 \%$ is required which could reasonably be achieved by a Series HEV for CAFE-weighted driving. However, a Series HEV could also easily miss this target. Thus, further investigation is required but at least the user knows that it is within the realm of possibility for a Series HEV to beat a P4 Parallel HEV. If the analysis had indicated that it was not reasonable to expect the Series HEV to out-perform the P4 Parallel HEV, the user could have saved time by eliminating the need to perform a more thorough analysis to compare the two powertrains. 


\section{Conclusions}

The global demand for energy, petroleum energy in particular, is increasing rapidly with the growing economies of countries like China, India and Brazil. As a result, Americans are increasingly subject to rising gas prices with no relief in sight. In the U.S. transportation sector, light-duty passenger vehicles account for about $58 \%$ of transportation energy use. Therefore incremental improvements in light-duty vehicle efficiency and energy use will significantly impact the overall landscape of energy use in America. The first step in this process is understanding automotive powertrains and the factors that influence automotive fuel economy. Additionally, the development of tools to accurately model fuel economy is critical to implementing advanced fuel economy improving technologies into production vehicles. This thesis has created such a tool, called VTool. This tool and its underlying method provide a simple means to model fuel economy to a reasonable degree of accuracy.

Literature Review: Several methods for calculating fuel consumption have already been established by many different authors. Some methods, like those of Sovran, Gantt, Hochgraf and Yanni, are simple enough to be useful as design and learning tools. Other modeling methods, like Baglione's method or PSAT/Autonomie, are much more complex and are meant for high-fidelity fuel consumption modeling. VTool, however, is a relatively simple model that can be used as a reality check against other models or as a sanity check against test data. This work builds directly off of the work done by Sovran and Gantt and segregates vehicle operation into propel, brake and idle cases and calculates tractive energy at the wheels using the Sovran coefficient method. The tool then explicitly calculates energy consumption and losses for each component in each powertrain, both before and after charging (if applicable). Building from Gantt's work, VTool also properly accounts for the effects of regen braking by explicitly separating regen energy capture and reuse into distinct cases for clarity and accuracy. VTool also goes a step further than Gantt and defines propel, brake and idle fuel consumption for Series and Parallel HEV powertrains.

Both Park and Boyd developed the concept of a recirculation efficiency for their works. This thesis builds on this concept by introducing a single parameter, the Power Split Fraction, that directly controls how much energy is diverted into the recirculation path. This parameter allows the user to explore tradeoffs in energy management strategies for Series or Parallel HEVs It provides the user with a simple way to examine an otherwise complex phenomenon that is entirely dependent upon control strategy. VTool also uses the concept of relating energy use back to fuel-equivalent energy to allow for direct comparison between otherwise dissimilar energy quantities. This method is used to translate captured regen brake energy into fuel-equivalent energy to directly evaluate the fuel savings of regen braking. It is also used to translate idle accessory use directly into fuel energy for evaluation of the impact of accessory load.

Modeling EV and HEV Energy Consumption: The tool first segregates vehicle operation into propel, brake and idle cases and calculates tractive energy at the wheels using the Sovran coefficient method. The tractive energy required to complete a cycle is independent of vehicle powertrain and depends only on the drive cycle and the vehicle glider properties. The tool then explicitly calculates energy consumption for four distinct powertrains: EV, Series HEV, P3/P4 Parallel HEV and P2 HEV. Losses are also calculated for each component in each powertrain, both before and after charging (if applicable). These calculations are done using energy-weighted component efficiencies. The EV model is the simplest in this thesis and can be used in conjunction with one of the HEV models to generate results for an EREV or PHEV. The EV model also establishes the regen brake case which is reused for each subsequent model. The Series HEV introduces more components, complexity and degrees of operational freedom. The Power Split Fraction is introduced here to control the amount of genset energy that is directly used for traction. Additionally, regen brake energy reuse is accounted for by calculating the fuel-equivalent energy credit that is received by capturing then reusing regen energy. These two concepts are also used for the 
two Parallel HEV powertrains presented. For the P3/P4 Parallel powertrain, the power split occurs at the drive axle instead of the HV bus. Additionally, the Parallel HEV has a shorter path from the fuel tank to the wheels with only 2 full-time losses compared to 4 for the Series HEV. However, the recirculation path is longer involving 6 losses compared to just two for the Series HEV. The P2 Parallel HEV is nearly identical to the P3/P4 Parallel HEV except that the engine driveline is not in the accessory energy path. The placement of the engine driveline also creates extra losses in the regen energy capture and reuse process.

Throughout the modeling process, VTool gives the user full access into the process to empower the user to develop a deeper understanding of the vehicle powertrain as a system. VTool offers 162 tunable parameters across 9 different standard drive cycles (18 per drive cycle) for each of these distinct powertrains. This extensive set of parameters gives the user exceptional freedom to tune VTool for various purposes: to represent a particular vehicle powertrain, to compare various different powertrain architectures or different components within a powertrain, to explore the limits of fuel economy and the sensitivity of component efficiencies within a powertrain, and to explore tradeoffs in some basic energy management strategies. Additionally, the ability to model multiple cycles allows the user to explore the effect of drive cycles on powertrain energy consumption and component efficiency. Standard drive cycle weightings, such as the EPA CAFE or 5-cycle weightings, can also be performed within VTool without the need for any post-processing. VTool also has the capability to calculate Utility Factor weighted energy consumption as well as the overall Well-to-Wheel effects of a given powertrain for a given cycle or cycle weighting.

Model Validation: The tool has been validated using on-road test data from the Emissions and Energy Consumption event of the 2011 EcoCAR competition. The E\&EC event was designed to be an on-road approximation of the CAFE cycle weighting and of the SAE J1711 method for calculating UF-weighted fuel economy for a PHEV. The E\&EC drive schedule overestimated the road load for UDDS by $7.4 \%$ and overestimated HwFET by $4.2 \%$ but was within $3 \%$ for the CAFE weighted road load. This shows that the E\&EC schedule was reasonably good in approximating the individual UDDS and HwFET cycles, but was very effective in approximating the overall CAFE weighting. The accuracy of the test method therefore lends confidence to the validation work performed.

The E\&EC test data was used to tune component parameters for an EV and a P4 Parallel HEV over the UDDS and HwFET cycles as well as the CAFE weighting. The net AC and DC energy consumption of the EV were validated to be within $1 \%$ of the actual CAFE weighted value. The fuel consumption of the P4 Parallel HEV was also validated to be within $1 \%$ of the actual CAFE weighted value. Additionally, the capability of VTool to perform UF-weighting was validated to be within $3 \%$ of the total UF-weighted CAFE energy consumption of the test vehicle. The validation work performed in this thesis is limited only to the EV and P4 Parallel models and only the UDDS and HwFET cycles. However, the successful validation serves as proof concept. Given the proper data, VTool could also be used to model other powertrains and other drive cycles such as US06, SC03 or LA92.

Recommendations for Future Work: Though much was accomplished in this thesis, there still remain some weakness and areas to expand and improve upon this work. Perhaps the most obvious is to expand the model to also include a Series-Parallel HEV. Many production HEVs, such as the Toyota Prius, operate as Series-Parallel HEVs so the inclusion of this powertrain architecture would be useful in applying the model to more production vehicles. Additionally, it would allow for additional exploration of the concept of the Power Split Fraction and its application to a more complicated powertrain. The Power Split Fraction in and of itself is another area for further exploration and improvement. Currently, there is no well-defined correlation between the Power Split Fraction and engine or generator efficiency. An example was included in this thesis that began to explore the mechanics of the Power Split Fraction, but 
further exploration is well warranted. The range of the Power Split Fraction is most certainly non-linear in its relation to engine efficiency, so it could be very interesting and useful to develop a map of engine efficiency versus Power Split Fraction that could be used as a guide for calibrating the two parameters.

It would also be very useful to develop a method by which to approximate component efficiencies without the need for test data. Certainly, another simulation program (like Autonomie) could be used for this purpose but that defeats the purpose of VTool being a simple model. Rather, the method to approximate component efficiencies should also be simple and focused on providing a 'ballpark' range for the component efficiency rather than a high-fidelity value. In seeking to achieve this goal, it could be useful to explore the idea of comparing the continuous power rating of a powertrain component (like a motor or engine) to the average power of the drive cycle to develop some kind of rough correlation. It may also be insightful to explore the concept of 'binning' the power used by the powertrain over the course of the cycle.

Conclusions: This thesis has researched automotive powertrain fuel economy and modeling methods, developed a new modeling method and packaged it into a tool. This method builds off of currently existing work from Sovran, and Gantt and draws inspiration from many others. It also introduces novel ideas while focusing on the fundamental objective of empowering the user to understand advanced vehicle powertrains and fuel economy. In conclusion, this thesis has accomplished its stated objectives and VTool has been presented, validated and demonstrated and is ready for general consumption. 


\section{References}

1. Green, D., Baker, H., and Plotkin, S., (2011), "Reducing Greenhouse Gas Emissions from U.S. Transportation," Prepared for the Pew Center for Global Climate Change.

2. Department of Energy, (2011), "Report on the First Quadrennial Technology Review," Department of Energy, Washington, DC.

3. Department of Transportation, (2003), Omnibus Household Survey, vol. 3(4). Fig 2. US Department of Transportation, Bureau of Transportation Statistics.

4. Pearre, N.S., et al., (2011), "Electric vehicles: How much range is required for a day's driving?," Transportation Research Part C: Emerging Technologies, Vol. 19, Issue 6, Dec. 2011, pp. 11711184.

5. Salisa, R., Zhang, N., and Zhu, G., (2011), "A Comparative Analysis of Fuel Economy and Emissions between a Conventional HEV and the UTS PHEV," Vehicular Technology, IEEE Transactions on, Vol. 60, No. 1, pp. 44-54 Jan. 2011. doi: 10.1109/TVT.2010.2091156

6. Miller, A., Holmes, G., Conlon, M., and Savagian, J., (2011), "The GM "Voltec" 4ET50 Multi-Mode Electric Transaxle", SAE Paper 2011-01-0887, SAE Int. J. Engines 4(1):1102-1114, 2011, doi:10.4271/2011-01-0887

7. Burke, A., (2007), "Batteries and Ultracapacitors for Electric, Hybrid, and Fuel Cell Vehicles," Proceedings of the IEEE, Vol. 95, No. 4, pp. 806-820, doi: 10.1109/JPROC.2007.892490

8. Vyas, A., Santini, J., and Johnson, R., (2009), "The Potential of Plug-in Hybrid Electric Vehicles to Reduce Petroleum Use: Issues Involved in Developing Reliable Estimates," Transportation Research Record: Journal of the Transportation Research Board, Vol 2139, pp. 55-63

9. Elgowainy, A., Han, J., Poch, L., Wang, M., Vyas, A., Mahalik, M., and Rousseau, A., (2010), "Wellto-Wheels Analysis of Energy Use and Greenhouse Gas Emissions of Plug-In Hybrid Electric Vehicles," Argonne National Labs, ANL/ESD/10-1, June 2010, http://www.osti.gov/bridge/product.biblio.jsp?query id=1\&page=0\&osti id=982352\&Row=1\&formna $\underline{\text { me=basicsearch.jsp }}$

10. Husain, I., (2011), "Electric and Hybrid Vehicles: Design Fundamentals," pp. 449-450, Taylor and Francis Group, Baton Rouge, FL.

$\begin{array}{llll}\text { 11. Powertrain } & \text { Systems } & \text { Analysis } & \text { Toolkit }\end{array}$ http://www.transportation.anl.gov/software/PSAT/index.html

12. Greenhouse Gasses, Regulated Emissions and Energy Use in Transportation Model (GREET), http://greet.es.anl.gov/

13. Brinkman, T. Weber, Wang, M., Darlington, T., (2005), "Well-to-Wheels Analysis of Advanced Fuel/Vehicle Systems - A North American Study of Energy Use, Greenhouse Gas Emissions, and Criteria Pollutant Emissions", Argonne Nation Labs. www.transportation.anl.gov/pdfs/TA/339.pdf

14. Gantt, L., Walsh, P., Alley, R., Bennett, J., King, J., Nelson, D., (2011), "Development and Validation of an E85 Range Extended Electric Vehicle", SAE Paper 2011-01-0912,SAE 2011 International World Congress, April 12-14, Detroit, Mi, doi:10.4271/2011-01-0912 
15. SAE International Surface Vehicle Recommended Practice, "Recommended Practice for Measuring the Exhaust Emissions and Fuel Economy of Hybrid-Electric Vehicles, Including Plug-in Hybrid Vehicles," SAE Standard J1711, Rev. Jun. 2010.

16. Alley, R., King, J., Gantt L., Walsh P., and Nelson D., (2012), "Refinement and Testing of a Split Parallel Extended Range Electric Vehicle", SAE Paper 2012-01-1196, SAE 2012 International World Congress, April 24-26, Detroit, Mi.

17. Alley, R., King, J., Manning, C., White, E., and Nelson D., (2012), "Results of 2011 EcoCAR Plug-in Hybrid Electric Vehicle On-Road Testing", Paper 6030594, EVS-26, The 26th International Battery, Hybrid and Fuel Cell Electric Vehicle Symposium and Exhibition, Los Angeles, California, May 6-9, 2012.

18. Douba, M. (2012), "Design of an On-Road PHEV Fuel Economy Testing Methodology with Built-In Utility Factor Distance Weighting", SAE Paper 2012-01-1194, SAE 2012 International World Congress, April 23-26, Detroit, Mi. doi:10.4271/2012-01-1194

19. Alley, R., King, J., Manning, C., White, E., and Nelson D., (2012), "Impact of US06 Drive Cycle on EcoCAR 2 Plug-in Hybrid Electric Vehicle Architecture Design and Component Sizing", Paper 6030593, EVS-26, The 26th International Battery, Hybrid and Fuel Cell Electric Vehicle Symposium and Exhibition, Los Angeles, California, May 6-9, 2012.

20. Alley, R., King, J., White, E., and Nelson D., (2012), "Hybrid Architecture Selection and Component Sizing to Reduce Emissions and Petroleum Energy Consumption", SAE Paper 2012-01-1195, SAE 2012 International World Congress, April 24-26, Detroit, Mi.

21. Sovran, G., Blaser, D., (2003), "A Contribution to Understanding Automotive Fuel Economy and Its Limits," SAE Paper 2003-01-2070, SAE Transactions, Washington, D.C., doi:10.4271/2003-01-2070

22. Sovran, G., Bohn, M., (1981), "Formulae for the Tractive-Energy Requirements of Vehicles Driving the EPA Schedules," SAE Paper 810184, SAE 1981 World Congress, Detroit, Michigan, USA, doi:10.4271/810184

23. Sovran, G., Blaser, D., (2006), "Quantifying the Potential Impacts of Regenerative Braking on a Vehicle's Tractive-Fuel Consumption for a U.S., European, and Japanese Driving Schedules," SAE Paper 2006-01-0664. SAE 2006 World Congress. Detroit, Michigan, USA, doi:10.4271/2006-010664

24. Gantt, L. (2011) "Energy Losses for Propelling and Braking Conditions of an Electric Vehicle." MS thesis, Virginia Tech, scholar.lib.vt.edu/theses/available/etd-05162011-220140/

25. Gantt, L., Perkins, D., Alley, R. and Nelson, D., (2011), "Regenerative Brake Energy Analysis for the VTREX Plug-in Hybrid Electric Vehicle", Vehicle Power and Propulsion Conference (VPPC), 2011 IEEE , September 7-9, 2011, Chicago, IL, doi: 10.1109/VPPC.2011.6043049.

26. Hochgraf, C., Duoba, M., (2010), "What if the Prius Wasn't a Hybrid? What if the Corolla Were? An Analysis Based on Vehicle Limited Fuel Consumption and Powertrain and Braking Efficiency," SAE Paper 2010-01-0834, SAE 2010 World Congress, Detroit, Michigan, USA, doi:10.4271/2010-010834 
27. Yanni, T., Venhovens, P., (2010), "Impact and Sensitivity of Vehicle Design Parameters on Fuel Economy Estimates," SAE Paper 2010-01-0734. SAE 2010 World Congress. Detroit, Michigan, USA, doi:10.4271/2010-01-0734

28. Duoba, M., Carlson, R., Bocci, D., (2009), "Calculating Results and Performance Parameters for PHEVs," SAE Paper 2009-01-1328. SAE 2009 World Congress. Detroit, Michigan, USA, doi:10.4271/2009-01-1328

29. Baglione, M., Duty, M., Pannone, G., (2007), "Vehicle System Energy Analysis Methodology and Tool for Determining Vehicle Subsystem Energy Supply and Demand," SAE Paper 2007-01-0398. SAE 2007 World Congress. Detroit, Michigan, USA, doi:10.4271/2007-01-0398

30. Park, J., Park Y., Park J., (2007), "Real-Time Powertrain Control Strategy for Series-Parallel Hybrid Electric Vehicles", SAE Paper 2007-01-3472, SAE 2007 Asia Pacific Automotive Engineering Conference, Aug 2007, Hollywood, CA.

31. Boyd, S., Nelson, D., (2008), "Hybrid Electric Vehicle Control Strategy Based on Power Loss Calculations", SAE Paper 2008-01-0084,SAE 2008 International World Congress, Detroit, Mi, doi:10.4271/2008-01-0084

32. G. Paganelli, S. Delpart, T. M. Guerra, J. Rimaux, and J. J. Santin, "Equivalent Consumption Minimization Strategy for Parallel Hybrid Powertrains," IEEE Vehicular Technology Conference (VTC), 2002 IEEE, May, 2002, Birmingham, AL, pp. 2076-2080. doi: 10.1109/VTC.2002.1002989.

33. Perkins, D., Gantt, L., Alley, R., Nelson, D., (2011), "An Assessment of Accessory Loads in a Hybrid Electric Vehicle", Vehicle Power and Propulsion Conference (VPPC), 2011 IEEE, September 7-9, 2011, Chicago, IL, doi: 10.1109/VPPC.2011.6043045. 


\section{APPENDIX A: Equations for the Propel Case (EV)}

This appendix contains the definitions for the component efficiencies used in the energy consumption equations developed for VTool. Some information may be duplicated in the body of this paper, but all relevant terms, definitions and equations have been reproduced here for convenience.

\begin{tabular}{|l|l|l|}
\hline Term & $\begin{array}{l}\text { Inherent } \\
\text { Sign }\end{array}$ & Definition \\
\hline$\left(E_{\text {batt,int }}\right)^{+}$ & + & Electrical energy inside the battery used during the propel case \\
\hline$\left(E_{\text {batt, }}\right)^{+}$ & + & Electrical energy out of the battery or at the terminals during the propel case \\
\hline$\left(E_{\text {acc }}\right)^{+}$ & + & Electrical energy siphoned off the HV bus for accessory loads during propel case \\
\hline$\left(E_{\text {mot,elec }}\right)^{+}$ & + & Electrical energy in to the motor for the propel case \\
\hline$\left(E_{\text {mot,mech }}\right)^{+}$ & + & Mechanical energy out of the motor for the propel case \\
\hline$\left(E_{t r}\right)^{+}$ & + & Tractive energy at the wheels for the propel case \\
\hline
\end{tabular}

\section{Efficiency Expressions:}

Equation 21: Motor driveline propel efficiency (EV, propel)

$$
\tilde{\eta}_{d l, m o t}^{+}=\frac{\left(E_{t r}\right)^{+}}{\left(E_{\text {mot }, \text { mech }}\right)^{+}}
$$

Equation 22: Motor propel efficiency (EV, propel)

$$
\tilde{\eta}_{\text {mot }}^{+}=\frac{\left(E_{\text {mot }, \text { mech }}\right)^{+}}{\left(E_{\text {mot }, \text { elec }}\right)^{+}}
$$

Equation 23: Battery discharge efficiency (EV, propel)

$$
\tilde{\eta}_{\text {batt }}^{+}=\frac{\left(E_{\text {batt }, T}\right)^{+}}{\left(E_{\text {batt }, \text { int }}\right)^{+}}
$$

Equation 28: Propel case powertrain efficiency (EV, propel)

$$
\tilde{\eta}_{p t}^{+}=\frac{\left(E_{t r}\right)^{+}}{\left(E_{\text {batt }, T}\right)^{+}}
$$

\section{Loss Expressions:}

Equation 129: Motor driveline losses (EV, propel)

$\left(E_{\text {dlmot }, \text { loss }}\right)^{+}=\left(E_{\text {mot }, \text { mech }}\right)^{+}-\left(E_{\text {tr }}\right)^{+}$

Equation 130: Motor losses (EV, propel)

$\left(E_{\text {mot }, \text { loss }}\right)^{+}=\left(E_{\text {mot,elec }}\right)^{+}-\left(E_{\text {mot }, \text { mech }}\right)^{+}$

Equation 131: Battery losses (EV, propel)

$$
\left(E_{\text {batt }, \text { loss }}\right)^{+}=\left(E_{\text {batt }, \text { int }}\right)^{+}-\left(E_{\text {batt }, T}\right)^{+}
$$


Equation 132: Propel powertrain losses (EV, propel)

$$
\begin{aligned}
& \left(E_{\text {pt loss }}\right)^{+}=\left(E_{\text {batt }, \text { int }}\right)^{+}-\left(E_{\text {Acc }}\right)^{+}-\left(E_{\text {tr }}\right)^{+} \\
& \text {Restated: } \\
& \left(E_{\text {pt,loss }}\right)^{+}=\left(E_{\text {batt }, \text { loss }}\right)^{+}+\left(E_{\text {mot }, \text { loss }}\right)^{+}+\left(E_{\text {dlmot }, \text { loss }}\right)^{+}
\end{aligned}
$$

\section{Conservation of Energy Expressions:}

Equation 24: Conservation of energy at the HV Bus (EV, propel)

$\left(E_{\text {batt }, T}\right)^{+}=\left(E_{\text {mot }, \text { elec }}\right)^{+}+\left(E_{\text {Acc }}\right)^{+}$

\section{Accessory Energy:}

Equation 25: Propel case accessory energy

$\left(E_{A c c}\right)^{+}=\tau\left(P_{A c c}\right)^{+}$

Developing Equation for Propel Case Energy Consumption (in terms of efficiencies):

$\left(E_{\text {batt }, \text { int }}\right)^{+}=\frac{\left(E_{\text {batt }, T}\right)^{+}}{\tilde{\eta}_{\text {batt }}^{+}}$

$\left(E_{\text {batt }, i n t}\right)^{+}=\frac{1}{\tilde{\eta}_{\text {batt }}^{+}}\left\{\left(E_{\text {Acc }}\right)^{+}+\left(E_{\text {mot }, e l e c}\right)^{+}\right\}$

$\left(E_{\text {batt }, \text { int }}\right)^{+}=\frac{1}{\tilde{\eta}_{\text {batt }}^{+}}\left\{\left(E_{\text {Acc }}\right)^{+}+\left[\frac{\left(E_{\text {mot }, \text { mech }}\right)^{+}}{\tilde{\eta}_{\text {mot }}^{+}}\right]\right\}$

Equation 26: Propel case energy consumption (EV, propel)

$\left(E_{\text {batt }, \text { int }}\right)^{+}=\frac{1}{\tilde{\eta}_{\text {batt }}^{+}}\left\{\left(E_{\text {Acc }}\right)^{+}+\left[\frac{\left(E_{t r}\right)^{+}}{\left(\tilde{\eta}_{\text {mot }}^{+}\right)\left(\tilde{\eta}_{d l, m o t}^{+}\right)}\right]\right\}$ 


\section{Developing Equation for Propel Case Energy Consumption (in terms of losses):}

$$
\begin{aligned}
& \left(E_{\text {batt }, \text { int }}\right)^{+}=\left(E_{\text {batt }, T}\right)^{+}+\left(E_{\text {batt }, \text { loss }}\right)^{+} \\
& \left(E_{\text {batt }, \text { int }}\right)^{+}=\left(E_{\text {mot }, \text { elec }}\right)^{+}+\left(E_{\text {Acc }}\right)^{+}+\left(E_{\text {batt }, \text { loss }}\right)^{+} \\
& \left(E_{\text {batt }, \text { int }}\right)^{+}=\left(E_{\text {mot }, t r}\right)^{+}+\left(E_{\text {Acc }}\right)^{+}+\left(E_{\text {batt }, \text { loss }}\right)^{+}+\left(E_{\text {mot }, l o s s}\right)^{+} \\
& \left(E_{\text {batt }, \text { int }}\right)^{+}=\left(E_{\text {tr }}\right)^{+}+\left(E_{\text {Acc }}\right)^{+}+\left(E_{\text {batt }, \text { loss }}\right)^{+}+\left(E_{\text {mot }, \text { loss }}\right)^{+}+\left(E_{\text {dlmot }, \text { loss }}\right)^{+}
\end{aligned}
$$

Equation 27: Propel case energy consumption in terms of losses (EV, propel)

$$
\left(E_{\text {batt }, \text { int }}\right)^{+}=\left(E_{\text {tr }}\right)^{+}+\left(E_{\text {Acc }}\right)^{+}+\left(E_{\text {batt }, \text { loss }}\right)^{+}+\left(E_{\text {mot }, \text { loss }}\right)^{+}+\left(E_{\text {dlmot }, \text { loss }}\right)^{+}
$$




\section{APPENDIX B: Equations for the Brake Case (EV)}

This appendix contains the definitions for the component efficiencies used in the energy consumption equations developed for VTool. Some information may be duplicated in the body of this paper, but all relevant terms, definitions and equations have been reproduced here for convenience.

\begin{tabular}{|l|l|l|}
\hline Term & $\begin{array}{l}\text { Inherent } \\
\text { Sign }\end{array}$ & Definition \\
\hline$\left(E_{t r}\right)^{-}$ & - & Tractive energy at the wheels for the brake case \\
\hline$\left(E_{t r, \zeta}\right)^{-}$ & - & Tractive energy in to driveline after effect of regen fraction \\
\hline$\left(E_{\text {mot,mech }}\right)^{-}$ & - & Mechanical energy in to the motor for the brake case \\
\hline$\left(E_{\text {mot,elec }}\right)^{-}$ & - & Electrical energy out of the motor for the brake case \\
\hline$\left(E_{\text {acc }}\right)^{-}$ & + & Electrical energy siphoned off the HV bus for accessory loads during brake case \\
\hline$\left(E_{\text {batt, },}\right)^{-}$ & - & Electrical energy in to the battery or at the terminals during the brake case \\
\hline$\left(E_{\text {batt,int }}\right)^{-}$ & - & Electrical energy inside the battery captured during the brake case \\
\hline
\end{tabular}

\section{Efficiency Expressions:}

Equation 29: Regen brake fraction

$\tilde{\zeta}=\frac{\left(E_{t r, \zeta}\right)^{-}}{\left(E_{t r}\right)^{-}}$

Equation 133: Motor driveline regen efficiency (EV, brake)

$\tilde{\eta}_{d l, m o t}^{-}=\frac{\left(E_{m o t, m e c h}\right)^{-}}{\left(E_{t r, \zeta}\right)^{-}}$

Equation 33: Motor regen efficiency (EV, brake)

$\tilde{\eta}_{\text {mot }}^{-}=\frac{\left(E_{\text {mot }, \text { elec }}\right)^{-}}{\left(E_{\text {mot }, \text { mech }}\right)^{-}}$

Equation 134: Battery charge efficiency (EV, brake)

$\tilde{\eta}_{\text {batt }}^{-}=\frac{\left(E_{\text {batt }, \text { int }}\right)^{-}}{\left(E_{\text {batt }, T}\right)^{-}}$

Equation 35: Brake case powertrain efficiency (EV, brake)

$\tilde{\eta}_{p t}^{-}=\frac{\left(E_{b a t t, T}\right)^{-}}{\left(E_{t r}\right)^{-}}$ 


\section{Loss Expressions:}

Equation 135: Friction brake losses (EV, brake)

$\left(E_{\text {brk }, l o s s}\right)^{-}=\left(E_{t r, \zeta}\right)^{-}-\left(E_{t r}\right)^{-}$

Equation 136: Motor driveline losses (EV, brake)

$\left(E_{\text {dlmot }, l o s s}\right)^{-}=\left(E_{\text {mot }, \text { mech }}\right)^{-}-\left(E_{t r, \zeta}\right)^{-}$

Equation 137: Motor losses (EV, brake)

$\left(E_{\text {mot }, \text { loss }}\right)^{-}=\left(E_{\text {mot,elec }}\right)^{-}-\left(E_{\text {mot }, \text { mech }}\right)^{-}$

Equation 138: Battery losses (EV, brake)

$\left(E_{\text {batt }, \text { loss }}\right)^{-}=\left(E_{\text {batt }, \text { int }}\right)^{-}-\left(E_{\text {batt }, T}\right)^{-}$

Equation 139: Brake powertrain losses (EV, brake)

$\left(E_{p t, l o s s}\right)^{-}=\left(E_{\text {batt }, \text { int }}\right)^{-}-\left(E_{\text {Acc }}\right)^{-}-\left(E_{t r}\right)^{-}$

Restated:

$\left(E_{p t, l o s s}\right)^{-}=\left(E_{\text {batt }, \text { loss }}\right)^{-}+\left(E_{\text {mot }, \text { loss }}\right)^{-}+\left(E_{\text {dl,loss }}\right)^{-}+\left(E_{\text {brk }, \text { loss }}\right)^{-}$

\section{Conservation of Energy Expressions:}

Equation 30: Conservation of energy at the HV Bus (EV, brake)

$\left(E_{\text {batt }, T}\right)^{-}=\left(E_{\text {mot }, \text { elec }}\right)^{-}+\left(E_{\text {Acc }}\right)^{-}$

\section{Accessory Energy:}

Equation 31: Brake case accessory energy

$\left(E_{\text {Acc }}\right)^{-}=\left(T-\tau-t_{\text {idle }}\right)\left(P_{\text {Acc }}\right)^{-}$ 


\section{Developing Equation for Brake Case Energy Consumption (in terms of efficiencies):}

$$
\begin{aligned}
& \left(E_{\text {batt }, \text { int }}\right)^{-}=\tilde{\eta}_{\text {batt }}^{-} *\left(E_{\text {batt }, T}\right)^{-} \\
& \left(E_{\text {batt }, \text { int }}\right)^{-}=\tilde{\eta}_{\text {batt }}^{-}\left\{\left(E_{\text {mot,elec }}\right)^{-}+\left(E_{\text {Acc }}\right)^{-}\right\} \\
& \left(E_{\text {batt }, \text { int }}\right)^{-}=\tilde{\eta}_{\text {batt }}^{-}\left\{\left(E_{\text {mot }, \text { mech }}\right)^{-}\left(\tilde{\eta}_{\text {mot }}^{-}\right)+\left(E_{\text {Acc }}\right)^{-}\right\} \\
& \left(E_{\text {batt }, \text { int }}\right)^{-}=\tilde{\eta}_{\text {batt }}^{-}\left\{\left(E_{\text {tr }, \zeta}\right)^{-}\left(\tilde{\eta}_{\overline{d l}, \text { mot }}^{-}\right)\left(\tilde{\eta}_{\text {mot }}^{-}\right)+\left(E_{\text {Acc }}\right)^{-}\right\}
\end{aligned}
$$

Equation 32: Brake case energy consumption (EV, brake)

$\left(E_{\text {batt }, \text { int }}\right)^{-}=\tilde{\eta}_{\text {batt }}^{-}\left\{\left(E_{t r}\right)^{-}(\tilde{\zeta})\left(\tilde{\eta}_{d l, m o t}^{-}\right)\left(\tilde{\eta}_{\text {mot }}^{-}\right)+\left(E_{\text {Acc }}\right)^{-}\right\}$

\section{Developing Equation for Brake Case Energy Consumption (in terms of losses):}

$$
\begin{aligned}
& \left(E_{\text {batt }, \text { int }}\right)^{-}=\left(E_{\text {batt }, T}\right)^{-}+\left(E_{\text {batt }, \text { loss }}\right)^{-} \\
& \left(E_{\text {batt }, \text { int }}\right)^{-}=\left(E_{\text {mot }, \text { elec }}\right)^{-}+\left(E_{\text {Acc }}\right)^{-}+\left(E_{\text {batt }, \text { loss }}\right)^{-} \\
& \left(E_{\text {batt }, \text { int }}\right)^{-}=\left(E_{\text {mot }, t r}\right)^{-}+\left(E_{\text {Acc }}\right)^{-}+\left(E_{\text {batt }, \text { loss }}\right)^{-}+\left(E_{\text {mot }, \text { loss }}\right)^{-} \\
& \left(E_{\text {batt }, \text { int }}\right)^{-}=\left(E_{\text {tr, }, \zeta}\right)^{-}+\left(E_{\text {acc }}\right)^{-}+\left(E_{\text {batt,loss }}\right)^{-}+\left(E_{\text {mot }, \text { loss }}\right)^{-}+\left(E_{\text {dlmot }, \text { loss }}\right)^{-}
\end{aligned}
$$

Equation 34: Brake case energy consumption in terms of losses (EV, brake)

$$
\left(E_{\text {batt }, \text { int }}\right)^{-}=\left(E_{t r}\right)^{-}+\left(E_{\text {Acc }}\right)^{-}+\left(E_{\text {batt }, \text { loss }}\right)^{-}+\left(E_{\text {mot }, \text { loss }}\right)^{-}+\left(E_{\text {dlmot }, l o s s}\right)^{-}+\left(E_{\text {brk,loss }}\right)^{-}
$$




\section{APPENDIX C: Equations for the Idle Case (EV)}

This appendix contains the definitions for the component efficiencies used in the energy consumption equations developed for VTool. Some information may be duplicated in the body of this paper, but all relevant terms, definitions and equations have been reproduced here for convenience.

\begin{tabular}{|l|l|l|}
\hline Term & $\begin{array}{l}\text { Inherent } \\
\text { Sign }\end{array}$ & Definition \\
\hline$\left(E_{\text {batt,int }}\right)^{0}$ & + & Electrical energy inside the battery used during the idle case \\
\hline$\left(E_{\text {batt, }}\right)^{0}$ & + & Electrical energy out of the battery or at the terminals during idle case \\
\hline$\left(E_{\text {acc }}\right)^{0}$ & + & Electrical energy siphoned off the HV bus for accessory loads during idle case \\
\hline
\end{tabular}

\section{Efficiency Expressions:}

Equation 140: Battery discharge efficiency (EV, idle)

$$
\tilde{\eta}_{\text {batt }}^{0}=\frac{\left(E_{\text {batt }, T}\right)^{0}}{\left(E_{\text {batt }, \text { int }}\right)^{0}}=\tilde{\eta}_{\text {batt }}^{+}
$$

\section{Loss Expressions:}

Equation 141: Battery losses (EV, idle)

$$
\left(E_{\text {batt }, \text { loss }}\right)^{0}=\left(E_{\text {batt }, \text { int }}\right)^{0}-\left(E_{\text {batt }, T}\right)^{0}
$$

\section{Conservation of Energy Expressions:}

Equation 142: Conservation of energy at the HV Bus (EV, idle)

$\left(E_{\text {batt }, T}\right)^{0}=\left(E_{A c c}\right)^{0}$

\section{Accessory Energy:}

Equation 36: Idle accessory energy

$$
\left(E_{\text {Acc }}\right)^{0}=\left(t_{\text {idle }}\right)\left(P_{\text {Acc }}\right)^{0}
$$

\section{Developing Equation for Idle Case Energy Consumption (in terms of efficiencies):}

Equation 37: Idle case energy consumption (EV, idle)

$$
\left(E_{\text {batt }, \text { int }}\right)^{0}=\frac{\left(E_{\text {batt }, T}\right)^{0}}{\tilde{\eta}_{\text {batt }}^{0}}=\frac{\left(E_{\text {Acc }}\right)^{0}}{\tilde{\eta}_{\text {batt }}^{0}}
$$


Developing Equation for Idle Case Energy Consumption (in terms of losses):

Equation 143: Idle case energy consumption in terms of losses (EV, idle)

$\left(E_{\text {batt }, \text { int }}\right)^{0}=\left(E_{\text {batt }, T}\right)^{0}+\left(E_{\text {batt }, \text { loss }}\right)^{0}=\left(E_{\text {Acc }}\right)^{0}+\left(E_{\text {bat }, \text { loss }}\right)^{0}$ 


\section{APPENDIX D: Equations for the DCnet Case (EV)}

This appendix contains the definitions for the component efficiencies used in the energy consumption equations developed for VTool. Some information may be duplicated in the body of this paper, but all relevant terms, definitions and equations have been reproduced here for convenience.

\begin{tabular}{|l|l|l|}
\hline Term & $\begin{array}{l}\text { Inherent } \\
\text { Sign }\end{array}$ & Definition \\
\hline$\left(E_{\text {batt, },}\right)^{+}$ & + & Electrical energy out of the battery or at the terminals during the propel case \\
\hline$\left(E_{\text {batt, },}\right)^{-}$ & - & Electrical energy in to the battery or at the terminals during the brake case \\
\hline$\left(E_{\text {batt, } T}\right)^{0}$ & + & Electrical energy out of the battery or at the terminals during idle case \\
\hline$\left(E_{\text {batt, },}\right)^{D C n e t}$ & + & Net electrical energy at the terminals for a given drive cycle \\
\hline$\left(E_{\text {batt,int }}\right)^{+}$ & + & Electrical energy inside the battery used during the propel case \\
\hline$\left(E_{\text {batt, int }}\right)^{-}$ & - & Electrical energy inside the battery captured during the brake case \\
\hline$\left(E_{\text {batt,int }}\right)^{0}$ & + & Electrical energy inside the battery used during the idle case \\
\hline$\left(E_{\text {batt,int }}\right)^{D C n e t}$ & + & Net electrical energy inside the battery for a given drive cycle \\
\hline
\end{tabular}

\section{Efficiency Expressions:}

Equation 40: Net powertrain efficiency (EV, DCnet)

$\tilde{\eta}_{p t}^{\text {DCnet }}=\frac{\left(E_{\text {tr }}\right)^{\text {DCnet }}}{\left(E_{\text {batt }, T}\right)^{\text {DCnet }}}=\frac{\left(E_{t r}\right)^{+}+\left(E_{t r}\right)^{-}}{\left(E_{\text {batt }, T}\right)^{+}+\left(E_{\text {batt }, T}\right)^{-}+\left(E_{\text {batt }, T}\right)^{0}}$

Equation 28: Propel case powertrain efficiency (EV, propel)

$\tilde{\eta}_{p t}^{+}=\frac{\left(E_{t r}\right)^{+}}{\left(E_{\text {batt }, i n t}\right)^{+}}$

Equation 35: Brake case powertrain efficiency (EV, brake)

$\tilde{\eta}_{p t}^{-}=\frac{\left(E_{\text {batt }, \text { int }}\right)^{-}}{\left(E_{t r}\right)^{-}}$ 


\section{Loss Expressions:}

Equation 144: Net friction brake losses (EV, DCnet)

$\left(E_{\text {brk }, \text { loss }}\right)^{\text {DCnet }}=\left(E_{\text {brk }, \text { loss }}\right)^{-}$

Equation 145: Net motor driveline losses (EV, DCnet)

$\left(E_{d l, l o s s}\right)^{\text {DCnet }}=\left(E_{d l, l o s s}\right)^{+}+\left(E_{d l, l o s s}\right)^{-}$

Equation 146: Net motor losses (EV, DCnet)

$\left(E_{\text {mot }, \text { loss }}\right)^{\text {DCnet }}=\left(E_{\text {mot }, \text { loss }}\right)^{+}+\left(E_{\text {mot }, \text { loss }}\right)^{-}$

Equation 147: Net battery losses (EV, DCnet)

$\left(E_{\text {batt }, \text { loss }}\right)^{\text {DCnet }}=\left(E_{\text {batt }, \text { loss }}\right)^{+}+\left(E_{\text {batt }, \text { loss }}\right)^{-}+\left(E_{\text {batt }, \text { loss }}\right)^{0}$

Equation 42: Net powertrain losses (EV, DCnet)

$\left(E_{p t, l o s s}\right)^{\text {DCnet }}=\left(E_{p t, l o s s}\right)^{+}+\left(E_{p t, l o s s}\right)^{-}+\left(E_{\text {batt }, \text { loss }}\right)^{0}$

\section{Conservation of Energy Expressions:}

Equation 38: Net internal battery energy used (EV, DCnet)

$\left(E_{\text {batt }, \text { int }}\right)^{\text {DCnet }}=\left(E_{\text {batt }, \text { int }}\right)^{+}+\left(E_{\text {batt }, \text { int }}\right)^{-}+\left(E_{\text {bat }, \text { int }}\right)^{0}$

Equation 39: Net DC energy consumption (EV, DCnet)

$\left(E_{\text {batt }, T}\right)^{\text {DCnet }}=\left(E_{\text {batt }, T}\right)^{+}+\left(E_{\text {batt }, T}\right)^{-}+\left(E_{\text {batt }, T}\right)^{0}$

Equation 43: Overall energy balance (EV,DCnet)

$\left(E_{\text {batt }, \text { int }}\right)^{\text {DCnet }}=\left(E_{t r}\right)^{\text {DCnet }}+\left(E_{\text {Acc }}\right)^{D C n e t}+\left(E_{p t, \text { loss }}\right)^{D C n e t}$

\section{Accessory Energy:}

Equation 41: Net accessory energy

$\left(E_{A C C}\right)^{\text {DCnet }}=\left(E_{A C C}\right)^{+}+\left(E_{A C C}\right)^{-}+\left(E_{A C C}\right)^{0}=P_{A C C} * T$ 


\section{APPENDIX E: Equations for the Charge Case (EV)}

This appendix contains the definitions for the component efficiencies used in the energy consumption equations developed for VTool. Some information may be duplicated in the body of this paper, but all relevant terms, definitions and equations have been reproduced here for convenience.

\begin{tabular}{|l|l|l|}
\hline Term & $\begin{array}{l}\text { Inherent } \\
\text { Sign }\end{array}$ & Definition \\
\hline$\left(E_{\text {chrg,AC }}\right)^{\text {ACnet }}$ & + & Electrical energy (AC) from the grid in to the battery charger \\
\hline$\left(E_{\text {chrq, }}\right)^{C}$ & + & Electrical energy (DC) out of the battery charger \\
\hline$\left(E_{\text {acc }}\right)^{C}$ & + & Electrical energy siphoned off the HV bus for accessory loads during charge case \\
\hline$\left(E_{\text {batt, }, T}\right)^{C}$ & - & Electrical energy out of the battery or at the terminals during the charging case \\
\hline$\left(E_{\text {batt,int }}\right)^{\text {DCnet }}$ & + & Net electrical energy inside the battery for a given drive cycle \\
\hline$\left(E_{\text {batt,int }}\right)^{C}$ & - & Electrical energy required to replenish the battery pack after depletion \\
\hline$t_{\text {charge }}$ & + & Time in seconds required to replenish the battery energy expended over a cycle \\
\hline
\end{tabular}

\section{Efficiency Expressions:}

Equation 45: Battery charge efficiency (EV, charge)

$$
\tilde{\eta}_{\text {batt }}^{c}=-\frac{\left(E_{\text {batt }, \text { int }}\right)^{c}}{\left(E_{\text {batt }, T}\right)^{c}}=\frac{\left(E_{\text {batt }, \text { int }}\right)^{\text {DCnet }}}{\left(E_{\text {batt }, T}\right)^{c}} \approx \tilde{\eta}_{\text {batt }}^{-}
$$

Equation 46: Charger efficiency (EV, charge)

$$
\tilde{\eta}_{\text {chrgr }}^{c}=\frac{\left(E_{\text {chrg,DC }}\right)^{c}}{\left(E_{\text {chrg }, A C}\right)^{A C n e t}}
$$

Equation 49: Charging efficiency (EV, charge)

$$
\tilde{\eta}_{\text {chrgn }}^{c}=-\frac{\left(E_{\text {batt }, \text { int }}\right)^{c}}{\left(E_{\text {chrg, } A C}\right)^{A C n e t}}=\frac{\left(E_{\text {batt }, \text { int }}\right)^{\text {DCnet }}}{\left(E_{\text {chrg }, A C}\right)^{A C n e t}}
$$




\section{Loss Expressions:}

Equation 148: Battery losses (EV, charge)

$\left(E_{\text {batt }, \text { loss }}\right)^{c}=\left(E_{\text {batt }, \text { int }}\right)^{c}-\left(E_{\text {batt }, T}\right)^{C}$

Equation 149: Charger losses (EV, charge)

$\left(E_{\text {chrg }, \text { loss }}\right)^{C}=\left(E_{\text {chrg }, A C}\right)^{A C n e t}-\left(E_{\text {chrg }, D C}\right)^{C}$

Equation 150: Battery losses (EV, charge)

$\left(E_{\text {pt,loss }}\right)^{c}=\left(E_{\text {chrg }, A C}\right)^{\text {ACnet }}-\left(E_{\text {Acc }}\right)^{c}+\left(E_{\text {batt }, \text { int }}\right)^{c}$

Restated:

$\left(E_{\text {pt }, \text { loss }}\right)^{c}=\left(E_{\text {chrg, loss }}\right)^{c}+\left(E_{\text {batt }, \text { loss }}\right)^{c}$

\section{Conservation of Energy Expressions:}

Equation 44: Battery internal energy to be replenished (EV, charge)

$\left(E_{\text {batt }, \text { int }}\right)^{c}=-\left(E_{\text {batt }, \text { int }}\right)^{\text {DCnet }}$

Equation 151: Conservation of energy at the HV Bus (EV, charge)

$\left(E_{b a t t, T}\right)^{c}=\left(E_{A c c}\right)^{c}-\left(E_{c h r g, D C}\right)^{c}$

\section{Accessory Energy:}

Equation 47: Charge case accessory energy (EV, charge)

$\left(E_{\text {Acc }}\right)^{C}=\left(t_{\text {charge }}\right)\left(P_{\text {Acc }}\right)^{C}$

Equation 48: Time required for charging (EV, charge)

$\left(t_{\text {charge }}\right)=\frac{\left(E_{\text {batt }, \text { int }}\right)^{\text {DCnet }}}{\left(P_{\text {charger }}\right)^{C}}$ 


\section{Developing Equation for Charge Case Energy Consumption (in terms of efficiencies):}

$\left(E_{\text {chrg }, A C}\right)^{\text {ACnet }}=\frac{\left(E_{\text {chrg,DC }}\right)^{C}}{\tilde{\eta}_{\text {chrgr }}^{c}}$

$\left(E_{\text {chrg }, A C}\right)^{A C n e t}=\frac{\left(E_{\text {Acc }}\right)^{c}-\left(E_{\text {batt }, T}\right)^{c}}{\tilde{\eta}_{\text {chrgr }}^{c}}$

$\left(E_{\text {chrg }, A C}\right)^{A C n e t}=\frac{1}{\tilde{\eta}_{c h r g r}^{c}}\left[\left(E_{\text {Acc }}\right)^{c}-\frac{\left(E_{\text {batt }, \text { int }}\right)^{c}}{\tilde{\eta}_{\text {batt }}^{c}}\right]$

Equation 50: Charge case energy consumption (EV, charge)

$\left(E_{\text {chrg }, A C}\right)^{A C n e t}=\frac{1}{\tilde{\eta}_{\text {chrgr }}^{c}}\left[\left(E_{\text {Acc }}\right)^{c}+\frac{\left(E_{\text {batt }, \text { int }}\right)^{\text {DCnet }}}{\tilde{\eta}_{\text {batt }}^{c}}\right]$

Equation 51: Charge case energy consumption using charging process efficiency (EV, charge)

$\left(E_{\text {chrg }, A C}\right)^{\text {ACnet }}=\frac{\left(E_{\text {batt }, \text { int }}\right)^{\text {DCnet }}}{\tilde{\eta}_{\text {chrgn }}^{c}}$

\section{Developing Equation for Charge Case Energy Consumption (in terms of losses):}

$\left(E_{\text {chrg }, A C}\right)^{A C n e t}=\left(E_{\text {grid }, D C}\right)^{C}+\left(E_{\text {chrg }, \text { loss }}\right)^{c}$

$\left(E_{\text {chrg }, A C}\right)^{\text {ACnet }}=\left(E_{\text {batt }, T}\right)^{C}+\left(E_{\text {Acc }}\right)^{c}+\left(E_{\text {chrg }, \text { loss }}\right)^{c}$

Equation 52Equation 52: Charge case energy consumption in terms of losses (EV, charge)

$\left(E_{\text {chrg }, A C}\right)^{\text {ACnet }}=\left(E_{\text {batt }, \text { int }}\right)^{\text {DCnet }}+\left(E_{\text {Acc }}\right)^{c}+\left(E_{\text {chrg, loss }}\right)^{c}+\left(E_{\text {batt }, \text { loss }}\right)^{c}$ 


\section{APPENDIX F: Equations for the Propel Case (Series HEV)}

This appendix contains the definitions for the component efficiencies used in the energy consumption equations developed for VTool. Some information may be duplicated in the body of this paper, but all relevant terms, definitions and equations have been reproduced here for convenience.

\begin{tabular}{|l|l|l|}
\hline Term & $\begin{array}{l}\text { Inherent } \\
\text { Sign }\end{array}$ & Definition \\
\hline$\left(E_{\text {eng,fuel }}\right)^{+}$ & + & Fuel energy in to the engine during the propel case \\
\hline$\left(E_{\text {eng,mech }}\right)^{+}$ & + & Mechanical energy out of the engine during the propel case \\
\hline$\left(E_{\text {gen,elec }}\right)^{+}$ & + & Electrical energy out of the generator during the propel case \\
\hline$\left(E_{\text {gen,use }}\right)^{+}$ & + & Electrical generator energy directly used for propulsion \\
\hline$\left(E_{\text {gen,store }}\right)^{+ \text {gen }}$ & - & Electrical generator energy stored before being used for propulsion \\
\hline$\left(E_{\text {batt,int }}\right)^{\text {gen }}$ & - & Electrical energy inside the battery stored from excess generating \\
\hline$\left(E_{\text {batt,int }}\right)^{\text {prop }}$ & + & Electrical energy inside the battery to be used in propulsion \\
\hline$\left(E_{\text {batt,use }}\right)^{+ \text {prop }}$ & + & Electrical energy at the terminals to be used in propulsion \\
\hline$\left(E_{\text {acc }}\right)^{+}$ & + & Electrical energy siphoned off the HV bus for accessory loads during propel case \\
\hline$\left(E_{\text {mot,elec }}\right)^{+}$ & + & Electrical energy in to the motor for the propel case \\
\hline$\left(E_{\text {mot,mech }}\right)^{+}$ & + & Mechanical energy out of the motor for the propel case \\
\hline$\left(E_{\text {tr }}\right)^{+}$ & + & Tractive energy at the wheels for the propel case \\
\hline
\end{tabular}

\section{Efficiency Expressions:}

Equation 54: Power Split Fraction, genset energy directly used (Series, propel)

$$
\tilde{\xi}=\frac{\left(E_{\text {gen }, \text { use }}\right)^{+}}{\left(E_{\text {gen,elec }}\right)^{+}}
$$

Restated:

$\left(E_{\text {gen,use }}\right)^{+}=\tilde{\xi}\left(E_{\text {gen }, \text { elec }}\right)^{+}$

Equation 55: Power Split Fraction, genset energy recirculated (Series, propel)

$\left(E_{\text {gen }, \text { store }}\right)^{+ \text {gen }}=(-1)(1-\tilde{\xi})\left(E_{\text {gen }, \text { elec }}\right)^{+}$

Equation 152: Motor driveline propel efficiency (Series, propel)

$\tilde{\eta}_{d l, m o t}^{+}=\frac{\left(E_{t r}\right)^{+}}{\left(E_{\text {mot }, \text { mech }}\right)^{+}}$

Equation 153: Motor propel efficiency (Series, propel)

$$
\tilde{\eta}_{\text {mot }}^{+}=\frac{\left(E_{\text {mot }, \text { mech }}\right)^{+}}{\left(E_{\text {mot }, \text { elec }}\right)^{+}}
$$


Equation 154: Battery charge efficiency (Series, propel)

$\tilde{\eta}_{\text {batt }}^{+ \text {gen }}=\frac{\left(E_{\text {batt }, \text { int }}\right)^{+ \text {gen }}}{\left(E_{\text {gen,store }}\right)^{+ \text {gen }}} \approx \tilde{\eta}_{\text {batt }}^{-}$

Equation 155: Battery discharge efficiency (Series, propel)

$\tilde{\eta}_{\text {batt }}^{+ \text {prop }}=\frac{\left(E_{\text {batt }, \text { use }}\right)^{+ \text {prop }}}{\left(E_{\text {batt }, \text { int }}\right)^{+ \text {prop }}} \approx \tilde{\eta}_{\text {batt }}^{+}$

Equation 156: Generator propel efficiency (Series, propel)

$\tilde{\eta}_{\text {gen }}^{+}=\frac{\left(E_{\text {gen }, \text { elec }}\right)^{+}}{\left(E_{\text {eng,mech }}\right)^{+}}$

Equation 157: Engine propel efficiency (Series, propel)

$\tilde{\eta}_{\text {eng }}^{+}=\frac{\left(E_{\text {eng,mech }}\right)^{+}}{\left(E_{\text {eng,fuel }}\right)^{+}}$

Equation 60: Propel case powertrain efficiency (Series, propel)

$\tilde{\eta}_{p t}^{+}=\frac{\left(E_{\text {tr }}\right)^{+}}{\left(E_{\text {eng,fuel }}\right)^{+}}$

\section{Loss Expressions:}

Equation 158: Motor driveline losses (Series, propel)

$\left(E_{\text {dlmot }, \text { loss }}\right)^{+}=\left(E_{\text {mot }, \text { mech }}\right)^{+}-\left(E_{t r}\right)^{+}$

Equation 68: Motor losses (Series, propel)

$\left(E_{\text {mot }, \text { loss }}\right)^{+}=\left(E_{\text {mot }, \text { elec }}\right)^{+}-\left(E_{\text {mot }, \text { mech }}\right)^{+}$

Equation 159: Battery charge losses (Series, propel)

$\left(E_{\text {batt }, \text { loss }}\right)^{+ \text {gen }}=\left(E_{\text {batt }, \text { int }}\right)^{+ \text {gen }}-\left(E_{\text {gen,store }}\right)^{+ \text {gen }}$

Equation 160: Battery discharge losses (Series, propel)

$\left(E_{\text {batt }, \text { loss }}\right)^{+ \text {prop }}=\left(E_{\text {batt } \text { int }}\right)^{+ \text {prop }}-\left(E_{\text {batt }, \text { use }}\right)^{+ \text {prop }}$

Equation 161: Total battery losses (Series, propel)

$\left(E_{\text {batt }, \text { loss }}\right)^{+}=\left(E_{\text {batt }, \text { int }}\right)^{+}-\left(E_{\text {batt }, T}\right)^{+}$ 
Equation 162: Generator losses (Series, propel)

$\left(E_{\text {gen }, \text { loss }}\right)^{+}=\left(E_{\text {eng,mech }}\right)^{+}-\left(E_{\text {gen }, \text { elec }}\right)^{+}$

Equation 163: Engine losses (Series, propel)

$\left(E_{\text {eng }, \text { loss }}\right)^{+}=\left(E_{\text {eng,fuel }}\right)^{+}-\left(E_{\text {eng }, \text { mech }}\right)^{+}$

Equation 164: Propel powertrain losses (Series, propel)

$\left(E_{\text {pt,loss }}\right)^{+}=\left(E_{\text {eng,fuel }}\right)^{+}-\left(E_{\text {Acc }}\right)^{+}-\left(E_{\text {tr }}\right)^{+}$

Restated:

$\left(E_{\text {pt }, \text { loss }}\right)^{+}=\left(E_{\text {eng,loss }}\right)^{+}+\left(E_{\text {gen }, \text { loss }}\right)^{+}+\left(E_{\text {batt }, \text { loss }}\right)^{+}+\left(E_{\text {mot }, \text { loss }}\right)^{+}+\left(E_{\text {dl, loss }}\right)^{+}$

\section{Conservation of Energy Expressions:}

Equation 56: Conservation of energy at the HV bus (Series, propel)

$\left(E_{\text {gen }, \text { use }}\right)^{+}+\left(E_{\text {batt }, \text { use }}\right)^{+ \text {prop }}=\left(E_{\text {mot }, \text { elec }}\right)^{+}+\left(E_{\text {Acc }}\right)^{+}$

Equation 165: Conservation of energy for the Power Split Fraction (Series, propel)

$\left(E_{\text {gen,elec }}\right)^{+}=\left(E_{\text {gen }, \text { use }}\right)^{+}-\left(E_{\text {gen }, \text { store }}\right)^{+ \text {gen }}$

Equation 53: Conservation of energy in the battery to maintain charge balance (Series, propel)

$\left(E_{\text {batt }, \text { int }}\right)^{+ \text {prop }}=-\left(E_{\text {batt }, \text { int }}\right)^{+ \text {gen }}$

\section{Accessory Energy:}

Equation 25: Propel case accessory energy

$\left(E_{A c c}\right)^{+}=\tau\left(P_{A c c}\right)^{+}$

\section{Developing Equation for Power Split Fraction (in terms of efficiencies):}

- Storing Energy in the Battery During the Propel Case:

$\left(E_{\text {batt }, \text { int }}\right)^{+ \text {gen }}=\left(E_{\text {gen }, \text { store }}\right)^{+ \text {gen }}\left(\tilde{\eta}_{\text {batt }}^{+ \text {gen }}\right)$

Equation 166: Energy stored in the battery during times of surplus power generation (Series, propel)

$\left(E_{\text {batt }, \text { int }}\right)^{+ \text {gen }}=(-1)(1-\tilde{\xi})\left(E_{\text {gen,elec }}\right)^{+}\left(\tilde{\eta}_{\text {batt }}^{+ \text {gen }}\right)$ 


\section{- Using Energy from the Battery During the Propel Case:}

Equation 167: Energy used from the battery during shortages of genset power generation (Series, propel)

$$
\left(E_{\text {batt }, \text { int }}\right)^{+ \text {prop }}=\frac{\left(E_{\text {batt }, \text { use }}\right)^{+ \text {prop }}}{\tilde{\eta}_{\text {batt }}^{+ \text {prop }}}
$$

\section{- Conservation of Energy to Maintain Charge Balance:}

Equation 53: Conservation of energy in the battery to maintain charge balance (Series, propel)

$$
\begin{aligned}
& \left(E_{\text {batt }, \text { int }}\right)^{+ \text {prop }}=-\left(E_{\text {batt }, \text { int }}\right)^{+ \text {gen }} \\
& \frac{\left(E_{\text {batt }, \text { use }}\right)^{+ \text {prop }}}{\tilde{\eta}_{\text {batt }}^{+ \text {prop }}}=(1-\tilde{\xi})\left(E_{\text {gen,elec }}\right)^{+}\left(\tilde{\eta}_{\text {batt }}^{+ \text {gen }}\right)
\end{aligned}
$$

Equation 57: Energy from the battery into the HV bus during the propel case (Series, propel)

$$
\left(E_{\text {batt }, \text { use }}\right)^{+ \text {prop }}=(1-\tilde{\xi})\left(E_{\text {gen }, \text { elec }}\right)^{+}\left(\tilde{\eta}_{\text {batt }}^{+ \text {gen }}\right)\left(\tilde{\eta}_{\text {batt }}^{+ \text {prop }}\right)
$$

\section{- Conservation of Energy on the HV Bus:}

Equation 56: Conservation of energy at the HV bus (Series, propel)

$$
\begin{aligned}
& \left(E_{\text {gen }, \text { use }}\right)^{+}+\left(E_{\text {batt }, \text { use }}\right)^{+ \text {prop }}=\left(E_{\text {mot }, \text { elec }}\right)^{+}+\left(E_{\text {Acc }}\right)^{+} \\
& \left(E_{\text {gen }, \text { use }}\right)^{+}=\left(E_{\text {mot }, \text { elec }}\right)^{+}+\left(E_{\text {Acc }}\right)^{+}-\left(E_{\text {batt }, \text { use }}\right)^{+ \text {prop }} \\
& \left(E_{\text {gen }, \text { use }}\right)^{+}=\left(E_{\text {mot }, \text { elec }}\right)^{+}+\left(E_{\text {Acc }}\right)^{+}-(1-\tilde{\xi})\left(E_{\text {gen }, \text { elec }}\right)^{+}\left(\tilde{\eta}_{\text {batt }}^{+ \text {gen }}\right)\left(\tilde{\eta}_{\text {batt }}^{+ \text {prop }}\right) \\
& \xi\left(E_{\text {gen }, \text { elec }}\right)^{+}=\left(E_{\text {mot }, \text { elec }}\right)^{+}+\left(E_{\text {Acc }}\right)^{+}-(1-\tilde{\xi})\left(E_{\text {gen }, \text { elec }}\right)^{+}\left(\tilde{\eta}_{\text {batt }}^{+ \text {gen }}\right)\left(\tilde{\eta}_{\text {batt }}^{+ \text {prop }}\right) \\
& \xi\left(E_{\text {gen,elec }}\right)^{+}+(1-\tilde{\xi})\left(E_{\text {gen }, \text { elec }}\right)^{+}\left(\tilde{\eta}_{\text {batt }}^{+ \text {gen }}\right)\left(\tilde{\eta}_{\text {batt }}^{+ \text {prop }}\right)=\left(E_{\text {mot,elec }}\right)^{+}+\left(E_{\text {Acc }}\right)^{+} \\
& \left(E_{\text {gen }, \text { elec }}\right)^{+}\left[\tilde{\xi}+(1-\tilde{\xi})\left(\tilde{\eta}_{\text {batt }}^{+ \text {gen }}\right)\left(\tilde{\eta}_{\text {batt }}^{+ \text {prop }}\right)\right]=\left(E_{\text {mot }, \text { elec }}\right)^{+}+\left(E_{\text {Acc }}\right)^{+}
\end{aligned}
$$

Equation 58: Energy supplied by the generator (Series, propel) 


$$
\left(E_{\text {gen }, \text { elec }}\right)^{+}=\frac{\left(E_{\text {mot }, \text { elec }}\right)^{+}+\left(E_{\text {Acc }}\right)^{+}}{\tilde{\xi}+(1-\tilde{\xi})\left(\tilde{\eta}_{\text {batt }}^{+ \text {gen }}\right)\left(\tilde{\eta}_{\text {batt }}^{+ \text {prop }}\right)}
$$

\section{Developing Equation for Power Split Fraction (in terms of losses):}

Equation 165: Conservation of energy for the Power Split Fraction (Series, propel)

$$
\begin{aligned}
& \left(E_{\text {gen }, \text { use }}\right)^{+}+\left(E_{\text {batt }, \text { use }}\right)^{+ \text {prop }}=\left(E_{\text {mot }, \text { elec }}\right)^{+}+\left(E_{\text {Acc }}\right)^{+} \\
& \left(E_{\text {gen }, u s e}\right)^{+}=\left(E_{\text {mot }, e l e c}\right)^{+}+\left(E_{\text {Acc }}\right)^{+}-\left(E_{\text {batt }, \text { use }}\right)^{+ \text {prop }} \\
& \left(E_{\text {gen,use }}\right)^{+}=\left(E_{\text {mot }, \text { elec }}\right)^{+}+\left(E_{\text {Acc }}\right)^{+}-\left(E_{\text {batt }, \text { int }}\right)^{+}+\left(E_{\text {batt }, \text { loss }}\right)^{+ \text {prop }} \\
& \left(E_{\text {gen }, \text { use }}\right)^{+}=\left(E_{\text {mot,elec }}\right)^{+}+\left(E_{\text {Acc }}\right)^{+}-\left(E_{\text {gen,store }}\right)^{+ \text {gen }}+\left(E_{\text {batt }, \text { loss }}\right)^{+ \text {prop }}+\left(E_{\text {batt }, \text { loss }}\right)^{+ \text {gen }} \\
& \left(E_{\text {gen }, \text { use }}\right)^{+}=\left(E_{\text {mot }, \text { elec }}\right)^{+}+\left(E_{\text {Acc }}\right)^{+}-(1-\xi)\left(E_{\text {gen }, \text { elec }}\right)^{+}+\left(E_{\text {batt }, \text { loss }}\right)^{+} \\
& \tilde{\xi}\left(E_{\text {gen }, \text { elec }}\right)^{+}=\left(E_{\text {mot }, \text { elec }}\right)^{+}+\left(E_{\text {Acc }}\right)^{+}-(1-\xi)\left(E_{\text {gen,elec }}\right)^{+}+\left(E_{\text {batt }, \text { loss }}\right)^{+} \\
& \tilde{\xi}\left(E_{\text {gen }, \text { elec }}\right)^{+}+(1-\tilde{\xi})\left(E_{\text {gen }, \text { elec }}\right)^{+}=\left(E_{\text {mot }, \text { elec }}\right)^{+}+\left(E_{\text {Acc }}\right)^{+}+\left(E_{\text {batt }, \text { loss }}\right)^{+} \\
& \left(E_{\text {gen,elec }}\right)^{+}[\tilde{\xi}+(1-\tilde{\xi})]=\left(E_{\text {mot }, \text { elec }}\right)^{+}+\left(E_{\text {Acc }}\right)^{+}+\left(E_{\text {batt }, \text { loss }}\right)^{+} \\
& \left(E_{\text {gen }, \text { elec }}\right)^{+}=\frac{\left(E_{\text {mot }, \text { elec }}\right)^{+}+\left(E_{\text {Acc }}\right)^{+}+\left(E_{\text {batt }, \text { loss }}\right)^{+}}{[\tilde{\xi}+(1-\tilde{\xi})]}
\end{aligned}
$$

Equation 168: Energy supplied by the generator in terms of losses (Series, propel)

$$
\left(E_{\text {gen }, \text { elec }}\right)^{+}=\left(E_{\text {mot }, \text { elec }}\right)^{+}+\left(E_{\text {Acc }}\right)^{+}+\left(E_{\text {batt }, \text { loss }}\right)^{+}
$$




\section{Developing Equation for Propel Case Energy Consumption (in terms of efficiencies):}

$$
\begin{aligned}
& \left(E_{\text {eng,fuel }}\right)^{+}=\frac{\left(E_{\text {eng,mech }}\right)^{+}}{\tilde{\eta}_{\text {eng }}^{+}} \\
& \left(E_{\text {eng,fuel }}\right)^{+}=\frac{\left(E_{\text {gen }, \text { elec }}\right)^{+}}{\left(\tilde{\eta}_{\text {eng }}^{+}\right)\left(\tilde{\eta}_{\text {gen }}^{+}\right)} \\
& \left(E_{\text {eng,fuel }}\right)^{+}=\frac{1}{\left(\tilde{\eta}_{\text {eng }}^{+}\right)\left(\tilde{\eta}_{\text {gen }}^{+}\right)}\left[\frac{\left(E_{\text {mot,elec }}\right)^{+}+\left(E_{\text {Acc }}\right)^{+}}{\tilde{\xi}+(1-\tilde{\xi})\left(\tilde{\eta}_{\text {batt }}^{+ \text {gen }}\right)\left(\tilde{\eta}_{\text {batt }}^{+ \text {prop }}\right)}\right] \\
& \left(E_{\text {eng,fuel }}\right)^{+}=\frac{1}{\left(\tilde{\eta}_{\text {eng }}^{+}\right)\left(\tilde{\eta}_{\text {gen }}^{+}\right)\left(\tilde{\xi}+(1-\tilde{\xi})\left(\tilde{\eta}_{\text {batt }}^{+ \text {gen }}\right)\left(\tilde{\eta}_{\text {batt }}^{+ \text {prop }}\right)\right)}\left[\left(E_{\text {mot,elec }}\right)^{+}+\left(E_{\text {Acc }}\right)^{+}\right] \\
& \left(E_{\text {eng,fuel }}\right)^{+}=\frac{1}{\left(\tilde{\eta}_{\text {eng }}^{+}\right)\left(\tilde{\eta}_{\text {gen }}^{+}\right)\left(\tilde{\xi}+(1-\tilde{\xi})\left(\tilde{\eta}_{\text {batt }}^{+ \text {gen }}\right)\left(\tilde{\eta}_{\text {batt }}^{+ \text {prop }}\right)\right)}\left[\frac{\left(E_{\text {mot,tr }}\right)^{+}}{\tilde{\eta}_{\text {mot }}^{+}}+\left(E_{\text {Acc }}\right)^{+}\right]
\end{aligned}
$$

Equation 59: Propel case fuel energy consumption (Series, propel)

$$
\left(E_{\text {eng,fuel }}\right)^{+}=\frac{1}{\left(\tilde{\eta}_{\text {eng }}^{+}\right)\left(\tilde{\eta}_{\text {gen }}^{+}\right)\left(\tilde{\xi}+(1-\tilde{\xi})\left(\tilde{\eta}_{\text {batt }}^{+ \text {gen }}\right)\left(\tilde{\eta}_{\text {batt }}^{+ \text {prop }}\right)\right)}\left[\frac{\left(E_{\text {tr }}\right)^{+}}{\left(\tilde{\eta}_{\text {mot }}^{+}\right)\left(\tilde{\eta}_{\text {dl }}^{+}\right)}+\left(E_{\text {Acc }}\right)^{+}\right]
$$

\section{Developing Equation for Propel Case Energy Consumption (in terms of losses):}

$$
\begin{aligned}
\left(E_{\text {eng }, \text { fuel }}\right)^{+}= & \left(E_{\text {eng }, \text { mech }}\right)^{+}+\left(E_{\text {eng }, \text { loss }}\right)^{+} \\
\left(E_{\text {eng }, \text { fuel }}\right)^{+}= & \left(E_{\text {gen }, \text { elec }}\right)^{+}+\left(E_{\text {eng,loss }}\right)^{+}+\left(E_{\text {gen }, \text { loss }}\right)^{+} \\
\left(E_{\text {eng,fuel }}\right)^{+}= & \left(E_{\text {mot }, \text { elec }}\right)^{+}+\left(E_{\text {Acc }}\right)^{+}+\left(E_{\text {eng,loss }}\right)^{+}+\left(E_{\text {gen }, \text { loss }}\right)^{+}+\left(E_{\text {batt }, \text { loss }}\right)^{+} \\
\left(E_{\text {eng,fuel }}\right)^{+} & =\left(E_{\text {mot }, \text { mech }}\right)^{+}+\left(E_{\text {Acc }}\right)^{+}+\left(E_{\text {eng }, \text { loss }}\right)^{+}+\left(E_{\text {gen }, \text { loss }}\right)^{+}+\left(E_{\text {batt }, \text { loss }}\right)^{+} \\
& +\left(E_{\text {mot }, \text { loss }}\right)^{+}
\end{aligned}
$$

Equation 169: Propel case fuel energy consumption in terms of losses (Series, propel)

$$
\begin{aligned}
\left(E_{\text {eng,fuel }}\right)^{+}= & \left(E_{\text {tr }}\right)^{+}+\left(E_{\text {Acc }}\right)^{+}+\left(E_{\text {eng }, \text { loss }}\right)^{+}+\left(E_{\text {gen }, \text { loss }}\right)^{+}+\left(E_{\text {batt }, \text { loss }}\right)^{+}+\left(E_{\text {mot }, \text { loss }}\right)^{+} \\
& +\left(E_{\text {dl, loss }}\right)^{+}
\end{aligned}
$$




\section{APPENDIX G: Equations for the Brake Case (Series HEV)}

This appendix contains the definitions for the component efficiencies used in the energy consumption equations developed for VTool. The Brake case for the Series HEV is identical to an EV, so only select equations are replicated here. For full documentation of the brake case, see Appendix B.

\begin{tabular}{|l|l|l|}
\hline Term & $\begin{array}{l}\text { Inherent } \\
\text { Sign }\end{array}$ & Definition \\
\hline$\left(E_{\mathrm{tr}}\right)^{-}$ & - & Tractive energy at the wheels for the brake case \\
\hline$\left(\mathrm{E}_{\mathrm{tr}, \zeta}\right)^{-}$ & - & Tractive energy in to driveline after effect of regen fraction \\
\hline$\left(\mathrm{E}_{\text {mot,mech }}\right)^{-}$ & - & Mechanical energy in to the motor for the brake case \\
\hline$\left(\mathrm{E}_{\mathrm{mot}, \mathrm{elec}}\right)^{-}$ & - & Electrical energy out of the motor for the brake case \\
\hline$\left(\mathrm{E}_{\mathrm{acc}}\right)^{-}$ & + & Electrical energy siphoned off the HV bus for accessory loads during brake case \\
\hline$\left(\mathrm{E}_{\mathrm{batt}, \mathrm{T}}\right)^{-}$ & - & Electrical energy in to the battery or at the terminals during the brake case \\
\hline$\left(\mathrm{E}_{\mathrm{batt}, \mathrm{int}}\right)^{-}$ & - & Electrical energy inside the battery captured during the brake case \\
\hline
\end{tabular}

\section{Accessory Energy:}

Equation 31: Brake case accessory energy

$\left(E_{\text {Acc }}\right)^{-}=\left(T-\tau-t_{\text {idle }}\right)\left(P_{\text {Acc }}\right)^{-}$

Equation for Brake Case Energy Consumption (in terms of efficiencies):

Equation 32: Brake case energy consumption (EV, brake)

$\left(E_{\text {batt }, i n t}\right)^{-}=\tilde{\eta}_{\text {batt }}^{-}\left\{\left(E_{\text {tr }}\right)^{-}(\tilde{\zeta})\left(\tilde{\eta}_{d l, m o t}^{-}\right)\left(\tilde{\eta}_{\text {mot }}^{-}\right)+\left(E_{\text {Acc }}\right)^{-}\right\}$

Developing Equation for Brake Case Energy Consumption (in terms of losses):

Equation 34: Brake case energy consumption in terms of losses (EV, brake)

$$
\left(E_{\text {batt }, \text { int }}\right)^{-}=\left(E_{t r}\right)^{-}+\left(E_{\text {Acc }}\right)^{-}+\left(E_{\text {batt }, \text { loss }}\right)^{-}+\left(E_{\text {mot }, \text { loss }}\right)^{-}+\left(E_{\text {dlmot }, l o s s}\right)^{-}+\left(E_{\text {brk,loss }}\right)^{-}
$$




\section{APPENDIX H: Equations for Regen Energy Reuse (Series HEV)}

This appendix contains the definitions for the component efficiencies used in the energy consumption equations developed for VTool. Some information may be duplicated in the body of this paper, but all relevant terms, definitions and equations have been reproduced here for convenience.

\begin{tabular}{|l|l|l|}
\hline Term & $\begin{array}{l}\text { Inherent } \\
\text { Sign }\end{array}$ & Definition \\
\hline$\left(E_{\text {batt,int }}\right)^{-1+}$ & - & Electrical energy inside the battery captured during the brake case \\
\hline$\left(E_{\text {batt,int }}\right)^{-1+}$ & + & Net electrical energy inside the battery available to be reused for propulsion \\
\hline$\left(E_{\text {batt, } T}\right)^{-/+}$ & + & Net electrical at the battery terminals available to be reused for propulsion \\
\hline$\left(E_{\text {mot,elec }}\right)^{-1+}$ & + & Electrical energy in to the motor to be reused for propulsion \\
\hline$\left(E_{\text {mot,mech }}\right)^{-1+}$ & + & Mechanical energy out of the motor to be reused for propulsion \\
\hline$\left(E_{t r, \text { credit }}\right)^{-/+}$ & + & Tractive energy at the wheels from stored regen energy reused for propulsion \\
\hline
\end{tabular}

\section{Efficiency Expressions:}

Equation 170: Motor driveline efficiency (Series, regen reuse)

$\tilde{\eta}_{d l, m o t}^{-/+}=\frac{\left(E_{t r, \text { credit }}\right)^{-/+}}{\left(E_{\text {mot }, \text { mech }}\right)^{-/+}} \approx \tilde{\eta}_{d l, \text { mot }}^{+}$

Equation 62: Motor propel efficiency (Series, regen reuse)

$\tilde{\eta}_{\text {mot }}^{-/+}=\frac{\left(E_{\text {mot }, \text { mech }}\right)^{-/+}}{\left(E_{\text {mot }, \text { elec }}\right)^{-/+}} \approx \tilde{\eta}_{\text {mot }}^{+}$

Equation 171: Battery discharge efficiency (Series, regen reuse)

$\tilde{\eta}_{\text {batt }}^{-/+}=\frac{\left(E_{\text {batt }, T}\right)^{-/+}}{\left(E_{\text {batt }, \text { int }}\right)^{-/+}} \approx \tilde{\eta}_{\text {batt }}^{+}$

Equation 65: Powertrain efficiency for regen energy reuse (Series, regen reuse)

$\tilde{\eta}_{p t}^{-/+}=\frac{\left(E_{\text {tr,credit }}\right)^{-/+}}{\left(E_{\text {batt }, \text { int }}\right)^{-/+}}$

\section{Loss Expressions:}

Equation 172: Motor driveline losses (Series, regen reuse)

$\left(E_{\text {dlmot }, \text { loss }}\right)^{-/+}=\left(E_{\text {mot }, \text { mech }}\right)^{-/+}-\left(E_{\text {tr,credit }}\right)^{-/+}$

Equation 173: Motor losses (Series, regen reuse)

$\left(E_{\text {mot }, \text { loss }}\right)^{-/+}=\left(E_{\text {mot }, e l e c}\right)^{-/+}-\left(E_{\text {mot }, \text { mech }}\right)^{-/+}$

Equation 174: Battery losses (Series, regen reuse)

$\left(E_{\text {batt }, \text { loss }}\right)^{-/+}=\left(E_{\text {batt }, \text { int }}\right)^{-/+}-\left(E_{\text {batt }, T}\right)^{-/+}$ 
Equation 175: Propel powertrain losses (Series, regen reuse)

$\left(E_{p t, l o s s}\right)^{-/+}=\left(E_{\text {batt }, \text { int }}\right)^{-/+}-\left(E_{\text {tr,credit }}\right)^{-/+}$

Restated:

$\left(E_{\text {pt,loss }}\right)^{-/+}=\left(E_{\text {batt }, \text { loss }}\right)^{-/+}+\left(E_{\text {mot }, \text { loss }}\right)^{-/+}+\left(E_{\text {dlmot }, \text { loss }}\right)^{-/+}$

\section{Conservation of Energy Expressions:}

Equation 176: Conservation of energy at the HV bus (Series, regen reuse)

$\left(E_{\text {bat }, T}\right)^{-/+}=\left(E_{\text {mot }, e l e c}\right)^{-/+}$

Equation 61: Conservation of energy for regen energy capture and reuse (Series, regen reuse)

$\left(E_{\text {batt }, \text { int }}\right)^{-}=-\left(E_{\text {batt }, \text { int }}\right)^{-/+}$

\section{Developing Equation for Regen Energy Tractive Energy Credit (in terms of efficiencies):}

$\left(E_{\text {tr,credit }}\right)^{-/+}=\left(E_{\text {mot }, \text { mech }}\right)^{-/+}\left(\tilde{\eta}_{d l, \text { mot }}^{-/+}\right)$

$\left(E_{\text {tr,credit }}\right)^{-/+}=\left(E_{\text {mot,elec }}\right)^{-/+}\left(\tilde{\eta}_{\text {mot }}^{-/+}\right)\left(\tilde{\eta}_{\text {dl,mot }}^{-/+}\right)$

$\left(E_{\text {tr,credit }}\right)^{-/+}=\left(E_{\text {batt }, T}\right)^{-/+}\left(\tilde{\eta}_{\text {mot }}^{-/+}\right)\left(\tilde{\eta}_{d l, \text { mot }}^{-/+}\right)$

$\left(E_{\text {tr,credit }}\right)^{-/+}=\left(E_{\text {batt }, \text { int }}\right)^{-/+}\left(\tilde{\eta}_{\text {batt }}^{-/+}\right)\left(\tilde{\eta}_{\text {mot }}^{-/+}\right)\left(\tilde{\eta}_{\text {dl,mot }}^{-/+}\right)$

Equation 63: Equivalent tractive energy 'credit' from regen braking (Series, regen reuse)

$\left(E_{\text {tr,credit }}\right)^{-/+}=-\left(E_{\text {batt }, \text { int }}\right)^{-}\left(\tilde{\eta}_{\text {batt }}^{-/+}\right)\left(\tilde{\eta}_{\text {mot }}^{-/+}\right)\left(\tilde{\eta}_{\text {dl,mot }}^{-/+}\right)$

\section{Developing Equation for Regen Energy Tractive Energy Credit (in terms of losses):}

$\left(E_{\text {tr,credit }}\right)^{-/+}=\left(E_{\text {mot }, \text { mech }}\right)^{-/+}-\left(E_{\text {dlmot }, \text { loss }}\right)^{-/+}$

$\left(E_{\text {tr,credit }}\right)^{-/+}=\left(E_{\text {mot,elec }}\right)^{-/+}-\left(E_{\text {mot }, \text { loss }}\right)^{-/+}-\left(E_{\text {dlmot }, \text { loss }}\right)^{-/+}$

$\left(E_{\text {tr }, \text { credit }}\right)^{-/+}=\left(E_{\text {batt }, T}\right)^{-/+}-\left(E_{\text {mot }, \text { loss }}\right)^{-/+}-\left(E_{\text {dlmot }, \text { loss }}\right)^{-/+}$

$\left(E_{\text {tr,credit }}\right)^{-/+}=\left(E_{\text {batt }, \text { int }}\right)^{-/+}-\left(E_{\text {batt }, \text { loss }}\right)^{-/+}-\left(E_{\text {mot }, \text { loss }}\right)^{-/+}-\left(E_{\text {dlmot }, \text { loss }}\right)^{-/+}$

Equation 64: Equivalent tractive energy 'credit' from regen in terms of losses (Series, regen reuse) $\left(E_{\text {tr, credit }}\right)^{-/+}=\left(E_{\text {batt }, \text { int }}\right)^{-/+}-\left(E_{\text {pt,loss }}\right)^{-/+}=-\left(E_{\text {batt }, \text { int }}\right)^{-}-\left(E_{p t, l o s s}\right)^{-/+}$ 


\section{APPENDIX I: Equations for Fuel-Equivalent Regen Energy Credit (Series HEV)}

This appendix contains the definitions for the component efficiencies used in the energy consumption equations developed for VTool. Some information may be duplicated in the body of this paper, but all relevant terms, definitions and equations have been reproduced here for convenience.

\begin{tabular}{|c|c|c|}
\hline Term & $\begin{array}{l}\text { Inherent } \\
\text { Sign }\end{array}$ & Definition \\
\hline$\left(E_{\text {eng,fuel }}\right)^{\mathrm{EqFC}}$ & - & Theoretical fuel energy out of the engine to be used as fuel credit \\
\hline$\left(E_{\text {eng,mech }}\right)^{\mathrm{EqFC}}$ & - & Theoretical Mechanical energy in to the engine to be used as fuel credit \\
\hline$\left(E_{\text {gen,elec }}\right)^{\text {EqFC }}$ & - & Theoretical electrical energy in to the generator to be used as fuel credit \\
\hline$\left(\mathrm{E}_{\text {gen,store }}\right)^{\mathrm{EqFC}}$ & + & Theoretical electrical energy in to HV bus. Analog to $\left(\mathrm{E}_{g e n, \text { store }}\right)^{+\mathrm{gen}}$ \\
\hline$\left(\mathrm{E}_{\text {batt, int }}\right)^{-\mathrm{Eq} F \mathrm{C}}$ & + & Theoretical electrical energy inside the battery. Analog to $\left(E_{\text {batt,int }}\right)^{+g e n}$ \\
\hline$\left(E_{\text {batt,int }}\right)^{+E q F C}$ & - & Theoretical electrical energy inside the battery. Analog to $\left(E_{\text {batt,int }}\right)^{+p r o p}$ \\
\hline$\left(\mathrm{E}_{\text {batt use }}\right)^{\mathrm{EFCC}}$ & - & Theoretical electrical energy in to the battery. Analog to ( $\left.\mathrm{E}_{\text {batt use }}\right)^{+ \text {prop }}$ \\
\hline$\left(E_{\text {mot,elec }}\right)^{\mathrm{EqFC}}$ & - & Theoretical electrical energy out of the motor to be used as fuel credit \\
\hline$\left(\mathrm{E}_{\mathrm{mot}, \mathrm{mech}}\right)^{\mathrm{EqFC}}$ & - & Theoretical mechanical energy in to the motor to be used as fuel credit \\
\hline$\left(E_{t r, \text { credit }}\right)^{\text {EqFC }}$ & - & Theoretical tractive energy in to the wheels to be used as fuel credit \\
\hline$\left(\mathrm{E}_{\mathrm{tr}, \text { credit }}\right)^{-1+}$ & + & Tractive energy at the wheels from stored regen energy reused for propulsion \\
\hline
\end{tabular}

Efficiency Expressions: (same as propel case because theoretical powerflow emulates propel)

Equation 54: Power Split Fraction, genset energy directly used (Series, propel)

$\tilde{\xi}=\frac{\left(E_{\text {gen }, \text { use }}\right)^{+}}{\left(E_{\text {gen }, \text { elec }}\right)^{+}}$

Restated:

$\left(E_{\text {gen }, \text { use }}\right)^{+}=\tilde{\xi}\left(E_{\text {gen }, \text { elec }}\right)^{+}$

Equation 55: Power Split Fraction, genset energy recirculated (Series, propel)

$\left(E_{\text {gen }, \text { store }}\right)^{+ \text {gen }}=(-1)(1-\tilde{\xi})\left(E_{\text {gen }, \text { elec }}\right)^{+}$

Equation 152: Motor driveline propel efficiency (Series, propel)

$\tilde{\eta}_{d l, m o t}^{+}=\frac{\left(E_{t r}\right)^{+}}{\left(E_{\text {mot }, \text { mech }}\right)^{+}}$

Equation 153: Motor propel efficiency (Series, propel)

$\tilde{\eta}_{\text {mot }}^{+}=\frac{\left(E_{\text {mot }, \text { mech }}\right)^{+}}{\left(E_{\text {mot }, \text { elec }}\right)^{+}}$ 
Equation 154: Battery charge efficiency (Series, propel)

$\tilde{\eta}_{\text {batt }}^{+ \text {gen }}=\frac{\left(E_{\text {batt }, \text { int }}\right)^{+ \text {gen }}}{\left(E_{\text {gen,store }}\right)^{+g e n}} \approx \tilde{\eta}_{\text {batt }}^{-}$

Equation 155: Battery discharge efficiency (Series, propel)

$\tilde{\eta}_{\text {batt }}^{+ \text {prop }}=\frac{\left(E_{\text {batt }, \text { use }}\right)^{+ \text {prop }}}{\left(E_{\text {batt }, \text { int }}\right)^{+ \text {prop }}} \approx \tilde{\eta}_{\text {batt }}^{+}$

Equation 156: Generator propel efficiency (Series, propel)

$\tilde{\eta}_{\text {gen }}^{+}=\frac{\left(E_{\text {gen, }, \text { elec }}\right)^{+}}{\left(E_{\text {eng,mech }}\right)^{+}}$

Equation 157: Engine propel efficiency (Series, propel)

$\tilde{\eta}_{\text {eng }}^{+}=\frac{\left(E_{\text {eng,mech }}\right)^{+}}{\left(E_{\text {eng,fuel }}\right)^{+}}$

Equation 60: Propel case powertrain efficiency (Series, propel)

$\tilde{\eta}_{p t}^{+}=\frac{\left(E_{\text {tr }}\right)^{+}}{\left(E_{\text {eng,fuel }}\right)^{+}}$

\section{Loss Expressions:}

Equation 177: Motor driveline losses (Series, regen fuel credit)

$\left(E_{\text {dl,loss }}\right)^{E q F C}=\left(E_{\text {mot }, \text { mech }}\right)^{E q F C}-\left(E_{\text {tr, credit }}\right)^{E q F C}$

Equation 69: Motor losses (Series, regen fuel credit)

$\left(E_{\text {mot }, \text { loss }}\right)^{E q F C}=\left(E_{\text {mot,elec }}\right)^{E q F C}-\left(E_{\text {mot }, \text { mech }}\right)^{E q F C}$

Equation 178: Total battery losses (Series, regen fuel credit)

$\left(E_{\text {batt }, \text { loss }}\right)^{E q F C}=-\left(E_{\text {gen,store }}\right)^{E q F C}-\left(E_{\text {batt }, \text { use }}\right)^{E q F C}$

Equation 179: Generator losses (Series, regen fuel credit)

$\left(E_{\text {gen,loss }}\right)^{E q F C}=\left(E_{\text {eng,mech }}\right)^{E q F C}-\left(E_{\text {gen,elec }}\right)^{E q F C}$

Equation 180: Engine losses (Series, regen fuel credit)

$\left(E_{\text {eng,loss }}\right)^{E q F C}=\left(E_{\text {eng,fuel }}\right)^{E q F C}-\left(E_{\text {eng,mech }}\right)^{E q F C}$ 
Equation 181: Propel powertrain losses (Series, regen fuel credit)

$$
\begin{aligned}
& \left(E_{\text {pt,loss }}\right)^{E q F C}=\left(E_{\text {eng,fuel }}\right)^{E q F C}-\left(E_{\text {tr,credit }}\right)^{E q F C} \\
& \text { Restated: } \\
& \left(E_{\text {pt,loss }}\right)^{E q F C}=\left(E_{\text {eng }, \text { loss }}\right)^{E q F C}+\left(E_{\text {gen }, \text { loss }}\right)^{E q F C}+\left(E_{\text {batt }, \text { loss }}\right)^{E q F C}+\left(E_{\text {mot }, \text { loss }}\right)^{E q F C}+\left(E_{\text {dl,loss }}\right)^{E q F C}
\end{aligned}
$$

\section{Conservation of Energy Expressions:}

Equation 66: Conservation of energy for regen fuel credit (Series, regen fuel credit)

$\left(E_{\text {tr,credit }}\right)^{E q F C}=-\left(E_{\text {tr,credit }}\right)^{-/+}$

\section{Developing Equation for Fuel-Equivalent Regen Energy Credit (in terms of efficiencies):}

**Begin from Propel Case for CS Series HEV

Equation 59: Propel case fuel energy consumption (Series, propel)

$$
\begin{aligned}
& \left(E_{\text {eng,fuel }}\right)^{+}=\frac{1}{\left(\tilde{\eta}_{\text {eng }}^{+}\right)\left(\tilde{\eta}_{\text {gen }}^{+}\right)\left(\tilde{\xi}+(1-\tilde{\xi})\left(\tilde{\eta}_{\text {batt }}^{+ \text {gen }}\right)\left(\tilde{\eta}_{\text {batt }}^{+ \text {prop }}\right)\right)}\left[\frac{\left(E_{\text {tr }}\right)^{+}}{\left(\tilde{\eta}_{\text {mot }}^{+}\right)\left(\tilde{\eta}_{\text {dl }}^{+}\right)}+\left(E_{\text {Acc }}\right)^{+}\right] \\
& \left(E_{\text {eng,fuel }}\right)^{E q F C}=\frac{1}{\left(\tilde{\eta}_{\text {eng }}^{+}\right)\left(\tilde{\eta}_{\text {gen }}^{+}\right)\left(\xi+(1-\xi)\left(\tilde{\eta}_{\text {batt }}^{+ \text {gen }}\right)\left(\tilde{\eta}_{\text {batt }}^{+ \text {prop }}\right)\right)}\left[\frac{\left(E_{\text {tr,credit }}\right)^{E q F C}}{\left(\tilde{\eta}_{\text {mot }}^{+}\right)\left(\tilde{\eta}_{\text {dl }}^{+}\right)}+\left(F^{+}\right]\right.
\end{aligned}
$$

Equation 67: Fuel-equivalent regen energy 'credit' (Series, regen fuel credit)

$$
\left(E_{\text {eng,fuel }}\right)^{E q F C}=\frac{1}{\left(\tilde{\eta}_{\text {eng }}^{+}\right)\left(\tilde{\eta}_{\text {gen }}^{+}\right)\left(\tilde{\xi}+(1-\tilde{\xi})\left(\tilde{\eta}_{\text {batt }}^{+ \text {gen }}\right)\left(\tilde{\eta}_{\text {batt }}^{+ \text {prop }}\right)\right)}\left[\frac{-\left(E_{\text {tr,credit }}\right)^{-/+}}{\left(\tilde{\eta}_{\text {mot }}^{+}\right)\left(\tilde{\eta}_{\text {dl }}^{+}\right)}\right]
$$

\section{Developing Equation for Fuel-Equivalent Regen Energy Credit (in terms of losses):}

\section{**Begin from Propel Case for CS Series HEV}

Equation 169: Propel case fuel energy consumption in terms of losses (Series, propel)

$$
\begin{aligned}
\left(E_{\text {eng }, \text { fuel }}\right)^{+}= & \left(E_{\text {tr }}\right)^{+}+\left(E_{\text {Acc }}\right)^{+}+\left(E_{\text {eng }, \text { loss }}\right)^{+}+\left(E_{\text {gen }, \text { loss }}\right)^{+}+\left(E_{\text {batt }, \text { loss }}\right)^{+}+\left(E_{\text {mot }, \text { loss }}\right)^{+} \\
& +\left(E_{\text {dl }, \text { loss }}\right)^{+}
\end{aligned}
$$

Equation 182: Fuel-equivalent regen energy 'credit' (P3 Parallel, regen fuel credit)

$$
\begin{aligned}
\left(E_{\text {eng,fuel }}\right)^{E q F C} & =-\left(E_{\text {tr,credit }}\right)^{E q F C}+\left(E_{\text {eng,loss }}\right)^{E q F C}+\left(E_{\text {gen }, \text { loss }}\right)^{E q F C}+\left(E_{\text {batt }, \text { loss }}\right)^{E q F C} \\
& +\left(E_{\text {mot }, \text { loss }}\right)^{E q F C}+\left(E_{\text {dl, loss }}\right)^{E q F C}
\end{aligned}
$$




\section{APPENDIX J: Equations for the Idle Case (Series HEV)}

This appendix contains the definitions for the component efficiencies used in the energy consumption equations developed for VTool. Some information may be duplicated in the body of this paper, but all relevant terms, definitions and equations have been reproduced here for convenience.

\begin{tabular}{|l|l|l|}
\hline Term & $\begin{array}{l}\text { Inherent } \\
\text { Sign }\end{array}$ & Definition \\
\hline$\left(\mathrm{E}_{\text {eng,fuel }}\right)^{0}$ & + & Fuel energy in to the engine during the idle case \\
\hline$\left(\mathrm{E}_{\text {eng,mech }}\right)^{0}$ & + & Mechanical energy out of the engine during the idle case \\
\hline$\left(\mathrm{E}_{\text {gen,elec }}\right)^{0}$ & + & Electrical energy out of the generator during the idle case \\
\hline$\left(\mathrm{E}_{\text {acc }}\right)^{0}$ & + & Electrical energy siphoned off the HV bus for accessory loads during idle case \\
\hline
\end{tabular}

Efficiency Expressions: (same as propel case because theoretical powerflow emulates propel)

Equation 183: Generator efficiency (Series, idle)

$\tilde{\eta}_{\text {gen }}^{0}=\frac{\left(E_{\text {gen }, \text { elec }}\right)^{0}}{\left(E_{\text {eng,mech }}\right)^{0}} \approx \tilde{\eta}_{\text {gen }}^{+}$

Equation 184: Engine efficiency (Series, idle)

$\tilde{\eta}_{\text {eng }}^{0}=\frac{\left(E_{\text {eng,mech }}\right)^{0}}{\left(E_{\text {eng,fuel }}\right)^{0}} \approx \tilde{\eta}_{\text {eng }}^{+}$

\section{Loss Expressions:}

Equation 185: Generator losses (Series, idle)

$\left(E_{\text {gen,loss }}\right)^{0}=\left(E_{\text {eng }, \text { mech }}\right)^{0}-\left(E_{\text {gen }, \text { elec }}\right)^{0}$

Equation 186: Engine losses (Series, idle)

$\left(E_{\text {eng,loss }}\right)^{0}=\left(E_{\text {eng,fuel }}\right)^{0}-\left(E_{\text {eng, mech }}\right)^{0}$

\section{Conservation of Energy Expressions:}

Equation 187: Conservation of energy at the HV Bus (EV, idle)

$\left(E_{\text {gen }, e l e c}\right)^{0}=\left(E_{A c c}\right)^{0}$ 


\section{Accessory Energy:}

Equation 36: Idle accessory energy

$\left(E_{\text {Acc }}\right)^{0}=\left(t_{\text {idle }}\right)\left(P_{\text {Acc }}\right)^{0}$

Developing Equation for Idle Case Energy Consumption (in terms of efficiencies):

$\left(E_{\text {eng,fuel }}\right)^{0}=\frac{\left(E_{\text {eng,mech }}\right)^{0}}{\tilde{\eta}_{\text {eng }}^{0}}$

Equation 70: Idle case energy consumption (Series, idle)

$\left(E_{\text {eng,fuel }}\right)^{0}=\frac{\left(E_{\text {gen }, \text { elec }}\right)^{0}}{\left(\tilde{\eta}_{\text {eng }}^{0}\right)\left(\tilde{\eta}_{\text {gen }}^{0}\right)}=\frac{\left(E_{\text {Acc }}\right)^{0}}{\left(\tilde{\eta}_{\text {eng }}^{0}\right)\left(\tilde{\eta}_{\text {gen }}^{0}\right)}$

\section{Developing Equation for Idle Case Energy Consumption (in terms of losses):}

$\left(E_{\text {eng,fuel }}\right)^{0}=\left(E_{\text {eng,mech }}\right)^{0}+\left(E_{\text {eng,loss }}\right)^{0}$

$\left(E_{\text {eng,fuel }}\right)^{0}=\left(E_{\text {gen }, \text { elec }}\right)^{0}+\left(E_{\text {gen }, \text { loss }}\right)^{0}+\left(E_{\text {eng }, \text { loss }}\right)^{0}$

Equation 188: Idle case energy consumption in terms of losses (Series, idle)

$\left(E_{\text {eng }, \text { fuel }}\right)^{0}=\left(E_{\text {Acc }}\right)^{0}+\left(E_{\text {gen }, \text { loss }}\right)^{0}+\left(E_{\text {eng }, \text { loss }}\right)^{0}$ 


\section{APPENDIX K: Equations for the DCnet Case (Series HEV)}

This appendix contains the definitions for the component efficiencies used in the energy consumption equations developed for VTool. Some information may be duplicated in the body of this paper, but all relevant terms, definitions and equations have been reproduced here for convenience.

\begin{tabular}{|l|l|l|}
\hline Term & $\begin{array}{l}\text { Inherent } \\
\text { Sign }\end{array}$ & Definition \\
\hline$\left(E_{\text {batt,int }}\right)^{-1+}$ & - & Electrical energy inside the battery captured during the brake case \\
\hline$\left(E_{\text {batt,int }}\right)^{-+}$ & + & Net electrical energy inside the battery available to be reused for propulsion \\
\hline$\left(E_{\text {batt,int }}\right)^{+g e n}$ & - & Electrical energy inside the battery stored from excess generating \\
\hline$\left(E_{\text {batt,int }}\right)^{+ \text {prop }}$ & + & Electrical energy inside the battery to be used in propulsion \\
\hline$\left(E_{\text {batt,int }}\right)^{\text {DCnet }}$ & + & Net electrical energy inside the battery for a given drive cycle \\
\hline$\left(E_{\text {tr,credit }}\right)^{-1+}$ & + & Tractive energy at the wheels from stored regen energy reused for propulsion \\
\hline$\left(E_{\text {eng,fuel }}\right)^{+}$ & + & Fuel energy in to the engine during the propel case \\
\hline$\left(E_{\text {eng,fuel }}\right)^{0}$ & + & Fuel energy in to the engine during the idle case \\
\hline$\left(E_{\text {eng,fuel }}\right)^{\text {EqrC }}$ & - & Theoretical fuel energy out of the engine to be used as fuel credit \\
\hline$\left(E_{\text {tr }}\right)^{\text {bCnet }}$ & + & Net tractive energy at the wheels for a given drive cycle (Road Load $)$ \\
\hline
\end{tabular}

\section{Efficiency Expressions:}

Equation 75: Net powertrain efficiency (Series, DCnet)

$\tilde{\eta}_{p t}^{\text {DCnet }}=\frac{\left(E_{\text {tr }}\right)^{\text {DCnet }}}{\left(E_{\text {eng,fuel }}\right)^{\text {DCnet }}}$

Equation 60: Propel case powertrain efficiency (Series, propel)

$\tilde{\eta}_{p t, \text { eng }}^{+}=\frac{\left(E_{\text {tr }}\right)^{+}}{\left(E_{\text {eng,fuel }}\right)^{+}}$

Equation 65: Powertrain efficiency for regen energy reuse (Series, regen reuse)

$\tilde{\eta}_{p t, E V}^{-/+}=\frac{\left(E_{\text {tr,credit }}\right)^{-/+}}{\left(E_{\text {batt }, \text { int }}\right)^{-/+}}$

Equation 76: Net powertrain efficiency for combined propel operation (Series, DCnet)

$\tilde{\eta}_{\text {pt,propel }}^{\text {DCnet }}=\frac{\left(E_{\text {tr }}\right)^{+}+\left(E_{\text {tr,credit }}\right)^{E q F C}}{\left(E_{\text {eng,fuel }}\right)^{+}+\left(E_{\text {batt }, \text { int }}\right)^{-/+}}$

Equation 35: Brake case powertrain efficiency (EV, brake)

$\tilde{\eta}_{p t}^{-}=\frac{\left(E_{\text {batt }, \text { int }}\right)^{-}}{\left(E_{t r}\right)^{-}}$ 


\section{Loss Expressions:}

Equation 144: Net friction brake losses (EV, DCnet)

$\left(E_{\text {brk }, \text { loss }}\right)^{\text {DCnet }}=\left(E_{\text {brk }, \text { loss }}\right)^{-}$

Equation 189: Net motor driveline losses (Series, DCnet)

$\left(E_{d l, l o s s}\right)^{D C n e t}=\left(E_{d l, l o s s}\right)^{+}+\left(E_{d l, l o s s}\right)^{-}+\left(E_{d l, l o s s}\right)^{-/+}+\left(E_{d l, l o s s}\right)^{E q F C}$

Equation 190: Net motor losses (Series, DCnet)

$\left(E_{\text {mot,loss }}\right)^{\text {DCnet }}=\left(E_{\text {mot }, \text { loss }}\right)^{+}+\left(E_{\text {mot }, \text { loss }}\right)^{-}+\left(E_{\text {mot }, \text { loss }}\right)^{-/+}+\left(E_{\text {mot }, \text { loss }}\right)^{E q F C}$

Equation 73: Net battery losses (Series, DCnet)

$\left(E_{\text {batt }, \text { loss }}\right)^{\text {DCnet }}=\left(E_{\text {batt }, \text { loss }}\right)^{+}+\left(E_{\text {batt }, \text { loss }}\right)^{-}+\left(E_{\text {batt }, \text { loss }}\right)^{-/+}+\left(E_{\text {batt }, \text { loss }}\right)^{\text {EqFC }}$

Equation 191: Net generator losses (Series, DCnet)

$\left(E_{\text {gen,loss }}\right)^{\text {DCnet }}=\left(E_{\text {gen }, \text { loss }}\right)^{+}+\left(E_{\text {gen,loss }}\right)^{0}+\left(E_{\text {gen }, \text { loss }}\right)^{E q F C}$

Equation 192: Net engine losses (Series, DCnet)

$\left(E_{\text {eng, loss }}\right)^{\text {DCnet }}=\left(E_{\text {eng }, \text { loss }}\right)^{+}+\left(E_{\text {eng,loss }}\right)^{0}+\left(E_{\text {eng,loss }}\right)^{E q F C}$

Equation 74: Net powertrain losses (Series, DCnet)

$\left(E_{p t, l o s s}\right)^{\text {DCnet }}=\left(E_{p t, l o s s}\right)^{+}+\left(E_{p t, l o s s}\right)^{-}+\left(E_{p t, l o s s}\right)^{0}+\left(E_{p t, l o s s}\right)^{-/+}+\left(E_{p t, l o s s}\right)^{E q F C}$

\section{Conservation of Energy Expressions:}

Equation 61: Conservation of energy for regen energy capture and reuse (Series, regen reuse)

$\left(E_{\text {batt }, \text { int }}\right)^{-/+}+\left(E_{\text {batt }, \text { int }}\right)^{-}=0$

Equation 64: Equivalent tractive energy 'credit' from regen in terms of losses (Series, regen reuse)

$\left(E_{\text {batt }, \text { int }}\right)^{-/+}=\left(E_{\text {tr,credit }}\right)^{-/+}+\left(E_{\text {pt,loss }}\right)^{-/+}$

Equation 53: Conservation of energy in the battery to maintain charge balance (Series, propel)

$\left(E_{\text {batt }, \text { int }}\right)^{+ \text {prop }}+\left(E_{\text {batt }, \text { int }}\right)^{+ \text {gen }}=0$

Equation 77: Energy balance for overall charge balance (Series, DCnet)

$\left(E_{\text {batt }, \text { int }}\right)^{+ \text {prop }}+\left(E_{\text {batt }, \text { int }}\right)^{+ \text {gen }}+\left(E_{\text {batt,int }}\right)^{-}+\left(E_{\text {tr,credit }}\right)^{-/+}+\left(E_{\text {pt,loss }}\right)^{-/+}=0$ 
Equation 71: Total fuel energy calculated by case (Series, DCnet)

$\left(E_{\text {eng,fuel }}\right)^{\text {DCnet }}=\left(E_{\text {eng,fuel }}\right)^{+}+\left(E_{\text {eng,fuel }}\right)^{0}+\left(E_{\text {eng,fuel }}\right)^{E q F C}$

Equation 72: Total fuel energy calculated from overall energy balance (Series, DCnet)

$\left(E_{\text {eng,fuel }}\right)^{\text {DCnet }}=\left(E_{\text {tr }}\right)^{\text {DCnet }}+\left(E_{\text {ACC }}\right)^{\text {DCnet }}+\left(E_{\text {pt,loss }}\right)^{\text {DCnet }}$

\section{Accessory Energy:}

Equation 41: Net accessory energy

$\left(E_{A C C}\right)^{\text {DCnet }}=\left(E_{A C C}\right)^{+}+\left(E_{A C C}\right)^{-}+\left(E_{A C C}\right)^{0}=P_{A C C} * T$ 


\section{APPENDIX L: Equations for Tractive Energy in the Propel Case (P3/P4 Parallel HEV)}

This appendix contains the definitions for the component efficiencies used in the energy consumption equations developed for VTool. Some information may be duplicated in the body of this paper, but all relevant terms, definitions and equations have been reproduced here for convenience.

\begin{tabular}{|c|c|c|}
\hline Term & $\begin{array}{l}\text { Inherent } \\
\text { Sign }\end{array}$ & Definition \\
\hline$\left(E_{\text {eng,fuel }}\right)^{+t r}$ & + & Fuel energy in to the engine used for tractive power during the propel case \\
\hline$\left(\mathrm{E}_{\text {eng,mech }}\right)^{+}$ & + & Mechanical energy out of the engine during the propel case \\
\hline$\left(E_{\text {dleng,tr }}\right)^{+}$ & + & Tractive energy out of the engine driveline to the drive axle for the propel case \\
\hline$\left(E_{\text {eng,use }}\right)^{+}$ & + & Tractive energy from the engine directly used for propulsion \\
\hline$\left(\mathrm{E}_{\text {eng,store }}\right)^{+}$ & - & Tractive energy from the engine stored before being used for propulsion \\
\hline$\left(\mathrm{E}_{\mathrm{dlmot}, \mathrm{tr}}\right)^{+\mathrm{gen}}$ & - & Excess tractive energy in to the motor driveline from the drive axle to be stored \\
\hline$\left(E_{\text {mot,mech }}\right)^{+g e n}$ & - & Mechanical energy in to the motor to be stored for future propulsion \\
\hline$\left(E_{\text {mot,elec }}\right)^{\text {tgen }}$ & - & Electrical energy out of the motor to be stored \\
\hline$\left(\mathrm{E}_{\text {batt }} \mathrm{T}^{\text {tgen }}\right.$ & - & Electrical energy in to the battery at the terminals to be stored \\
\hline$\left(\mathrm{E}_{\text {batt.int }}\right)^{+ \text {gen }}$ & - & Electrical energy inside the battery stored from excess generating \\
\hline$\left(\mathrm{E}_{\text {batt, int }}\right)^{+ \text {prop }}$ & + & Electrical energy inside the battery to be used in propulsion \\
\hline$\left(\mathrm{E}_{\text {batt }, T}\right)^{+ \text {prop }}$ & + & Electrical energy out of the battery at the terminals to be used \\
\hline$\left(E_{\text {mot,elec }}\right)^{+ \text {prop }}$ & + & Electrical energy in to the motor to be used \\
\hline$\left(E_{\text {mot,mech }}\right)^{+ \text {prop }}$ & + & Mechanical energy out of the motor to be used for propulsion \\
\hline$\left(E_{\text {dlmot,tr }}\right)^{+ \text {prop }}$ & + & Surplus tractive energy out of the motor $\mathrm{D} / \mathrm{L}$ from the drive axle to be used \\
\hline$\left(E_{t r}\right)^{+}$ & + & Tractive energy at the wheels for the propel case \\
\hline
\end{tabular}

\section{Efficiency Expressions:}

Equation 78: Power Split Fraction, engine energy directly used (P3 Parallel, propel)

$$
\begin{gathered}
\tilde{\xi}=\frac{\left(E_{\text {eng,use }}\right)^{+}}{\left(E_{\text {dleng,tr }}\right)^{+}} \\
\text {Restated: } \\
\left(E_{\text {eng }, \text { use }}\right)^{+}=\tilde{\xi}\left(E_{\text {dleng,tr }}\right)^{+}
\end{gathered}
$$

Equation 79: Power Split Fraction, engine energy recirculated (P3 Parallel, propel)

$\left(E_{\text {eng,store }}\right)^{+}=\left(E_{\text {dlmot }, \text { tr }}\right)^{+ \text {gen }}=(-1)(1-\xi)\left(E_{\text {dleng,tr }}\right)^{+}$

Equation 193: Engine propel efficiency (P3 Parallel, propel)

$$
\tilde{\eta}_{\text {eng }}^{+}=\frac{\left(E_{\text {eng, mech }}\right)^{+}}{\left(E_{\text {eng,fuel }}\right)^{+t r}}
$$


Equation 194: Engine driveline propel efficiency (P3 Parallel, propel)

$\tilde{\eta}_{\text {dl,eng }}^{+}=\frac{\left(E_{\text {dleng,tr }}\right)^{+}}{\left(E_{\text {eng,mech }}\right)^{+}}$

Equation 195: Motor driveline brake efficiency (P3 Parallel, propel)

$\tilde{\eta}_{d l, m o t}^{+g e n}=\frac{\left(E_{\text {mot }, \text { mech }}\right)^{+g e n}}{\left(E_{d l m o t, t r}\right)^{+g e n}} \approx \tilde{\eta}_{d l, m o t}^{-}$

Equation 196: Motor brake efficiency (P3 Parallel, propel)

$\tilde{\eta}_{\text {mot }}^{+ \text {gen }}=\frac{\left(E_{\text {mot }, \text { elec }}\right)^{+ \text {gen }}}{\left(E_{\text {mot }, \text { mech }}\right)^{+g e n}} \approx \tilde{\eta}_{\text {mot }}^{-}$

Equation 197: Battery charge efficiency (P3 Parallel, propel)

$\tilde{\eta}_{\text {batt }}^{+g e n}=\frac{\left(E_{\text {batt }, \text { int }}\right)^{+g e n}}{\left(E_{\text {batt }, T}\right)^{+g e n}} \approx \tilde{\eta}_{\text {batt }}^{-}$

Equation 198: Battery discharge efficiency (P3 Parallel, propel)

$\tilde{\eta}_{\text {batt }}^{+ \text {prop }}=\frac{\left(E_{\text {batt }, T}\right)^{+ \text {prop }}}{\left(E_{\text {batt }, \text { int }}\right)^{+ \text {prop }}} \approx \tilde{\eta}_{\text {batt }}^{+}$

Equation 199: Motor propel efficiency (P3 Parallel, propel)

$\tilde{\eta}_{\text {mot }}^{+ \text {prop }}=\frac{\left(E_{\text {mot }, \text { mech }}\right)^{+ \text {prop }}}{\left(E_{\text {mot }, \text { elec }}\right)^{+ \text {prop }}} \approx \tilde{\eta}_{\text {mot }}^{+}$

Equation 200: Motor driveline propel efficiency (P3 Parallel, propel)

$\tilde{\eta}_{d l, m o t}^{+ \text {prop }}=\frac{\left(E_{d l m o t, t r}\right)^{+ \text {prop }}}{\left(E_{\text {mot }, \text { mech }}\right)^{+ \text {prop }}} \approx \tilde{\eta}_{d l, m o t}^{+}$

Equation 84: Power Split Fraction energy recirculation efficiency (P3 Parallel, propel)

$\tilde{\eta}_{\text {recirc }}^{+}=\left(\tilde{\eta}_{\text {dl,mot }}^{+ \text {gen }}\right)\left(\tilde{\eta}_{\text {mot }}^{+ \text {gen }}\right)\left(\tilde{\eta}_{\text {batt }}^{+ \text {gen }}\right)\left(\tilde{\eta}_{\text {batt }}^{+ \text {prop }}\right)\left(\tilde{\eta}_{\text {mot }}^{+ \text {prop }}\right)\left(\tilde{\eta}_{\text {dl,mot }}^{+ \text {prop }}\right)$

Equation 88: Propel case powertrain efficiency for tractive energy (P3 Parallel, propel)

$\tilde{\eta}_{p t}^{+t r}=\frac{\left(E_{\text {tr }}\right)^{+}}{\left(E_{\text {eng,fuel }}\right)^{+t r}}$ 


\section{Loss Expressions:}

Equation 201: Engine losses for tractive energy (P3 Parallel, propel)

$\left(E_{\text {eng,loss }}\right)^{+t r}=\left(E_{\text {eng,fuel }}\right)^{+t r}-\left(E_{\text {eng,mech }}\right)^{+}$

Equation 202: Engine driveline losses for tractive energy (P3 Parallel, propel)

$\left(E_{\text {dleng, loss }}\right)^{+t r}=\left(E_{\text {eng,mech }}\right)^{+}-\left(E_{\text {dleng,tr }}\right)^{+}$

Equation 203: Motor driveline generate losses for tractive energy (P3 Parallel, propel)

$\left(E_{d l m o t, l o s s}\right)^{+g e n}=\left(E_{\text {mot }, \text { mech }}\right)^{+g e n}-\left(E_{d l m o t, t r}\right)^{+g e n}$

Equation 204: Motor generate losses for tractive energy (P3 Parallel, propel)

$\left(E_{\text {mot }, \text { loss }}\right)^{+ \text {gen }}=\left(E_{\text {mot }, \text { elec }}\right)^{+ \text {gen }}-\left(E_{\text {mot }, \text { mech }}\right)^{+ \text {gen }}$

Equation 205: Battery charge losses for tractive energy (P3 Parallel, propel)

$\left(E_{\text {batt }, \text { loss }}\right)^{+ \text {gen }}=\left(E_{\text {batt }, \text { int }}\right)^{+ \text {gen }}-\left(E_{\text {batt }, T}\right)^{+g e n}$

Equation 206: Battery discharge losses for tractive energy (P3 Parallel, propel)

$\left(E_{\text {batt }, \text { loss }}\right)^{+ \text {prop }}=\left(E_{\text {batt }, \text { int }}\right)^{+ \text {prop }}-\left(E_{\text {batt }, T}\right)^{+ \text {prop }}$

Equation 207: Motor propel losses for tractive energy (P3 Parallel, propel)

$\left(E_{\text {mot }, \text { loss }}\right)^{+ \text {prop }}=\left(E_{\text {mot }, \text { elec }}\right)^{+ \text {prop }}-\left(E_{\text {mot }, \text { mech }}\right)^{+ \text {prop }}$

Equation 208: Motor driveline propel losses for tractive energy (P3 Parallel, propel)

$\left(E_{\text {dlmot }, l o s s}\right)^{+ \text {prop }}=\left(E_{\text {mot }, \text { mech }}\right)^{+ \text {prop }}-\left(E_{\text {dlmot }, t r}\right)^{+ \text {prop }}$

Equation 209: Motor driveline total losses for tractive energy (P3 Parallel, propel)

$\left(E_{\text {dlmot }, \text { loss }}\right)^{+t r}=\left(E_{\text {dlmot }, \text { loss }}\right)^{+ \text {gen }}+\left(E_{\text {dlmot }, \text { loss }}\right)^{+ \text {prop }}$

Equation 210: Motor total losses for tractive energy (P3 Parallel, propel)

$\left(E_{\text {mot,loss }}\right)^{+t r}=\left(E_{\text {mot,loss }}\right)^{+ \text {gen }}+\left(E_{\text {mot,loss }}\right)^{+ \text {prop }}$

Equation 211: Battery total losses for tractive energy (P3 Parallel, propel)

$\left(E_{\text {batt }, \text { loss }}\right)^{+t r}=\left(E_{\text {batt }, \text { loss }}\right)^{+ \text {gen }}+\left(E_{\text {batt }, \text { loss }}\right)^{+ \text {prop }}$

Equation 212: Powertrain losses for tractive energy (P3 Parallel, propel)

$\left(E_{\text {pt loss }}\right)^{+t r}=\left(E_{\text {eng,loss }}\right)^{+t r}+\left(E_{\text {dleng,loss }}\right)^{+t r}+\left(E_{\text {dlmot }, \text { loss }}\right)^{+t r}+\left(E_{\text {mot }, \text { loss }}\right)^{+t r}+\left(E_{\text {batt }, \text { loss }}\right)^{+t r}$

Restated:

$\left(E_{p t, l o s s}\right)^{+t r}=\left(E_{\text {eng,fuel }}\right)^{+t r}-\left(E_{t r}\right)^{+}$ 


\section{Conservation of Energy Expressions:}

Equation 85: Conservation of energy 'inside' the drive axle (P3 Parallel, propel)

$\left(E_{\text {tr }}\right)^{+}=\left(E_{\text {eng }, u s e}\right)^{+}+\left(E_{\text {dlmot }, t r}\right)^{+ \text {prop }}$

Equation 213: Conservation of energy at or 'outside' the drive axle (P3 Parallel, propel)

$\left(E_{\text {tr }}\right)^{+}=\left(E_{\text {dleng,tr }}\right)^{+}+\left(E_{\text {dlmot }, \text { tr }}\right)^{+ \text {prop }}+\left(E_{\text {dlmot }, t r}\right)^{+g e n}$

Equation 214: Conservation of energy at the HV bus while charging (P3 Parallel, propel)

$\left(E_{\text {batt }, T}\right)^{+ \text {gen }}=\left(E_{\text {mot }, \text { elec }}\right)^{+ \text {gen }}$

Equation 215: Conservation of energy at the HV bus while discharging (P3 Parallel, propel) $\left(E_{\text {batt }, T}\right)^{+ \text {prop }}=\left(E_{\text {mot,elec }}\right)^{+ \text {prop }}$

Equation 216: Conservation of energy for the Power Split Fraction (P3 Parallel, propel) $\left(E_{\text {dleng,tr }}\right)^{+}=\left(E_{\text {eng,use }}\right)^{+}-\left(E_{\text {dlmot }, t r}\right)^{+g e n}$

Equation 82: Conservation of energy in the battery to maintain charge balance (P3 Parallel, propel) $\left(E_{\text {batt }, \text { int }}\right)^{+ \text {prop }}=-\left(E_{\text {batt }, \text { int }}\right)^{+ \text {gen }}$

\section{Developing Equation for Power Split Fraction (in terms of efficiencies):}

- Storing Energy in the Battery During the Propel Case:

$$
\begin{aligned}
& \left(E_{\text {batt }, \text { int }}\right)^{+ \text {gen }}=\left(E_{\text {batt }, T}\right)^{+ \text {gen }}\left(\tilde{\eta}_{\text {batt }}^{+ \text {gen }}\right) \\
& \left(E_{\text {batt }, \text { int }}\right)^{+ \text {gen }}=\left(E_{\text {mot }, \text { elec }}\right)^{+ \text {gen }}\left(\tilde{\eta}_{\text {batt }}^{+ \text {gen }}\right) \\
& \left(E_{\text {batt }, \text { int }}\right)^{+ \text {gen }}=\left(E_{\text {mot }, \text { mech }}\right)^{+ \text {gen }}\left(\tilde{\eta}_{\text {mot }}^{\text {gen }}\right)\left(\tilde{\eta}_{\text {batt }}^{+ \text {gen }}\right) \\
& \left(E_{\text {batt }, \text { int }}\right)^{+ \text {gen }}=\left(E_{\text {dlmot }, t r}\right)^{+ \text {gen }}\left(\tilde{\eta}_{\text {dl,mot }}^{+ \text {gen }}\right)\left(\tilde{\eta}_{\text {mot }}^{+ \text {gen }}\right)\left(\tilde{\eta}_{\text {batt }}^{+ \text {gen }}\right)
\end{aligned}
$$

Equation 80: Energy stored in the battery during times of surplus power generation (P3 Parallel, propel)

$$
\left(E_{\text {batt }, \text { int }}\right)^{+g e n}=(-1)(1-\xi)\left(E_{\text {dleng,tr }}\right)^{+}\left(\tilde{\eta}_{\text {dl,mot }}^{+ \text {gen }}\right)\left(\tilde{\eta}_{\text {mot }}^{+g e n}\right)\left(\tilde{\eta}_{\text {batt }}^{+ \text {gen }}\right)
$$




\section{- Using Energy from the Battery During the Propel Case:}

$$
\begin{aligned}
& \left(E_{\text {batt }, \text { int }}\right)^{+ \text {prop }}=\frac{\left(E_{\text {batt }, T}\right)^{+ \text {prop }}}{\tilde{\eta}_{\text {batt }}^{+ \text {prop }}} \\
& \left(E_{\text {batt }, \text { int }}\right)^{+ \text {prop }}=\frac{\left(E_{\text {mot,elec }}\right)^{+ \text {prop }}}{\tilde{\eta}_{\text {batt }}^{+ \text {prop }}} \\
& \left(E_{\text {batt }, \text { int }}\right)^{+ \text {prop }}=\frac{\left(E_{\text {mot }, \text { mech }}\right)^{+ \text {prop }}}{\left(\tilde{\eta}_{\text {batt }}^{+ \text {prop }}\right)\left(\tilde{\eta}_{\text {mot }}^{+ \text {prop }}\right)}
\end{aligned}
$$

Equation 81: Energy used from the battery during shortages of power generation (P3 Parallel, propel)

$$
\left(E_{\text {bat }, \text { int }}\right)^{+ \text {prop }}=\frac{\left(E_{\text {dlmot }, \text { tr }}\right)^{+ \text {prop }}}{\left(\tilde{\eta}_{\text {batt }}^{+ \text {prop }}\right)\left(\tilde{\eta}_{\text {mot }}^{+ \text {prop }}\right)\left(\tilde{\eta}_{\text {dl,mot }}^{+ \text {prop }}\right)}
$$

\section{- Conservation of Energy to Maintain Charge Balance:}

Equation 82: Conservation of energy in the battery to maintain charge balance (P3 Parallel, propel)

$$
\begin{aligned}
& \left(E_{\text {batt,int }}\right)^{+ \text {prop }}=-\left(E_{\text {batt }, \text { int }}\right)^{+ \text {gen }} \\
& (-1)(1-\xi)\left(E_{\text {dleng,tr }}\right)^{+}\left(\tilde{\eta}_{d l, m o t}^{+g e n}\right)\left(\tilde{\eta}_{m o t}^{+g e n}\right)\left(\tilde{\eta}_{\text {batt }}^{+ \text {gen }}\right)=\frac{-\left(E_{\text {dlmot }, t r}\right)^{+ \text {prop }}}{\left(\tilde{\eta}_{\text {batt }}^{+ \text {prop }}\right)\left(\tilde{\eta}_{\text {mot }}^{+ \text {prop }}\right)\left(\tilde{\eta}_{d l, m o t}^{+ \text {prop }}\right)} \\
& (1-\xi)\left(E_{\text {dleng,tr }}\right)^{+}\left(\tilde{\eta}_{d l, m o t}^{+g e n}\right)\left(\tilde{\eta}_{\text {mot }}^{+g e n}\right)\left(\tilde{\eta}_{\text {batt }}^{+g e n}\right)=\frac{\left(E_{\text {dlmot }, t r}\right)^{+ \text {prop }}}{\left(\tilde{\eta}_{\text {batt }}^{+ \text {prop }}\right)\left(\tilde{\eta}_{\text {mot }}^{+ \text {prop }}\right)\left(\tilde{\eta}_{d l, m o t}^{+ \text {prop }}\right)} \\
& \left(E_{\text {dleng,tr }}\right)^{+}=\frac{\left(E_{\text {dlmot,tr }}\right)^{+ \text {prop }}}{(1-\xi)\left(\tilde{\eta}_{d l, m o t}^{+g e n}\right)\left(\tilde{\eta}_{\text {mot }}^{+g e n}\right)\left(\tilde{\eta}_{\text {batt }}^{+g e n}\right)\left(\tilde{\eta}_{\text {batt }}^{+ \text {prop }}\right)\left(\tilde{\eta}_{\text {mot }}^{+ \text {prop }}\right)\left(\tilde{\eta}_{d l, m o t}^{+ \text {prop }}\right)} \\
& \left(E_{\text {dleng,tr }}\right)^{+}=\frac{\left(E_{\text {dlmot }, \text { tr }}\right)^{+ \text {prop }}}{(1-\xi)\left(\tilde{\eta}_{\text {recirc }}^{+}\right)} \\
& \left(E_{\text {dleng,tr }}\right)^{+}(1-\xi)\left(\tilde{\eta}_{\text {recirc }}^{+}\right)=\left(E_{\text {dlmot }, t r}\right)^{+ \text {prop }}
\end{aligned}
$$

Equation 83: Energy from the motor onto the drive axle during the propel case (P3 Parallel, propel)

$$
\left(E_{\text {dlmot }, \text { tr }}\right)^{+ \text {prop }}=\left(E_{\text {dleng,tr }}\right)^{+}(1-\xi)\left(\tilde{\eta}_{\text {recirc }}^{+}\right)
$$




\section{- Conservation of Energy at the drive axle:}

Equation 85: Conservation of energy 'inside' the drive axle (P3 Parallel, propel)

$$
\begin{aligned}
& \left(E_{\text {tr }}\right)^{+}=\left(E_{\text {eng }, \text { use }}\right)^{+}+\left(E_{\text {dlmot }, t r}\right)^{+ \text {prop }} \\
& \left(E_{\text {eng }, \text { use }}\right)^{+}=\left(E_{\text {tr }}\right)^{+}-\left(E_{\text {dlmot }, t r}\right)^{+ \text {prop }} \\
& \xi\left(E_{\text {dleng,tr }}\right)^{+}=\left(E_{\text {tr }}\right)^{+}-\left[\left(E_{\text {dleng,tr }}\right)^{+}(1-\xi)\left(\tilde{\eta}_{\text {recirc }}^{+}\right)\right] \\
& \xi\left(E_{\text {dleng,tr }}\right)^{+}+\left(E_{\text {dleng,tr }}\right)^{+}(1-\xi)\left(\tilde{\eta}_{\text {recirc }}^{+}\right)=\left(E_{\text {tr }}\right)^{+} \\
& \left(E_{\text {dleng,tr }}\right)^{+}\left[\xi+(1-\xi)\left(\tilde{\eta}_{\text {recirc }}^{+}\right)\right]=\left(E_{\text {tr }}\right)^{+}
\end{aligned}
$$

Equation 86: Energy supplied by the engine during the propel case (P3 Parallel, propel)

$$
\left(E_{\text {dleng,tr }}\right)^{+}=\frac{\left(E_{\text {tr }}\right)^{+}}{\xi+(1-\xi)\left(\tilde{\eta}_{\text {recirc }}^{+}\right)}
$$

\section{Developing Equation for Propel Case Tractive Energy Consumption (in terms of efficiencies):}

$$
\begin{aligned}
& \left(E_{\text {eng,fuel }}\right)^{+t r}=\frac{\left(E_{\text {eng,mech }}\right)^{+}}{\tilde{\eta}_{\text {eng }}^{+}} \\
& \left(E_{\text {eng,fuel }}\right)^{+t r}=\frac{\left(E_{\text {dleng,tr }}\right)^{+}}{\left(\tilde{\eta}_{\text {eng }}^{+}\right)\left(\tilde{\eta}_{\text {dl,eng }}^{+}\right)} \\
& \left(E_{\text {eng,fuel }}\right)^{+t r}=\frac{\left(E_{\text {tr }}\right)^{+}}{\left[\xi+(1-\xi)\left(\tilde{\eta}_{\text {recirc }}^{+}\right)\right]\left(\tilde{\eta}_{\text {eng }}^{+}\right)\left(\tilde{\eta}_{\text {dl,eng }}^{+}\right)}
\end{aligned}
$$

Equation 87: Propel case tractive fuel energy consumption (P3 Parallel, propel)

$$
\left(E_{\text {eng,fuel }}\right)^{+t r}=\frac{\left(E_{\text {tr }}\right)^{+}}{\left[\xi+(1-\xi)\left(\tilde{\eta}_{\text {recirc }}^{+}\right)\right]\left(\tilde{\eta}_{\text {eng }}^{+}\right)\left(\tilde{\eta}_{\text {dl,eng }}^{+}\right)}
$$




\section{APPENDIX M: Equations for Accessory Energy in the Propel Case (P3/P4 Parallel HEV)}

This appendix contains the definitions for the component efficiencies used in the energy consumption equations developed for VTool. Some information may be duplicated in the body of this paper, but all relevant terms, definitions and equations have been reproduced here for convenience.

\begin{tabular}{|l|l|l|}
\hline Term & $\begin{array}{l}\text { Inherent } \\
\text { Sign }\end{array}$ & Definition \\
\hline$\left(E_{\text {eng,fuel }}\right)^{+ \text {acc }}$ & + & Fuel energy in to the engine used for accessory power \\
\hline$\left(E_{\text {eng,mech }}\right)^{\text {+acc }}$ & + & Mechanical energy out of the engine to be used for accessory power \\
\hline$\left(E_{\text {dleng,tr }}\right)^{\text {tacc }}$ & + & Tractive energy out of the engine driveline to be used for accessory power \\
\hline$\left(E_{\text {dlmot,tr }}\right)^{\text {acc }}$ & - & Tractive energy in to the motor driveline to be used for accessory power \\
\hline$\left(E_{\text {mot,mech }}\right)^{\text {+acc }}$ & - & Mechanical energy in to the motor to be used for accessory power \\
\hline$\left(E_{\text {mot,elec }}\right)^{\text {+acc }}$ & - & Electrical energy out of the motor to be used for accessory power \\
\hline$\left(E_{\text {acc }}\right)^{+}$ & + & Electrical energy siphoned off the HV bus for accessory loads during propel case \\
\hline
\end{tabular}

\section{Efficiency Expressions:}

Equation 217: Engine propel efficiency for accessory load (P3 Parallel, propel)

$\tilde{\eta}_{\text {eng }}^{+a c c}=\frac{\left(E_{\text {eng,mech }}\right)^{+a c c}}{\left(E_{\text {eng,fuel }}\right)^{+a c c}} \approx \tilde{\eta}_{\text {eng }}^{+}$

Equation 218: Engine driveline propel efficiency for accessory load (P3 Parallel, propel)

$\tilde{\eta}_{\text {dl,eng }}^{+a c c}=\frac{\left(E_{\text {dleng,tr }}\right)^{+a c c}}{\left(E_{\text {eng,mech }}\right)^{+a c c}} \approx \tilde{\eta}_{\text {dl,eng }}^{+}$

Equation 219: Motor driveline brake efficiency for accessory load (P3 Parallel, propel)

$\tilde{\eta}_{d l, m o t}^{+a c c}=\frac{\left(E_{\text {mot }, \text { mech }}\right)^{+a c c}}{\left(E_{\text {dlmot }, t r}\right)^{+a c c}} \approx \tilde{\eta}_{d l, m o t}^{-}$

Equation 220: Motor brake efficiency for accessory load (P3 Parallel, propel)

$\tilde{\eta}_{\text {mot }}^{+a c c}=\frac{\left(E_{\text {mot }, \text { elec }}\right)^{+a c c}}{\left(E_{\text {mot }, \text { mech }}\right)^{+a c c}} \approx \tilde{\eta}_{\text {mot }}^{-}$

Equation 90: Propel case powertrain accessory efficiency for accessory load (P3 Parallel, propel)

$\tilde{\eta}_{p t}^{+a c c}=\frac{\left(E_{\text {Acc }}\right)^{+}}{\left(E_{\text {eng,fuel }}\right)^{+a c c}}$ 


\section{Loss Expressions:}

Equation 221: Engine losses for accessory load (P3 Parallel, propel)

$\left(E_{\text {eng }, \text { loss }}\right)^{+a c c}=\left(E_{\text {eng,fuel }}\right)^{+a c c}-\left(E_{\text {eng,mech }}\right)^{+a c c}$

Equation 222: Engine driveline losses for accessory load (P3 Parallel, propel)

$\left(E_{\text {dleng,loss }}\right)^{+a c c}=\left(E_{\text {eng }, \text { mech }}\right)^{+a c c}-\left(E_{\text {dleng,tr }}\right)^{+a c c}$

Equation 223: Motor driveline generate losses for accessory load (P3 Parallel, propel)

$\left(E_{\text {dlmot }, l o s s}\right)^{+a c c}=\left(E_{\text {mot }, \text { mech }}\right)^{+a c c}-\left(E_{\text {dlmot }, t r}\right)^{+a c c}$

Equation 224: Motor generate losses for accessory load (P3 Parallel, propel)

$\left(E_{\text {mot }, \text { loss }}\right)^{+a c c}=\left(E_{\text {mot }, \text { elec }}\right)^{+a c c}-\left(E_{\text {mot }, \text { mech }}\right)^{+a c c}$

Equation 225: Powertrain losses for accessory load (P3 Parallel, propel)

$\left(E_{\text {pt }, \text { loss }}\right)^{+a c c}=\left(E_{\text {eng,loss }}\right)^{+a c c}+\left(E_{\text {dleng,loss }}\right)^{+a c c}+\left(E_{\text {dlmot }, \text { loss }}\right)^{+a c c}+\left(E_{\text {mot }, \text { loss }}\right)^{+a c c}$

Restated:

$\left(E_{\text {pt,loss }}\right)^{+a c c}=\left(E_{\text {eng,fuel }}\right)^{+a c c}-\left(E_{\text {Acc }}\right)^{+}$

\section{Conservation of Energy Expressions:}

Equation 226: Conservation of energy at the drive axle for accessory load (P3 Parallel, propel)

$\left(E_{\text {dleng,tr }}\right)^{+a c c}=-\left(E_{\text {dlmot }, t r}\right)^{+a c c}$

Equation 227: Conservation of energy at the HV bus for accessory load (P3 Parallel, propel)

$\left(E_{\text {Acc }}\right)^{+}=-\left(E_{\text {mot }, \text { elec }}\right)^{+a c c}$

\section{Accessory Energy:}

Equation 25: Propel case accessory energy

$\left(E_{A c C}\right)^{+}=\tau\left(P_{A c C}\right)^{+}$ 
Developing Equation for Propel Case Accessory Energy Consumption (in terms of efficiencies):

$$
\begin{aligned}
& \left(E_{\text {eng,fuel }}\right)^{+a c c}=\frac{\left(E_{\text {eng,mech }}\right)^{+a c c}}{\tilde{\eta}_{\text {eng }}^{+}} \\
& \left(E_{\text {eng,fuel }}\right)^{+a c c}=\frac{\left(E_{\text {dleng,tr }}\right)^{+a c c}}{\left(\tilde{\eta}_{\text {eng }}^{+}\right)\left(\tilde{\eta}_{\text {dl,eng }}^{+}\right)} \\
& \left(E_{\text {eng,fuel }}\right)^{+a c c}=-\frac{\left(E_{\text {dlmot }, t r}\right)^{+a c c}}{\left(\tilde{\eta}_{\text {eng }}^{+}\right)\left(\tilde{\eta}_{\text {dl,eng }}^{+}\right)} \\
& \left(E_{\text {eng,fuel }}\right)^{+a c c}=-\frac{\left(E_{\text {mot,mech }}\right)}{\left(\tilde{\eta}_{\text {eng }}^{+}\right)\left(\tilde{\eta}_{\text {dl,eng }}^{+}\right)\left(\tilde{\eta}_{d l, m o t}^{+}\right)} \\
& \left(E_{\text {eng,fuel }}\right)^{+a c c}=-\frac{\left(E_{\text {mot }, \text { elec }}\right)^{+a c c}}{\left(\tilde{\eta}_{\text {eng }}^{+}\right)\left(\tilde{\eta}_{\text {dl,eng }}^{+}\right)\left(\tilde{\eta}_{\text {dl,mot }}^{+}\right)\left(\tilde{\eta}_{\text {mot }}^{+}\right)}
\end{aligned}
$$

Equation 89: Propel case accessory fuel energy consumption (P3 Parallel, propel)

$$
\left(E_{\text {eng,fuel }}\right)^{+a c c}=\frac{\left(E_{\text {Acc }}\right)^{+}}{\left(\tilde{\eta}_{\text {eng }}^{+}\right)\left(\tilde{\eta}_{d l, \text { eng }}^{+}\right)\left(\tilde{\eta}_{d l, m o t}^{+}\right)\left(\tilde{\eta}_{\text {mot }}^{+}\right)}
$$




\section{APPENDIX N: Equations for Total Energy in the Propel Case (P3/P4 Parallel HEV)}

This appendix contains the definitions for the component efficiencies used in the energy consumption equations developed for VTool. Some information may be duplicated in the body of this paper, but all relevant terms, definitions and equations have been reproduced here for convenience.

\begin{tabular}{|l|l|l|}
\hline Term & $\begin{array}{l}\text { Inherent } \\
\text { Sign }\end{array}$ & Definition \\
\hline$\left(E_{\text {eng,fuel }}\right)^{+ \text {acc }}$ & + & Fuel energy in to the engine used for accessory power \\
\hline$\left(E_{\text {eng,fuel }}\right)^{\text {tr }}$ & + & Fuel energy in to the engine used for tractive power \\
\hline$\left(E_{\text {dleng,tr }}\right)^{+a c c}$ & + & Tractive energy out of the engine driveline to be used for accessory power \\
\hline$\left(E_{\text {dlmot,tr }}\right)^{\text {tacc }}$ & - & Tractive energy in to the motor driveline to be used for accessory power \\
\hline$\left(E_{\text {mot,mech }}\right)^{\text {+acc }}$ & - & Mechanical energy in to the motor to be used for accessory power \\
\hline$\left(E_{\text {mot,elec }}\right)^{\text {+acc }}$ & - & Electrical energy out of the motor to be used for accessory power \\
\hline$\left(E_{\text {acc }}\right)^{+}$ & + & Electrical energy siphoned off the HV bus for accessory loads during propel case \\
\hline
\end{tabular}

\section{Efficiency Expressions:}

Equation 95: Propel case ICE powertrain efficiency (P3 Parallel, propel)

$$
\tilde{\eta}_{p t, I C E}^{+}=\frac{\left(E_{\text {tr }}\right)^{+}}{\left(E_{\text {eng,fuel }}\right)^{+}}
$$

\section{Loss Expressions:}

Equation 228: Engine total losses (P3 Parallel, propel)

$\left(E_{\text {eng,loss }}\right)^{+}=\left(E_{\text {eng }, \text { loss }}\right)^{+t r}+\left(E_{\text {eng, loss }}\right)^{+a c c}$

Equation 229: Engine driveline total losses (P3 Parallel, propel)

$\left(E_{\text {dleng }, \text { loss }}\right)^{+}=\left(E_{\text {dleng, loss }}\right)^{+t r}+\left(E_{\text {dleng, loss }}\right)^{+a c c}$

Equation 230: Motor driveline total losses (P3 Parallel, propel)

$\left(E_{\text {dlmot }, \text { loss }}\right)^{+}=\left(E_{\text {dlmot }, \text { loss }}\right)^{+t r}+\left(E_{\text {dlmot }, l o s s}\right)^{+a c c}$

Equation 93: Motor total losses (P3 Parallel, propel)

$\left(E_{\text {mot }, \text { loss }}\right)^{+}=\left(E_{\text {mot }, \text { loss }}\right)^{+t r}+\left(E_{\text {mot }, l o s s}\right)^{+a c c}$

Equation 231: Battery total losses (P3 Parallel, propel)

$\left(E_{\text {batt }, \text { loss }}\right)^{+}=\left(E_{\text {batt }, \text { loss }}\right)^{+t r}$

Equation 94: Total powertrain losses for accessory load (P3 Parallel, propel)

$\left(E_{\text {pt }, \text { loss }}\right)^{+}=\left(E_{\text {eng,loss }}\right)^{+}+\left(E_{\text {dleng,loss }}\right)^{+}+\left(E_{\text {dlmot }, \text { loss }}\right)^{+}+\left(E_{\text {mot }, \text { loss }}\right)^{+}+\left(E_{\text {bat }, \text { loss }}\right)^{+}$ 


\section{Conservation of Energy Expressions:}

Equation 91: Total fuel energy required for the propel case (P3 Parallel, propel)

$$
\left(E_{\text {eng,fuel }}\right)^{+}=\left(E_{\text {eng,fuel }}\right)^{+t r}+\left(E_{\text {eng,fuel }}\right)^{+ \text {acc }}
$$

\section{Developing Equation for Propel Case Total Energy Consumption (in terms of efficiencies):}

$$
\left(E_{\text {eng,fuel }}\right)^{+}=\left(E_{\text {eng,fuel }}\right)^{+t r}+\left(E_{\text {eng,fuel }}\right)^{+a c c}
$$

Equation 92: Propel case total fuel energy consumption (P3 Parallel, propel)

$$
\left(E_{\text {eng,fuel }}\right)^{+}=\frac{\left(E_{\text {tr }}\right)^{+}}{\left[\xi+(1-\xi)\left(\tilde{\eta}_{\text {recirc }}^{+}\right)\right]\left(\tilde{\eta}_{\text {eng }}^{+}\right)\left(\tilde{\eta}_{\text {dl,eng }}^{+}\right)}+\frac{\left(E_{\text {Acc }}\right)^{+}}{\left(\tilde{\eta}_{\text {eng }}^{+}\right)\left(\tilde{\eta}_{\text {dl,eng }}^{+}\right)\left(\tilde{\eta}_{\text {dl,mot }}^{+}\right)\left(\tilde{\eta}_{\text {mot }}^{+}\right)}
$$




\section{APPENDIX O: Equations for the Brake Case (P3/P4 Parallel HEV)}

This appendix contains the definitions for the component efficiencies used in the energy consumption equations developed for VTool. The Brake case for the P3/P4 Parallel HEV is identical to an EV, so only select equations are replicated here. For full documentation of the brake case, see Appendix B.

\begin{tabular}{|l|l|l|}
\hline Term & $\begin{array}{l}\text { Inherent } \\
\text { Sign }\end{array}$ & Definition \\
\hline$\left(E_{t r}\right)^{-}$ & - & Tractive energy at the wheels for the brake case \\
\hline$\left(E_{t r, \zeta}\right)^{-}$ & - & Tractive energy in to driveline after effect of regen fraction \\
\hline$\left(E_{\text {mot,mech }}\right)^{-}$ & - & Mechanical energy in to the motor for the brake case \\
\hline$\left(E_{\text {mot,elec }}\right)^{-}$ & - & Electrical energy out of the motor for the brake case \\
\hline$\left(E_{\text {acc }}\right)^{-}$ & + & Electrical energy siphoned off the HV bus for accessory loads during brake case \\
\hline$\left(E_{\text {batt, }, T}\right)^{-}$ & - & Electrical energy in to the battery or at the terminals during the brake case \\
\hline$\left(E_{\text {batt,int }}\right)^{-}$ & - & Electrical energy inside the battery captured during the brake case \\
\hline
\end{tabular}

\section{Accessory Energy:}

Equation 31: Brake case accessory energy

$\left(E_{\text {Acc }}\right)^{-}=\left(T-\tau-t_{\text {idle }}\right)\left(P_{\text {Acc }}\right)^{-}$

Equation for Brake Case Energy Consumption (in terms of efficiencies):

Equation 32: Brake case energy consumption (EV, brake)

$\left(E_{\text {batt }, \text { int }}\right)^{-}=\tilde{\eta}_{\text {batt }}^{-}\left\{\left(E_{t r}\right)^{-}(\tilde{\zeta})\left(\tilde{\eta}_{d l, m o t}^{-}\right)\left(\tilde{\eta}_{\text {mot }}^{-}\right)+\left(E_{\text {Acc }}\right)^{-}\right\}$

Developing Equation for Brake Case Energy Consumption (in terms of losses):

Equation 34: Brake case energy consumption in terms of losses (EV, brake)

$$
\left(E_{\text {batt }, \text { int }}\right)^{-}=\left(E_{\text {tr }}\right)^{-}+\left(E_{\text {Acc }}\right)^{-}+\left(E_{\text {batt }, \text { loss }}\right)^{-}+\left(E_{\text {mot }, \text { loss }}\right)^{-}+\left(E_{\text {dlmot }, \text { loss }}\right)^{-}+\left(E_{\text {brk }, \text { loss }}\right)^{-}
$$




\section{APPENDIX P: Equations for Regen Energy Reuse (P3/P4 Parallel HEV)}

This appendix contains the definitions for the component efficiencies used in the energy consumption equations developed for VTool. Some information may be duplicated in the body of this paper, but all relevant terms, definitions and equations have been reproduced here for convenience. The EV powertrain for the P3/P4 Parallel HEV is identical to the Series HEV so the equations in this section exactly duplicate the equations in Appendix $\mathrm{H}$.

\begin{tabular}{|l|l|l|}
\hline Term & $\begin{array}{l}\text { Inherent } \\
\text { Sign }\end{array}$ & Definition \\
\hline$\left(E_{\text {batt,int }}\right)^{-1+}$ & - & Electrical energy inside the battery captured during the brake case \\
\hline$\left(E_{\text {batt, int }}\right)^{-1+}$ & + & Net electrical energy inside the battery available to be reused for propulsion \\
\hline$\left(E_{\text {batt, }}\right)^{-1+}$ & + & Net electrical at the battery terminals available to be reused for propulsion \\
\hline$\left(E_{\text {mot,elec }}\right)^{-1+}$ & + & Electrical energy in to the motor to be reused for propulsion \\
\hline$\left(E_{\text {mot,mech }}\right)^{-1+}$ & + & Mechanical energy out of the motor to be reused for propulsion \\
\hline$\left(E_{t r, \text { credit }}\right)^{-1+}$ & + & Tractive energy at the wheels from stored regen energy reused for propulsion \\
\hline
\end{tabular}

\section{Efficiency Expressions:}

Equation 232: Motor driveline efficiency (P3 Parallel, regen reuse)

$$
\tilde{\eta}_{d l, m o t}^{-/+}=\frac{\left(E_{t r, \text { credit }}\right)^{-/+}}{\left(E_{\text {mot }, \text { mech }}\right)^{-/+}} \approx \tilde{\eta}_{d l, m o t}^{+}
$$

Equation 233: Motor propel efficiency (P3 Parallel, regen reuse)

$$
\tilde{\eta}_{\text {mot }}^{-/+}=\frac{\left(E_{\text {mot }, \text { mech }}\right)^{-/+}}{\left(E_{\text {mot }, \text { elec }}\right)^{-/+}} \approx \tilde{\eta}_{\text {mot }}^{+}
$$

Equation 234: Battery discharge efficiency (P3 Parallel, regen reuse)

$$
\tilde{\eta}_{\text {batt }}^{-/+}=\frac{\left(E_{\text {batt }, T}\right)^{-/+}}{\left(E_{\text {batt }, \text { int }}\right)^{-/+}} \approx \tilde{\eta}_{\text {batt }}^{+}
$$

Equation 97: Powertrain efficiency for regen energy reuse (P3 Parallel, regen reuse)

$$
\tilde{\eta}_{p t, E V}^{-/+}=\frac{\left(E_{t r, c r e d i t}\right)^{-/+}}{\left(E_{\text {batt }, i n t}\right)^{-/+}}
$$

\section{Loss Expressions:}

Equation 235: Motor driveline losses (P3 Parallel, regen reuse)

$\left(E_{\text {dlmot }, \text { loss }}\right)^{-/+}=\left(E_{\text {mot }, \text { mech }}\right)^{-/+}-\left(E_{\text {tr,credit }}\right)^{-/+}$

Equation 236: Motor losses (P3 Parallel, regen reuse)

$\left(E_{\text {mot }, \text { loss }}\right)^{-/+}=\left(E_{\text {mot }, \text { elec }}\right)^{-/+}-\left(E_{\text {mot }, \text { mech }}\right)^{-/+}$

Equation 237: Battery losses (P3 Parallel, regen reuse) 
$\left(E_{\text {batt }, \text { loss }}\right)^{-/+}=\left(E_{\text {batt }, \text { int }}\right)^{-/+}-\left(E_{\text {batt }, T}\right)^{-/+}$

Equation 238: Propel powertrain losses (P3 Parallel, regen reuse)

$\left(E_{\text {pt,loss }}\right)^{-/+}=\left(E_{\text {batt }, \text { int }}\right)^{-/+}-\left(E_{\text {tr,credit }}\right)^{-/+}$

Restated:

$\left(E_{p t, l o s s}\right)^{-/+}=\left(E_{\text {batt }, \text { loss }}\right)^{-/+}+\left(E_{\text {mot }, \text { loss }}\right)^{-/+}+\left(E_{\text {dlmot }, \text { loss }}\right)^{-/+}$

\section{Conservation of Energy Expressions:}

Equation 239: Conservation of energy at the HV bus (P3 Parallel, regen reuse)

$\left(E_{\text {bat }, T}\right)^{-/+}=\left(E_{\text {mot }, e l e c}\right)^{-/+}$

Equation 240: Conservation of energy for regen energy capture and reuse (P3 Parallel, regen reuse)

$\left(E_{\text {batt }, \text { int }}\right)^{-}=-\left(E_{\text {batt }, \text { int }}\right)^{-/+}$

\section{Developing Equation for Regen Energy Tractive Energy Credit (in terms of efficiencies):}

$$
\begin{aligned}
& \left(E_{\text {tr,credit }}\right)^{-/+}=\left(E_{\text {mot }, \text { mech }}\right)^{-/+}\left(\tilde{\eta}_{\text {dl,mot }}^{-/+}\right) \\
& \left(E_{\text {tr,credit }}\right)^{-/+}=\left(E_{\text {mot }, \text { elec }}\right)^{-/+}\left(\tilde{\eta}_{\text {mot }}^{-/+}\right)\left(\tilde{\eta}_{d l, m o t}^{-/+}\right) \\
& \left(E_{\text {tr,credit }}\right)^{-/+}=\left(E_{\text {batt }, T}\right)^{-/+}\left(\tilde{\eta}_{\text {mot }}^{-/+}\right)\left(\tilde{\eta}_{\text {dl,mot }}^{-/+}\right) \\
& \left(E_{\text {tr,credit }}\right)^{-/+}=\left(E_{\text {batt }, \text { int }}\right)^{-/+}\left(\tilde{\eta}_{\text {batt }}^{-/+}\right)\left(\tilde{\eta}_{\text {mot }}^{-/+}\right)\left(\tilde{\eta}_{\text {dl,mot }}^{-/+}\right)
\end{aligned}
$$

Equation 96: Equivalent tractive energy 'credit' from regen braking (P3 Parallel, regen reuse)

$$
\left(E_{\text {tr }, \text { credit }}\right)^{-/+}=-\left(E_{\text {batt }, \text { int }}\right)^{-}\left(\tilde{\eta}_{\text {batt }}^{-/+}\right)\left(\tilde{\eta}_{\text {mot }}^{-/+}\right)\left(\tilde{\eta}_{\text {dl,mot }}^{-/+}\right)
$$

\section{Developing Equation for Regen Energy Tractive Energy Credit (in terms of losses):}

$$
\begin{aligned}
& \left(E_{\text {tr,credit }}\right)^{-/+}=\left(E_{\text {mot }, \text { mech }}\right)^{-/+}-\left(E_{\text {dlmot }, \text { loss }}\right)^{-/+} \\
& \left(E_{\text {tr,credit }}\right)^{-/+}=\left(E_{\text {mot }, \text { elec }}\right)^{-/+}-\left(E_{\text {mot }, \text { loss }}\right)^{-/+}-\left(E_{\text {dlmot }, \text { loss }}\right)^{-/+} \\
& \left(E_{\text {tr,credit }}\right)^{-/+}=\left(E_{\text {batt }, T}\right)^{-/+}-\left(E_{\text {mot }, \text { loss }}\right)^{-/+}-\left(E_{\text {dlmot }, \text { loss }}\right)^{-/+} \\
& \left(E_{\text {tr, credit }}\right)^{-/+}=\left(E_{\text {batt }, \text { int }}\right)^{-/+}-\left(E_{\text {batt }, \text { loss }}\right)^{-/+}-\left(E_{\text {mot }, \text { loss }}\right)^{-/+}-\left(E_{\text {dlmot }, \text { loss }}\right)^{-/+}
\end{aligned}
$$

Equation 241: Equivalent tractive energy 'credit' from regen in terms of losses (P3 Parallel, regen reuse) $\left(E_{\text {tr, credit }}\right)^{-/+}=\left(E_{\text {bat }, \text { int }}\right)^{-/+}-\left(E_{\text {pt,loss }}\right)^{-/+}=-\left(E_{\text {bat }, \text { int }}\right)^{-}-\left(E_{p t, \text { loss }}\right)^{-/+}$ 


\section{APPENDIX Q: Equations for Fuel-Equivalent Regen Energy Credit (P3/P4 Parallel HEV)}

This appendix contains the definitions for the component efficiencies used in the energy consumption equations developed for VTool. Some information may be duplicated in the body of this paper, but all relevant terms, definitions and equations have been reproduced here for convenience.

\begin{tabular}{|c|c|c|}
\hline Term & $\begin{array}{l}\text { Inherent } \\
\text { Sign }\end{array}$ & Definition \\
\hline$\left(E_{\text {eng,fuel }}\right)^{\text {EqFC }}$ & - & Theoretical fuel energy out of the engine to be used as fuel credit \\
\hline$\left(E_{\text {eng,mech }}\right)^{\text {EqFC }}$ & - & Theoretical mechanical energy in to the engine to be used as fuel credit \\
\hline$\left(\mathrm{E}_{\text {dleng,tr }}\right)^{\mathrm{EqFC}}$ & - & Theoretical tractive energy in to the engine driveline to be used as fuel credit \\
\hline$\left(E_{d l m o t, t r}\right)^{-E q F C}$ & + & Theoretical tractive energy out of the motor driveline. Analog to $\left(\mathrm{E}_{\text {dlmot,tr }}\right)^{+ \text {gen }}$ \\
\hline$\left(E_{\text {mot,mech }}\right)^{-\mathrm{EqFC}}$ & + & Theoretical mechanical energy out of the motor. Analog to $\left(E_{\text {mot,mech }}\right)^{\text {tgen }}$ \\
\hline$\left(\mathrm{E}_{\text {mot,elec }}\right)^{-\mathrm{EqFC}}$ & + & Theoretical electrical energy in to the motor. Analog to $\left(\mathrm{E}_{\text {mot,elec }}\right)^{\text {+gen }}$ \\
\hline 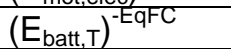 & + & Theoretical electrical energy at the battery terminals. Analog to $\left(E_{\text {batt }, T}\right)^{+g e n}$ \\
\hline$\left(E_{\text {batt,int }}\right)^{\text {-EqFC }}$ & + & Theoretical electrical energy inside the battery. Analog to $\left(\mathrm{E}_{\text {batt,int }}\right)^{\text {tgen }}$ \\
\hline$\left(\mathrm{E}_{\text {batt, int }}\right)^{+\mathrm{EqFC}}$ & - & Theoretical electrical energy inside the battery. Analog to $\left(E_{\text {batt,int }}\right)^{+p r o p}$ \\
\hline$\left(E_{\text {batt }, T}\right)^{+E q F C}$ & - & Theoretical electrical energy at the battery terminals. Analog to $\left(E_{\text {batt, }, T}\right)^{+p r o p}$ \\
\hline$\left(\mathrm{E}_{\text {mot,elec }}\right)^{+\mathrm{EqFC}}$ & - & Theoretical electrical energy in to the motor. Analog to $\left(E_{\text {mot,elec }}\right)^{+ \text {prop }}$ \\
\hline$\left(\mathrm{E}_{\mathrm{mot}, \mathrm{mech}}\right)^{+\mathrm{EqFC}}$ & - & Theoretical mechanical energy out of the motor. Analog to $\left(E_{\text {mot,mech }}\right)^{+p r o p}$ \\
\hline$\left(E_{d l m o t, t r}\right)^{+E q F C}$ & - & Theoretical tractive energy out of the motor driveline. Analog to $\left(E_{d l m o t, t r}\right)^{+p r o p}$ \\
\hline$\left(E_{\text {tr,credit }}\right)^{\text {EqFC }}$ & - & Theoretical tractive energy in to the wheels to be used as fuel credit \\
\hline$\left(E_{t r, c r e d i t}\right)^{-1+}$ & + & Tractive energy at the wheels from stored regen energy reused for propulsion \\
\hline
\end{tabular}

Efficiency Expressions: (same as propel case because theoretical powerflow emulates propel)

Equation 78: Power Split Fraction, engine energy directly used (P3 Parallel, propel)

$$
\begin{gathered}
\tilde{\xi}=\frac{\left(E_{\text {eng,use }}\right)^{+}}{\left(E_{\text {dleng,tr }}\right)^{+}} \\
\text {Restated: } \\
\left(E_{\text {eng,use }}\right)^{+}=\tilde{\xi}\left(E_{\text {dleng,tr }}\right)^{+}
\end{gathered}
$$

Equation 79: Power Split Fraction, engine energy recirculated (P3 Parallel, propel)

$\left(E_{\text {eng }, \text { store }}\right)^{+}=\left(E_{\text {dlmot }, \text { tr }}\right)^{+ \text {gen }}=(-1)(1-\xi)\left(E_{\text {dleng,tr }}\right)^{+}$

Equation 193: Engine propel efficiency (P3 Parallel, propel)

$\tilde{\eta}_{\text {eng }}^{+}=\frac{\left(E_{\text {eng,mech }}\right)^{+}}{\left(E_{\text {eng,fuel }}\right)^{+}}$

Equation 194: Engine driveline propel efficiency (P3 Parallel, propel)

$\tilde{\eta}_{\text {dl,eng }}^{+}=\frac{\left(E_{\text {dleng,tr }}\right)^{+}}{\left(E_{\text {eng,mech }}\right)^{+}}$ 
Equation 195: Motor driveline brake efficiency (P3 Parallel, propel)

$\tilde{\eta}_{d l, m o t}^{+g e n}=\frac{\left(E_{\text {mot }, \text { mech }}\right)^{+g e n}}{\left(E_{\text {dlmot }, t r}\right)^{+g e n}} \approx \tilde{\eta}_{d l, m o t}^{-}$

Equation 196: Motor brake efficiency (P3 Parallel, propel)

$\tilde{\eta}_{\text {mot }}^{+ \text {gen }}=\frac{\left(E_{\text {mot }, \text { elec }}\right)^{+ \text {gen }}}{\left(E_{\text {mot }, \text { mech }}\right)^{+g e n}} \approx \tilde{\eta}_{\text {mot }}^{-}$

Equation 197: Battery charge efficiency (P3 Parallel, propel)

$\tilde{\eta}_{\text {batt }}^{+g e n}=\frac{\left(E_{\text {batt }, \text { int }}\right)^{+g e n}}{\left(E_{\text {batt }, T}\right)^{+g e n}} \approx \tilde{\eta}_{\text {batt }}^{-}$

Equation 198: Battery discharge efficiency (P3 Parallel, propel)

$\tilde{\eta}_{\text {batt }}^{+ \text {prop }}=\frac{\left(E_{\text {batt }, T}\right)^{+ \text {prop }}}{\left(E_{\text {batt }, \text { int }}\right)^{+ \text {prop }}} \approx \tilde{\eta}_{\text {batt }}^{+}$

Equation 199: Motor propel efficiency (P3 Parallel, propel)

$\tilde{\eta}_{\text {mot }}^{+ \text {prop }}=\frac{\left(E_{\text {mot, mech }}\right)^{+ \text {prop }}}{\left(E_{\text {mot }, \text { elec }}\right)^{+ \text {prop }}} \approx \tilde{\eta}_{\text {mot }}^{+}$

Equation 200: Motor driveline propel efficiency (P3 Parallel, propel)

$\tilde{\eta}_{d l, m o t}^{+ \text {prop }}=\frac{\left(E_{d l m o t, t r}\right)^{+ \text {prop }}}{\left(E_{\text {mot,mech }}\right)^{+ \text {prop }}} \approx \tilde{\eta}_{d l, m o t}^{+}$

Equation 84: Power Split Fraction energy recirculation efficiency (P3 Parallel, propel)

$\tilde{\eta}_{\text {recirc }}^{+}=\left(\tilde{\eta}_{d l, \text { mot }}^{+ \text {gen }}\right)\left(\tilde{\eta}_{\text {mot }}^{+ \text {gen }}\right)\left(\tilde{\eta}_{\text {batt }}^{+ \text {gen }}\right)\left(\tilde{\eta}_{\text {batt }}^{+ \text {prop }}\right)\left(\tilde{\eta}_{\text {mot }}^{+ \text {prop }}\right)\left(\tilde{\eta}_{d l, m o t}^{+ \text {prop }}\right)$

Equation 88: Propel case powertrain efficiency for tractive energy (P3 Parallel, propel)

$\tilde{\eta}_{p t}^{+t r}=\frac{\left(E_{\text {tr }}\right)^{+}}{\left(E_{\text {eng,fuel }}\right)^{+}}$ 


\section{Loss Expressions:}

Equation 242: Engine losses for tractive energy (P3 Parallel, propel)

$\left(E_{\text {eng }, \text { loss }}\right)^{E q F C}=\left(E_{\text {eng,fuel }}\right)^{E q F C}-\left(E_{\text {eng,mech }}\right)^{E q F C}$

Equation 243: Engine driveline losses for tractive energy (P3 Parallel, propel)

$\left(E_{\text {dleng, loss }}\right)^{E q F C}=\left(E_{\text {eng,mech }}\right)^{E q F C}-\left(E_{\text {dleng,tr }}\right)^{E q F C}$

Equation 244: Motor driveline generate losses for tractive energy (P3 Parallel, propel)

$\left(E_{\text {dlmot }, l o s s}\right)^{-E q F C}=\left(E_{\text {mot }, \text { mech }}\right)^{-E q F C}-\left(E_{\text {dlmot }, t r}\right)^{-E q F C}$

Equation 245: Motor generate losses for tractive energy (P3 Parallel, propel)

$\left(E_{\text {mot }, \text { loss }}\right)^{-E q F C}=\left(E_{\text {mot }, \text { elec }}\right)^{-E q F C}-\left(E_{\text {mot }, \text { mech }}\right)^{-E q F C}$

Equation 246: Battery charge losses for tractive energy (P3 Parallel, propel)

$\left(E_{\text {batt }, l o s s}\right)^{-E q F C}=\left(E_{\text {batt }, \text { int }}\right)^{-E q F C}-\left(E_{\text {batt }, T}\right)^{-E q F C}$

Equation 247: Battery discharge losses for tractive energy (P3 Parallel, propel)

$\left(E_{\text {batt }, l o s s}\right)^{+E q F C}=\left(E_{\text {batt }, \text { int }}\right)^{+E q F C}-\left(E_{\text {batt }, T}\right)^{+E q F C}$

Equation 248: Motor propel losses for tractive energy (P3 Parallel, propel)

$\left(E_{\text {mot }, \text { loss }}\right)^{+E q F C}=\left(E_{\text {mot }, \text { elec }}\right)^{+E q F C}-\left(E_{\text {mot }, \text { mech }}\right)^{+E q F C}$

Equation 249: Motor driveline propel losses for tractive energy (P3 Parallel, propel)

$\left(E_{\text {dlmot }, l o s s}\right)^{+E q F C}=\left(E_{\text {mot }, \text { mech }}\right)^{+E q F C}-\left(E_{\text {dlmot }, t r}\right)^{+E q F C}$

Equation 250: Motor driveline total losses for tractive energy (P3 Parallel, propel)

$\left(E_{\text {dlmot }, l o s s}\right)^{E q F C}=\left(E_{\text {dlmot }, l o s s}\right)^{-E q F C}+\left(E_{\text {dlmot }, l o s s}\right)^{+E q F C}$

Equation 251: Motor total losses for tractive energy (P3 Parallel, propel)

$\left(E_{m o t, l o s s}\right)^{E q F C}=\left(E_{m o t, l o s s}\right)^{-E q F C}+\left(E_{m o t, l o s s}\right)^{+E q F C}$

Equation 252: Battery total losses for tractive energy (P3 Parallel, propel)

$\left(E_{\text {batt }, \text { loss }}\right)^{E q F C}=\left(E_{\text {batt }, \text { loss }}\right)^{-E q F C}+\left(E_{\text {batt }, \text { loss }}\right)^{+E q F C}$

Equation 253: Powertrain losses for tractive energy (P3 Parallel, propel)

$\left(E_{\text {pt,loss }}\right)^{E q F C}=\left(E_{\text {eng,fuel }}\right)^{E q F C}-\left(E_{\text {tr,credit }}\right)^{E q F C}$

\section{Conservation of Energy Expressions:}

Equation 254: Conservation of energy for regen fuel credit (P3 Parallel, propel)

$\left(E_{\text {tr,credit }}\right)^{E q F C}=-\left(E_{\text {tr,credit }}\right)^{-/+}$ 


\section{Developing Equation for Fuel-Equivalent Regen Energy Credit (in terms of efficiencies):}

\section{**Begin from Propel Case for CS P3/P4 Parallel HEV}

Equation 92: Propel case total fuel energy consumption (P3 Parallel, propel)

$$
\begin{aligned}
& \left(E_{\text {eng,fuel }}\right)^{+}=\frac{\left(E_{\text {tr }}\right)^{+}}{\left[\xi+(1-\xi)\left(\tilde{\eta}_{\text {recirc }}^{+}\right)\right]\left(\tilde{\eta}_{\text {eng }}^{+}\right)\left(\tilde{\eta}_{\text {dl,eng }}^{+}\right)}+\frac{\left(E_{\text {Acc }}\right)^{+}}{\left(\tilde{\eta}_{\text {eng }}^{+}\right)\left(\tilde{\eta}_{\text {dl,eng }}^{+}\right)\left(\tilde{\eta}_{\text {dl,mot }}^{+}\right)\left(\tilde{\eta}_{\text {mot }}^{+}\right)} \\
& \left(E_{\text {eng,fuel }}\right)^{E q F C}=\frac{\left(E_{\text {tr,credit }}\right)^{-/+}}{\left[\xi+(1-\xi)\left(\tilde{\eta}_{\text {recirc }}^{+}\right)\right]\left(\tilde{\eta}_{\text {eng }}^{+}\right)\left(\tilde{\eta}_{\text {dl,eng }}^{+}\right)}+\frac{)^{+}}{\left(\tilde{n}^{+}\right)\left(\tilde{n}^{+}\right)\left(\tilde{\eta}^{+}\right)}
\end{aligned}
$$

Equation 98: Fuel-equivalent regen energy 'credit' (P3 Parallel, regen fuel credit)

$$
\left(E_{\text {eng,fuel }}\right)^{E q F C}=\frac{-\left(E_{\text {tr,credit }}\right)^{E q F C}}{\left[\xi+(1-\xi)\left(\tilde{\eta}_{\text {recirc }}^{+}\right)\right]\left(\tilde{\eta}_{\text {eng }}^{+}\right)\left(\tilde{\eta}_{\text {dl,eng }}^{+}\right)}
$$




\section{APPENDIX R: Equations for the Idle Case (P3/P4 Parallel HEV)}

This appendix contains the definitions for the component efficiencies used in the energy consumption equations developed for VTool. Some information may be duplicated in the body of this paper, but all relevant terms, definitions and equations have been reproduced here for convenience.

\begin{tabular}{|l|l|l|}
\hline Term & $\begin{array}{l}\text { Inherent } \\
\text { Sign }\end{array}$ & Definition \\
\hline$\left(E_{\text {eng,fuel }}\right)^{0}$ & + & Fuel energy in to the engine for during the idle case \\
\hline$\left(E_{\text {eng,mech }}\right)^{0}$ & + & Mechanical energy out of the engine for during the idle case \\
\hline$\left(E_{\text {dleng,tr }}\right)^{0}$ & + & Tractive energy out of the engine driveline for the idle case \\
\hline$\left(E_{\text {dlmot,tr }}\right)^{0}$ & - & Tractive energy in to the motor driveline during the idle case \\
\hline$\left(E_{\text {mot,mech }}\right)^{0}$ & - & Mechanical energy in to the motor during the idle case \\
\hline$\left(E_{\text {mot,elec }}\right)^{0}$ & - & Electrical energy out of the motor during the idle case \\
\hline$\left(E_{\text {acc }}\right)^{0}$ & + & Electrical energy siphoned off the HV bus for accessory loads during the idle case \\
\hline
\end{tabular}

Efficiency Expressions: (same as propel case because theoretical powerflow emulates propel)

Equation 255: Engine efficiency (P3 Parallel, idle)

$\tilde{\eta}_{\text {eng }}^{0}=\frac{\left(E_{\text {eng,mech }}\right)^{0}}{\left(E_{\text {eng,fuel }}\right)^{0}} \approx \tilde{\eta}_{\text {eng }}^{+}$

Equation 256: Engine driveline efficiency (P3 Parallel, idle)

$\tilde{\eta}_{\text {dl,eng }}^{0}=\frac{\left(E_{\text {dleng,tr }}\right)^{0}}{\left(E_{\text {eng,mech }}\right)^{0}} \approx \tilde{\eta}_{\text {dl,eng }}^{+}$

Equation 257: Motor driveline efficiency (P3 Parallel, idle)

$\tilde{\eta}_{d l, m o t}^{0}=\frac{\left(E_{\text {mot,mech }}\right)^{0}}{\left(E_{\text {dlmot }, t r}\right)^{0}} \approx \tilde{\eta}_{d l, m o t}^{+}$

Equation 258: Motor efficiency (P3 Parallel, idle)

$\tilde{\eta}_{\text {mot }}^{0}=\frac{\left(E_{\text {mot }, \text { elec }}\right)^{0}}{\left(E_{\text {mot }, \text { mech }}\right)^{0}} \approx \tilde{\eta}_{\text {mot }}^{+}$

\section{Loss Expressions:}

Equation 259: Engine losses (P3 Parallel, idle)

$\left(E_{\text {eng,loss }}\right)^{0}=\left(E_{\text {eng,fuel }}\right)^{0}-\left(E_{\text {eng,mech }}\right)^{0}$

Equation 260: Engine driveline losses (P3 Parallel, idle)

$\left(E_{\text {dleng,loss }}\right)^{0}=\left(E_{\text {eng }, \text { mech }}\right)^{0}-\left(E_{\text {dleng,tr }}\right)^{0}$

Equation 261: Motor driveline losses (P3 Parallel, idle) 
$\left(E_{\text {dlmot }, \text { loss }}\right)^{0}=\left(E_{\text {mot }, \text { mech }}\right)^{0}-\left(E_{\text {dlmot }, t r}\right)^{0}$

Equation 262: Motor losses (P3 Parallel, idle)

$\left(E_{\text {mot }, \text { loss }}\right)^{0}=\left(E_{\text {mot,elec }}\right)^{0}-\left(E_{\text {mot }, \text { mech }}\right)^{0}$

Equation 263: Powertrain losses (P3 Parallel, idle)

$\left(E_{\text {pt,loss }}\right)^{0}=\left(E_{\text {eng,fuel }}\right)^{0}-\left(E_{\text {Acc }}\right)^{0}$

\section{Conservation of Energy Expressions:}

Equation 264: Conservation of energy at the HV Bus (P3 Parallel, idle)

$\left(E_{\text {Acc }}\right)^{0}=-\left(E_{\text {mot }, \text { elec }}\right)^{0}$

Equation 265: Conservation of energy at the drive axle (P3 Parallel, idle)

$\left(E_{\text {dleng }, t r}\right)^{0}=-\left(E_{\text {dlmot }, t r}\right)^{0}$

\section{Accessory Energy:}

Equation 36: Idle accessory energy

$\left(E_{\text {Acc }}\right)^{0}=\left(t_{\text {idle }}\right)\left(P_{\text {Acc }}\right)^{0}$

\section{Developing Equation for Idle Case Energy Consumption (in terms of efficiencies):}

$$
\begin{aligned}
& \left(E_{\text {eng,fuel }}\right)^{0}=\frac{\left(E_{\text {eng,mech }}\right)^{0}}{\tilde{\eta}_{\text {eng }}^{0}} \\
& \left(E_{\text {eng,fuel }}\right)^{0}=\frac{\left(E_{\text {dleng,tr }}\right)^{0}}{\left(\tilde{\eta}_{\text {eng }}^{0}\right)\left(\tilde{\eta}_{\text {dl,eng }}^{0}\right)} \\
& \left(E_{\text {eng,fuel }}\right)^{0}=\frac{-\left(E_{\text {dlmot }, \text { tr }}\right)^{0}}{\left(\tilde{\eta}_{\text {eng }}^{0}\right)\left(\tilde{\eta}_{\text {dl,eng }}^{0}\right)} \\
& \left(E_{\text {eng,fuel }}\right)^{0}=\frac{-\left(E_{\text {mot,mech }}\right)^{0}}{\left(\tilde{\eta}_{\text {eng }}^{0}\right)\left(\tilde{\eta}_{\text {dl,eng }}^{0}\right)\left(\tilde{\eta}_{d l, m o t}^{0}\right)}
\end{aligned}
$$

Equation 99: Idle case energy consumption (P3 Parallel, idle)

$$
\left(E_{\text {eng,fuel }}\right)^{0}=\frac{-\left(E_{\text {mot,elec }}\right)^{0}}{\left(\tilde{\eta}_{\text {eng }}^{0}\right)\left(\tilde{\eta}_{d l, \text { eng }}^{0}\right)\left(\tilde{\eta}_{d l, m o t}^{0}\right)\left(\tilde{\eta}_{m o t}^{0}\right)}=\frac{\left(E_{\text {Acc }}\right)^{0}}{\left(\tilde{\eta}_{\text {eng }}^{0}\right)\left(\tilde{\eta}_{d l, e n g}^{0}\right)\left(\tilde{\eta}_{d l, m o t}^{0}\right)\left(\tilde{\eta}_{m o t}^{0}\right)}
$$




\section{Developing Equation for Idle Case Energy Consumption (in terms of losses):}

$$
\begin{aligned}
& \left(E_{\text {eng,fuel }}\right)^{0}=\left(E_{\text {eng, mech }}\right)^{0}+\left(E_{\text {eng,loss }}\right)^{0} \\
& \left(E_{\text {eng,fuel }}\right)^{0}=\left(E_{\text {dleng, } t r}\right)^{0}+\left(E_{\text {dleng,loss }}\right)^{0}+\left(E_{\text {eng }, \text { loss }}\right)^{0} \\
& \left(E_{\text {eng,fuel }}\right)^{0}=-\left(E_{\text {dlmot }, \text { tr }}\right)^{0}+\left(E_{\text {dleng,loss }}\right)^{0}+\left(E_{\text {eng }, \text { loss }}\right)^{0} \\
& \left(E_{\text {eng }, f u e l}\right)^{0}=-\left(E_{\text {mot }, \text { mech }}\right)^{0}+\left(E_{\text {dlmot }, \text { loss }}\right)^{0}+\left(E_{\text {dleng,loss }}\right)^{0}+\left(E_{\text {eng }, \text { loss }}\right)^{0} \\
& \left(E_{\text {eng }, \text { fuel }}\right)^{0}=-\left(E_{\text {mot }, \text { elec }}\right)^{0}+\left(E_{\text {mot }, \text { loss }}\right)^{0}+\left(E_{\text {dlmot }, \text { loss }}\right)^{0}+\left(E_{\text {dleng,loss }}\right)^{0}+\left(E_{\text {eng, loss }}\right)^{0}
\end{aligned}
$$

Equation 266: Idle case energy consumption in terms of losses (P3 Parallel, idle)

$$
\left(E_{\text {eng }, \text { fuel }}\right)^{0}=\left(E_{\text {Acc }}\right)^{0}+\left(E_{\text {mot }, \text { loss }}\right)^{0}+\left(E_{\text {dlmot }, \text { loss }}\right)^{0}+\left(E_{\text {dleng,loss }}\right)^{0}+\left(E_{\text {eng }, \text { loss }}\right)^{0}
$$




\section{APPENDIX S: Equations for the DCnet Case (P3/P4 Parallel HEV)}

This appendix contains the definitions for the component efficiencies used in the energy consumption equations developed for VTool. Some information may be duplicated in the body of this paper, but all relevant terms, definitions and equations have been reproduced here for convenience.

\begin{tabular}{|c|c|c|}
\hline Term & $\begin{array}{l}\text { Inherent } \\
\text { Sign }\end{array}$ & Definition \\
\hline$\left(E_{\text {batt,int }}\right)^{-}$ & - & Electrical energy inside the battery captured during the brake case \\
\hline$\left(E_{\text {batt,int }}\right)^{-1+}$ & + & Net electrical energy inside the battery available to be reused for propulsion \\
\hline$\left(\mathrm{E}_{\text {batt,int }}\right)^{+ \text {gen }}$ & - & Electrical energy inside the battery stored from excess generating \\
\hline$\left(\mathrm{E}_{\text {batt,int }}\right)^{+ \text {prop }}$ & + & Electrical energy inside the battery to be used in propulsion \\
\hline$\left(\mathrm{E}_{\text {batt,int }}\right)^{\mathrm{DCnet}}$ & + & Net electrical energy inside the battery for a given drive cycle \\
\hline$\left(E_{t r, \text { credit }}\right)^{-1+}$ & + & Tractive energy at the wheels from stored regen energy reused for propulsion \\
\hline$\left(E_{\text {eng,fuel }}\right)^{+}$ & + & Fuel energy in to the engine during the propel case \\
\hline$\left(E_{\text {eng,fuel }}\right)^{0}$ & + & Fuel energy in to the engine during the idle case \\
\hline$\left(E_{\text {eng.fuel }}\right)^{\text {EqFC }}$ & - & Theoretical fuel energy out of the engine to be used as fuel credit \\
\hline$\left(E_{t r}\right)^{B}$ & + & Net tractive energy at the wheels for a given drive cycle (Road Load) \\
\hline
\end{tabular}

\section{Efficiency Expressions:}

Equation 103: Net powertrain efficiency (P3 Parallel, DCnet)

$\tilde{\eta}_{p t}^{\text {DCnet }}=\frac{\left(E_{\text {tr }}\right)^{\text {DCnet }}}{\left(E_{\text {eng,fuel }}\right)^{\text {DCnet }}}$

Equation 95: Propel case ICE powertrain efficiency (P3 Parallel, propel)

$\tilde{\eta}_{p t, I C E}^{+}=\frac{\left(E_{\text {tr }}\right)^{+}}{\left(E_{\text {eng,fuel }}\right)^{+}}$

Equation 97: Powertrain efficiency for regen energy reuse (P3 Parallel, regen reuse)

$\tilde{\eta}_{p t, E V}^{-/+}=\frac{\left(E_{\text {tr,credit }}\right)^{-/+}}{\left(E_{\text {batt }, \text { int }}\right)^{-/+}}$

Equation 104: Net powertrain efficiency for combined propel operation (P3 Parallel, DCnet)

$\tilde{\eta}_{\text {pt,propel }}^{\text {DCnet }}=\frac{\left(E_{\text {tr }}\right)^{+}+\left(E_{\text {tr,credit }}\right)^{E q F C}}{\left(E_{\text {eng,fuel }}\right)^{+}+\left(E_{\text {batt }, \text { int }}\right)^{-/+}}$

Equation 35: Brake case powertrain efficiency (EV, brake)

$\tilde{\eta}_{p t}^{-}=\frac{\left(E_{\text {batt }, i n t}\right)^{-}}{\left(E_{t r}\right)^{-}}$ 


\section{Loss Expressions:}

Equation 144: Net friction brake losses (EV, DCnet)

$\left(E_{\text {brk }, \text { loss }}\right)^{\text {DCnet }}=\left(E_{\text {brk, loss }}\right)^{-}$

Equation 267: Net engine driveline losses (P3 Parallel, DCnet)

$\left(E_{\text {dleng, loss }}\right)^{\text {DCnet }}=\left(E_{\text {dleng, loss }}\right)^{+}+\left(E_{\text {dleng,loss }}\right)^{-}+\left(E_{\text {dleng, loss }}\right)^{0}+\left(E_{\text {dleng,loss }}\right)^{E q F C}$

Equation 268: Net motor driveline losses (P3 Parallel, DCnet)

$\left(E_{\text {dlmot }, \text { loss }}\right)^{\text {DCnet }}=\left(E_{\text {dlmot }, \text { loss }}\right)^{+}+\left(E_{\text {dlmot }, \text { loss }}\right)^{-}+\left(E_{\text {dlmot }, \text { loss }}\right)^{-/+}+\left(E_{\text {dlmot }, \text { loss }}\right)^{0}+\left(E_{\text {mot }, \text { loss }}\right)^{E q F C}$

Equation 269: Net motor losses (P3 Parallel, DCnet)

$\left(E_{\text {mot }, \text { loss }}\right)^{\text {DCnet }}=\left(E_{\text {mot,loss }}\right)^{+}+\left(E_{\text {mot }, \text { loss }}\right)^{-}+\left(E_{\text {mot }, \text { loss }}\right)^{-/+}+\left(E_{\text {mot }, \text { loss }}\right)^{0}+\left(E_{\text {mot }, \text { loss }}\right)^{E q F C}$

Equation 270: Net battery losses (P3 Parallel, DCnet)

$\left(E_{\text {batt }, \text { loss }}\right)^{\text {DCnet }}=\left(E_{\text {batt }, \text { loss }}\right)^{+}+\left(E_{\text {batt }, \text { loss }}\right)^{-}+\left(E_{\text {batt }, \text { loss }}\right)^{-/+}+\left(E_{\text {batt }, \text { loss }}\right)^{0}+\left(E_{\text {batt }, \text { loss }}\right)^{\text {EqFC }}$

Equation 271: Net engine losses (P3 Parallel, DCnet)

$\left(E_{\text {eng,loss }}\right)^{\text {DCnet }}=\left(E_{\text {eng,loss }}\right)^{+}+\left(E_{\text {eng,loss }}\right)^{0}+\left(E_{\text {eng,loss }}\right)^{E q F C}$

Equation 102: Net powertrain losses (P3 Parallel, DCnet)

$\left(E_{p t, l o s s}\right)^{\text {DCnet }}=\left(E_{p t, l o s s}\right)^{+}+\left(E_{p t, l o s s}\right)^{-}+\left(E_{p t, l o s s}\right)^{-/+}+\left(E_{p t, l o s s}\right)^{0}+\left(E_{p t, l o s s}\right)^{E q F C}$

\section{Conservation of Energy Expressions:}

Equation 240: Conservation of energy for regen energy capture and reuse (P3 Parallel, regen reuse)

$\left(E_{\text {batt }, \text { int }}\right)^{-/+}+\left(E_{\text {batt }, \text { int }}\right)^{-}=0$

Equation 241: Equivalent tractive energy 'credit' from regen in terms of losses (P3 Parallel, regen reuse)

$\left(E_{\text {batt }, \text { int }}\right)^{-/+}=\left(E_{\text {tr,credit }}\right)^{-/+}+\left(E_{\text {pt,loss }}\right)^{-/+}$

Equation 82: Conservation of energy in the battery to maintain charge balance (P3 Parallel, propel)

$\left(E_{\text {batt }, \text { int }}\right)^{+ \text {prop }}+\left(E_{\text {batt }, \text { int }}\right)^{+ \text {gen }}=0$

Equation 272: Energy balance for overall charge balance (P3 Parallel, DCnet)

$\left(E_{\text {batt }, \text { int }}\right)^{+ \text {prop }}+\left(E_{\text {batt }, \text { int }}\right)^{+ \text {gen }}+\left(E_{\text {batt,int }}\right)^{-}+\left(E_{\text {tr,credit }}\right)^{-/+}+\left(E_{\text {pt,loss }}\right)^{-/+}=0$ 
Equation 100: Total fuel energy calculated by case (P3 Parallel, DCnet)

$\left(E_{\text {eng,fuel }}\right)^{\text {DCnet }}=\left(E_{\text {eng,fuel }}\right)^{+}+\left(E_{\text {eng,fuel }}\right)^{0}+\left(E_{\text {eng,fuel }}\right)^{E q F C}$

Equation 101: Total fuel energy calculated from overall energy balance (P3 Parallel, DCnet) $\left(E_{\text {eng,fuel }}\right)^{\text {DCnet }}=\left(E_{\text {tr }}\right)^{\text {DCnet }}+\left(E_{A C C}\right)^{\text {DCnet }}+\left(E_{\text {pt,loss }}\right)^{\text {DCnet }}$

\section{Accessory Energy:}

Equation 41: Net accessory energy

$\left(E_{A C C}\right)^{\text {DCnet }}=\left(E_{A C C}\right)^{+}+\left(E_{A C C}\right)^{-}+\left(E_{A C C}\right)^{0}=P_{A C C} * T$ 


\section{APPENDIX T: Equations for Tractive Energy in the Propel Case (P2 Parallel HEV)}

This appendix contains the definitions for the component efficiencies used in the energy consumption equations developed for VTool. Some information may be duplicated in the body of this paper, but all relevant terms, definitions and equations have been reproduced here for convenience.

\begin{tabular}{|c|c|c|}
\hline Term & $\begin{array}{l}\text { Inherent } \\
\text { Sign }\end{array}$ & Definition \\
\hline$\left(E_{\text {eng,fuel }}\right)^{+t r}$ & + & Fuel energy in to the engine used for tractive power during the propel case \\
\hline$\left(\mathrm{E}_{\text {eng,mech }}\right)^{+}$ & + & Mechanical energy out of the engine during the propel case \\
\hline$\left(\mathrm{E}_{\text {eng.use }}\right)^{+}$ & + & Tractive energy from the engine directly used for propulsion \\
\hline$\left(\mathrm{E}_{\text {eng,store }}\right)^{+}$ & - & Tractive energy from the engine stored before being used for propulsion \\
\hline$\left(\mathrm{E}_{\mathrm{dlm} \text { mot,tr }}\right)^{+ \text {tgen }}$ & - & Excess tractive energy in to the motor driveline from the drive axle to be stored \\
\hline$\left(\mathrm{E}_{\text {mot,mech }}\right)^{+\mathrm{gen}}$ & - & Mechanical energy in to the motor to be stored for future propulsion \\
\hline$\left(E_{\text {mot,elec }}\right)^{+ \text {gen }}$ & - & Electrical energy out of the motor to be stored \\
\hline$\left(E_{\text {batt }, T}\right)^{+ \text {gen }}$ & - & Electrical energy in to the battery at the terminals to be stored \\
\hline$\left(\mathrm{E}_{\text {batt,int }}\right)^{+ \text {gen }}$ & - & Electrical energy inside the battery stored from excess generating \\
\hline$\left(\mathrm{E}_{\text {batt, int }}\right)^{+ \text {prop }}$ & + & Electrical energy inside the battery to be used in propulsion \\
\hline$\left(\mathrm{E}_{\text {batt }, \mathrm{T}}\right)^{+ \text {prop }}$ & + & Electrical energy out of the battery at the terminals to be used \\
\hline$\left(E_{\text {mot,elec }}\right)^{+ \text {prop }}$ & + & Electrical energy in to the motor to be used \\
\hline$\left(E_{\text {mot,mech }}\right)^{+ \text {prop }}$ & + & Mechanical energy out of the motor to be used for propulsion \\
\hline$\left(E_{\text {dlmot,tr }}\right)^{+ \text {prop }}$ & + & Surplus tractive energy out of the motor $\mathrm{D} / \mathrm{L}$ from the drive axle to be used \\
\hline$\left(\mathrm{E}_{\mathrm{e} \& \mathrm{~m}, \mathrm{mech}}\right)^{+}$ & + & Sum of mechanical energy from engine and motor in to engine driveline \\
\hline$\left(E_{t r}\right)^{+}$ & + & Tractive energy at the wheels for the propel case \\
\hline
\end{tabular}

\section{Efficiency Expressions:}

Equation 105: Power Split Fraction, engine energy directly used (P2 Parallel, propel)

$$
\xi=\frac{\left(E_{\text {eng,use }}\right)^{+}}{\left(E_{\text {eng, mech }}\right)^{+}}
$$

Restated:

$$
\left(E_{\text {eng }, \text { use }}\right)^{+}=\tilde{\xi}\left(E_{\text {eng,mech }}\right)^{+}
$$

Equation 106: Power Split Fraction, engine energy recirculated (P2 Parallel, propel)

$$
\left(E_{\text {eng }, \text { store }}\right)^{+}=\left(E_{\text {dlmot }, t r}\right)^{+ \text {gen }}=(-1)(1-\xi)\left(E_{\text {eng,mech }}\right)^{+}
$$

Equation 273: Engine propel efficiency (P2 Parallel, propel)

$$
\tilde{\eta}_{\text {eng }}^{+}=\frac{\left(E_{\text {eng,mech }}\right)^{+}}{\left(E_{\text {eng,fuel }}\right)^{+t r}}
$$

Equation 274: Engine driveline propel efficiency (P2 Parallel, propel)

$$
\tilde{\eta}_{\text {dl,eng }}^{+}=\frac{\left(E_{\text {dleng,tr }}\right)^{+}}{\left(E_{\text {e\&m,mech }}\right)^{+}}
$$


Equation 275: Motor driveline brake efficiency (P2 Parallel, propel)

$\tilde{\eta}_{d l, m o t}^{+g e n}=\frac{\left(E_{\text {mot }, \text { mech }}\right)^{+g e n}}{\left(E_{\text {dlmot }, t r}\right)^{+g e n}} \approx \tilde{\eta}_{d l, m o t}^{-}$

Equation 276: Motor brake efficiency (P2 Parallel, propel)

$\tilde{\eta}_{\text {mot }}^{+ \text {gen }}=\frac{\left(E_{\text {mot }, \text { elec }}\right)^{+ \text {gen }}}{\left(E_{\text {mot }, \text { mech }}\right)^{+g e n}} \approx \tilde{\eta}_{\text {mot }}^{-}$

Equation 277: Battery charge efficiency (P2 Parallel, propel)

$\tilde{\eta}_{\text {batt }}^{+g e n}=\frac{\left(E_{\text {batt }, i n t}\right)^{+g e n}}{\left(E_{\text {batt }, T}\right)^{+g e n}} \approx \tilde{\eta}_{\text {batt }}^{-}$

Equation 278: Battery discharge efficiency (P2 Parallel, propel)

$\tilde{\eta}_{\text {batt }}^{+ \text {prop }}=\frac{\left(E_{\text {batt }, T}\right)^{+ \text {prop }}}{\left(E_{\text {batt }, i n t}\right)^{+ \text {prop }}} \approx \tilde{\eta}_{\text {batt }}^{+}$

Equation 279: Motor propel efficiency (P2 Parallel, propel)

$\tilde{\eta}_{\text {mot }}^{+ \text {prop }}=\frac{\left(E_{\text {mot }, \text { mech }}\right)^{+ \text {prop }}}{\left(E_{\text {mot }, \text { elec }}\right)^{+ \text {prop }}} \approx \tilde{\eta}_{\text {mot }}^{+}$

Equation 280: Motor driveline propel efficiency (P2 Parallel, propel)

$\tilde{\eta}_{d l, m o t}^{+ \text {prop }}=\frac{\left(E_{\text {dlmot }, t r}\right)^{+ \text {prop }}}{\left(E_{\text {mot }, \text { mech }}\right)^{+ \text {prop }}} \approx \tilde{\eta}_{d l, m o t}^{+}$

Equation 108: Power Split Fraction energy recirculation efficiency (P2 Parallel, propel)

$\tilde{\eta}_{\text {recirc }}^{+}=\left(\tilde{\eta}_{\text {dl,mot }}^{+ \text {gen }}\right)\left(\tilde{\eta}_{\text {mot }}^{+ \text {gen }}\right)\left(\tilde{\eta}_{\text {batt }}^{+ \text {gen }}\right)\left(\tilde{\eta}_{\text {batt }}^{+ \text {prop }}\right)\left(\tilde{\eta}_{\text {mot }}^{+ \text {prop }}\right)\left(\tilde{\eta}_{d l, m o t}^{+ \text {prop }}\right)$

Equation 109: Propel case powertrain efficiency for tractive energy (P2 Parallel, propel)

$\tilde{\eta}_{p t}^{+t r}=\frac{\left(E_{\text {tr }}\right)^{+}}{\left(E_{\text {eng,fuel }}\right)^{+}}$ 


\section{Loss Expressions:}

Equation 281: Engine losses for tractive energy (P2 Parallel, propel)

$\left(E_{\text {eng,loss }}\right)^{+t r}=\left(E_{\text {eng,fuel }}\right)^{+t r}-\left(E_{\text {eng,mech }}\right)^{+}$

Equation 282: Engine driveline losses for tractive energy (P2 Parallel, propel)

$\left(E_{\text {dleng,loss }}\right)^{+t r}=\left(E_{\text {e\&m,mech }}\right)^{+}-\left(E_{\text {tr }}\right)^{+}$

Equation 283: Motor driveline generate losses for tractive energy (P2 Parallel, propel)

$\left(E_{d l m o t, l o s s}\right)^{+g e n}=\left(E_{\text {mot }, \text { mech }}\right)^{+g e n}-\left(E_{d l m o t, t r}\right)^{+g e n}$

Equation 284: Motor generate losses for tractive energy (P2 Parallel, propel)

$\left(E_{\text {mot }, \text { loss }}\right)^{+ \text {gen }}=\left(E_{\text {mot }, \text { elec }}\right)^{+ \text {gen }}-\left(E_{\text {mot }, \text { mech }}\right)^{+ \text {gen }}$

Equation 285: Battery charge losses for tractive energy (P2 Parallel, propel)

$\left(E_{\text {batt }, \text { loss }}\right)^{+ \text {gen }}=\left(E_{\text {batt }, \text { int }}\right)^{+ \text {gen }}-\left(E_{\text {batt }, T}\right)^{+ \text {gen }}$

Equation 286: Battery discharge losses for tractive energy (P2 Parallel, propel)

$\left(E_{\text {batt }, \text { loss }}\right)^{+ \text {prop }}=\left(E_{\text {batt }, \text { int }}\right)^{+ \text {prop }}-\left(E_{\text {batt }, T}\right)^{+ \text {prop }}$

Equation 287: Motor propel losses for tractive energy (P2 Parallel, propel)

$\left(E_{\text {mot }, \text { loss }}\right)^{+ \text {prop }}=\left(E_{\text {mot }, \text { elec }}\right)^{+ \text {prop }}-\left(E_{\text {mot }, \text { mech }}\right)^{+ \text {prop }}$

Equation 288: Motor driveline propel losses for tractive energy (P2 Parallel, propel)

$\left(E_{\text {dlmot }, l o s s}\right)^{+ \text {prop }}=\left(E_{\text {mot }, \text { mech }}\right)^{+ \text {prop }}-\left(E_{\text {dlmot }, t r}\right)^{+ \text {prop }}$

Equation 289: Motor driveline total losses for tractive energy (P2 Parallel, propel)

$\left(E_{\text {dlmot }, \text { loss }}\right)^{+t r}=\left(E_{\text {dlmot }, \text { loss }}\right)^{+ \text {gen }}+\left(E_{\text {dlmot }, \text { loss }}\right)^{+ \text {prop }}$

Equation 290: Motor total losses for tractive energy (P2 Parallel, propel)

$\left(E_{\text {mot }, l o s s}\right)^{+t r}=\left(E_{\text {mot }, \text { loss }}\right)^{+ \text {gen }}+\left(E_{\text {mot }, l o s s}\right)^{+ \text {prop }}$

Equation 291: Battery total losses for tractive energy (P2 Parallel, propel)

$\left(E_{\text {batt }, \text { loss }}\right)^{+t r}=\left(E_{\text {batt }, \text { loss }}\right)^{+ \text {gen }}+\left(E_{\text {batt }, \text { loss }}\right)^{+ \text {prop }}$

Equation 292: Powertrain losses for tractive energy (P2 Parallel, propel)

$\left(E_{\text {pt,loss }}\right)^{+t r}=\left(E_{\text {eng,loss }}\right)^{+t r}+\left(E_{\text {dleng,loss }}\right)^{+t r}+\left(E_{\text {dlmot,loss }}\right)^{+t r}+\left(E_{\text {mot }, \text { loss }}\right)^{+t r}+\left(E_{\text {batt }, \text { loss }}\right)^{+t r}$

Restated:

$\left(E_{p t, l o s s}\right)^{+t r}=\left(E_{\text {eng,fuel }}\right)^{+t r}-\left(E_{t r}\right)^{+}$ 


\section{Conservation of Energy Expressions:}

Equation 293: Conservation of energy 'inside' the pre-trans node (P2 Parallel, propel)

$\left(E_{\text {e\&m,mech }}\right)^{+}=\left(E_{\text {eng }, \text { use }}\right)^{+}+\left(E_{\text {dlmot }, t r}\right)^{+ \text {prop }}$

Equation 294: Conservation of energy at or 'outside' the pre-trans node (P2 Parallel, propel)

$\left(E_{\text {e\&m,mech }}\right)^{+}=\left(E_{\text {eng }, \text { mech }}\right)^{+}+\left(E_{\text {dlmot }, t r}\right)^{+ \text {prop }}+\left(E_{\text {dlmot }, t r}\right)^{+g e n}$

Equation 295: Conservation of energy at the HV bus while charging (P2 Parallel, propel)

$\left(E_{\text {batt }, T}\right)^{+g e n}=\left(E_{\text {mot }, \text { elec }}\right)^{+g e n}$

Equation 296: Conservation of energy at the HV bus while discharging (P2 Parallel, propel) $\left(E_{\text {batt }, T}\right)^{+ \text {prop }}=\left(E_{\text {mot,elec }}\right)^{+ \text {prop }}$

Equation 297: Conservation of energy for the Power Split Fraction (P2 Parallel, propel) $\left(E_{\text {dleng,tr }}\right)^{+}=\left(E_{\text {eng,use }}\right)^{+}-\left(E_{\text {dlmot }, t r}\right)^{+g e n}$

Equation 298: Conservation of energy in the battery to maintain charge balance (P2 Parallel, propel) $\left(E_{\text {batt }, \text { int }}\right)^{+ \text {prop }}=-\left(E_{\text {batt }, \text { int }}\right)^{+ \text {gen }}$

\section{Developing Equation for Power Split Fraction (in terms of efficiencies):}

- Storing Energy in the Battery During the Propel Case:

$$
\begin{aligned}
& \left(E_{\text {batt }, \text { int }}\right)^{+g e n}=\left(E_{\text {batt }, T}\right)^{+g e n}\left(\tilde{\eta}_{\text {batt }}^{+ \text {gen }}\right) \\
& \left(E_{\text {batt }, \text { int }}\right)^{+g e n}=\left(E_{\text {mot }, \text { elec }}\right)^{+g e n}\left(\tilde{\eta}_{\text {batt }}^{+ \text {gen }}\right) \\
& \left(E_{\text {batt }, \text { int }}\right)^{+g e n}=\left(E_{\text {mot }, \text { mech }}\right)^{+g e n}\left(\tilde{\eta}_{\text {mot }}^{+g e n}\right)\left(\tilde{\eta}_{\text {batt }}^{+g e n}\right) \\
& \left(E_{\text {batt }, \text { int }}\right)^{+g e n}=\left(E_{\text {dlmot }, t r}\right)^{+g e n}\left(\tilde{\eta}_{\text {dl,mot }}^{+ \text {gen }}\right)\left(\tilde{\eta}_{\text {mot }}^{+ \text {gen }}\right)\left(\tilde{\eta}_{\text {batt }}^{+g e n}\right)
\end{aligned}
$$

Equation 299: Energy stored in the battery during times of surplus power generation (P2 Parallel, propel)

$$
\left(E_{\text {batt }, \text { int }}\right)^{+ \text {gen }}=(-1)(1-\xi)\left(E_{\text {eng,mech }}\right)^{+}\left(\tilde{\eta}_{d l, m o t}^{+g e n}\right)\left(\tilde{\eta}_{\text {mot }}^{+g e n}\right)\left(\tilde{\eta}_{\text {batt }}^{+g e n}\right)
$$




\section{- Using Energy from the Battery During the Propel Case:}

$$
\begin{aligned}
& \left(E_{\text {batt }, \text { int }}\right)^{+ \text {prop }}=\frac{\left(E_{\text {batt }, T}\right)^{+ \text {prop }}}{\tilde{\eta}_{\text {batt }}^{+ \text {prop }}} \\
& \left(E_{\text {batt }, \text { int }}\right)^{+ \text {prop }}=\frac{\left(E_{\text {mot }, \text { elec }}\right)^{+ \text {prop }}}{\tilde{\eta}_{\text {batt }}^{+ \text {prop }}} \\
& \left(E_{\text {batt }, \text { int }}\right)^{+ \text {prop }}=\frac{\left(E_{\text {mot }, \text { mech }}\right)^{+ \text {prop }}}{\left(\tilde{\eta}_{\text {batt }}^{+ \text {prop }}\right)\left(\tilde{\eta}_{\text {mot }}^{+ \text {prop }}\right)}
\end{aligned}
$$

Equation 300: Energy used from the battery during shortages of power generation (P2 Parallel, propel)

$$
\left(E_{\text {batt }, \text { int }}\right)^{+ \text {prop }}=\frac{\left(E_{\text {dlmot }, \text { tr }}\right)^{+ \text {prop }}}{\left(\tilde{\eta}_{\text {batt }}^{+ \text {prop }}\right)\left(\tilde{\eta}_{\text {mot }}^{+ \text {prop }}\right)\left(\tilde{\eta}_{\text {dl,mot }}^{+ \text {prop }}\right)}
$$

\section{- Conservation of Energy to Maintain Charge Balance:}

Equation 298: Conservation of energy in the battery to maintain charge balance (P2 Parallel, propel)

$$
\begin{aligned}
& \left(E_{\text {batt }, \text { int }}\right)^{+ \text {prop }}=-\left(E_{\text {batt }, \text { int }}\right)^{+ \text {gen }} \\
& (-1)(1-\xi)\left(E_{\text {eng,mech }}\right)^{+}\left(\tilde{\eta}_{d l, m o t}^{+g e n}\right)\left(\tilde{\eta}_{\text {mot }}^{+g e n}\right)\left(\tilde{\eta}_{\text {batt }}^{+g e n}\right)=\frac{-\left(E_{\text {dlmot,tr }}\right)^{+ \text {prop }}}{\left(\tilde{\eta}_{\text {batt }}^{+ \text {prop }}\right)\left(\tilde{\eta}_{\text {mot }}^{+ \text {prop }}\right)\left(\tilde{\eta}_{d l, m o t}^{+ \text {prop }}\right)} \\
& (1-\xi)\left(E_{\text {eng,mech }}\right)^{+}\left(\tilde{\eta}_{\text {dl,mot }}^{+ \text {gen }}\right)\left(\tilde{\eta}_{\text {mot }}^{+g e n}\right)\left(\tilde{\eta}_{\text {batt }}^{+g e n}\right)=\frac{\left(E_{\text {dlmot,tr }}\right)^{+ \text {prop }}}{\left(\tilde{\eta}_{\text {batt }}^{+ \text {prop }}\right)\left(\tilde{\eta}_{\text {mot }}^{+ \text {prop }}\right)\left(\tilde{\eta}_{d l, m o t}^{+ \text {prop }}\right)} \\
& \left(E_{\text {eng,mech }}\right)^{+}=\frac{\left(E_{\text {dlmot,tr }}\right)^{+ \text {prop }}}{(1-\xi)\left(\tilde{\eta}_{d l, m o t}^{+g e n}\right)\left(\tilde{\eta}_{\text {mot }}^{+g e n}\right)\left(\tilde{\eta}_{\text {batt }}^{+g e n}\right)\left(\tilde{\eta}_{\text {batt }}^{+ \text {prop }}\right)\left(\tilde{\eta}_{\text {mot }}^{+ \text {prop }}\right)\left(\tilde{\eta}_{d l, m o t}^{+ \text {prop }}\right)} \\
& \left(E_{\text {eng,mech }}\right)^{+}=\frac{\left(E_{\text {dlmot }, t r}\right)^{+ \text {prop }}}{(1-\xi)\left(\tilde{\eta}_{\text {recirc }}^{+}\right)} \\
& \left(E_{\text {eng }, \text { mech }}\right)^{+}(1-\xi)\left(\tilde{\eta}_{\text {recirc }}^{+}\right)=\left(E_{\text {dlmot }, t r}\right)^{+ \text {prop }}
\end{aligned}
$$

Equation 301: Energy from the motor onto the drive axle during the propel case (P2 Parallel, propel)

$\left(E_{\text {dlmot }, t r}\right)^{+ \text {prop }}=\left(E_{\text {eng,mech }}\right)^{+}(1-\xi)\left(\tilde{\eta}_{\text {recirc }}^{+}\right)$ 


\section{- Conservation of Energy at the pre-trans node:}

Equation 293: Conservation of energy 'inside' the pre-trans node (P2 Parallel, propel)

$$
\begin{aligned}
& \left(E_{\text {e\&m,mech }}\right)^{+}=\left(E_{\text {eng,use }}\right)^{+}+\left(E_{\text {dlmot }, \text { tr }}\right)^{+ \text {prop }} \\
& \left(E_{\text {eng,use }}\right)^{+}=\left(E_{\text {e\&m,mech }}\right)^{+}-\left(E_{\text {dlmot }, \text { tr }}\right)^{+ \text {prop }} \\
& \xi\left(E_{\text {eng,mech }}\right)^{+}=\left(E_{\text {e\&m,mech }}\right)^{+}-\left[\left(E_{\text {eng,mech }}\right)^{+}(1-\xi)\left(\tilde{\eta}_{\text {recirc }}^{+}\right)\right] \\
& \xi\left(E_{\text {eng,mech }}\right)^{+}+\left(E_{\text {eng,mech }}\right)^{+}(1-\xi)\left(\tilde{\eta}_{\text {recirc }}^{+}\right)=\left(E_{\text {e\&m,mech }}\right)^{+} \\
& \left(E_{\text {eng,mech }}\right)^{+}\left[\xi+(1-\xi)\left(\tilde{\eta}_{\text {recirc }}^{+}\right)\right]=\left(E_{\text {e\&m,mech }}\right)^{+} \\
& \left(E_{\text {eng,mech }}\right)^{+}=\frac{\left(E_{\text {e\&m,mech }}\right)^{+}}{\xi+(1-\xi)\left(\tilde{\eta}_{\text {recirc }}^{+}\right)}
\end{aligned}
$$

Equation 302: Energy supplied by the engine during the propel case (P2 Parallel, propel)

$$
\left(E_{\text {eng,mech }}\right)^{+}=\frac{\left(E_{\text {tr }}\right)^{+}}{\left[\xi+(1-\xi)\left(\tilde{\eta}_{\text {recirc }}^{+}\right)\right]\left(\tilde{\eta}_{\text {dl,eng }}^{+}\right)}
$$

\section{Developing Equation for Propel Case Tractive Energy Consumption (in terms of efficiencies):}

$\left(E_{\text {eng,fuel }}\right)^{+t r}=\frac{\left(E_{\text {eng,mech }}\right)^{+}}{\tilde{\eta}_{\text {eng }}^{+}}$

Equation 107: Propel case tractive fuel energy consumption (P2 Parallel, propel)

$$
\left(E_{\text {eng,fuel }}\right)^{+t r}=\frac{\left(E_{\text {tr }}\right)^{+}}{\left[\xi+(1-\xi)\left(\tilde{\eta}_{\text {recirc }}^{+}\right)\right]\left(\tilde{\eta}_{\text {eng }}^{+}\right)\left(\tilde{\eta}_{\text {dl,eng }}^{+}\right)}
$$




\section{APPENDIX U: Equations for Accessory Energy in the Propel Case (P2 Parallel HEV)}

This appendix contains the definitions for the component efficiencies used in the energy consumption equations developed for VTool. Some information may be duplicated in the body of this paper, but all relevant terms, definitions and equations have been reproduced here for convenience.

\begin{tabular}{|l|l|l|}
\hline Term & $\begin{array}{l}\text { Inherent } \\
\text { Sign }\end{array}$ & Definition \\
\hline$\left(E_{\text {en,fuel }}\right)^{+ \text {acc }}$ & + & Fuel energy in to the engine used for accessory power \\
\hline$\left(E_{\text {eng,mech }}\right)^{\text {acc }}$ & + & Mechanical energy out of the engine to be used for accessory power \\
\hline$\left(E_{\text {dlmot,tr }}\right)^{\text {tacc }}$ & - & Tractive energy in to the motor driveline to be used for accessory power \\
\hline$\left(E_{\text {mot,mech }}\right)^{\text {tacc }}$ & - & Mechanical energy in to the motor to be used for accessory power \\
\hline$\left(E_{\text {mot,elec }}\right)^{+ \text {acc }}$ & - & Electrical energy out of the motor to be used for accessory power \\
\hline$\left(E_{\text {acc }}\right)^{+}$ & + & Electrical energy siphoned off the HV bus for accessory loads during propel case \\
\hline$\left(E_{\text {eng,fuel }}\right)^{+ \text {acc }}$ & + & Fuel energy in to the engine used for accessory power \\
\hline
\end{tabular}

\section{Efficiency Expressions:}

Equation 303: Engine propel efficiency for accessory load (P2 Parallel, propel)

$\tilde{\eta}_{\text {eng }}^{+a c c}=\frac{\left(E_{\text {eng,mech }}\right)^{+a c c}}{\left(E_{\text {eng,fuel }}\right)^{+a c c}} \approx \tilde{\eta}_{\text {eng }}^{+}$

Equation 304: Motor driveline brake efficiency for accessory load (P2 Parallel, propel)

$\tilde{\eta}_{d l, m o t}^{+a c c}=\frac{\left(E_{\text {mot }, \text { mech }}\right)^{+a c c}}{\left(E_{d l m o t, t r}\right)^{+a c c}} \approx \tilde{\eta}_{d l, m o t}^{-}$

Equation 305: Motor brake efficiency for accessory load (P2 Parallel, propel)

$\tilde{\eta}_{\text {mot }}^{+ \text {acc }}=\frac{\left(E_{\text {mot }, \text { elec }}\right)^{+a c c}}{\left(E_{\text {mot }, \text { mech }}\right)^{+a c c}} \approx \tilde{\eta}_{\text {mot }}^{-}$

Equation 111: Propel case powertrain accessory efficiency for accessory load (P2 Parallel, propel)

$\tilde{\eta}_{p t}^{+a c c}=\frac{\left(E_{\text {Acc }}\right)^{+}}{\left(E_{\text {eng,fuel }}\right)^{+a c c}}$

\section{Loss Expressions:}

Equation 306: Engine losses for accessory load (P2 Parallel, propel)

$\left(E_{\text {eng }, \text { loss }}\right)^{+a c c}=\left(E_{\text {eng,fuel }}\right)^{+a c c}-\left(E_{\text {eng,mech }}\right)^{+a c c}$

Equation 307: Motor driveline generate losses for accessory load (P2 Parallel, propel)

$\left(E_{\text {dlmot }, l o s s}\right)^{+a c c}=\left(E_{\text {mot }, \text { mech }}\right)^{+a c c}-\left(E_{\text {dlmot }, t r}\right)^{+a c c}$ 
Equation 308: Motor generate losses for accessory load (P2 Parallel, propel)

$\left(E_{\text {mot }, \text { loss }}\right)^{+a c c}=\left(E_{\text {mot }, \text { elec }}\right)^{+a c c}-\left(E_{\text {mot }, \text { mech }}\right)^{+a c c}$

Equation 309: Powertrain losses for accessory load (P2 Parallel, propel)

$\left(E_{\text {pt,loss }}\right)^{+a c c}=\left(E_{\text {eng,loss }}\right)^{+a c c}+\left(E_{\text {dlmot }, l o s s}\right)^{+a c c}+\left(E_{\text {mot }, l o s s}\right)^{+a c c}$

Restated:

$\left(E_{\text {pt,loss }}\right)^{+a c c}=\left(E_{\text {eng,fuel }}\right)^{+a c c}-\left(E_{\text {Acc }}\right)^{+}$

\section{Conservation of Energy Expressions:}

Equation 310: Conservation of energy at the pre-trans node for accessory load (P2 Parallel, propel)

$\left(E_{\text {eng }, \text { mech }}\right)^{+a c c}=-\left(E_{\text {dlmot }, t r}\right)^{+a c c}$

Equation 311: Conservation of energy at the HV bus for accessory load (P2 Parallel, propel)

$\left(E_{\text {Acc }}\right)^{+}=-\left(E_{\text {mot }, \text { elec }}\right)^{+a c c}$

\section{Accessory Energy:}

Equation 25: Propel case accessory energy

$\left(E_{A c c}\right)^{+}=\tau\left(P_{A c c}\right)^{+}$

Developing Equation for Propel Case Accessory Energy Consumption (in terms of efficiencies):

$$
\begin{aligned}
& \left(E_{\text {eng,fuel }}\right)^{+a c c}=\frac{\left(E_{\text {eng,mech }}\right)^{+a c c}}{\tilde{\eta}_{\text {eng }}^{+}} \\
& \left(E_{\text {eng,fuel }}\right)^{+a c c}=-\frac{\left(E_{\text {dlmot }, \text { tr }}\right)^{+a c c}}{\left(\tilde{\eta}_{\text {eng }}^{+}\right)} \\
& \left(E_{\text {eng,fuel }}\right)^{+a c c}=-\frac{\left(E_{\text {mot }, \text { mech }}\right)^{+a c c}}{\left(\tilde{\eta}_{\text {eng }}^{+}\right)\left(\tilde{\eta}_{\text {dl,mot }}^{+}\right)} \\
& \left(E_{\text {eng,fuel }}\right)^{+a c c}=-\frac{\left(E_{\text {mot,elec }}\right)^{+a c c}}{\left(\tilde{\eta}_{\text {eng }}^{+}\right)\left(\tilde{\eta}_{\text {dl,mot }}^{+}\right)\left(\tilde{\eta}_{\text {mot }}^{+}\right)}
\end{aligned}
$$

Equation 110: Propel case accessory fuel energy consumption (P2 Parallel, propel)

$$
\left(E_{\text {eng,fuel }}\right)^{+a c c}=\frac{\left(E_{\text {Acc }}\right)^{+}}{\left(\tilde{\eta}_{\text {eng }}^{+}\right)\left(\tilde{\eta}_{d l, m o t}^{+}\right)\left(\tilde{\eta}_{m o t}^{+}\right)}
$$




\section{APPENDIX V: Equations for Total Energy in the Propel Case (P2 Parallel HEV)}

This appendix contains the definitions for the component efficiencies used in the energy consumption equations developed for VTool. Some information may be duplicated in the body of this paper, but all relevant terms, definitions and equations have been reproduced here for convenience.

\begin{tabular}{|l|l|l|}
\hline Term & $\begin{array}{l}\text { Inherent } \\
\text { Sign }\end{array}$ & Definition \\
\hline$\left(E_{\text {en,fuel }}\right)^{+ \text {acc }}$ & + & Fuel energy in to the engine used for accessory power \\
\hline$\left(E_{\text {eng,fuel }}\right)^{+ \text {tr }}$ & + & Fuel energy in to the engine used for tractive power \\
\hline$\left(E_{\text {dleng,tr }}\right)^{\text {tacc }}$ & + & Tractive energy out of the engine driveline to be used for accessory power \\
\hline$\left(E_{\text {dlmot,tr }}\right)^{+a c c}$ & - & Tractive energy in to the motor driveline to be used for accessory power \\
\hline$\left(E_{\text {mot,mech }}\right)^{+ \text {acc }}$ & - & Mechanical energy in to the motor to be used for accessory power \\
\hline$\left(E_{\text {mot,elec }}\right)^{\text {tacc }}$ & - & Electrical energy out of the motor to be used for accessory power \\
\hline$\left(E_{\text {acc }}\right)^{+}$ & + & Electrical energy siphoned off the HV bus for accessory loads during propel case \\
\hline
\end{tabular}

\section{Efficiency Expressions:}

Equation 113: Propel case powertrain efficiency (P2 Parallel, propel)

$$
\tilde{\eta}_{p t, I C E}^{+}=\frac{\left(E_{t r}\right)^{+}}{\left(E_{\text {eng,fuel }}\right)^{+}}
$$

\section{Loss Expressions:}

Equation 312: Engine total losses (P2 Parallel, propel)

$\left(E_{\text {eng, loss }}\right)^{+}=\left(E_{\text {eng, loss }}\right)^{+t r}+\left(E_{\text {eng,loss }}\right)^{+ \text {acc }}$

Equation 313: Engine driveline total losses (P2 Parallel, propel)

$\left(E_{\text {dleng, loss }}\right)^{+}=\left(E_{\text {dleng,loss }}\right)^{+t r}$

Equation 314: Motor driveline total losses (P2 Parallel, propel)

$\left(E_{\text {dlmot }, \text { loss }}\right)^{+}=\left(E_{\text {dlmot }, \text { loss }}\right)^{+t r}+\left(E_{\text {dlmot }, l o s s}\right)^{+a c c}$

Equation 315: Motor total losses (P2 Parallel, propel)

$\left(E_{\text {mot }, \text { loss }}\right)^{+}=\left(E_{\text {mot }, l o s s}\right)^{+t r}+\left(E_{m o t, l o s s}\right)^{+a c c}$

Equation 316: Battery total losses (P2 Parallel, propel)

$\left(E_{\text {batt }, \text { loss }}\right)^{+}=\left(E_{\text {batt }, \text { loss }}\right)^{+t r}$

Equation 317: Powertrain losses for accessory load (P2 Parallel, propel)

$\left(E_{\text {pt }, \text { loss }}\right)^{+}=\left(E_{\text {eng,loss }}\right)^{+}+\left(E_{\text {dleng,loss }}\right)^{+}+\left(E_{\text {dlmot }, \text { loss }}\right)^{+}+\left(E_{\text {mot }, \text { loss }}\right)^{+}+\left(E_{\text {bat }, \text { loss }}\right)^{+}$ 


\section{Conservation of Energy Expressions:}

Equation 318: Total fuel energy required for the propel case (P2 Parallel, propel)

$$
\left(E_{\text {eng,fuel }}\right)^{+}=\left(E_{\text {eng,fuel }}\right)^{+t r}+\left(E_{\text {eng,fuel }}\right)^{+ \text {acc }}
$$

\section{Developing Equation for Propel Case Total Energy Consumption (in terms of efficiencies):}

$$
\left(E_{\text {eng,fuel }}\right)^{+}=\left(E_{\text {eng,fuel }}\right)^{+t r}+\left(E_{\text {eng,fuel }}\right)^{+a c c}
$$

Equation 112: Propel case total fuel energy consumption (P2 Parallel, propel)

$$
\left(E_{\text {eng,fuel }}\right)^{+}=\frac{\left(E_{\text {tr }}\right)^{+}}{\left[\xi+(1-\xi)\left(\tilde{\eta}_{\text {recirc }}^{+}\right)\right]\left(\tilde{\eta}_{\text {eng }}^{+}\right)\left(\tilde{\eta}_{\text {dl,eng }}^{+}\right)}+\frac{\left(E_{\text {Acc }}\right)^{+}}{\left(\tilde{\eta}_{\text {eng }}^{+}\right)\left(\tilde{\eta}_{\text {dl,mot }}^{+}\right)\left(\tilde{\eta}_{\text {mot }}^{+}\right)}
$$




\section{APPENDIX W: Equations for the Brake Case (P2 Parallel HEV)}

This appendix contains the definitions for the component efficiencies used in the energy consumption equations developed for VTool. The Brake case for the P2 Parallel HEV is nearly identical to an EV, so only select equations are replicated here. The only difference is the addition of an engine driveline loss in the energy consumption equation. For full documentation of the brake case, see Appendix B.

\begin{tabular}{|l|l|l|}
\hline Term & $\begin{array}{l}\text { Inherent } \\
\text { Sign }\end{array}$ & Definition \\
\hline$\left(E_{t r}\right)^{-}$ & - & Tractive energy at the wheels for the brake case \\
\hline$\left(E_{t r, \zeta}\right)^{-}$ & - & Tractive energy in to driveline after effect of regen fraction \\
\hline$\left(E_{\text {mot,mech }}\right)^{-}$ & - & Mechanical energy in to the motor for the brake case \\
\hline$\left(E_{\text {mot,elec }}\right)^{-}$ & - & Electrical energy out of the motor for the brake case \\
\hline$\left(E_{\text {acc }}\right)^{-}$ & + & Electrical energy siphoned off the HV bus for accessory loads during brake case \\
\hline$\left(E_{\text {batt, },}\right)^{-}$ & - & Electrical energy in to the battery or at the terminals during the brake case \\
\hline$\left(E_{\text {batt,int }}\right)^{-}$ & - & Electrical energy inside the battery captured during the brake case \\
\hline
\end{tabular}

\section{Accessory Energy:}

Equation 31: Brake case accessory energy

$\left(E_{\text {Acc }}\right)^{-}=\left(T-\tau-t_{\text {idle }}\right)\left(P_{\text {Acc }}\right)^{-}$

Equation for Brake Case Energy Consumption (in terms of efficiencies):

Equation 32: Brake case energy consumption (EV, brake)

$\left(E_{\text {batt }, \text { int }}\right)^{-}=\tilde{\eta}_{\text {batt }}^{-}\left\{\left(E_{t r}\right)^{-}(\tilde{\zeta})\left(\tilde{\eta}_{d l, m o t}^{-}\right)\left(\tilde{\eta}_{\text {mot }}^{-}\right)+\left(E_{\text {Acc }}\right)^{-}\right\}$

*Adjust for extra driveline loss in regen energy flow path:

Equation 114: Propel case accessory fuel energy consumption (P2 Parallel, brake)

$\left(E_{\text {batt }, \text { int }}\right)^{-}=\tilde{\eta}_{\text {batt }}^{-}\left\{\left(E_{\text {tr }}\right)^{-}(\tilde{\zeta})\left(\tilde{\eta}_{\overline{d l}, m o t}^{-}\right)\left(\tilde{\eta}_{d l, e n g}^{-}\right)\left(\tilde{\eta}_{m o t}^{-}\right)+\left(E_{\text {Acc }}\right)^{-}\right\}$

Developing Equation for Brake Case Energy Consumption (in terms of losses):

Equation 34: Brake case energy consumption in terms of losses (EV, brake)

$\left(E_{\text {batt }, \text { int }}\right)^{-}=\left(E_{\text {tr }}\right)^{-}+\left(E_{\text {Acc }}\right)^{-}+\left(E_{\text {batt }, \text { loss }}\right)^{-}+\left(E_{\text {mot }, \text { loss }}\right)^{-}+\left(E_{\text {dlmot }, \text { loss }}\right)^{-}+\left(E_{\text {brk,loss }}\right)^{-}$

*Adjust for extra driveline loss in regen energy flow path:

Equation 319: Propel case accessory fuel energy consumption (P2 Parallel, brake)

$\left(E_{\text {batt }, \text { int }}\right)^{-}=\left(E_{\text {tr }}\right)^{-}+\left(E_{\text {Acc }}\right)^{-}+\left(E_{\text {batt }, \text { loss }}\right)^{-}+\left(E_{\text {mot }, \text { loss }}\right)^{-}+\left(E_{\text {dlmot }, \text { loss }}\right)^{-}+\left(E_{\text {dleng,loss }}\right)^{-}$ $+\left(E_{\text {brk }, l o s s}\right)^{-}$ 


\section{APPENDIX X: Equations for Regen Energy Reuse (P2 Parallel HEV)}

This appendix contains the definitions for the component efficiencies used in the energy consumption equations developed for VTool. Some information may be duplicated in the body of this paper, but all relevant terms, definitions and equations have been reproduced here for convenience.

\begin{tabular}{|l|l|l|}
\hline Term & $\begin{array}{l}\text { Inherent } \\
\text { Sign }\end{array}$ & Definition \\
\hline$\left(\mathrm{E}_{\text {batt,int }}\right)^{-}$ & - & Electrical energy inside the battery captured during the brake case \\
\hline$\left(\mathrm{E}_{\text {batt,int }}\right)^{-1+}$ & + & Net electrical energy inside the battery available to be reused for propulsion \\
\hline$\left(\mathrm{E}_{\text {batt, } \mathrm{T}}\right)^{-/+}$ & + & Net electrical at the battery terminals available to be reused for propulsion \\
\hline$\left(\mathrm{E}_{\text {mot,elec }}\right)^{-1+}$ & + & Electrical energy in to the motor to be reused for propulsion \\
\hline$\left(\mathrm{E}_{\text {mot,mech }}\right)^{-/+}$ & + & Mechanical energy out of the motor to be reused for propulsion \\
\hline$\left(\mathrm{E}_{\mathrm{dlmot}, \mathrm{tr}}\right)^{-/+}$ & + & Surplus tractive energy out of the motor D/L from the drive axle to be reused \\
\hline$\left(\mathrm{E}_{\text {e\&m,mech }}\right)^{-/+}$ & + & Sum of Mechanical energy from engine and motor in to engine driveline \\
\hline$\left(\mathrm{E}_{\mathrm{tr}, \mathrm{credit}}\right)^{-/+}$ & + & Tractive energy at the wheels from stored regen energy reused for propulsion \\
\hline
\end{tabular}

\section{Efficiency Expressions:}

Equation 320: Engine driveline efficiency (P2 Parallel, regen reuse)

$\tilde{\eta}_{d l, e n g}^{-/+}=\frac{\left(E_{\text {tr,credit }}\right)^{-/+}}{\left(E_{e \& m, \text { mech }}\right)^{-/+}} \approx \tilde{\eta}_{d l, e n g}^{+}$

Equation 321: Motor driveline efficiency (P2 Parallel, regen reuse)

$\tilde{\eta}_{d l, m o t}^{-/+}=\frac{\left(E_{d l m o t, t r}\right)^{-/+}}{\left(E_{m o t, m e c h}\right)^{-/+}} \approx \tilde{\eta}_{d l, m o t}^{+}$

Equation 322: Motor propel efficiency (P2 Parallel, regen reuse)

$\tilde{\eta}_{\text {mot }}^{-/+}=\frac{\left(E_{\text {mot }, \text { mech }}\right)^{-/+}}{\left(E_{\text {mot }, \text { elec }}\right)^{-/+}} \approx \tilde{\eta}_{\text {mot }}^{+}$

Equation 323: Battery discharge efficiency (P2 Parallel, regen reuse)

$\tilde{\eta}_{\text {batt }}^{-/+}=\frac{\left(E_{\text {batt }, T}\right)^{-/+}}{\left(E_{\text {batt }, \text { int }}\right)^{-/+}} \approx \tilde{\eta}_{\text {batt }}^{+}$

Equation 167: Energy used from the battery during shortages of genset power generation (Series, propel)

$\tilde{\eta}_{p t, E V}^{-/+}=\frac{\left(E_{\text {tr, credit }}\right)^{-/+}}{\left(E_{\text {batt }, \text { int }}\right)^{-/+}}$ 


\section{Loss Expressions:}

Equation 324: Engine driveline losses (P2 Parallel, regen reuse)

$\left(E_{\text {dleng,loss }}\right)^{-/+}=\left(E_{\text {e\&m,mech }}\right)^{-/+}-\left(E_{\text {tr,credit }}\right)^{-/+}$

Equation 325: Motor driveline losses (P2 Parallel, regen reuse)

$\left(E_{\text {dlmot }, \text { loss }}\right)^{-/+}=\left(E_{\text {mot }, \text { mech }}\right)^{-/+}-\left(E_{\text {dlmot }, t r}\right)^{-/+}$

Equation 326: Motor losses (P2 Parallel, regen reuse)

$\left(E_{\text {mot }, \text { loss }}\right)^{-/+}=\left(E_{\text {mot }, \text { elec }}\right)^{-/+}-\left(E_{\text {mot }, \text { mech }}\right)^{-/+}$

Equation 327: Battery losses (P2 Parallel, regen reuse)

$\left(E_{\text {batt }, \text { loss }}\right)^{-/+}=\left(E_{\text {batt }, \text { int }}\right)^{-/+}-\left(E_{\text {batt }, T}\right)^{-/+}$

Equation 328: Propel powertrain losses (P2 Parallel, regen reuse)

$\left(E_{\text {pt,loss }}\right)^{-/+}=\left(E_{\text {batt }, \text { int }}\right)^{-/+}-\left(E_{\text {tr,credit }}\right)^{-/+}$

Restated:

$\left(E_{\text {pt }, \text { loss }}\right)^{-/+}=\left(E_{\text {batt }, \text { loss }}\right)^{-/+}+\left(E_{\text {mot }, \text { loss }}\right)^{-/+}+\left(E_{\text {dlmot }, \text { loss }}\right)^{-/+}+\left(E_{\text {dleng }, \text { loss }}\right)^{-/+}$

\section{Conservation of Energy Expressions:}

Equation 329: Conservation of energy at the HV bus (P2 Parallel, regen reuse)

$\left(E_{\text {batt }, T}\right)^{-/+}=\left(E_{\text {mot }, e l e c}\right)^{-/+}$

Equation 330: Conservation of energy for regen energy capture and reuse (P2 Parallel, regen reuse)

$\left(E_{\text {batt }, \text { int }}\right)^{-}=-\left(E_{\text {batt }, \text { int }}\right)^{-/+}$

Equation 331: Conservation of energy at the pre-trans node (P2 Parallel, regen reuse)

$\left(E_{e \& m, m e c h}\right)^{-/+}=\left(E_{\text {dlmot }, t r}\right)^{-/+}$ 


\section{Developing Equation for Regen Energy Tractive Energy Credit (in terms of efficiencies):}

$$
\begin{aligned}
& \left(E_{\text {tr,credit }}\right)^{-/+}=\left(E_{\text {e\&m,mech }}\right)^{-/+}\left(\tilde{\eta}_{\text {dl,eng }}^{-/+}\right) \\
& \left(E_{\text {tr,credit }}\right)^{-/+}=\left(E_{\text {dlmot }, \mathrm{tr}}\right)^{-/+}\left(\tilde{\eta}_{\text {dl,eng }}^{-/+}\right) \\
& \left(E_{\text {tr,credit }}\right)^{-/+}=\left(E_{\text {mot,mech }}\right)^{-/+}\left(\tilde{\eta}_{d l, \text { mot }}^{-/+}\right)\left(\tilde{\eta}_{\text {dl,eng }}^{-/+}\right) \\
& \left(E_{\text {tr,credit }}\right)^{-/+}=\left(E_{\text {mot }, \text { elec }}\right)^{-/+}\left(\tilde{\eta}_{\text {mot }}^{-/+}\right)\left(\tilde{\eta}_{d l, \text { mot }}^{-/+}\right)\left(\tilde{\eta}_{d l, \text { eng }}^{-/+}\right) \\
& \left(E_{\text {tr,credit }}\right)^{-/+}=\left(E_{\text {batt }, T}\right)^{-/+}\left(\tilde{\eta}_{\text {mot }}^{-/+}\right)\left(\tilde{\eta}_{d l, \text { mot }}^{-/+}\right)\left(\tilde{\eta}_{\text {dl,eng }}^{-/+}\right) \\
& \left(E_{\text {tr,credit }}\right)^{-/+}=\left(E_{\text {batt }, \text { int }}\right)^{-/+}\left(\tilde{\eta}_{\text {batt }}^{-/+}\right)\left(\tilde{\eta}_{\text {mot }}^{-/+}\right)\left(\tilde{\eta}_{\text {dl,mot }}^{-/+}\right)\left(\tilde{\eta}_{d l, \text { eng }}^{-/+}\right)
\end{aligned}
$$

Equation 115: Equivalent tractive energy 'credit' from regen braking (P2 Parallel, regen reuse)

$$
\left(E_{\text {tr,credit }}\right)^{-/+}=-\left(E_{\text {batt }, \text { int }}\right)^{-}\left(\tilde{\eta}_{\text {batt }}^{-/+}\right)\left(\tilde{\eta}_{\text {mot }}^{-/+}\right)\left(\tilde{\eta}_{d l, m o t}^{-/+}\right)\left(\tilde{\eta}_{d l, e n g}^{-/+}\right)
$$

\section{Developing Equation for Regen Energy Tractive Energy Credit (in terms of losses):}

$$
\begin{aligned}
& \left(E_{\text {tr,credit }}\right)^{-/+}=\left(E_{\text {e\&m,mech }}\right)^{-/+}-\left(E_{\text {dleng,loss }}\right)^{-/+} \\
& \left(E_{\text {tr,credit }}\right)^{-/+}=\left(E_{\text {dlmot }, \text { tr }}\right)^{-/+}-\left(E_{\text {dleng,loss }}\right)^{-/+} \\
& \left(E_{\text {tr,credit }}\right)^{-/+}=\left(E_{\text {mot }, \text { mech }}\right)^{-/+}-\left(E_{\text {dlmot }, \text { loss }}\right)^{-/+}-\left(E_{\text {dleng,loss }}\right)^{-/+} \\
& \left(E_{\text {tr,credit }}\right)^{-/+}=\left(E_{\text {mot }, \text { elec }}\right)^{-/+}-\left(E_{\text {dlmot }, \text { loss }}\right)^{-/+}-\left(E_{\text {dleng,loss }}\right)^{-/+}-\left(E_{\text {dleng,loss }}\right)^{-/+} \\
& \left(E_{\text {tr,credit }}\right)^{-/+}=\left(E_{\text {batt }, T}\right)^{-/+}-\left(E_{\text {mot }, \text { loss }}\right)^{-/+}-\left(E_{\text {dlmot }, \text { loss }}\right)^{-/+}-\left(E_{\text {dleng }, \text { loss }}\right)^{-/+} \\
& \left(E_{\text {tr, credit }}\right)^{-/+} \\
& =\left(E_{\text {batt }, \text { int }}\right)^{-/+}-\left(E_{\text {batt }, \text { loss }}\right)^{-/+}-\left(E_{\text {mot }, \text { loss }}\right)^{-/+}-\left(E_{\text {dlmot }, \text { loss }}\right)^{-/+} \\
& -\left(E_{\text {dleng, loss }}\right)^{-/+} \\
& \left(E_{\text {tr }, \text { credit }}\right)^{-/+}=\left(E_{\text {batt }, \text { int }}\right)^{-/+}-\left(E_{p t, l o s s}\right)^{-/+}
\end{aligned}
$$

Equation 332: Equivalent tractive energy 'credit' from regen in terms of losses (P2 Parallel, regen reuse) $\left(E_{\text {tr }, \text { credit }}\right)^{-/+}=\left(E_{\text {bat }, \text { int }}\right)^{-/+}-\left(E_{\text {pt,loss }}\right)^{-/+}=-\left(E_{\text {bat }, \text { int }}\right)^{-}-\left(E_{p t, l o s s}\right)^{-/+}$ 


\section{APPENDIX Y: Equations for Fuel-Equivalent Regen Energy Credit (P2 Parallel HEV)}

This appendix contains the definitions for the component efficiencies used in the energy consumption equations developed for VTool. Some information may be duplicated in the body of this paper, but all relevant terms, definitions and equations have been reproduced here for convenience.

\begin{tabular}{|c|c|c|}
\hline Term & $\begin{array}{l}\text { Inherent } \\
\text { Sign }\end{array}$ & Definition \\
\hline$\left(E_{\text {eng,fuel }}\right)^{\text {EqFC }}$ & - & Theoretical fuel energy out of the engine to be used as fuel credit \\
\hline$\left(E_{\text {eng,mech }}\right)^{\mathrm{EqFC}}$ & - & Theoretical mechanical energy in to the engine to be used as fuel credit \\
\hline$\left(E_{d l m o t, t r}\right)^{- \text {-EqFC }}$ & + & Theoretical tractive energy out of the motor driveline. Analog to $\left(\mathrm{E}_{\mathrm{dlmot}, \mathrm{tr}}\right)^{+\mathrm{gen}}$ \\
\hline$\left(E_{\text {mot,mech }}\right)^{-\mathrm{EqFC}}$ & + & Theoretical mechanical energy out of the motor. Analog to $\left(\mathrm{E}_{\text {mot,mech }}\right)^{+g e n}$ \\
\hline$\left(E_{\text {mot,elec }}\right)^{-\mathrm{EqFC}}$ & + & Theoretical electrical energy in to the motor. Analog to $\left(\mathrm{E}_{\text {motelec }}\right)^{\text {tgen }}$ \\
\hline$\left(E_{\text {batt }, T}\right)^{- \text {-EqFC }}$ & + & Theoretical electrical energy at the battery terminals. Analog to $\left(E_{b a t t},\right)^{+g e n}$ \\
\hline$\left(E_{\text {batt, int }}\right)^{-E q F C}$ & + & Theoretical electrical energy inside the battery. Analog to $\left(\mathrm{E}_{\text {batt.int }}\right)^{+ \text {tgen }}$ \\
\hline$\left(\mathrm{E}_{\text {batt,int }}\right)^{+\mathrm{EqFC}}$ & - & Theoretical electrical energy inside the battery. Analog to $\left(E_{\text {batt,int }}\right)^{\text {+prop }}$ \\
\hline 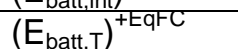 & - & Theoretical electrical energy at the battery terminals. Analog to $\left(E_{\text {batt }, T}\right)^{+p r o p}$ \\
\hline$\left(\mathrm{E}_{\text {mot,elec }}\right)^{+\mathrm{EqFC}}$ & - & Theoretical electrical energy in to the motor. Analog to $\left(E_{\text {mot.elec }}\right)^{\text {+prop }}$ \\
\hline$\left(E_{\text {mot,mech }}\right)^{+E q F C}$ & - & Theoretical mechanical energy out of the motor. Analog to $\left(E_{\text {mot.mech }}\right)^{+ \text {pro }}$ \\
\hline$\left(\mathrm{E}_{\mathrm{dlmot}, \mathrm{tr}}\right)^{+\mathrm{EqFC}}$ & - & Theoretical tractive energy out of the motor driveline. Analog to $\left(\mathrm{E}_{\mathrm{dlm} m \mathrm{~m}, \mathrm{tr}}\right)^{+\mathrm{p}}$ \\
\hline$\left(\mathrm{E}_{\mathrm{e} \& \mathrm{~m}, \mathrm{mech}}\right)^{\mathrm{EqFC}}$ & + & Theoretical mechanical energy in from engine driveline to be used as fuel credit \\
\hline$\left(E_{\text {tr.credit }}\right)^{\text {tatrC }}$ & - & Theoretical tractive energy in to the wheels to be used as fuel credit \\
\hline$\left(E_{t r, c r e d i t}\right)^{-1+}$ & + & Tractive energy at the wheels from stored regen energy reused for propulsion \\
\hline$\left(E_{\text {dleng,tr }}\right)^{\text {EqFC }}$ & - & Theoretical tractive energy in to the engine driveline to be used as fuel credit \\
\hline
\end{tabular}

Efficiency Expressions: (same as propel case because theoretical powerflow emulates propel)

Equation 105: Power Split Fraction, engine energy directly used (P2 Parallel, propel)

$\xi=\frac{\left(E_{\text {eng }, \text { use }}\right)^{+}}{\left(E_{\text {eng }, \text { mech }}\right)^{+}}$

Restated:

$\left(E_{\text {eng }, \text { use }}\right)^{+}=\tilde{\xi}\left(E_{\text {eng }, \text { mech }}\right)^{+}$

Equation 106: Power Split Fraction, engine energy recirculated (P2 Parallel, propel)

$\left(E_{\text {eng,store }}\right)^{+}=\left(E_{\text {dlmot }, \text { tr }}\right)^{+ \text {gen }}=(-1)(1-\xi)\left(E_{\text {eng,mech }}\right)^{+}$

Equation 273: Engine propel efficiency (P2 Parallel, propel)

$\tilde{\eta}_{\text {eng }}^{+}=\frac{\left(E_{\text {eng,mech }}\right)^{+}}{\left(E_{\text {eng,fuel }}\right)^{+t r}}$

Equation 274: Engine driveline propel efficiency (P2 Parallel, propel)

$\tilde{\eta}_{\text {dl,eng }}^{+}=\frac{\left(E_{\text {dleng,tr }}\right)^{+}}{\left(E_{\text {e\&m,mech }}\right)^{+}}$ 
Equation 275: Motor driveline brake efficiency (P2 Parallel, propel)

$\tilde{\eta}_{d l, m o t}^{+g e n}=\frac{\left(E_{\text {mot }, \text { mech }}\right)^{+g e n}}{\left(E_{\text {dlmot }, t r}\right)^{+g e n}} \approx \tilde{\eta}_{d l, m o t}^{-}$

Equation 276: Motor brake efficiency (P2 Parallel, propel)

$\tilde{\eta}_{\text {mot }}^{+ \text {gen }}=\frac{\left(E_{\text {mot }, \text { elec }}\right)^{+ \text {gen }}}{\left(E_{\text {mot }, \text { mech }}\right)^{+g e n}} \approx \tilde{\eta}_{\text {mot }}^{-}$

Equation 277: Battery charge efficiency (P2 Parallel, propel)

$\tilde{\eta}_{\text {batt }}^{+g e n}=\frac{\left(E_{\text {batt }, i n t}\right)^{+g e n}}{\left(E_{\text {batt }, T}\right)^{+g e n}} \approx \tilde{\eta}_{\text {batt }}^{-}$

Equation 278: Battery discharge efficiency (P2 Parallel, propel)

$\tilde{\eta}_{\text {batt }}^{+ \text {prop }}=\frac{\left(E_{\text {batt }, T}\right)^{+ \text {prop }}}{\left(E_{\text {batt }, i n t}\right)^{+ \text {prop }}} \approx \tilde{\eta}_{\text {batt }}^{+}$

Equation 279: Motor propel efficiency (P2 Parallel, propel)

$\tilde{\eta}_{\text {mot }}^{+ \text {prop }}=\frac{\left(E_{\text {mot }, \text { mech }}\right)^{+ \text {prop }}}{\left(E_{\text {mot }, \text { elec }}\right)^{+ \text {prop }}} \approx \tilde{\eta}_{\text {mot }}^{+}$

Equation 280: Motor driveline propel efficiency (P2 Parallel, propel)

$\tilde{\eta}_{d l, m o t}^{+ \text {prop }}=\frac{\left(E_{d l m o t, t r}\right)^{+ \text {prop }}}{\left(E_{\text {mot }, \text { mech }}\right)^{+ \text {prop }}} \approx \tilde{\eta}_{d l, m o t}^{+}$

Equation 108: Power Split Fraction energy recirculation efficiency (P2 Parallel, propel)

$\tilde{\eta}_{\text {recirc }}^{+}=\left(\tilde{\eta}_{\text {dl,mot }}^{+ \text {gen }}\right)\left(\tilde{\eta}_{\text {mot }}^{+ \text {gen }}\right)\left(\tilde{\eta}_{\text {batt }}^{+ \text {gen }}\right)\left(\tilde{\eta}_{\text {batt }}^{+ \text {prop }}\right)\left(\tilde{\eta}_{\text {mot }}^{+ \text {prop }}\right)\left(\tilde{\eta}_{d l, m o t}^{+ \text {prop }}\right)$

Equation 109: Propel case powertrain efficiency for tractive energy (P2 Parallel, propel)

$\tilde{\eta}_{p t}^{+t r}=\frac{\left(E_{\text {tr }}\right)^{+}}{\left(E_{\text {eng,fuel }}\right)^{+}}$ 


\section{Loss Expressions:}

Equation 333: Engine losses for tractive energy (P2 Parallel, propel)

$\left(E_{\text {eng }, \text { loss }}\right)^{E q F C}=\left(E_{\text {eng,fuel }}\right)^{E q F C}-\left(E_{\text {eng,mech }}\right)^{E q F C}$

Equation 334: Engine driveline losses for tractive energy (P2 Parallel, propel)

$\left(E_{\text {dleng }, l o s s}\right)^{E q F C}=\left(E_{\text {e\&m,mech }}\right)^{E q F C}-\left(E_{\text {tr }}\right)^{E q F C}$

Equation 335: Motor driveline generate losses for tractive energy (P2 Parallel, propel)

$\left(E_{\text {dlmot }, l o s s}\right)^{-E q F C}=\left(E_{\text {mot }, \text { mech }}\right)^{-E q F C}-\left(E_{\text {dlmot }, t r}\right)^{-E q F C}$

Equation 336: Motor generate losses for tractive energy (P2 Parallel, propel)

$\left(E_{\text {mot }, \text { loss }}\right)^{-E q F C}=\left(E_{\text {mot }, e l e c}\right)^{-E q F C}-\left(E_{\text {mot }, \text { mech }}\right)^{-E q F C}$

Equation 337: Battery charge losses for tractive energy (P2 Parallel, propel)

$\left(E_{\text {batt }, l o s s}\right)^{-E q F C}=\left(E_{\text {batt }, \text { int }}\right)^{-E q F C}-\left(E_{\text {batt }, T}\right)^{-E q F C}$

Equation 338: Battery discharge losses for tractive energy (P2 Parallel, propel)

$\left(E_{\text {batt }, l o s s}\right)^{+E q F C}=\left(E_{\text {batt }, \text { int }}\right)^{+E q F C}-\left(E_{\text {batt }, T}\right)^{+E q F C}$

Equation 339: Motor propel losses for tractive energy (P2 Parallel, propel)

$\left(E_{\text {mot }, \text { loss }}\right)^{+E q F C}=\left(E_{\text {mot }, \text { elec }}\right)^{+E q F C}-\left(E_{\text {mot }, \text { mech }}\right)^{+E q F C}$

Equation 340: Motor driveline propel losses for tractive energy (P2 Parallel, propel)

$\left(E_{\text {dlmot }, l o s s}\right)^{+E q F C}=\left(E_{\text {mot }, \text { mech }}\right)^{+E q F C}-\left(E_{\text {dlmot }, t r}\right)^{+E q F C}$

Equation 341: Motor driveline total losses for tractive energy (P2 Parallel, propel)

$\left(E_{\text {dlmot }, l o s s}\right)^{E q F C}=\left(E_{\text {dlmot }, l o s s}\right)^{-E q F C}+\left(E_{\text {dlmot }, l o s s}\right)^{+E q F C}$

Equation 342: Motor total losses for tractive energy (P2 Parallel, propel)

$\left(E_{m o t, l o s s}\right)^{E q F C}=\left(E_{m o t, l o s s}\right)^{-E q F C}+\left(E_{m o t, l o s s}\right)^{+E q F C}$

Equation 343: Battery total losses for tractive energy (P2 Parallel, propel)

$\left(E_{\text {batt }, \text { loss }}\right)^{E q F C}=\left(E_{\text {batt }, \text { loss }}\right)^{-E q F C}+\left(E_{\text {batt }, \text { loss }}\right)^{+E q F C}$

Equation 344: Powertrain losses for tractive energy (P2 Parallel, propel)

$\left(E_{\text {pt,loss }}\right)^{E q F C}=\left(E_{\text {eng,fuel }}\right)^{E q F C}-\left(E_{\text {tr,credit }}\right)^{E q F C}$

\section{Conservation of Energy Expressions:}

Equation 345: Conservation of energy for regen fuel credit (P2 Parallel, propel)

$\left(E_{\text {tr,credit }}\right)^{E q F C}=-\left(E_{\text {tr,credit }}\right)^{-/+}$ 


\section{Developing Equation for Fuel-Equivalent Regen Energy Credit (in terms of efficiencies):}

\section{**Begin from Propel Case for CS P2 Parallel HEV}

Equation 112: Propel case total fuel energy consumption (P2 Parallel, propel)

$$
\begin{aligned}
& \left(E_{\text {eng,fuel }}\right)^{+}=\frac{\left(E_{\text {tr }}\right)^{+}}{\left[\xi+(1-\xi)\left(\tilde{\eta}_{\text {recirc }}^{+}\right)\right]\left(\tilde{\eta}_{\text {eng }}^{+}\right)\left(\tilde{\eta}_{\text {dl,eng }}^{+}\right)}+\frac{\left(E_{\text {Acc }}\right)^{+}}{\left(\tilde{\eta}_{\text {eng }}^{+}\right)\left(\tilde{\eta}_{\text {dl,mot }}^{+}\right)\left(\tilde{\eta}_{\text {mot }}^{+}\right)} \\
& \left(E_{\text {eng,fuel }}\right)^{E q F C}=\frac{\left(E_{\text {tr,credit }}\right)^{-/+}}{\left[\xi+(1-\xi)\left(\tilde{\eta}_{\text {recirc }}^{+}\right)\right]\left(\tilde{\eta}_{\text {eng }}^{+}\right)\left(\tilde{\eta}_{\text {dl,eng }}^{+}\right)}+\frac{\left(\tilde{\eta}^{+}\right)\left(\tilde{n}^{+}\right)\left(\tilde{\eta}^{+}\right)}{\left(\frac{1}{(\xi)}\right.}
\end{aligned}
$$

Equation 117: Fuel-equivalent regen energy 'credit' (P2 Parallel, idle)

$$
\left(E_{\text {eng,fuel }}\right)^{E q F C}=\frac{-\left(E_{\text {tr,credit }}\right)^{E q F C}}{\left[\xi+(1-\xi)\left(\tilde{\eta}_{\text {recirc }}^{+}\right)\right]\left(\tilde{\eta}_{\text {eng }}^{+}\right)\left(\tilde{\eta}_{\text {dl,eng }}^{+}\right)}
$$




\section{APPENDIX Z: Equations for the Idle Case (P2 Parallel HEV)}

This appendix contains the definitions for the component efficiencies used in the energy consumption equations developed for VTool. Some information may be duplicated in the body of this paper, but all relevant terms, definitions and equations have been reproduced here for convenience.

\begin{tabular}{|l|l|l|}
\hline Term & $\begin{array}{l}\text { Inherent } \\
\text { Sign }\end{array}$ & Definition \\
\hline$\left(E_{\text {eng,fuel }}\right)^{0}$ & + & Fuel energy in to the engine during the idle case \\
\hline$\left(E_{\text {eng,mech }}\right)^{0}$ & + & Mechanical energy out of the engine during the idle case \\
\hline$\left(E_{\text {dlmot,tr }}\right)^{0}$ & - & Tractive energy in to the motor driveline during the idle case \\
\hline$\left(E_{\text {mot,mech }}\right)^{0}$ & - & Mechanical energy in to the motor during the idle case \\
\hline$\left(E_{\text {mot,elec }}\right)^{0}$ & - & Electrical energy out of the motor during the idle case \\
\hline$\left(E_{\text {acc }}\right)^{0}$ & + & Electrical energy siphoned off the HV bus for accessory loads during the idle case \\
\hline
\end{tabular}

Efficiency Expressions: (same as propel case because theoretical powerflow emulates propel)

Equation 346: Engine efficiency (P2 Parallel, idle)

$\tilde{\eta}_{\text {eng }}^{0}=\frac{\left(E_{\text {eng,mech }}\right)^{0}}{\left(E_{\text {eng,fuel }}\right)^{0}} \approx \tilde{\eta}_{\text {eng }}^{+}$

Equation 347: Motor driveline efficiency (P2 Parallel, idle)

$\tilde{\eta}_{d l, m o t}^{0}=\frac{\left(E_{\text {mot }, \text { mech }}\right)^{0}}{\left(E_{\text {dlmot }, t r}\right)^{0}} \approx \tilde{\eta}_{d l, m o t}^{+}$

Equation 348: Motor efficiency (P2 Parallel, idle)

$\tilde{\eta}_{\text {mot }}^{0}=\frac{\left(E_{\text {mot }, \text { elec }}\right)^{0}}{\left(E_{\text {mot }, \text { mech }}\right)^{0}} \approx \tilde{\eta}_{\text {mot }}^{+}$

\section{Loss Expressions:}

Equation 349: Engine losses (P2 Parallel, idle)

$\left(E_{\text {eng }, \text { loss }}\right)^{0}=\left(E_{\text {eng,fuel }}\right)^{0}-\left(E_{\text {eng }, \text { mech }}\right)^{0}$

Equation 350: Motor driveline losses (P2 Parallel, idle)

$\left(E_{\text {dlmot }, \text { loss }}\right)^{0}=\left(E_{\text {mot }, \text { mech }}\right)^{0}-\left(E_{\text {dlmot }, t r}\right)^{0}$

Equation 351: Motor losses (P2 Parallel, idle)

$\left(E_{\text {mot }, \text { loss }}\right)^{0}=\left(E_{\text {mot }, e l e c}\right)^{0}-\left(E_{\text {mot }, \text { mech }}\right)^{0}$

Equation 352: Powertrain losses (P2 Parallel, idle)

$\left(E_{\text {pt,loss }}\right)^{0}=\left(E_{\text {eng,fuel }}\right)^{0}-\left(E_{\text {Acc }}\right)^{0}$ 


\section{Conservation of Energy Expressions:}

Equation 353: Conservation of energy at the HV Bus (P2 Parallel, idle)

$\left(E_{\text {Acc }}\right)^{0}=-\left(E_{\text {mot }, \text { elec }}\right)^{0}$

Equation 354: Conservation of energy at the pre-trans node axle (P2 Parallel, idle)

$$
\left(E_{\text {eng,mech }}\right)^{0}=-\left(E_{\text {dlmot,tr }}\right)^{0}
$$

\section{Accessory Energy:}

Equation 36: Idle accessory energy

$$
\left(E_{\text {Acc }}\right)^{0}=\left(t_{\text {idle }}\right)\left(P_{\text {Acc }}\right)^{0}
$$

\section{Developing Equation for Idle Case Energy Consumption (in terms of efficiencies):}

$$
\begin{aligned}
& \left(E_{\text {eng,fuel }}\right)^{0}=\frac{\left(E_{\text {eng,mech }}\right)^{0}}{\tilde{\eta}_{\text {eng }}^{0}}=\frac{-\left(E_{\text {dlmot }, \text { rr }}\right)^{0}}{\left(\tilde{\eta}_{\text {eng }}^{0}\right)} \\
& \left(E_{\text {eng,fuel }}\right)^{0}=\frac{-\left(E_{\text {mot }, \text { mech }}\right)^{0}}{\left(\tilde{\eta}_{\text {eng }}^{0}\right)\left(\tilde{\eta}_{\text {dl,mot }}^{0}\right)}
\end{aligned}
$$

Equation 118: Idle case energy consumption (P2 Parallel, idle)

$$
\left(E_{\text {eng,fuel }}\right)^{0}=\frac{-\left(E_{\text {mot }, \text { elec }}\right)^{0}}{\left(\tilde{\eta}_{\text {eng }}^{0}\right)\left(\tilde{\eta}_{\text {dl,mot }}^{0}\right)\left(\tilde{\eta}_{\text {mot }}^{0}\right)}=\frac{\left(E_{\text {Acc }}\right)^{0}}{\left(\tilde{\eta}_{\text {eng }}^{0}\right)\left(\tilde{\eta}_{\text {dl,mot }}^{0}\right)\left(\tilde{\eta}_{\text {mot }}^{0}\right)}
$$

\section{Developing Equation for Idle Case Energy Consumption (in terms of losses):}

$$
\begin{aligned}
& \left(E_{\text {eng,fuel }}\right)^{0}=\left(E_{\text {eng,mech }}\right)^{0}+\left(E_{\text {eng,loss }}\right)^{0}=-\left(E_{\text {dlmot }, t r}\right)^{0}+\left(E_{\text {eng,loss }}\right)^{0} \\
& \left(E_{\text {eng,fuel }}\right)^{0}=-\left(E_{\text {mot }, \text { mech }}\right)^{0}+\left(E_{\text {dlmot }, \text { loss }}\right)^{0}+\left(E_{\text {eng,loss }}\right)^{0} \\
& \left(E_{\text {eng,fuel }}\right)^{0}=-\left(E_{\text {mot }, \text { elec }}\right)^{0}+\left(E_{\text {mot }, \text { loss }}\right)^{0}+\left(E_{\text {dlmot }, \text { loss }}\right)^{0}+\left(E_{\text {eng,loss }}\right)^{0}
\end{aligned}
$$

Equation 355: Idle case energy consumption in terms of losses (P2 Parallel, idle)

$$
\left(E_{\text {eng,fuel }}\right)^{0}=\left(E_{\text {Acc }}\right)^{0}+\left(E_{\text {mot }, \text { loss }}\right)^{0}+\left(E_{\text {dlmot }, \text { loss }}\right)^{0}+\left(E_{\text {eng,loss }}\right)^{0}
$$




\section{APPENDIX AA: Equations for the DCnet Case (P2 Parallel HEV)}

This appendix contains the definitions for the component efficiencies used in the energy consumption equations developed for VTool. Some information may be duplicated in the body of this paper, but all relevant terms, definitions and equations have been reproduced here for convenience.

\begin{tabular}{|c|c|c|}
\hline Term & $\begin{array}{l}\text { Inherent } \\
\text { Sign }\end{array}$ & Definition \\
\hline$\left(E_{\text {batt,int }}\right)^{-}$ & - & Electrical energy inside the battery captured during the brake case \\
\hline$\left(E_{\text {batt,int }}\right)^{-1+}$ & + & Net electrical energy inside the battery available to be reused for propulsion \\
\hline$\left(\mathrm{E}_{\text {batt,int }}\right)^{+ \text {gen }}$ & - & Electrical energy inside the battery stored from excess generating \\
\hline$\left(\mathrm{E}_{\text {batt,int }}\right)^{+ \text {prop }}$ & + & Electrical energy inside the battery to be used in propulsion \\
\hline$\left(\mathrm{E}_{\text {batt,int }}\right)^{\mathrm{DCnet}}$ & + & Net electrical energy inside the battery for a given drive cycle \\
\hline$\left(E_{t r, \text { credit }}\right)^{-1+}$ & + & Tractive energy at the wheels from stored regen energy reused for propulsion \\
\hline$\left(E_{\text {eng,fuel }}\right)^{+}$ & + & Fuel energy in to the engine during the propel case \\
\hline$\left(E_{\text {eng,fuel }}\right)^{0}$ & + & Fuel energy in to the engine during the idle case \\
\hline$\left(E_{\text {eng.fuel }}\right)^{\text {EqFC }}$ & - & Theoretical fuel energy out of the engine to be used as fuel credit \\
\hline$\left(E_{t r}\right)^{B}$ & + & Net tractive energy at the wheels for a given drive cycle (Road Load) \\
\hline
\end{tabular}

\section{Efficiency Expressions:}

Equation 122: Net powertrain efficiency (P2 Parallel, DCnet)

$\tilde{\eta}_{p t}^{\text {DCnet }}=\frac{\left(E_{\text {tr }}\right)^{\text {DCnet }}}{\left(E_{\text {eng,fuel }}\right)^{\text {DCnet }}}$

Equation 113: Propel case powertrain efficiency (P2 Parallel, propel)

$\tilde{\eta}_{p t, I C E}^{+}=\frac{\left(E_{\text {tr }}\right)^{+}}{\left(E_{\text {eng,fuel }}\right)^{+}}$

Equation 116: Powertrain efficiency for regen energy reuse (P2 Parallel, regen reuse)

$\tilde{\eta}_{p t, E V}^{-/+}=\frac{\left(E_{\text {tr,credit }}\right)^{-/+}}{\left(E_{\text {batt }, \text { int }}\right)^{-/+}}$

Equation 123: Net powertrain efficiency for combined propel operation (P2 Parallel, DCnet)

$\tilde{\eta}_{\text {pt,propel }}^{\text {DCnet }}=\frac{\left(E_{\text {tr }}\right)^{+}+\left(E_{\text {tr,credit }}\right)^{E q F C}}{\left(E_{\text {eng,fuel }}\right)^{+}+\left(E_{\text {batt }, \text { int }}\right)^{-/+}}$

Equation 35: Brake case powertrain efficiency (EV, brake)

$\tilde{\eta}_{p t}^{-}=\frac{\left(E_{\text {batt }, i n t}\right)^{-}}{\left(E_{\text {tr }}\right)^{-}}$ 


\section{Loss Expressions:}

Equation 144: Net friction brake losses (EV, DCnet)

$\left(E_{\text {brk }, \text { loss }}\right)^{\text {DCnet }}=\left(E_{\text {brk, loss }}\right)^{-}$

Equation 356: Net engine driveline losses (P2 Parallel, DCnet)

$\left(E_{\text {dleng, loss }}\right)^{\text {DCnet }}=\left(E_{\text {dleng,loss }}\right)^{+}+\left(E_{\text {dleng, loss }}\right)^{-}+\left(E_{\text {dleng, loss }}\right)^{-/+}+\left(E_{\text {dleng,loss }}\right)^{E q F C}$

Equation 357: Net motor driveline losses (P2 Parallel, DCnet)

$\left(E_{\text {dlmot }, \text { loss }}\right)^{\text {DCnet }}=\left(E_{\text {dlmot }, \text { loss }}\right)^{+}+\left(E_{\text {dlmot }, \text { loss }}\right)^{-}+\left(E_{\text {dlmot }, \text { loss }}\right)^{-/+}+\left(E_{\text {dlmot,loss }}\right)^{0}+\left(E_{\text {mot }, \text { loss }}\right)^{E q F C}$

Equation 358: Net motor losses (P2 Parallel, DCnet)

$\left(E_{\text {mot }, \text { loss }}\right)^{\text {DCnet }}=\left(E_{\text {mot,loss }}\right)^{+}+\left(E_{\text {mot }, \text { loss }}\right)^{-}+\left(E_{\text {mot }, \text { loss }}\right)^{-/+}+\left(E_{\text {mot }, \text { loss }}\right)^{0}+\left(E_{\text {mot }, \text { loss }}\right)^{E q F C}$

Equation 359: Net battery losses (P2 Parallel, DCnet)

$\left(E_{\text {batt }, \text { loss }}\right)^{\text {DCnet }}=\left(E_{\text {batt }, \text { loss }}\right)^{+}+\left(E_{\text {batt }, \text { loss }}\right)^{-}+\left(E_{\text {batt }, \text { loss }}\right)^{-/+}+\left(E_{\text {batt }, \text { loss }}\right)^{0}+\left(E_{\text {batt }, \text { loss }}\right)^{E q F C}$

Equation 360: Net engine losses (P2 Parallel, DCnet)

$\left(E_{\text {eng, loss }}\right)^{\text {DCnet }}=\left(E_{\text {eng }, \text { loss }}\right)^{+}+\left(E_{\text {eng,loss }}\right)^{0}+\left(E_{\text {eng,loss }}\right)^{E q F C}$

Equation 121: Net powertrain losses (P2 Parallel, DCnet)

$\left(E_{p t, l o s s}\right)^{\text {DCnet }}=\left(E_{p t, l o s s}\right)^{+}+\left(E_{p t, l o s s}\right)^{-}+\left(E_{p t, l o s s}\right)^{-/+}+\left(E_{p t, l o s s}\right)^{0}+\left(E_{p t, l o s s}\right)^{E q F C}$

\section{Conservation of Energy Expressions:}

Equation 330: Conservation of energy for regen energy capture and reuse (P2 Parallel, regen reuse)

$\left(E_{\text {batt }, \text { int }}\right)^{-/+}+\left(E_{\text {batt }, \text { int }}\right)^{-}=0$

Equation 332: Equivalent tractive energy 'credit' from regen in terms of losses (P2 Parallel, regen reuse)

$\left(E_{\text {batt }, \text { int }}\right)^{-/+}=\left(E_{\text {tr,credit }}\right)^{-/+}+\left(E_{\text {pt,loss }}\right)^{-/+}$

Equation 298: Conservation of energy in the battery to maintain charge balance (P2 Parallel, propel)

$\left(E_{\text {batt }, \text { int }}\right)^{+ \text {prop }}+\left(E_{\text {batt }, \text { int }}\right)^{+ \text {gen }}=0$

Equation 361: Energy balance for overall charge balance (P2 Parallel, DCnet)

$\left(E_{\text {batt }, \text { int }}\right)^{+ \text {prop }}+\left(E_{\text {batt }, \text { int }}\right)^{+ \text {gen }}+\left(E_{\text {batt,int }}\right)^{-}+\left(E_{\text {tr,credit }}\right)^{-/+}+\left(E_{\text {pt,loss }}\right)^{-/+}=0$ 
Equation 119: Total fuel energy calculated by case (P2 Parallel, DCnet)

$\left(E_{\text {eng,fuel }}\right)^{\text {DCnet }}=\left(E_{\text {eng,fuel }}\right)^{+}+\left(E_{\text {eng,fuel }}\right)^{0}+\left(E_{\text {eng,fuel }}\right)^{E q F C}$

Equation 120: Total fuel energy calculated from overall energy balance (P2 Parallel, DCnet) $\left(E_{\text {eng,fuel }}\right)^{\text {DCnet }}=\left(E_{\text {tr }}\right)^{\text {DCnet }}+\left(E_{A C C}\right)^{\text {DCnet }}+\left(E_{\text {pt }, \text { loss }}\right)^{\text {DCnet }}$

\section{Accessory Energy:}

Equation 41: Net accessory energy

$\left(E_{A C C}\right)^{\text {DCnet }}=\left(E_{A C C}\right)^{+}+\left(E_{A C C}\right)^{-}+\left(E_{A C C}\right)^{0}=P_{A C C} * T$ 


\section{APPENDIX BB: Power Split Fraction Case Study Model Parameters}

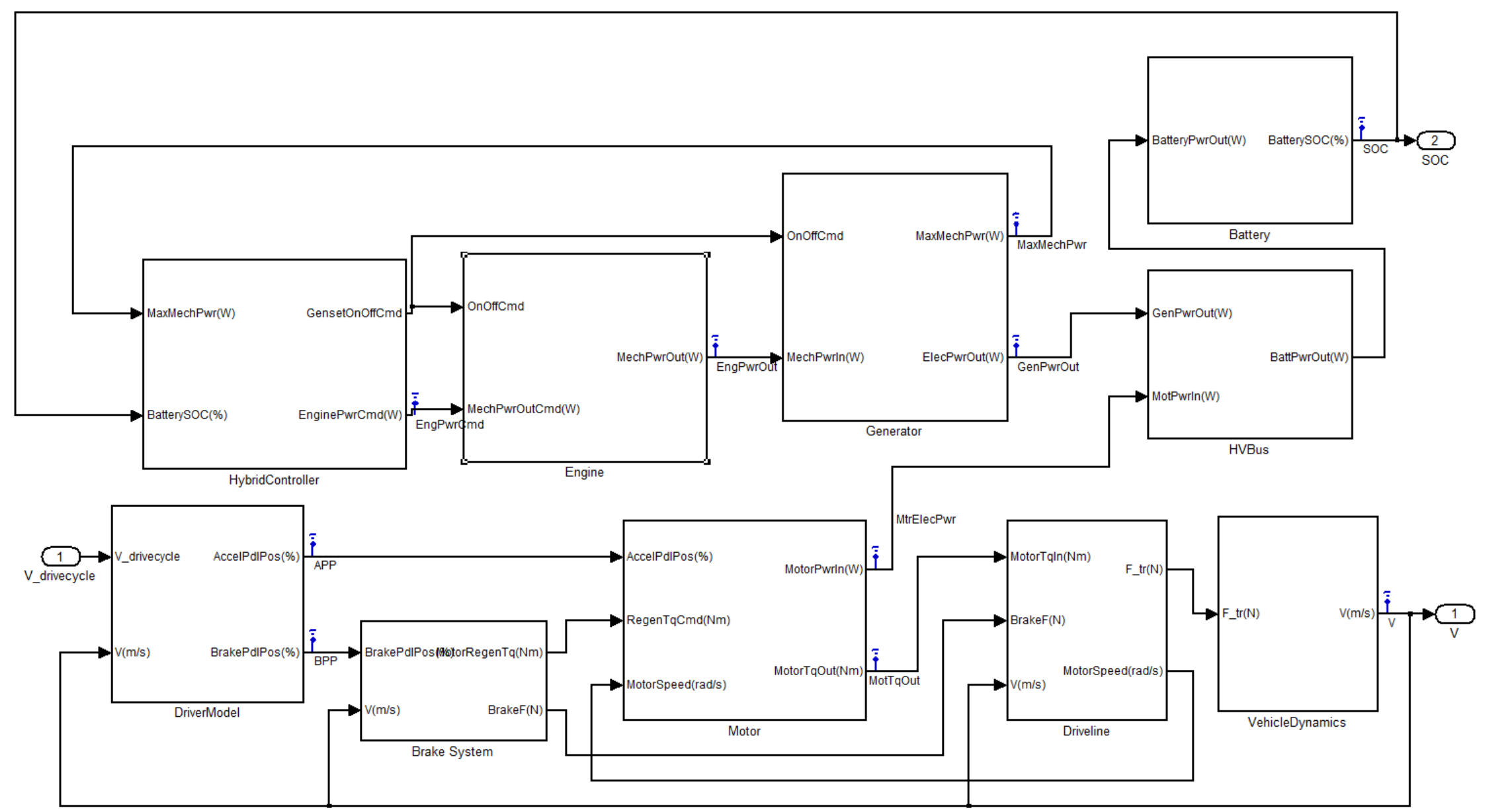

Figure 48: Top-level diagram of the Simulink model used in the Power Split Fraction case study 
Table 45: Vehicle glider properties and other properties

\begin{tabular}{|c|c|c|}
\hline \multicolumn{3}{|c|}{ Vehicle Glider Properties and Other Properties } \\
\hline Parameter & Units & Value \\
\hline $\mathrm{C}_{\mathrm{rr}}$ & {$[---]$} & 0.01 \\
\hline $\mathrm{C}_{\mathrm{d}} \mathrm{A}$ & {$\left[\mathrm{m}^{2}\right]$} & 0.76 \\
\hline Mass (M) & $\mathrm{kg}$ & 2150 \\
\hline Mi & $\mathrm{kg}$ & $2150 * 1.04$ \\
\hline grade: & {$[---]$} & $0 \%$ \\
\hline Rho & {$\left[\mathrm{kg} / \mathrm{m}^{3}\right]$} & 1.2 \\
\hline Gravity & {$\left[\mathrm{m} / \mathrm{s}^{2}\right]$} & 9.8 \\
\hline Regen Brake Fraction & {$[---]$} & 0.55 \\
\hline \multicolumn{3}{|c|}{ Battery Model Parameters } \\
\hline Energy Capacity & {$[\mathrm{kWh}]$} & 8.8 \\
\hline SOC max & [\%] & 95 \\
\hline SOC min & [\%] & 30 \\
\hline Voc & [V] & 340 \\
\hline Rint & [ohm] & 0.14 \\
\hline Acc_elec & [W] & 600 \\
\hline \multicolumn{3}{|c|}{ Engine Model Parameters } \\
\hline Peak Power (mech) & {$[\mathrm{kW}]$} & 130 \\
\hline Cont Power (mech) & {$[\mathrm{kW}]$} & 25 \\
\hline Min Power (mech) & {$[\mathrm{kW}]$} & *varies* \\
\hline $\mathrm{CO}$ & [W] & 6760 \\
\hline C1 & {$[---]$} & 1.32 \\
\hline $\mathrm{C} 2$ & {$[1 / \mathrm{W}]$} & $4.2 \times 10^{-6}$ \\
\hline \multicolumn{3}{|c|}{ Generator Model Parameters } \\
\hline Peak Power (mech) & {$[\mathrm{kW}]$} & 50 \\
\hline Cont Power (mech) & {$[\mathrm{kW}]$} & 25 \\
\hline Min Power (mech) & {$[\mathrm{kW}]$} & "varies* \\
\hline $\mathrm{CO}$ & {$[\mathrm{W}]$} & 600 \\
\hline C1 & {$[---]$} & 0.036 \\
\hline $\mathrm{C} 2$ & {$[1 / \mathrm{W}]$} & $1.3 \times 10^{-6}$ \\
\hline \multicolumn{3}{|c|}{ Motor Model Parameters ( } \\
\hline Max Torque & {$[\mathrm{Nm}]$} & 300 \\
\hline Max Power & {$[\mathrm{kW}]$} & 125 \\
\hline $\mathrm{kc}$ & & 0.12 \\
\hline ki & & 0.01 \\
\hline kw & & $1.2 \times 10^{-5}$ \\
\hline \multicolumn{3}{|c|}{ Motor Driveline Model Parameters } \\
\hline Spin Loss & {$[\mathrm{Nm}]$} & 3 \\
\hline Gear Ratio & {$[---]$} & 7.17 \\
\hline$r_{w}$ & [m] & 0.324 \\
\hline
\end{tabular}




\section{APPENDIX CC: Power Split Fraction Case Study Model Parameters}

The results for the 4 highway cases are shown below in Figure 49, Figure 50, and Figure 51. Each case shows the results for 2 back-to-back HWFET cycles. This was done to show the effect of the initial SOC, which was always higher than the target. As a result, the battery charged less during the first cycle. It is also interesting to note that in case 4 , the generator reaches its continuous power level every time it comes on. Because the results were identical for cases 2 and 3, one graph was used for both cases.

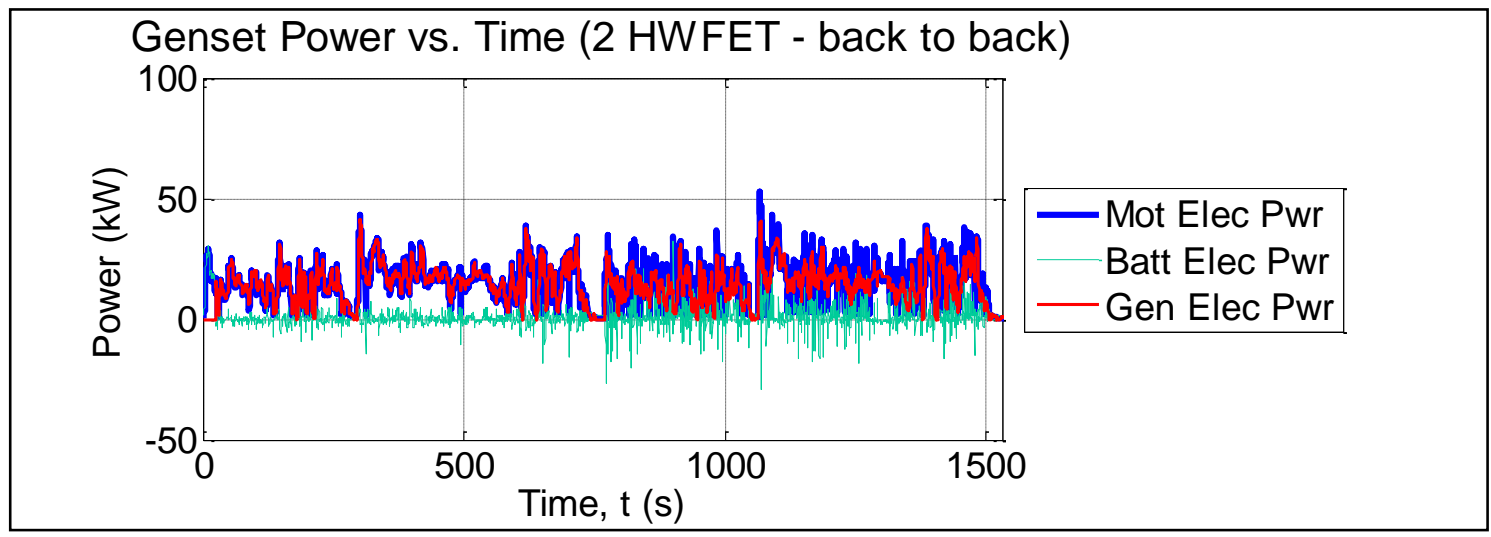

Figure 49: Power Split Fraction case study, HwFET, case 1, near load-following, $\xi=0.96$

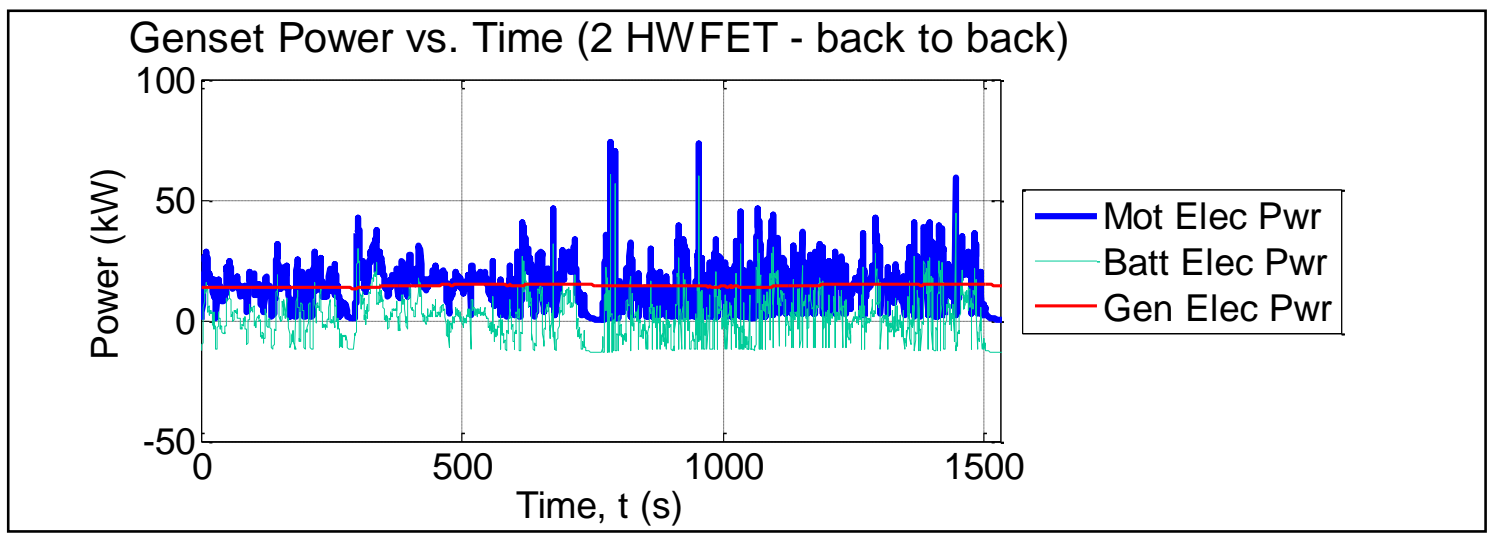

Figure 50: Power Split Fraction case study, HwFET, case 2 and case 3, average power, $\xi=0.81$

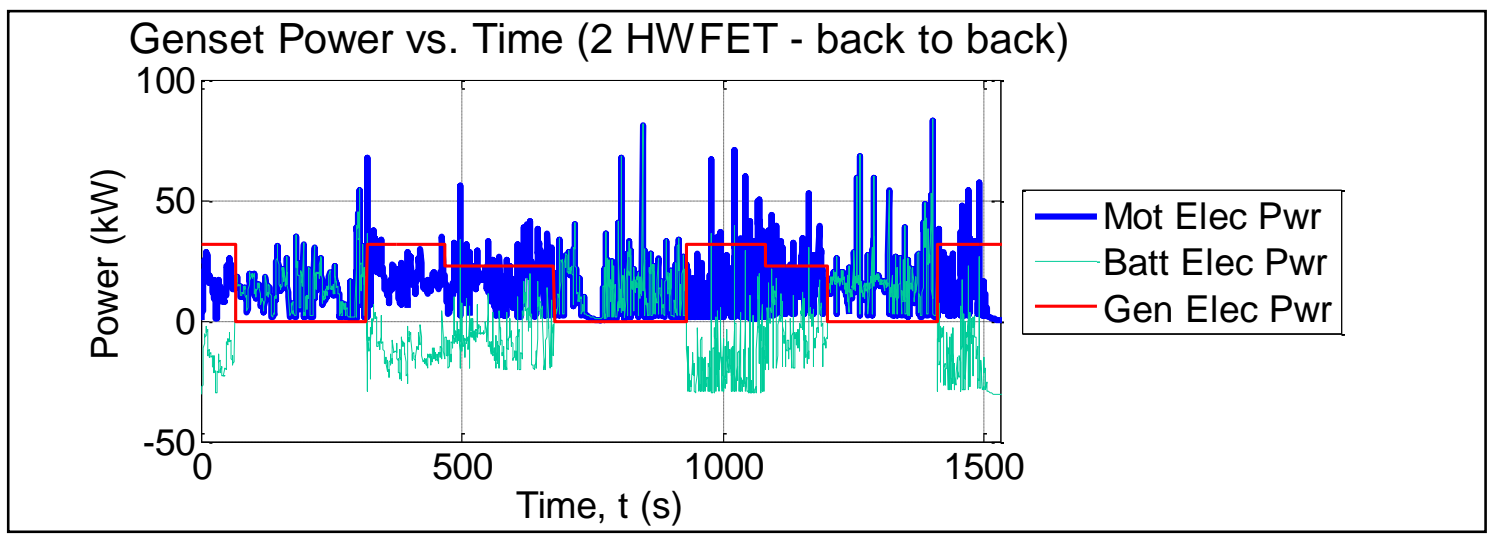

Figure 51: Power Split Fraction case study, HwFET, case 4, average power, $\xi=0.59$ 


\section{APPENDIX DD: Summary of Tunable Parameters Used in VTool}

This appendix contains the final values of all component parameters calibrated or estimated during the course of this thesis. The values for UDDS and HwFET cycles were calibrated using on-road test data (see Section 4). The remaining values were estimated using component efficiency models from an independently developed excel-based $1 \mathrm{~Hz}$ model.

Table 46: Summary of all VTool tunable parameters calibrated using E\&EC data and other data

\begin{tabular}{|l|l|l|l|l|l|l|l|l|}
\hline Parameter & Symbol & UDDS & 505 & 867 & HwFET & US06 & $\begin{array}{l}\text { US06 } \\
\text { City }\end{array}$ & $\begin{array}{l}\text { US06 } \\
\text { Hwy }\end{array}$ \\
\hline Motor Driveline (propel) & $\left(\eta_{\mathrm{dl}, \mathrm{mot}}\right)^{+}$ & $91 \%$ & $91 \%$ & $91 \%$ & $91 \%$ & $91 \%$ & $91 \%$ & $91 \%$ \\
\hline Motor Driveline (brake) & $\left(\eta_{\mathrm{dl}, \mathrm{mot}}\right)^{-}$ & $91 \%$ & $91 \%$ & $91 \%$ & $91 \%$ & $91 \%$ & $91 \%$ & $91 \%$ \\
\hline Engine Driveline (propel) & $\left(\eta_{\mathrm{dl}, \mathrm{eng}}\right)^{+}$ & $88 \%$ & $88 \%$ & $88 \%$ & $88 \%$ & $88 \%$ & $88 \%$ & $88 \%$ \\
\hline Engine Driveline (brake) & $\left(\eta_{\mathrm{dl}, \mathrm{eng}}\right)^{-}$ & $88 \%$ & $88 \%$ & $88 \%$ & $88 \%$ & $88 \%$ & $88 \%$ & $88 \%$ \\
\hline Engine-Motor Driveline & $\left(\eta_{\mathrm{dl}, \mathrm{e}-\mathrm{m}}\right)^{+}$ & $98 \%$ & $98 \%$ & $98 \%$ & $98 \%$ & $98 \%$ & $98 \%$ & $98 \%$ \\
\hline Motor/Inverter (propel) & $\left(\eta_{\text {mot }}\right)^{+}$ & $84 \%$ & $85 \%$ & $82 \%$ & $79 \%$ & $86 \%$ & $85 \%$ & $87 \%$ \\
\hline Motor/Inverter (brake) & $\left(\eta_{\text {mot }}\right)^{-}$ & $86 \%$ & $87 \%$ & $85 \%$ & $88 \%$ & $88 \%$ & $89 \%$ & $87 \%$ \\
\hline Generator & $\left(\eta_{\text {gen }}\right)^{+}$ & $86 \%$ & $86 \%$ & $86 \%$ & $86 \%$ & $86 \%$ & $86 \%$ & $86 \%$ \\
\hline Engine (Series HEV) & $\left(\eta_{\text {eng,ser }}\right)^{+}$ & $35 \%$ & $35 \%$ & $35 \%$ & $35 \%$ & $35 \%$ & $35 \%$ & $35 \%$ \\
\hline Engine (Parallel HEV) & $\left(\eta_{\text {eng,par }}\right)^{+}$ & $24 \%$ & $24 \%$ & $24 \%$ & $26 \%$ & $26 \%$ & $26 \%$ & $24 \%$ \\
\hline Battery (propel) & $\left(\eta_{\text {batt }}\right)^{+}$ & $99 \%$ & $99 \%$ & $99 \%$ & $99 \%$ & $99 \%$ & $99 \%$ & $99 \%$ \\
\hline Battery (brake) & $\left(\eta_{\text {batt }}\right)^{-}$ & $99 \%$ & $99 \%$ & $99 \%$ & $99 \%$ & $99 \%$ & $99 \%$ & $99 \%$ \\
\hline Charger & $\left(\eta_{\text {chrgr }}\right)^{c}$ & $92 \%$ & $92 \%$ & $92 \%$ & $92 \%$ & $92 \%$ & $92 \%$ & $92 \%$ \\
\hline Charging & $\left(\eta_{\text {chrgn }}\right)^{\mathrm{C}}$ & $80 \%$ & $80 \%$ & $80 \%$ & $80 \%$ & $80 \%$ & $80 \%$ & $80 \%$ \\
\hline Regen Fraction & $\zeta$ & $75 \%$ & $75 \%$ & $75 \%$ & $75 \%$ & $75 \%$ & $75 \%$ & $75 \%$ \\
\hline Power Split Fraction & $\xi$ & $74 \%$ & $74 \%$ & $74 \%$ & $76 \%$ & $74 \%$ & $78 \%$ & $80 \%$ \\
\hline Accessory Load (W) & $P_{\text {acc }}$ & 600 & 600 & 600 & 600 & 600 & 600 & 600 \\
\hline Charging Acc'y Load $(\mathrm{W})$ & $\mathrm{P}_{\text {acc,c }}$ & 102 & 102 & 102 & 102 & 102 & 102 & 102 \\
\hline
\end{tabular}




\section{APPENDIX EE: Summary of VTool Results for EV and P3/P4 Parallel HEV models}

This appendix contains the final values of all component parameters calibrated or estimated during the course of this thesis. The values for UDDS and HwFET cycles were calibrated using on-road test data (see Section 4). The remaining values were estimated using component efficiency models from an independently developed excel-based $1 \mathrm{~Hz}$ model.

Table 47: Summary of propel and DCnet case results for the EV model

\begin{tabular}{|c|c|c|c|c|c|c|c|}
\hline & \multicolumn{7}{|c|}{ Summary of Drive Cycle Models - Electric Vehicle } \\
\hline & $\left(E_{\text {grid, } A C}\right)^{A C n e t}$ & $\left(E_{\text {batt }, T}\right)^{\mathrm{DCnet}}$ & $\left(E_{t r}\right)^{\text {DCnet }}$ & $\left(E_{\mathrm{tr}}\right)^{+}$ & $\left(E_{t r}\right)^{-}$ & $\left(\eta_{\mathrm{pt}}\right)^{\mathrm{DCnet}}$ & $\left(E_{p t, \text { loss }}\right)^{\text {DCnet }}$ \\
\hline & [AC Wh/mi] & [DC Wh/mi] & {$[\mathrm{Wh} / \mathrm{mi}]$} & [Wh/mi] & [Wh/mi] & [\%] & [Wh/mi] \\
\hline UDDS & 423 & 334 & 151 & 297 & -146 & $45 \%$ & 157 \\
\hline 505 & 443 & 350 & 180 & 314 & -135 & $51 \%$ & 152 \\
\hline 867 & 411 & 324 & 125 & 281 & -156 & $39 \%$ & 166 \\
\hline HwFET & 446 & 353 & 226 & 259 & -33 & $64 \%$ & 118 \\
\hline US06 & 612 & 483 & 290 & 438 & -148 & $60 \%$ & 187 \\
\hline US06 City & 746 & 586 & 207 & 637 & -430 & $35 \%$ & 366 \\
\hline US06 Hwy & 570 & 450 & 313 & 381 & -68 & $69 \%$ & 133 \\
\hline
\end{tabular}

Table 48: Summary of results for the brake case for the P3/P4 Parallel HEV model

\begin{tabular}{|c|c|c|c|c|c|c|}
\hline & \multicolumn{6}{|c|}{ Regen Brake Summary - Electric Vehicle } \\
\hline & $\left(E_{\text {batt,int }}\right)^{-}$ & $\left(E_{t r}\right)^{-}$ & $\left(E_{\text {brake,loss }}\right)^{-}$ & $\left(\eta_{\mathrm{pt}}\right)^{-}$ & $\left(E_{\text {eng,fuel }}\right)^{\text {EqFC }}$ & $\zeta$ \\
\hline & [DC Wh/mi] & [Wh/mi] & [Wh/mi] & {$[\%]$} & {$[\mathrm{Wh} / \mathrm{mi}]$} & [\%] \\
\hline UDDS & -77 & -146 & 36 & $52 \%$ & -77 & 0.75 \\
\hline 505 & -73 & -135 & 34 & $55 \%$ & -73 & 0.75 \\
\hline 867 & -79 & -156 & 39 & $51 \%$ & -79 & 0.75 \\
\hline HwFET & -18 & -33 & 8 & $55 \%$ & -18 & 0.75 \\
\hline US06 & -85 & -148 & 37 & $57 \%$ & -85 & 0.75 \\
\hline US06 City & -250 & -430 & 108 & $58 \%$ & -250 & 0.75 \\
\hline US06 Hwy & -38 & -68 & 17 & $57 \%$ & -38 & 0.75 \\
\hline
\end{tabular}


Table 49: Summary of propel and DCnet case results for the P3/P4 Parallel HEV model

\begin{tabular}{|c|c|c|c|c|c|c|c|}
\hline & \multicolumn{7}{|c|}{ Summary of Drive Cycle Models - CS P3/P4 Parallel HEV } \\
\hline & $\left(E_{\text {eng,fuel }}\right)^{\mathrm{DCnet}}$ & $\left(E_{\text {eng,fuel }}\right)^{\mathrm{DCnet}}$ & $\left(E_{t r}\right)^{D C n e t}$ & $\left(E_{t r}\right)^{+}$ & $\left(E_{t r}\right)^{-}$ & $\left(\eta_{p t}\right)^{D C n e t}$ & $\left(\eta_{\text {eng,par }}\right)^{+}$ \\
\hline & {$[\mathrm{Wh} / \mathrm{mi}]$} & [mpg] & [Wh/mi] & [Wh/mi] & {$[\mathrm{Wh} / \mathrm{mi}]$} & [\%] & [\%] \\
\hline UDDS & 1405 & 23 & 151 & 297 & -146 & $11 \%$ & $24 \%$ \\
\hline 505 & 1469 & 22 & 180 & 314 & -135 & $12 \%$ & $24 \%$ \\
\hline 867 & 1353 & 24 & 125 & 281 & -156 & $9 \%$ & $24 \%$ \\
\hline HwFET & 1261 & 26 & 226 & 259 & -33 & $18 \%$ & $26 \%$ \\
\hline US06 & 1857 & 17 & 290 & 438 & -148 & $16 \%$ & $26 \%$ \\
\hline US06 City & 2205 & 15 & 207 & 637 & -430 & $9 \%$ & $26 \%$ \\
\hline US06 Hwy & 1643 & 20 & 192 & 382 & -190 & $12 \%$ & $27 \%$ \\
\hline
\end{tabular}

Table 50: Summary of results for brake case and Power Split Fraction for the P3/P4 Parallel HEV model

\begin{tabular}{|c|c|c|c|c|c|c|c|c|c|}
\hline & \multicolumn{9}{|c|}{ Regen Brake and Power Split Fraction Summary - CS P3/P4 Parallel HEV } \\
\hline & $\left(E_{\text {batt,int }}\right)^{-}$ & $\left(E_{t r}\right)^{-}$ & $\left(E_{\text {brake,loss }}\right)^{-}$ & $\left(\eta_{\mathrm{pt}}\right)^{-}$ & $\left(E_{\text {eng,fuel }}\right)^{\text {EqFC }}$ & $\zeta$ & $\left(E_{\text {batt,int }}\right)^{+ \text {prop }}$ & $\xi$ & $\left(\eta_{\text {recirc }}\right)^{+}$ \\
\hline & [DC Wh/mi] & [Wh/mi] & [Wh/mi] & [\%] & [Wh/mi] & [\%] & [Wh/mi] & [\%] & [\%] \\
\hline UDDS & -77 & -146 & 36 & $52 \%$ & -307 & 0.75 & 0.75 & 0.74 & $59 \%$ \\
\hline 505 & -73 & -135 & 34 & $55 \%$ & -297 & 0.75 & 0.75 & 0.74 & $60 \%$ \\
\hline 867 & -79 & -156 & 39 & $51 \%$ & -312 & 0.75 & 0.75 & 0.74 & $57 \%$ \\
\hline HwFET & -18 & -33 & 8 & $55 \%$ & -62 & 0.75 & 0.75 & 0.76 & $56 \%$ \\
\hline US06 & -85 & -148 & 37 & $57 \%$ & -320 & 0.75 & 0.75 & 0.74 & $61 \%$ \\
\hline US06 City & -250 & -430 & 108 & $58 \%$ & -913 & 0.75 & 0.75 & 0.78 & $61 \%$ \\
\hline US06 Hwy & -38 & -68 & 17 & $57 \%$ & -138 & 0.75 & 0.75 & 0.8 & $61 \%$ \\
\hline
\end{tabular}




\section{APPENDIX FF: Citations for Copyrighted Works}

This appendix contains a citation and/or fair use determination for each figure or table used in this thesis.

1. Figure 1: U.S. transportation energy use and $\mathrm{CO} 2$ emissions, (public domain [1]), page 1

Citation:

Green, D., Baker, H., and Plotkin, S., (2011), "Reducing Greenhouse Gas Emissions from U.S. Transportation," Prepared for the Pew Center for Global Climate Change.

Fair Use Consideration:

Works by the U.S. government are not eligible for U.S. copyright protection.

2. Figure 2: Trends in U.S. consumption, production, and net imports of liquid fuels (public domain [2]), page 2

Citation:

Department of Energy, (2011), "Report on the First Quadrennial Technology Review," Department of Energy, Washington, DC.

Fair Use Consideration:

Works by the U.S. government are not eligible for U.S. copyright protection.

3. Figure 3: Typical one-way commute of the average U.S. resident, (public domain [3]), page 3

Citation:

Department of Transportation, (2003), Omnibus Household Survey, vol. 3(4). Fig 2. US Department of Transportation, Bureau of Transportation Statistics.

Fair Use Consideration:

Works by the U.S. government are not eligible for U.S. copyright protection.

4. Figure 4: Average daily mileage distribution, (used with permission of Elsevier [4]), page 4

Citation:

Pearre, N.S., et al., (2011), "Electric vehicles: How much range is required for a day's driving?," Transportation Research Part C: Emerging Technologies, Vol. 19, Issue 6, Dec. 2011, pp. 11711184.

Fair Use Consideration:

Used with permission from Elsevier Journal. License agreement attached in Appendix 
5. Figure 5: Fraction of the fleet appropriate for varying vehicle ranges, (used with permission of Elsevier [4]), page 4

Citation:

Same as Figure 5.

Fair Use Consideration:

Used with permission from Elsevier Journal. License agreement attached.

6. Figure 7: Series hybrid architecture design and characteristics (public domain [9]), page 7

Citation:

Elgowainy, A., Han, J., Poch, L., Wang, M., Vyas, A., Mahalik, M., and Rousseau, A., (2010), "Well-to-Wheels Analysis of Energy Use and Greenhouse Gas Emissions of Plug-In Hybrid Electric Vehicles," Argonne National Labs, ANL/ESD/10-1, June 2010, http://www.osti.gov/bridge/product.biblio.jsp?query_id=1\&page=0\&osti_id=982352\&Row=1\&form name=basicsearch.jsp

Fair Use Consideration:

Works by the U.S. government are not eligible for U.S. copyright protection.

7. Figure 8: Parallel hybrid architecture design and characteristics (public domain [9]), page 7

Citation:

Same as Figure 7.

Fair Use Consideration:

Works by the U.S. government are not eligible for U.S. copyright protection.

8. Figure 9: Series-Parallel hybrid architecture design and characteristics Figure 9: Series-Parallel hybrid architecture design and characteristics (public domain [9]), page 7

Citation:

Same as Figure 7.

Fair Use Consideration:

Works by the U.S. government are not eligible for U.S. copyright protection. 